Fall 1974

\title{
1974 Miracle Yearbook
}

\section{Cedarville College}

Follow this and additional works at: https://digitalcommons.cedarville.edu/yearbooks

Part of the Higher Education Commons, Organizational Communication Commons, and the Public Relations and Advertising Commons

\section{Recommended Citation}

Cedarville College, "1974 Miracle Yearbook" (1974). Yearbooks. 51.

https://digitalcommons.cedarville.edu/yearbooks/51

This Book is brought to you for free and open access by DigitalCommons@Cedarville, a service of the Centennial Library. It has been accepted for inclusion in Yearbooks by an authorized administrator of DigitalCommons@Cedarville. For more information, please contact digitalcommons@cedarville.edu. 


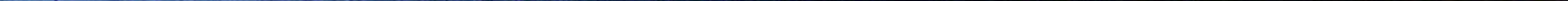

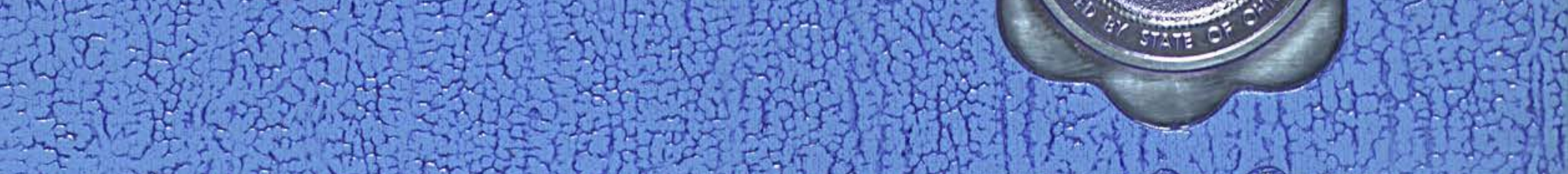

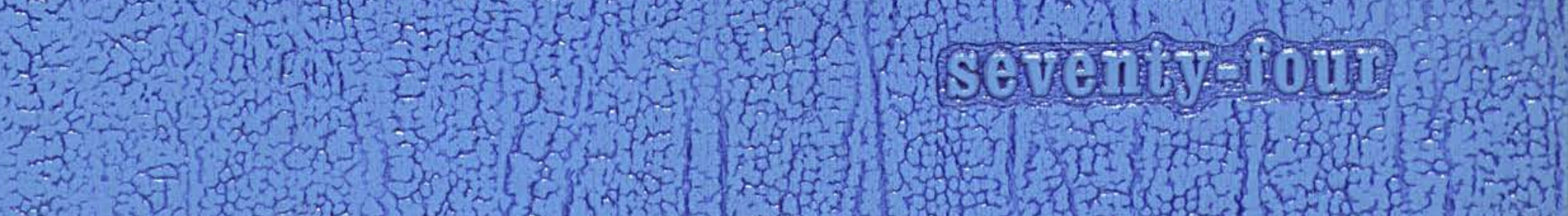

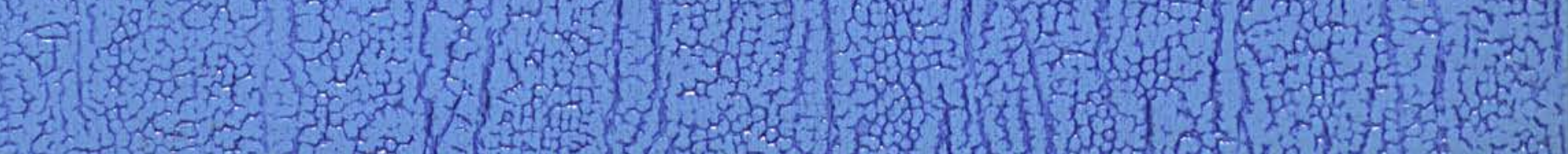







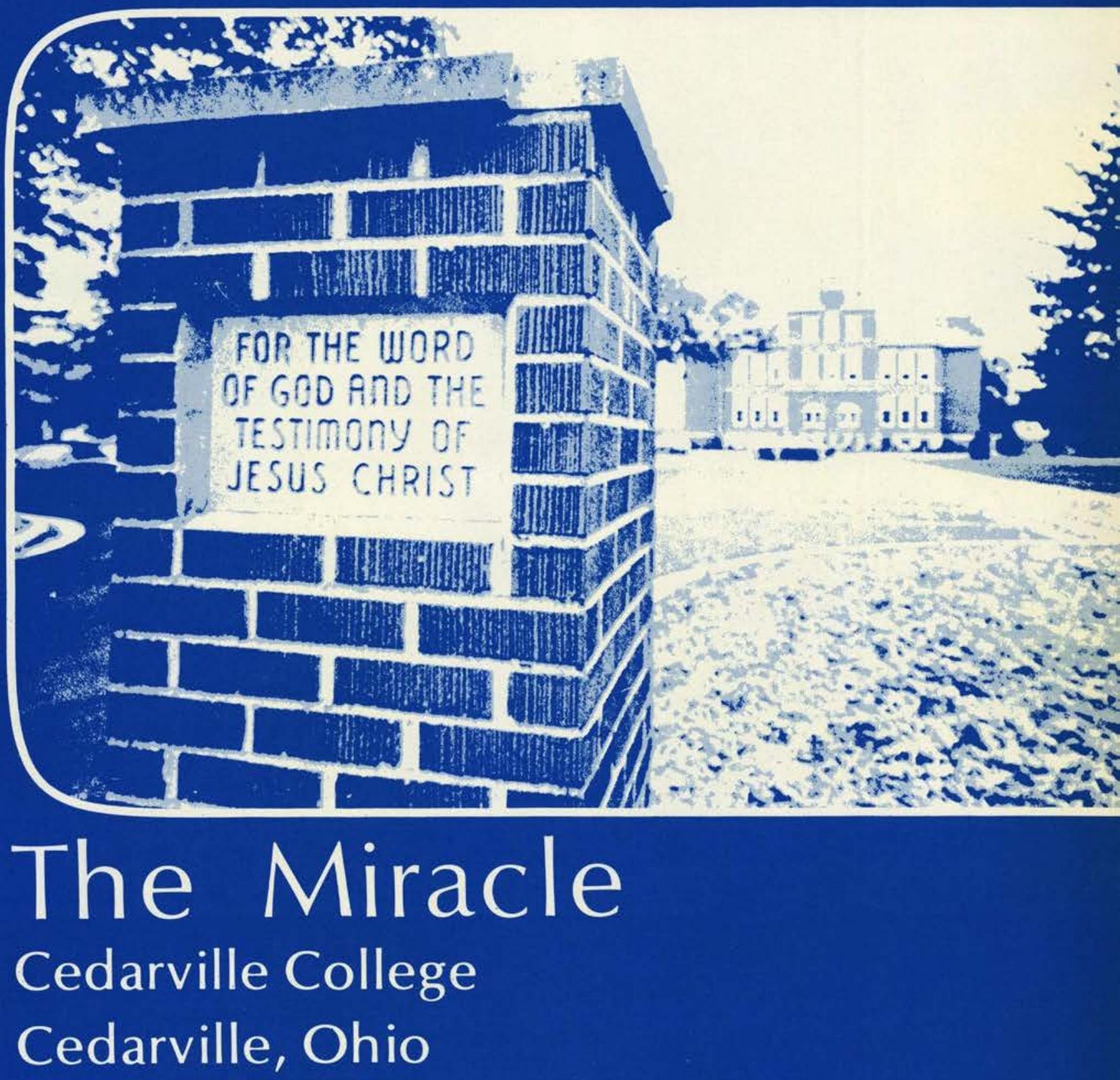

The Miracle 


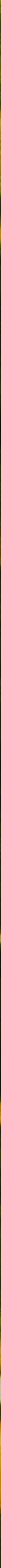




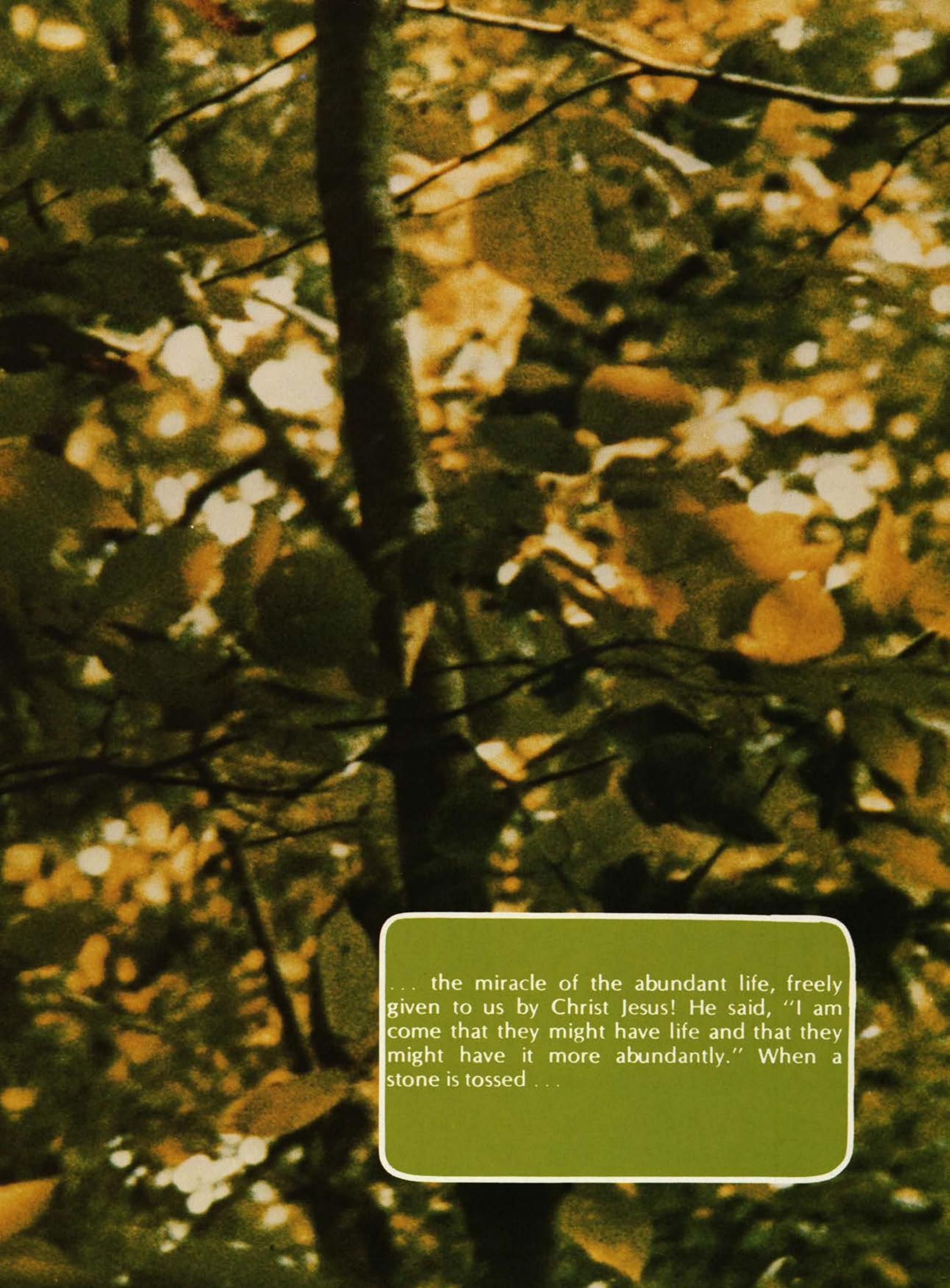



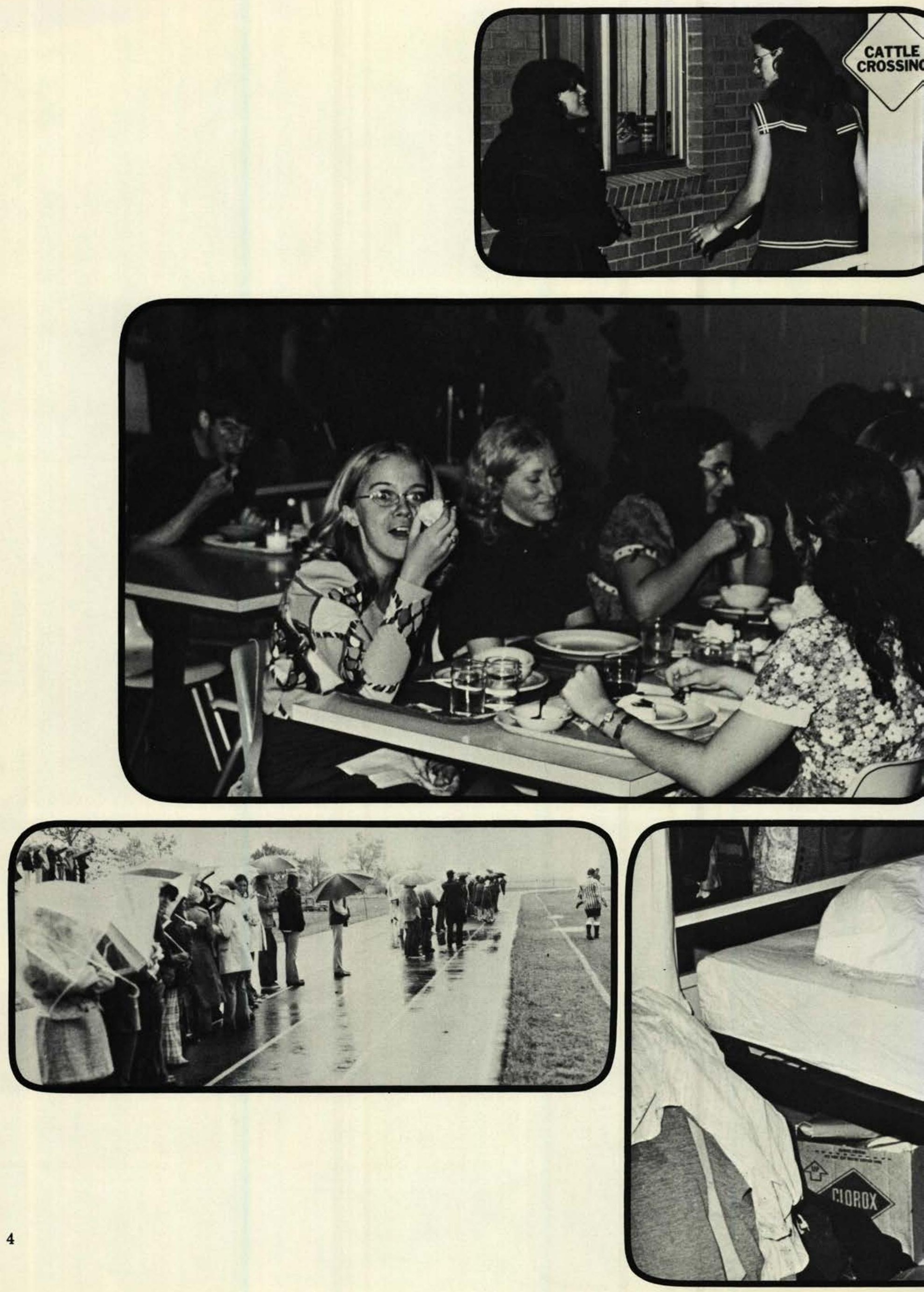
. into a still pond, waves of enlarging circles ripple the surface. The abundant life is forever broadening into greater circles of personal growth, wisdom, fellowship, harmony, and the deep peace and joy of God revealing himself to us.

"He that believeth on the Son hath everlasting life."
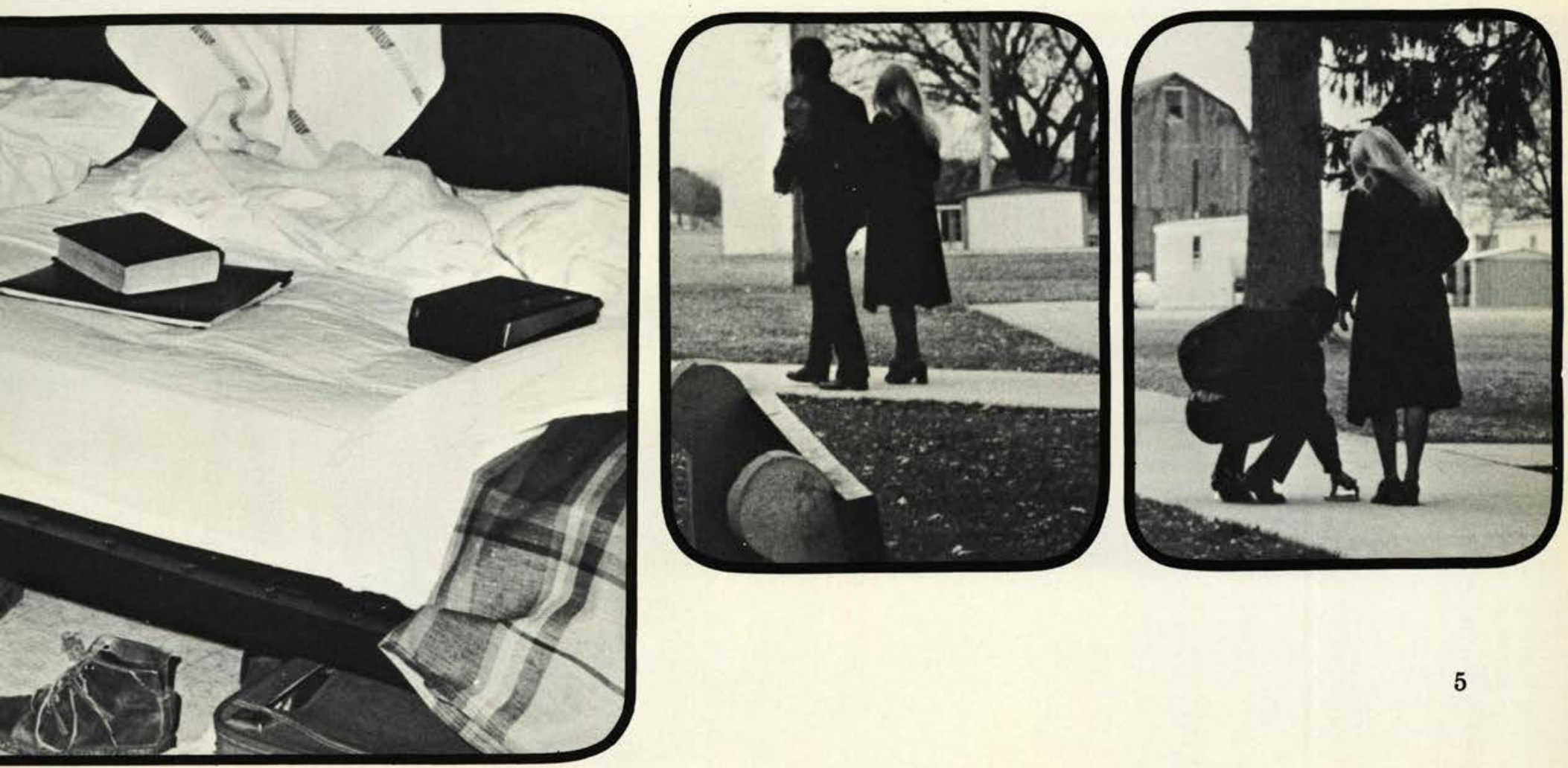

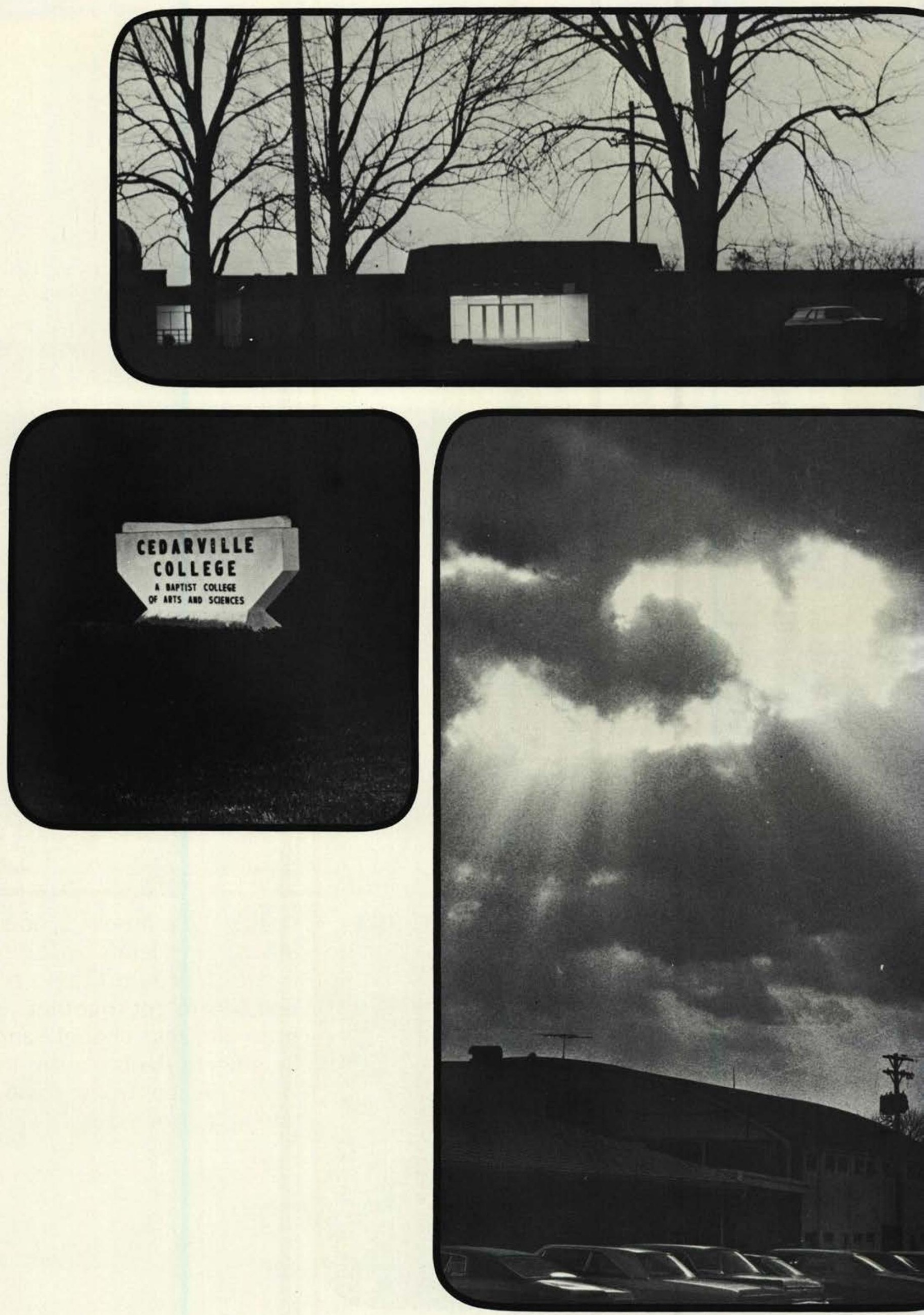

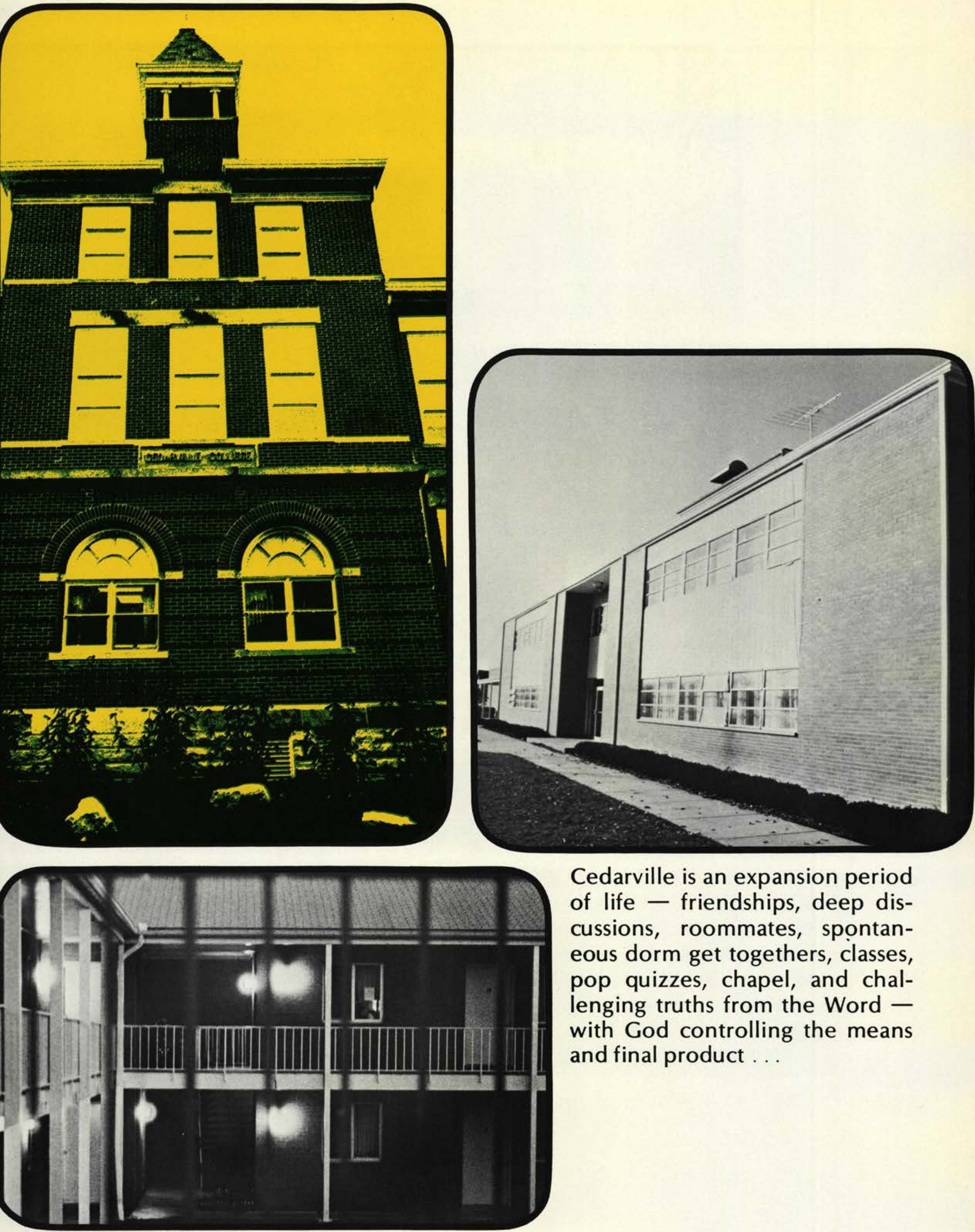

Cedarville is an expansion period of life - friendships, deep discussions, roommates, spontaneous dorm get togethers, classes, pop quizzes, chapel, and challenging truths from the Word with God controlling the means and final product ... 

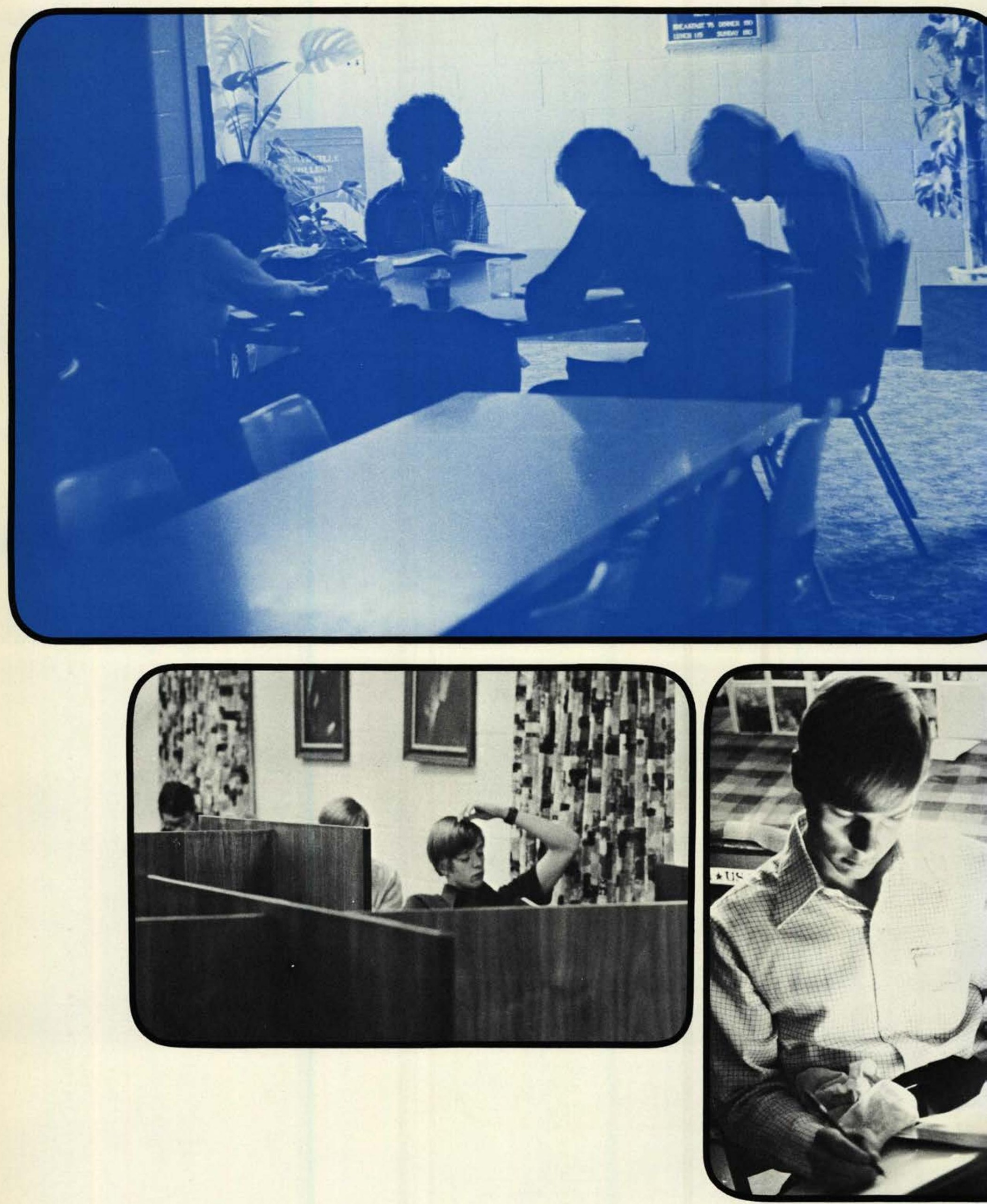

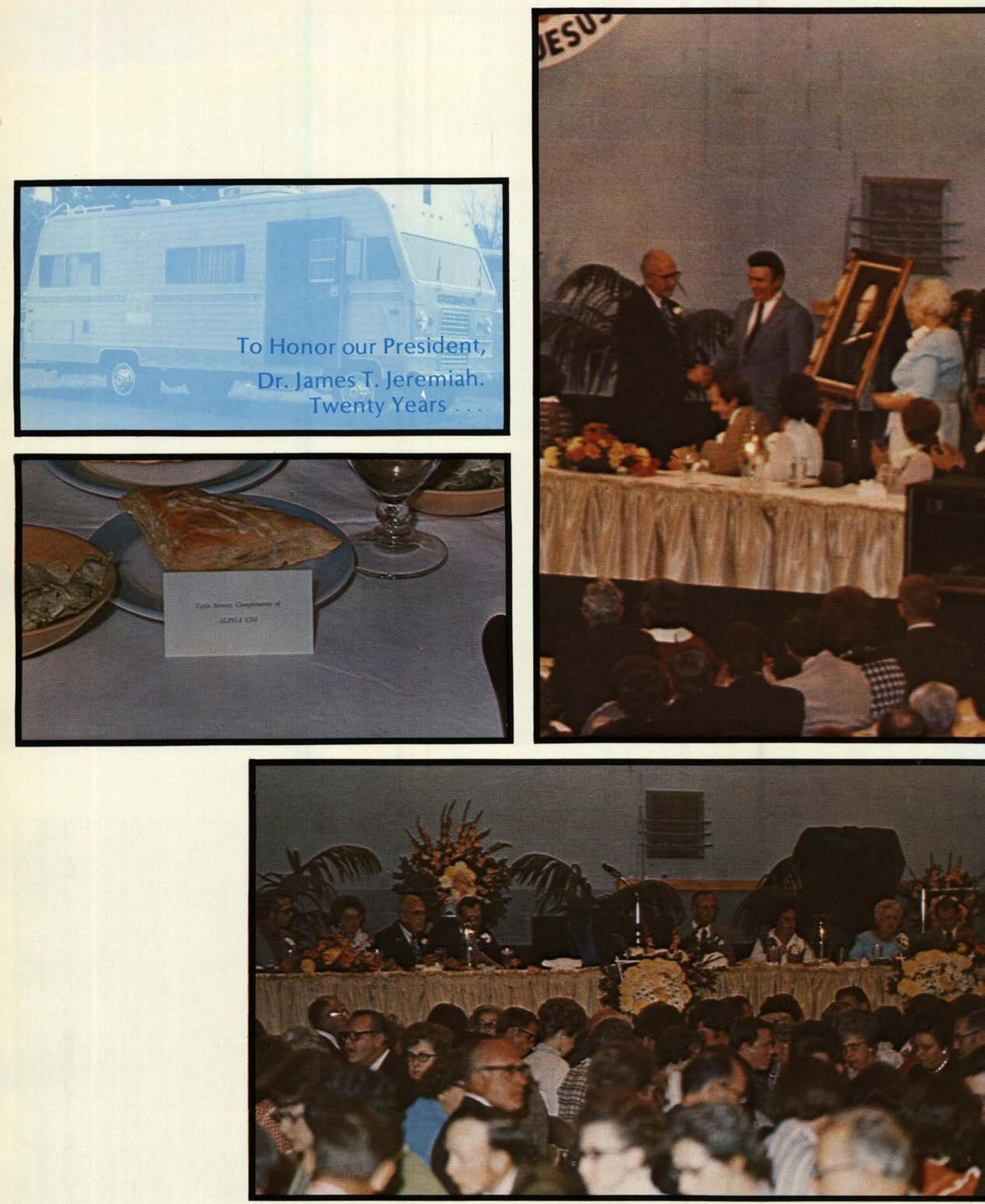
a tiny school of less than 200 to over 1000 today - we have seen the blessing of God on our college under Dr. Jeremiah's leadership.

To commemorate his second decade of service, a banquet was given in the fall. The president was presented with a mobile vacation home by the faculty and friends and honored with a painting of himself.
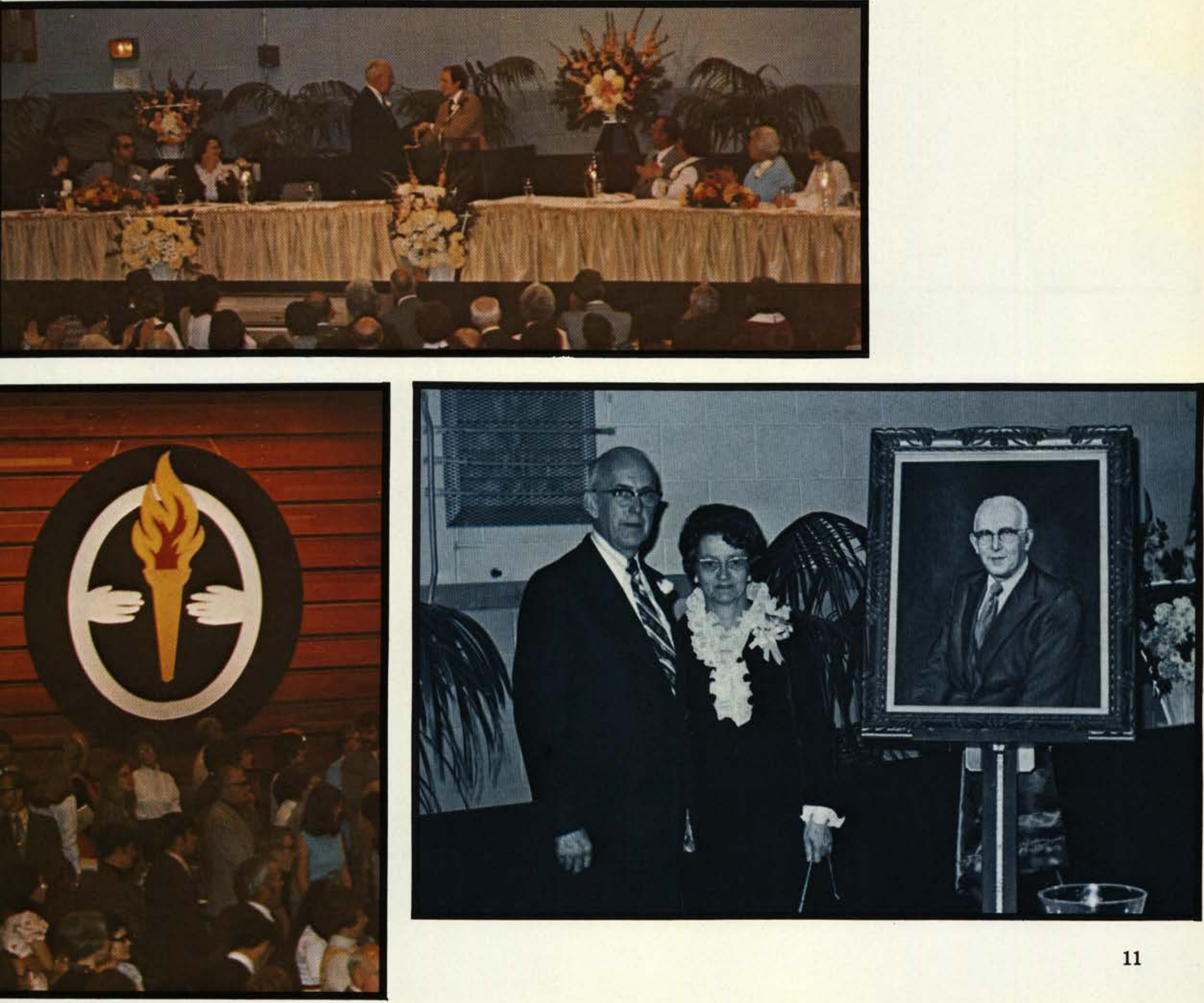


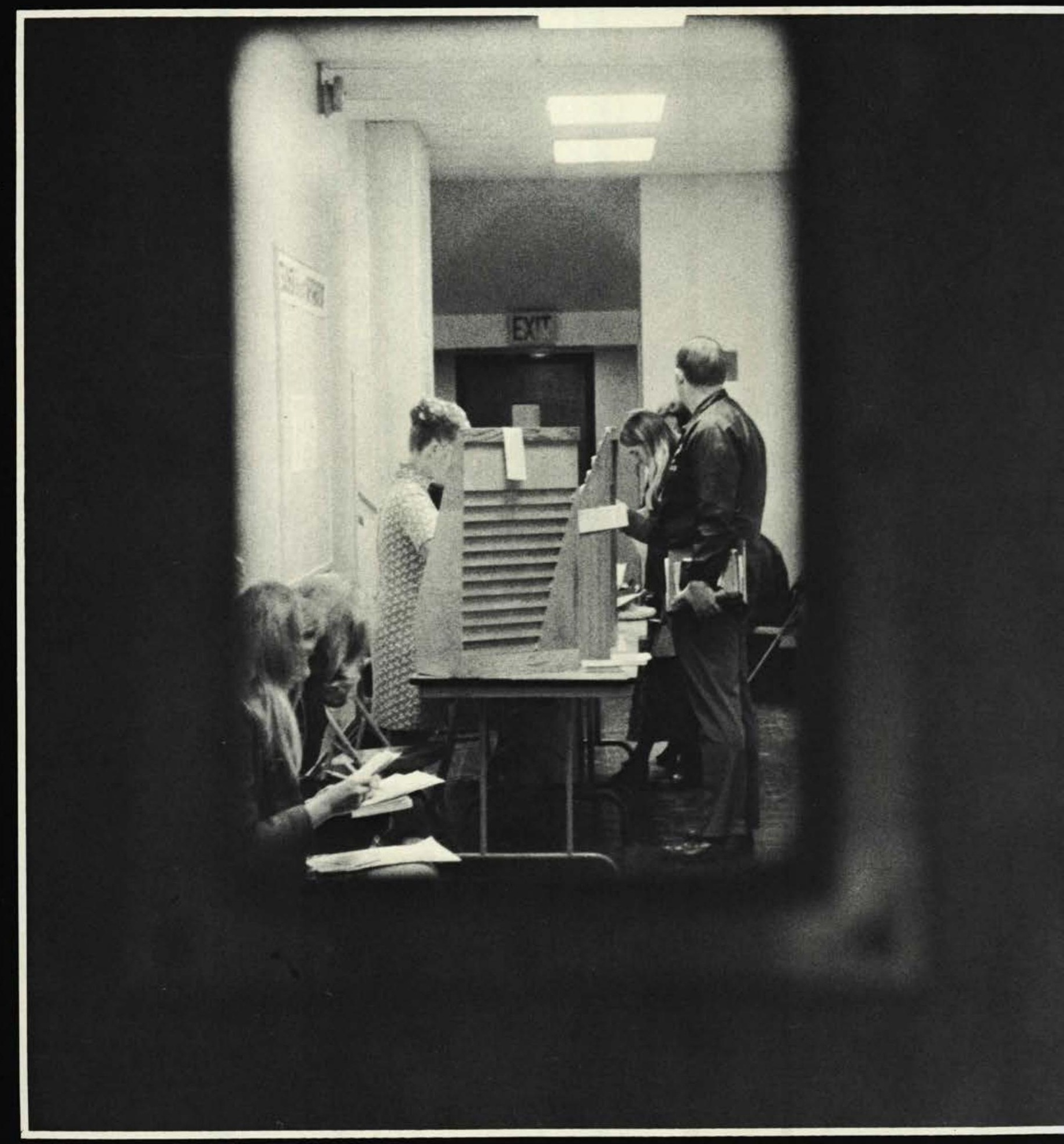



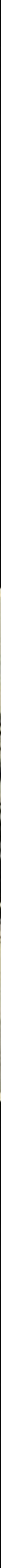

\section{Registration}




\section{Pi Delta Says "Meet Cedarville"}
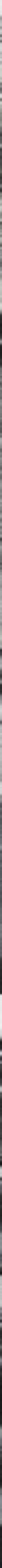

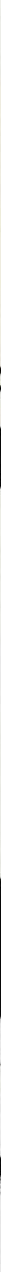

5:00 a.m. ... Get 'em up! Up Freshmen, up! Bobbie Sox and beanies, Calisthenics and jogging, Laughter across the black, still lake. Uh ... Would you sign my pizzaboard? Ring around the rosie. Kangaroo Court! Freshmen are dumb! Sophomores are great!! Freshmen are dumb! Sophomores are great!!
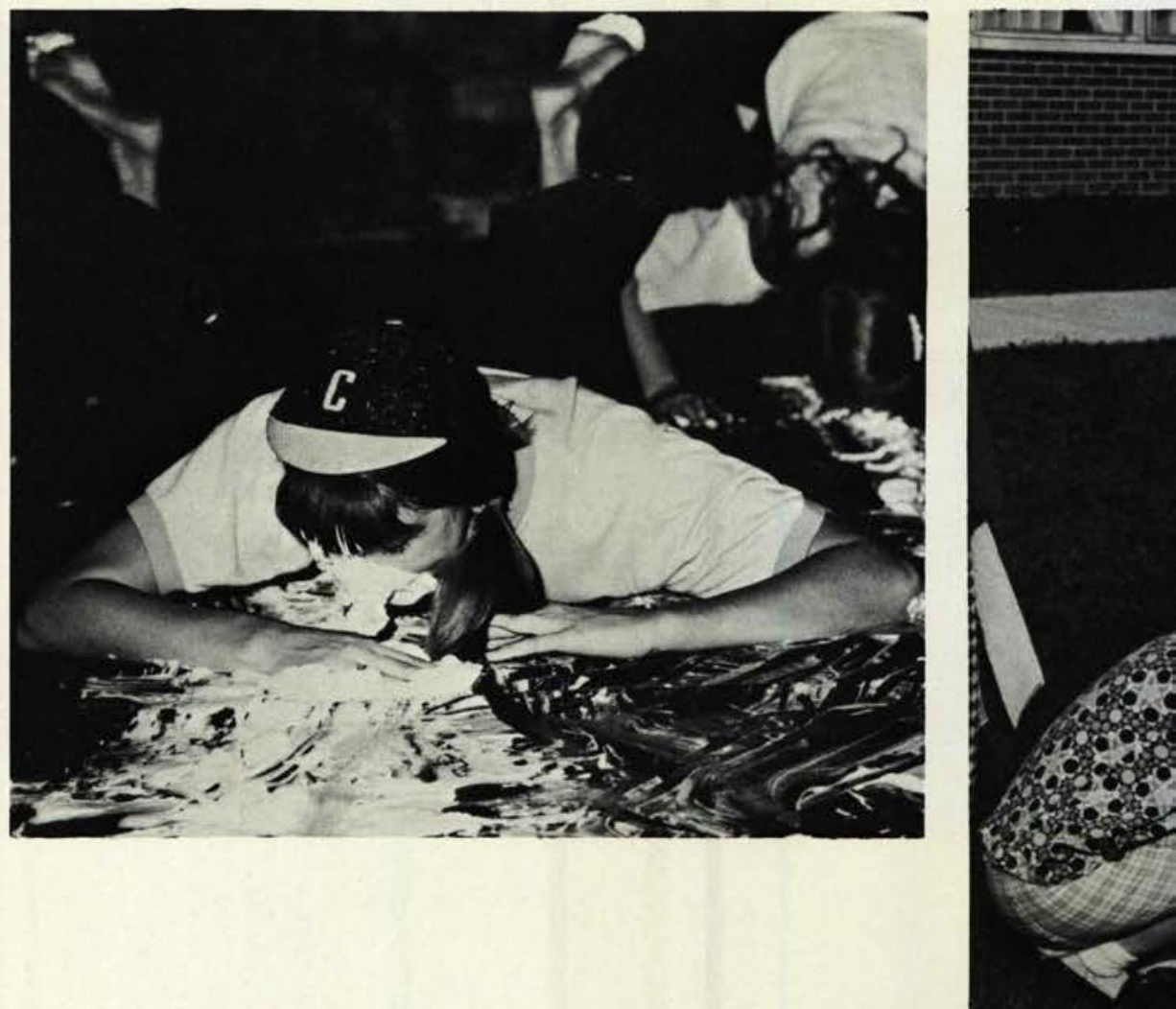


$$
\frac{5}{1+2} \frac{6}{0}
$$

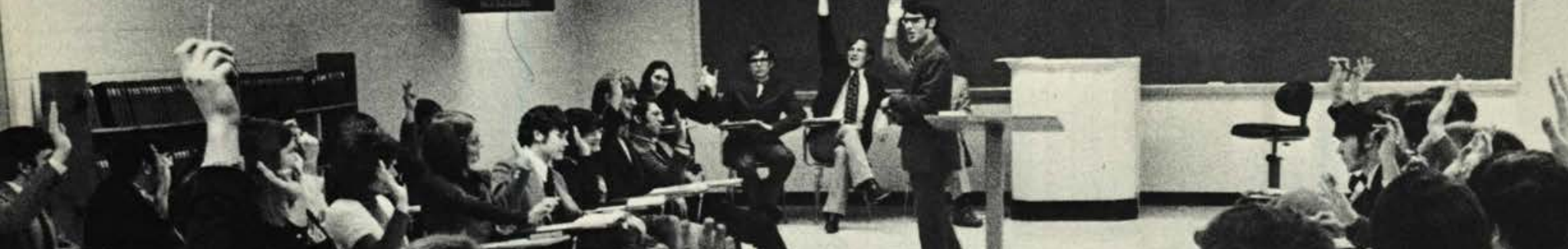
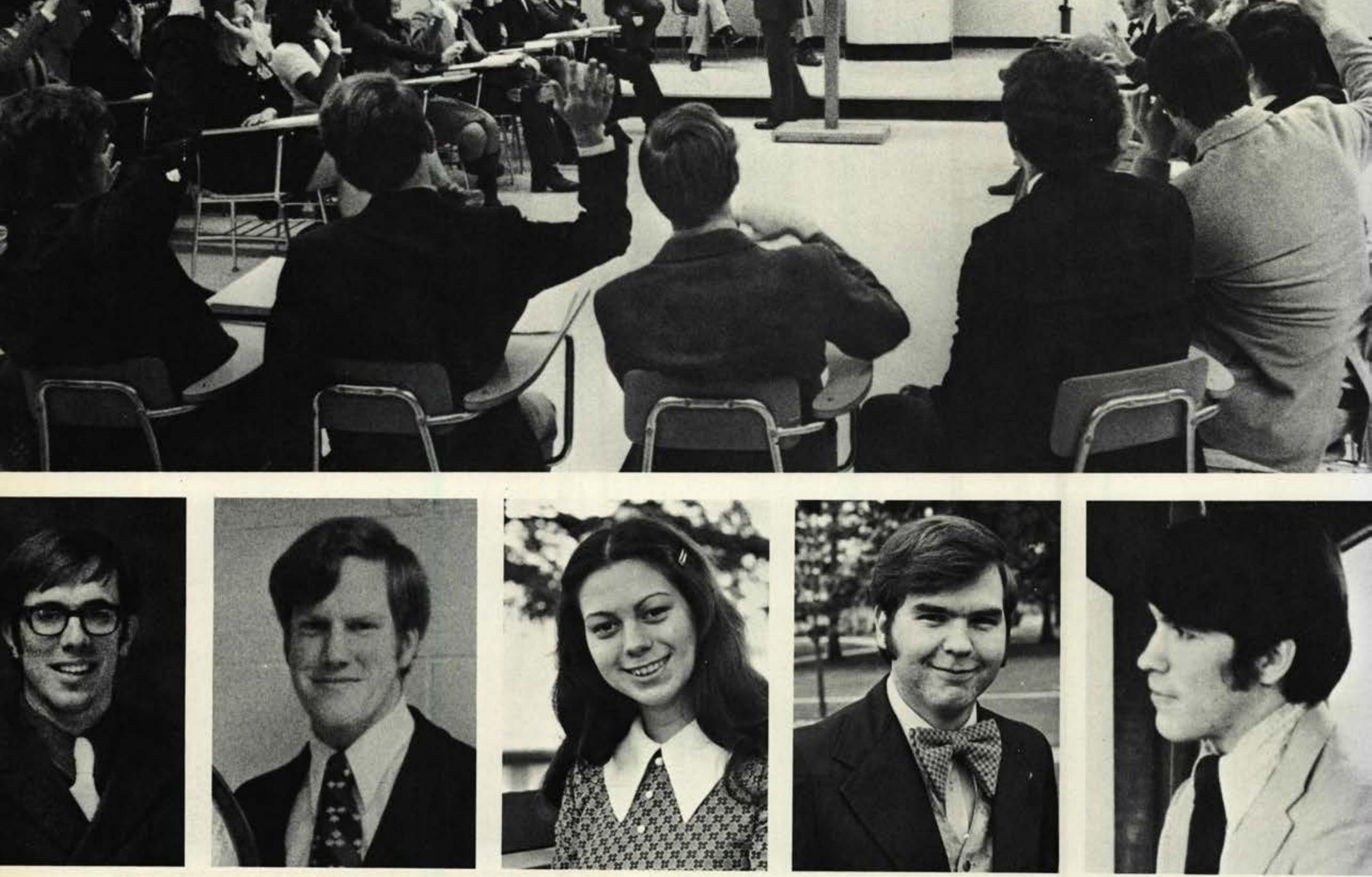

\section{Student Senate}

eff Kistler, SBP Chair.

Steve Overholt, Chaplain

Diane Jacobs, Sect.

Charles Sheppard, Tres.

Bob Mahl, V. Pres

Dan Estes, Pres.
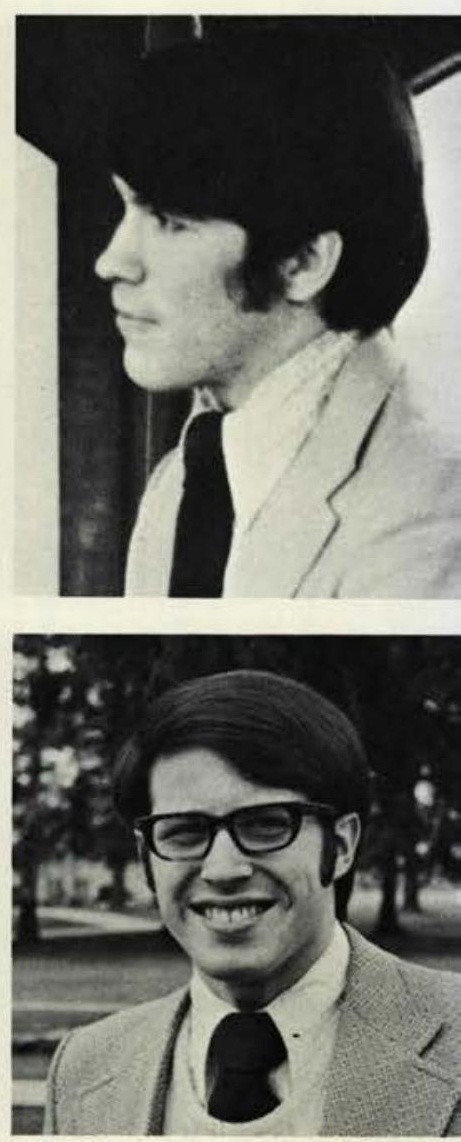
BACK ROW: D. Estes, B. Mahl, D. Schroeder, K. Brock, D Romaine, J. Bowen, S. Overholt, K. Mclntosh, D. Jacobs, R. Gruenberg, L. Severs, S. Cressman, J. Goetz, D. Stemen, J. Kistler, D. Stewart, T. Gifford, Dean Rickard, R. McGhee, FRONT ROW: M. Johnson, S. Young, S. Gazdik, D. Twigg, D. Eggleston, J. Young, D. Dear, P. Donelson, S. Estes, K. Lancaster, S. Gons, D. Deland, J. St. Clair, M. Seeley, C. Carr, B. Davison.
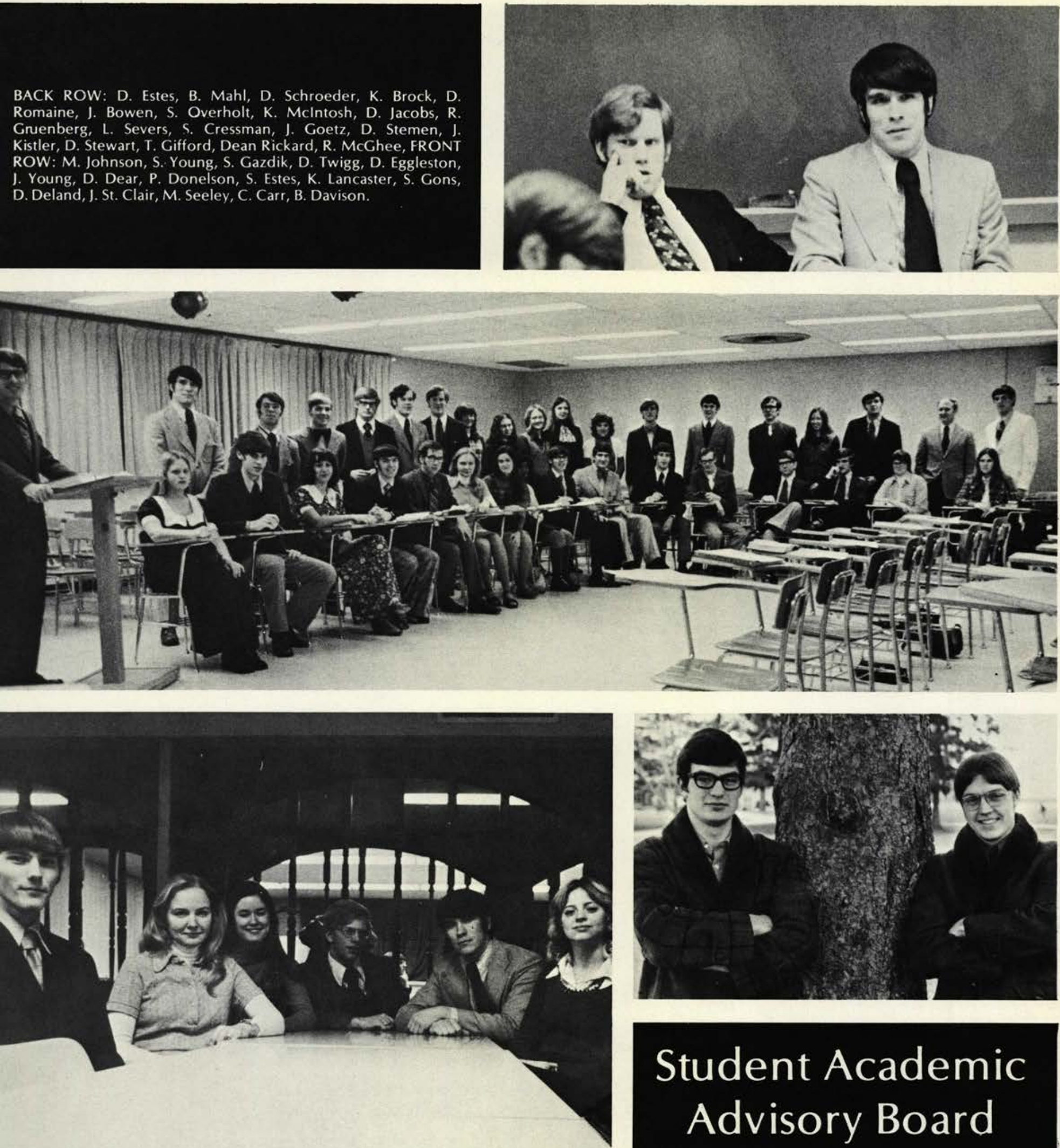

\section{Student Academic Advisory Board}

B. Klett, Biblical Ed.; J. Long, Lang. \& Lit.; D. Dear El. Ed.; B. Sampson, Soc. Sci.; B. Mahl, Speech; S. Holwerda, Phys. Ed.; D. Nicholas, Math \& Science; B. Geisman, Bus. Ad. Not Pictured: B. Luketic, Psvch.; N. Tallman, Music. 


\section{Fall Bible Conference}

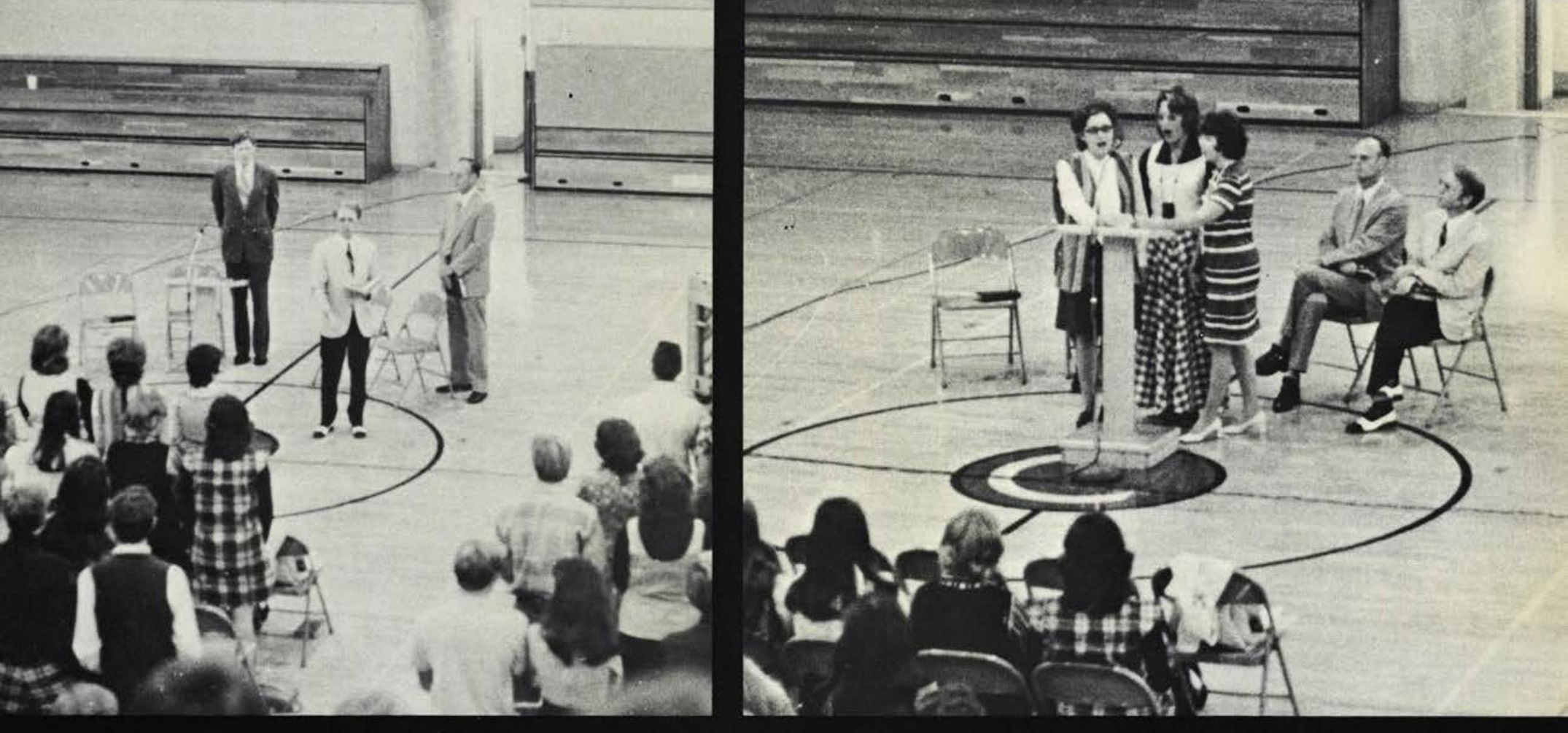

8

$n^{8} \times 1=$

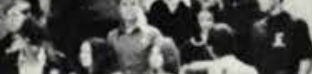
G 15 ondaris? I 1

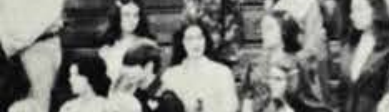
1. -15 Thon -2 in 4

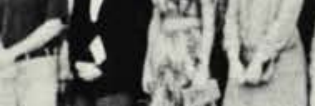
1. (1) si .

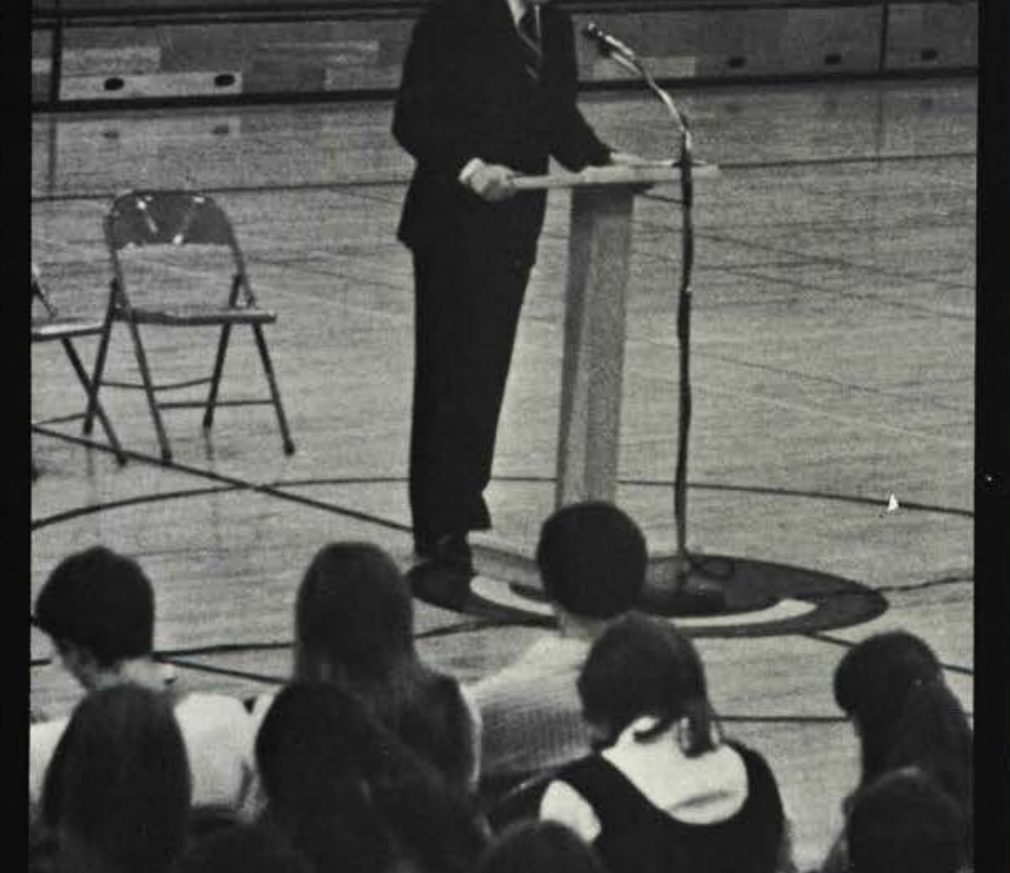




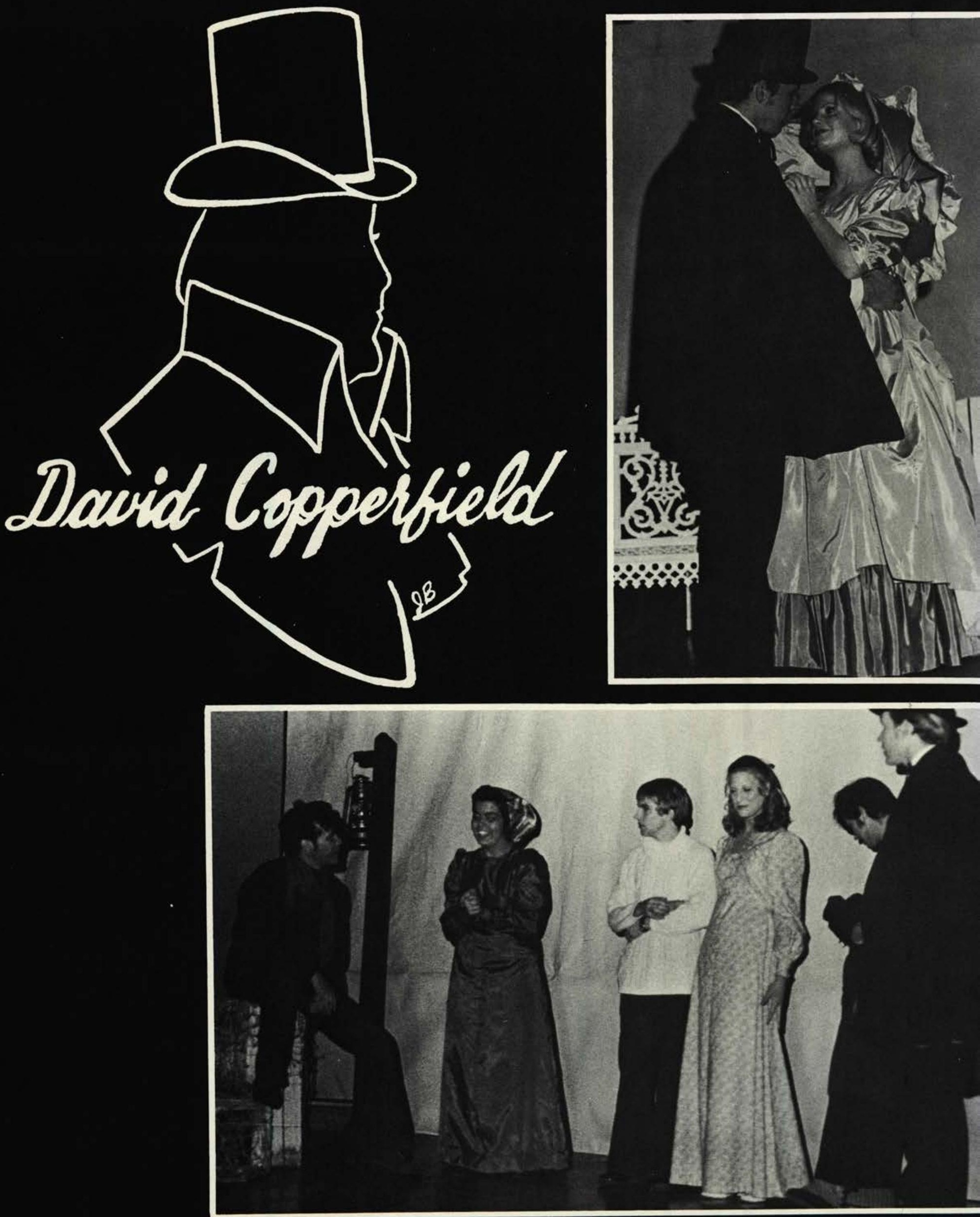



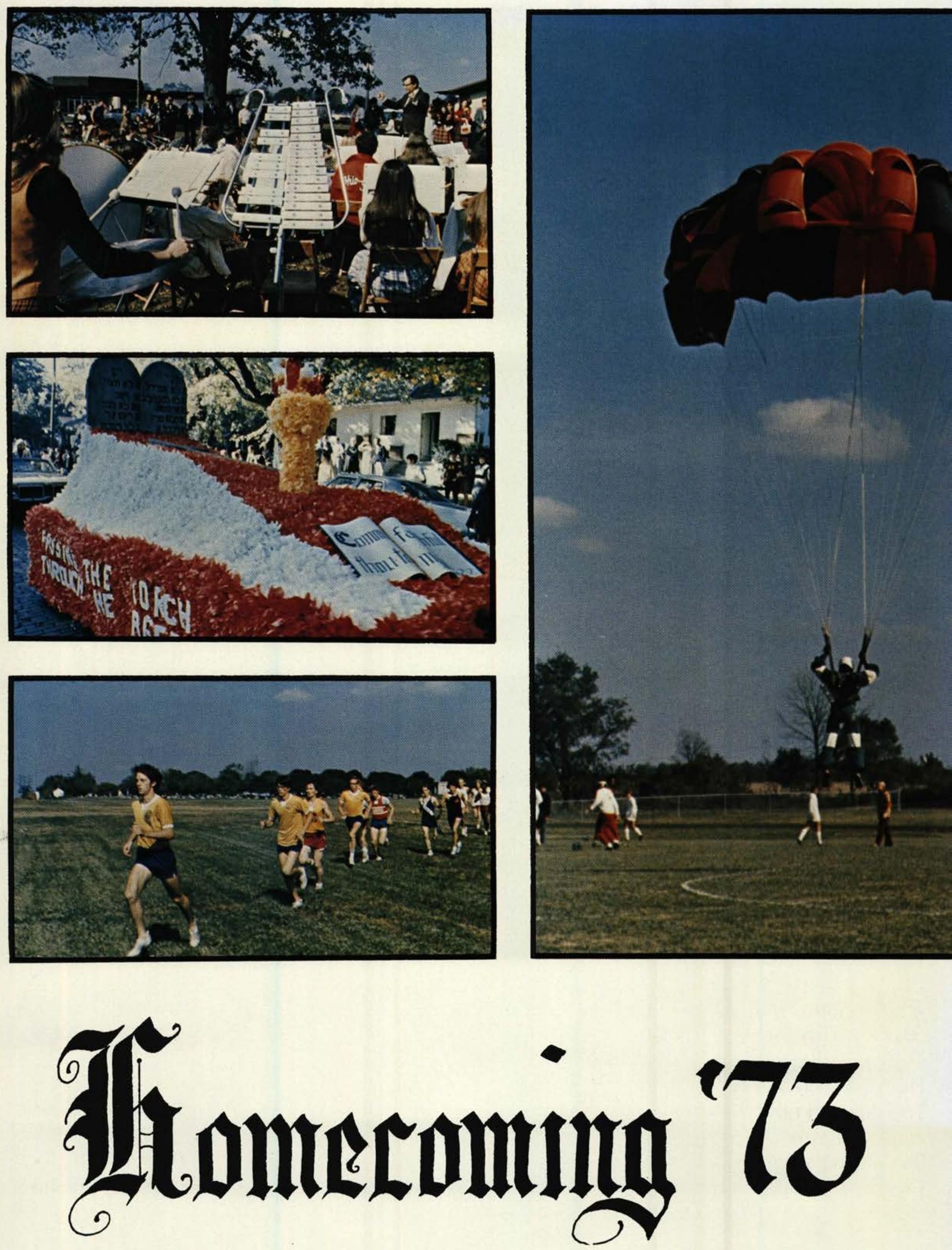

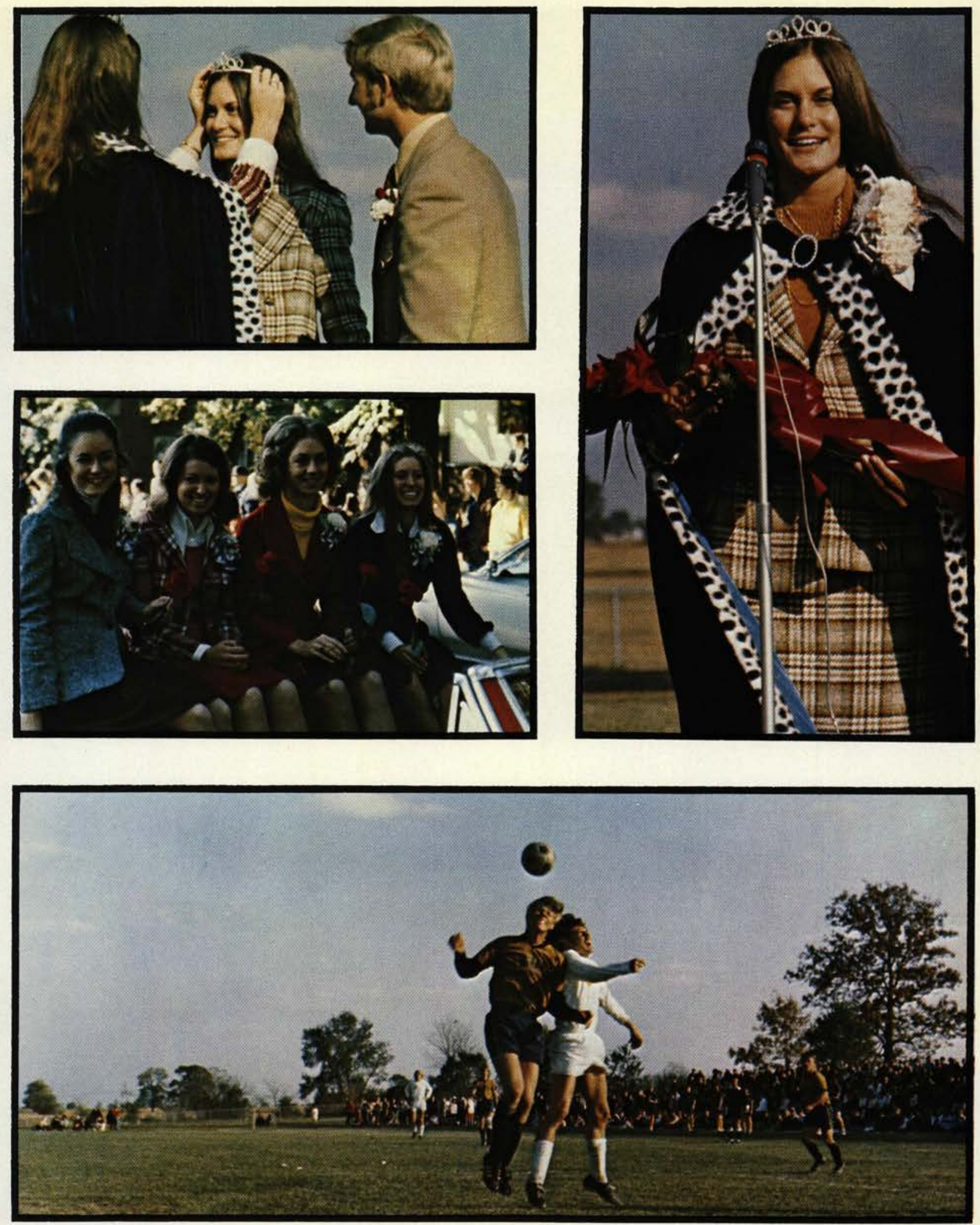

The big game ...

Sunshine on the new field ...

Homecoming Queen. Peggy Wagner The crowd ... faculty kids all over ....

Halftime activities...

The Alumni take the float competition ...

Homecoming Court: Debbie Dear A new queen ... But it's the same story ... Becky Lamb Cedarville always wins! Mindy Andes Carolyn Sapp 

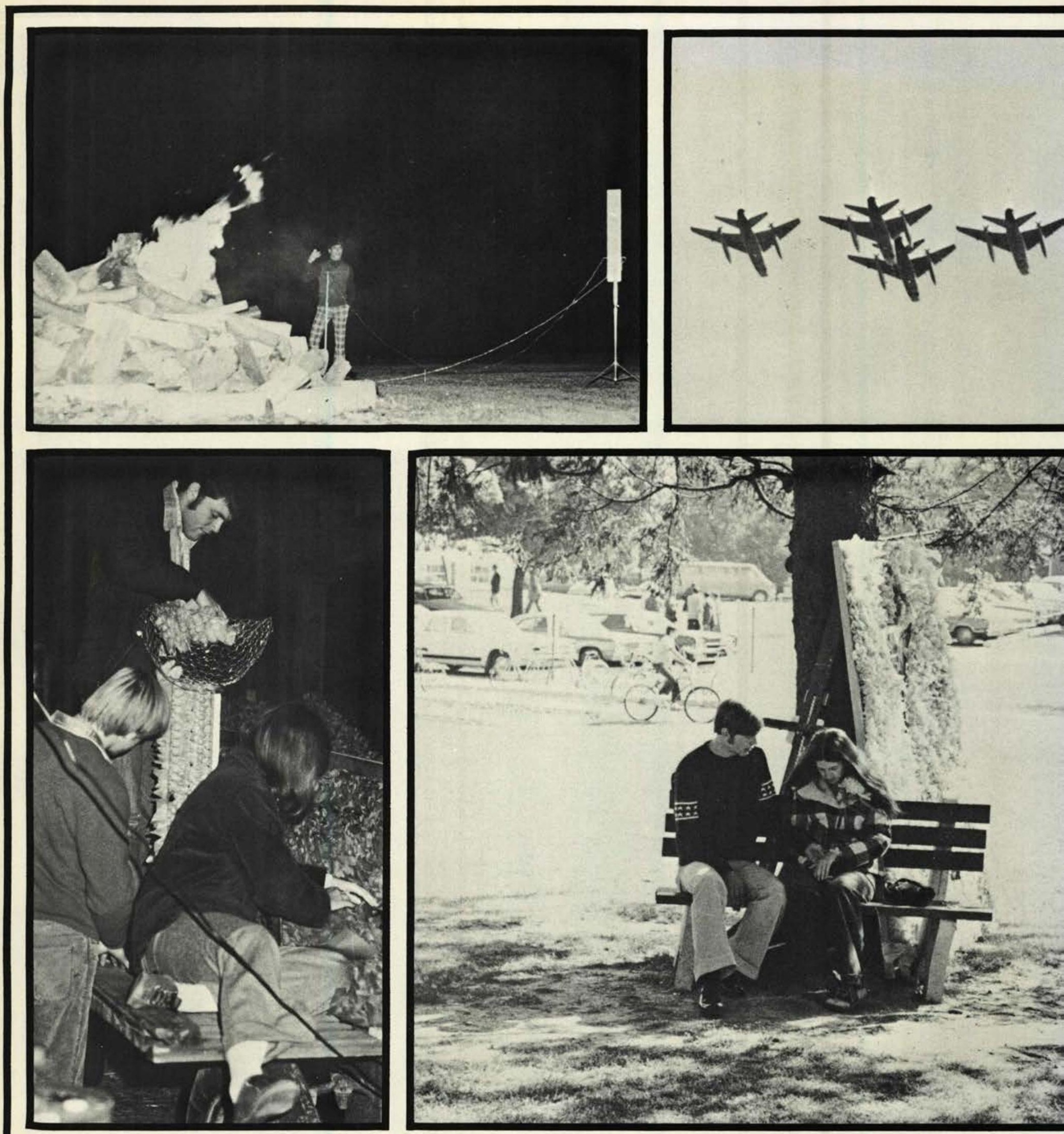

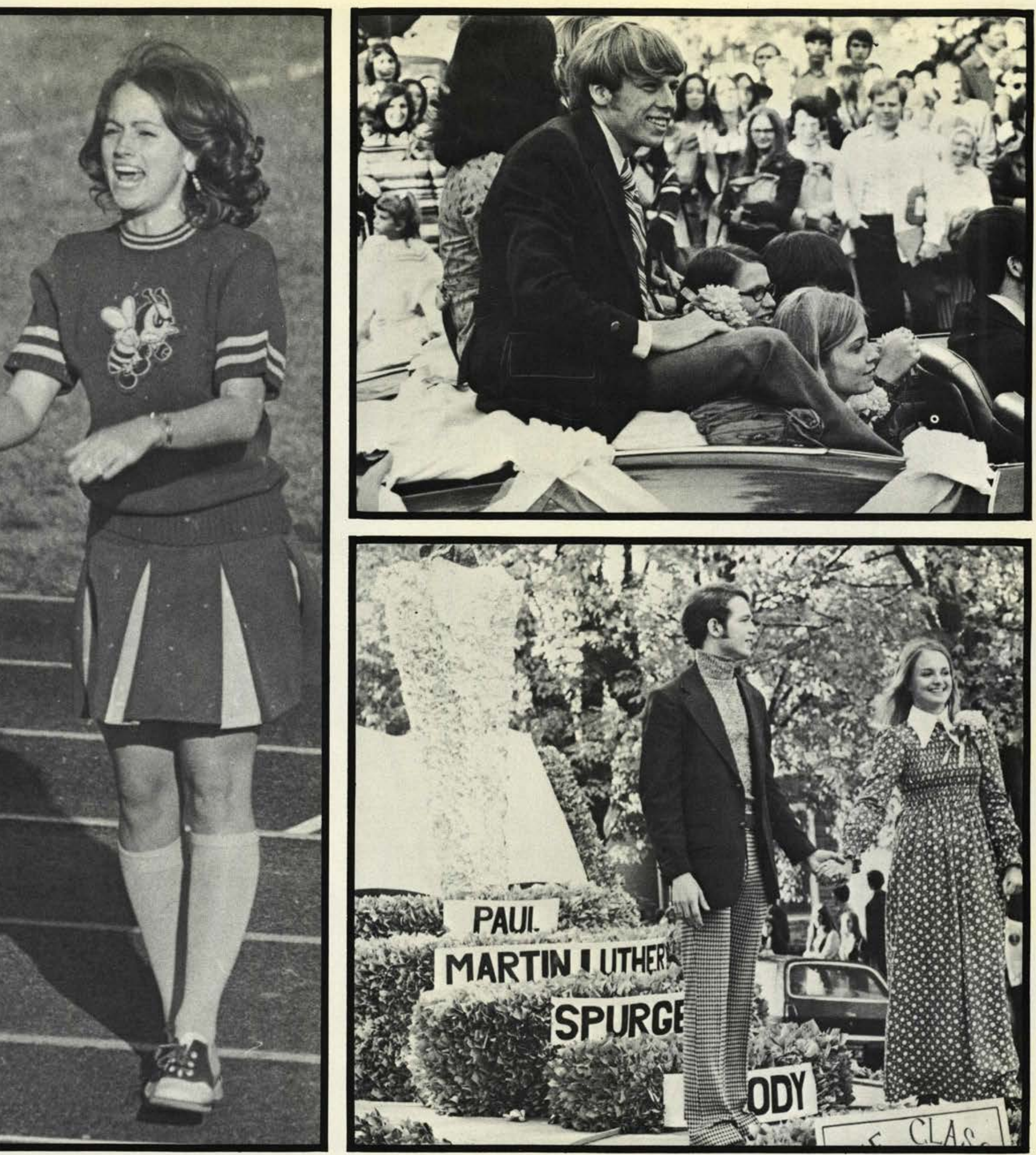

Back to the 'ville Seeing old friends Exchanging news "Have you seen ... ?" "He hasn't changed a bit." Homecoming .. Guess it will never really change. 
STARTING FROM BACK: Janet Carr, Debbie Butcher, Alice Miller, Pam Burtner, Sherry Chamblin, Steve Holman, Dan Bowman, Lois Matheny, Barb Loach, Ellen Spaulding, Pat Henry, Steve Wilson, Joel Harriman, Esther Lightcap, Carolyn Hayes, Mary Glenn, Mindy Andes, Charlotte Rowland, Dora Ewing, Georgeanna Axiotis, Bev Davidson, Mary Pruden, Marcia Houck, Dawna Robb, Ray Warwick, Peggy Wagner, Susan Seldon, Clancy Parker, Linda Murray, Robert Fisher, Steven Koon, Bob Gillespie, George Sperry, Barb Cooper, Dennis Twigg, Jackie Spinks, Tim Pasma, Paul Bowers, Becky Lamb, Dave True, Alan Dunn, Ellen Taft, Sue Johnson, Bill Giesman, Rodney Blystone, John Street, Wes Johnson, Douglas Schroeder.
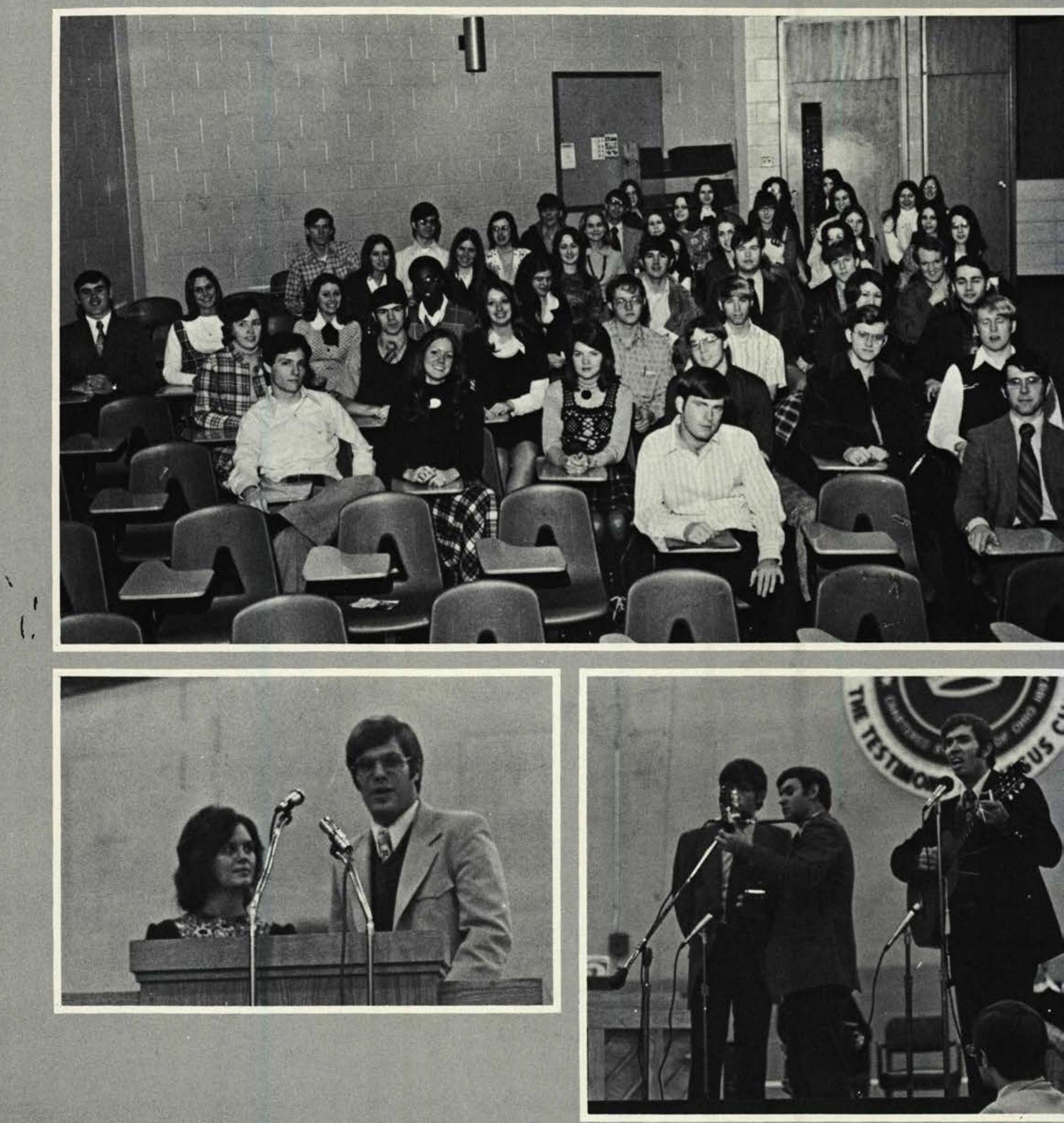
$-70^{2}$
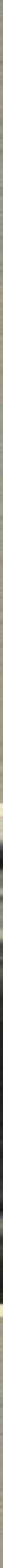

Swordbearers:

A Conference

in the Fall 


\section{Fall M.I.S. Banquet}

STANDING: Dr. Callan, K. Strawn, D. Arthur, D. Twigg, B. Potter, D. Douglas, C. Heslep, B. Cooper, C. Towle, E. Schultz, D. Jones, B. Kemble, N. Walter, L. Hinds, L. Mitchell, C. Parker, B. Lamb, D. Eggleston, D. Dear, C. Larson, B. Ziegler, D. Weaver, V. Weah, T. Kaufman, D. Brannon, J. Weah, D. Jewell, L. Bollback, Mrs. Johnson; KNEELING: Pastor Green, D. Hickey, R. Clark, D. Johnson, D. Schroeder, M. Mawhorter, P. Mc-

Donald, Dr. Johnson.
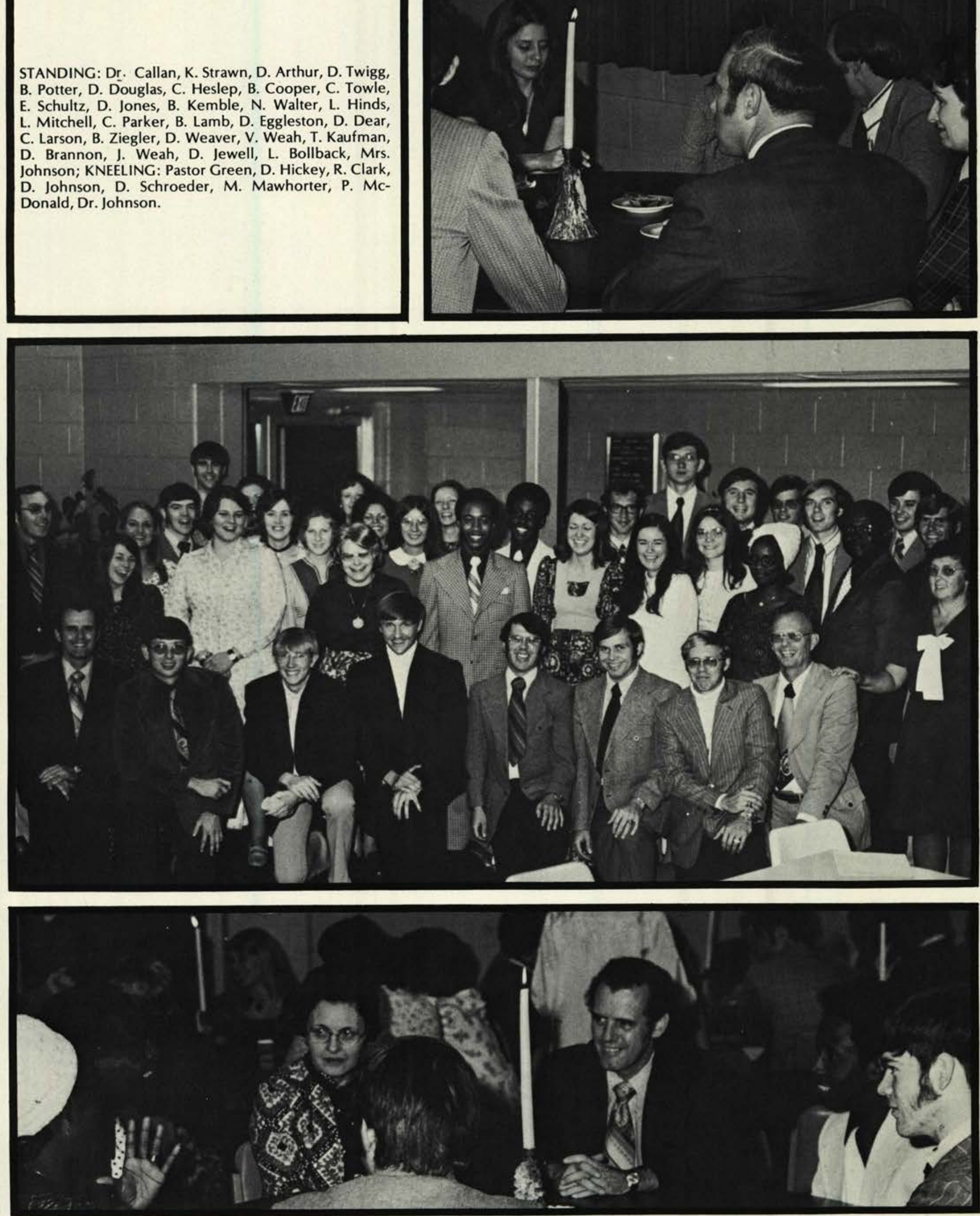


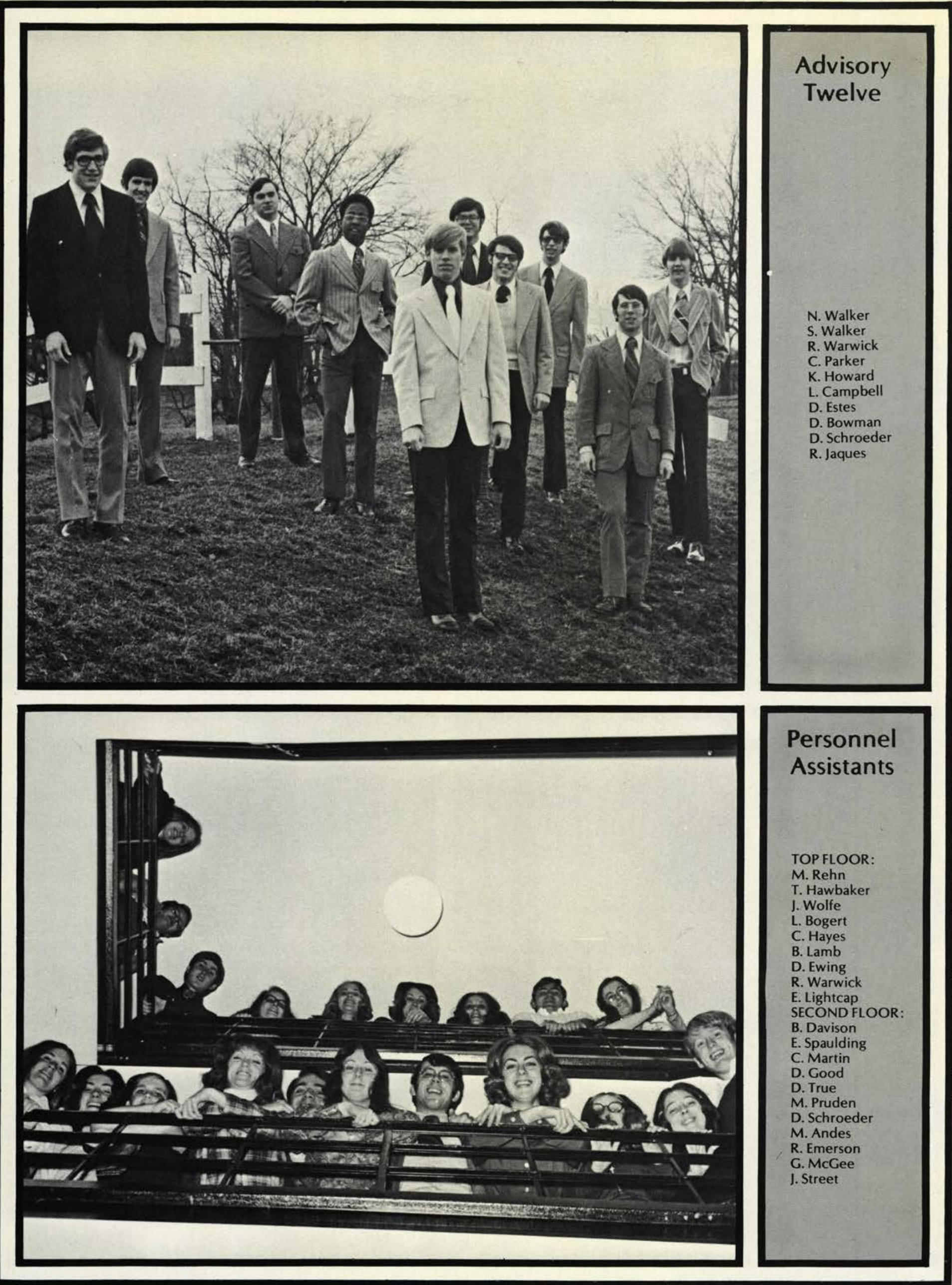



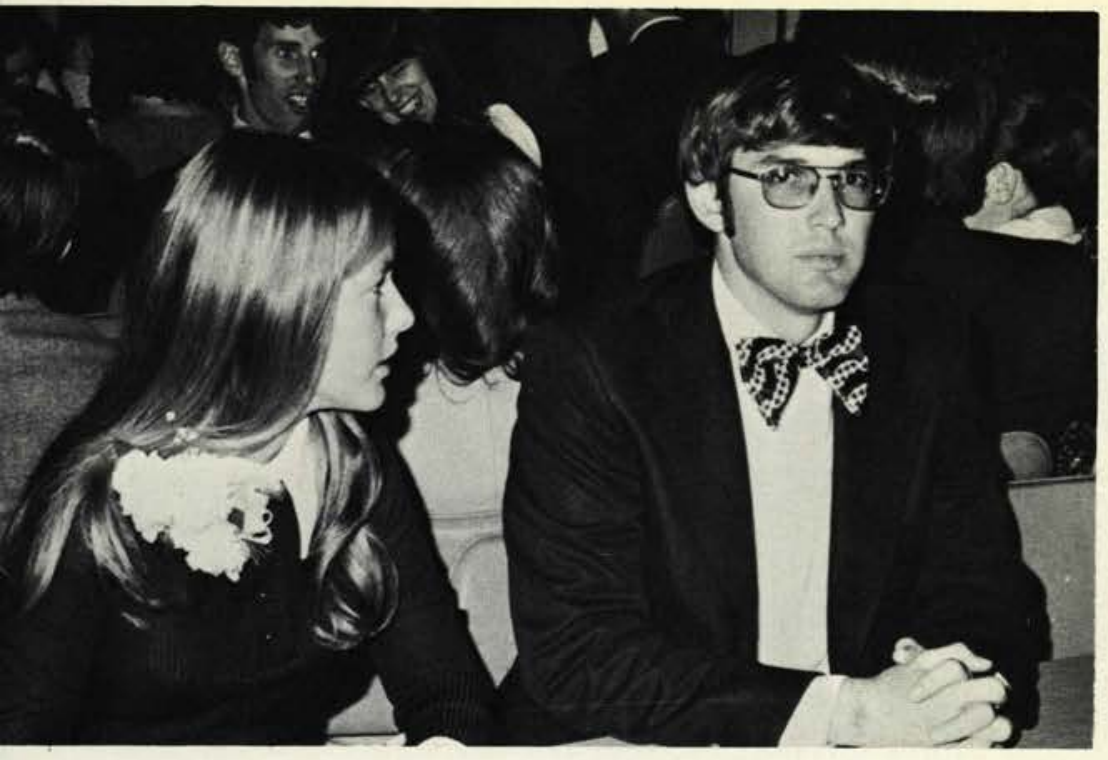

Alpha Chi Presents ...

Alpha Chi presents.

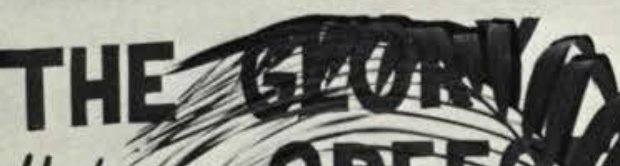

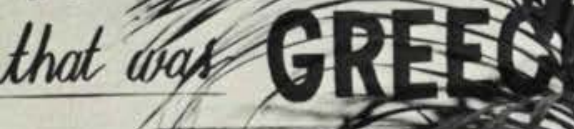

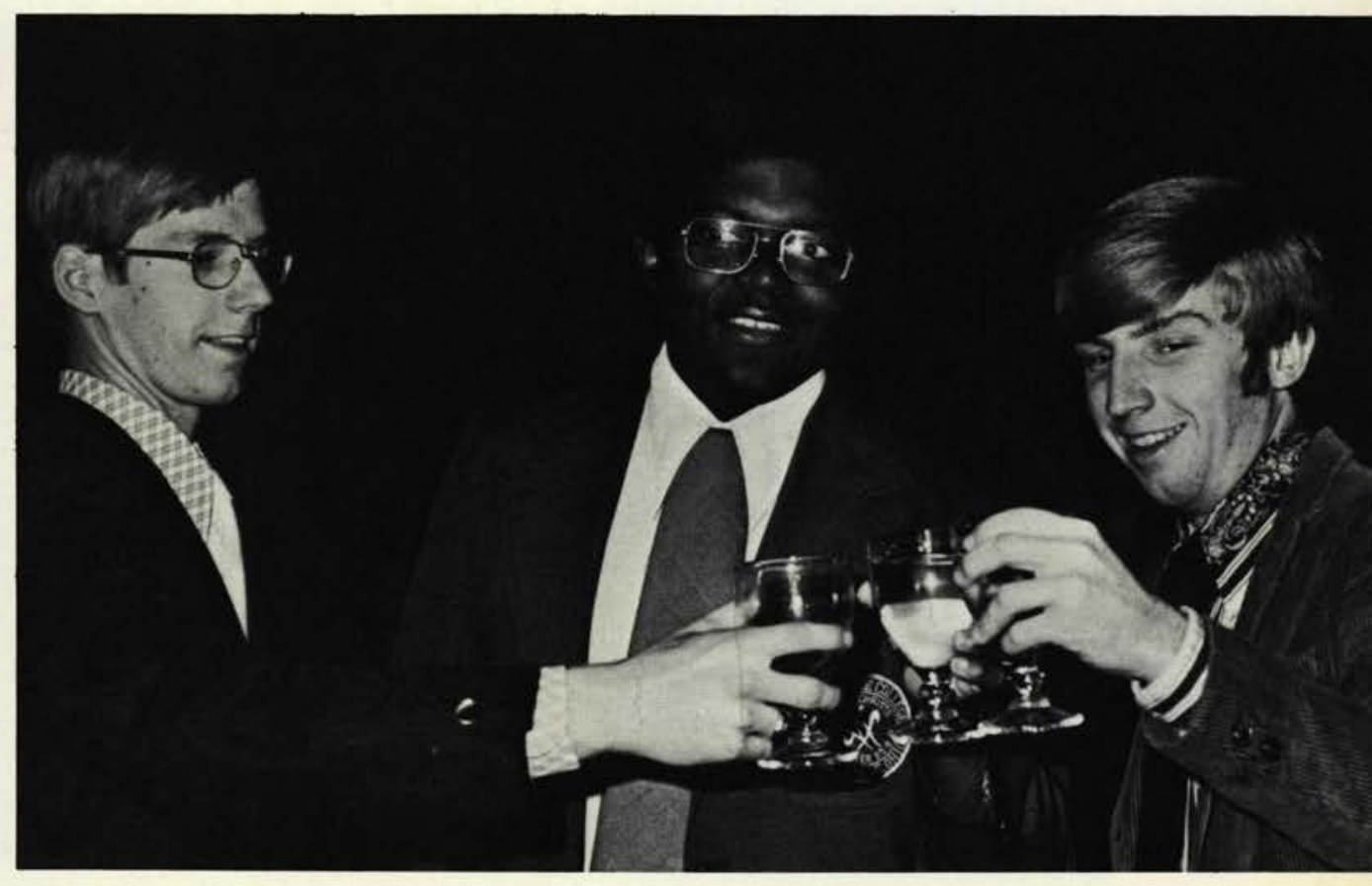

The Glory

That Was Greece

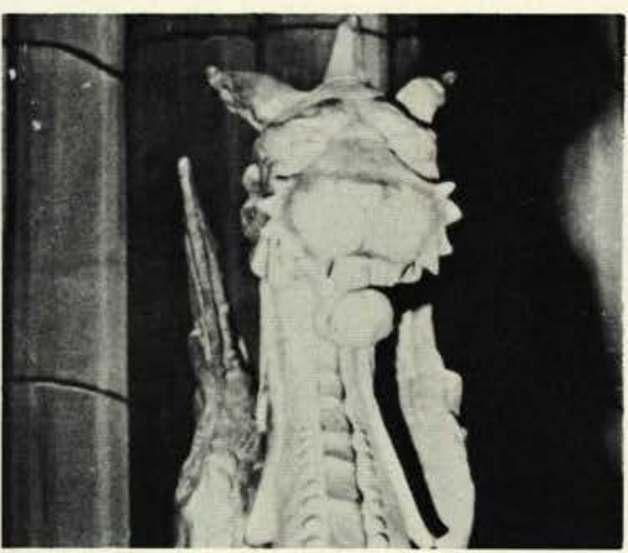



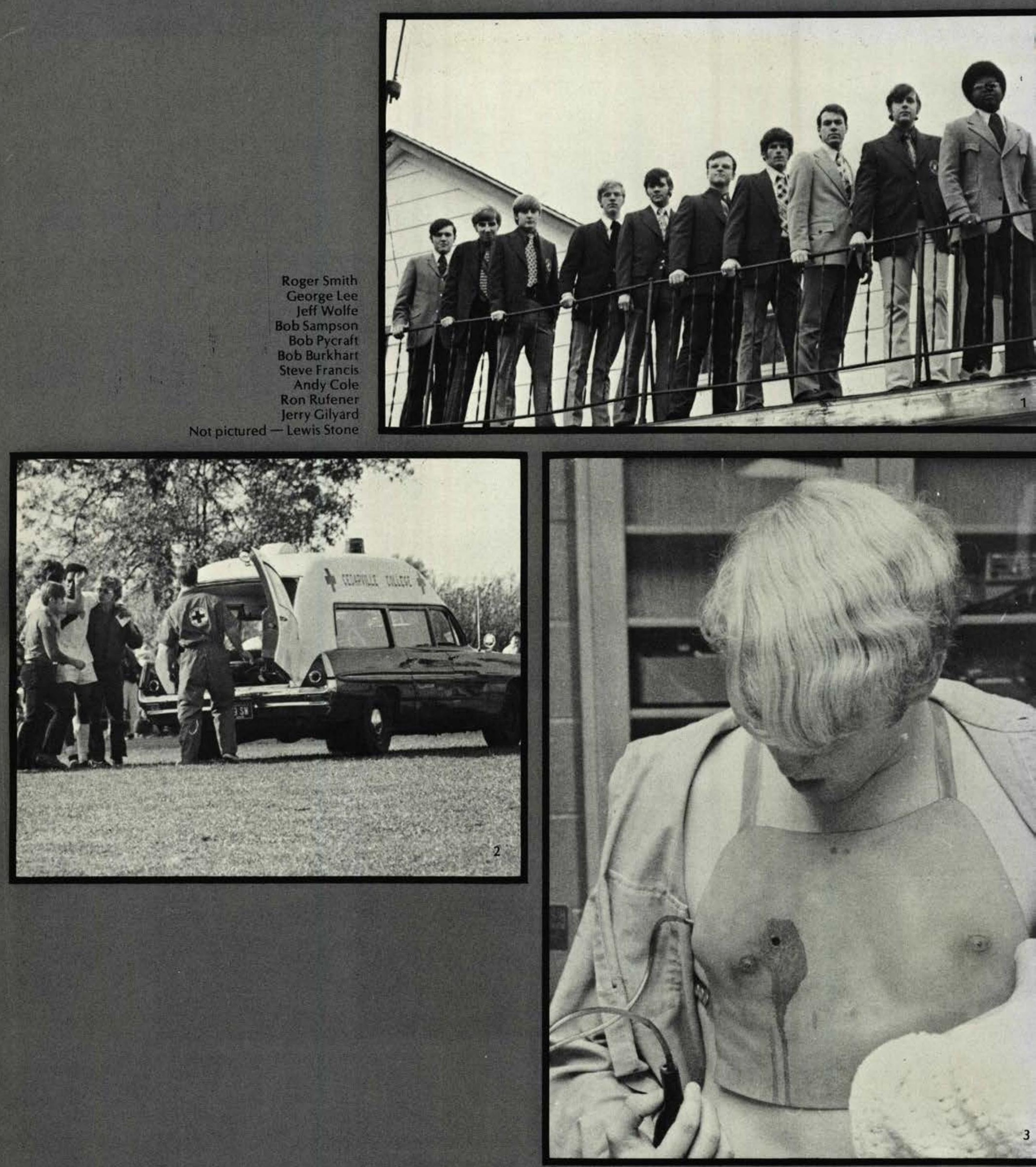


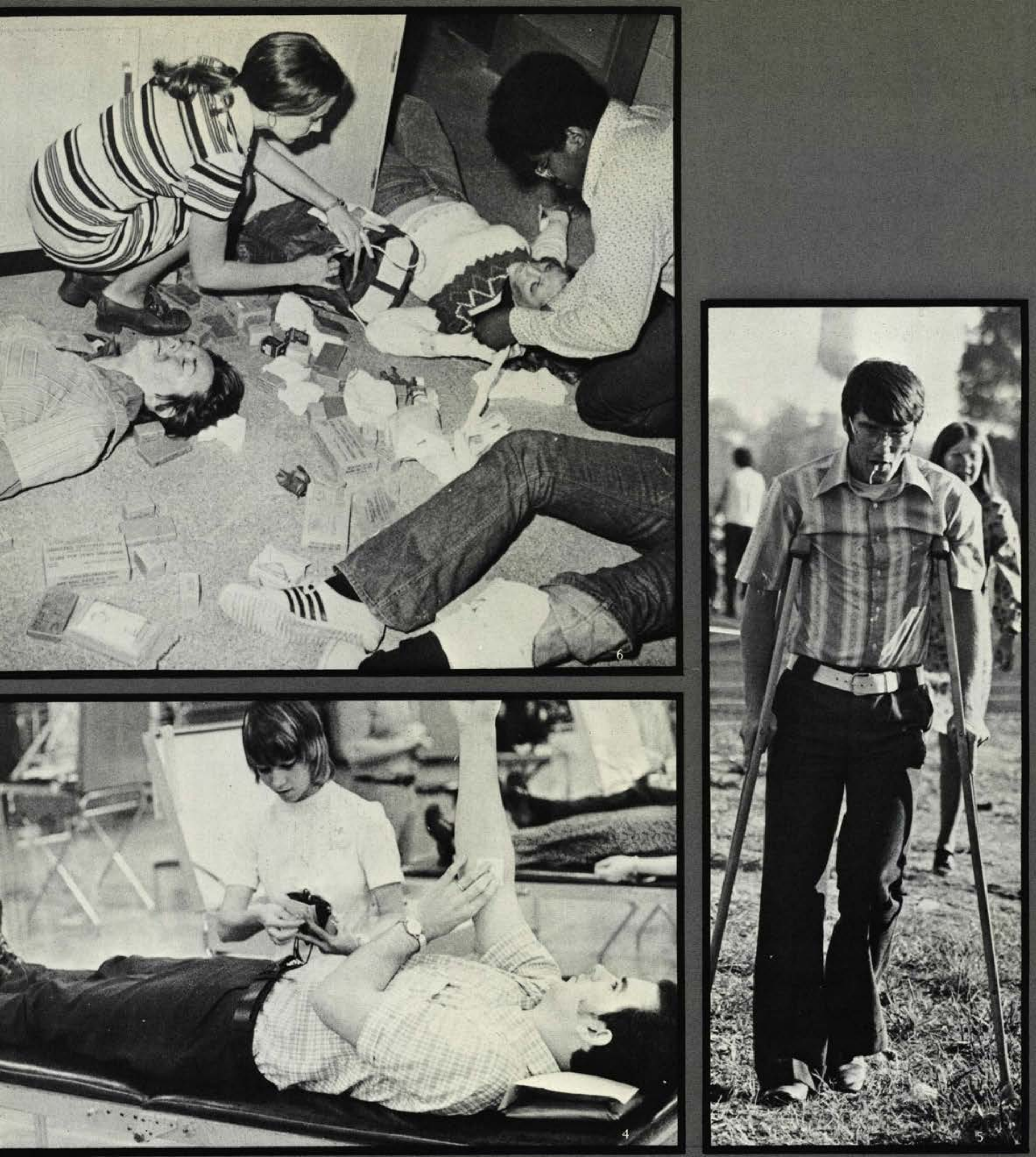

1 Getting the squad together

Injury interrupts a soccer game.

Rubber bullet hole, at a mock disaster:

4 Sharing life, giving blood.

A familiar sight around Cedarville this year - CRUTCHES

6 Attendants come to aid at mock disaster. 

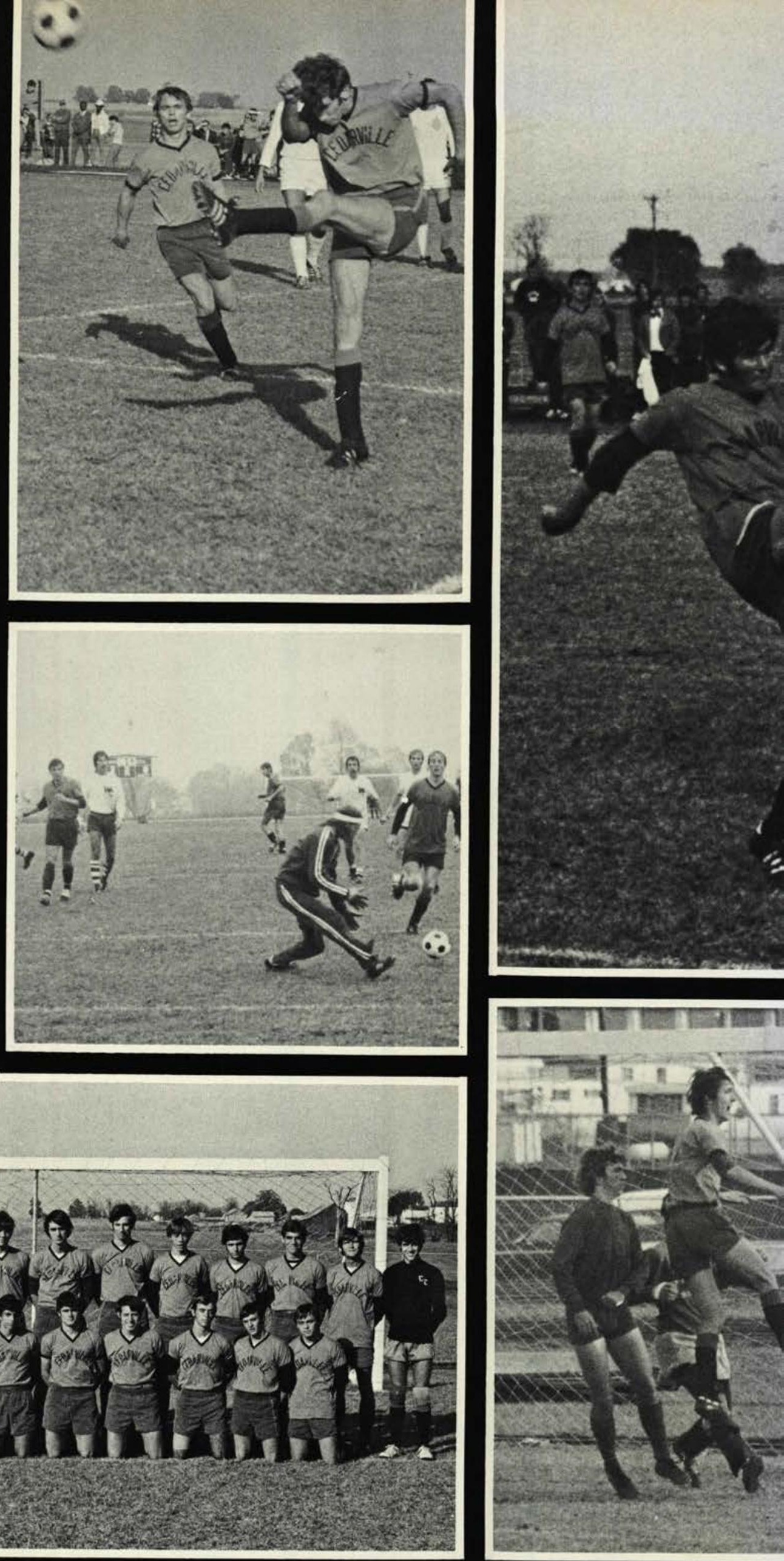

BACK ROW: Coach Berry, R. Profant, B. Luketic, K. Howard, J. Mitchell, T. Hlad, T. Knowles, H. Diaz, J. Cavey, G. Trittipoe, M. Ziegler, L. Bollback, T. Hollev, FRONT ROW: T. Cook, D. Delancev, J. Wyman, D. Hare, J. Battaglia, J. Bartemus, D. Estes, P. Kester, J. Jenkins, P. Mc Millen 

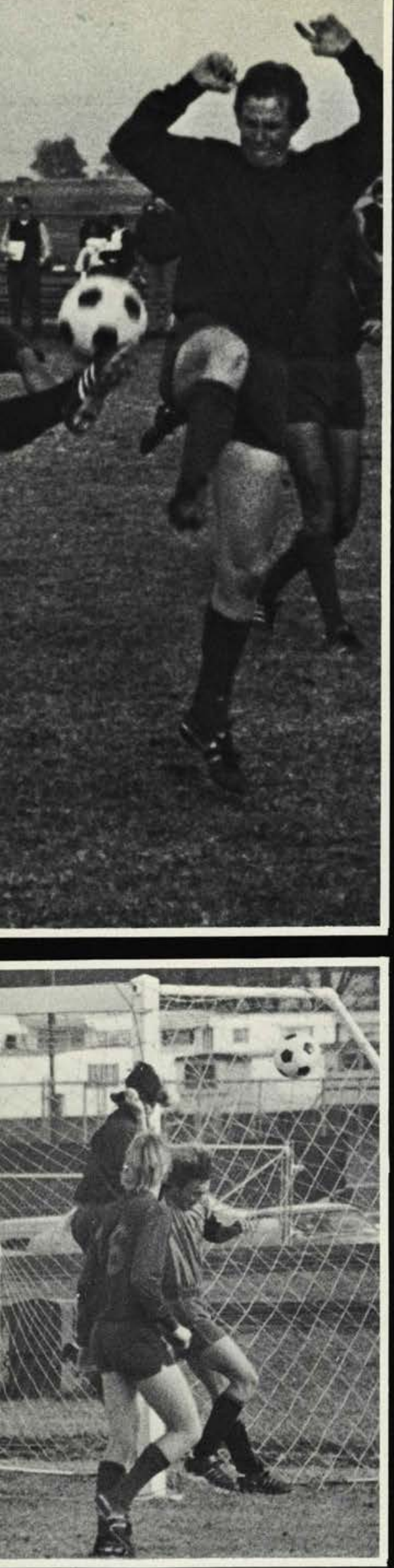

\section{(S) (a) (G) (G) 足}
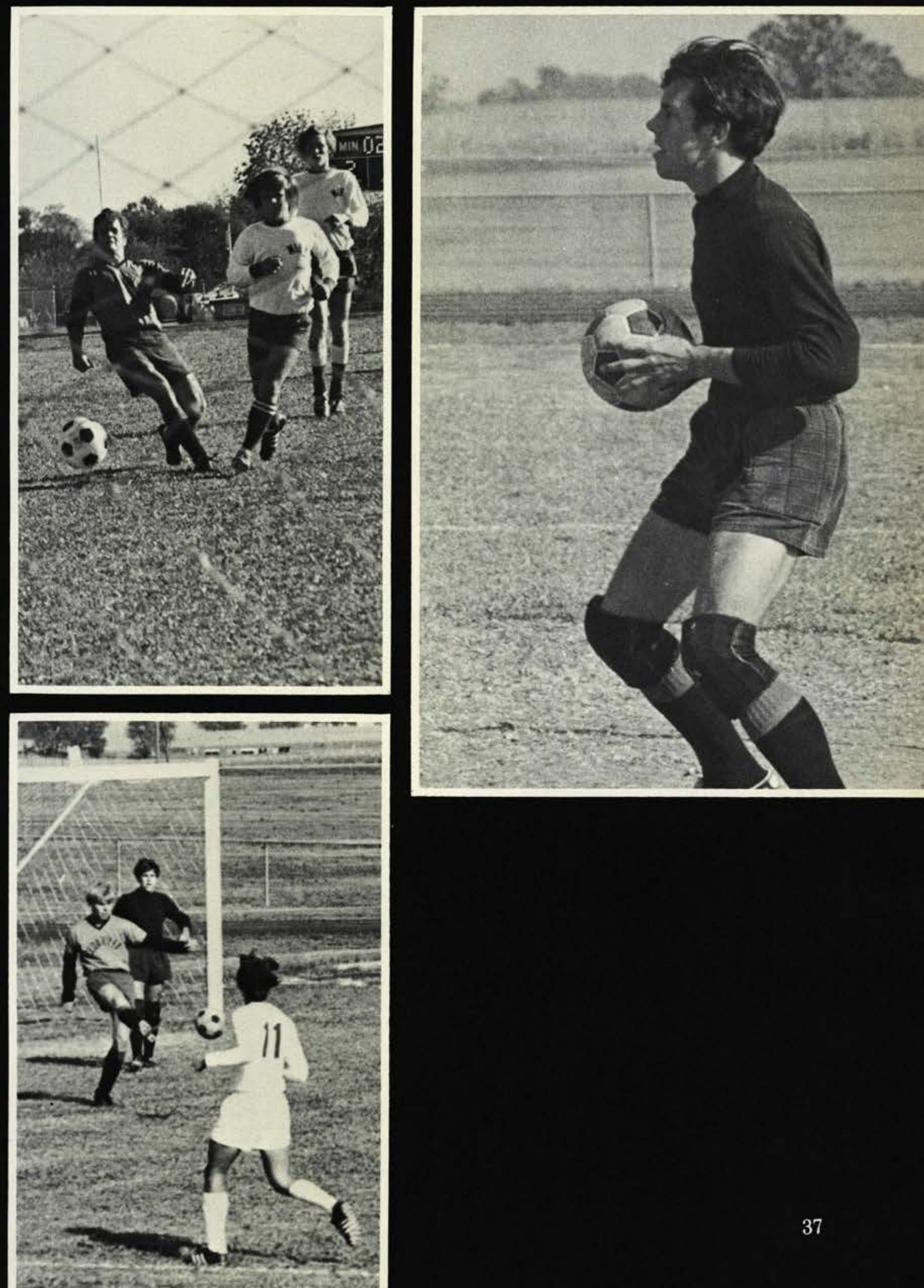


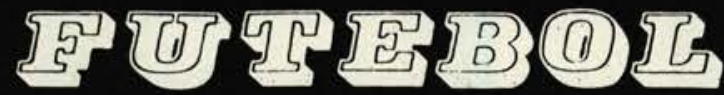
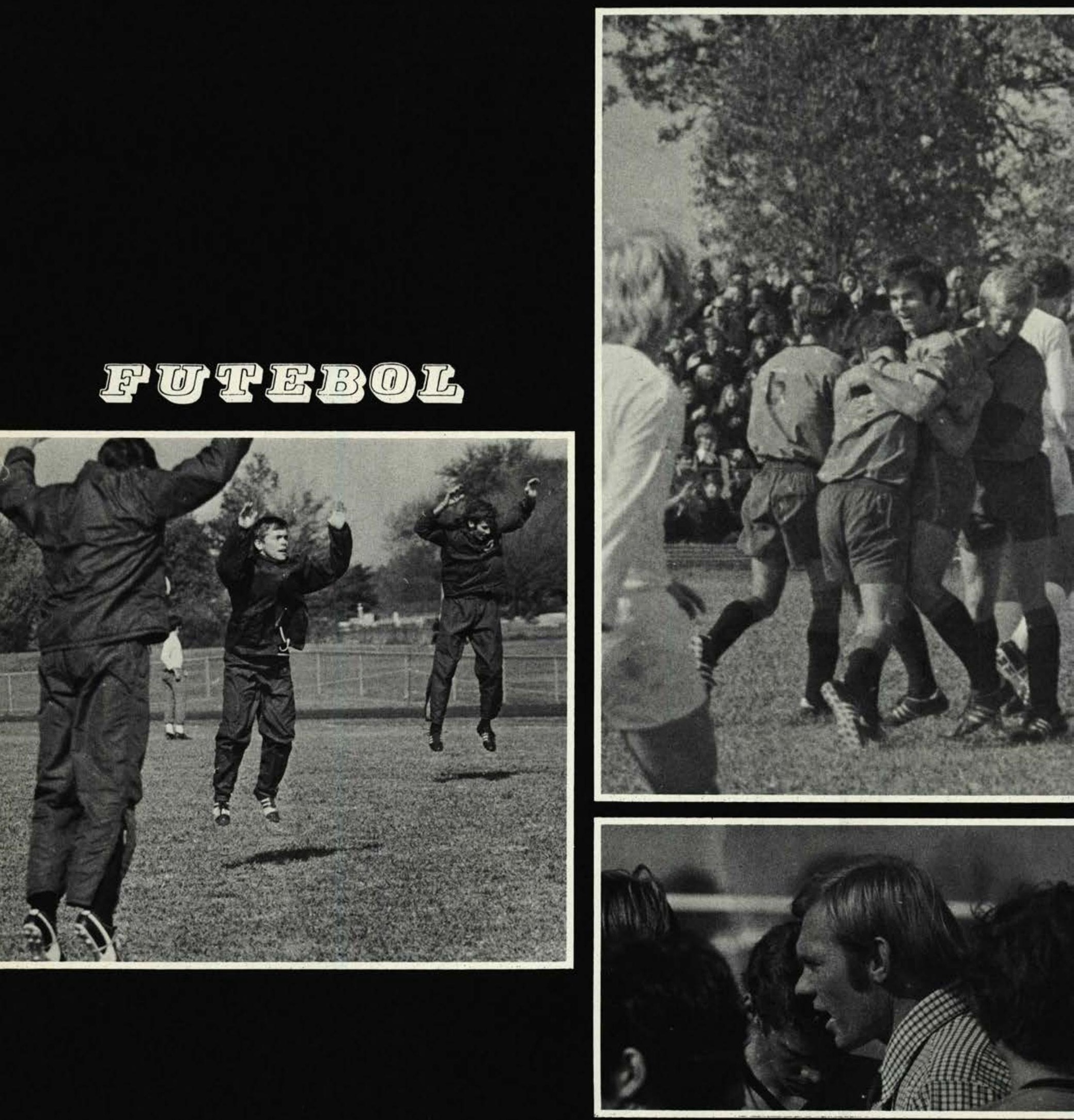
FRONT ROW: Ron Miller, Paul Beck, Jim Chambers, Paul Budnik BACK ROW: Ron Thomas, Dave Wishart, Bill Fenton, Tom Burnette

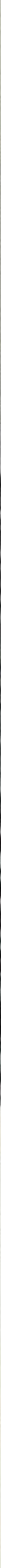



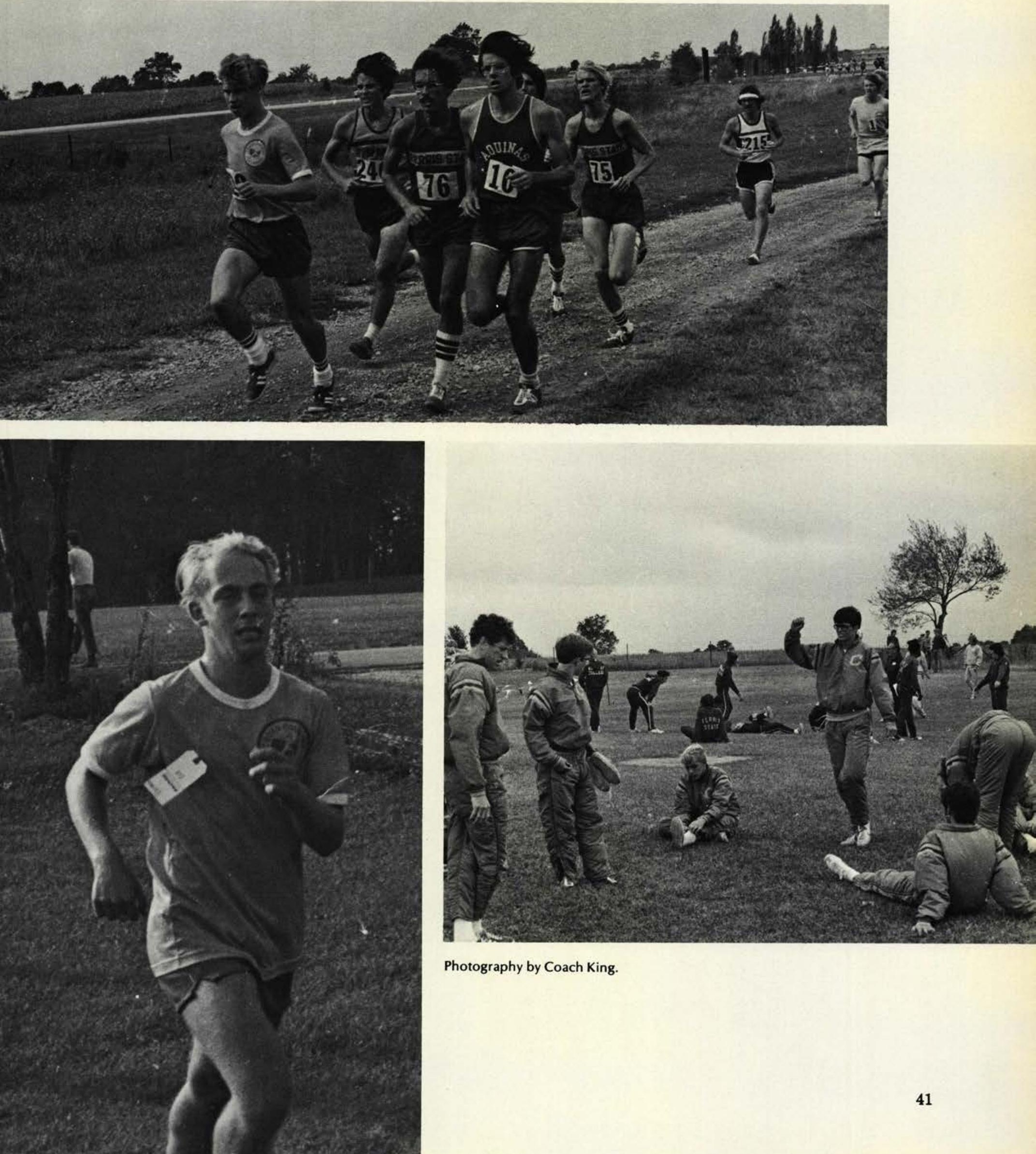

Photography by Coach King. 


\section{Women's Fall Sports - Field Hockey}

Varsity Volleyball: Marianne Frauenknecht, Jenny O'Neil, Linda Osborne, Denise DeMars, Sandy Howerda, Sally Ori-

hood, Cheryl Heslep, Sandy Finch, Laureen Clark, Coach Kearney.
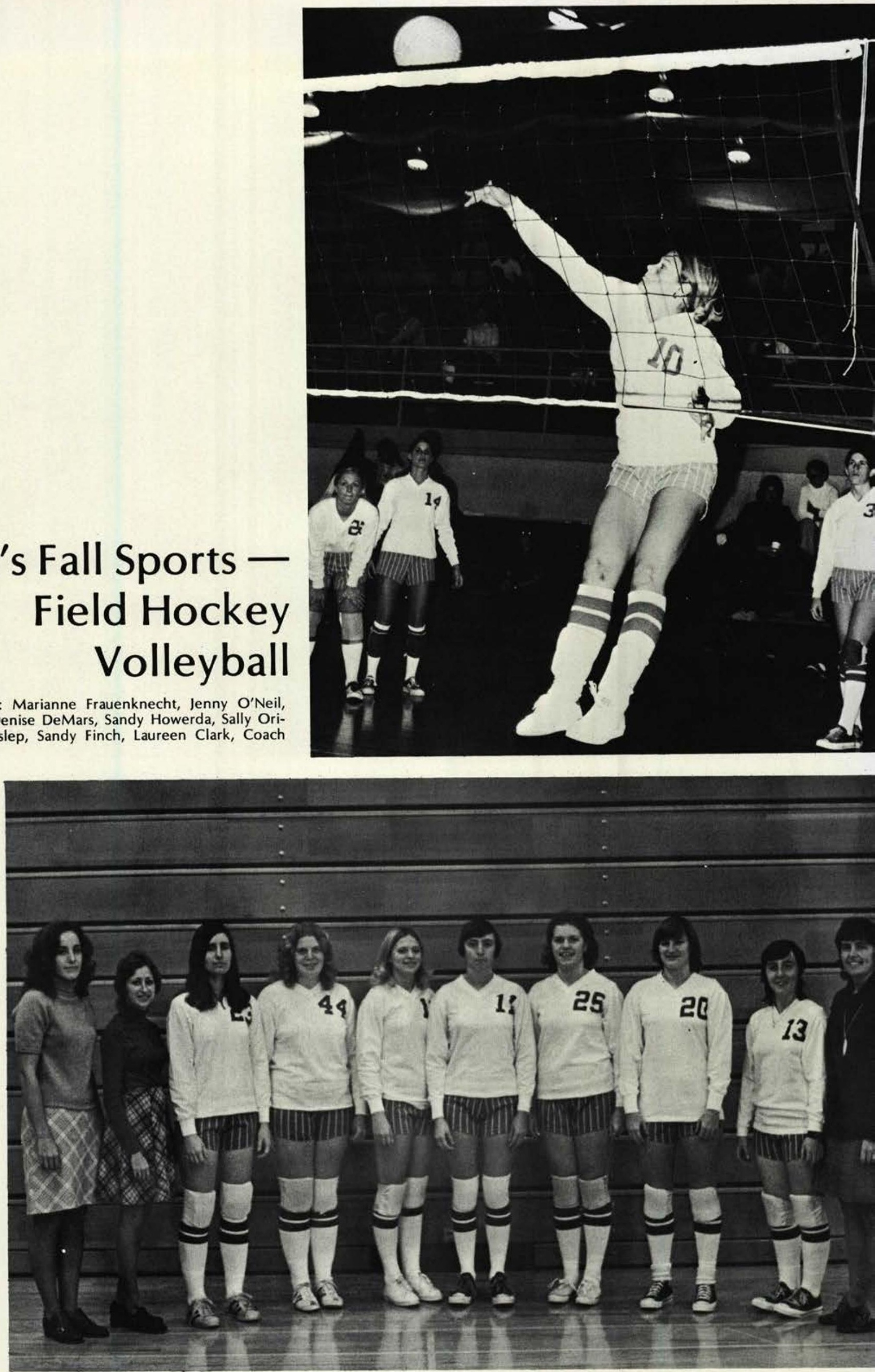
Field Hockey: Coach Kearney, Dawna Robb, Eunice Merrill, Chris Tompkins, Sally Orihood, Bertha Carter, Valerie Hook, Joy Erikson Debbie Stewart, Cindy Wiggins, FRONT, Laureen Clark, Margo Axiotis, Nancy Towle, Jenny O'Neil, Cindy Young.
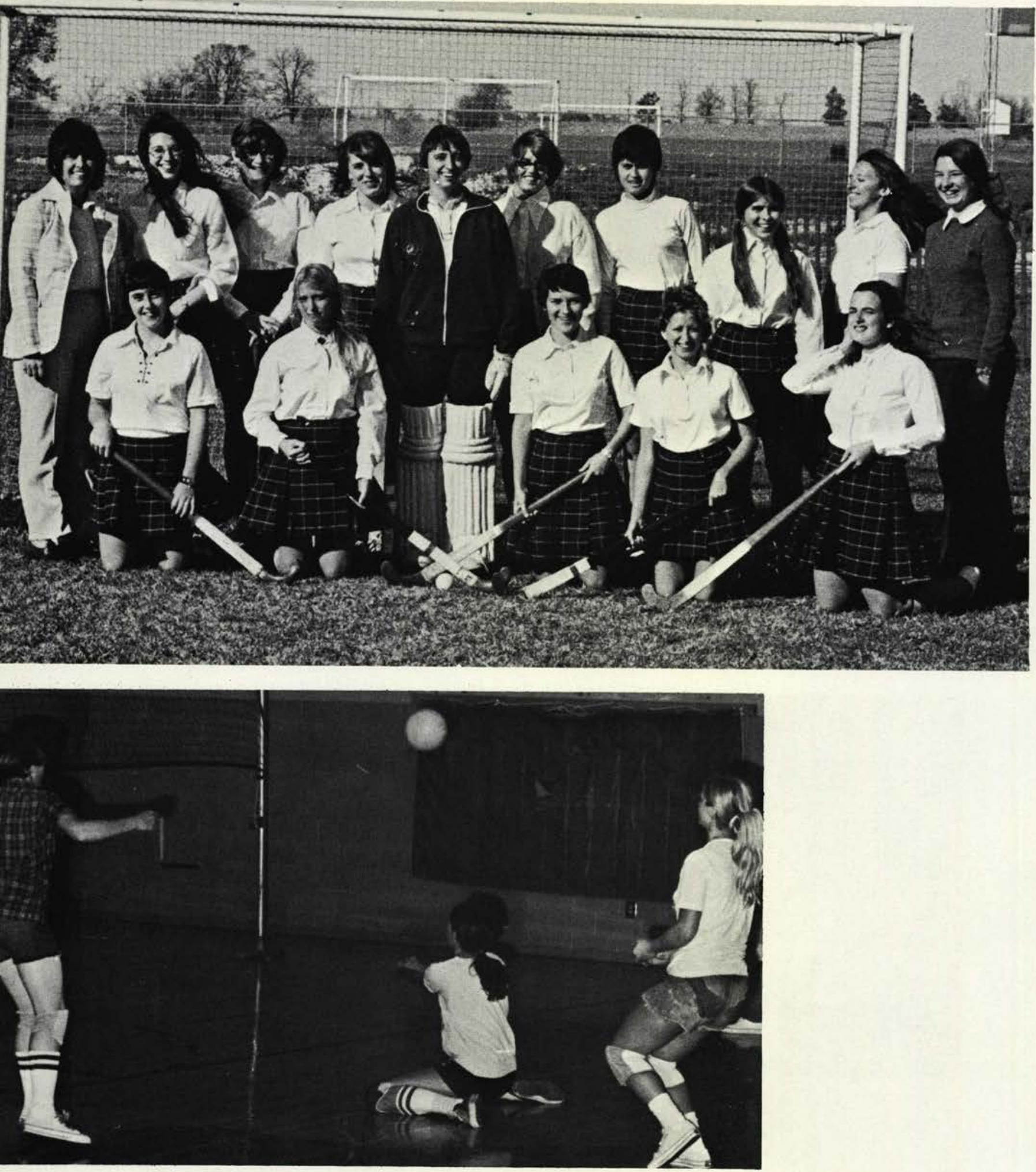

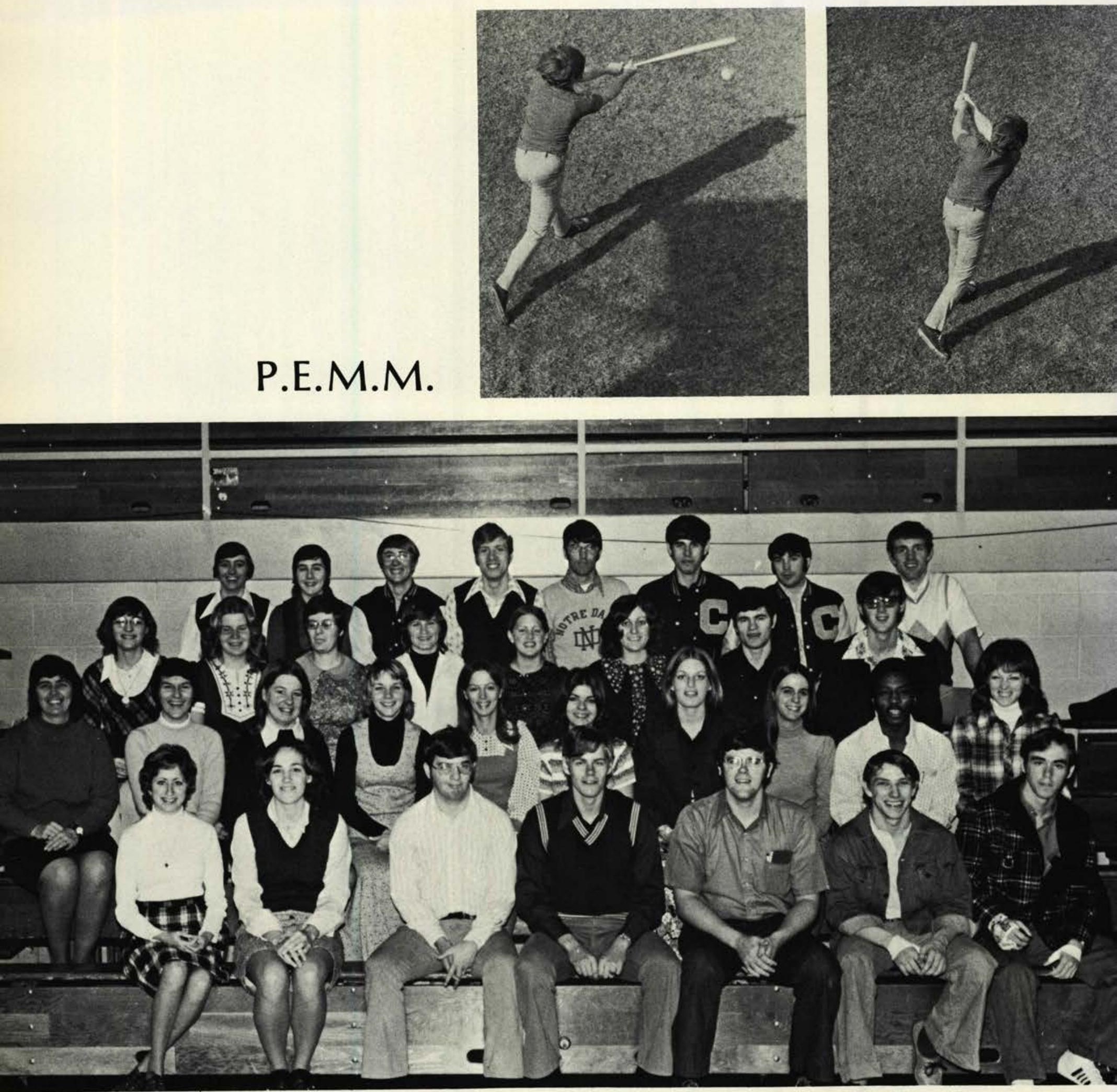

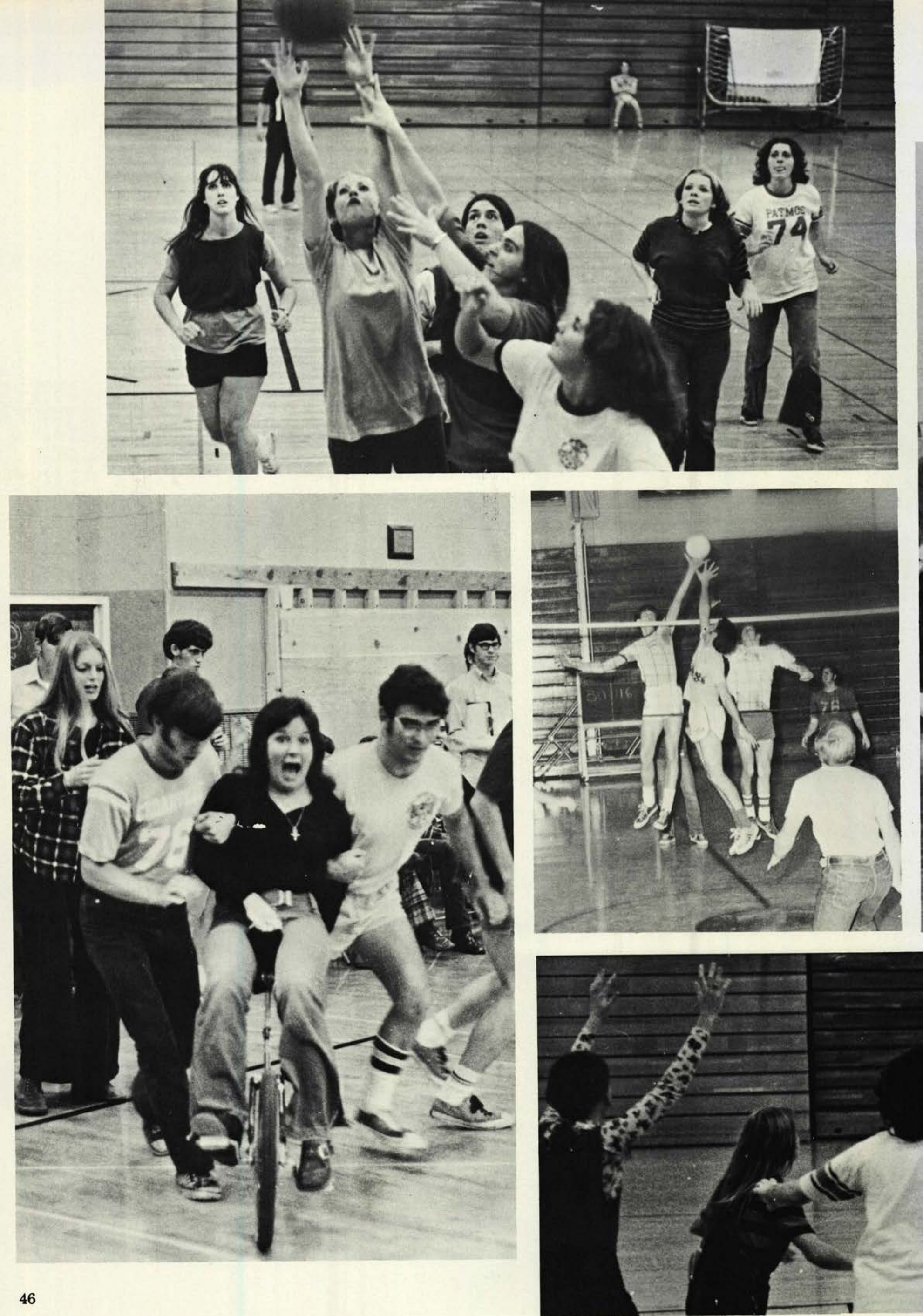

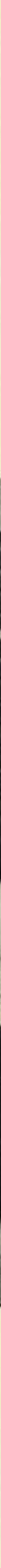

\section{All Sports Night}

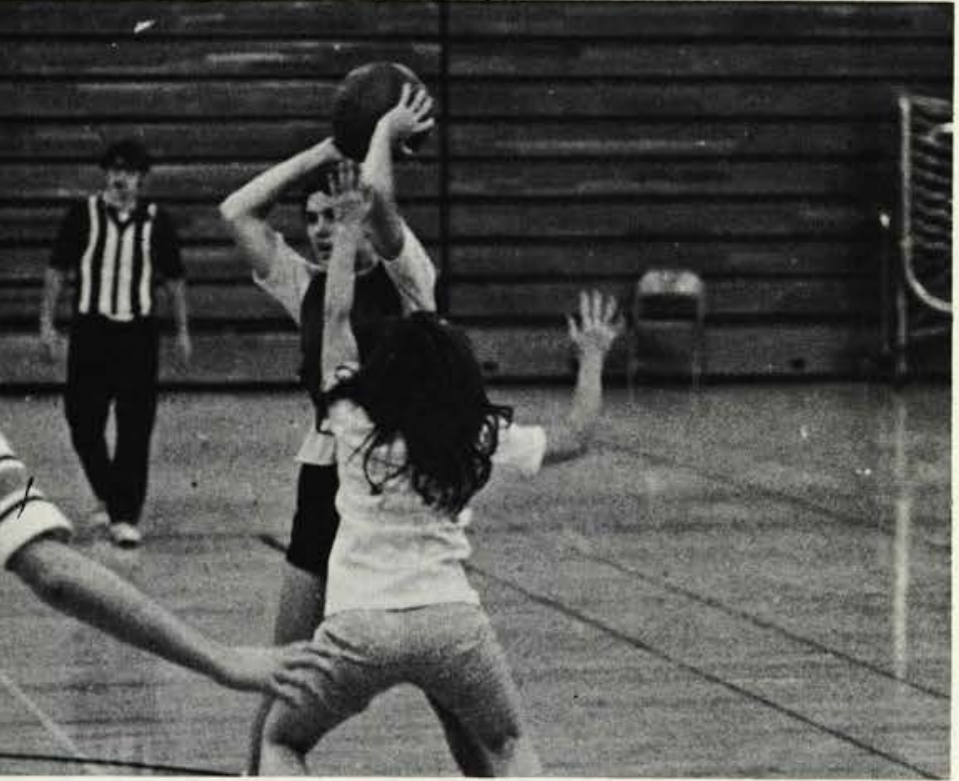




\section{Who's Who} in American Colleges and Universities

Becky Lamb 1. Georgeanna Axiotis 2. Sharon Gazdik 3. Rex Rogers 4. Linden Kirby 5. Peggy Wagner 6. Esther Lightcap 7.

Debra Dear 8. Holly Jenkin 9 . Molly Rehn 10. Janet Long 11.

Darla Jewell 12. Barrett Luketic 13. Jayne Eberling 14.

Not Pictured:

Don Eggleston Richard McGee Nancy Tallmon
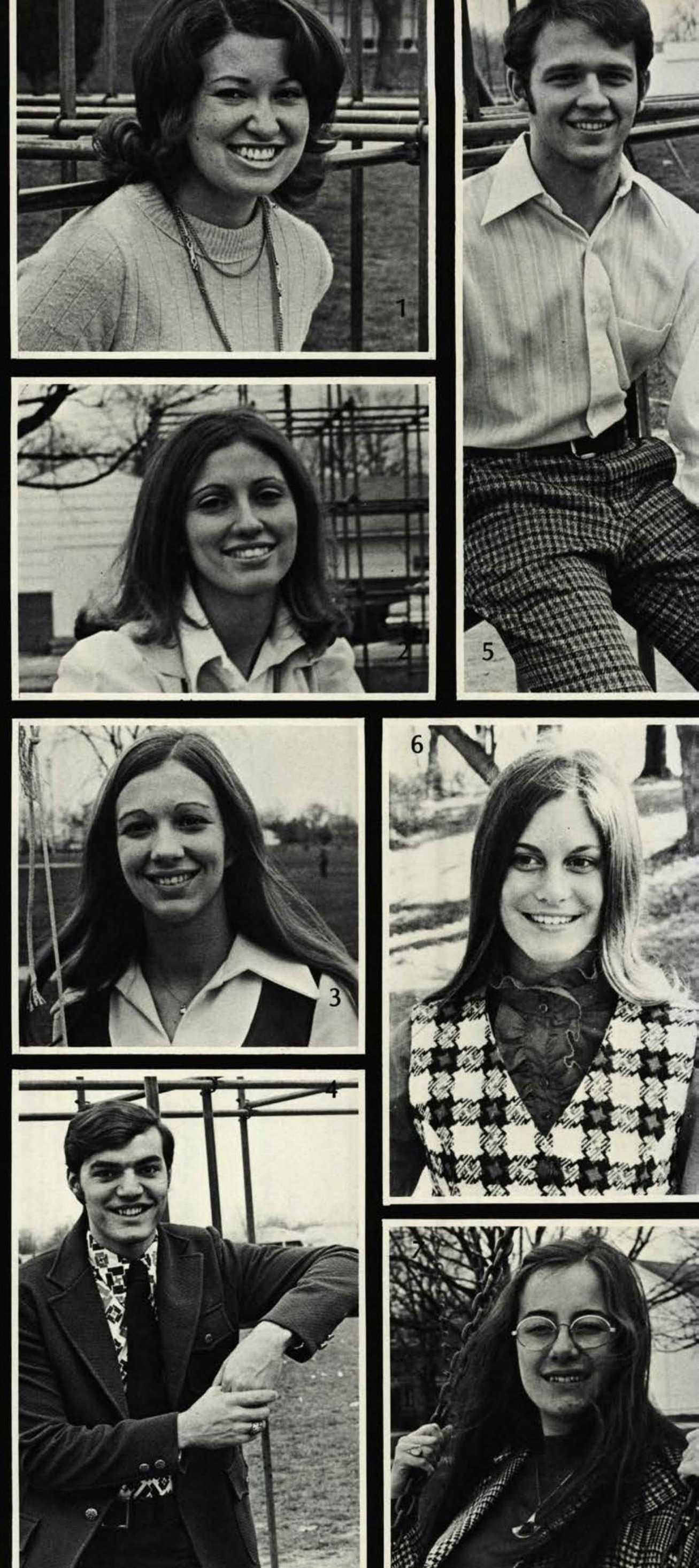


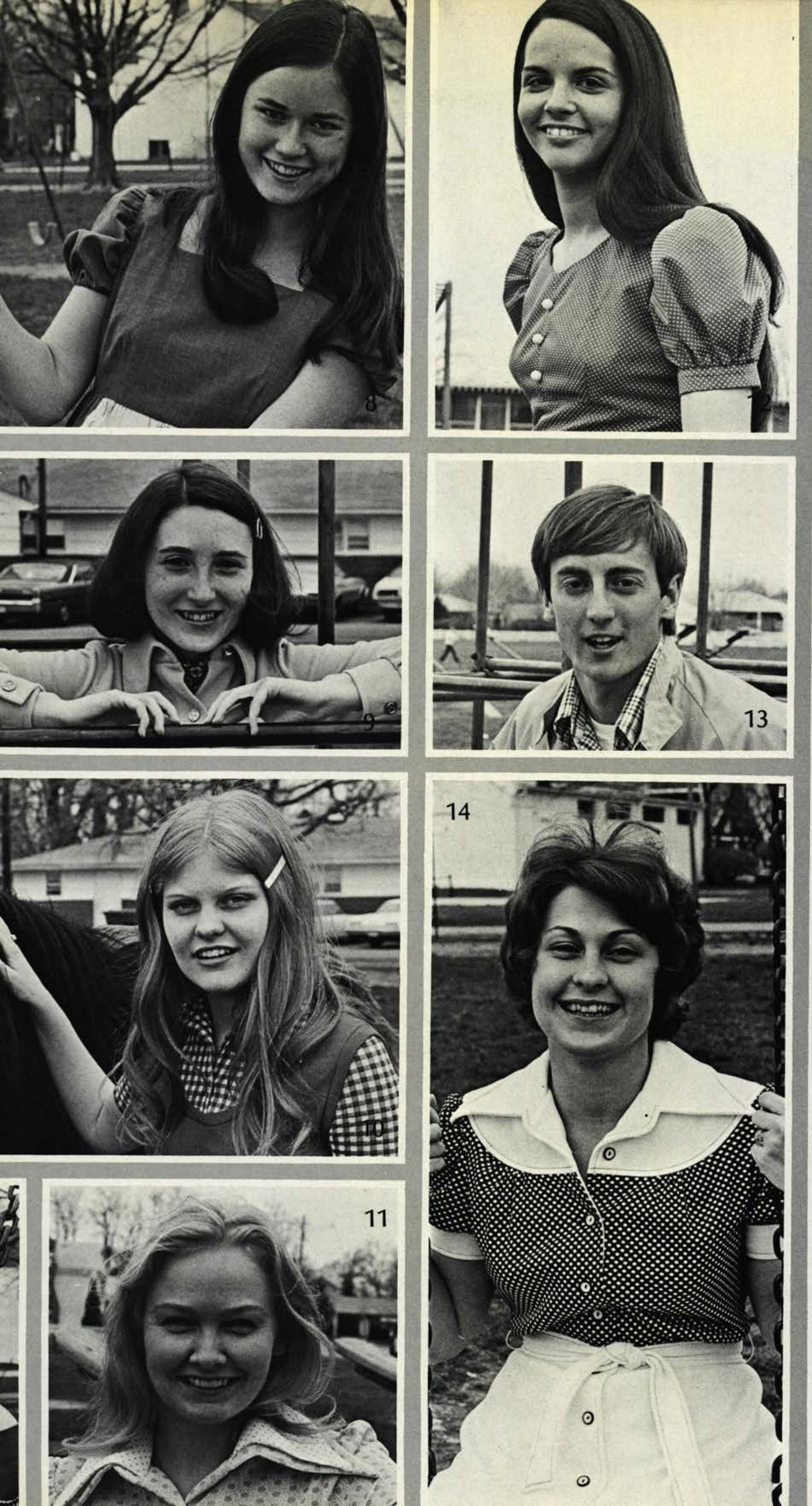



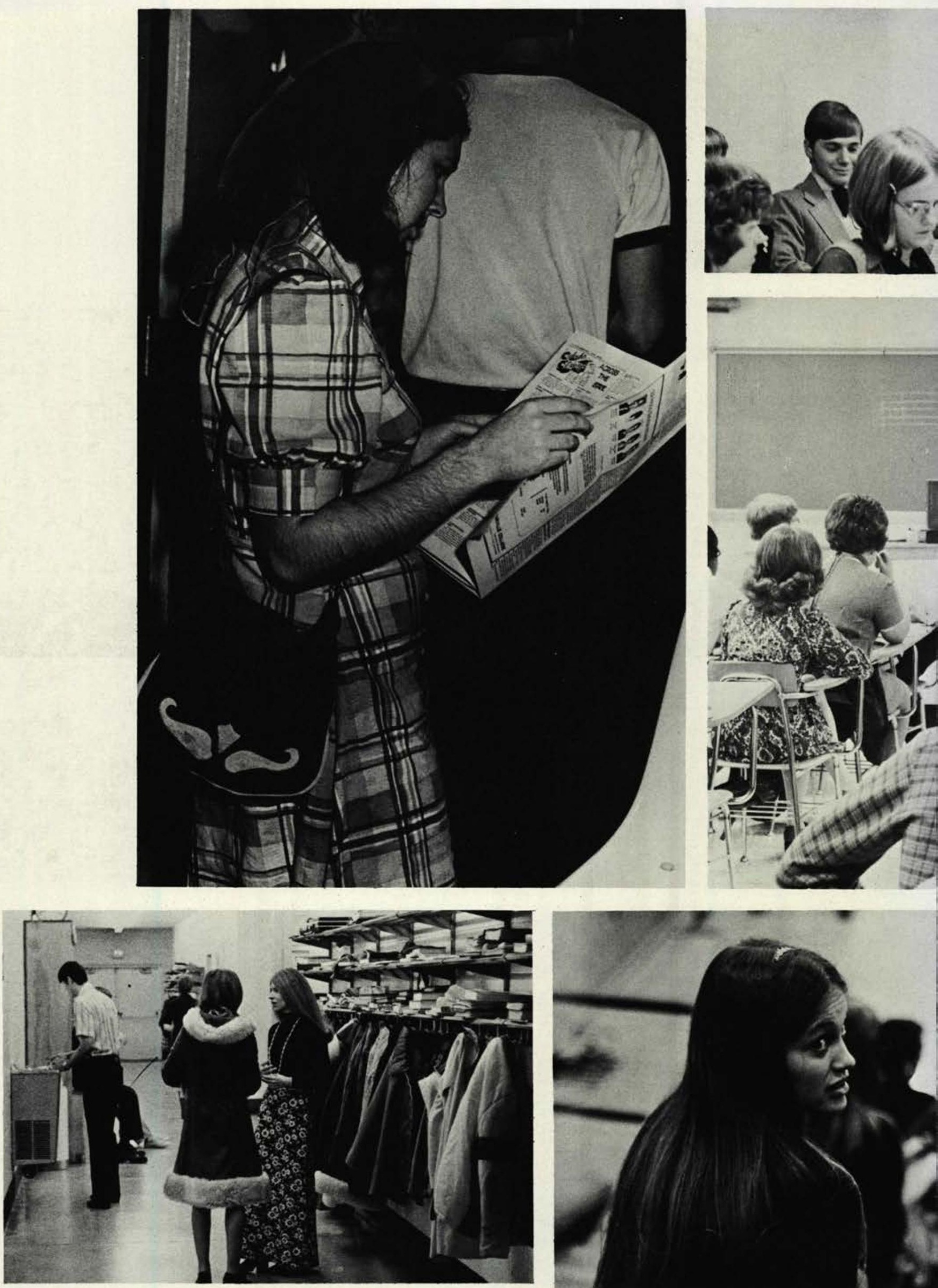

College Life in General ... 


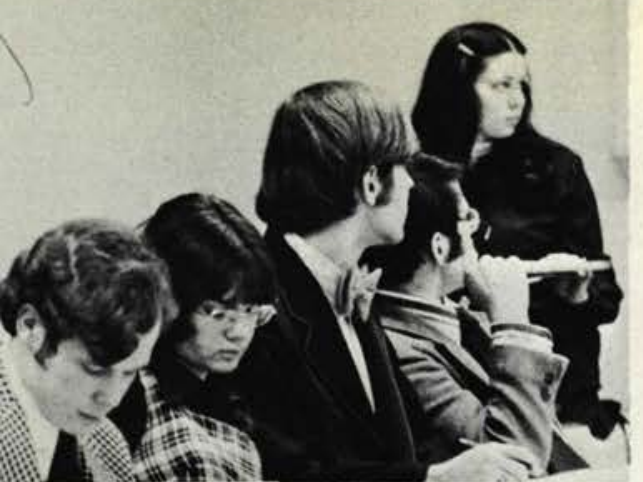

\section{$\Theta$}

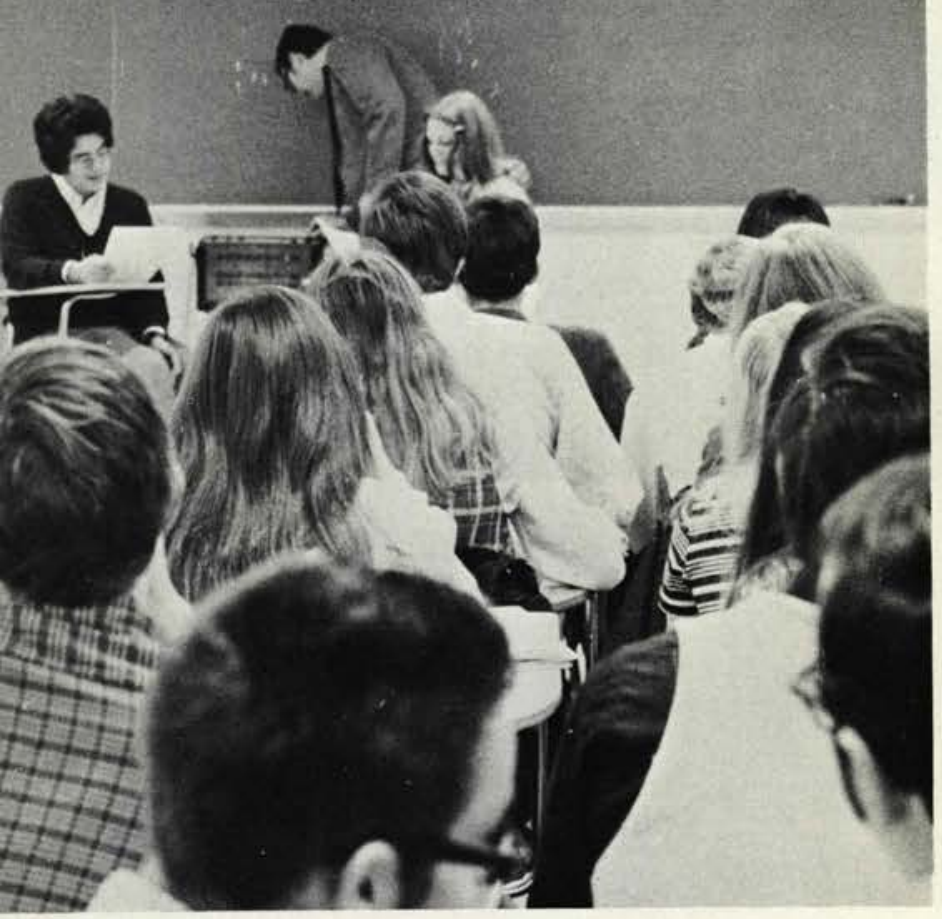

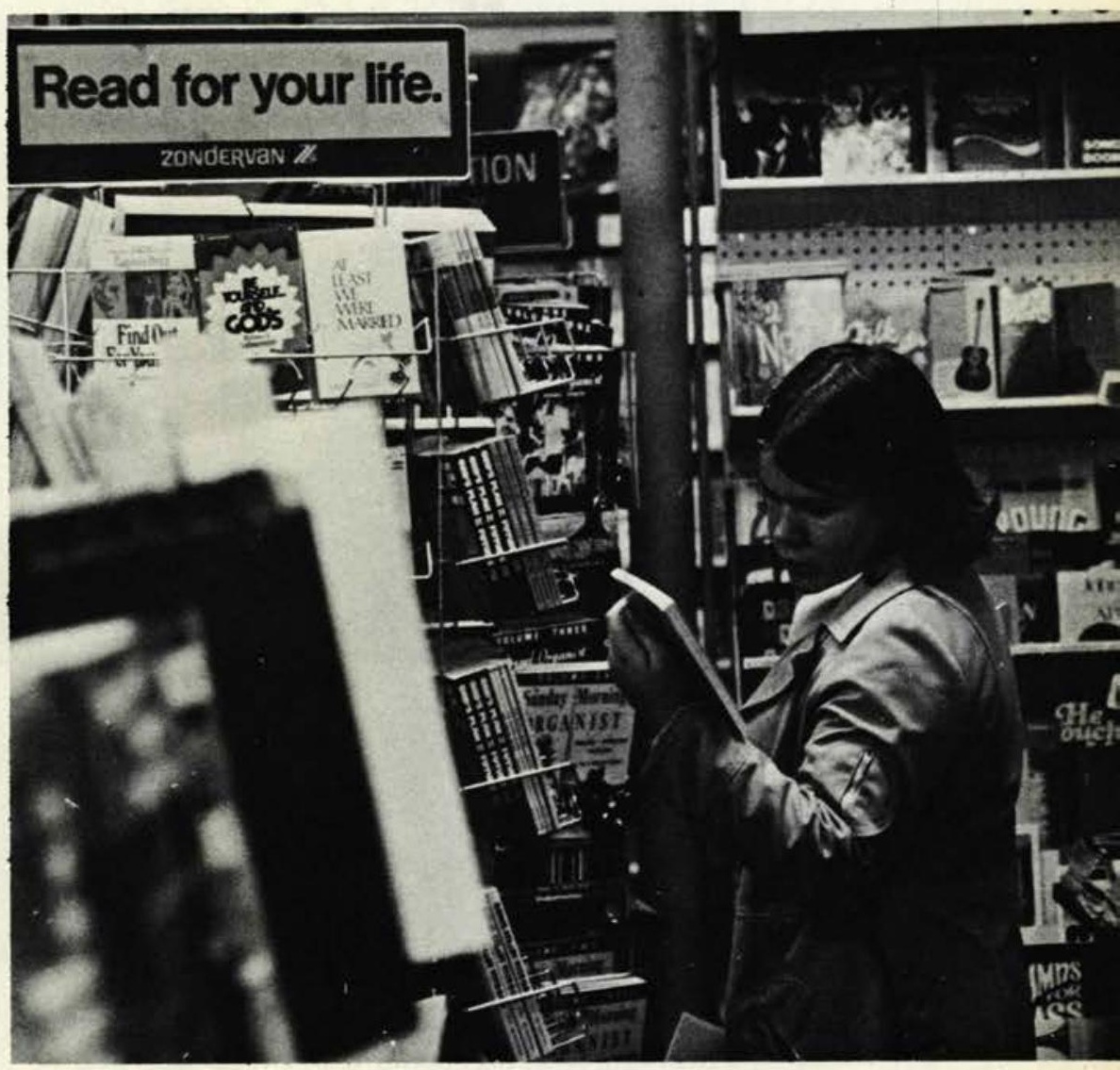

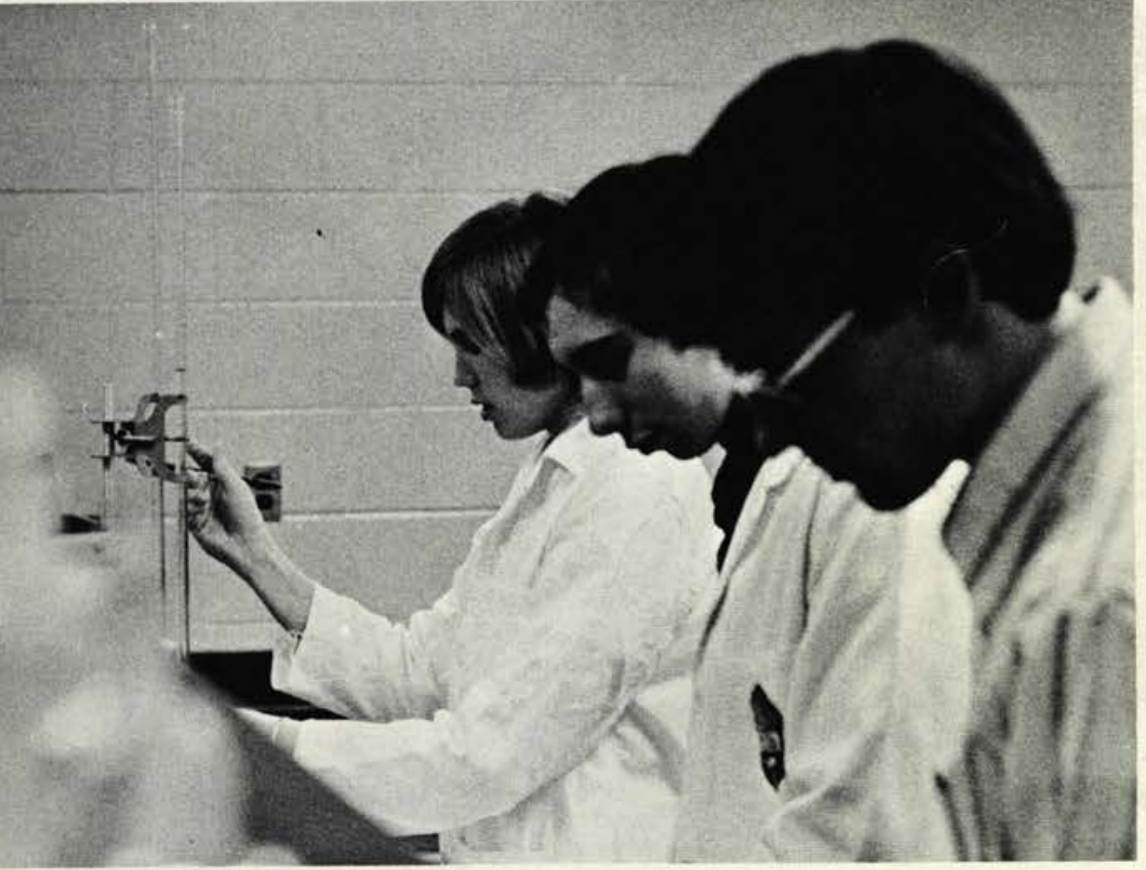




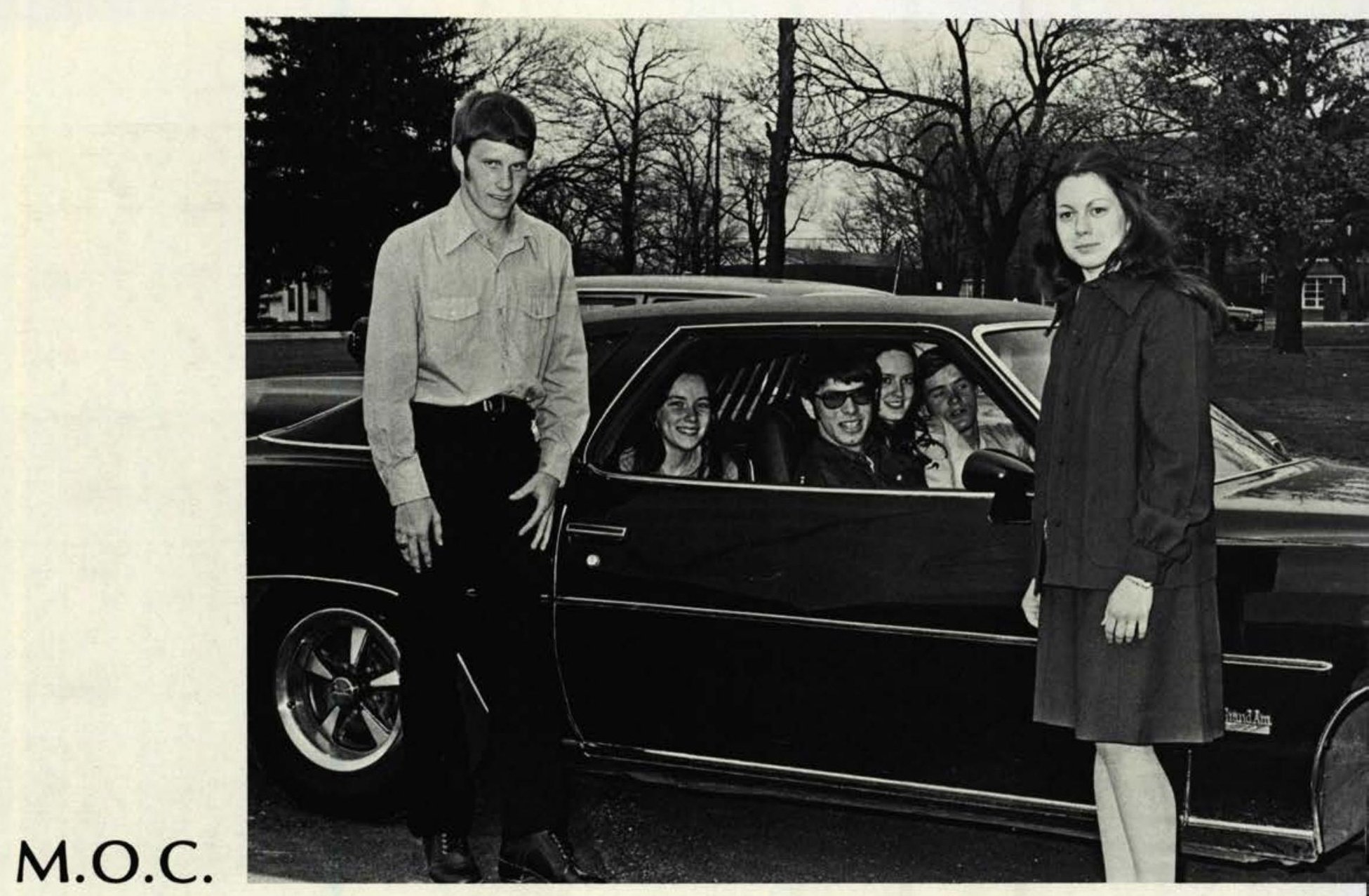

Terry Howdyshell, Cindy Powers, Dan Bowman, Cindi Porter, Brad Cronbaugh, Diane Jacobs.
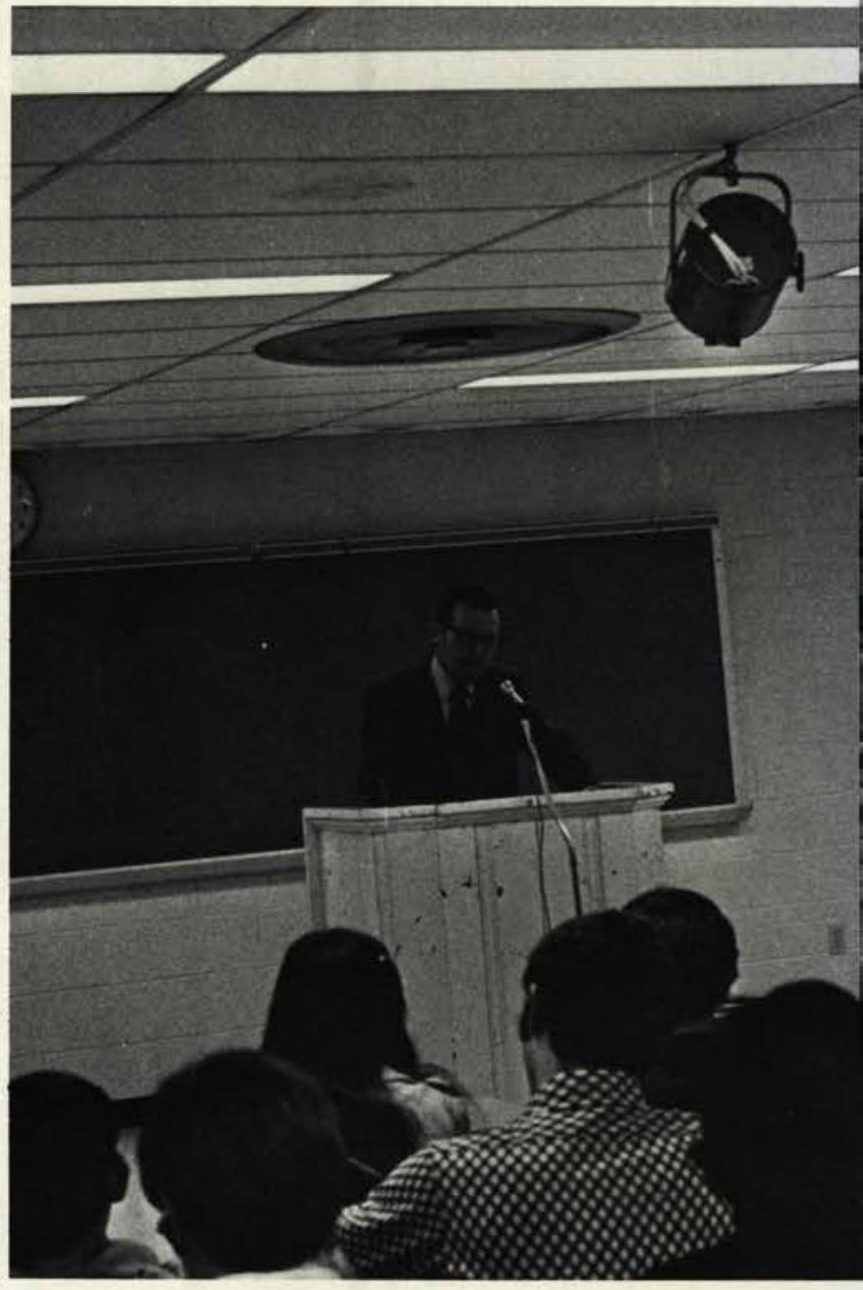
Bill Potter, Dan Steere, Don Yeaton, Esther Lightcap, Karen Chrestensen, Brad Cronbaugh, Ellen Taft, Don Eggleston, Sue Johnson.
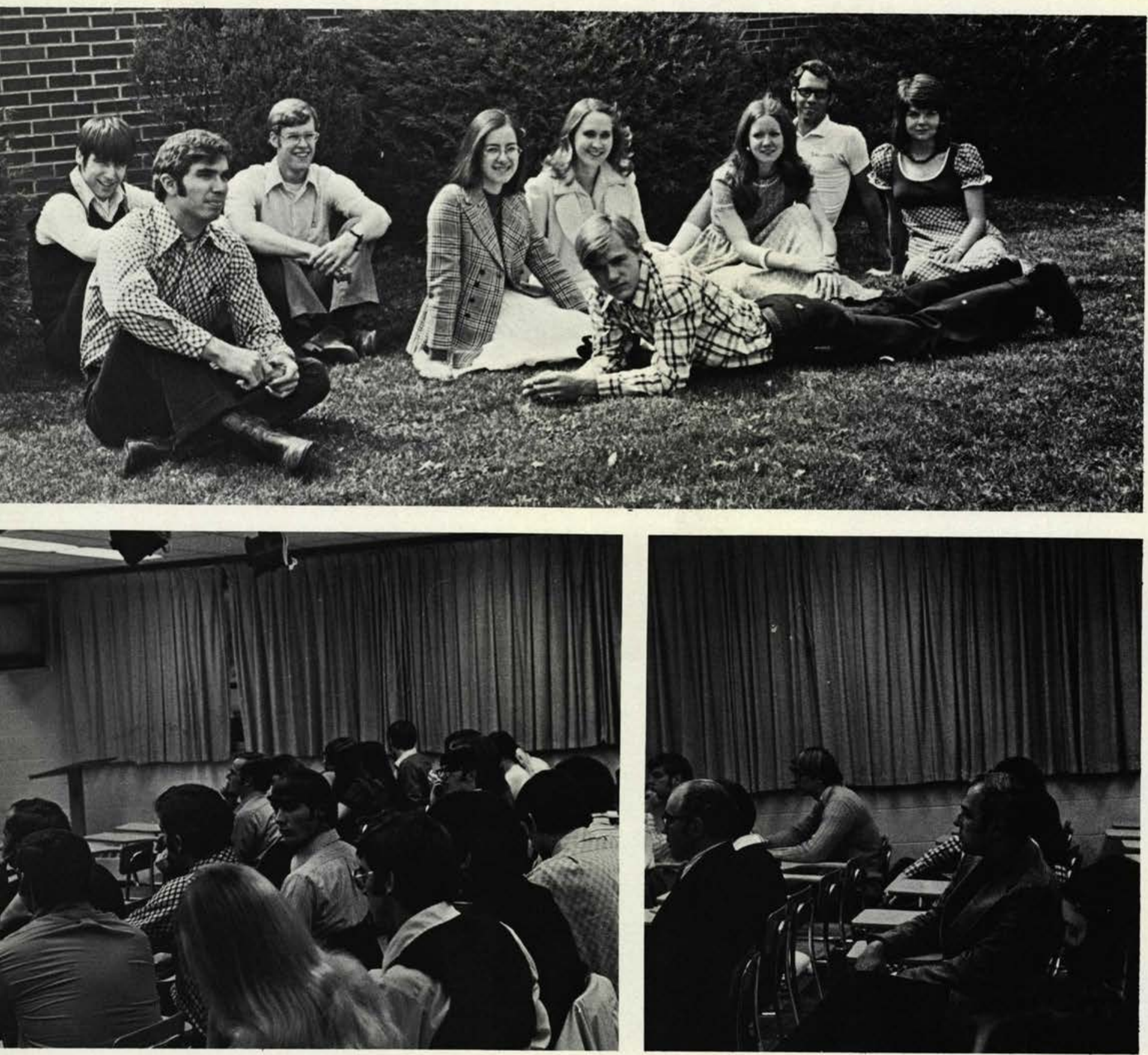

\section{Timalathians}




\section{FAR ENSTERT FAKTASY}

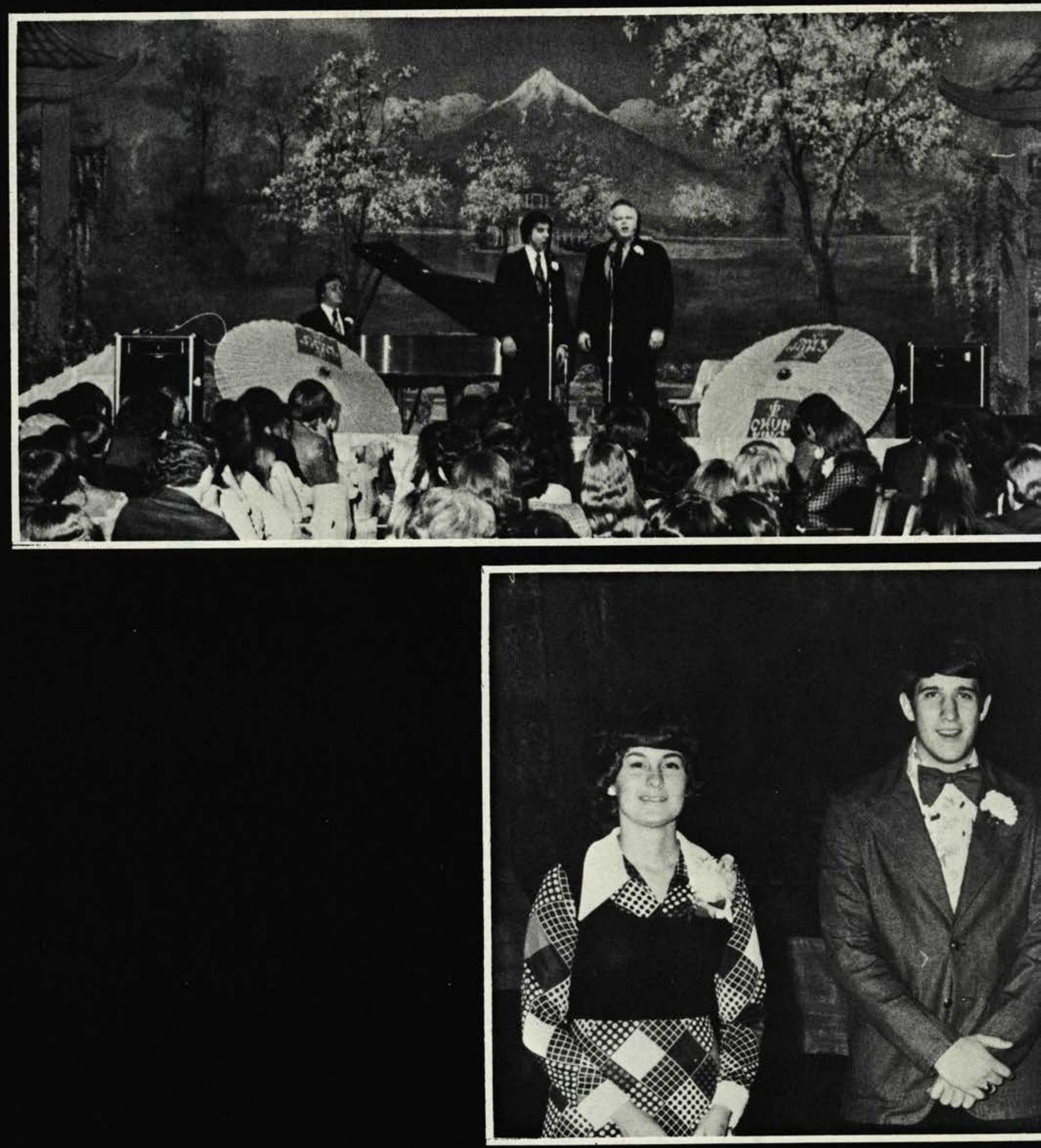



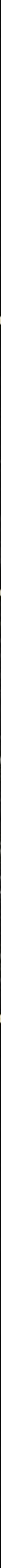


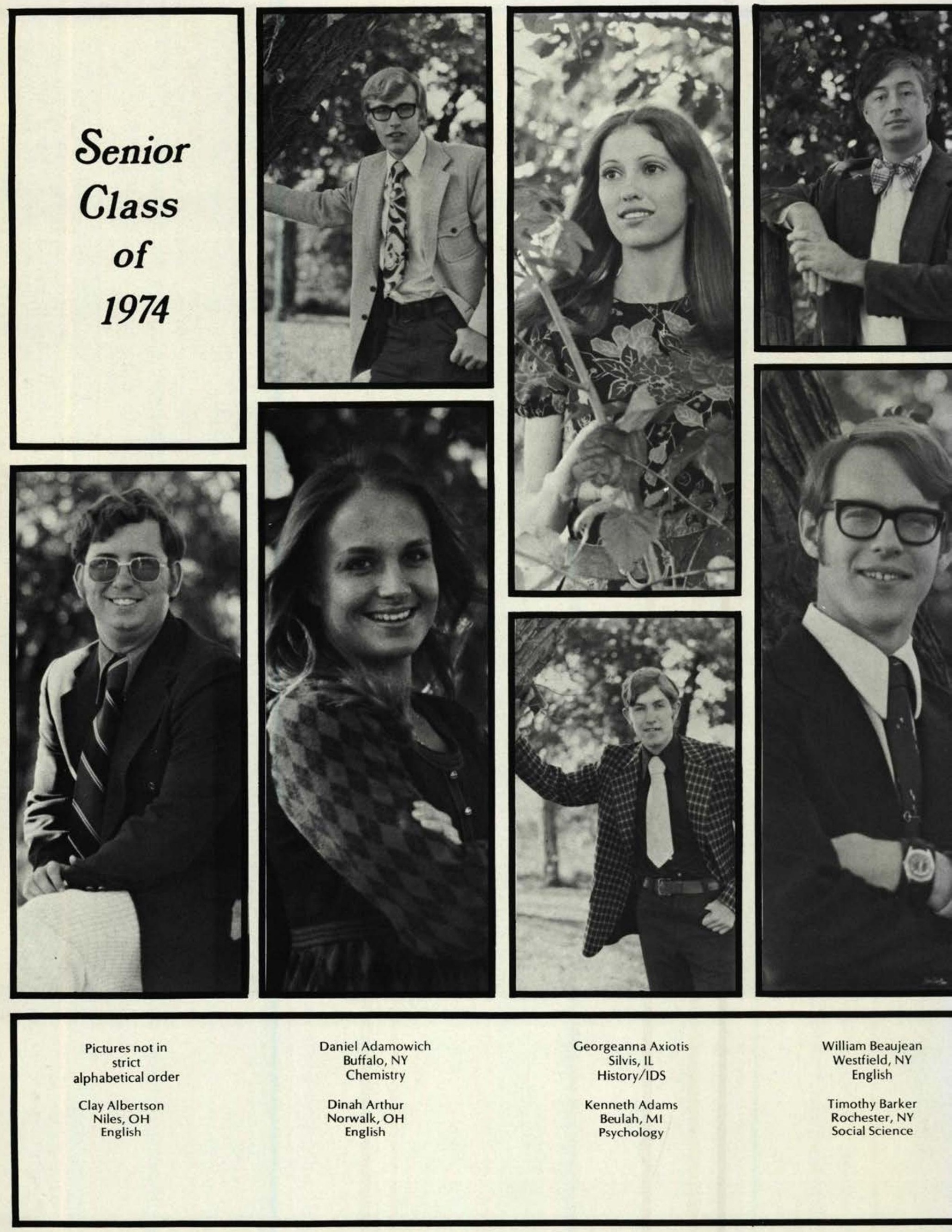



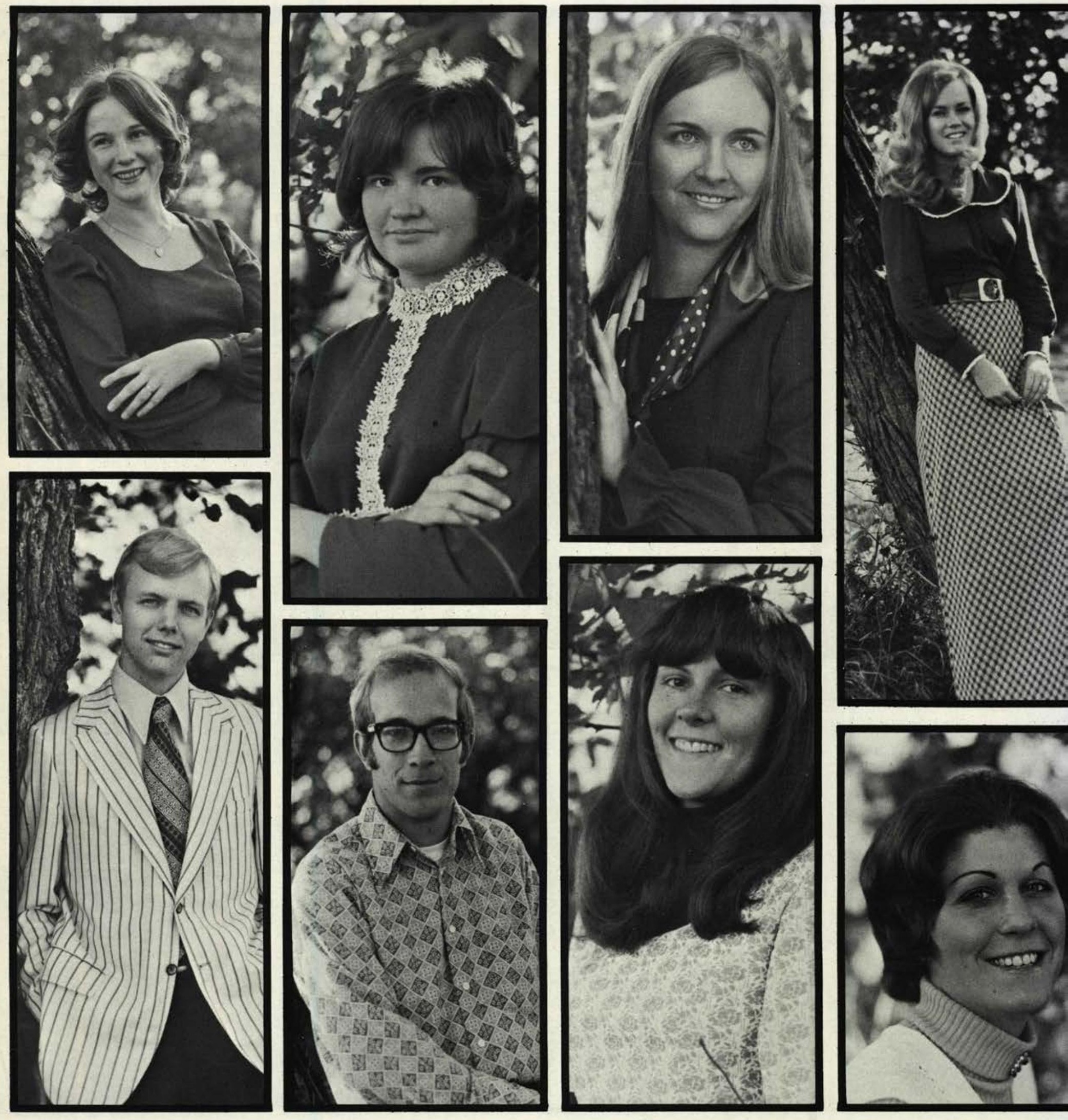

Cheryl Huff Bradford

Rochester, NY

English

Gary Brock

Columbus, $\mathrm{OH}$

Speech
Bonnie Busho

Cedarville, $\mathrm{OH}$

English

Craig Busho

Cedarville, $\mathrm{OH}$

Bible
Debbie'Byers

Boonville, IN

Bible

Joleen Byers

Pella, LA

Elementary Education
Kathy Burkhard

Ft. Lauderdale, FL

Music

Barbara Campbell

Absecon, NJ

Psychology 

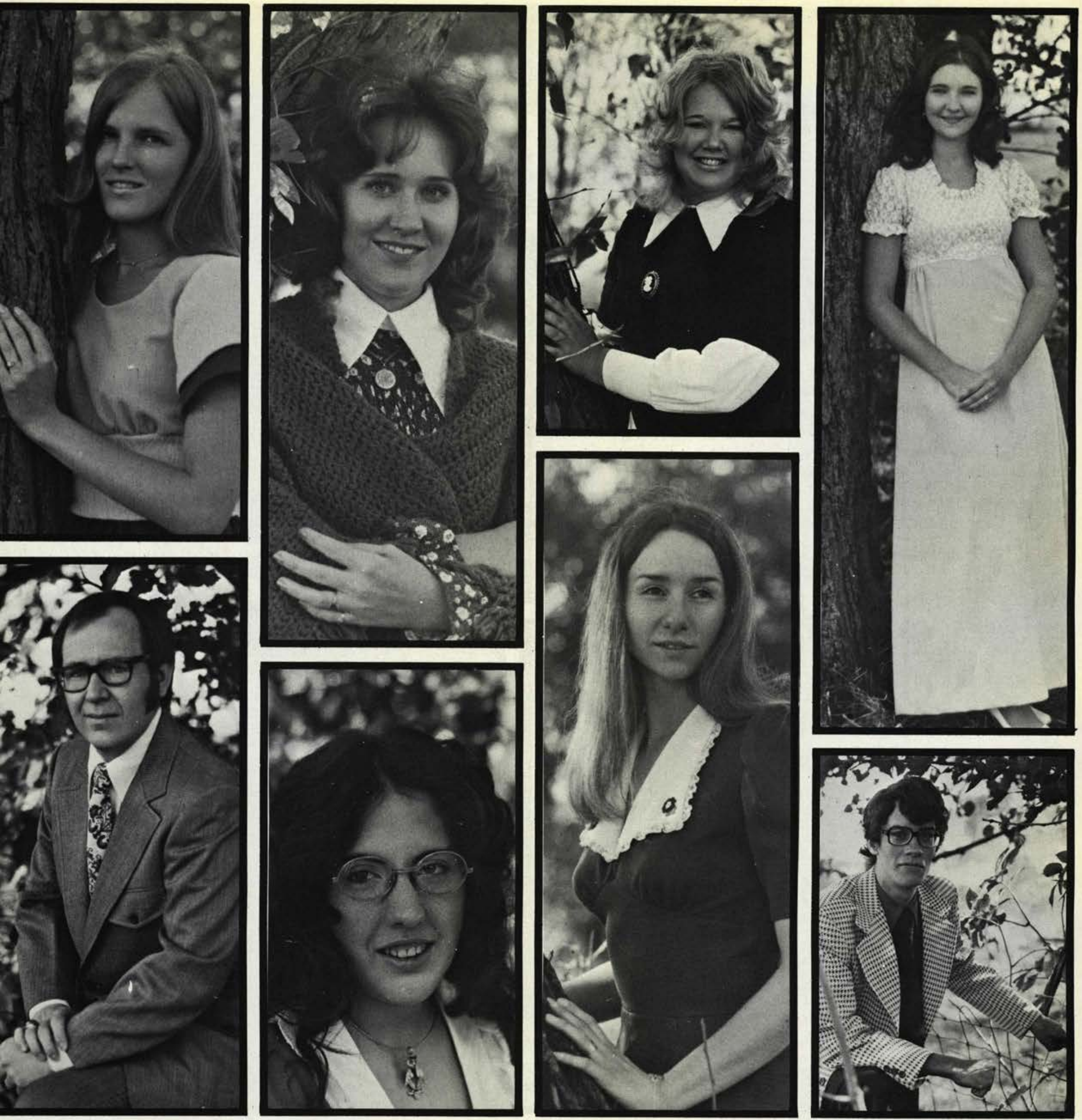

Ellen Collison

Cedarville, $\mathrm{OH}$

Elementary Education

Kenneth Carlton

Harvey, IL

Elementary Education
Karen Chrestensen

Berea, $\mathrm{OH}$

Biblical Education

Jackie Cline

Rockbridge, $\mathrm{OH}$

Biology
Diane Craig

Flushing, MI

Physical Education

Nancy Cleckner
Salem, $\mathrm{OH}$

Music Education
Shirley Carpenter

Springfield, $\mathrm{OH}$

Elementary Education/

Bookkeeping

David Cotnoir

St. Theresa, Quebec

Social Science 

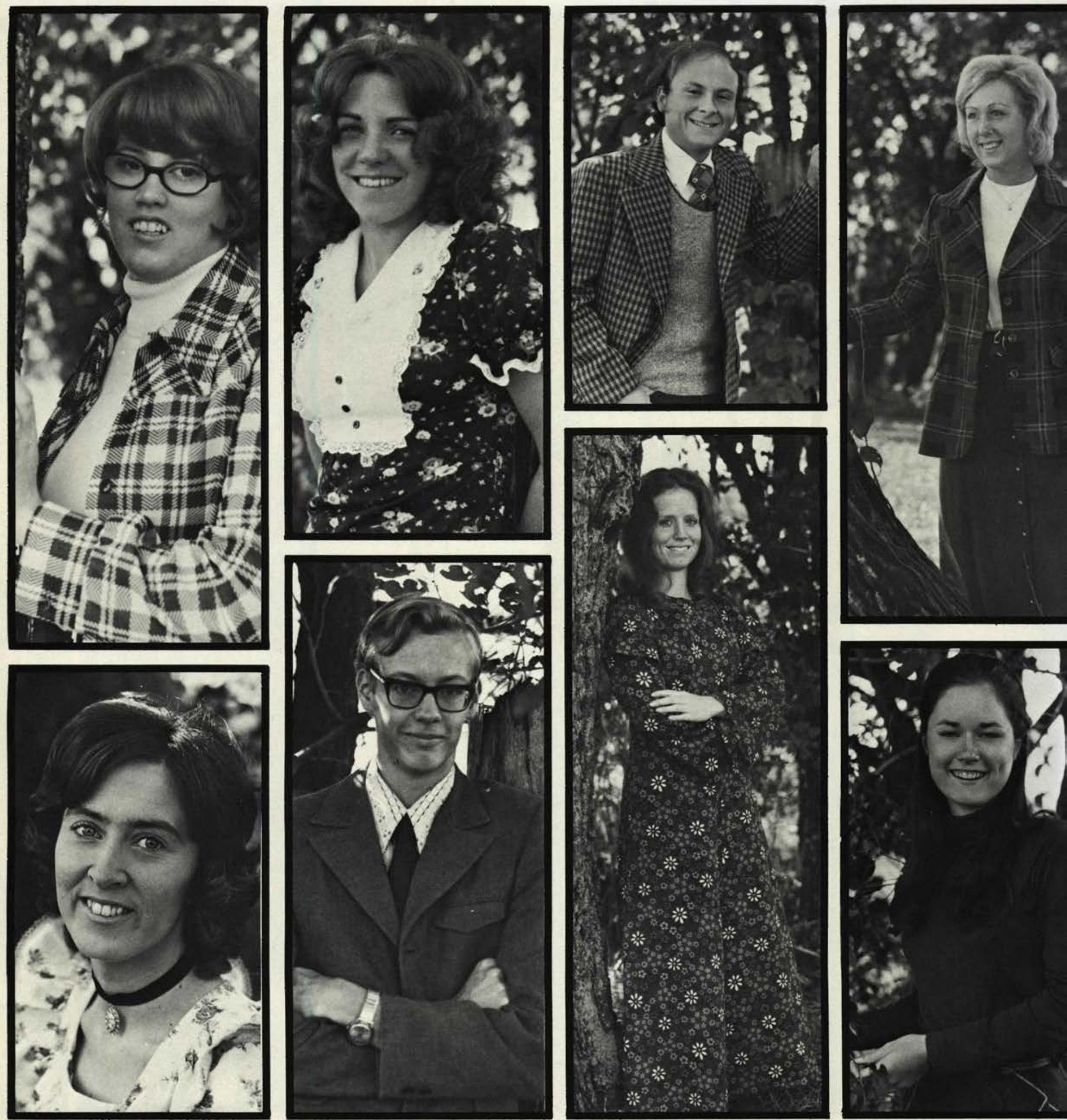

Bertha Carter

Connecticut, $\mathrm{OH}$

Social Science

Barbara Cooper

N. Tonnawanda, NY

Bible
Sherrill Cressman

Delphos, $\mathrm{OH}$

Physical Education

Douglas DeLand

Dresden, NY

Math
Eric Cuenin

Amhurst, $\mathrm{OH}$

Physical Education

Joyce DeBruine

Battle Creek, MI

Bible
Kathy Dalton

Wakeman, $\mathrm{OH}$

Elementary Education

Debra Dear

Gap, PA

Elementary Education 

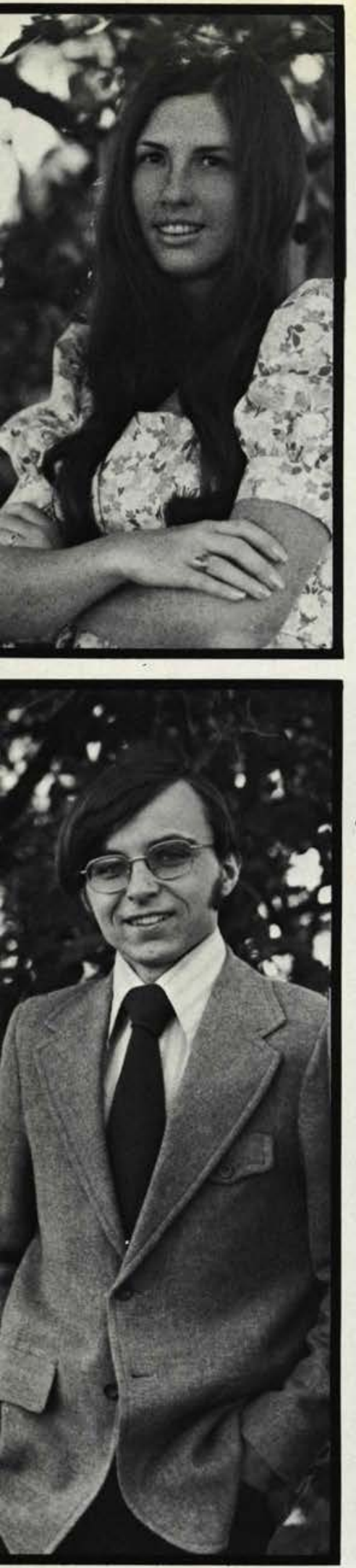
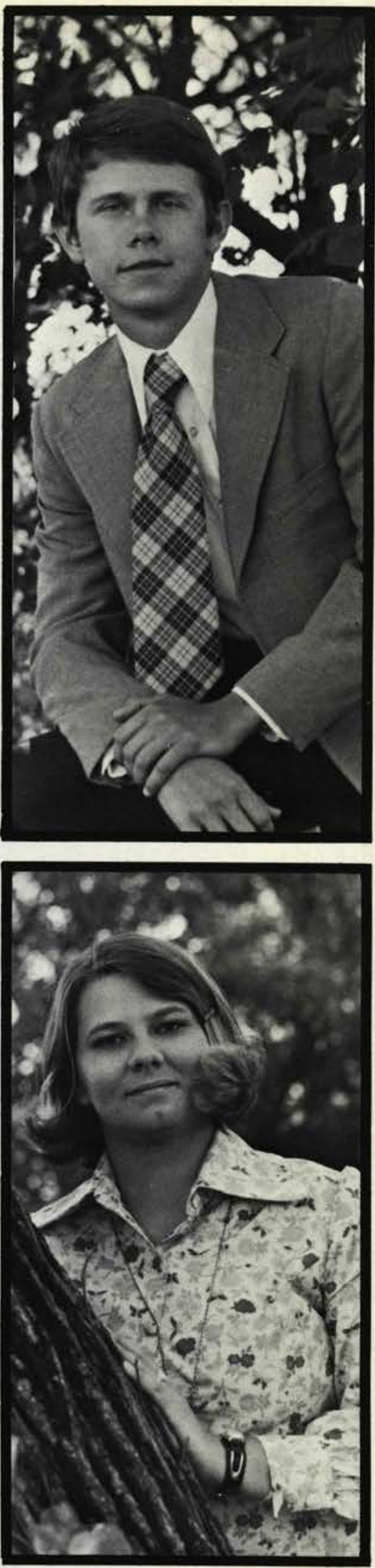
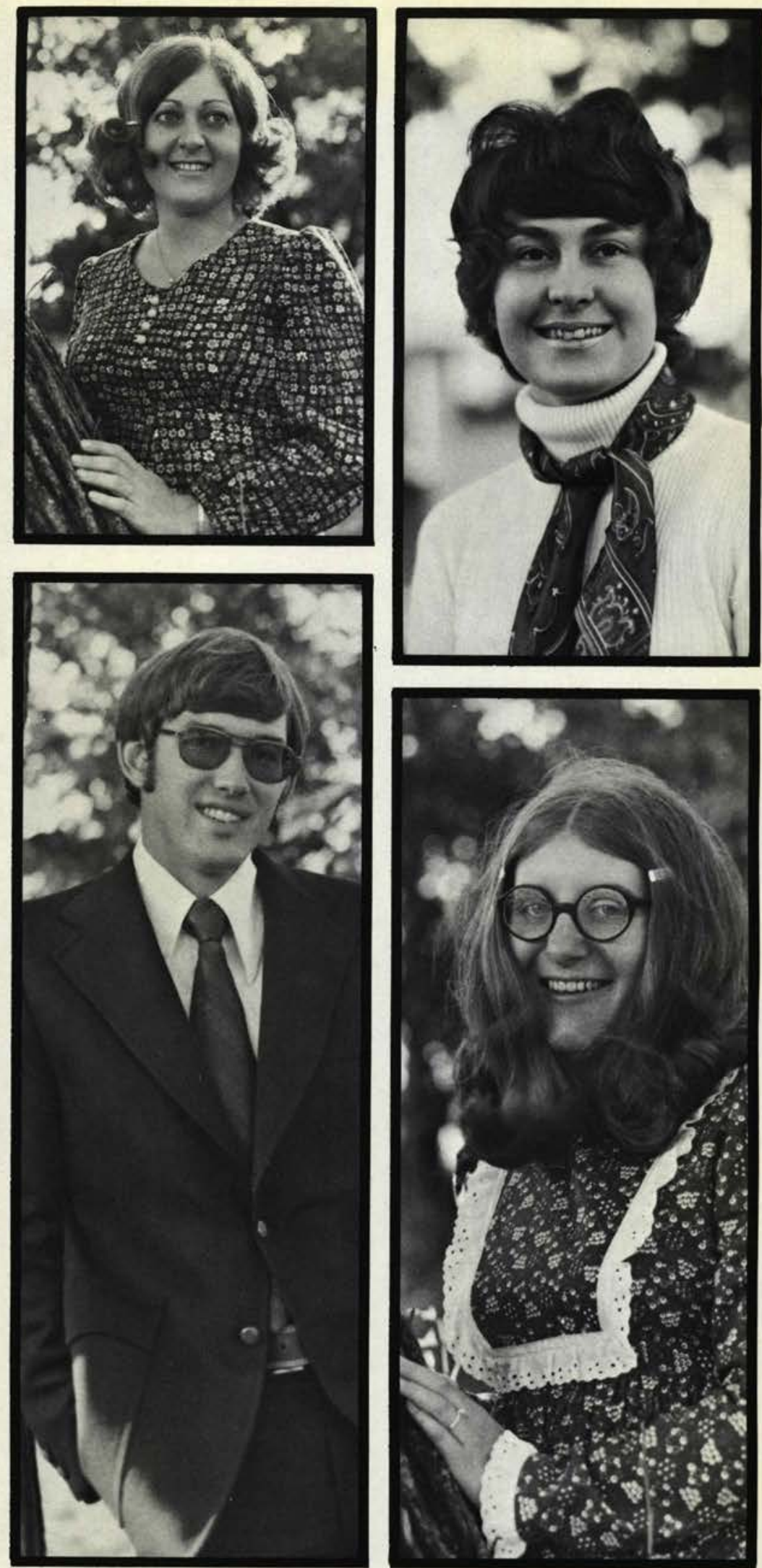

Carol Demo

Dublin, $\mathrm{OH}$

Bible

Harry Dilcher

Elba, NY

Biology/Chemistry
Charles Dolph

Bellefontaine, $\mathrm{OH}$

Psychology

Diana Douglass

Ava, NY

Bible
Denise Edwards

Albion, IA

Physical Education

Daniel Ehnis

Brooklyn, MI

Psychology
Jayne Eberling

Springfield, $\mathrm{OH}$

English

Ruth Emerson

Prattsburg, NY

Business Administration 

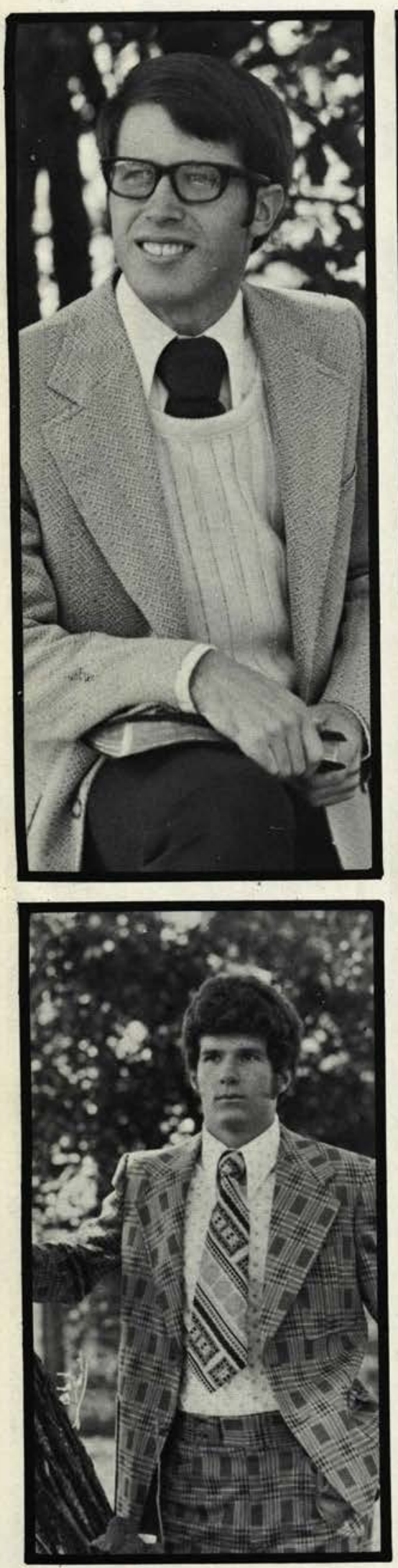
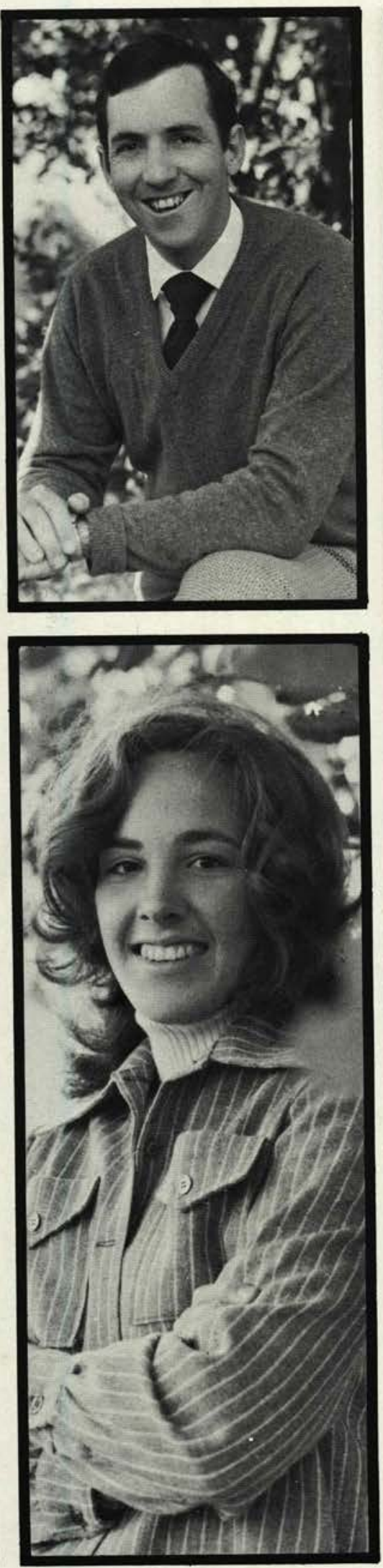
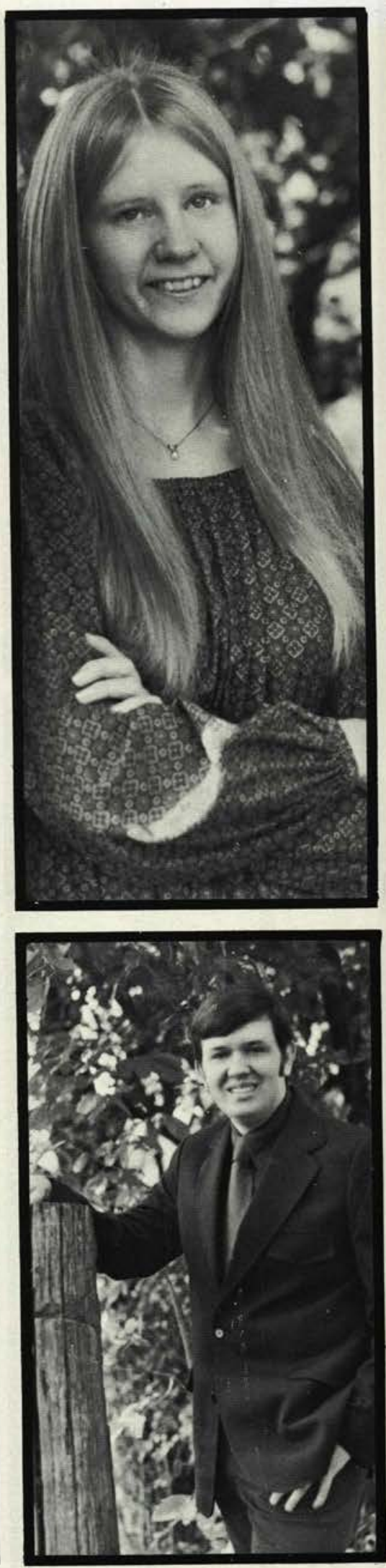

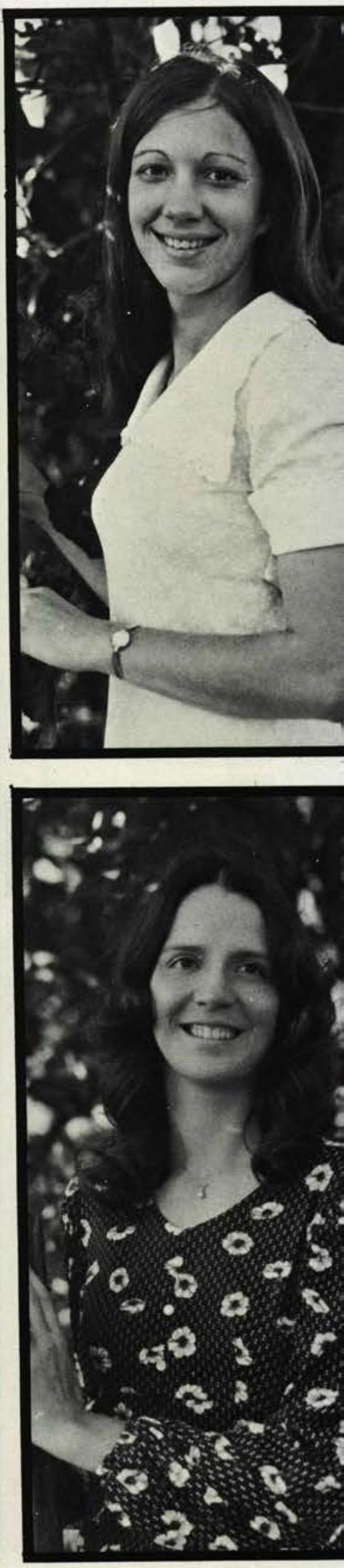

Daniel Estes Endicott, NY Pre-Sem./Eng.

Steve Francis Kansas City, KS Business
Donald Gates

Dayton, $\mathrm{OH}$

Pre-Seminary

Marianne Frauenknecht

Morrow, $\mathrm{OH}$

Physical Education
Carolyn Goodlande Riverside; IA

Elementary Education

Ted Gifford
West Union, OH
Speech/English

Speech/English

Sharon Gazdik Shawinigan-Sud, Quebec Math

Sarah Griffeth

Cedarville, $\mathrm{OH}$

Elementary Education 

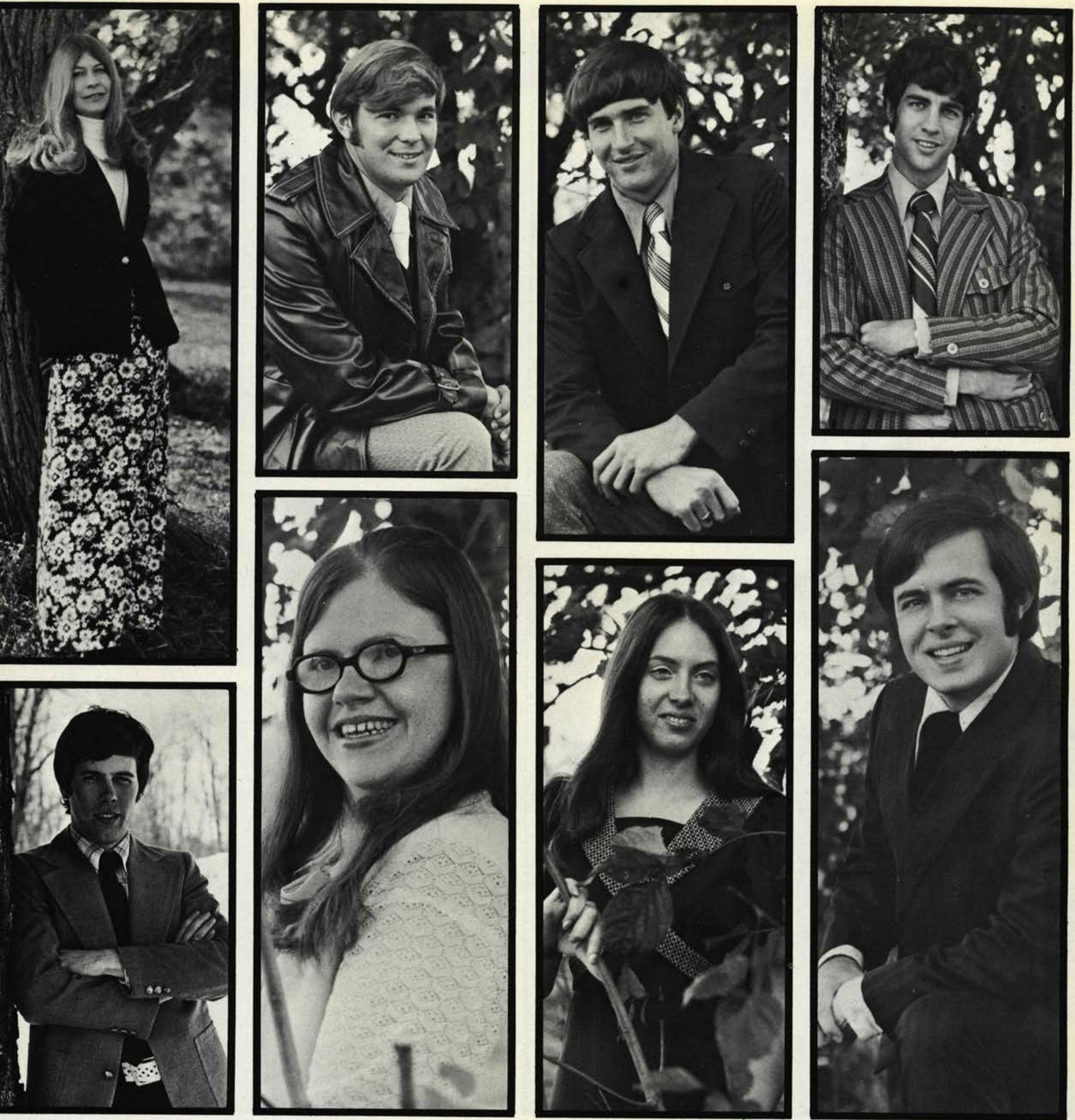

Suzanne Hale

East Alton, II

Music

Steve Gruber

Cedarville, $\mathrm{OH}$

Social Science
Jerold Griswold

Covington, IN

Math

Jackie Hargis

Wakeman, $\mathrm{OH}$

Business Adm.
Rod Hansen

Mt. Pleasant, MI

Physical Education

Sherlyn Hansen

Mt. Pleasant, MI Math
Terry Holley

Twinsburg, $\mathrm{OH}$

Bible

Don Harmon

Cedarville, $\mathrm{OH}$

Speech 

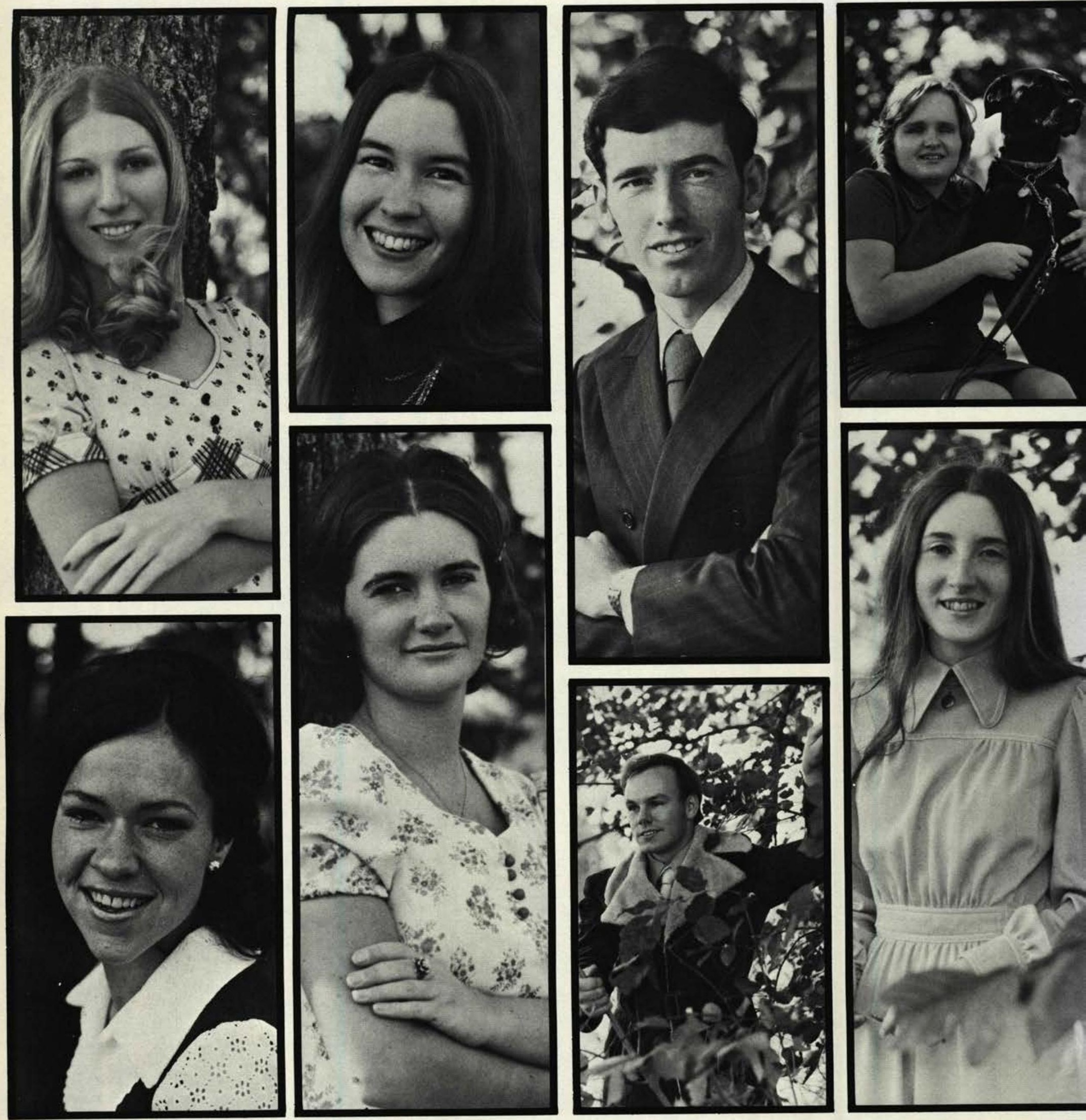

Debbie Hostetler

Smithville, $\mathrm{OH}$

Springfield, $\mathrm{OH}$

Elementary Education

Kathy Howell

Garrettsville, $\mathrm{OH}$

Music
Elementary Education

Debbie Holt

Mentor, $\mathrm{OH}$

Elementary Education
Clyde Hughes

South Charleston, $\mathrm{OH}$

Bible

Doug Hudson

Springfield, $\mathrm{OH}$

Psychology
April Jenkins Springfield, $\mathrm{OH}$

Psychology

Holly Jenkin

Lyndhurst, $\mathrm{OH}$

English 

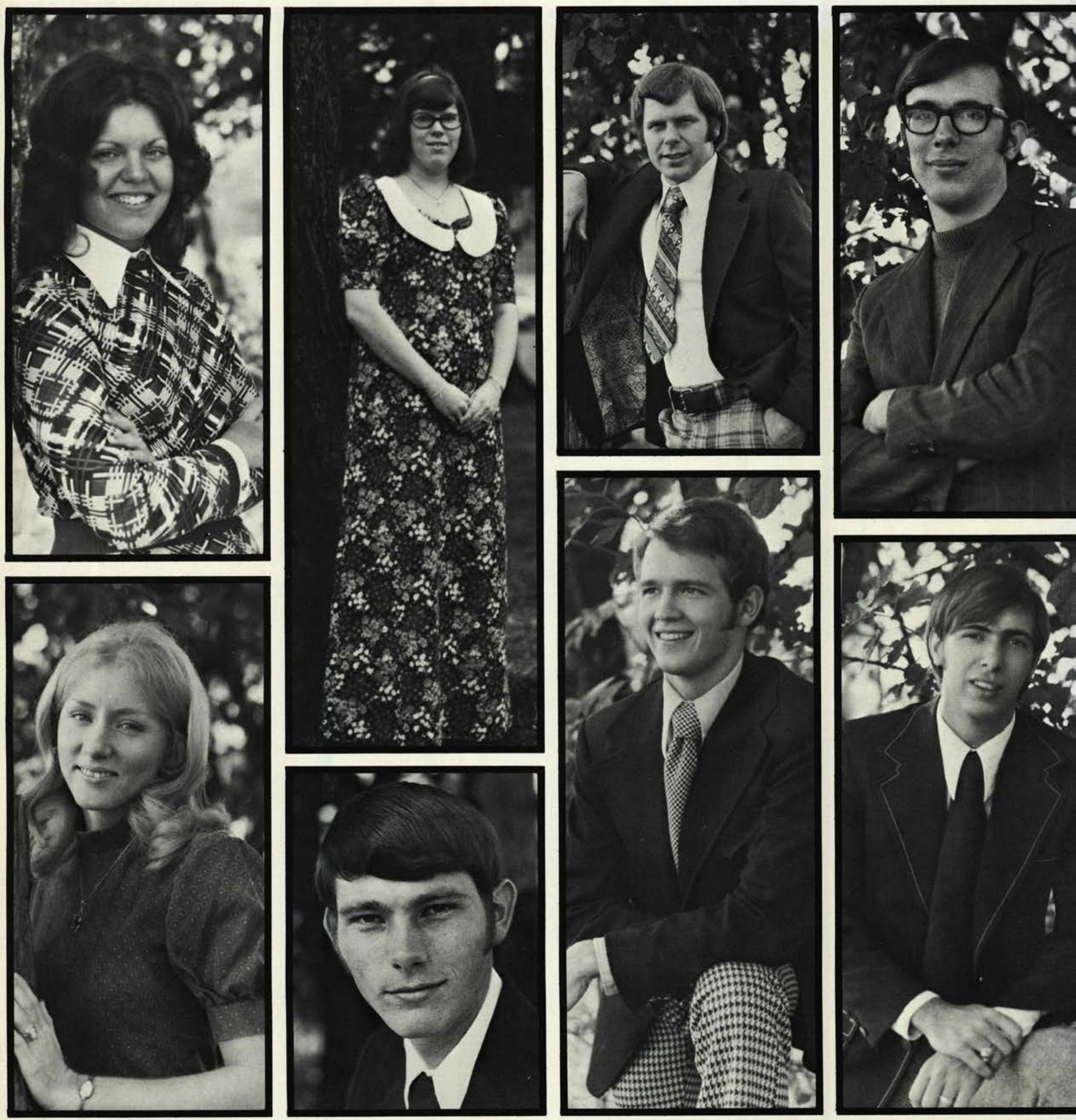

Becky Kemble

Elyria, $\mathrm{OH}$

Psychology

Karen Kennedy

Cornillus, NY

Biology
Nanci Layne

Gallopolis, $\mathrm{OH}$

Elementary Education

Bruce Klett

Boise, ID

Pre-Seminary
Jerry Kinniburgh

Marion, MT

Business Adm.

Linden Kirby

Prospect, $\mathrm{OH}$

IDS
Jeff Kistler

Hadden Heights, N

Pre-Seminary

Tim Lee

Trenton, NJ

Physical Education 

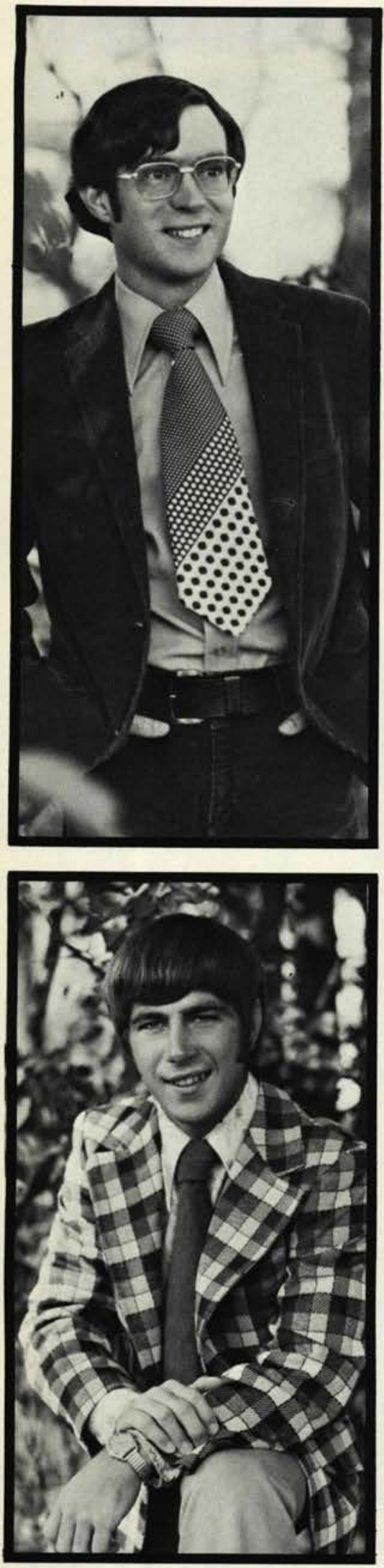
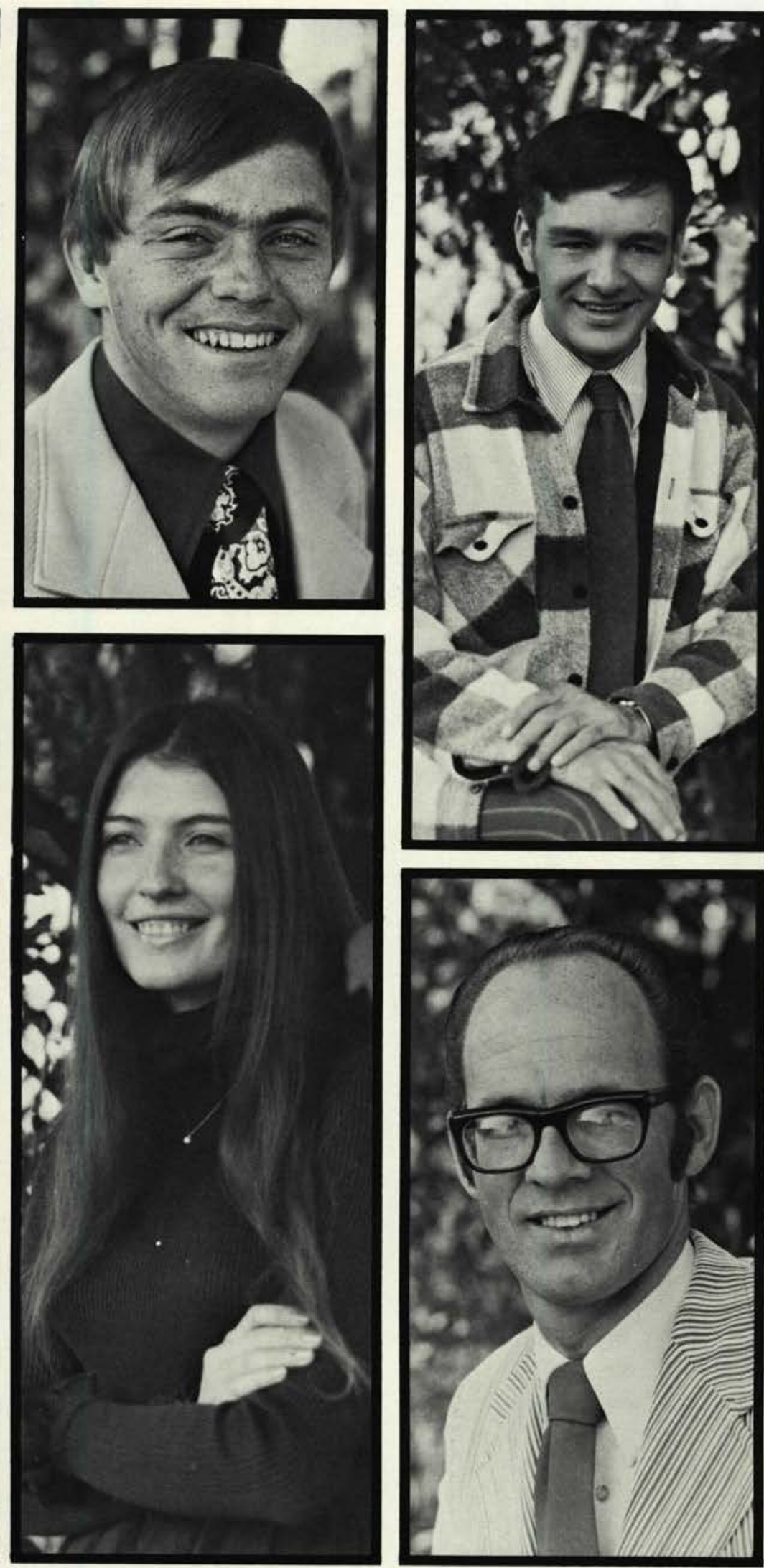
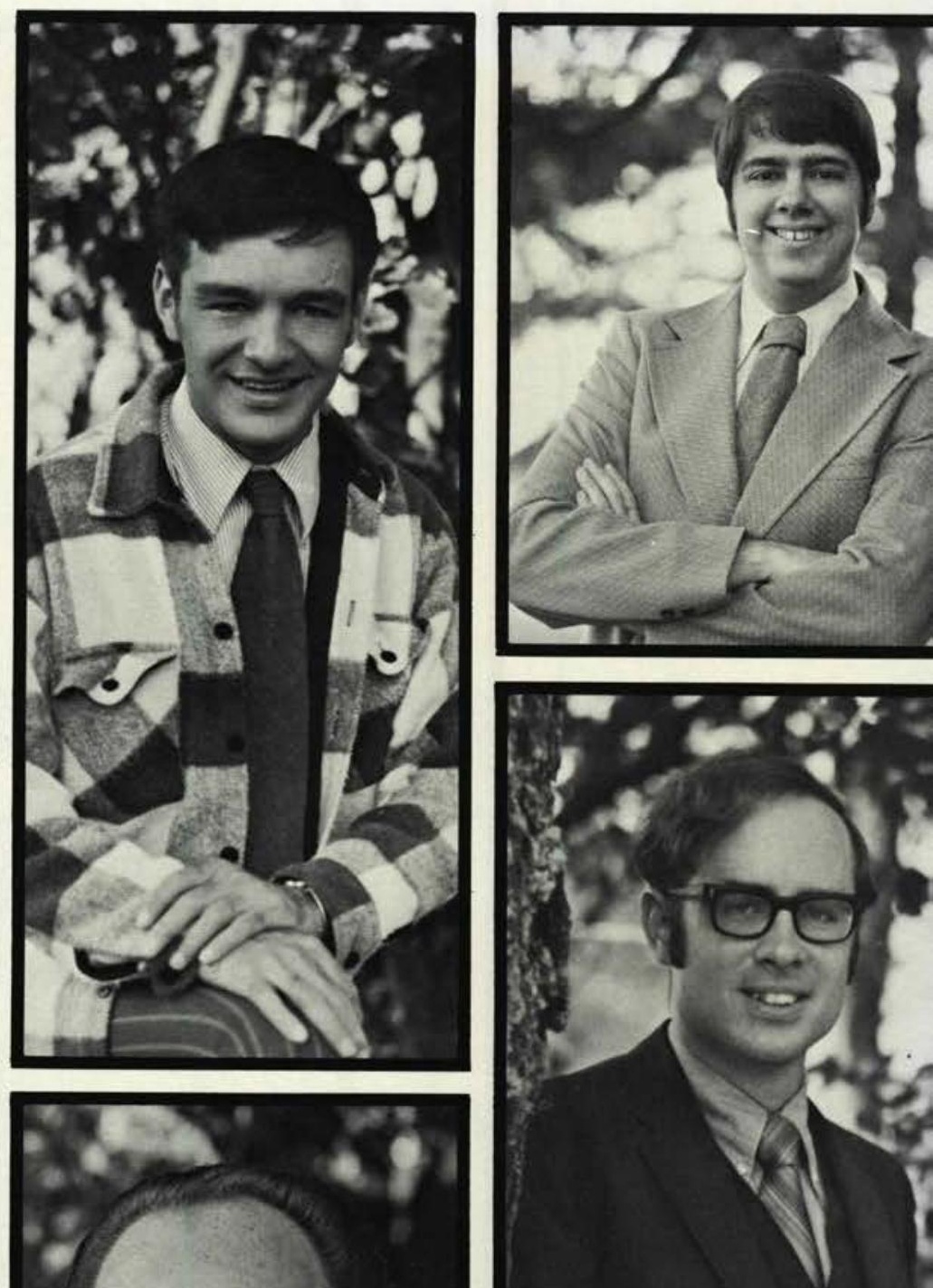

Don McKanna

Fort Jennings, $\mathrm{OH}$ Elementary Education

\section{Steve McCallister \\ Columbus, $\mathrm{OH}$} Elementary Education
Phil McMillan Washington, D.C. Bible

Diana McCarty

Cavfield, $\mathrm{OH}$

Psychology
Bill McCormick

Cedarville, $\mathrm{OH}$ Math

Chris Meenach

Cedarville, $\mathrm{OH}$

Elementary Education
Rick McIntosh

Cedarville

Broadcasting/Speech

Richard Meloy

Oradell, NJ

Business Adm. 

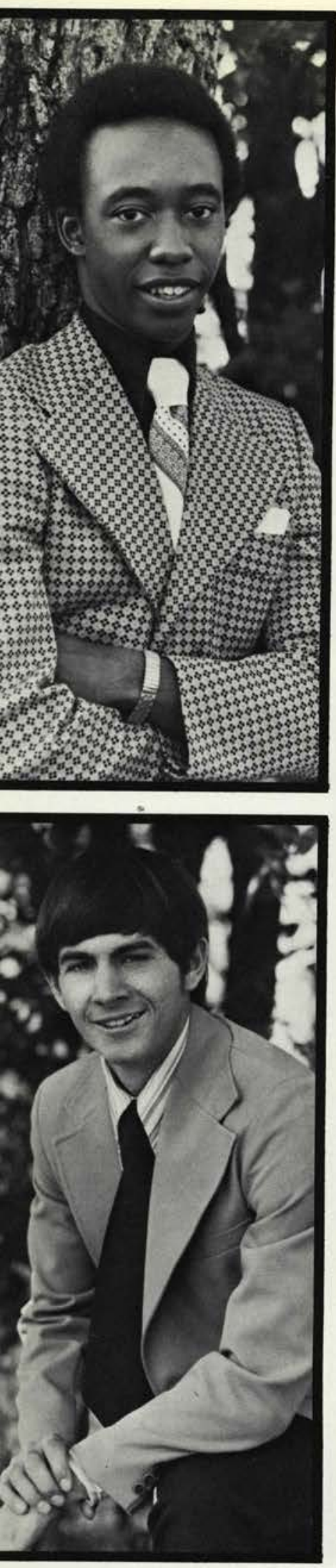
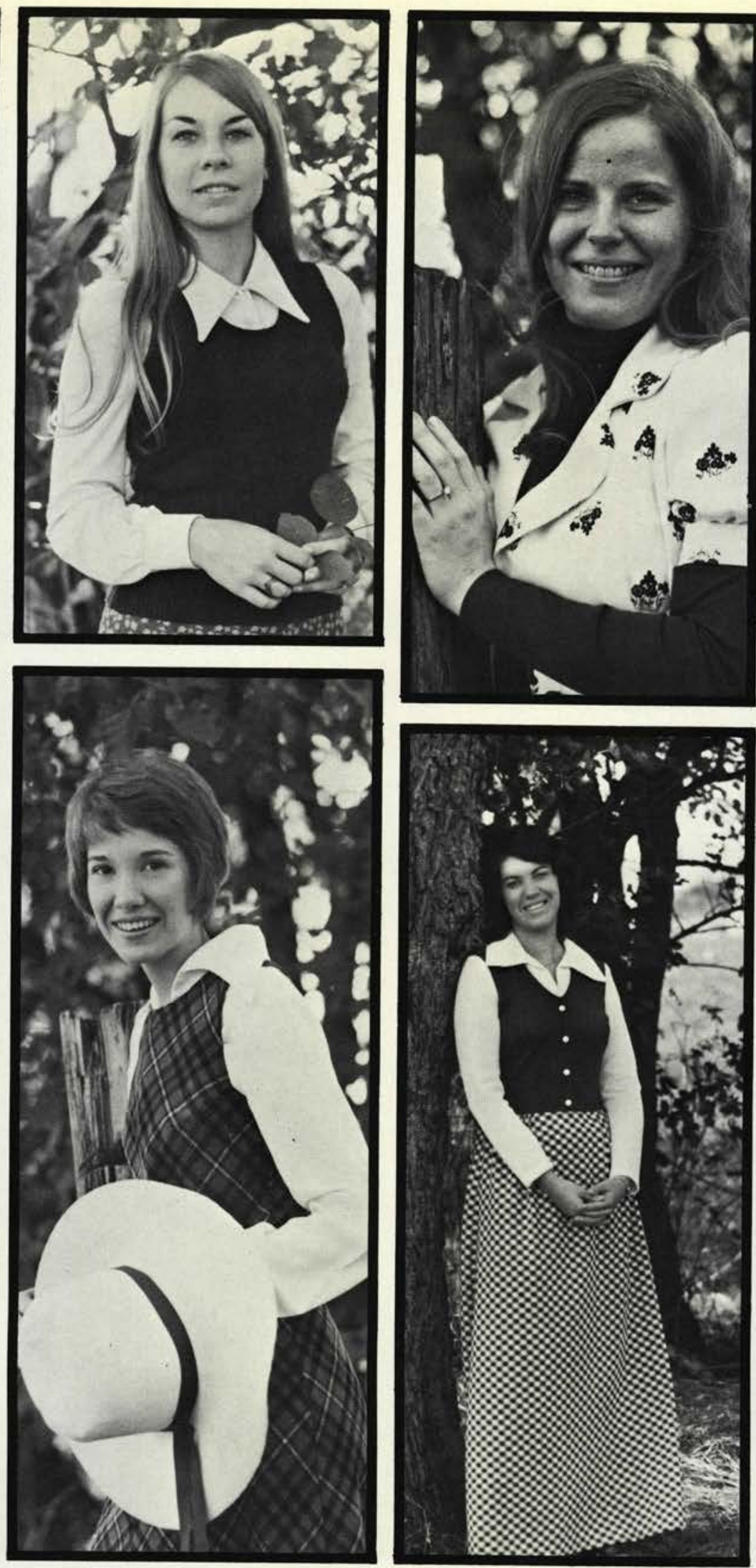
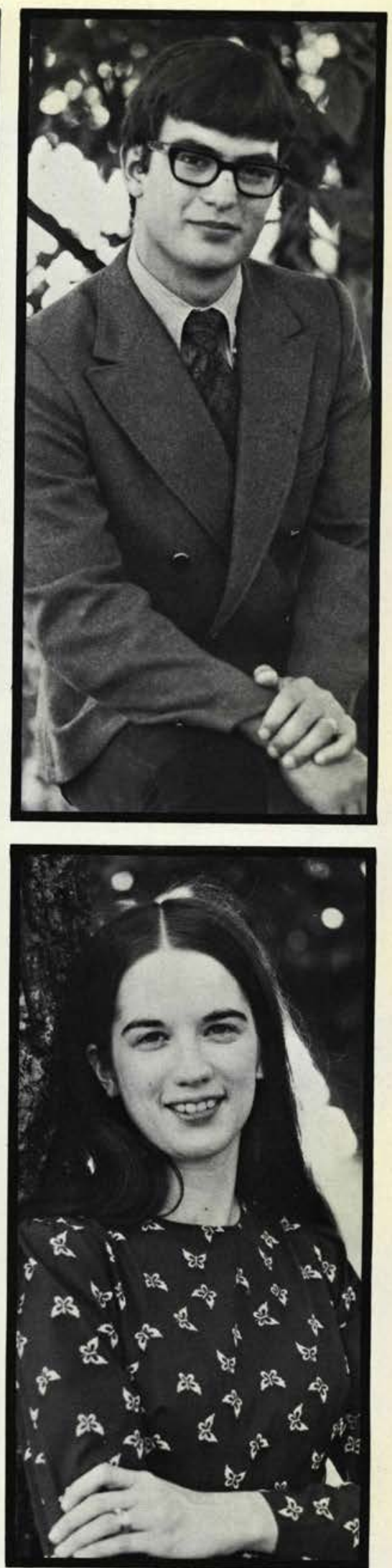

Lynn Mitchell

Cedarville, $\mathrm{OH}$

Elementary Education

Steve Millikan
Bothell, WA

Music
Sally Mitchell

Cedarville, $\mathrm{OH}$

Music

Ranelle Nabring

Cedarville, $\mathrm{OH}$

English
Karen Mulder

Lansing, MI

Speech

Carolyn Newman

West Union, $\mathrm{OH}$

Business Adm.
Dave Nicholas

Cedarville, $\mathrm{OH}$

Math

Luann Nicholas

Cedarville, $\mathrm{OH}$

Social Science 

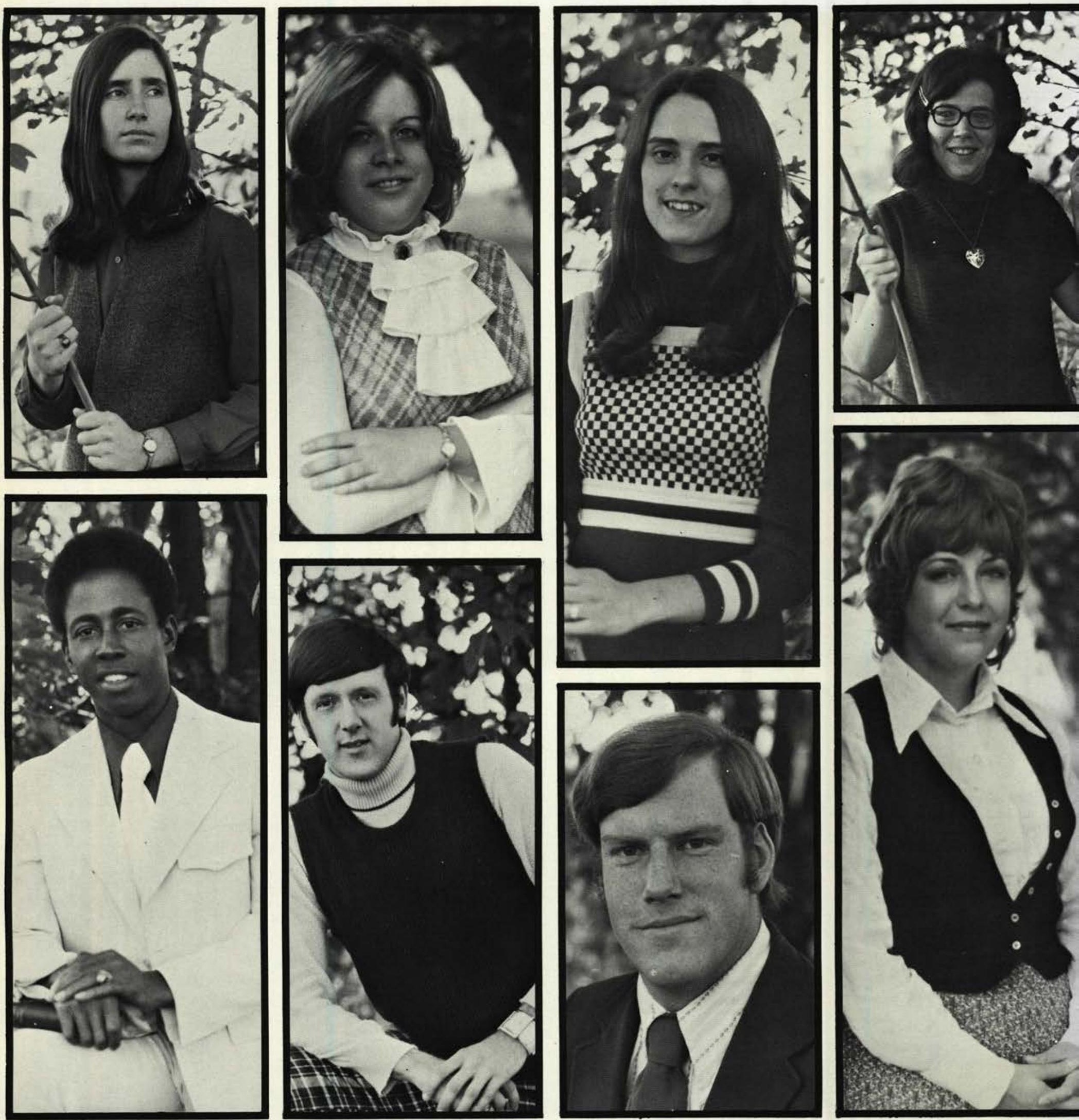

Linda Osborn

Springfield, $\mathrm{OH}$

Physical Education

Clarence Parker

Portsmouth, $\mathrm{OH}$

Pre-Seminary
Linda Pauley

Pana, IL

Elementary Education

Barton Otto

Concord, MI

Pre-Sem./IDS
Becky Overholt

Hazel Park, MI

Elementary Education

Steve Overholt

Hazel Park, MI

History
Gloria Parker

Berea, $\mathrm{OH}$

Elementary Education

Gale Portz

Greenville, PA

Psychology 

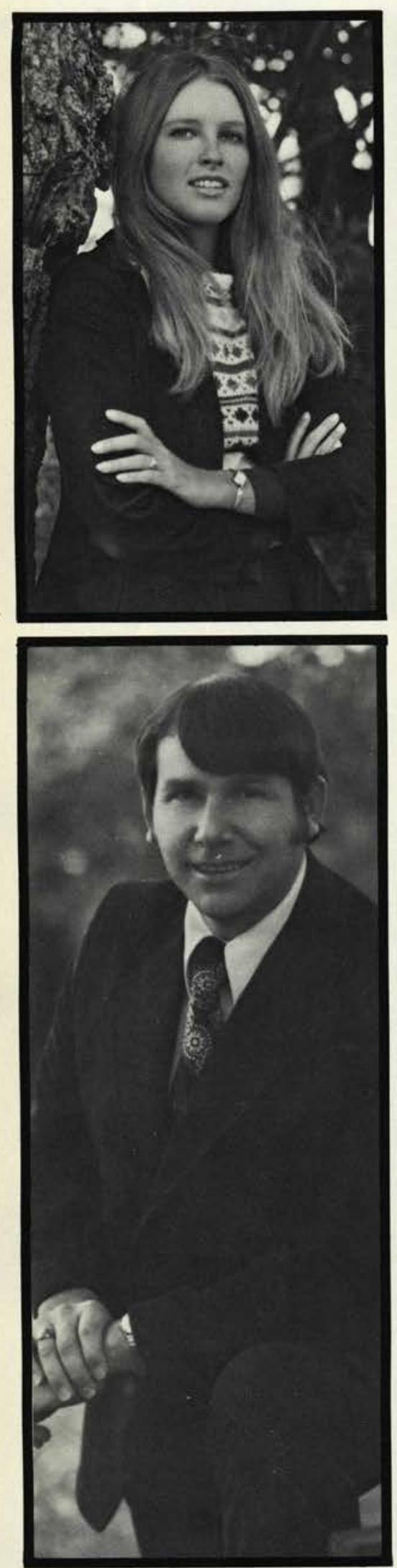
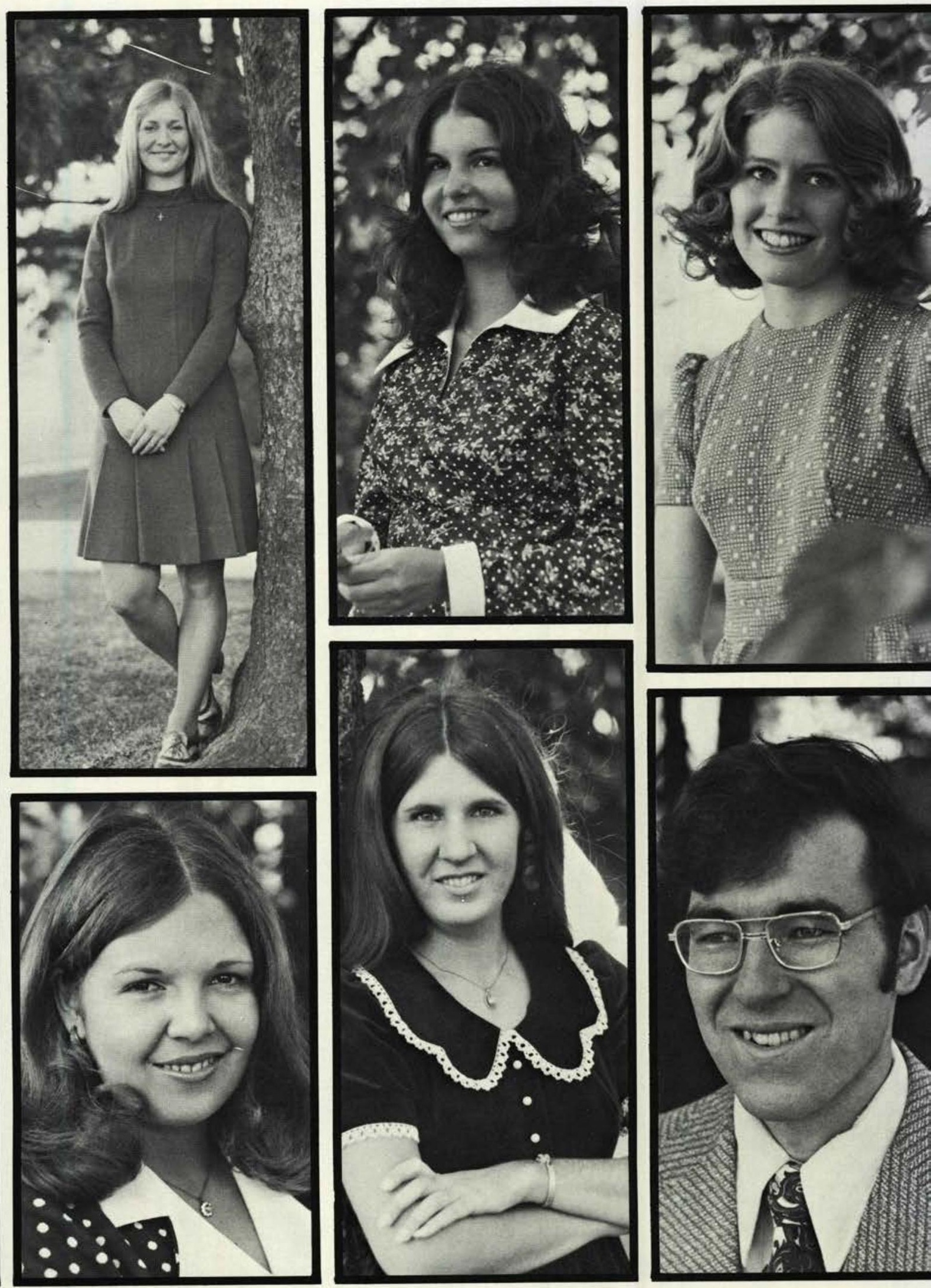
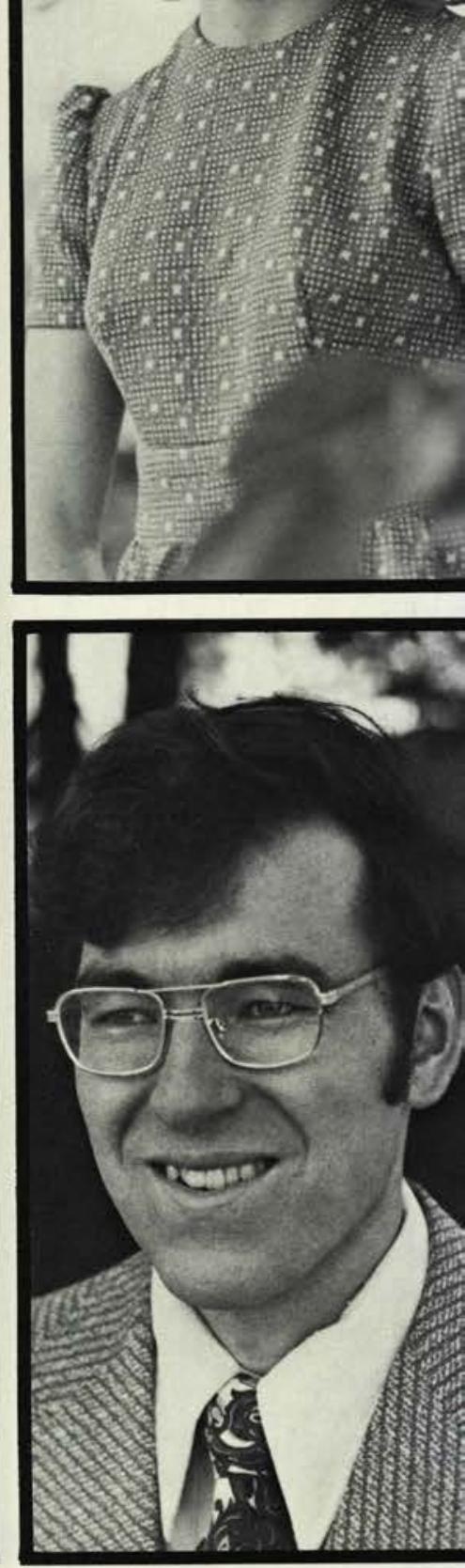

Fay Salomon Hamburg, NY Psychology

Ken Sanders Cedarville, $\mathrm{OH}$

Bible
Kathy Scott

Clifton, $\mathrm{OH}$

Bible Education

Linda Severs

Hamilton Square, NJ

Elementary Education
Sue Saunders

Port Jervis, NY

Elementary Education

Rennee Seigneur

Stryker, $\mathrm{OH}$

Elementary Education
Sue Selden

Huntsburg, $\mathrm{OH}$

English

Ron Schroeder

Royal Oak, MI

Pre-Seminary 

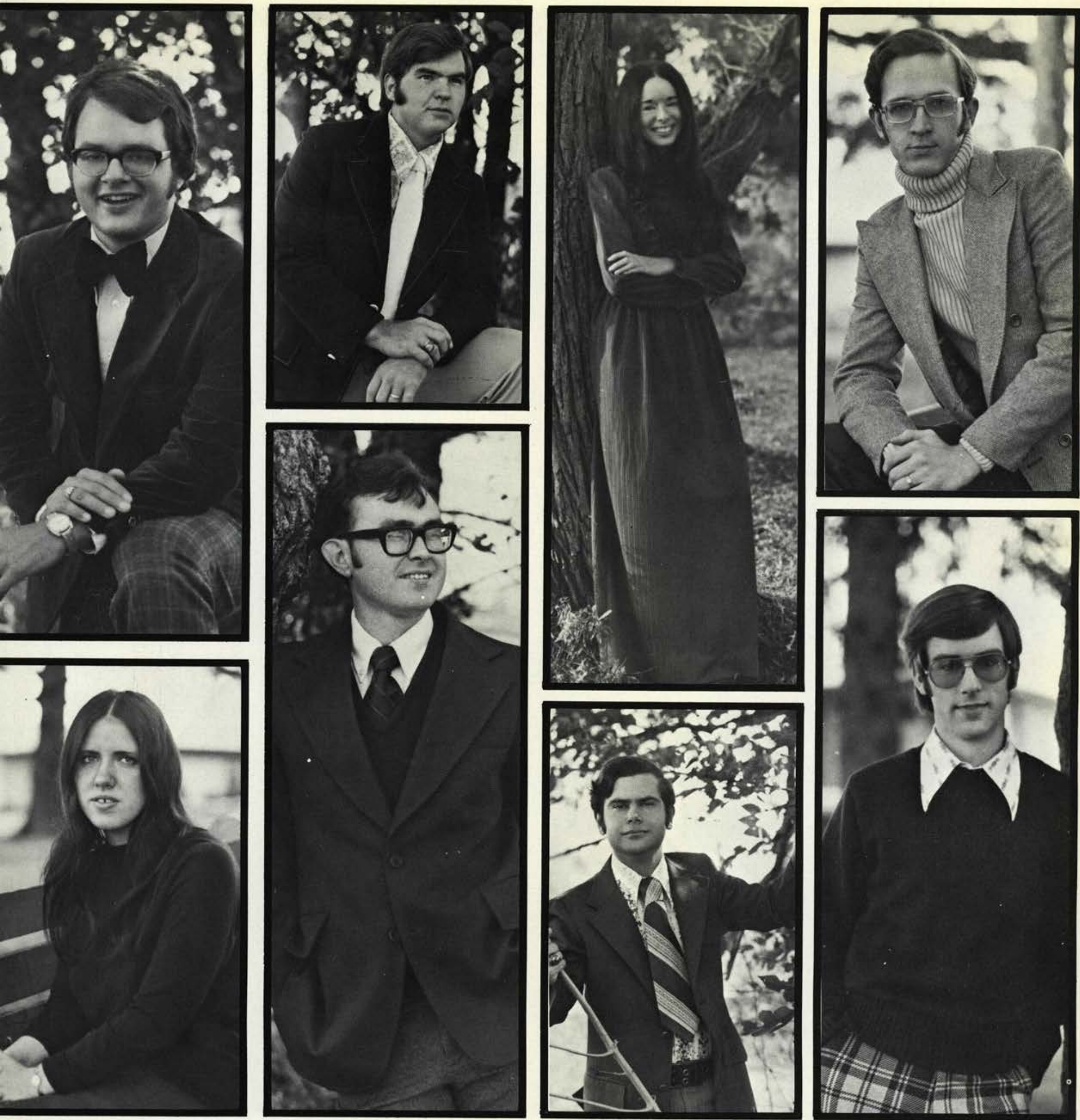

Richard Shaw

Naples, FL

Psychology

Cathy Sharp

Jonesville, MI

Biology
Charlie Sheppard

Haddon Heights, NJ History

Dean Shugars

Jamestown, $\mathrm{OH}$

Elementary Education
Debbie Sneller

Columbus, $\mathrm{OH}$

Elementary Education

Kevin Sims

Waterloo, LA

History
Greg Smith

Cedarville, $\mathrm{OH}$

Psych./English

Steve Spencer

Delevan, NY

IDS 

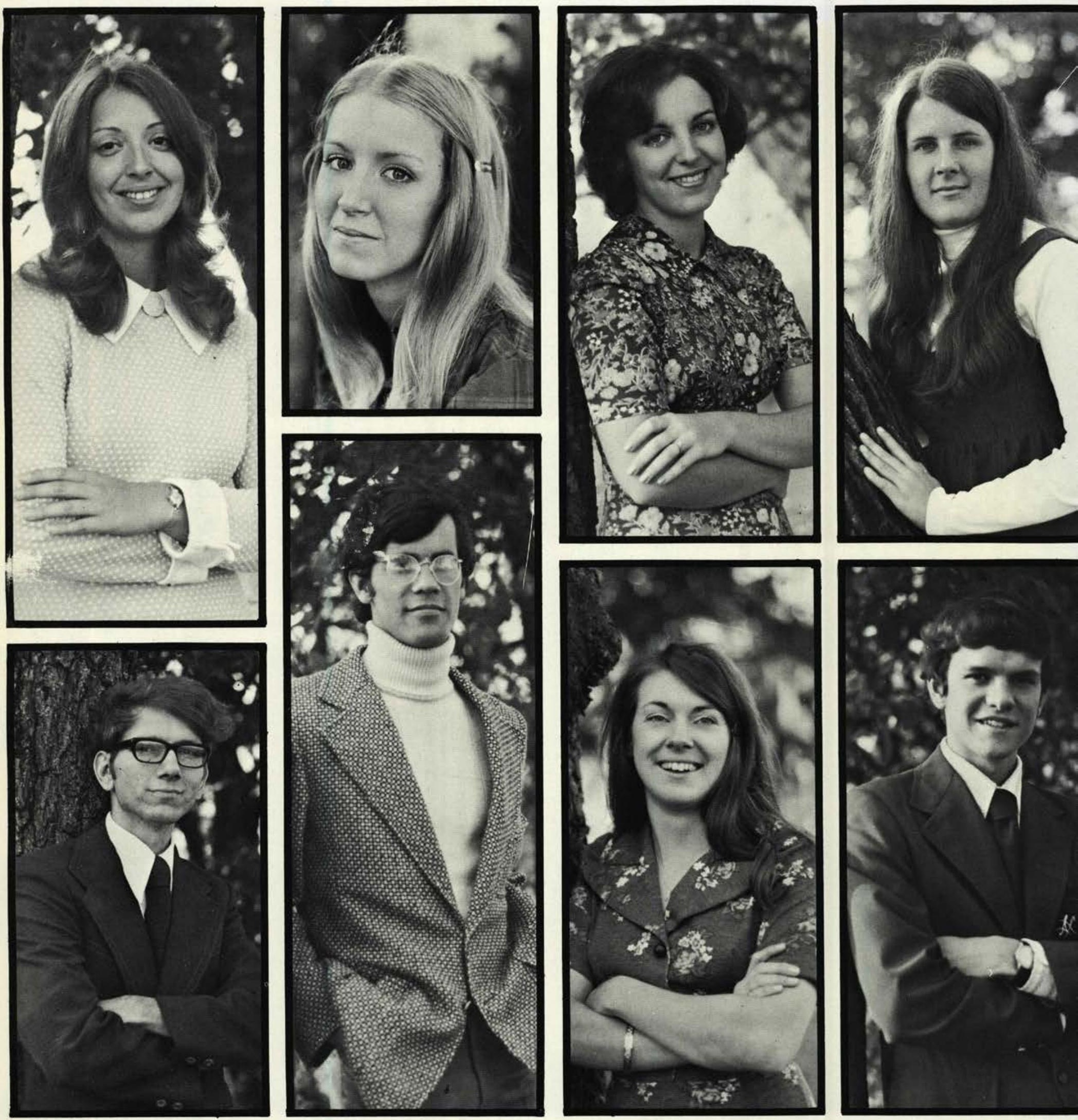

Jackie Spinks

Sherman, NY

Behavioral Science

Jeff St. Clair

Cedarville, $\mathrm{OH}$

Speech
Cheryl Taylor

Brooklyn, MI

Elementary Education

Larry St. Lawrence

Minneapolis, MN

Political Science

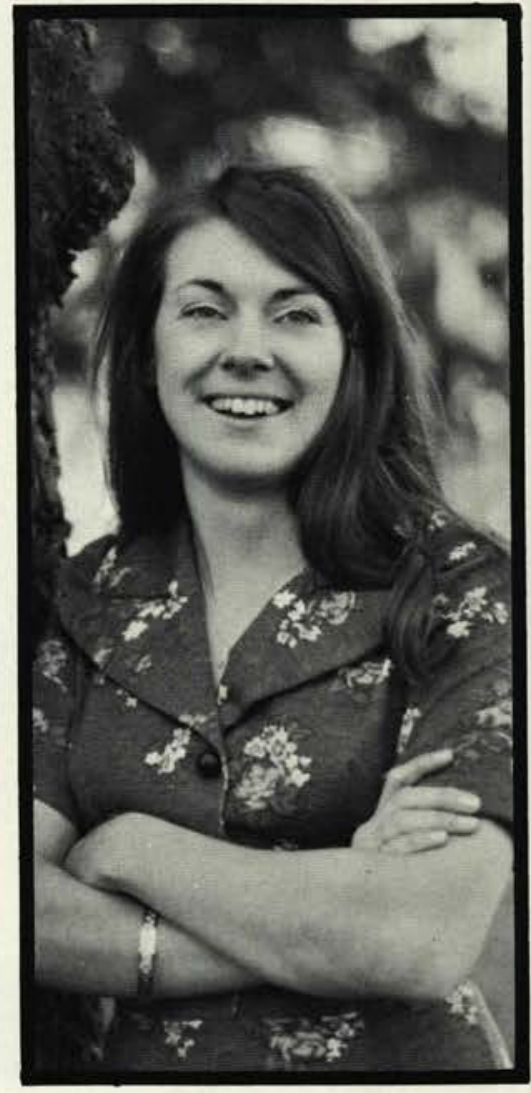

Sarah Stone

St. Albans, WV

Social Science

Kathy Strawn

Maryland Heights, MO

Elementary Education
Nancy Tallman

Creston, IA

Music

Dan Steman

Fort Wayne, IN

Psychology 

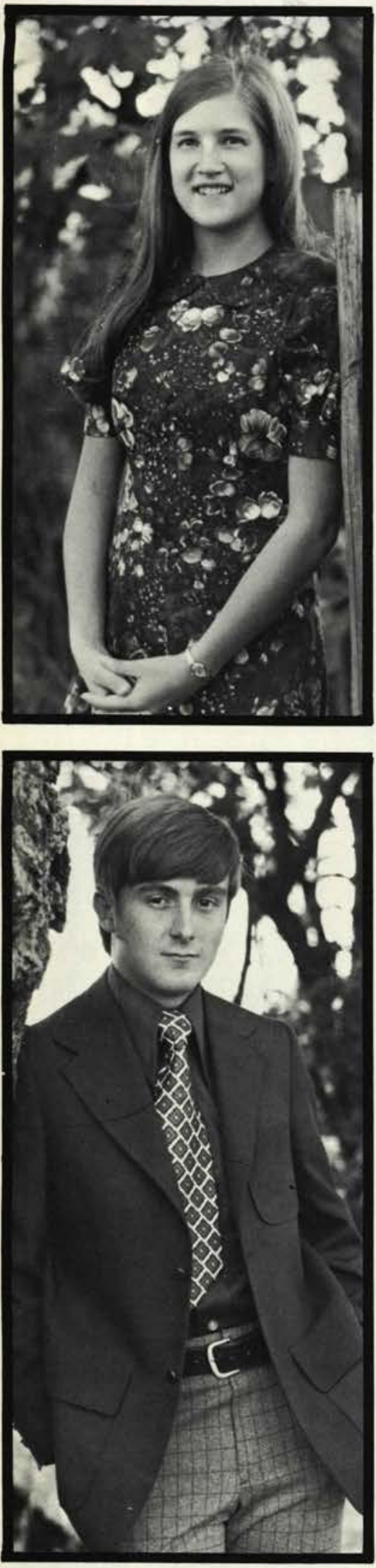
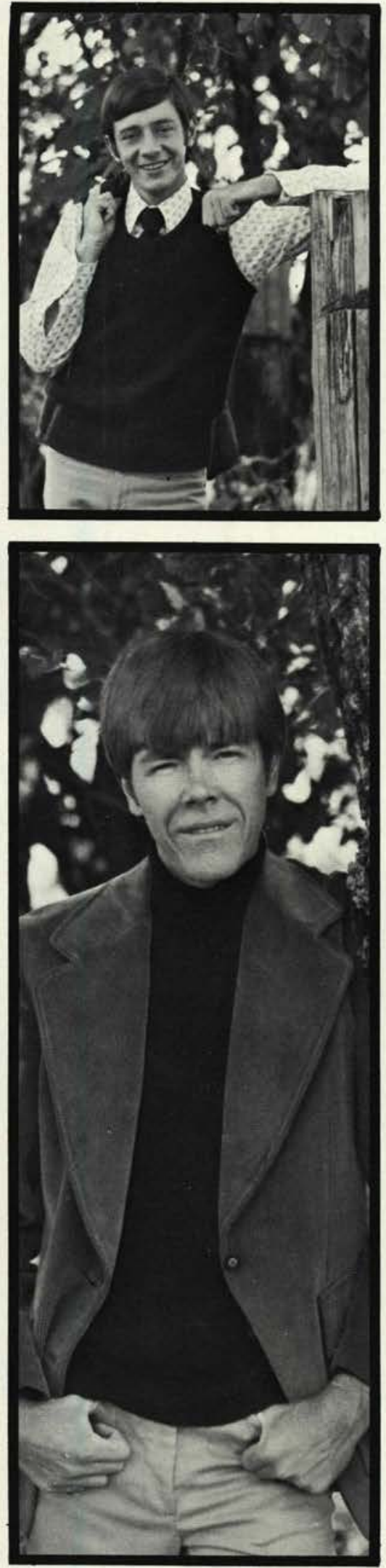
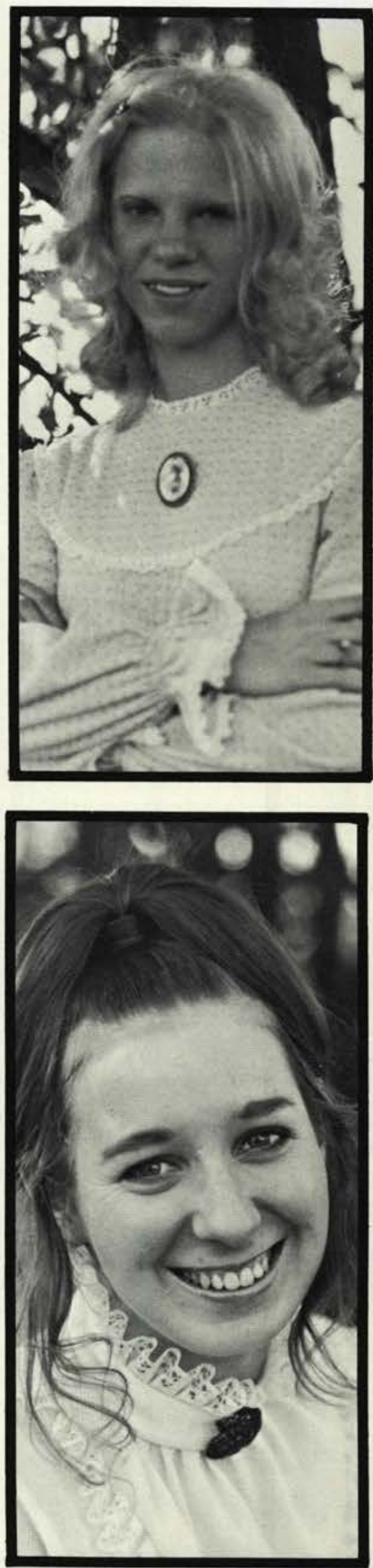
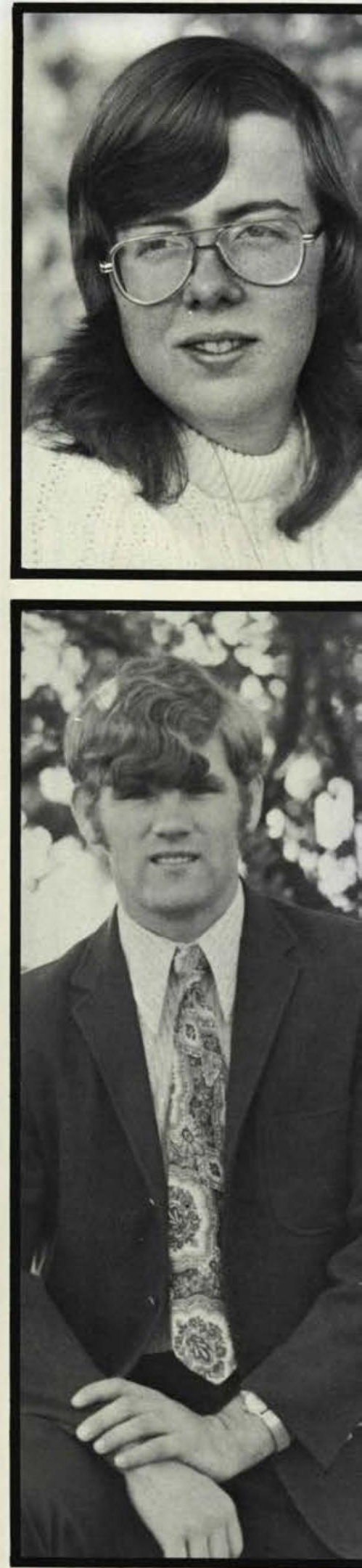

Brenda Will

Forestville, NY

Elementary Education

$$
\begin{aligned}
& \text { Jeff Wolfe } \\
& \text { East Gary, IN }
\end{aligned}
$$

History
Howard Wolff

Millersburg, $\mathrm{OH}$

Pre-Seminary

Kevin Wolf
Littleton, CO
Business, Adm.
Debbie Wood

Curwensville, PA

Elementary Education

Wendy Work

Cedarville, $\mathrm{OH}$

Psychology
Pamela Williams

Springfield, $\mathrm{OH}$

Psychology

Ivan Work

Coudersport, PA

Chemistry 

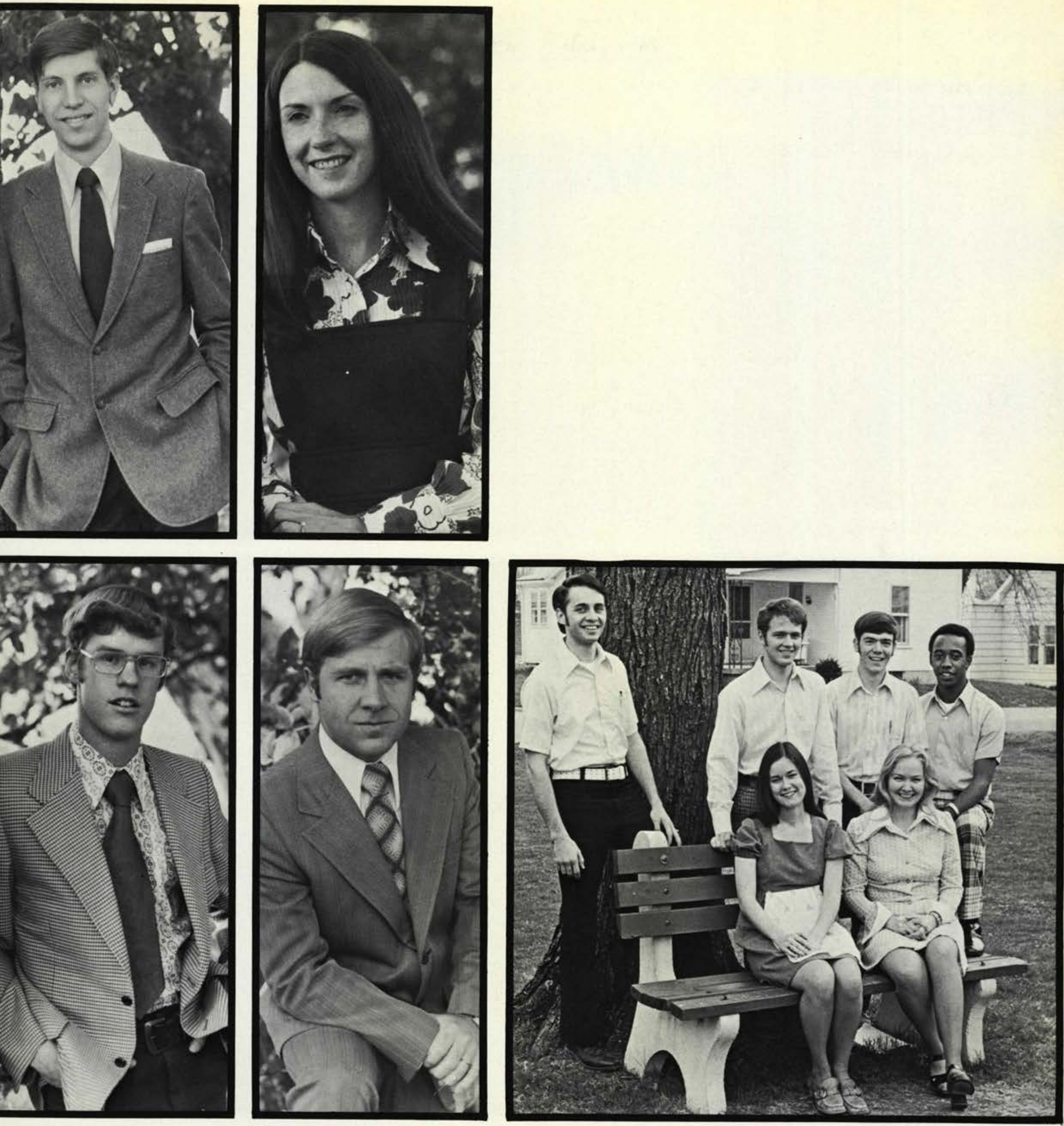

Bill Wymer

Warren, Oh

Pre-Seminary

Don Yeaton

Grossville, NH

Pre-Seminary
Janet Young

Waterloo, lowa

Elementary Education

Mr. Jim Phipps

Senior Class Advisor
Class Officers: David True, Pres., Linden Kirby, V.P., Jackie Spinks, Sec. Dennis Twigg, Treas., Janet Long, Rep., Debbie Dear, Rep., Lynn Mitchell, Chaplain. 

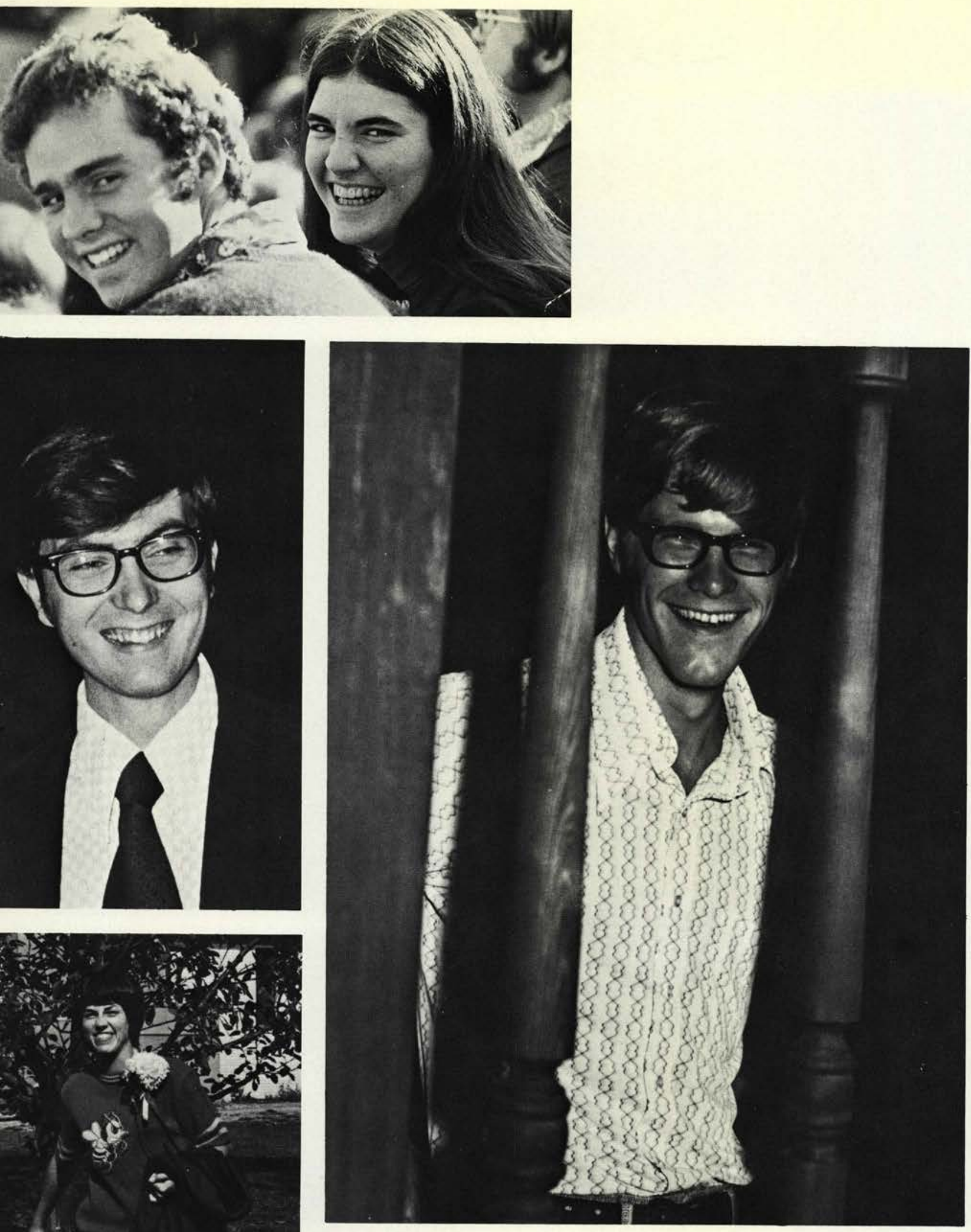

... the Happy Side of Life!

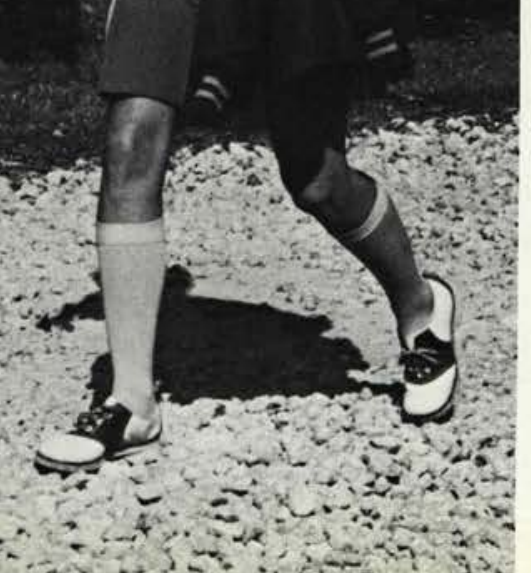




\section{Winter's Tce-Gapades}
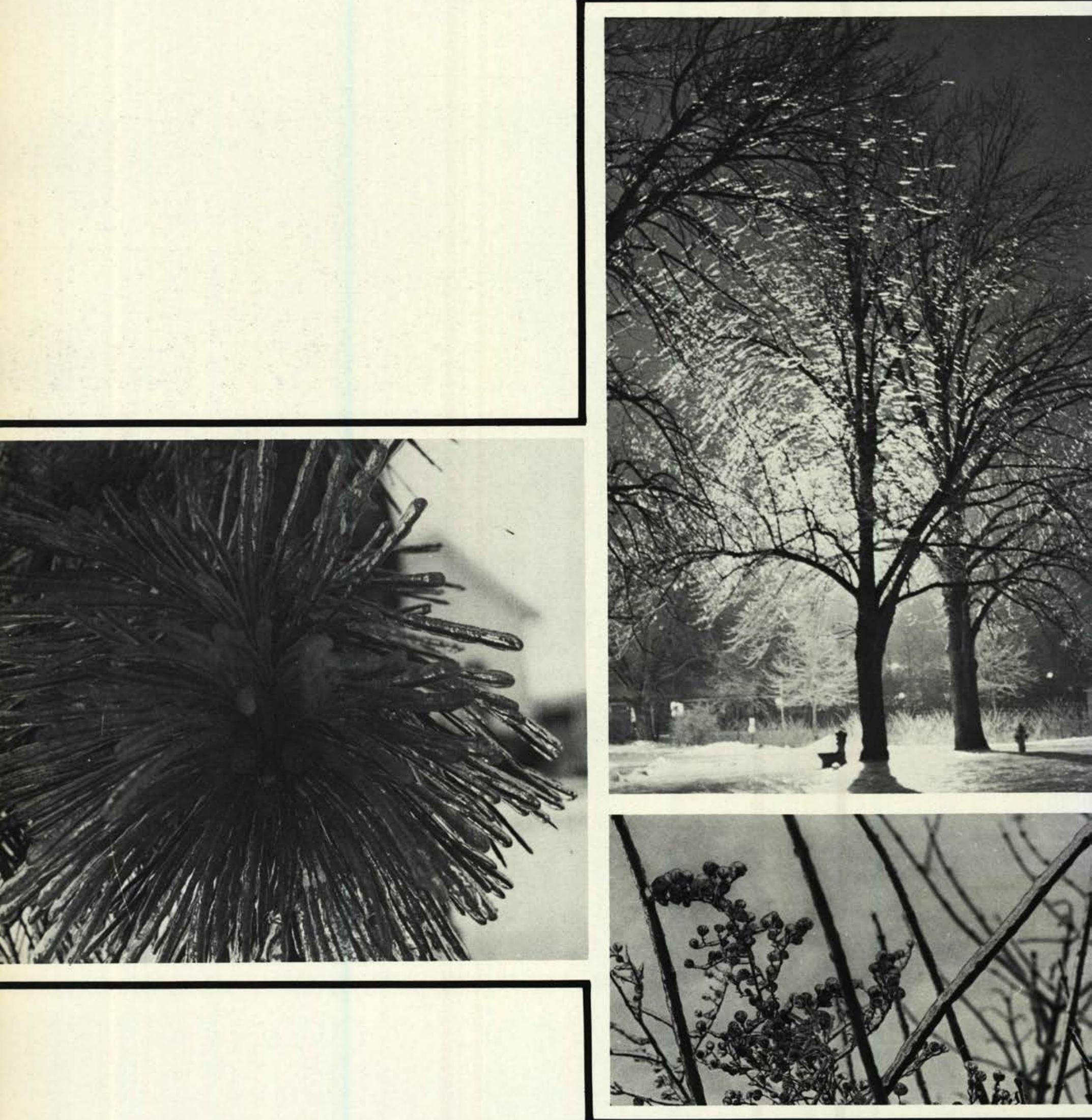



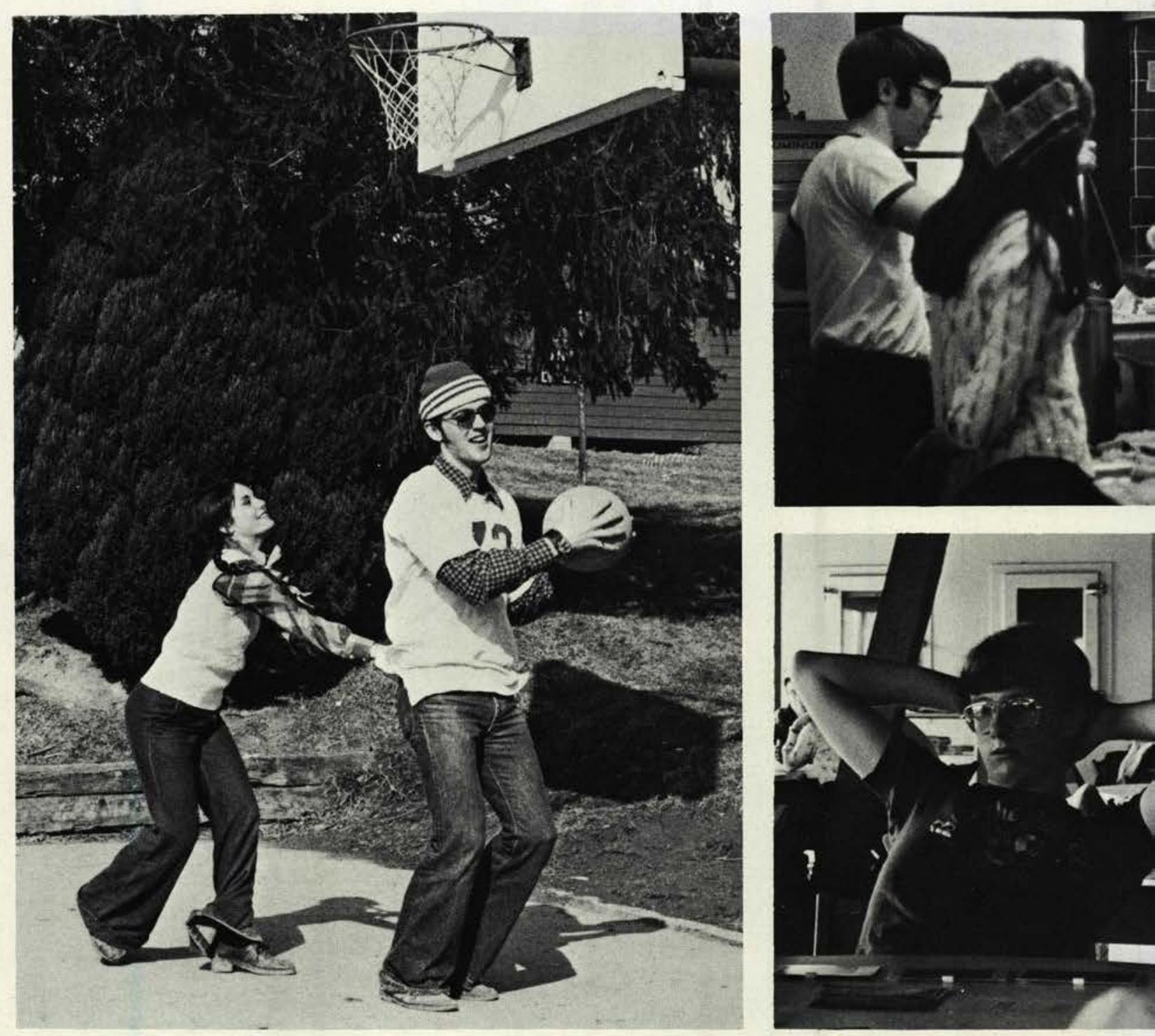

AX Retreat

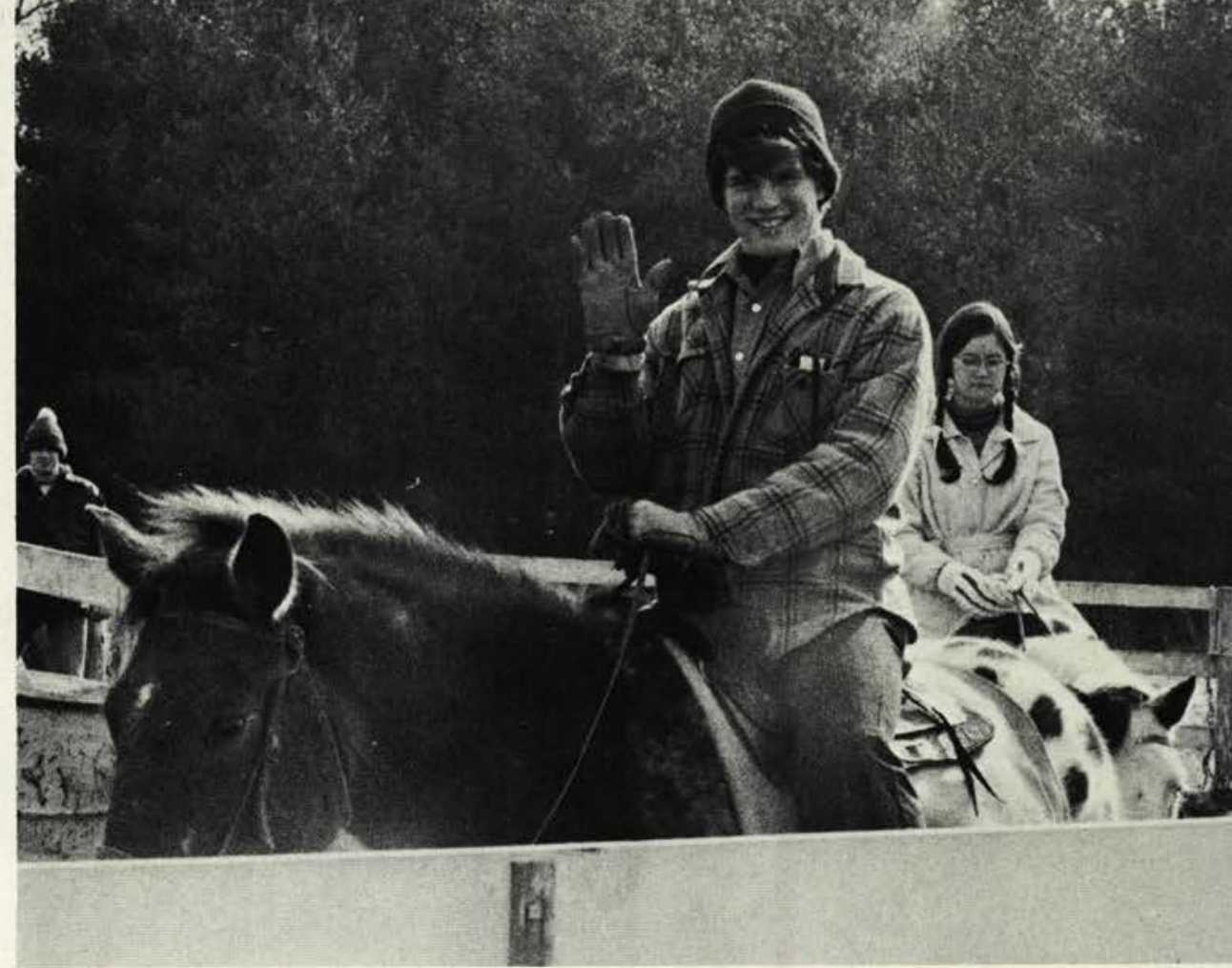



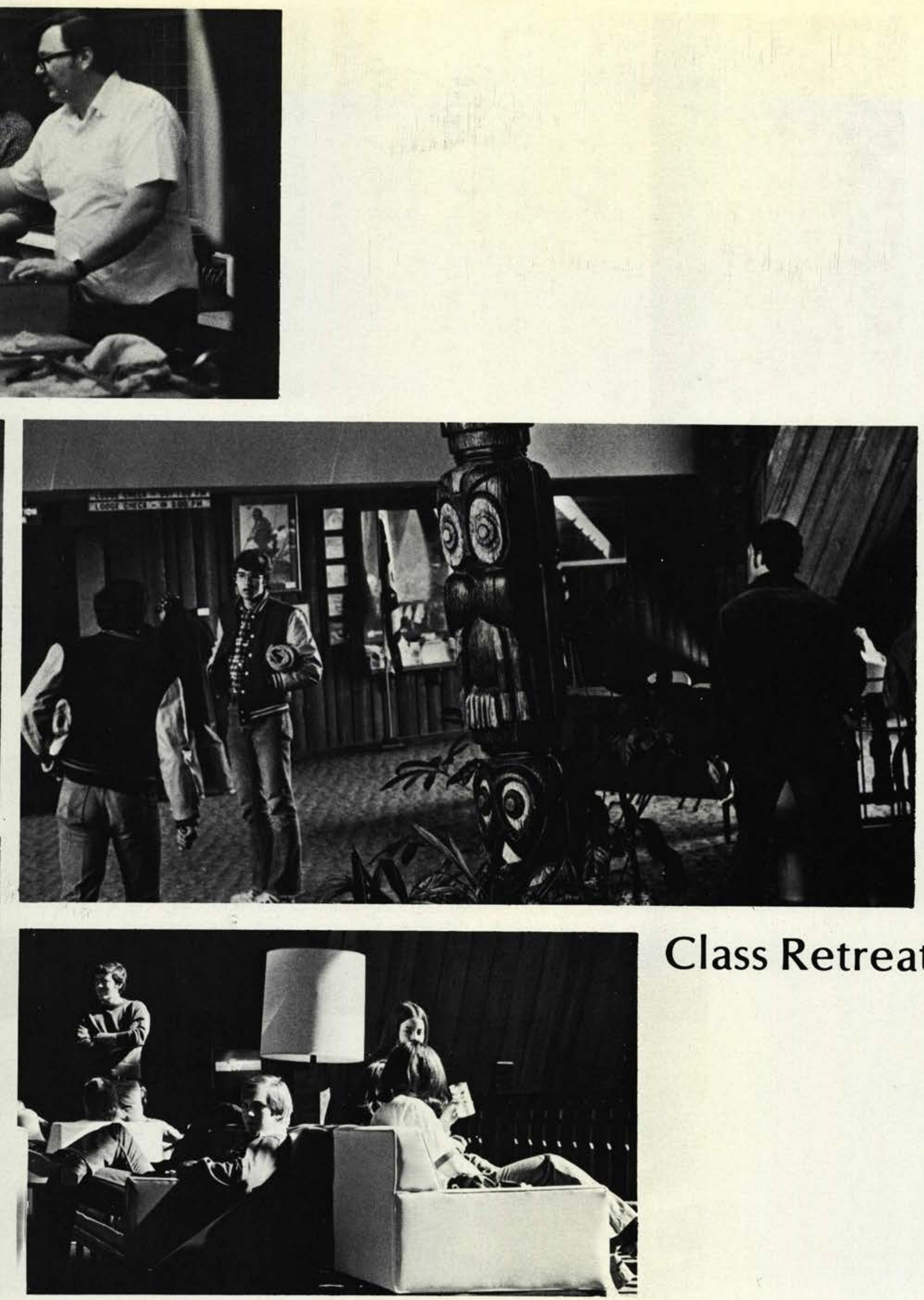

\section{Class Retreats}



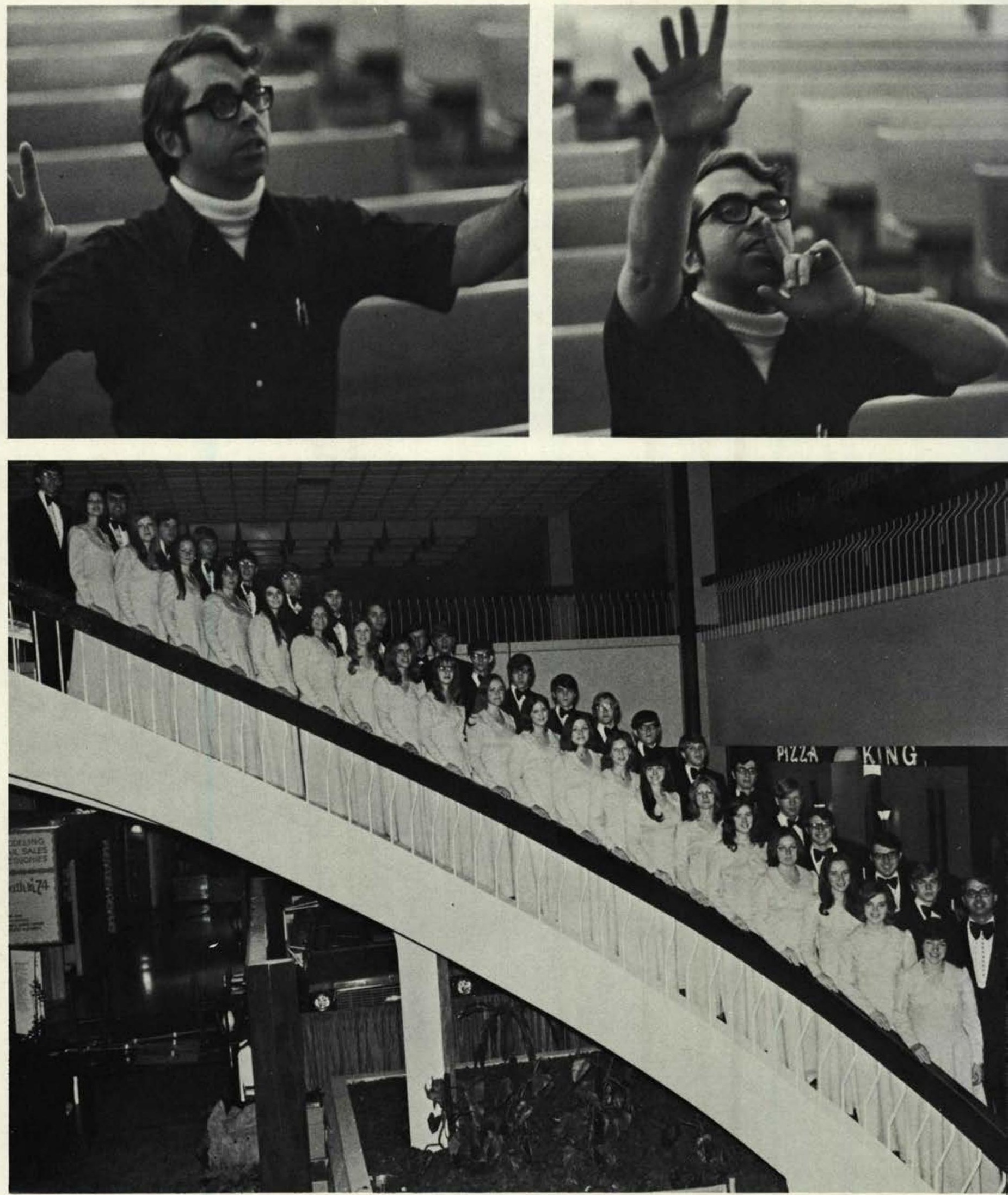

Concert Choir: FRONT ROW, Georgeanna Axiotis, Cindy Mally, Ellen Taft, Deanna Wagnitz, Linda Carlton, Becky Kemble, Elaine Moore, Debbie Gidley, Debbie Ward, Linda Mayo, Debbie Banks, Sharon Franks, Carol Towle, Carol Keller, Andrea Rouch, Wendy Montgomery, Sandy Soliday, Cindy Powers, Nancy Price, Kathy Howell, BACK ROW, Bob Melford, Steve Lantz, Dan Ritenburg, John Walborn, Steve Estes, Dan Jones, John Jeffords, Dave True, Jim Douglas, Dave Frederick, Tim VanLoon, Mark Sigmund, Bob Young, Doug Wilson, Don Criss, Doug Wing, Ron Bechtel, Tom Thomas, Dan Estes, Maynard Smart, Tony Wilson, Mr. David Matson, Director. 

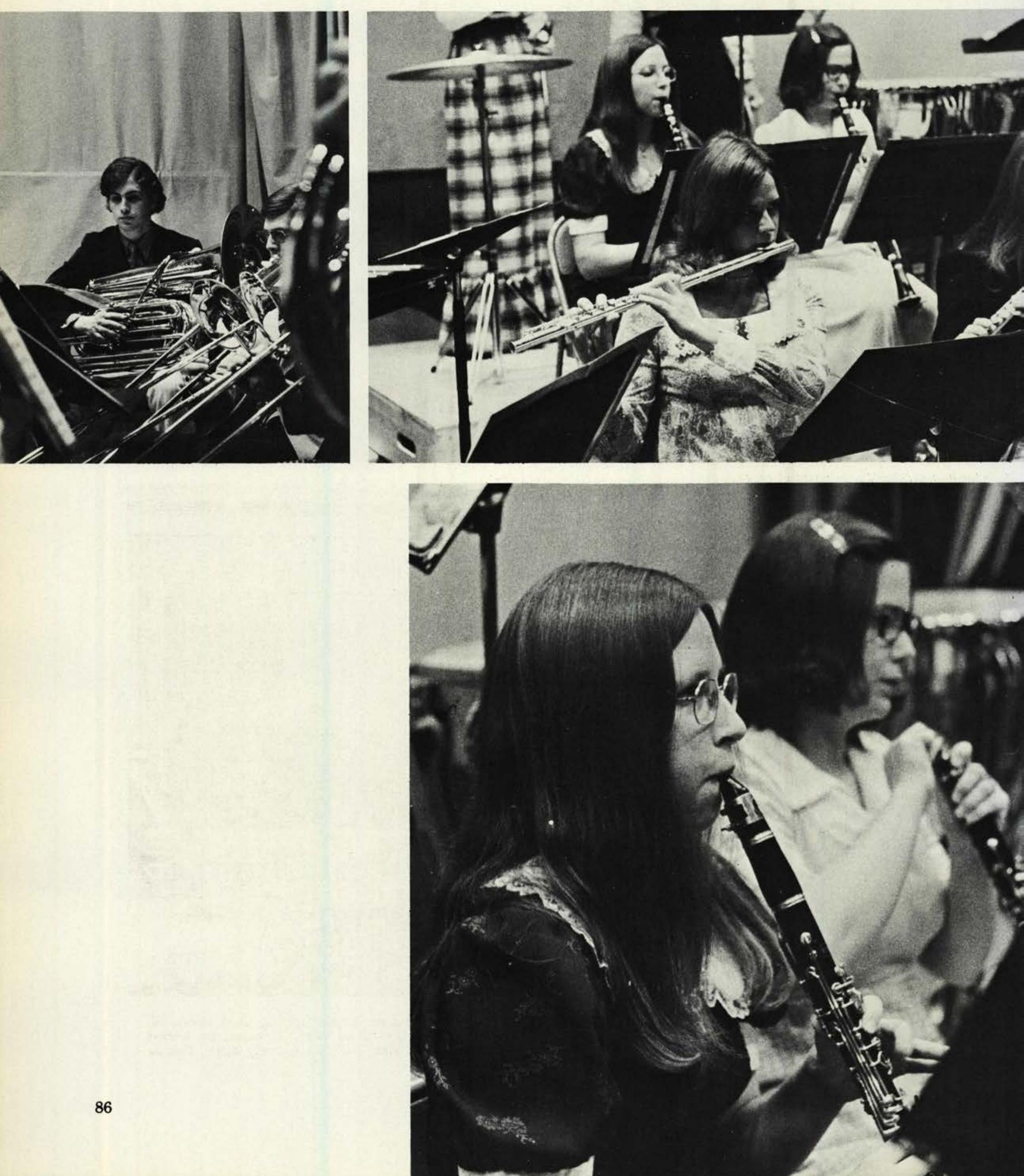

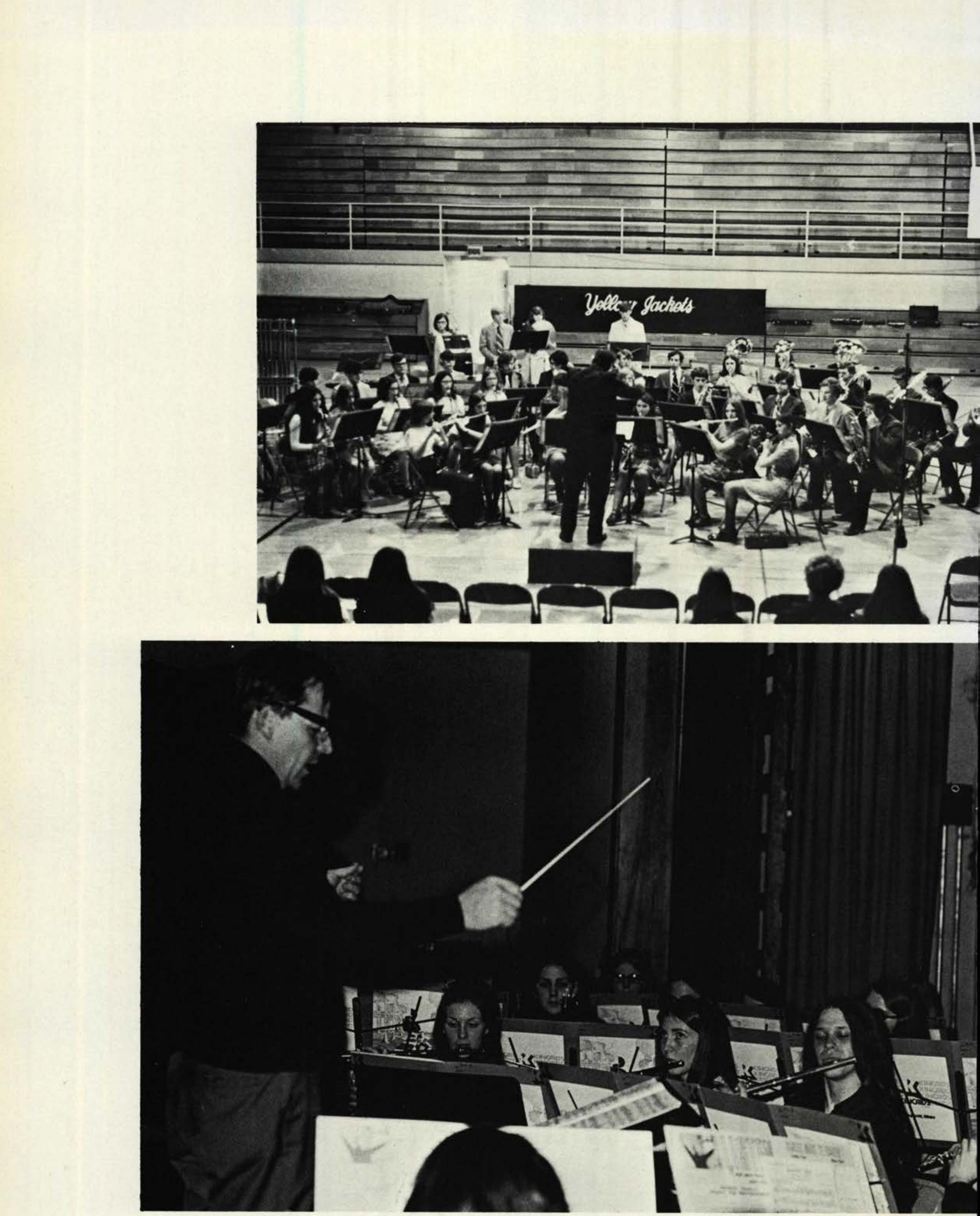

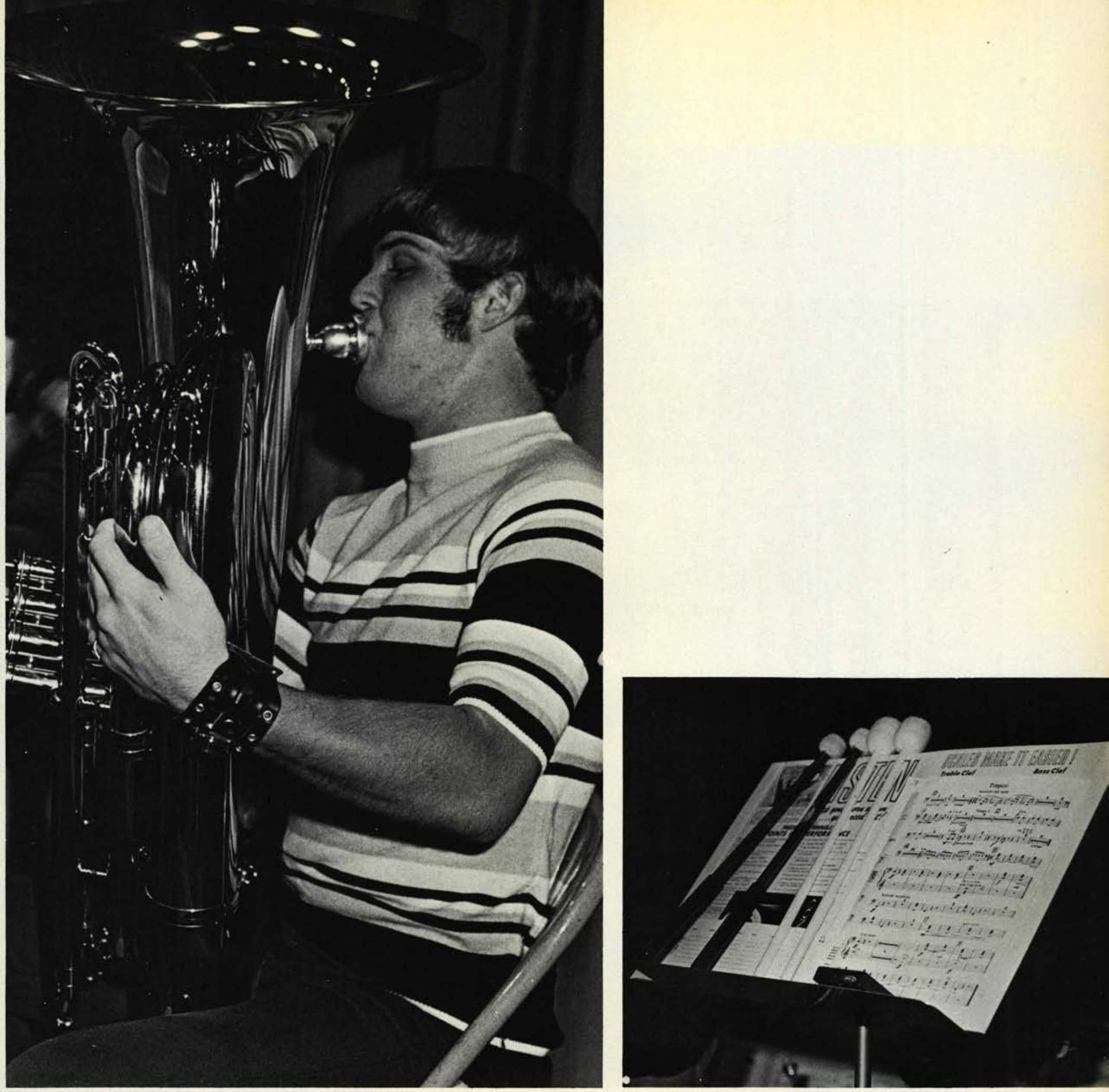

\section{Cedarville College Band}




\section{Choralaires}

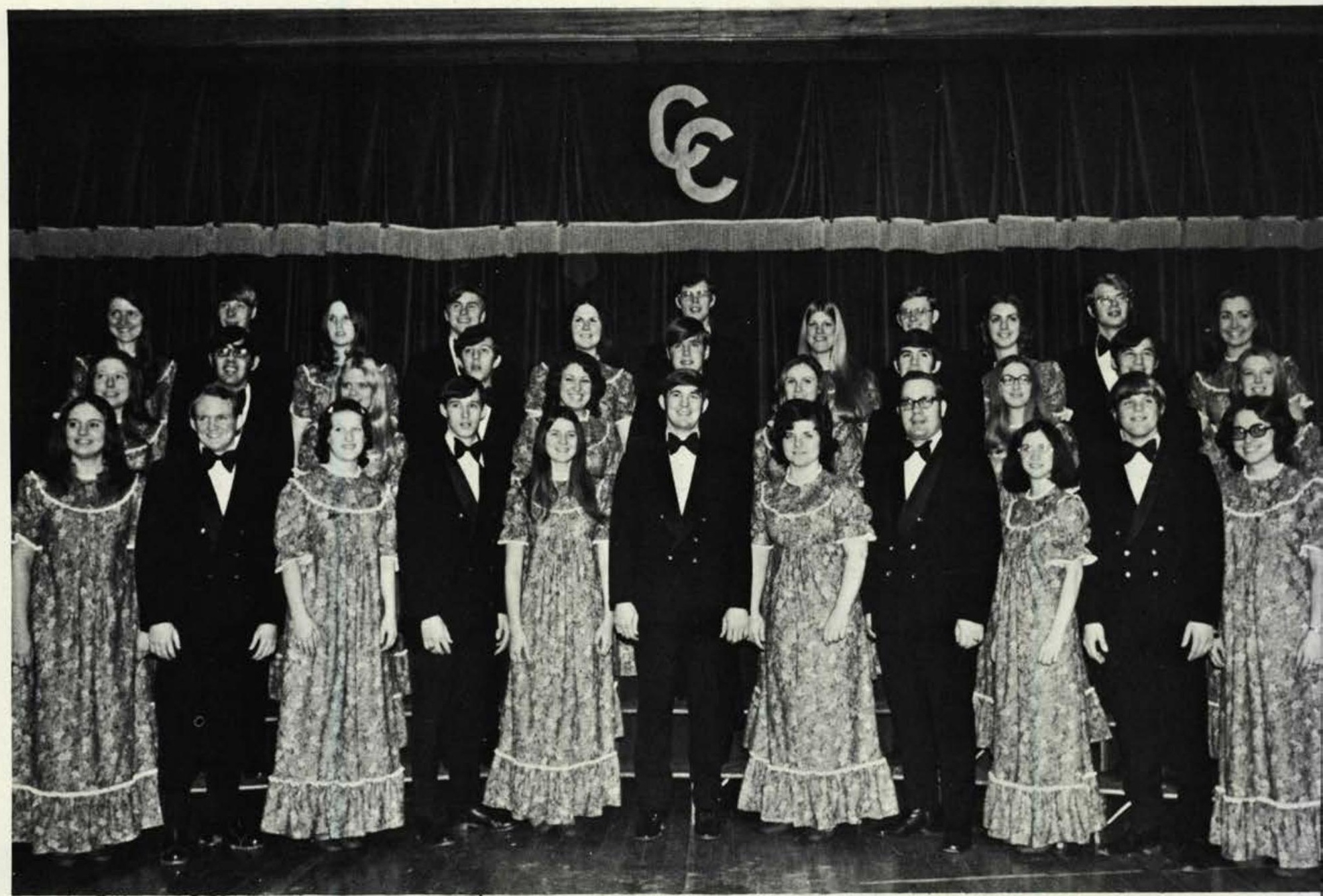

Kathy Hulsman, George Sperry, Alice Manwaring, Dale Wright, Mary Griffith, Jerry Griswold, Sharon Bartemus, Ron Jutton, Leah Bathurst, Ken Harris, Ferris Ometz, Donna Stevens, John Simons, Pam Romaine, Jeff Zinn, Becky Lamb, Jim Goetz, Nancy Taylor, Bob Fisher, Chris Giesel, Rich Regling, Lynn Anderson, Terry Baker, Tim Miller, Dwight Strickland, Jim Gilbert, Ruth McCauley, Rodney Blystone, Mindy Andes, Duane Mawhorter, Tery Smith. 


\section{The Printed Page... The Library}
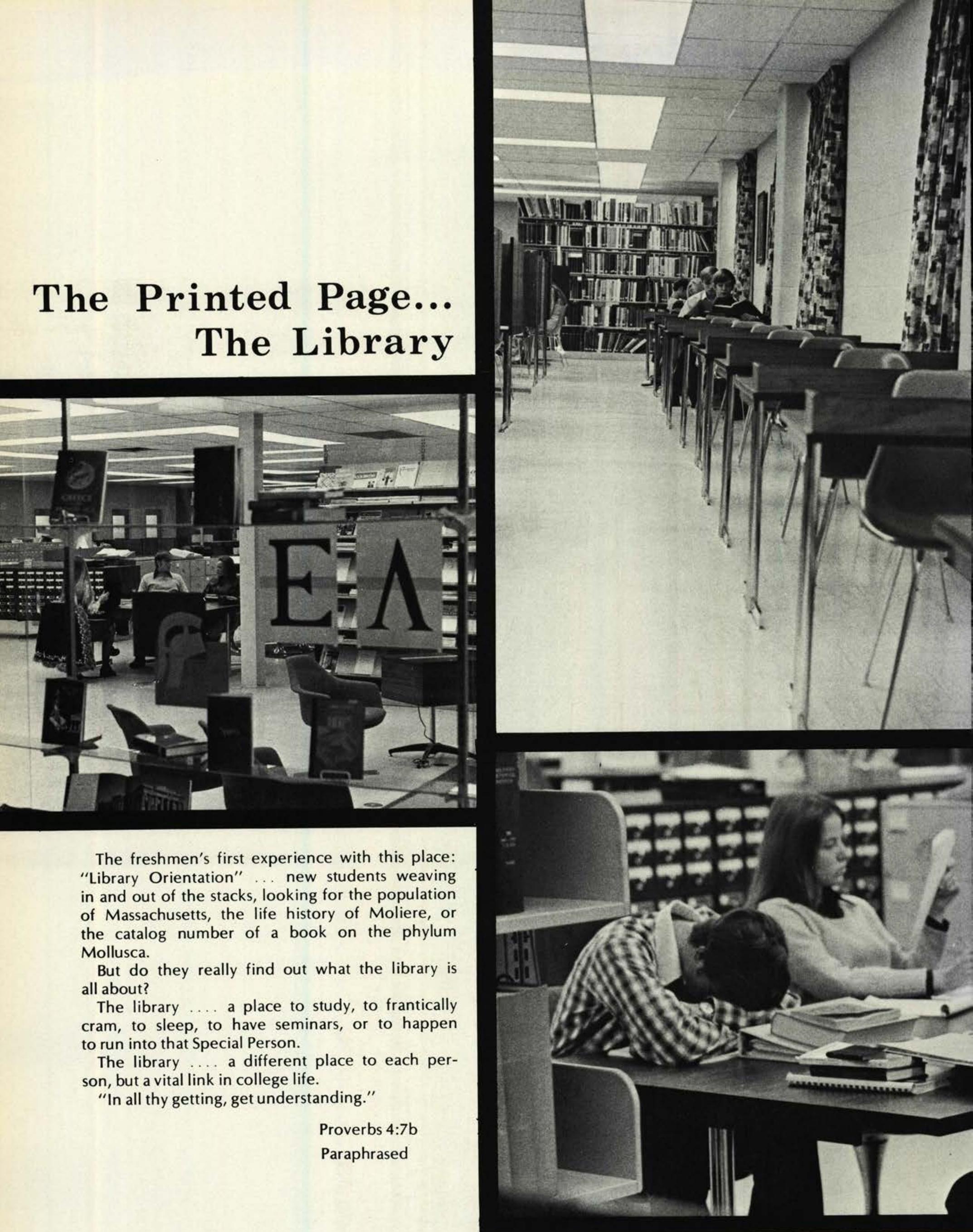


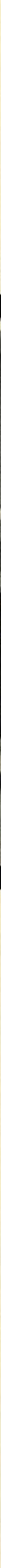




\section{Faculty}

Mead C. Armstrong Professor of Bible

Jean Fisher Associate Professor of Christian Education

James M. Grier Assistant Professor of Philosophy

Richard T. McIntosh Associate Professor of Bible

Robert G. Gromacki Professor of Bible and Greek

George L. Lawlor

Professor of Bible and Greek

Jack R. Riggs Professor of Bible

James E. Seaman Assistant Professor of Marketing and Management
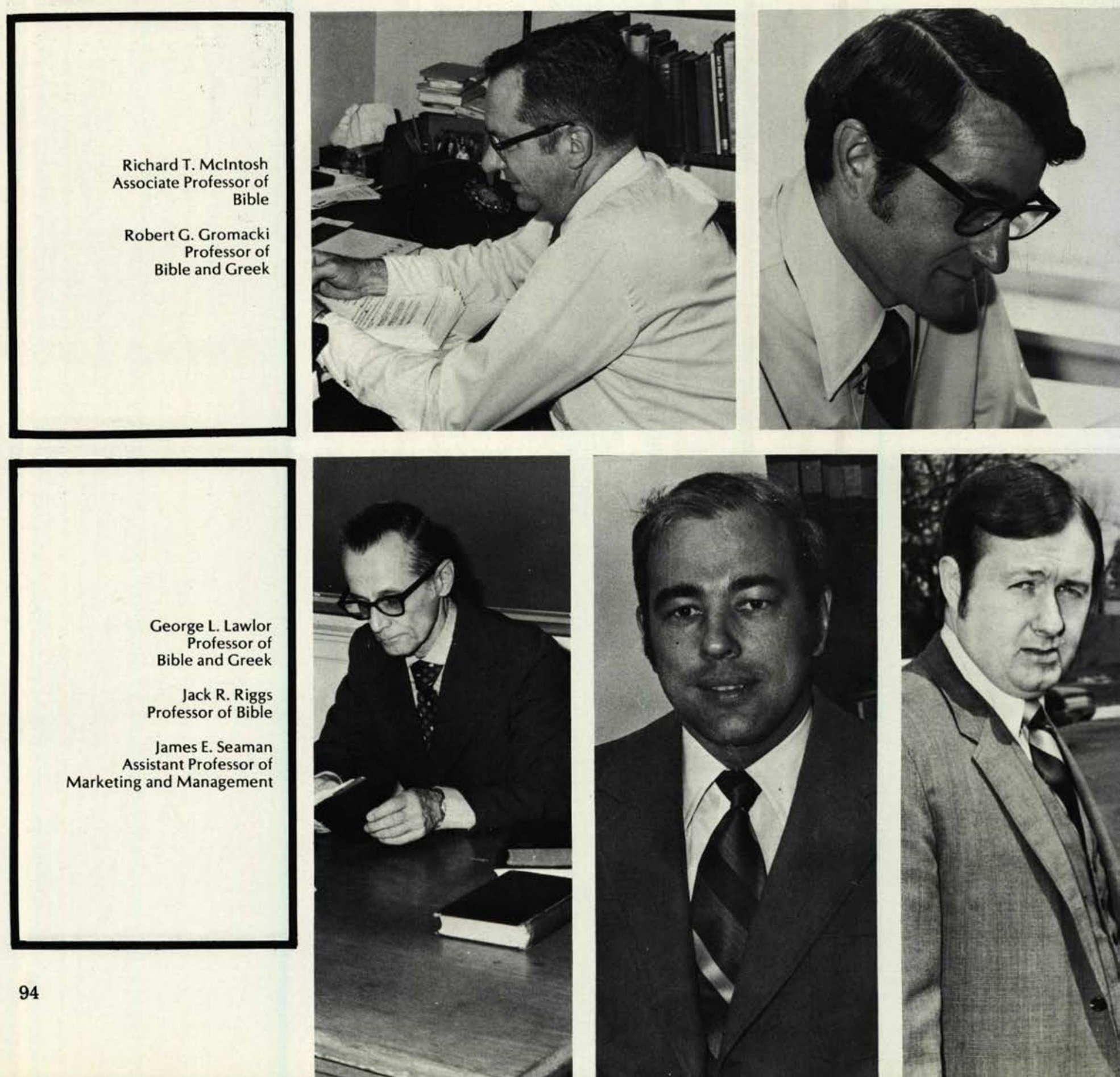

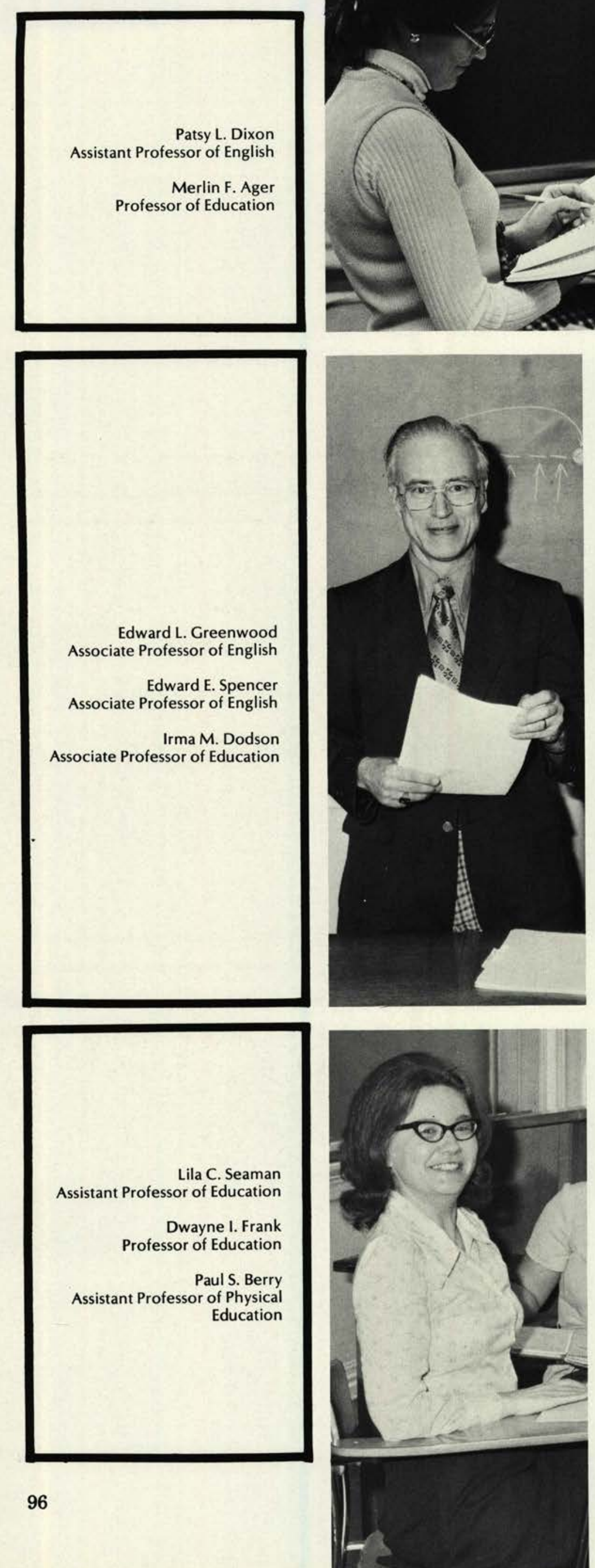
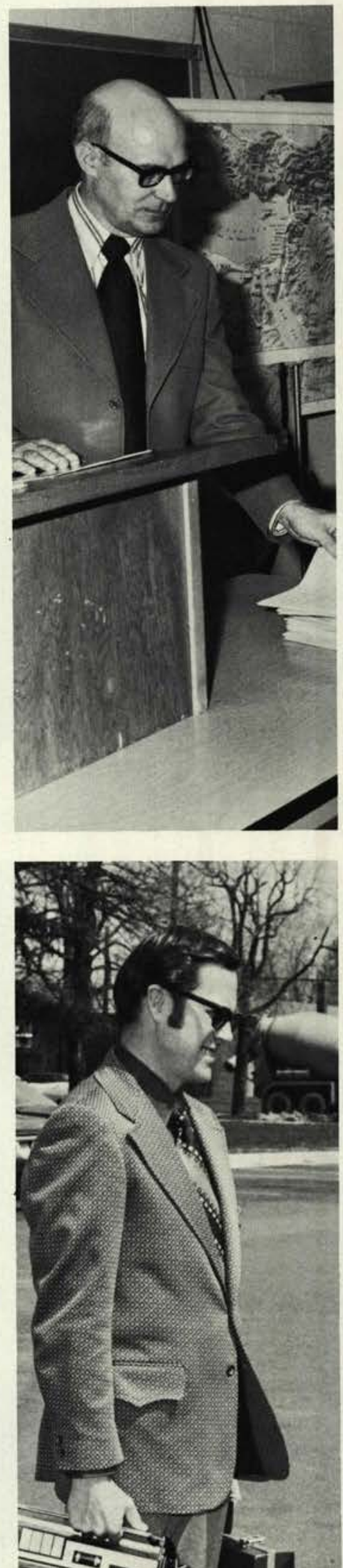
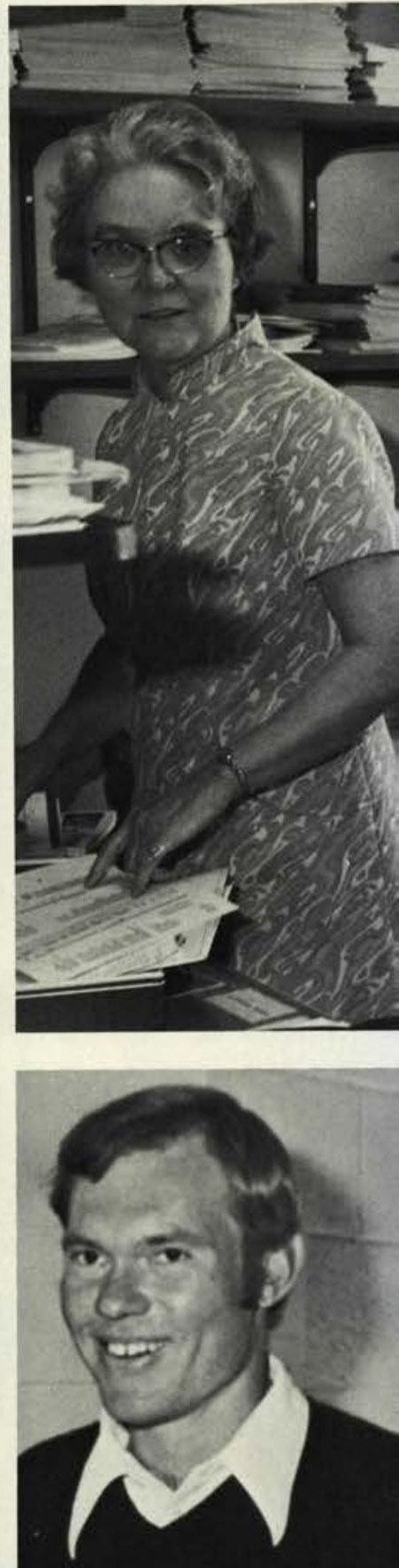


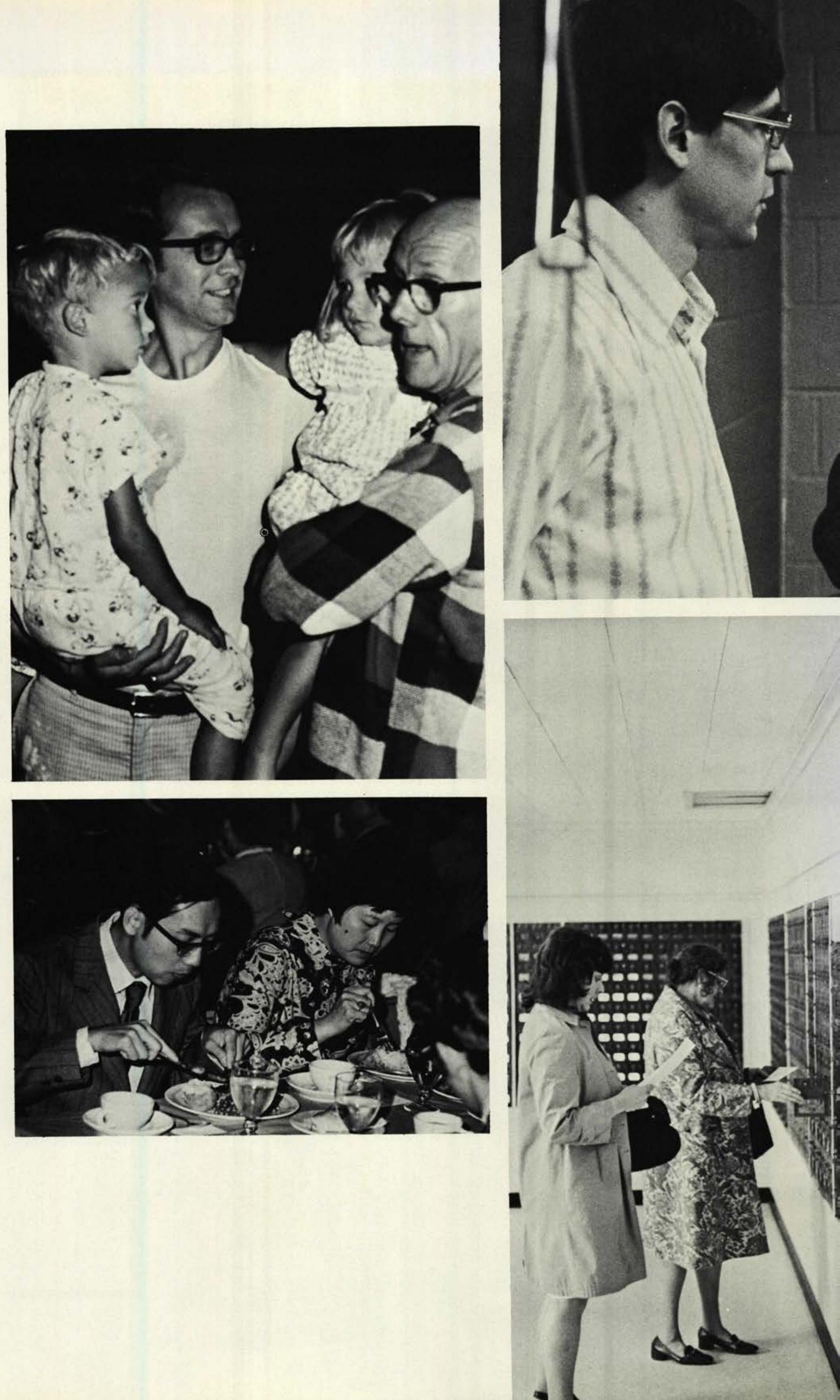


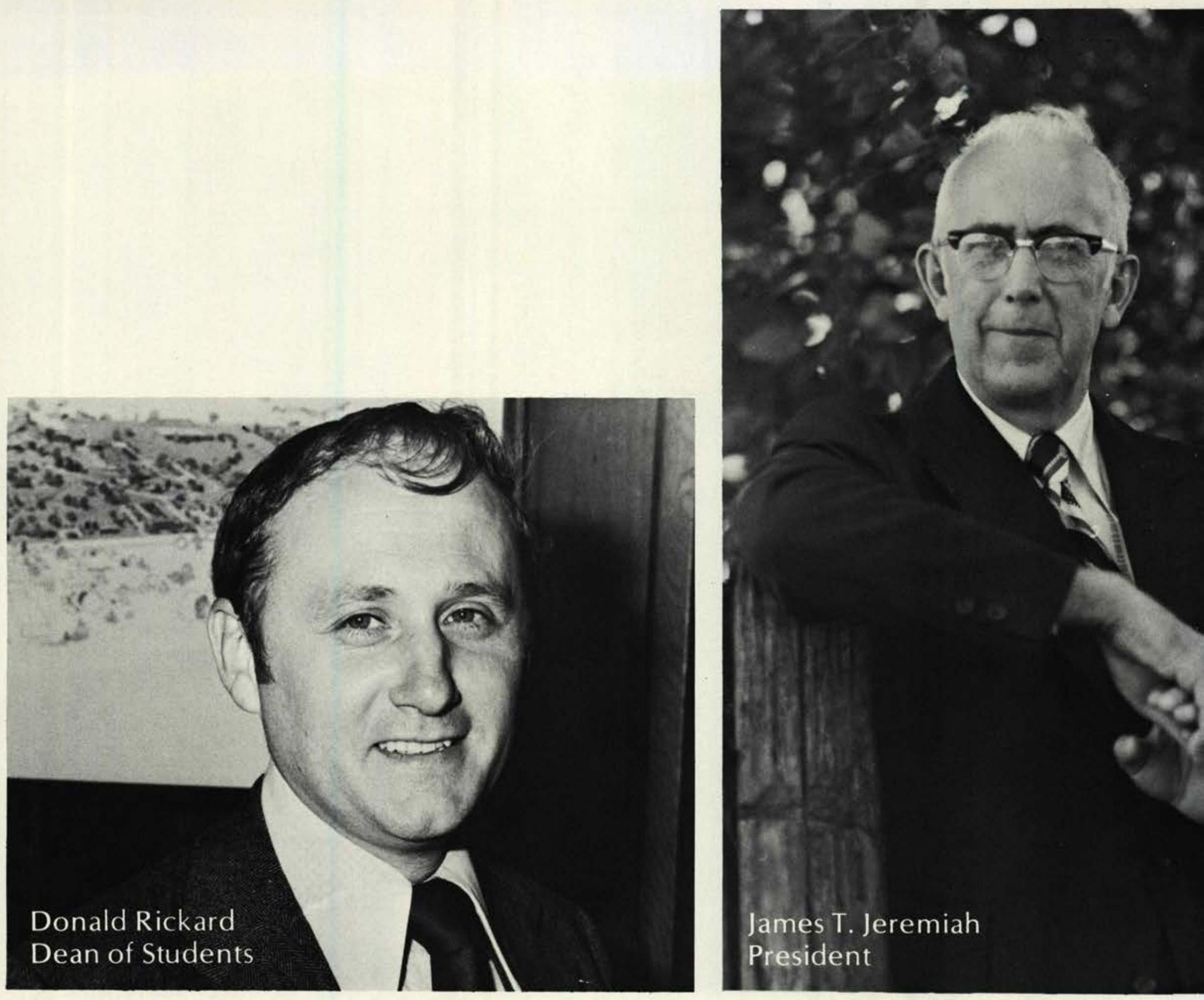

\section{Administration}

\section{Kenneth St. Clair}

Business Manager
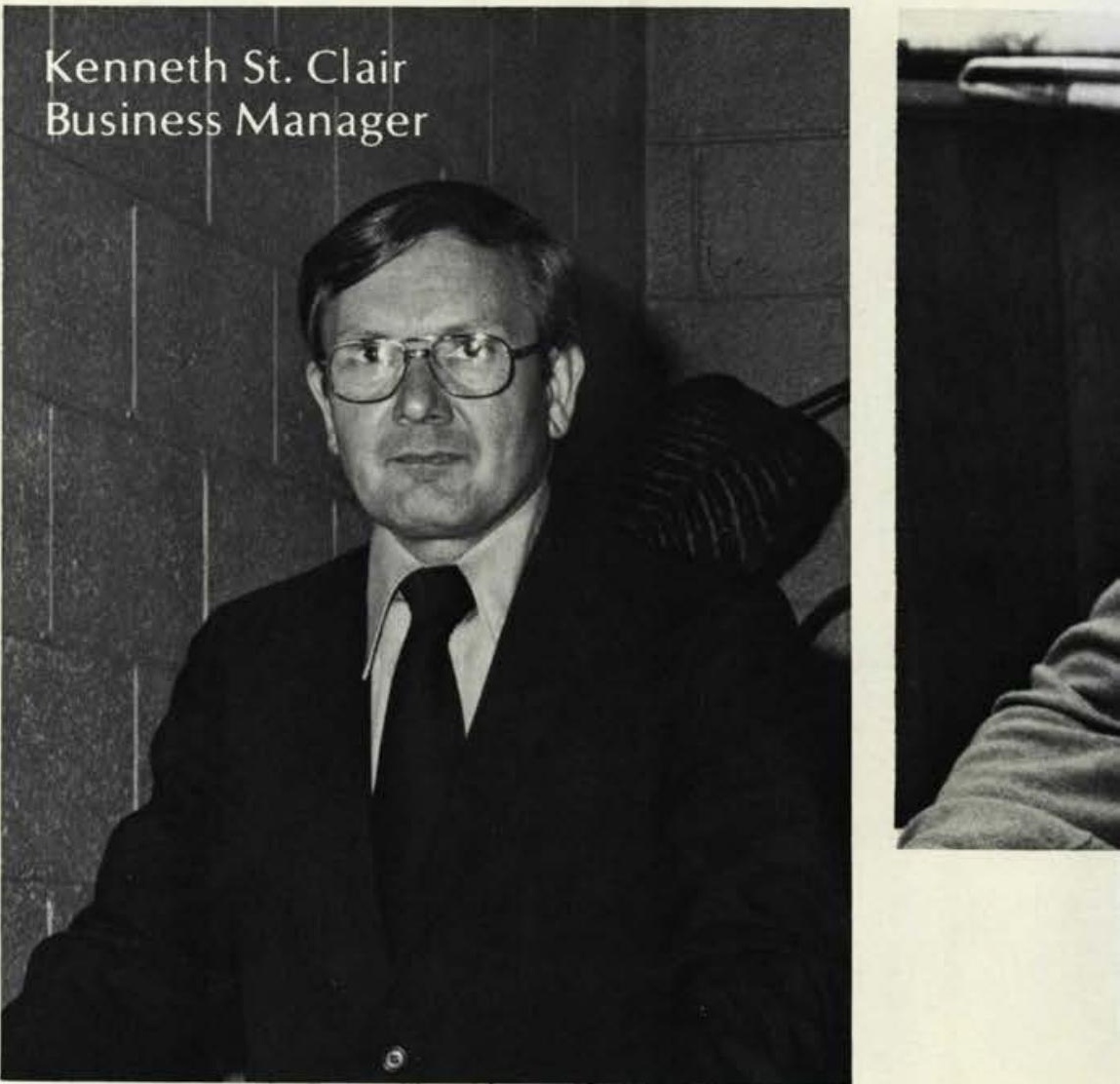


\section{Administration}
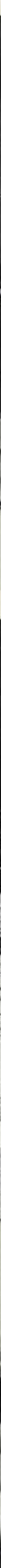

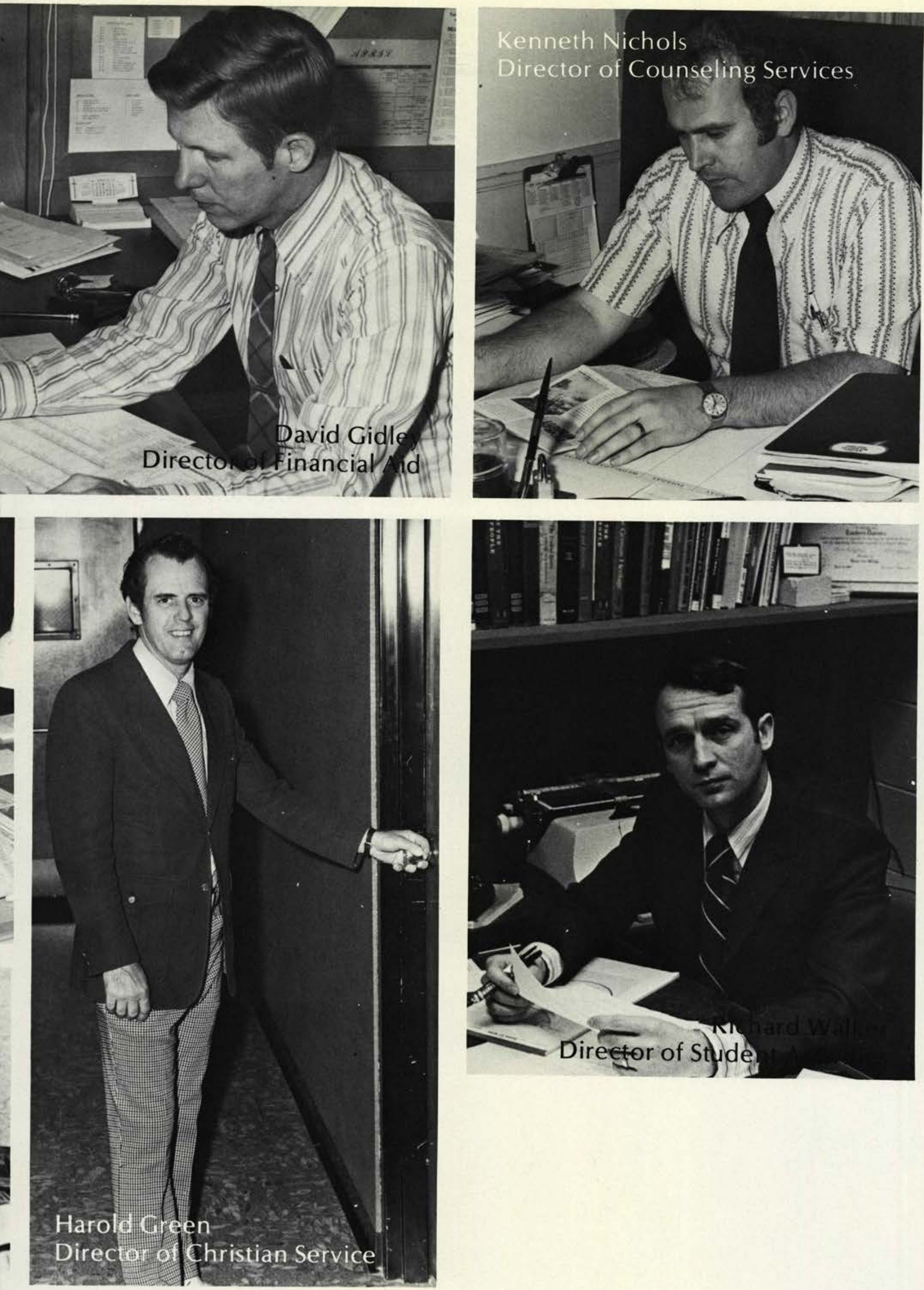

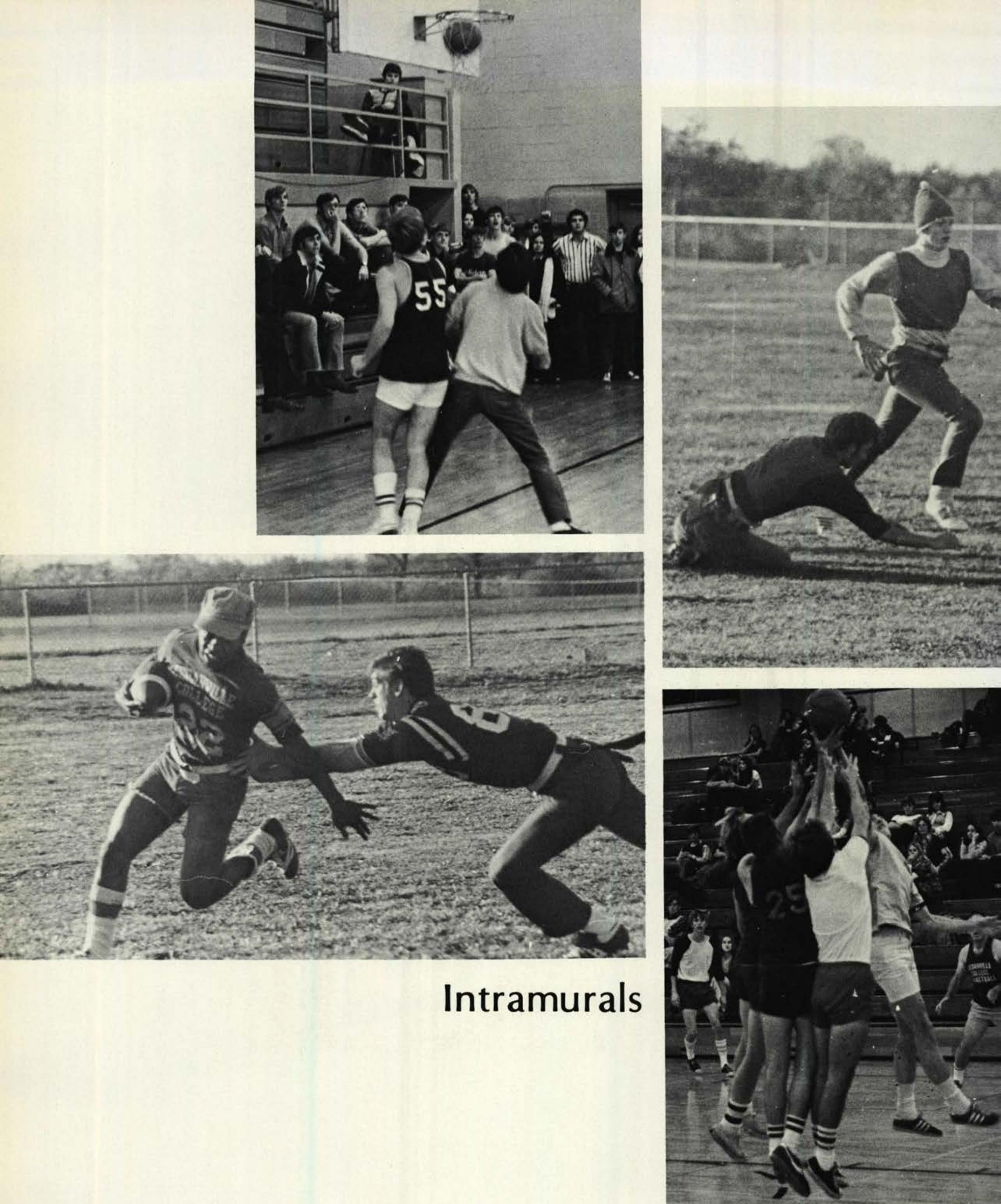

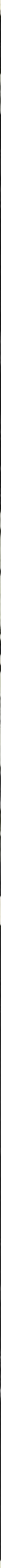

Varsity Team: Bruce Spencer, Jon Myers, Dale Thatcher, Bill Potter, Martin Book, Steve Young, Sam McGinnis, John Eissens, Dan Coomes, Ric Watson, Greg Webster, Not Pictured: Tom Burnette, Rock Thacker, Charlie Shepherd, mgr.

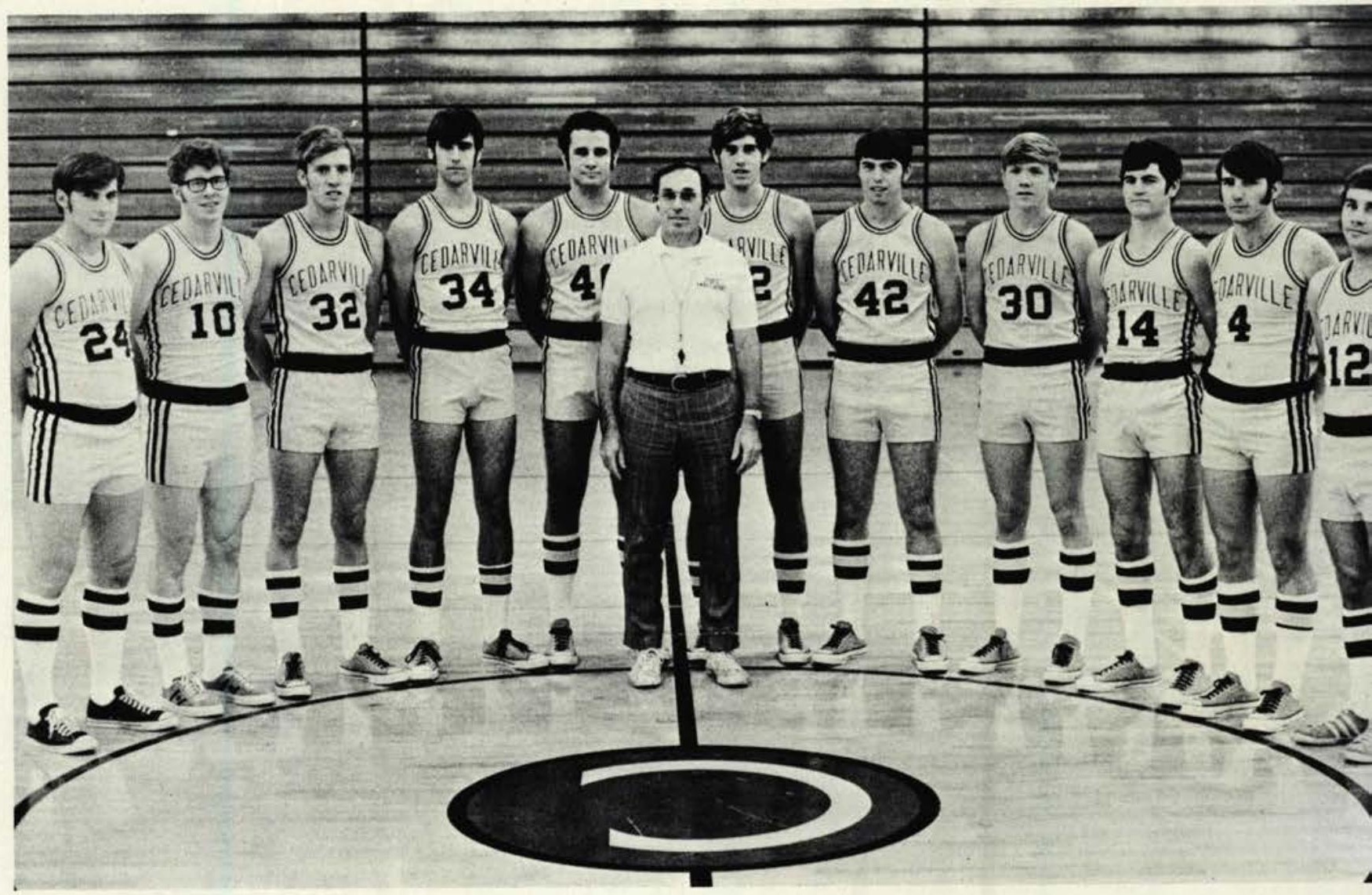

Varsity Basketball Scores, 1973-1974

$\begin{array}{ll}\text { Cedarville } & 83 \\ \text { Cedarville } & 88 \\ \text { Cedarville } & 97 \\ \text { Cedarville } & 93 \\ \text { Cedarville } & 75 \\ \text { Cedarville } & 82 \\ \text { Cedarville } & 73 \\ \text { Cedarville } & 94 \\ \text { Cedarville } & 77 \\ \text { Cedarville } & 80 \\ \text { Cedarville } & 83 \\ \text { Cedarville } & 84 \\ \text { Cedarville } & 82 \\ \text { Cedarville } & 60 \\ \text { Cedarville } & 68 \\ \text { Cedarville } & 79 \\ \text { Cedarville } & 78 \\ \text { Cedarville } & 69 \\ \text { Cedarville } & 66 \\ \text { Cedarville } & 77 \\ \text { Cedarville } & 81 \\ \text { Cedarville } & 77 \\ \text { Cedarville } & 78 \\ \text { Cedarville } & 65 \\ \text { Cedarville } & 89 \\ \text { Cedarville } & 75\end{array}$

Letourneau

Barrington

Trinity

Indiana-Purdue

Bluffton

Rio Grande

Marion

Tiffin

St. Francis

Ft. Wayne Bible

Tiffin

Wilberforce

Malone

Ohio Dominican

Central State

Urbana

Wilberforce

Rio Grande

Walsh

Ohio Dominican

Urbana

Walsh

Grace

Malone

Wilmington

Wright State

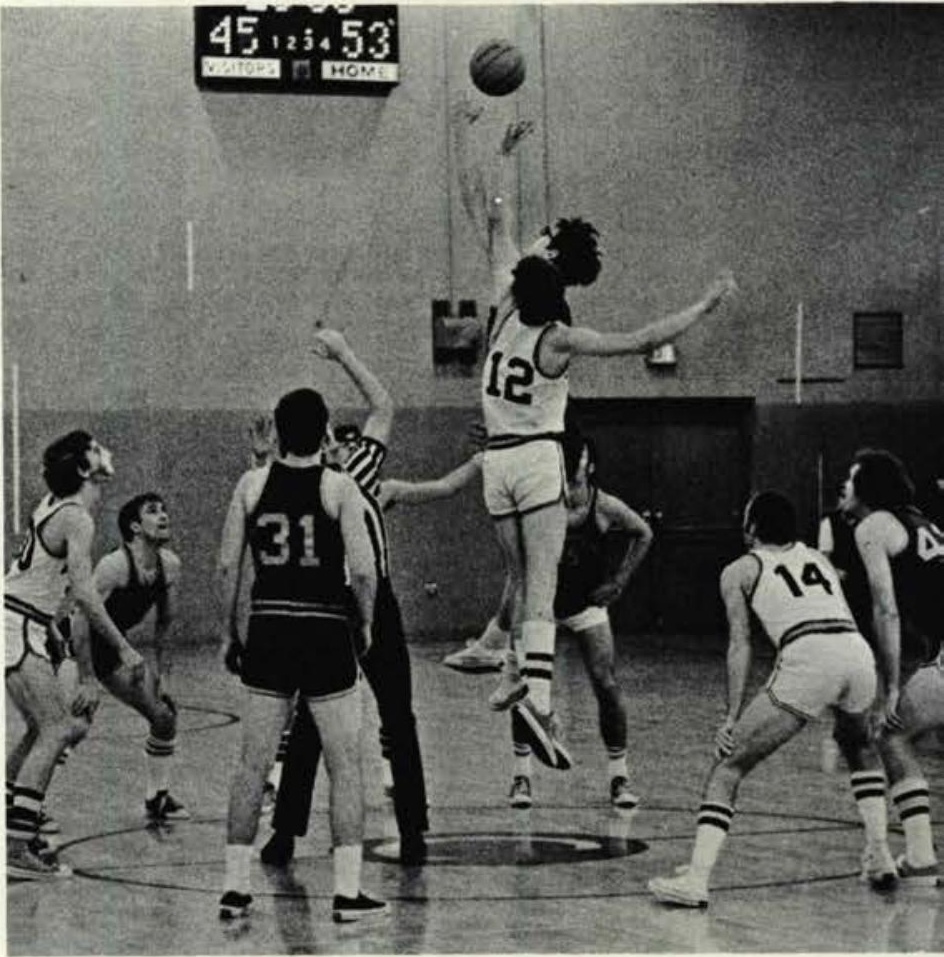

Basketball '74 

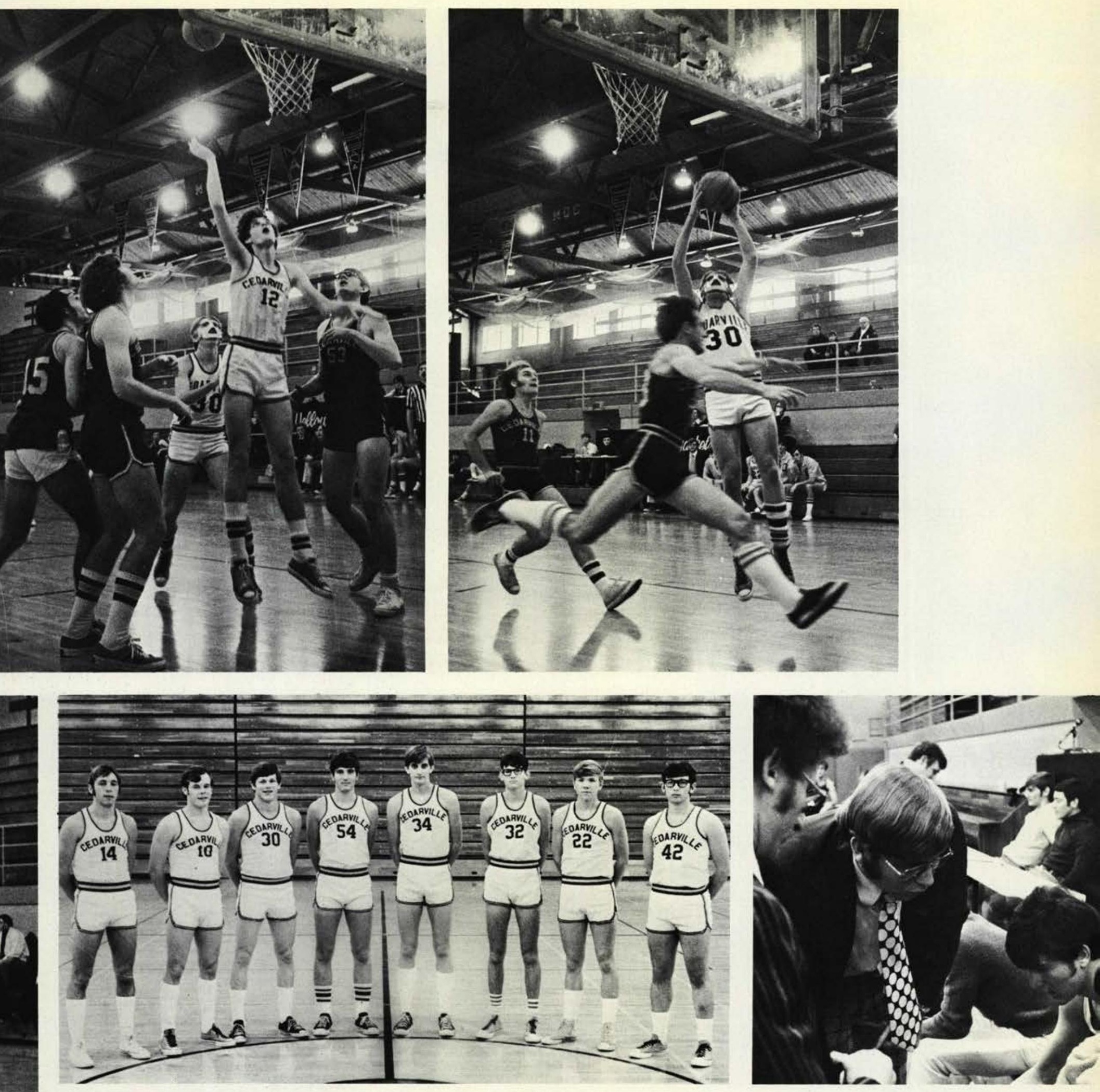

J. V. Team: Sonny Tudor, Jeff Bowen, Steve Smith, Chip Bernhard, Rock Thacker, Jim Reed, John Eissens, Dave Hickey. Not Pictured: Dave DeLancy, Tom Burnett, Coach McDowell, Charlie Shepherd, mgr. 

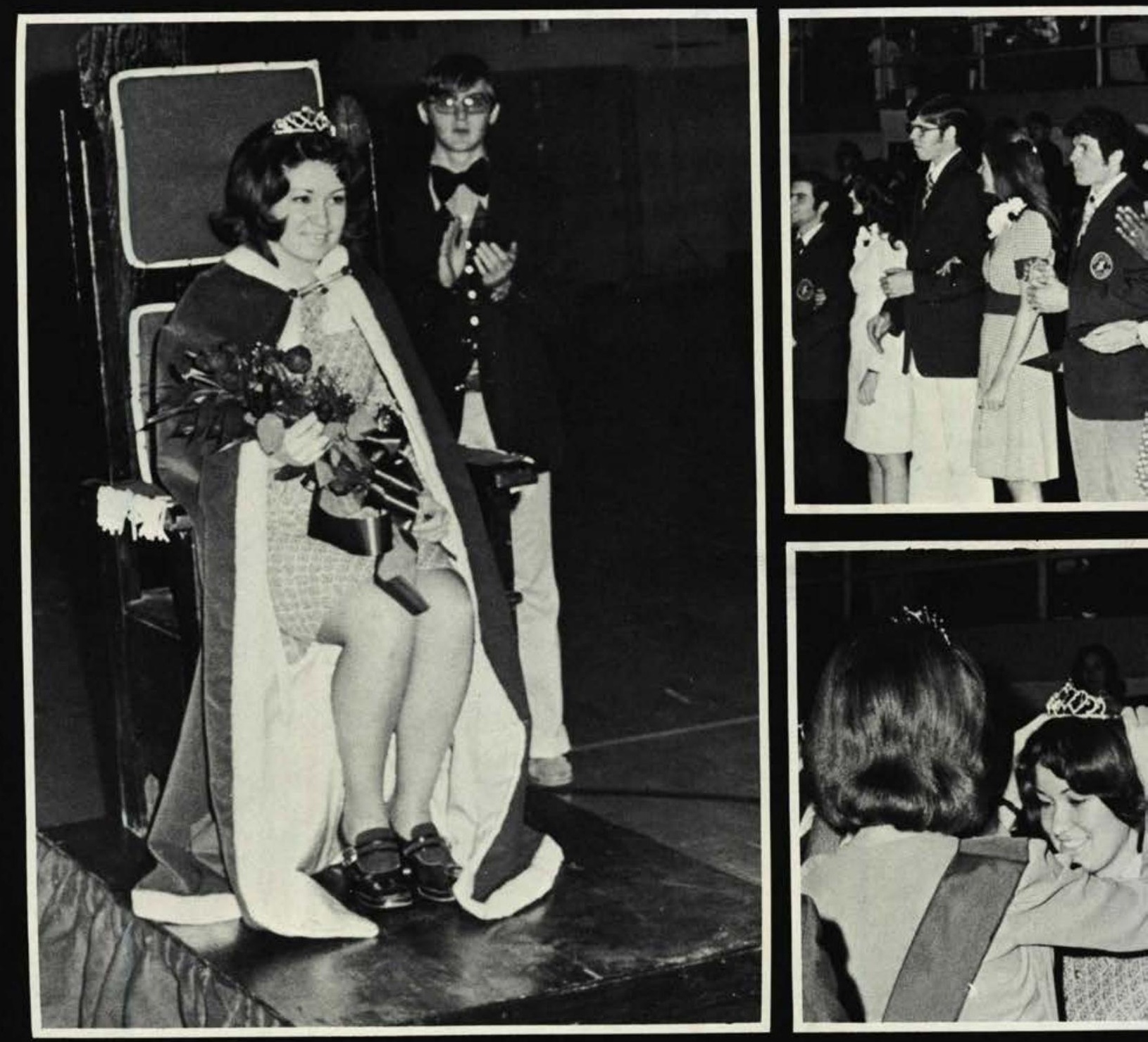

AX Sweetheart

Becky Lamb
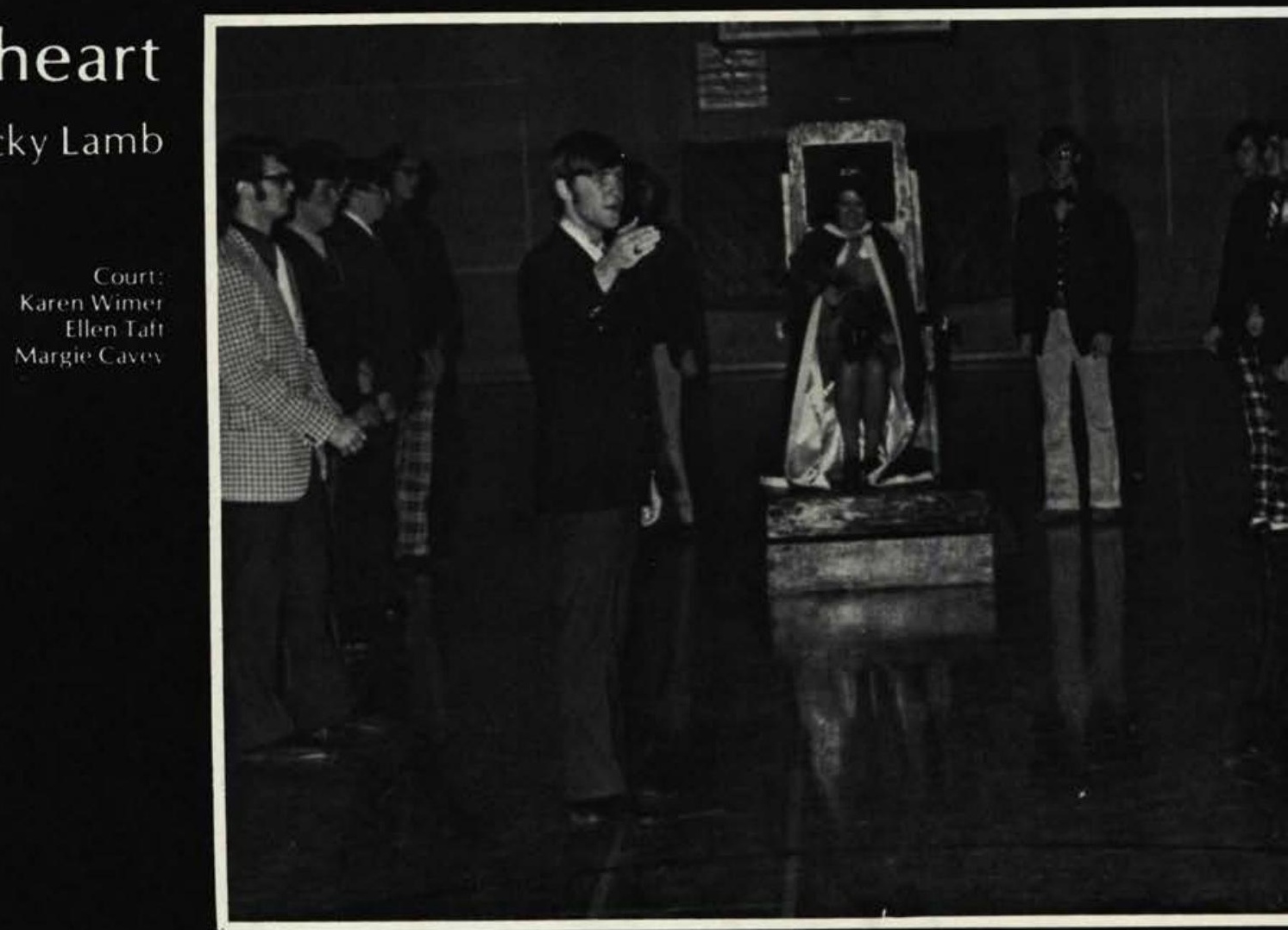


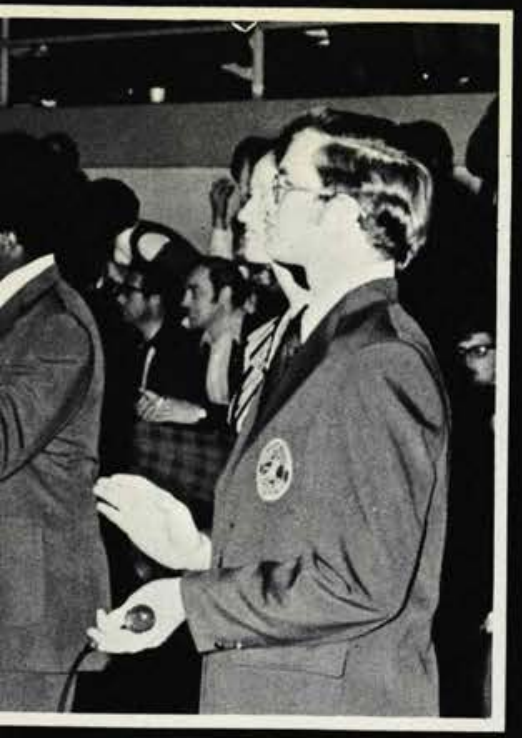

Bob Sampson, Steve Francis, I im Barker, Andy Cole, Earl Albertson, Jon Mitchell, Jim Goet/, Nate Walker Maik Seeley, Marlin Cayton, Iarry Strong, Glenn Fawcett, Doug Schroeder, Dennis Iwigg, Bruce William Roger Smith, Don Davis, Iim Grabianowski, Jim Delange, Roy Perkins, Chuck Elliot, Jim Douglas, Dave True, Bob Burkhart, Dan Estes, Randy Douglas Jim Hawbaker, Tom Pycraft, Jim Hints, Sam Kester, Ken Howard, Steve Sheetz, Hank Diaz, Jon Bearham, Ron Rufener, Kevin Sims, Dave Cole, George Lee, Larry Barnett, Jerr Gilyard, Jeff Wolfe
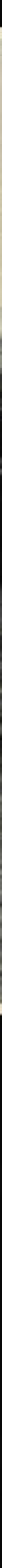

Alpha Chi 

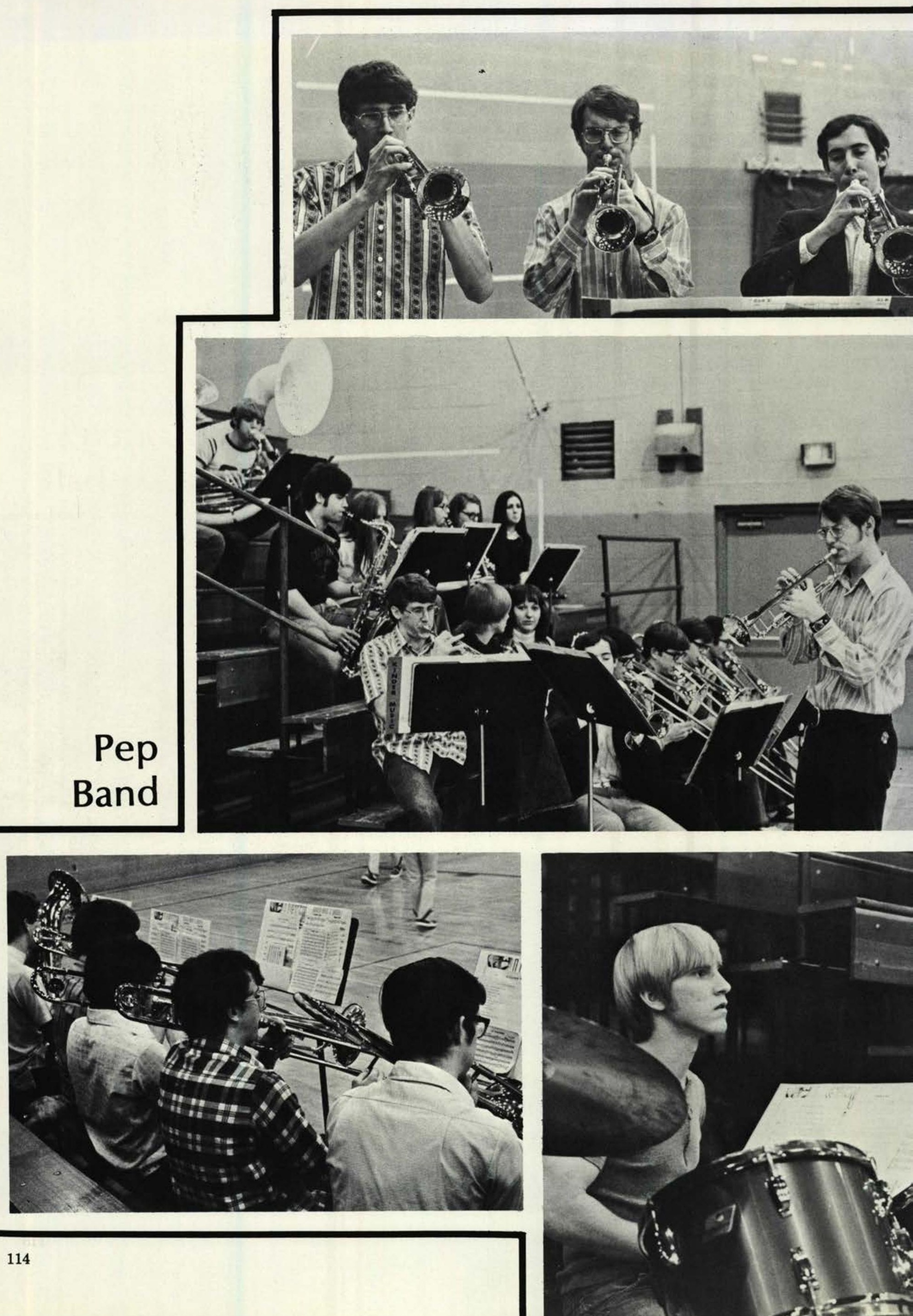
VARSITY WOMEN'S BASKETBALL SCHEDULE

$\begin{array}{ll}\text { Cedarville } & 68 \\ \text { Cedarville } & 33 \\ \text { Cedarville } & 73 \\ \text { Cedarville } & 55 \\ \text { Cedarville } & 46 \\ \text { Cedarville } & 42 \\ \text { Cedarville } & 34 \\ \text { Cedarville } & 46 \\ \text { Cedarville } & 43 \\ \text { Cedarville } & 43 \\ \text { Cedarville } & 41 \\ \text { Cedarville } & 65 \\ \text { Cedarville } & 50 \\ \text { Cedarville } & 69 \\ \text { Cedarville } & 60 \\ & \\ \text { Cedarville } & 52 \\ \text { Cedarville } & 63 \\ \text { Cedarville } & 41 \\ \text { Cedarville } & 42\end{array}$

Wright State

Wittenberg

Manchester (IN)

Miami

Bowling Green

Ohio State

Western Michigan

Ohio Dominican

Dayton

Ashland

Wooster

Mt. St. Joe

Dayton

Central State

State Tournament

Cincinnati

$\begin{array}{ll}52 & \text { Dayton } \\ 63 & \text { Miami } \\ 41 & \text { Bowling Green } \\ 42 & \text { Ohio University }\end{array}$

4th in State

Ohio University

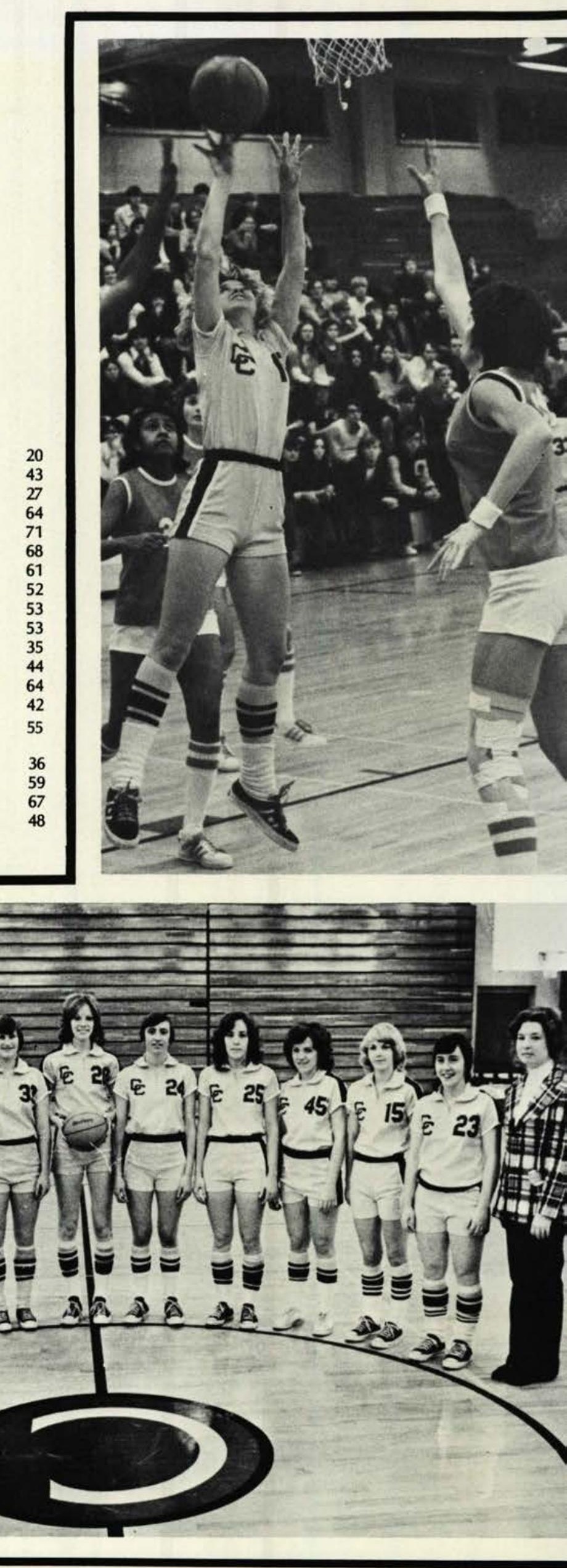

Coach Jeremiah

Debbie Jones, Mgr.

Nancy Towle

Denise Edwards

Sandy Holwerda

Sandy Finch

Vicki Jordon

Sally Orihood

Marianne Frauenknech

Sherrill Cressman

Sherry Burns

Laureen Clark

Cindy Wiggins, Mgr.

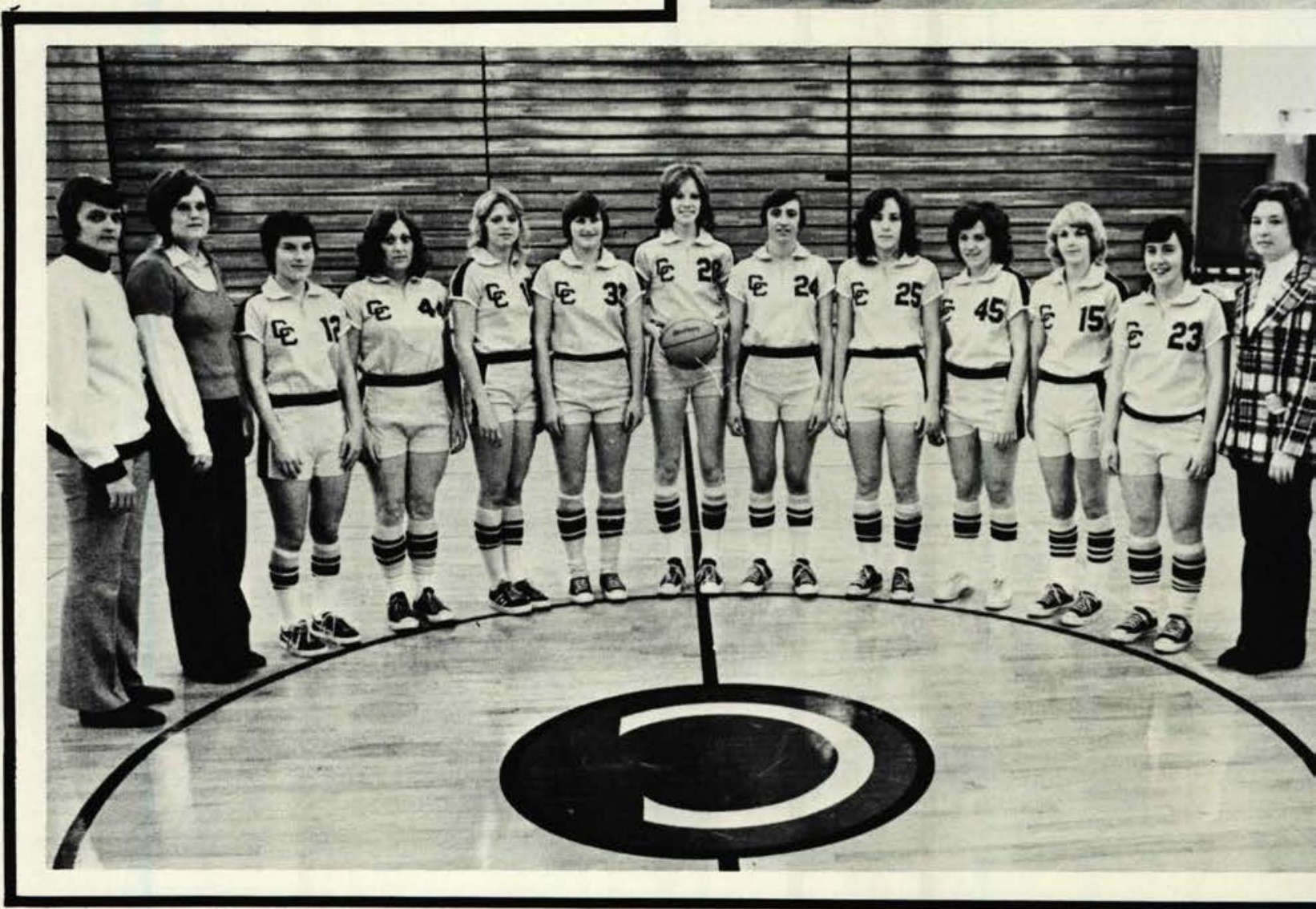



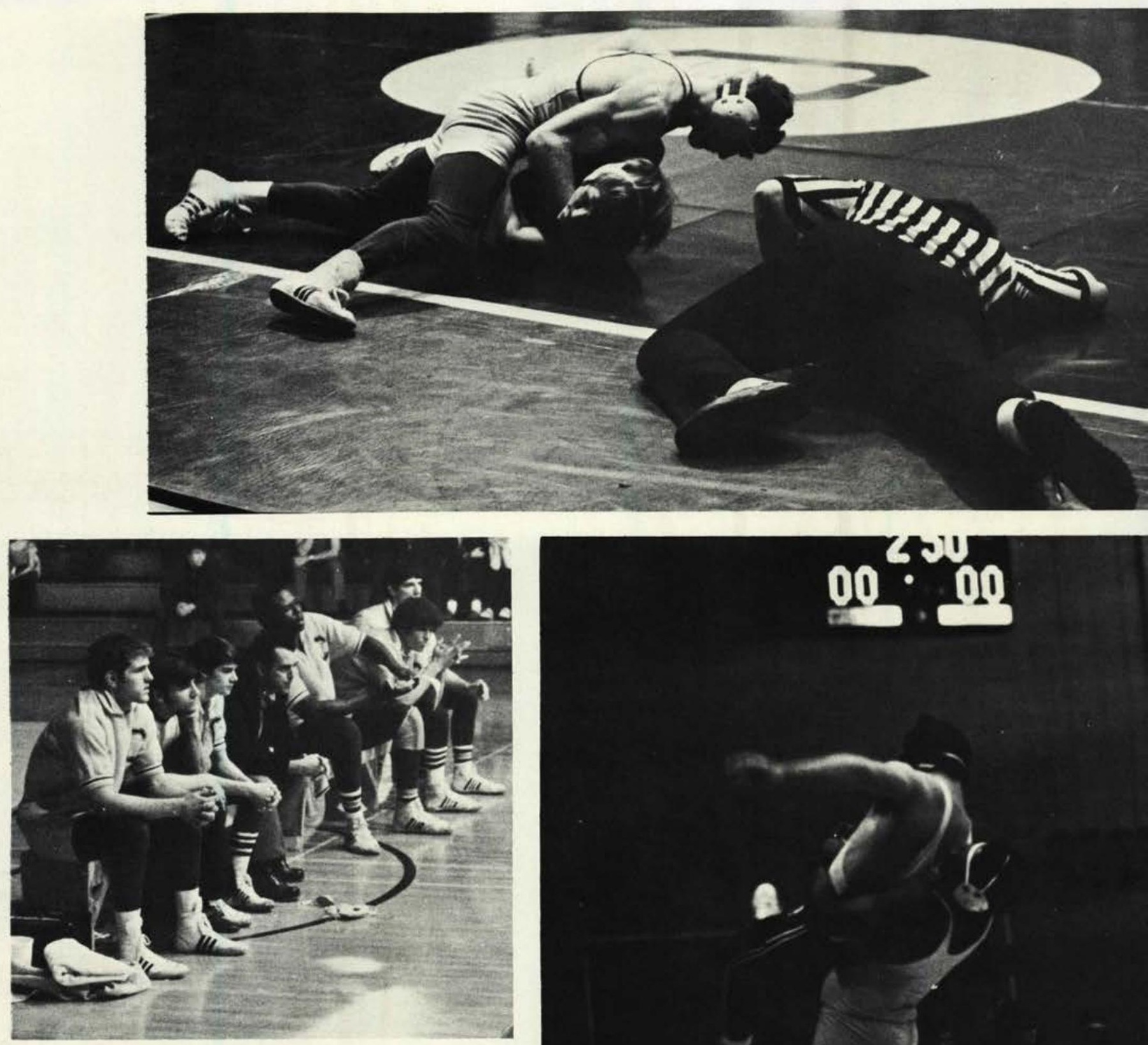

Wrestling
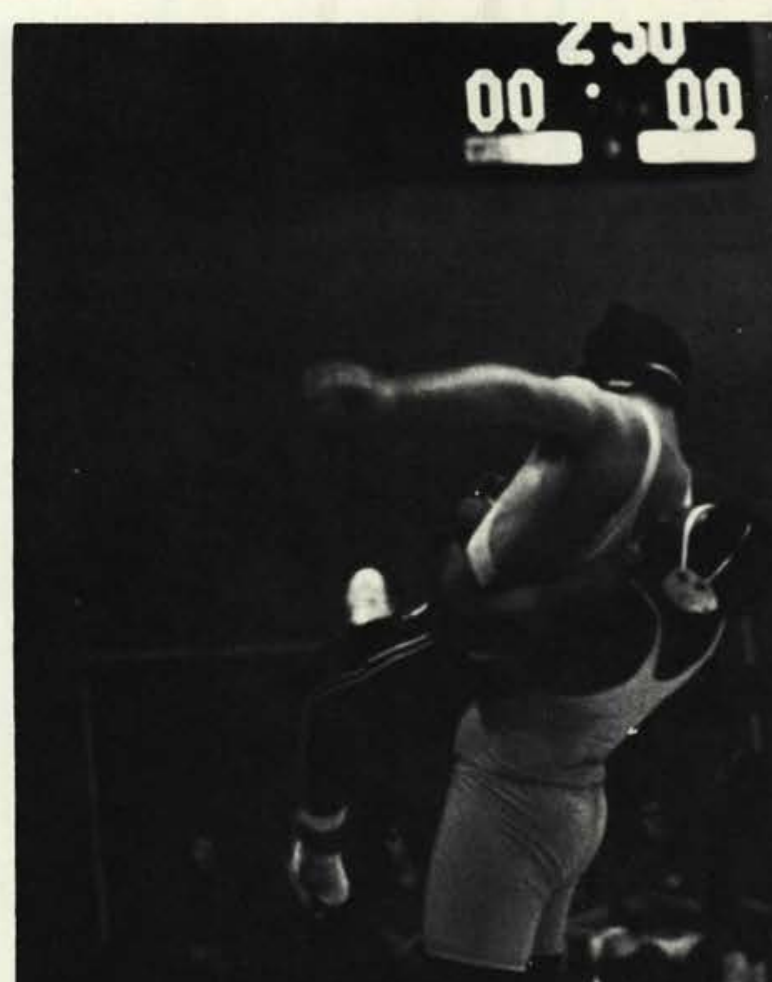

3) in 3

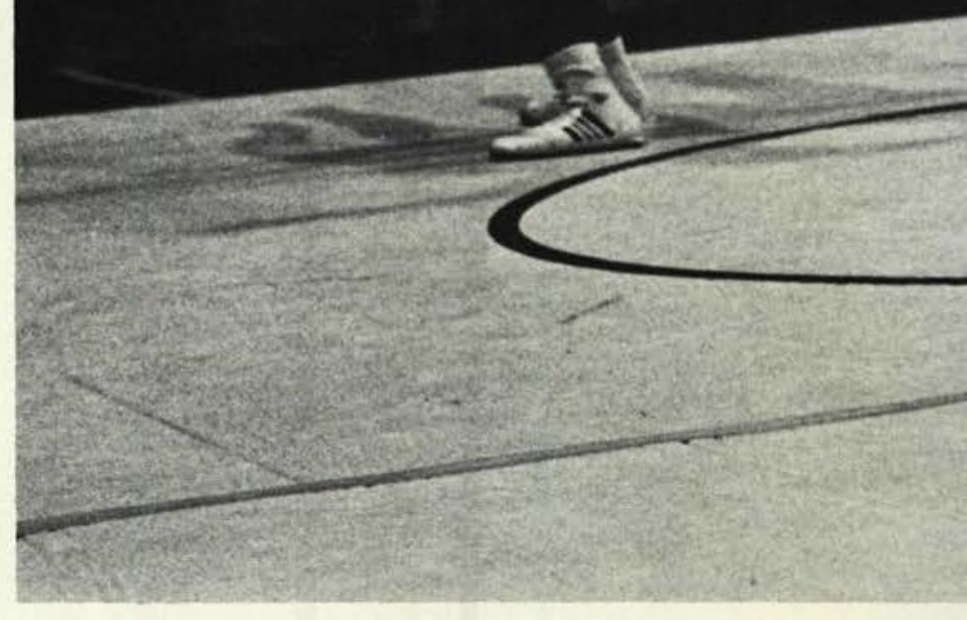



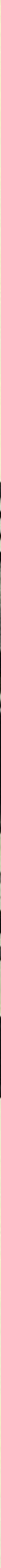

Cedarville

Cedarville

Cedarville

Cedarville

Cedarville

Cedarville

Cedarville

Cedarville

Cedarville

Cedarville

Cedarville

Cedarville

Cedarville

Cedarville

Cedarville

Cedarville

Taylor University

Wheaton College

MOC

NAIA District \#22

NAIA Nationals
Wrestling Schedule

Delta
Hilldale
Wilberforce
Olivet
Malone
Northern
Dayton
Urbana
Moorehead St. (Ky.)
Eastern Ky.
Wright State
Wilmington
Huntington
Findlay
No. Ky. St. U.
Centre (Ky.)
ents
3rd of 8
10th of 14
3rd
4th
Vic Millard 5th




\section{Varsity C}
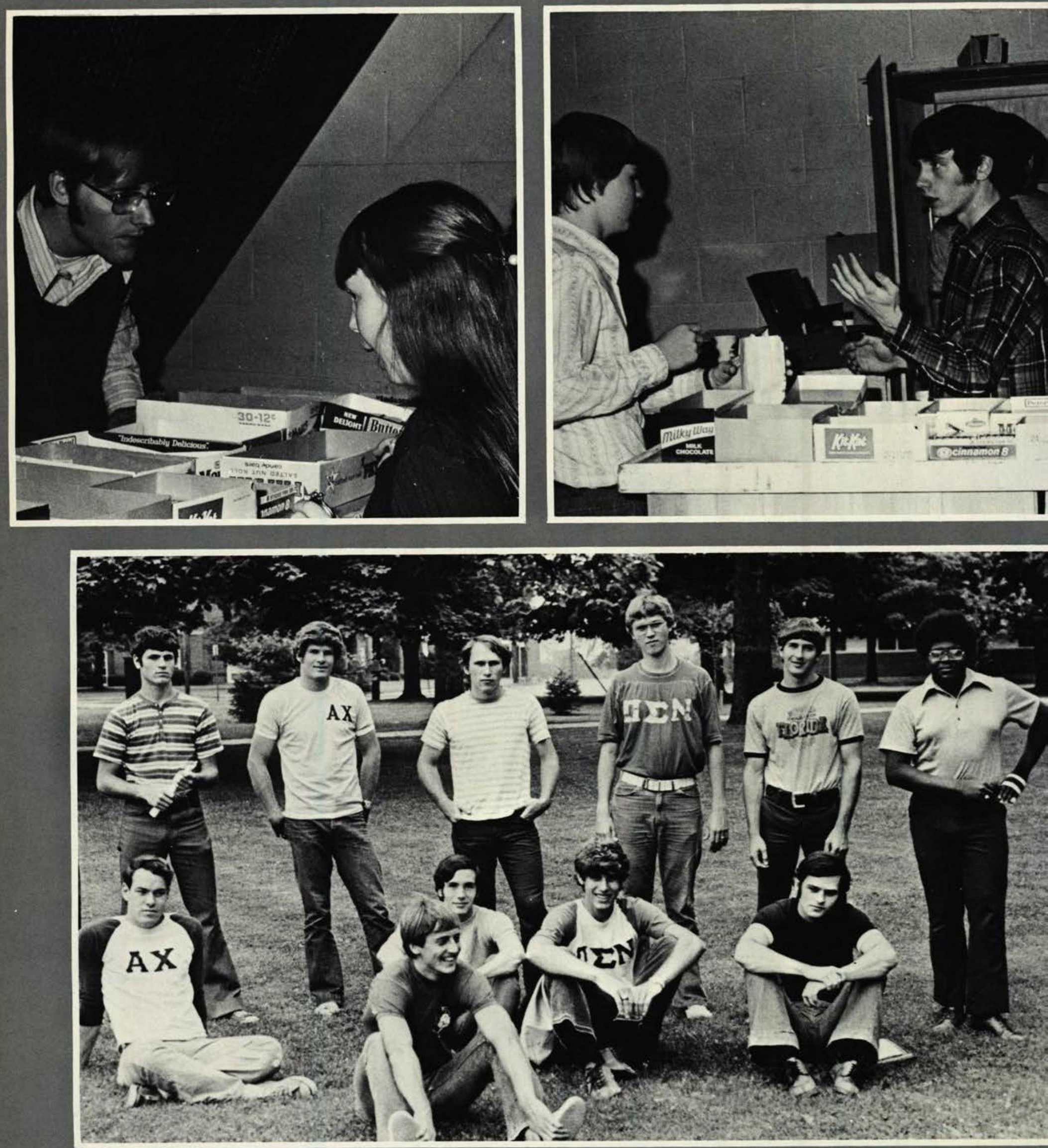

SEATED: Andy Cole, Barrett Luketic, Paul Beck, Steve Young, Dennis Lowstetter STANDING: Dan Coomes, Steve Francis, Tom Hopewell, Kirk Martin, Rod Hansen, Jerry Gilyard 

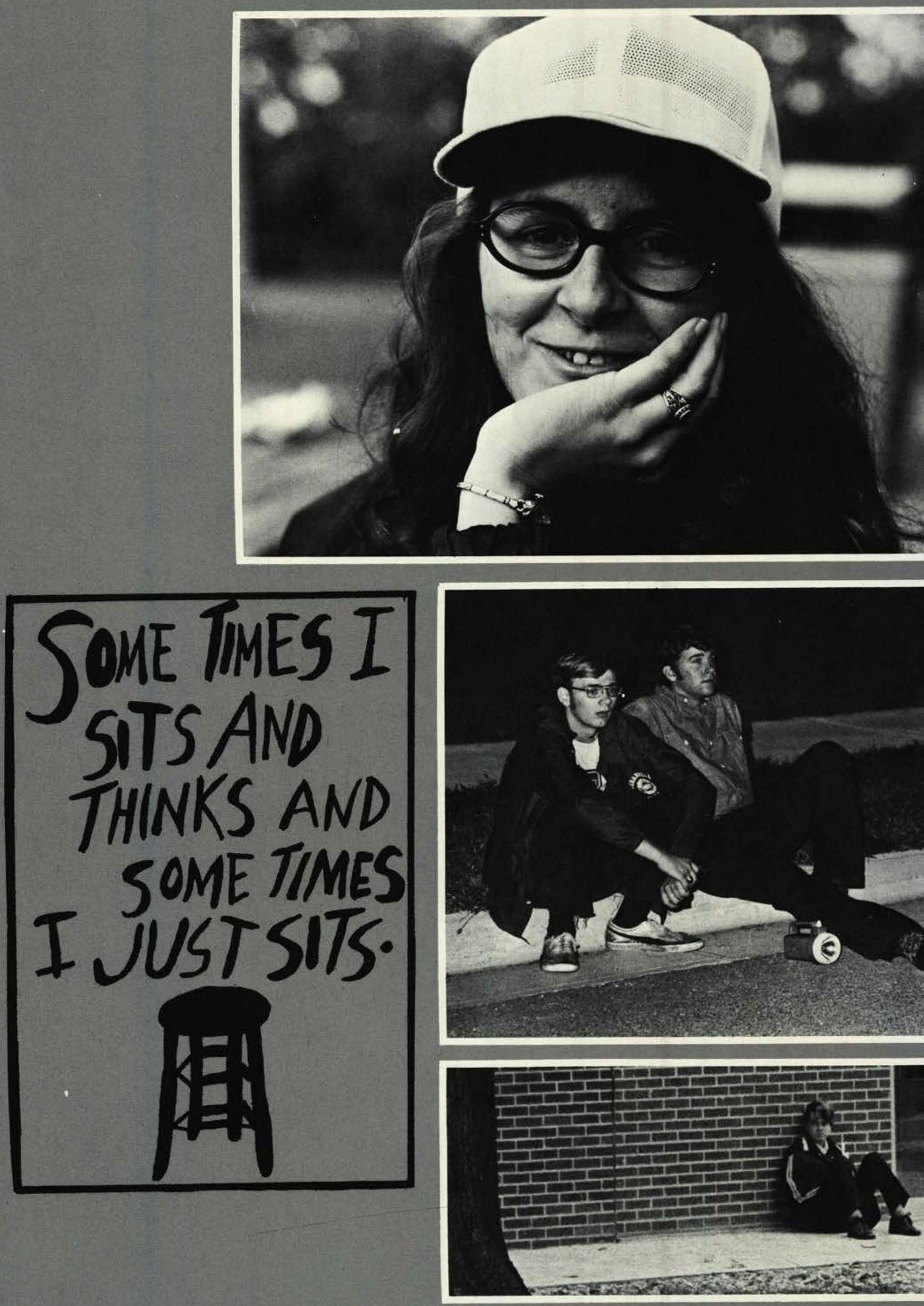


\section{"Spectating"}

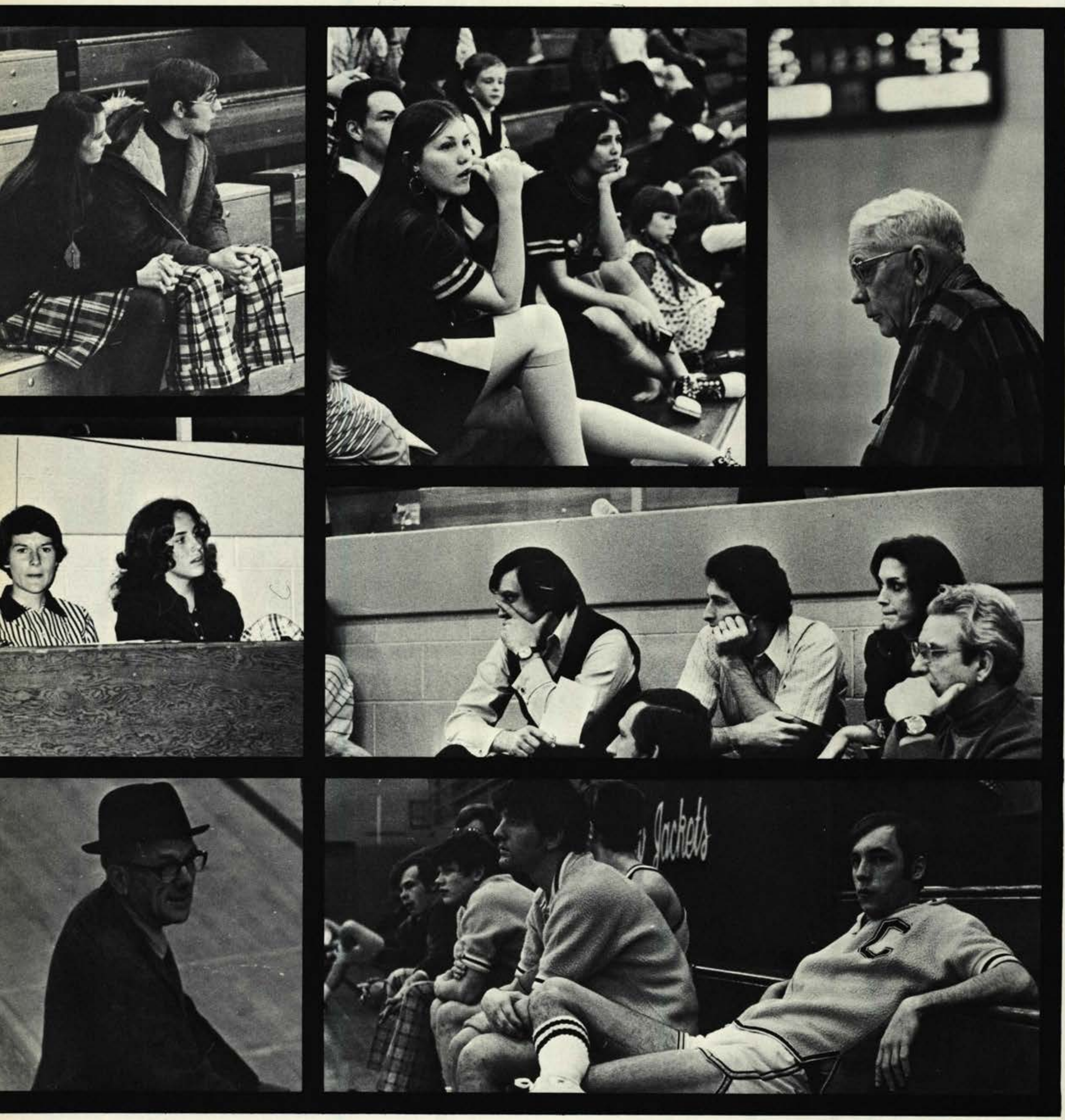




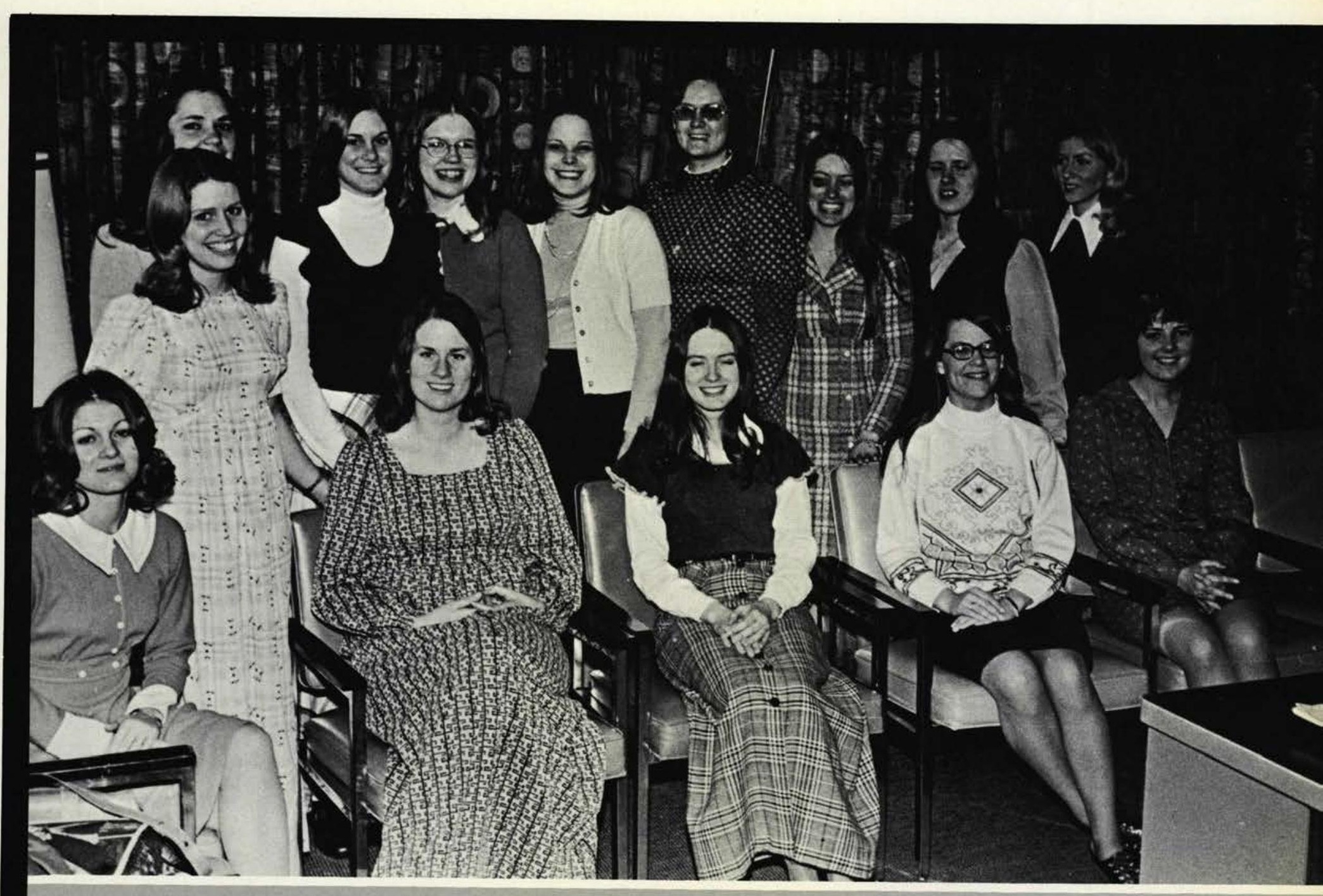
Connie Stolzfus, Sue Seldon, Bev Ziegler, Peg Wagner, Deb Dudley, Deb Conrad, Lois Matheny, Cindy Porter, Deb Jones, Carolyn
Sapp, Peg Temple, Cathy Sharp, Karen Kennedy, Penny Linger.

\section{Kappa Delta Chi}




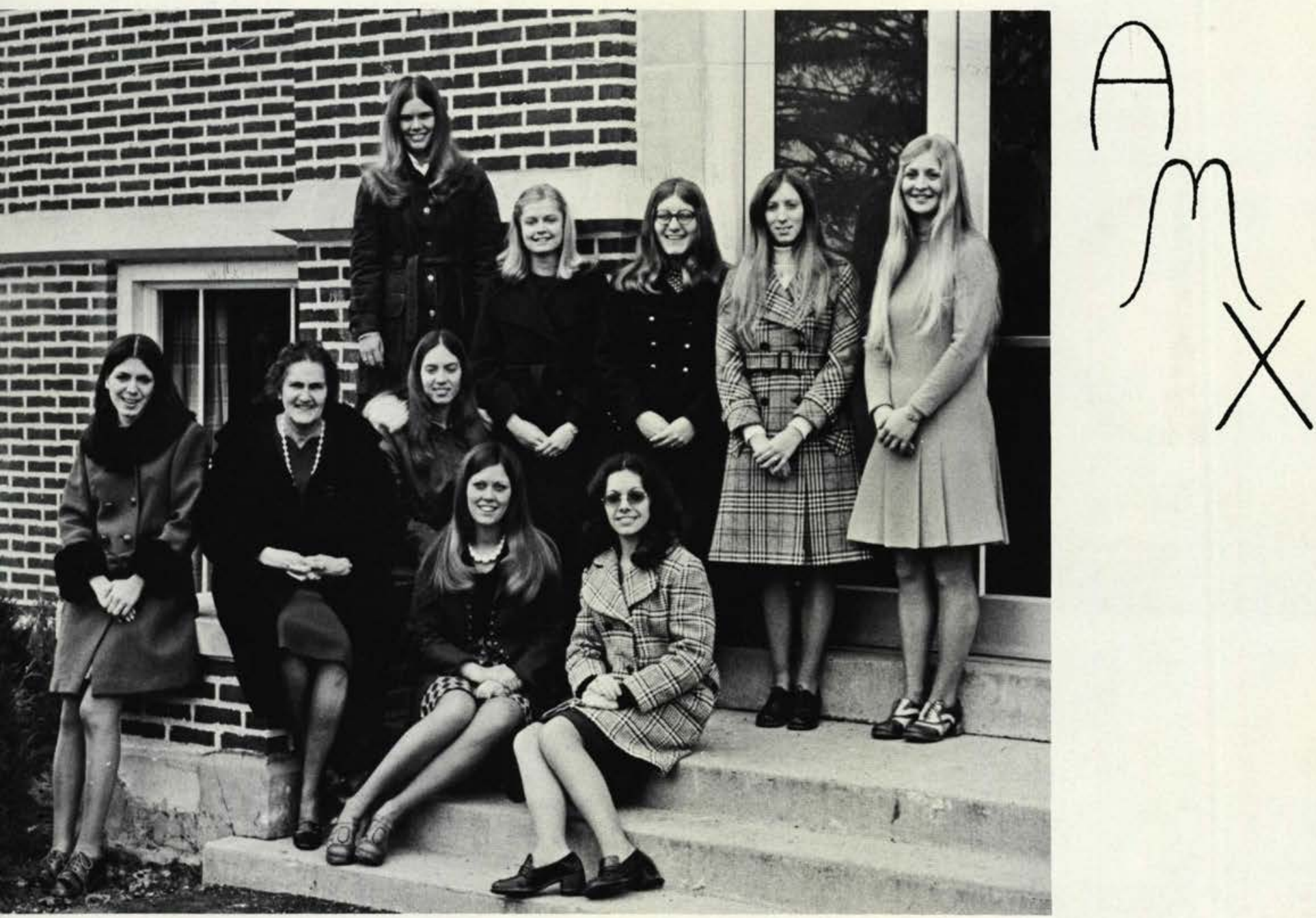

Laura Sollenne, Mrs. Printy, Bev Davison, Sherlyn Hansen, Judy Cline, Sandra Stachler, Darlene Jones, Ruth Emerson, Patsy Northcutt, Kathy L. Scott. 

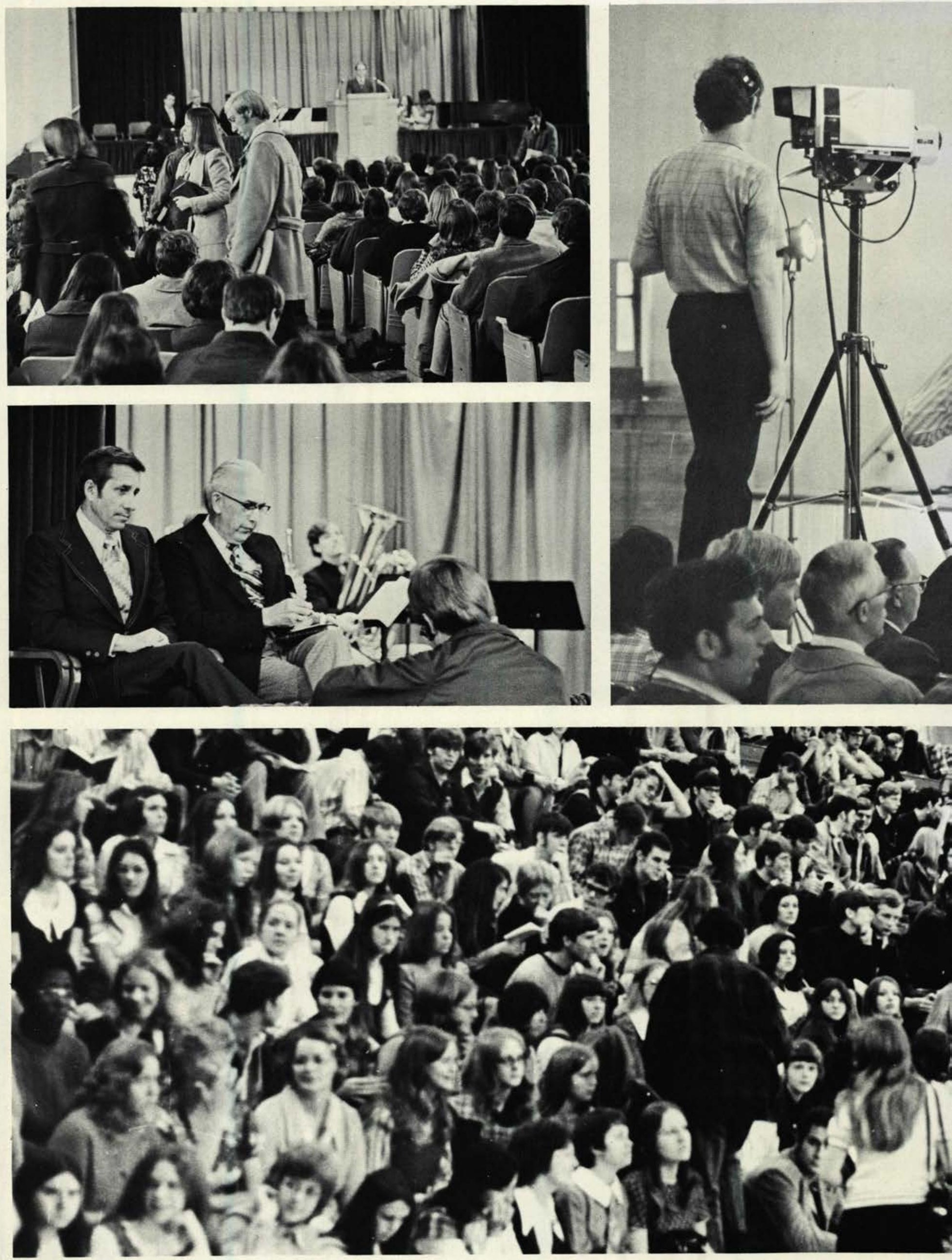

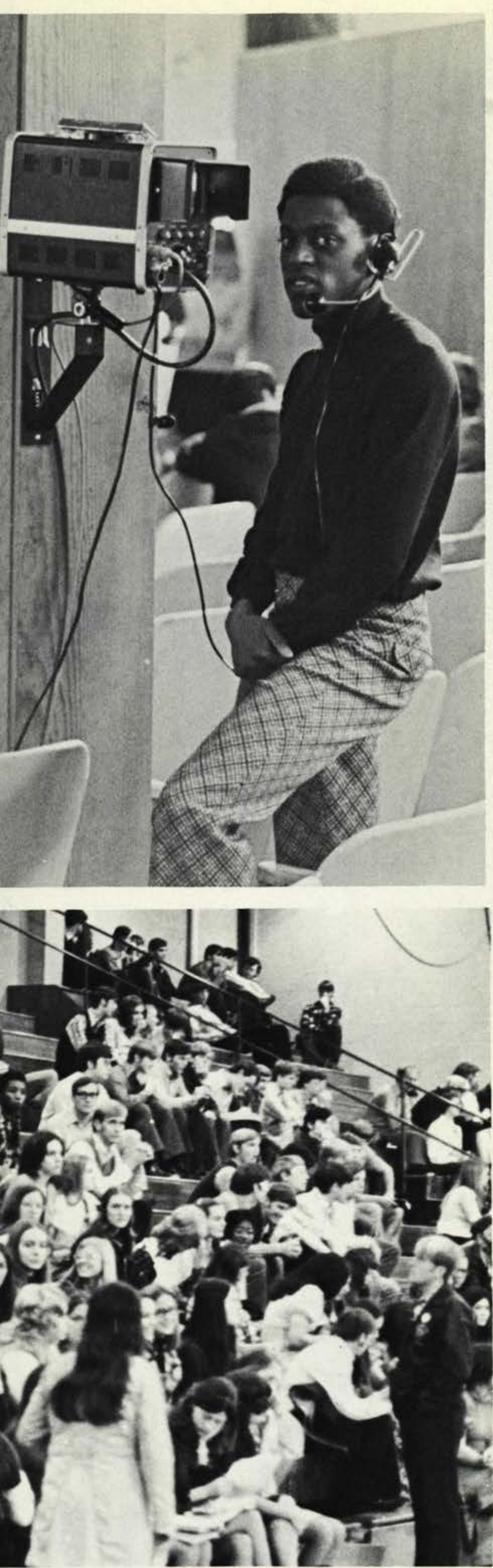

\section{Pausing to Ponder ...}

And can it be that I should gain

An int'rest in the Savior's blood?

Died $\mathrm{He}$ for me, who caused His pain?

For me, who Him to death pursued?

Amazing love!

How can it be that Thou, my God, shouldst die for me?

No condemnation now I dread,

I am my Lord's and He is mine;

Alive in Him, my living Head,

And clothed in righteousness divine.

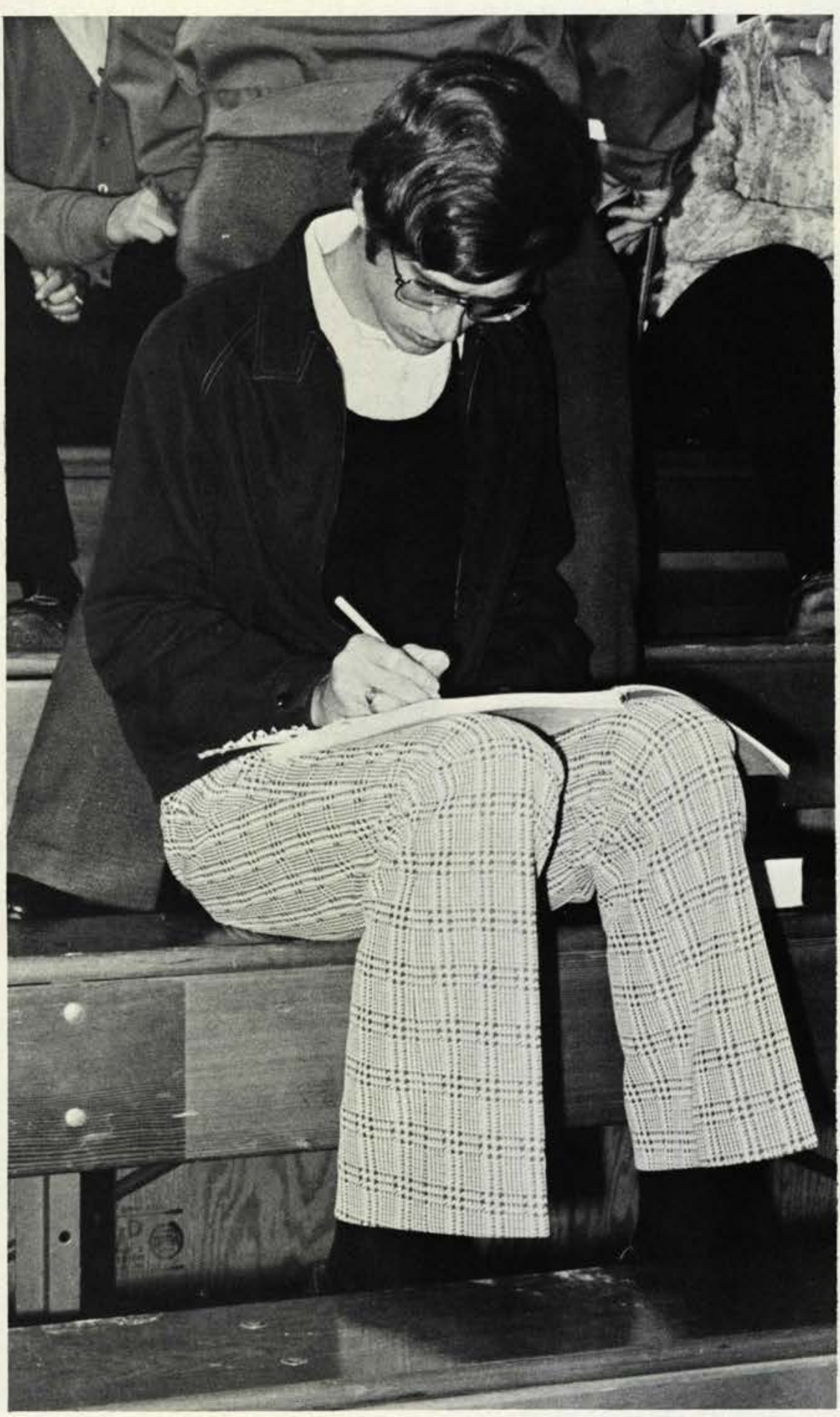




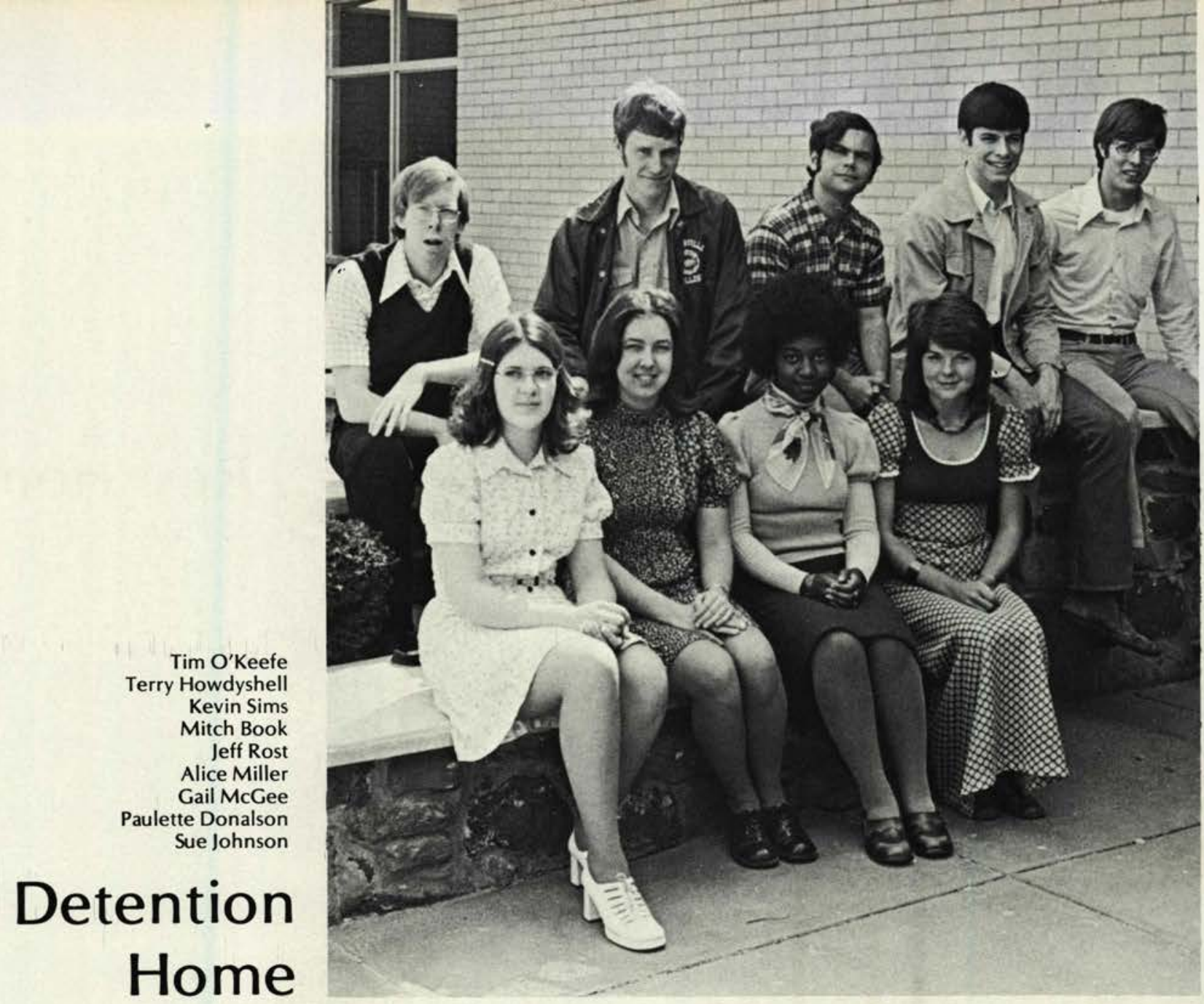

\section{Campus Evangelism}

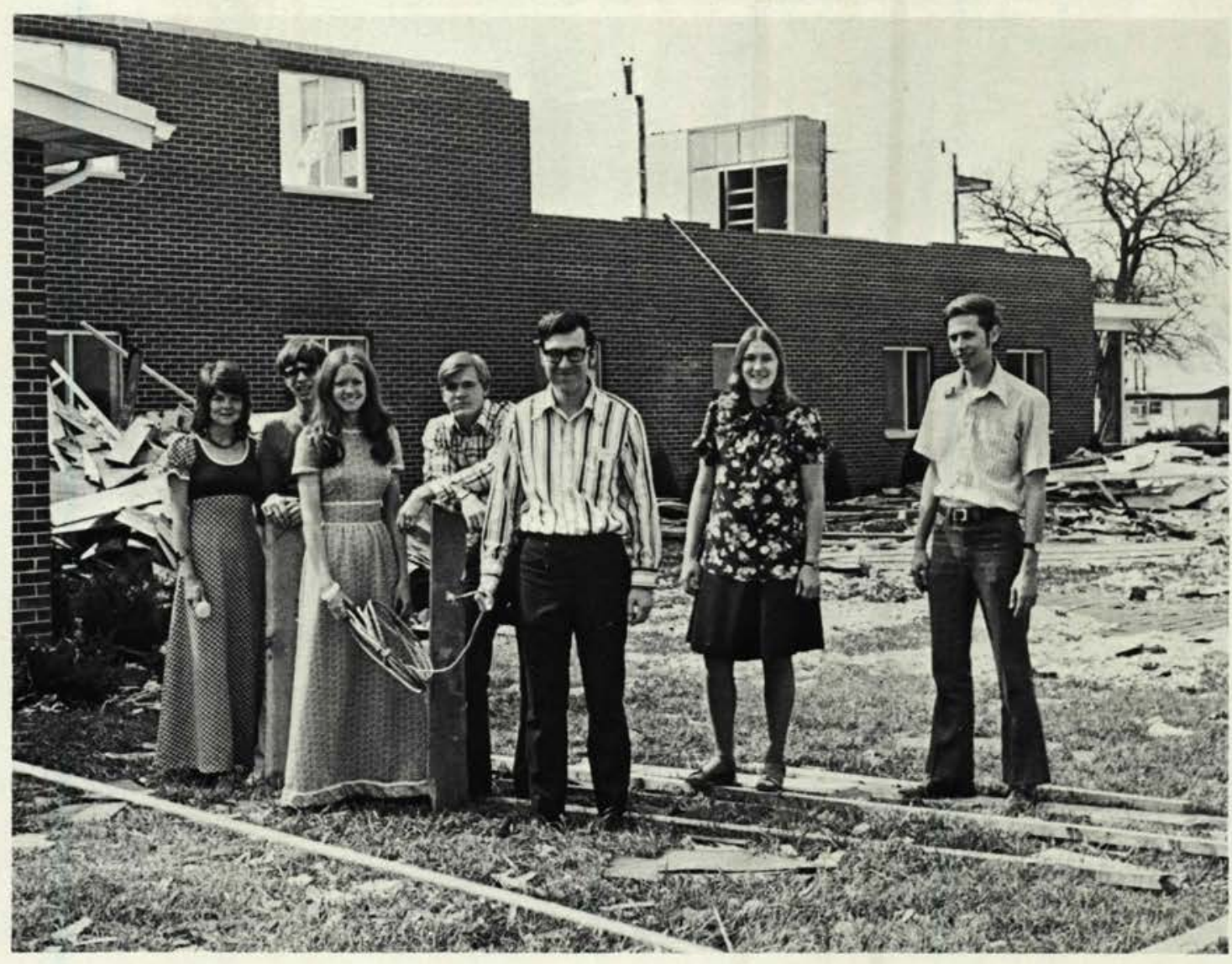

Sue Johnson, Dan Bowman, Ellen Taft, Brad Cronbaugh, Ron Schroeder, Carla Morley, Dale Woolard 


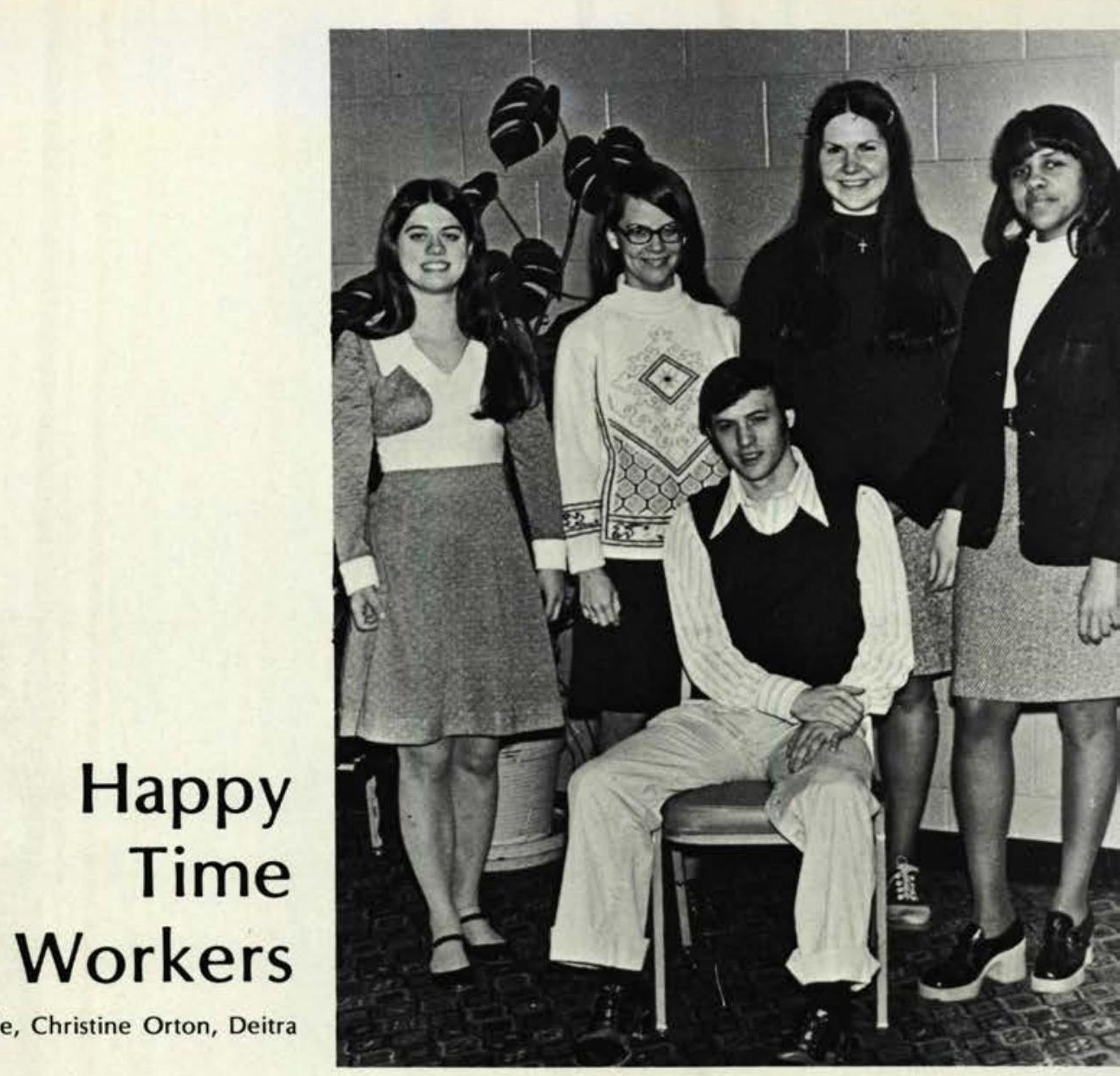

Roma Mack, Peg Temple, Christine Orton, Deitra Marshall, Joe Jenkins.

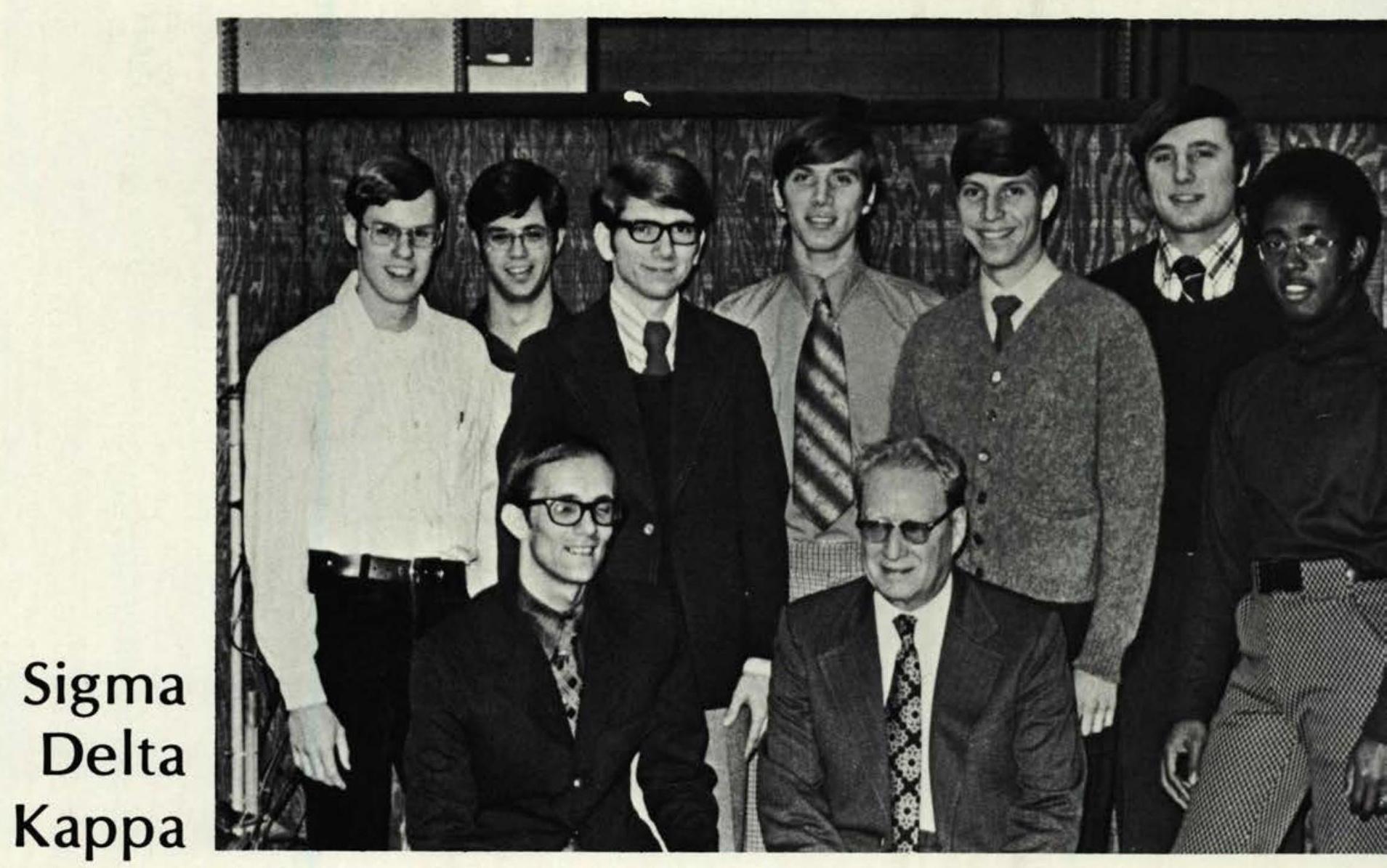

Don Yeaton, Rick Lautzenheiser, Jeff St. Clair, Randy Douglass, Bill Wymer, Doug Weaver, Clancy Parker, SEATED, Martin Cossins, Dr. Armstrong, Advisor. 


\section{Junior Church Workers}

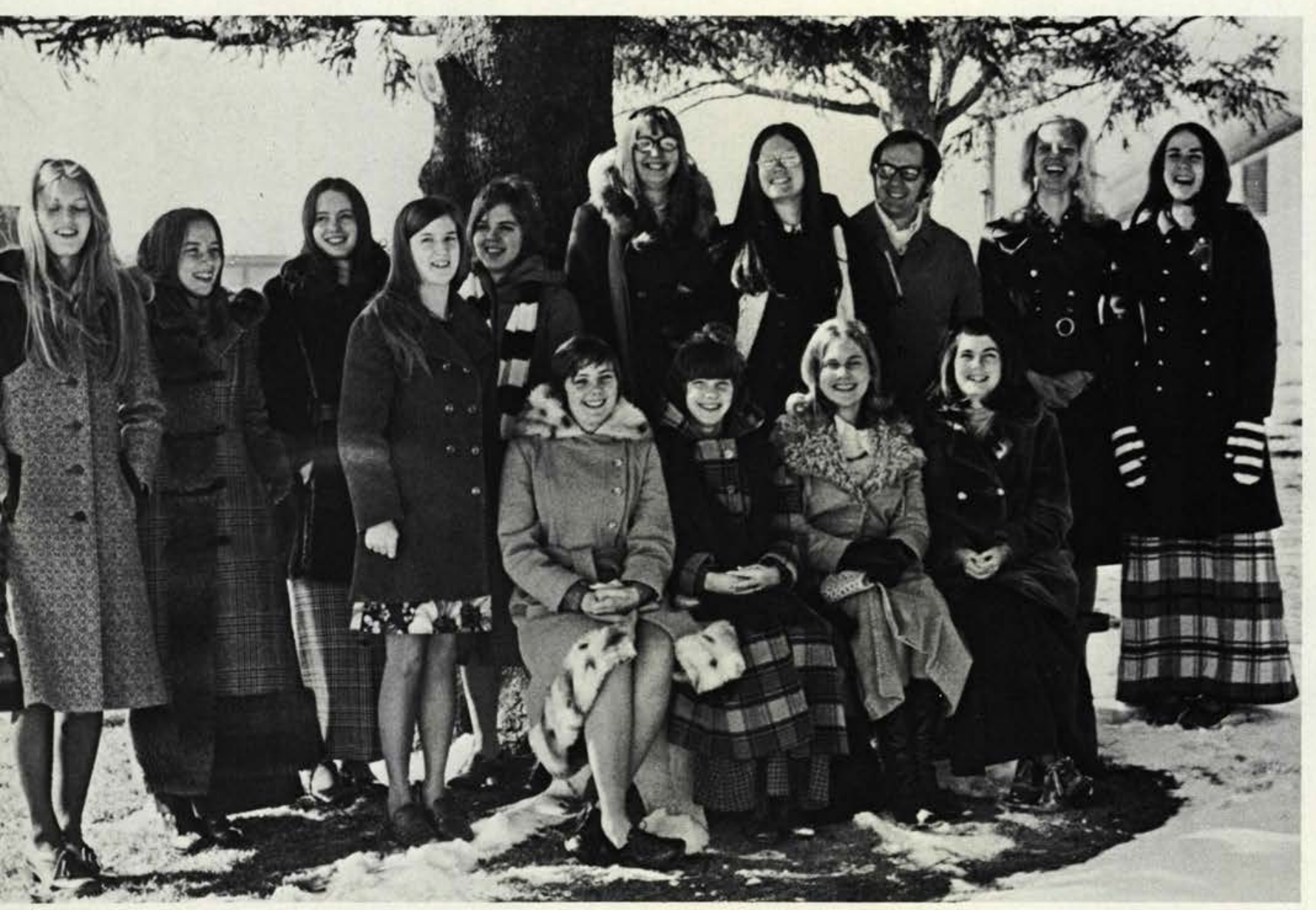

Karen Namy, Cindy Powers, Cindy Porter, Brenda Will, Nancy Price, Sally Brueser, Diane Humphres, Ken Carlton, Debbie Wood, Sally Price, SEATED, Penny Linger, Teresa Stewart, Carolyn Hayes, Carol Ockert. 


\section{ВEACH EVANGELISM}

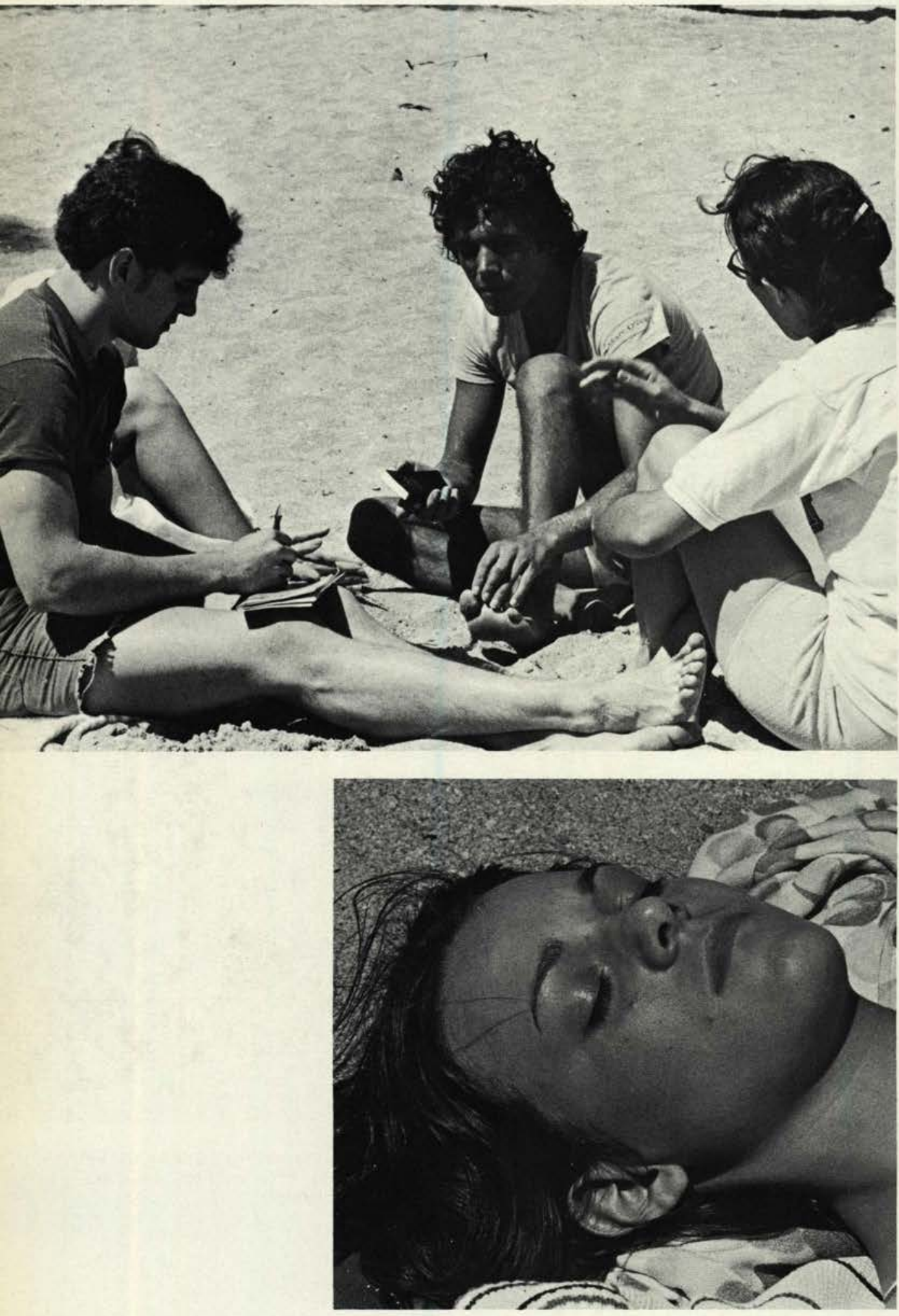


Photography by Pastor Green.
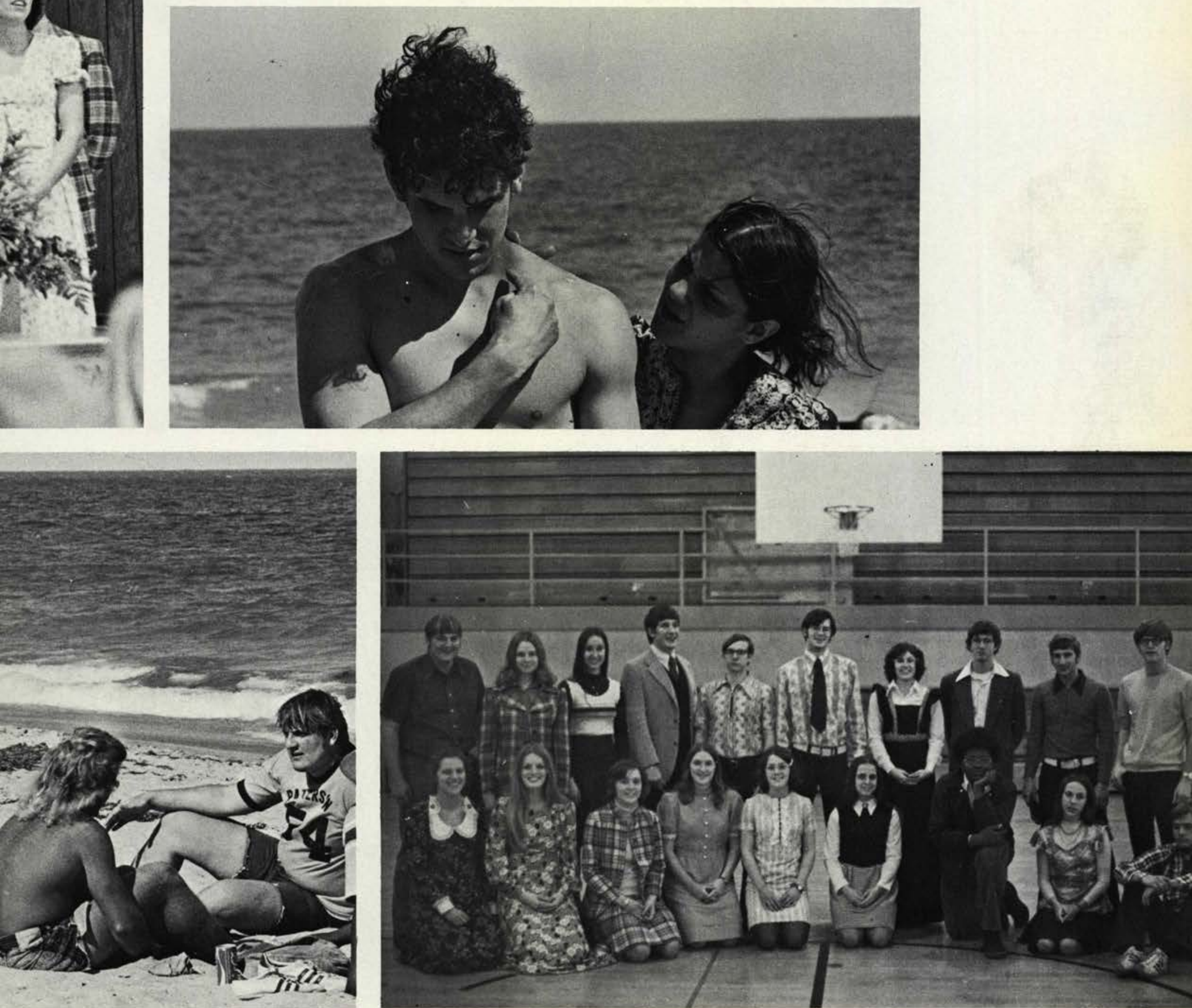

FRONT, Cheryl Heslep, Barb Cooper, Carla Morley, Michelle Meeker, Carol Phillips, Jerry Gilyard, Sue Peacock, Brad Cronbaugh, BACK, Dean McGee, Kathy Hepworth, Shelly Knowles, Kirby Lancaster, Paul Smith, Rod Robertson, Bobbi Litts, Marc Bresson, Harold Burkhalter, Terry Mudder. 


\section{Spring Swordbearers Teams}

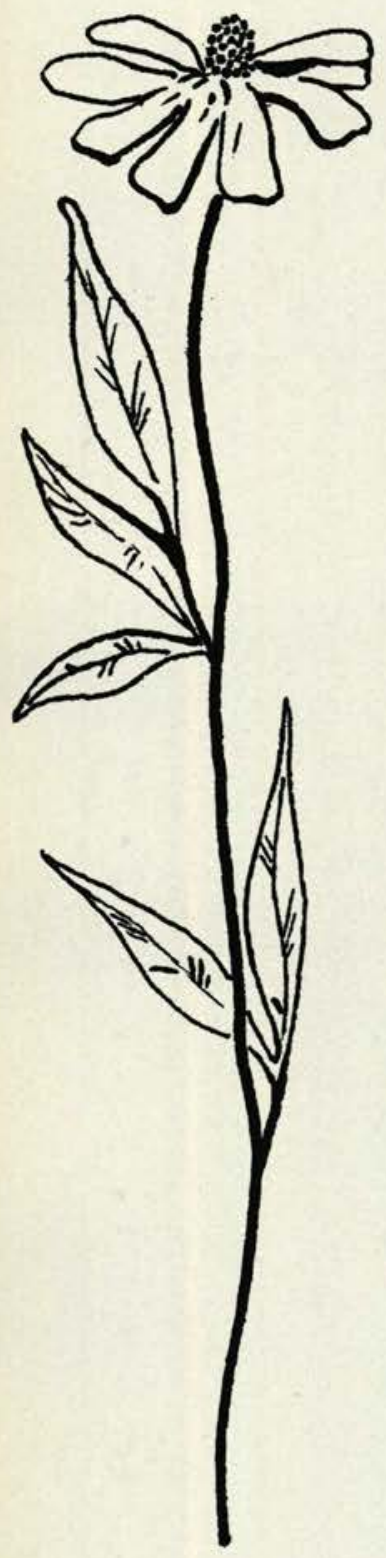

SWORDBEARERS EAST BACK:

Lewis Stone

John Street

Phil West

Lee Campbell

Dennis Twigg

FRONT:

Lois Jarvis

Janice Ortiz

Ellen Spaulding

Jackie Spinks

Sue Gazdik

Mary Glenn

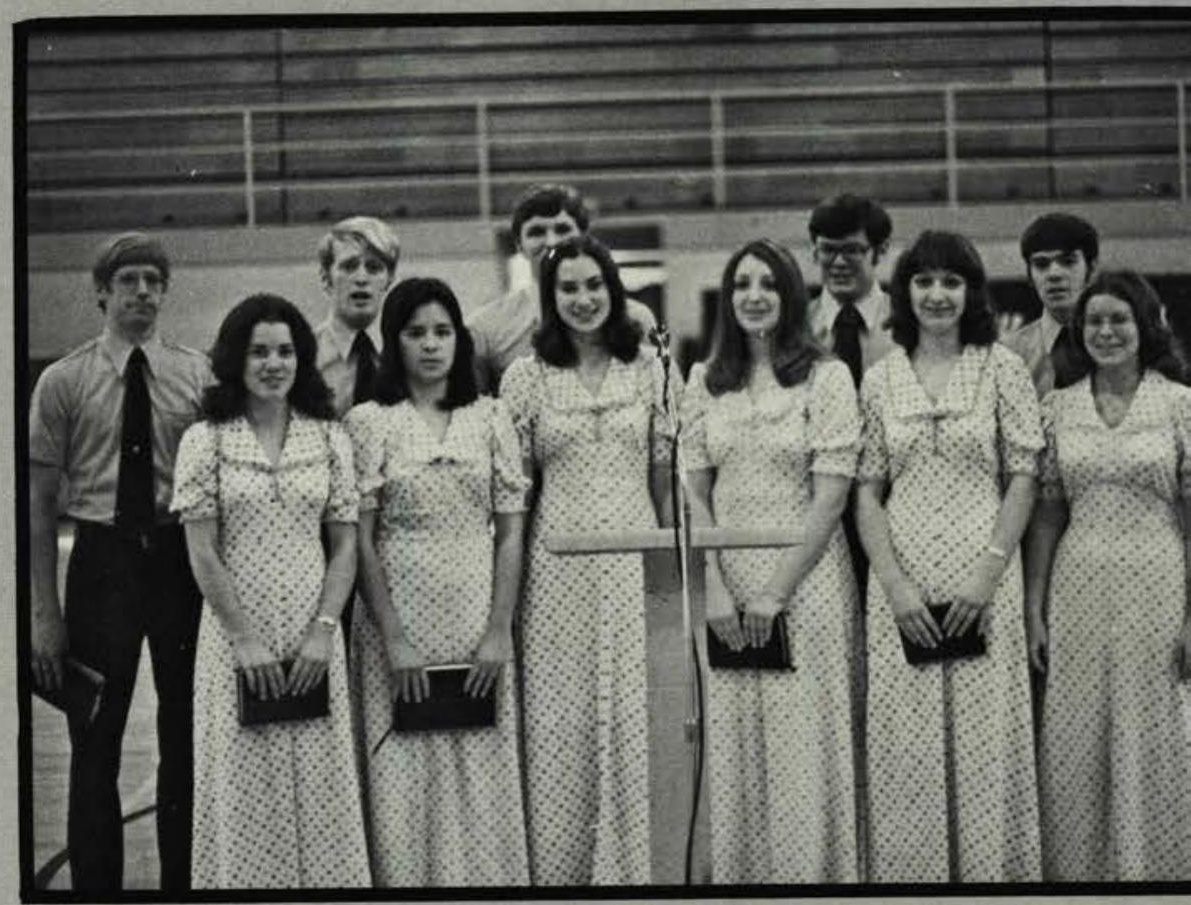

SWORDBEARERS WEST

BACK:

Carolyn Hayes

Jan Dixon

Ron Thomas

Suzanne Hale

Paul Bowers

Kathi Artrip

Debbie Dillsworth

FRONT:

Randy Rose

Esther Lightcap

Bill Giesman

Not Pictured:

Gary Percesepe

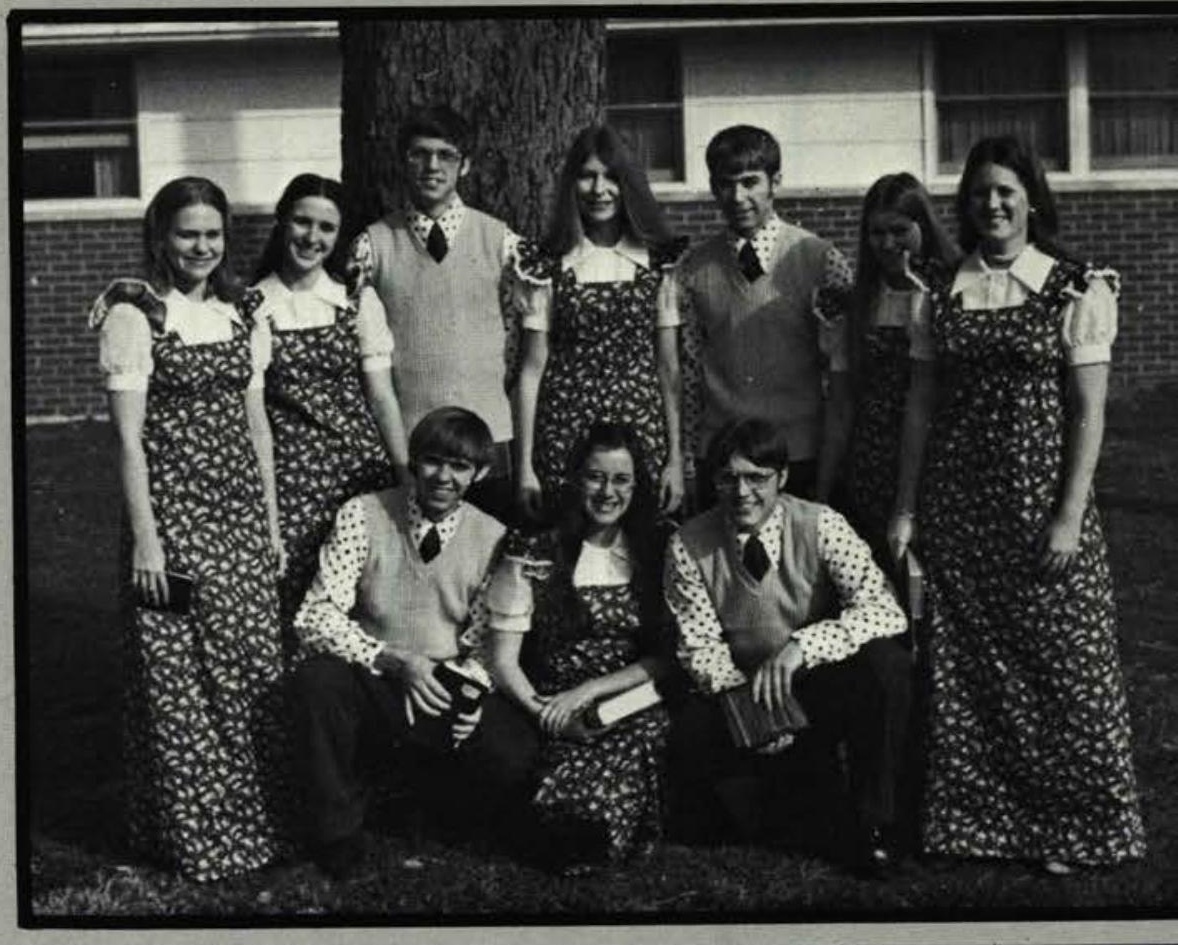




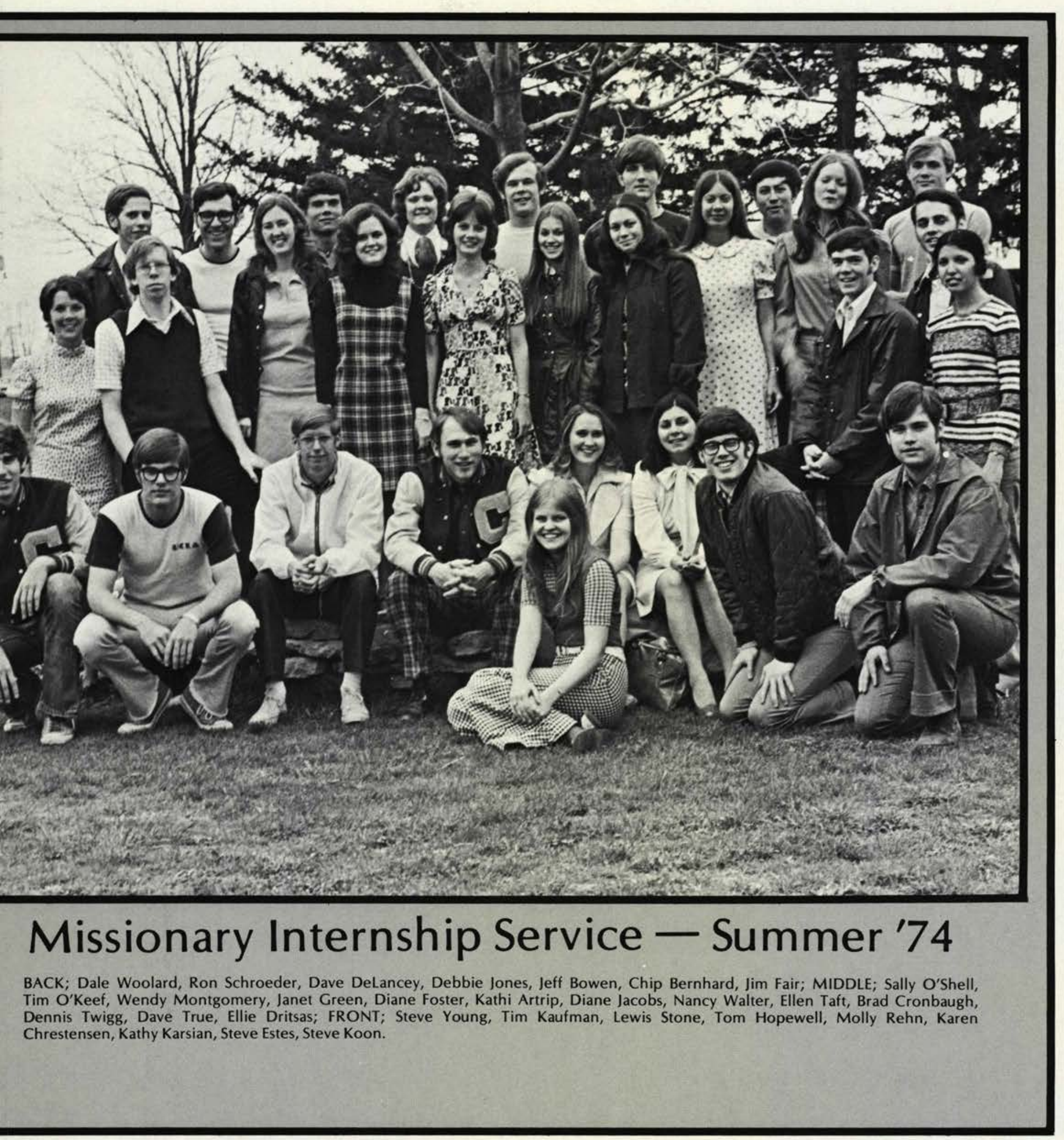




\section{O.S.S.O. Morning}

FRONT; Dora Ewing, Sharon Totman, Cindy Banks, Shelley Knowles, Sherry Cowell, BACK; Irene Buisch, Doug DeLand, Dan Adamowich, Hank Diaz, Paul Thorsell, Chuck Elliott, Tim Gabler.
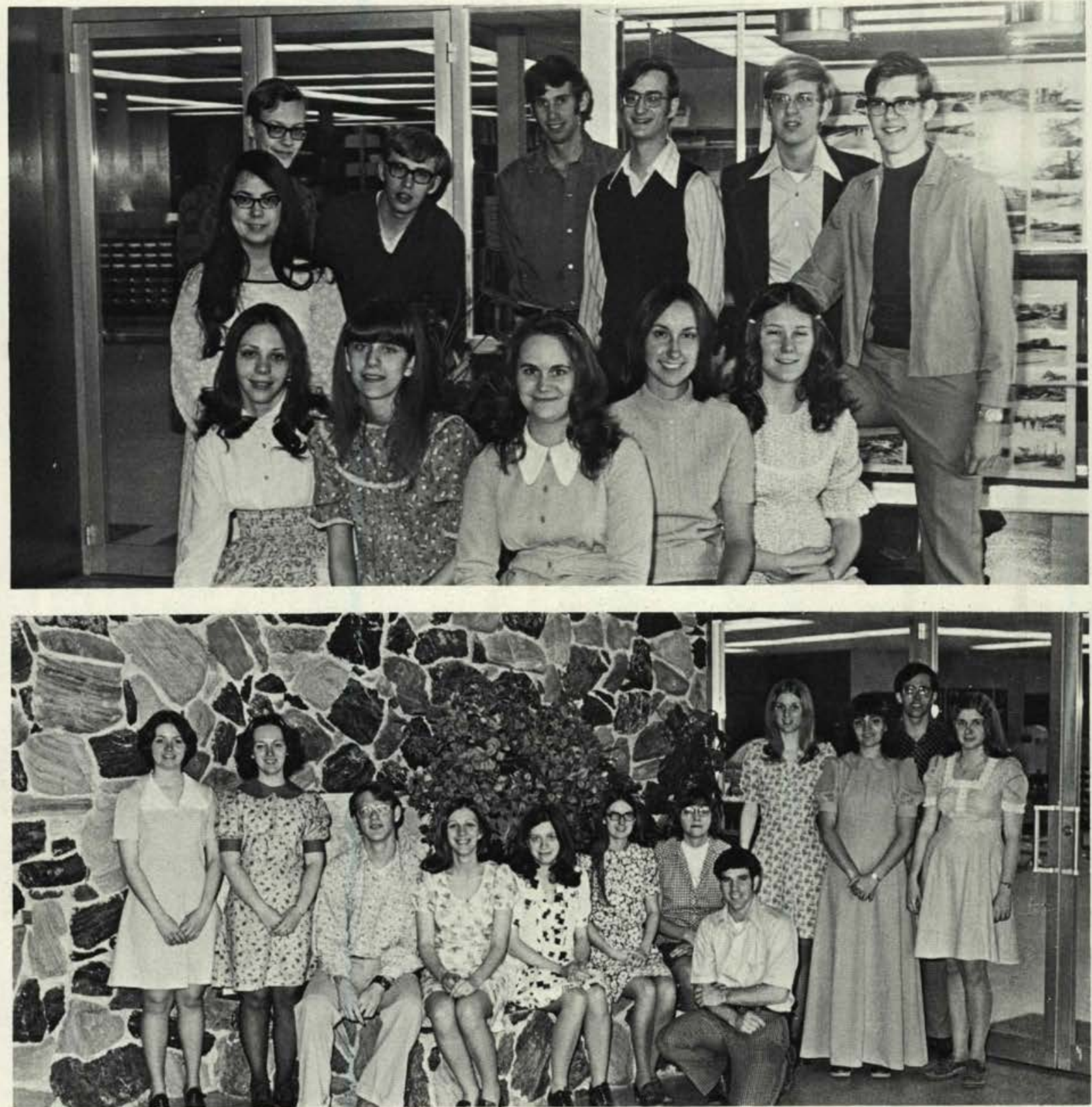

O.S.S.O. Becky Sininger, Sara Browning, Tim Evening Barker, Sharon Gazdik, Staranna Glo-

Harold Pulver, Chris Taylor, Brenda Finch, Doug Hilleary, Elaine Howard.
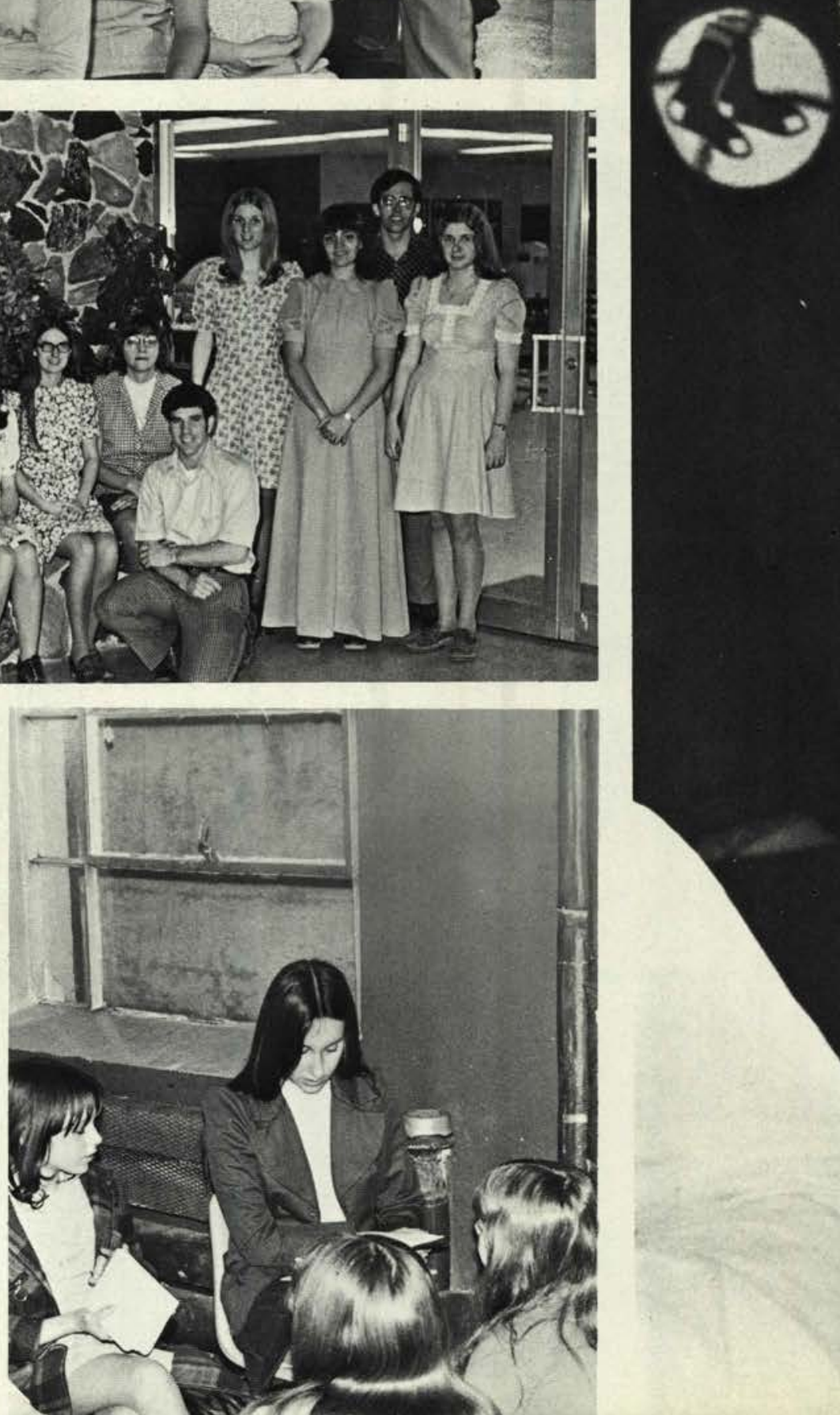



\section{How Shall They Hear?}

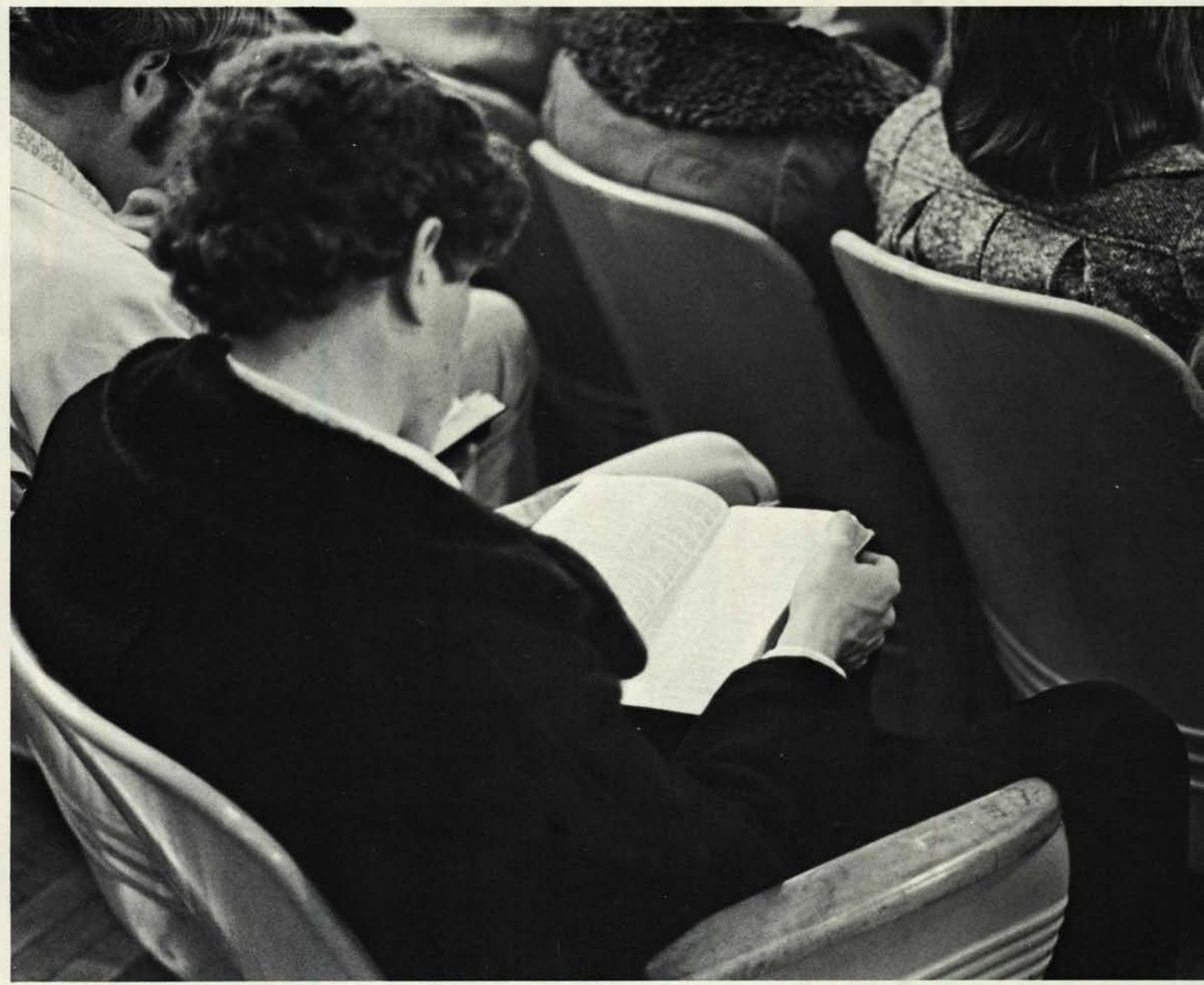

15th Annual Missionary Conference 

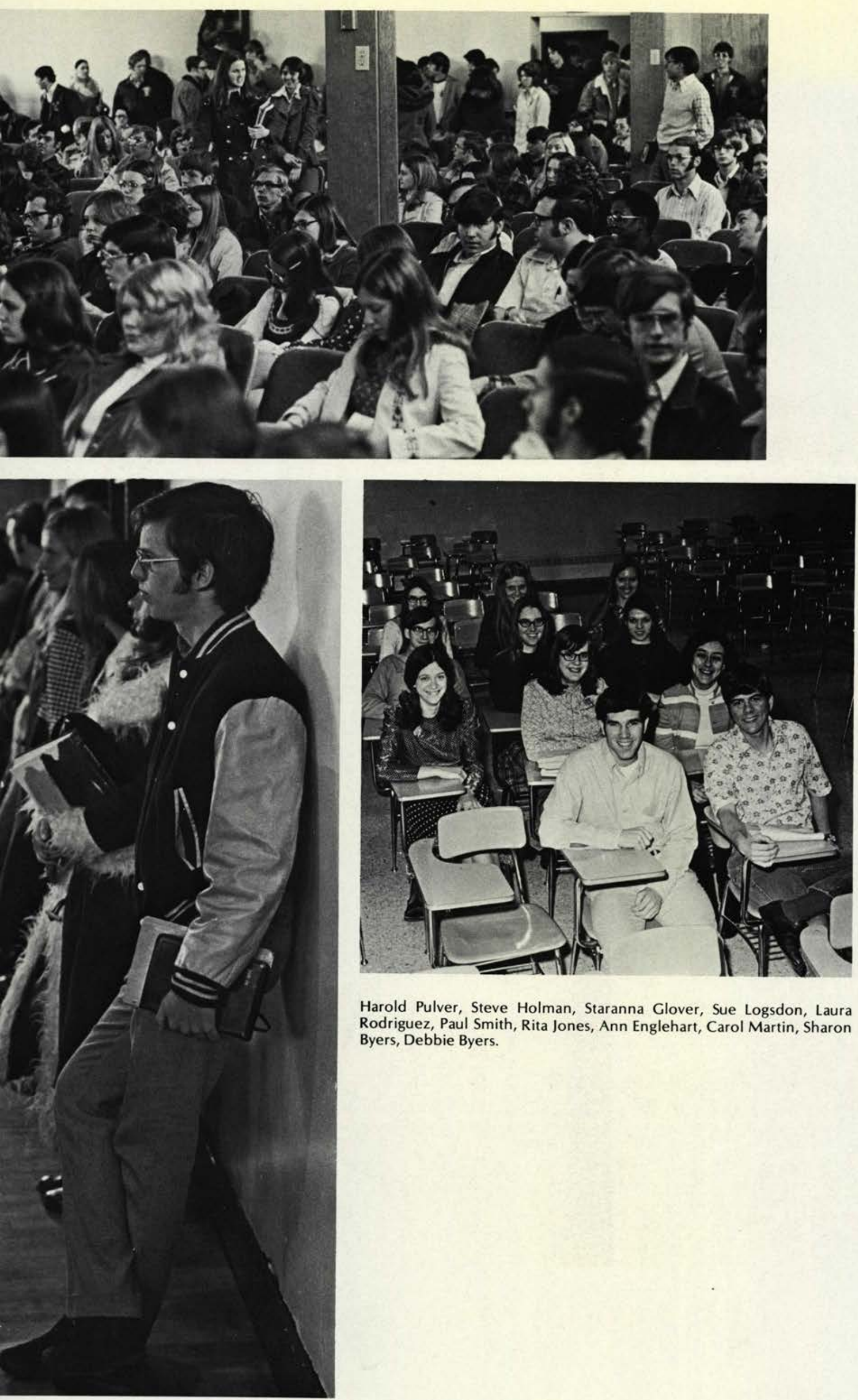

F.W.M.

Harold Pulver, Steve Holman, Staranna Glover, Sue Logsdon, Laura Rodriguez, Paul Smith, Rita Jones, Ann Englehart, Carol Martin, Sharon Byers, Debbie Byers. 


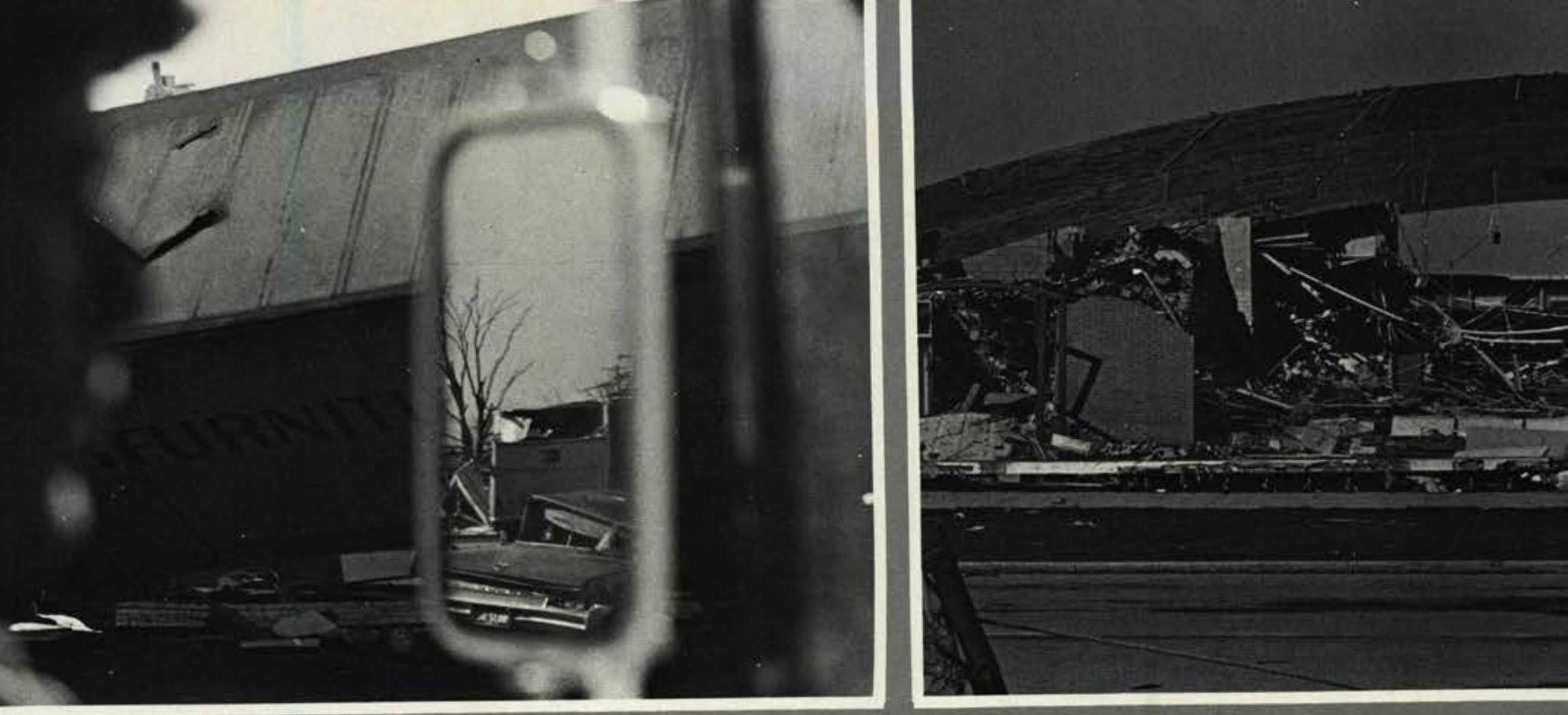




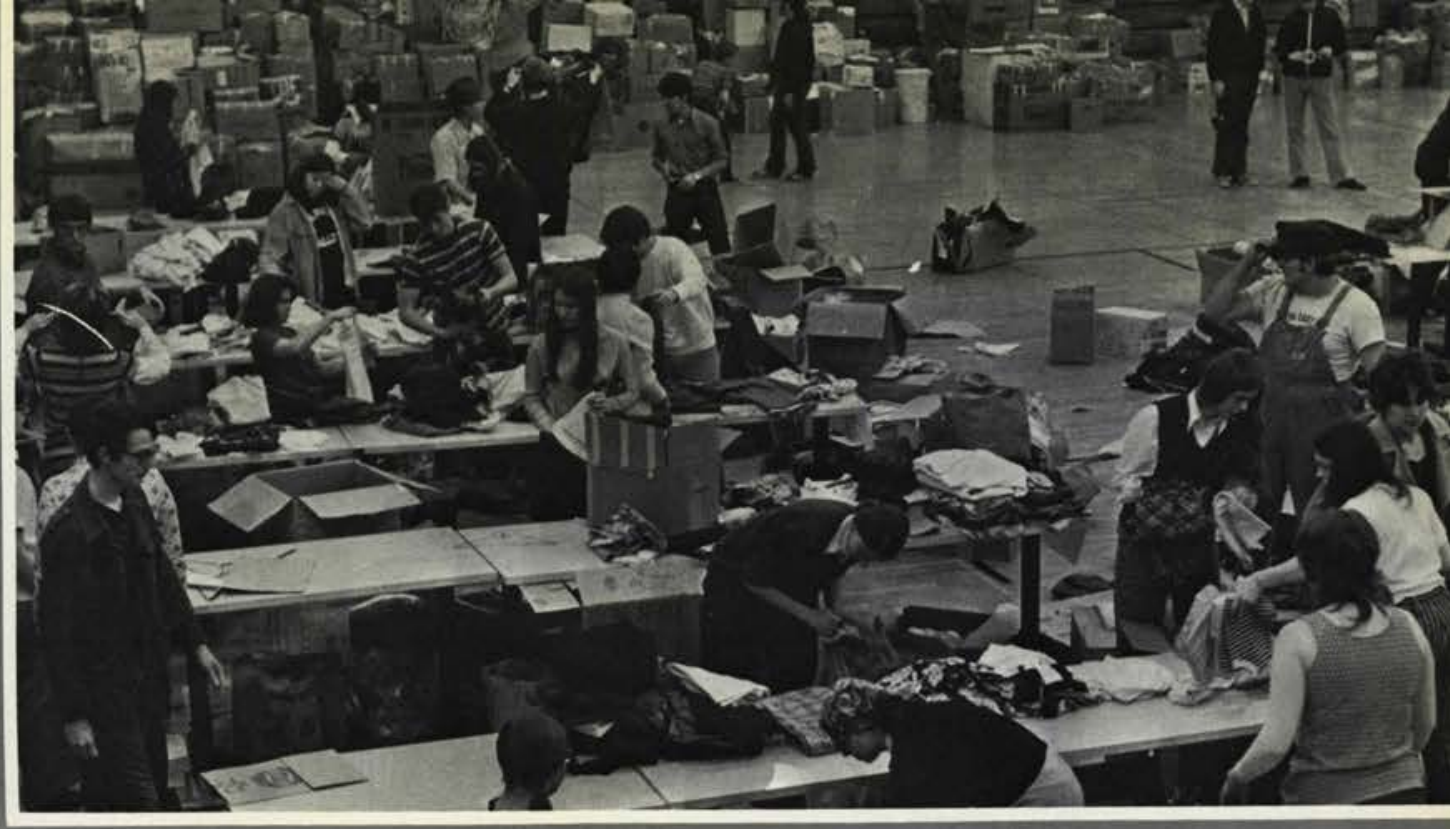

it left half of Xenia as twisted debris. We at Cedarville ex-

tended a helping hand where we could, to relieve distress and to bear witness for Christ.

"It is of the Lord's mercies that we are not consumed, because his compassions fail not.

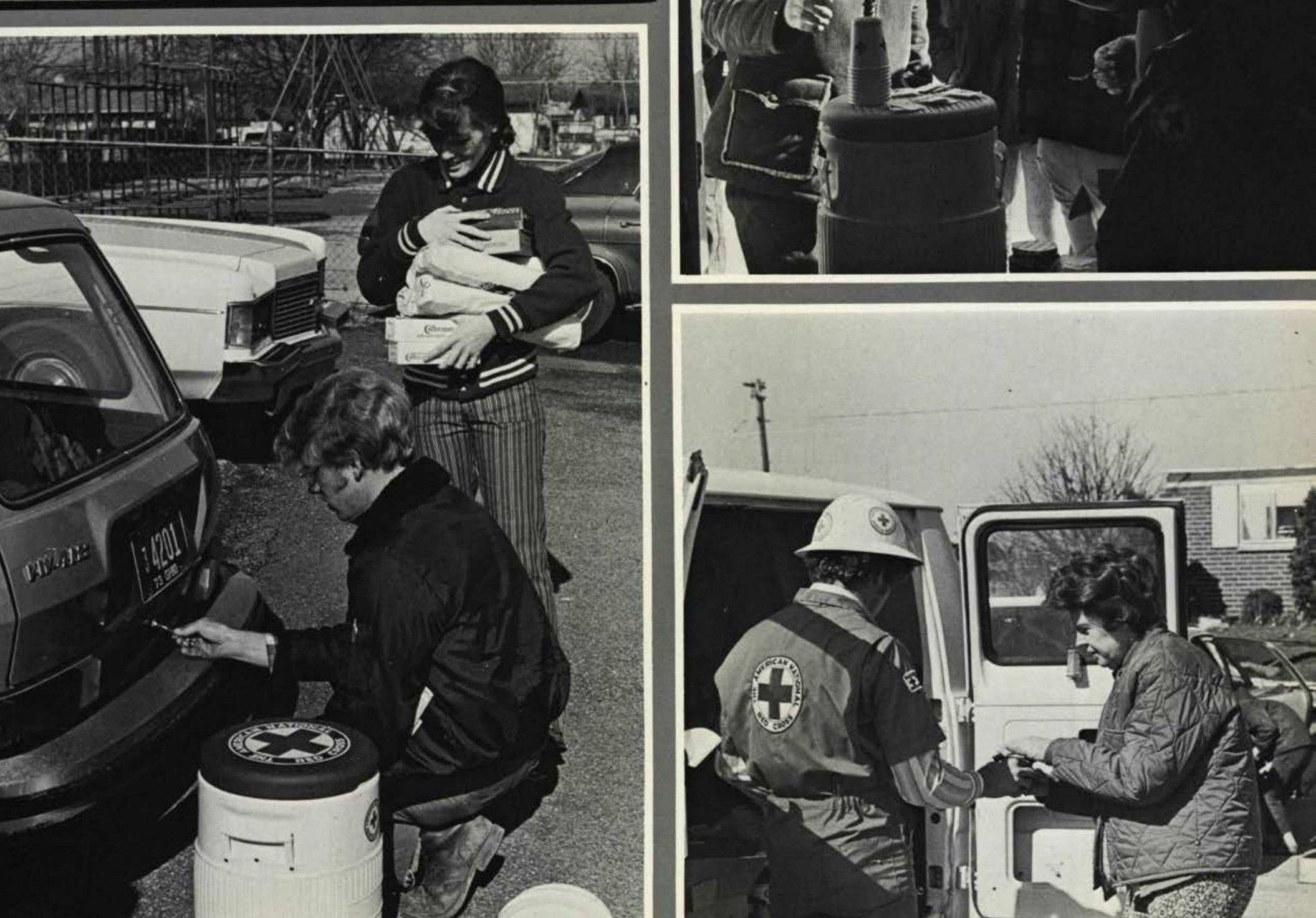


Mike Niles, Randy McFarland, Dan Rosecrans, Sharon Franks, Mark Seeley, Paul Bowers, Tim Detweiler, Jeff St. Clair, Bob Mohat, Debbie Vansickle, Wayne Shepherd (Program Director), Bill Nash, Kevin Fields (Student Production Director), Not Pictured - Paul Gathany (General Manager).

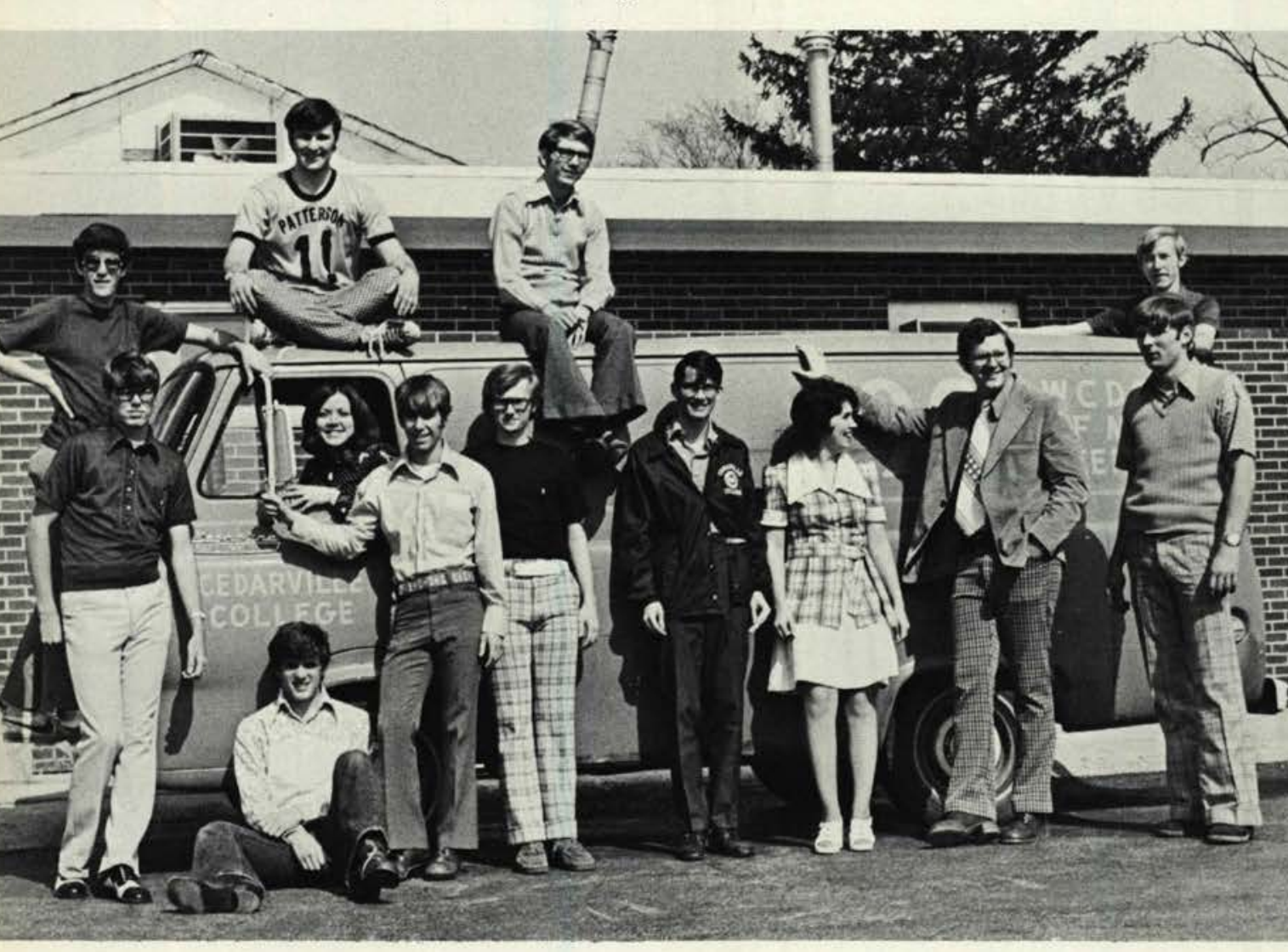

\section{WCDR Staff}

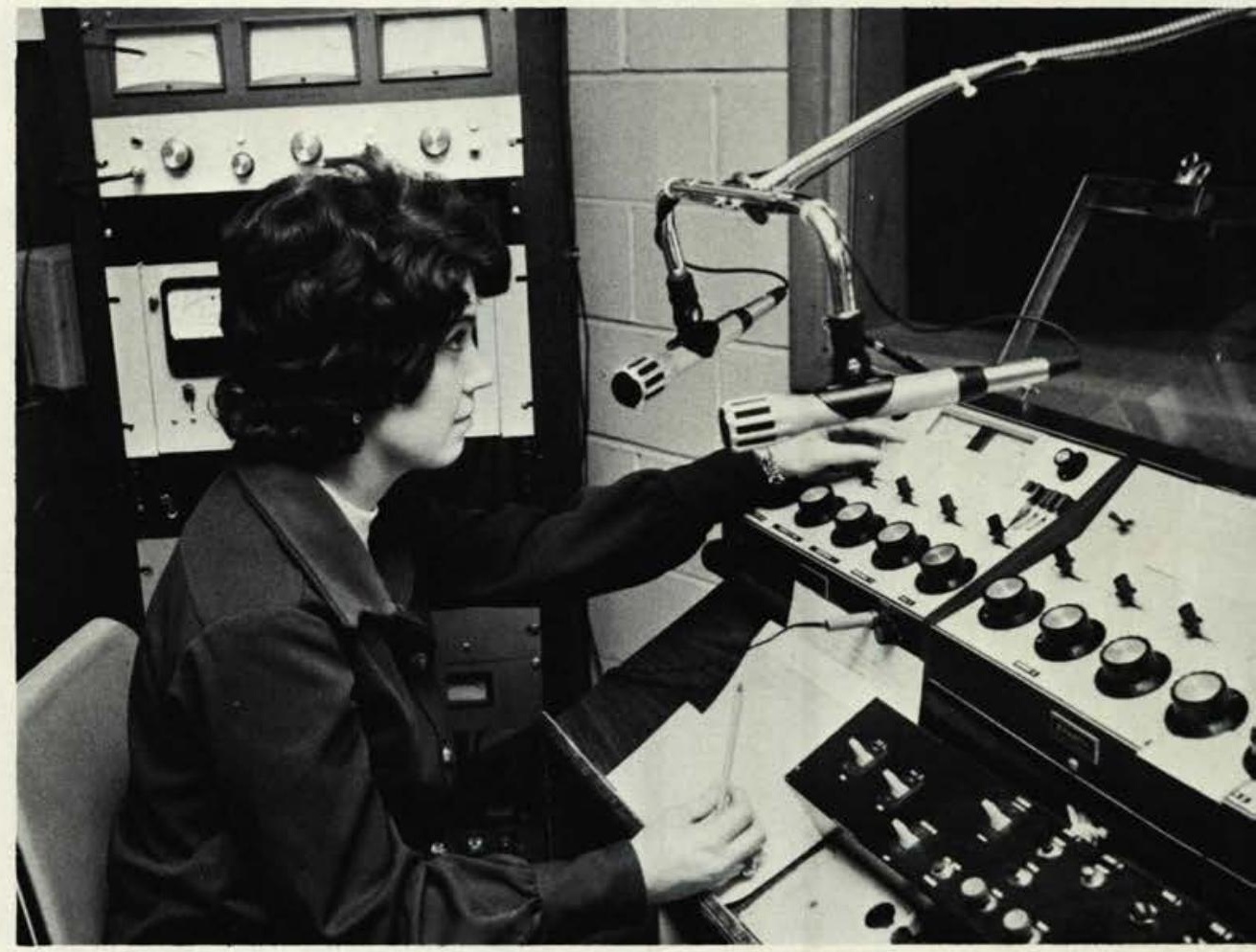



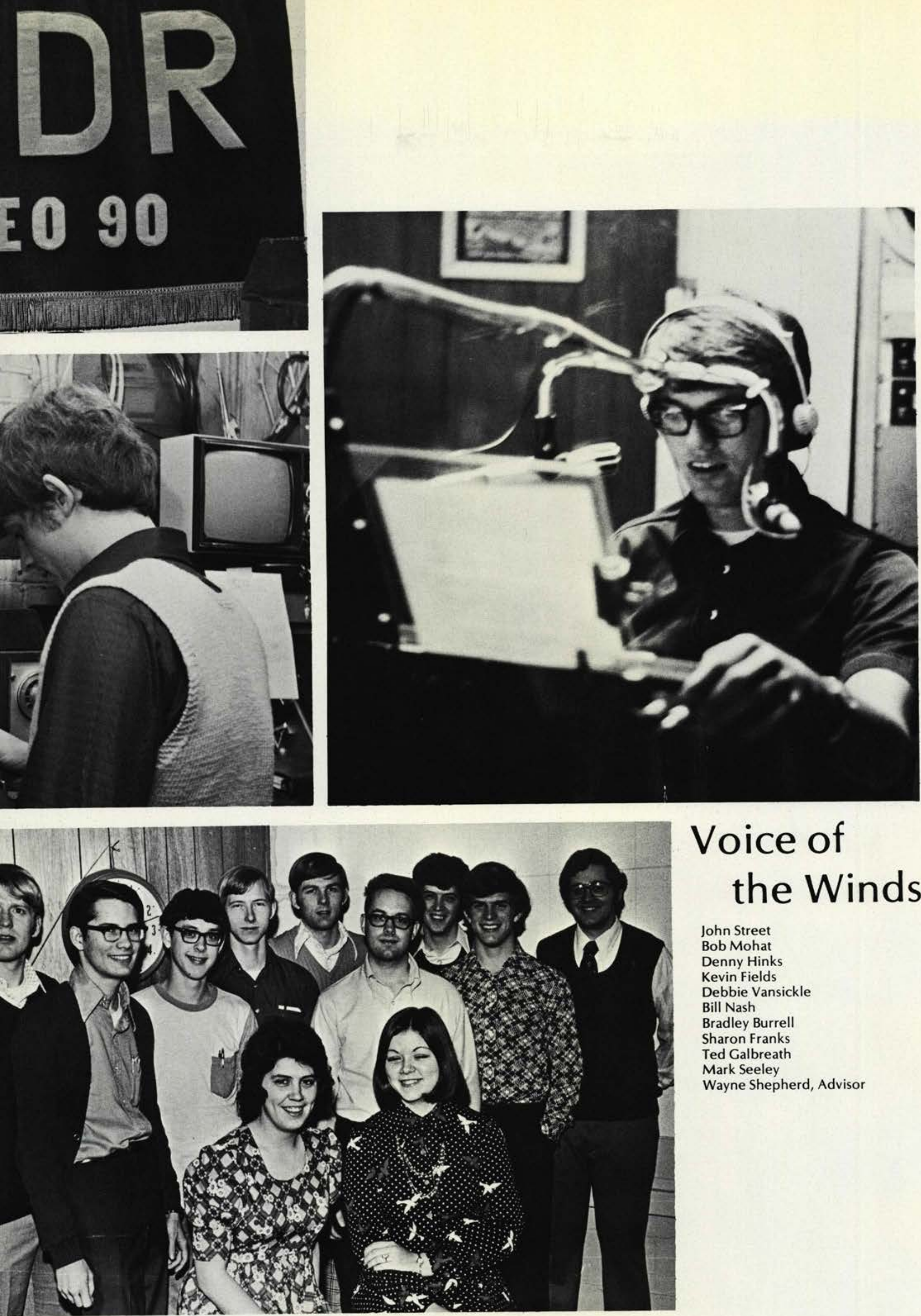

\section{Voice of} the Winds

John Street

Bob Mohat

Denny Hinks

Kevin Fields

Debbie Vansickle

Bill Nash

Bradley Burrell

Sharon Franks

Ted Galbreath

Mark Seeley

Wayne Shepherd, Advisor 

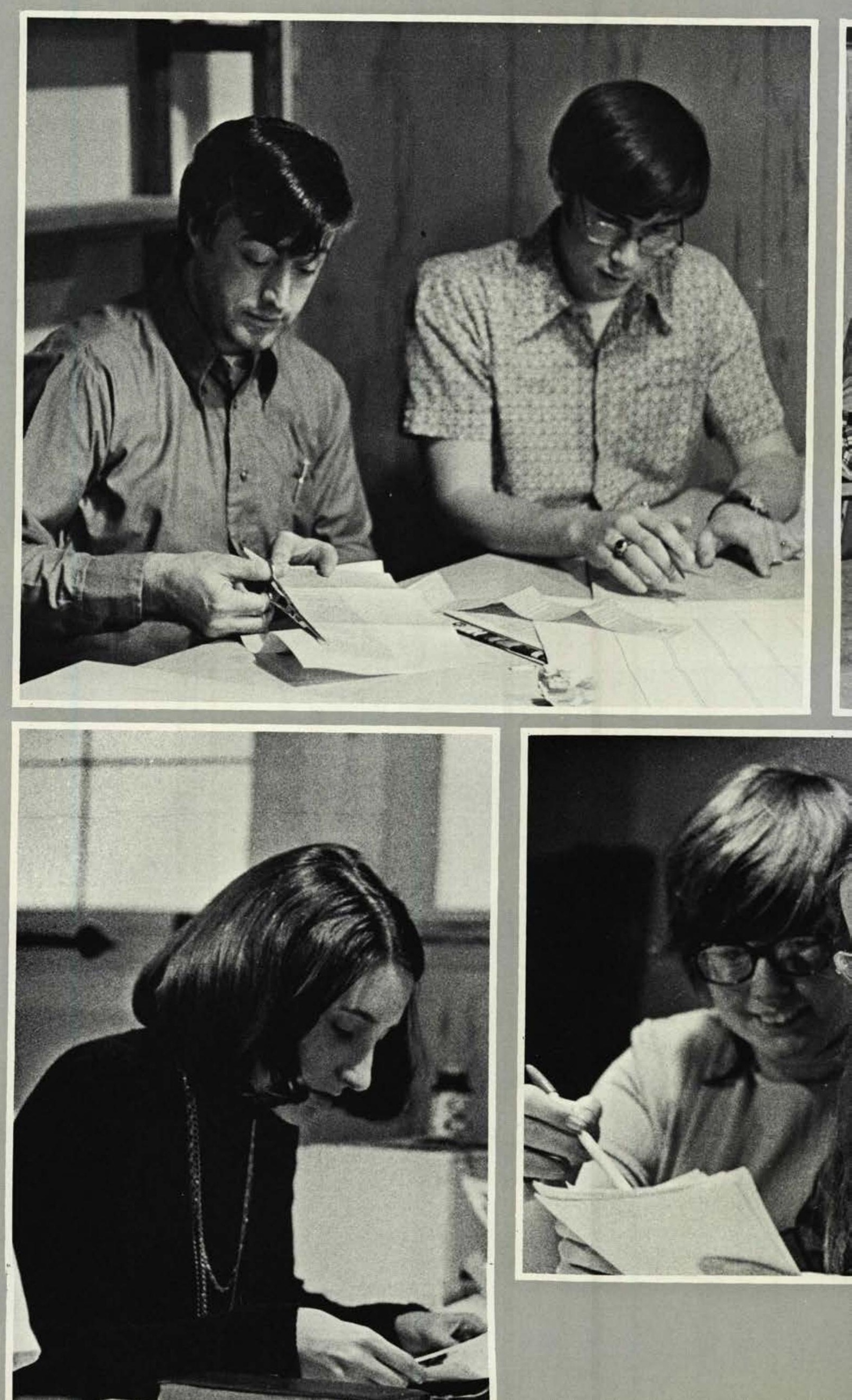

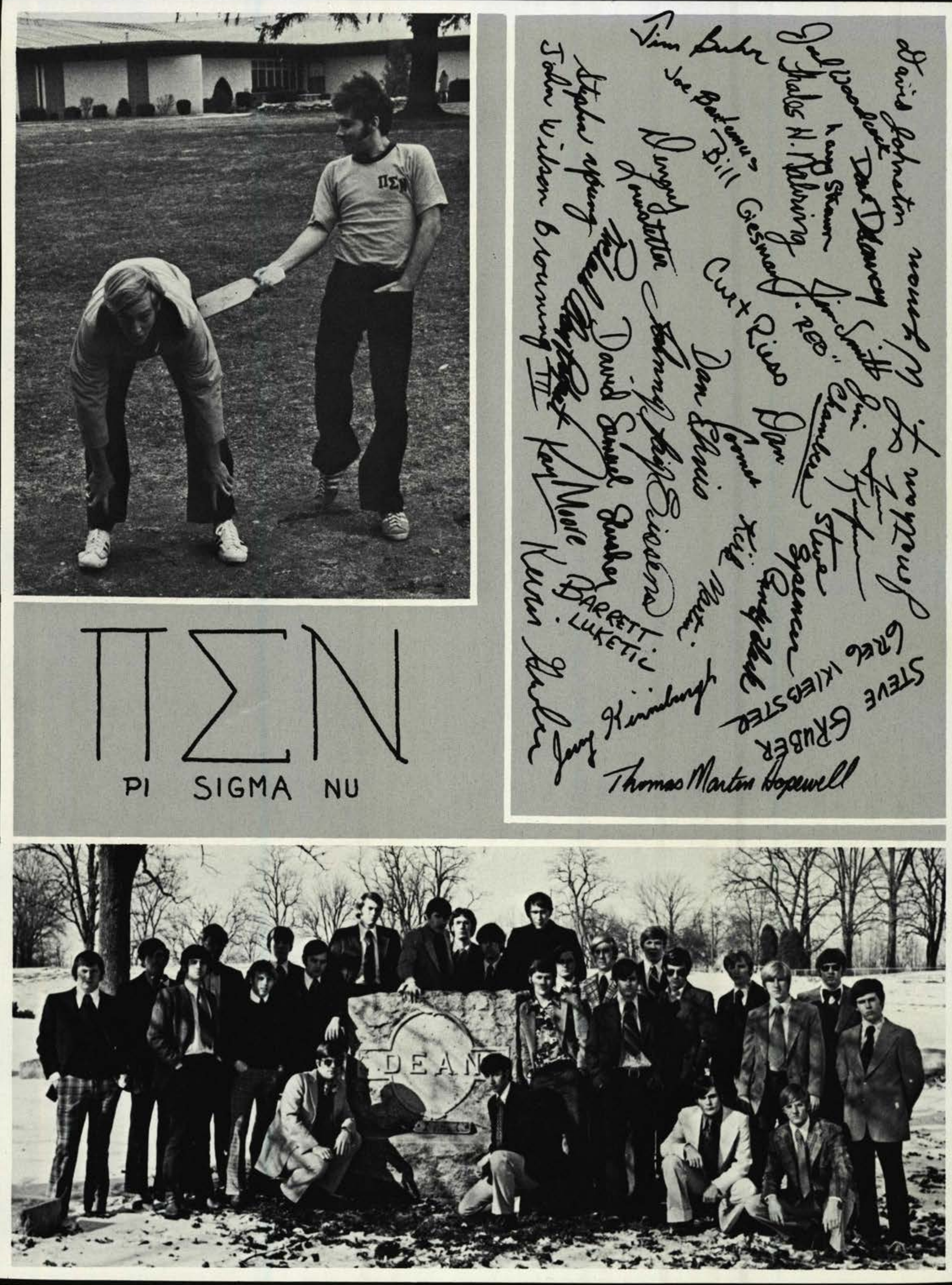


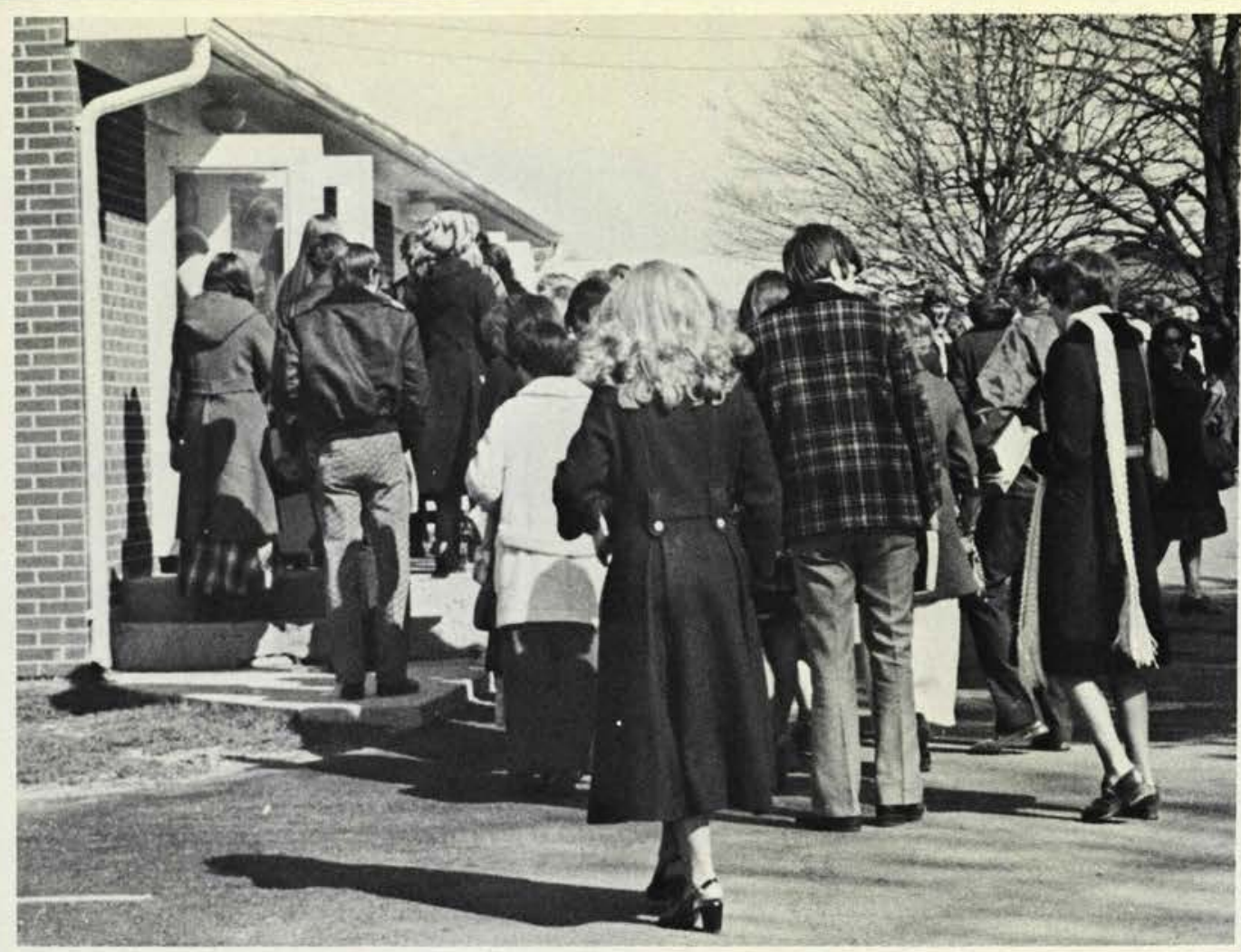

Post Office
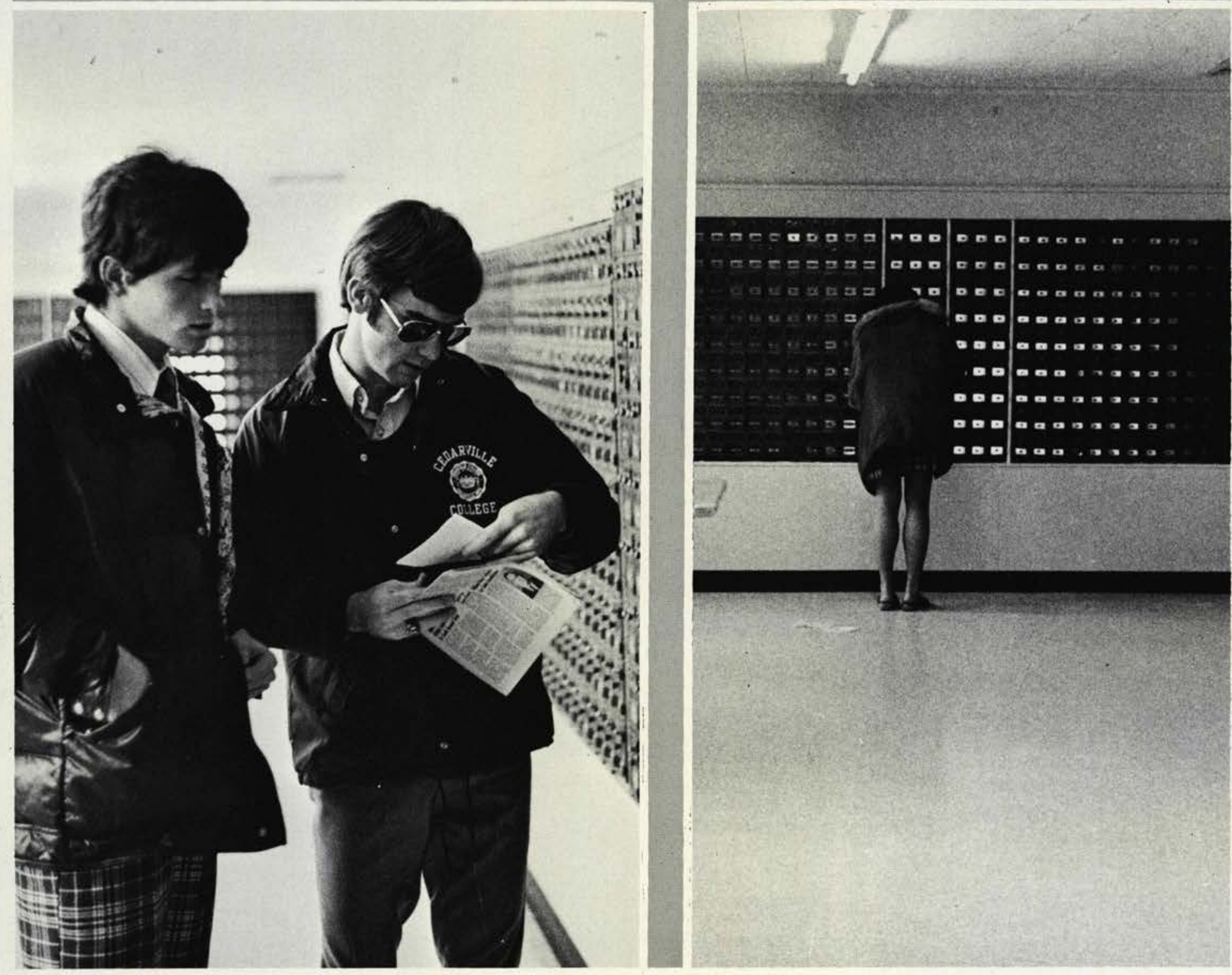

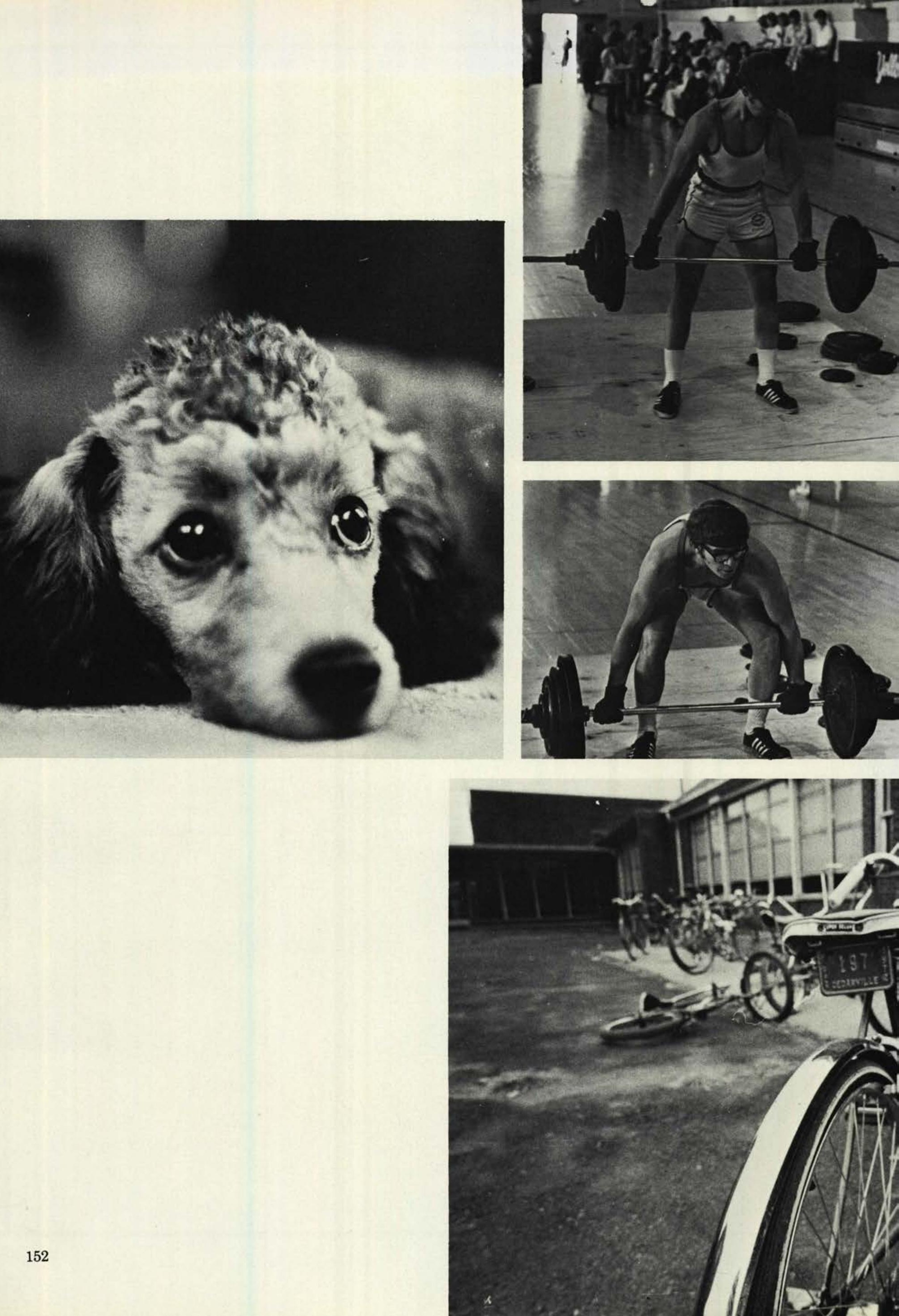

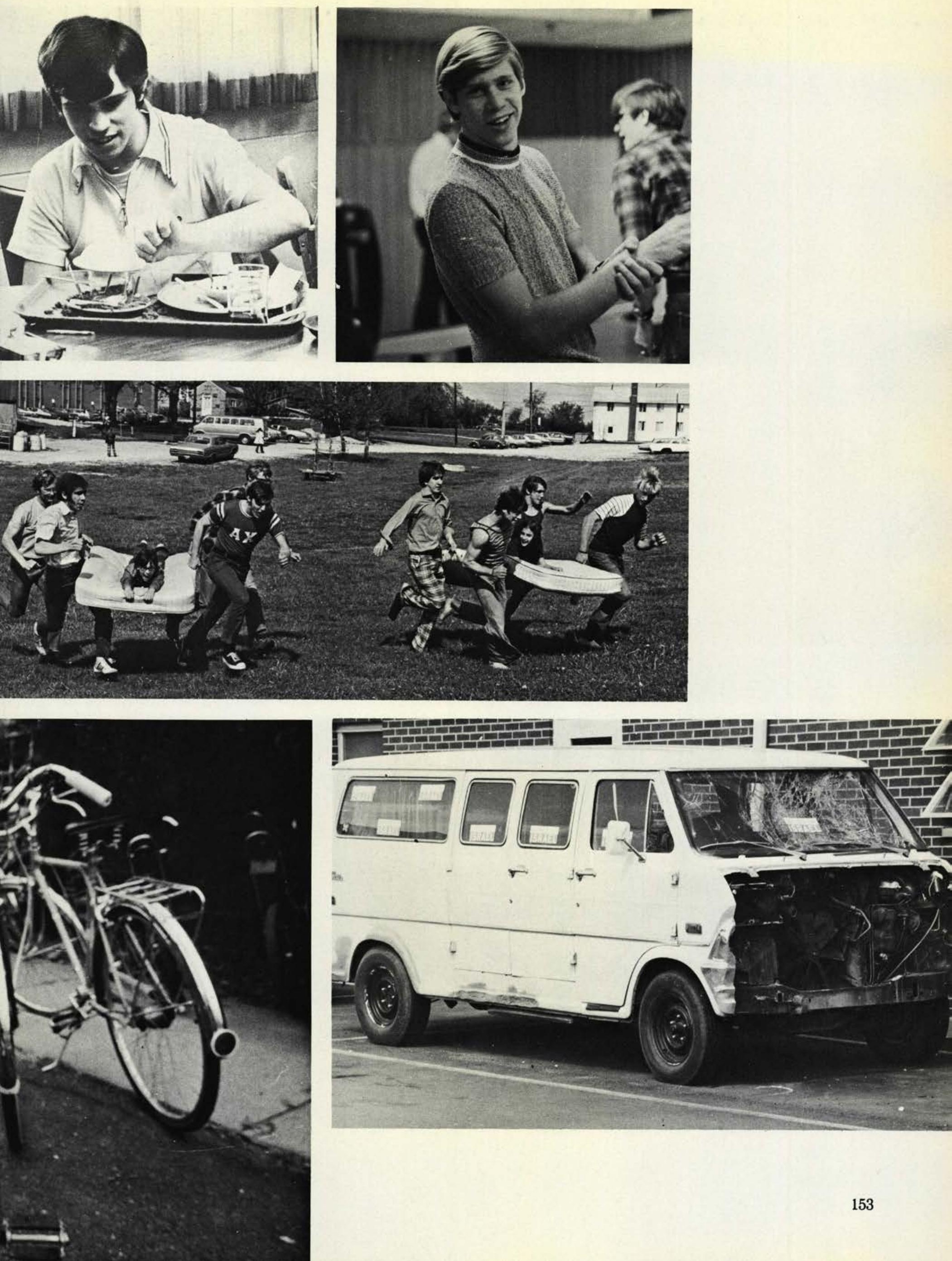
Coach Murdoch, Dave DeLancy, Wes Johnson, Chuck Neuhaus, Sam Kesler, Bill Williams, Kirk Martin, Steve 'Spud' Millikan, Eric Cuenin, George Hoffman, Don Eggleston, Alan Edlund.
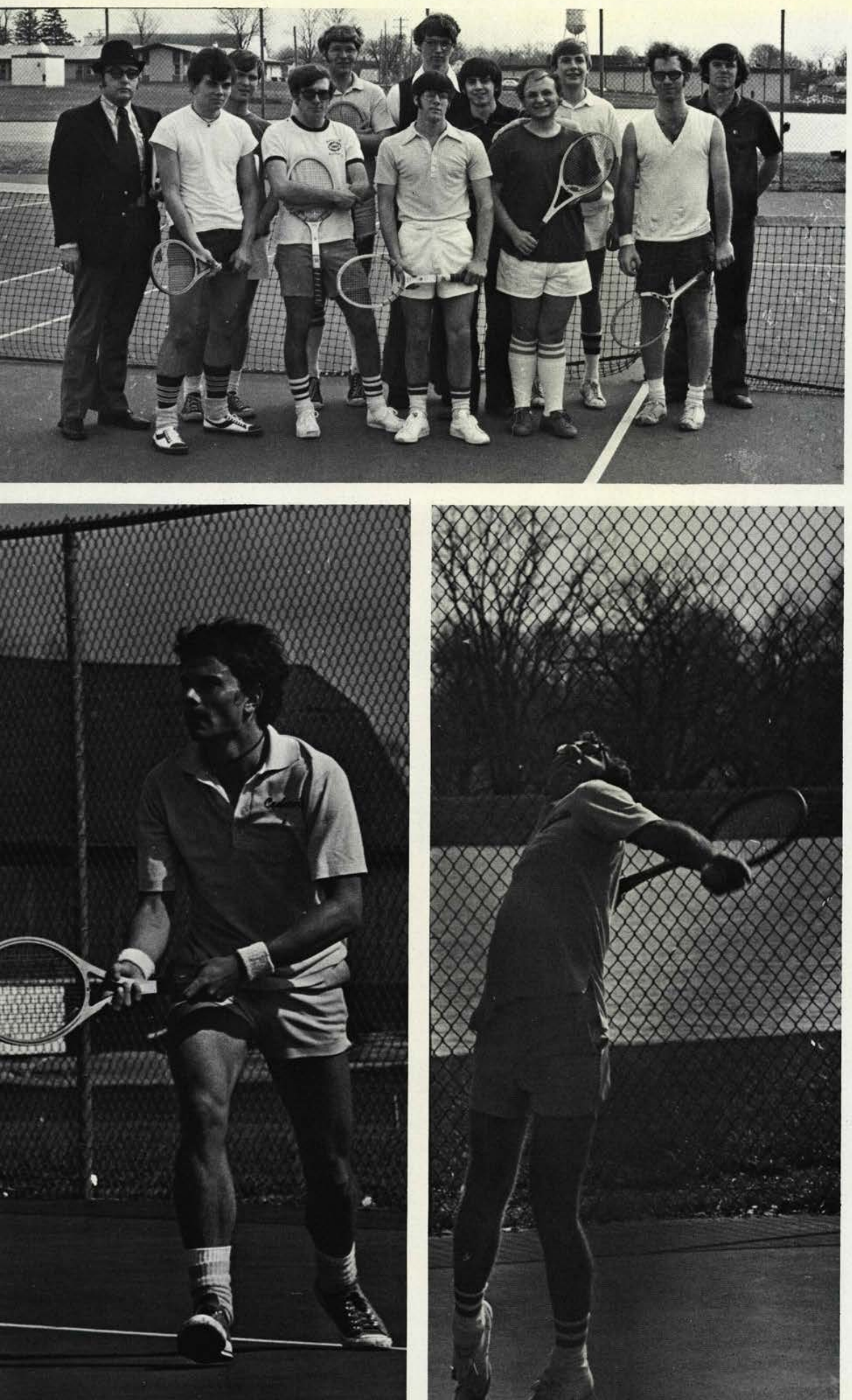

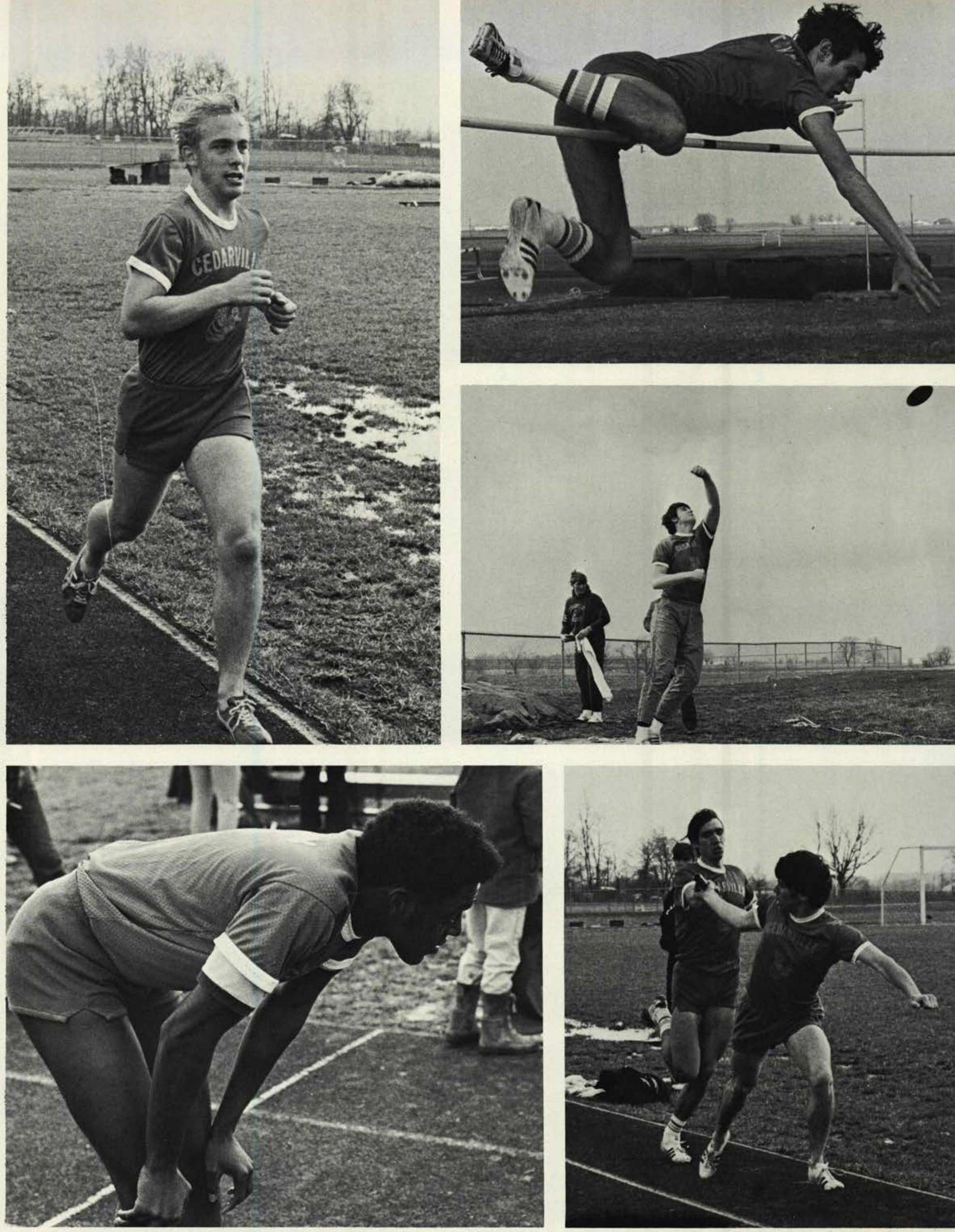

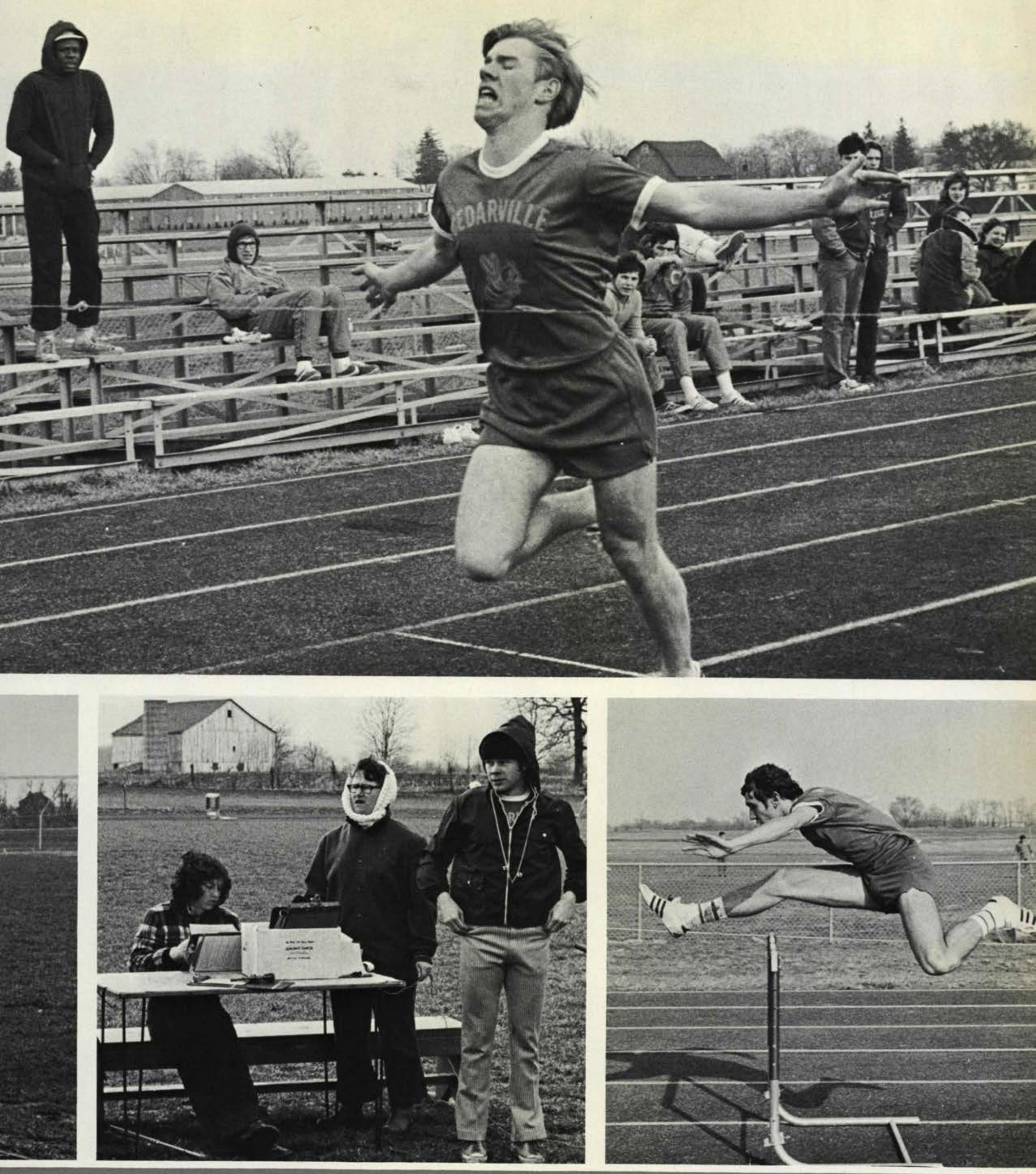

Track'74 


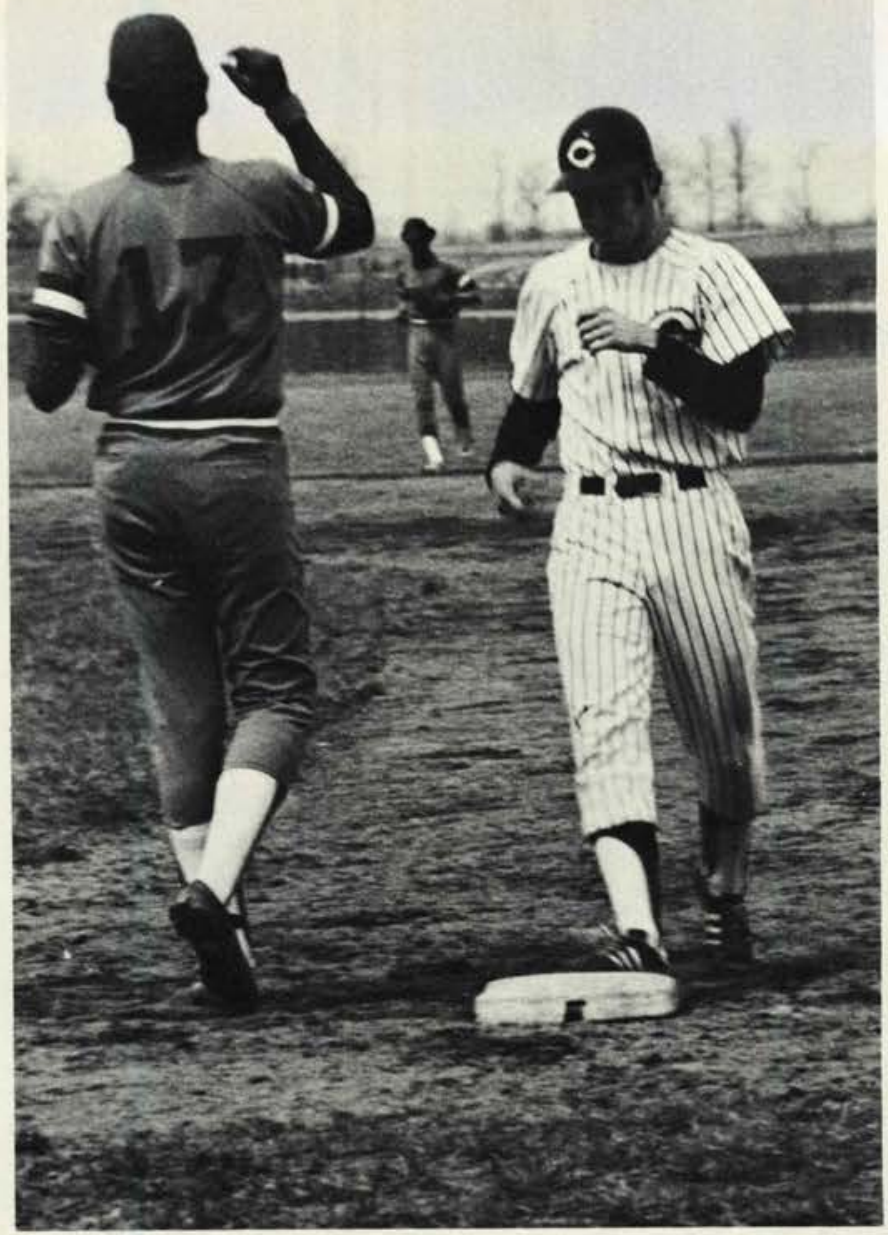

\section{Baseball '74}
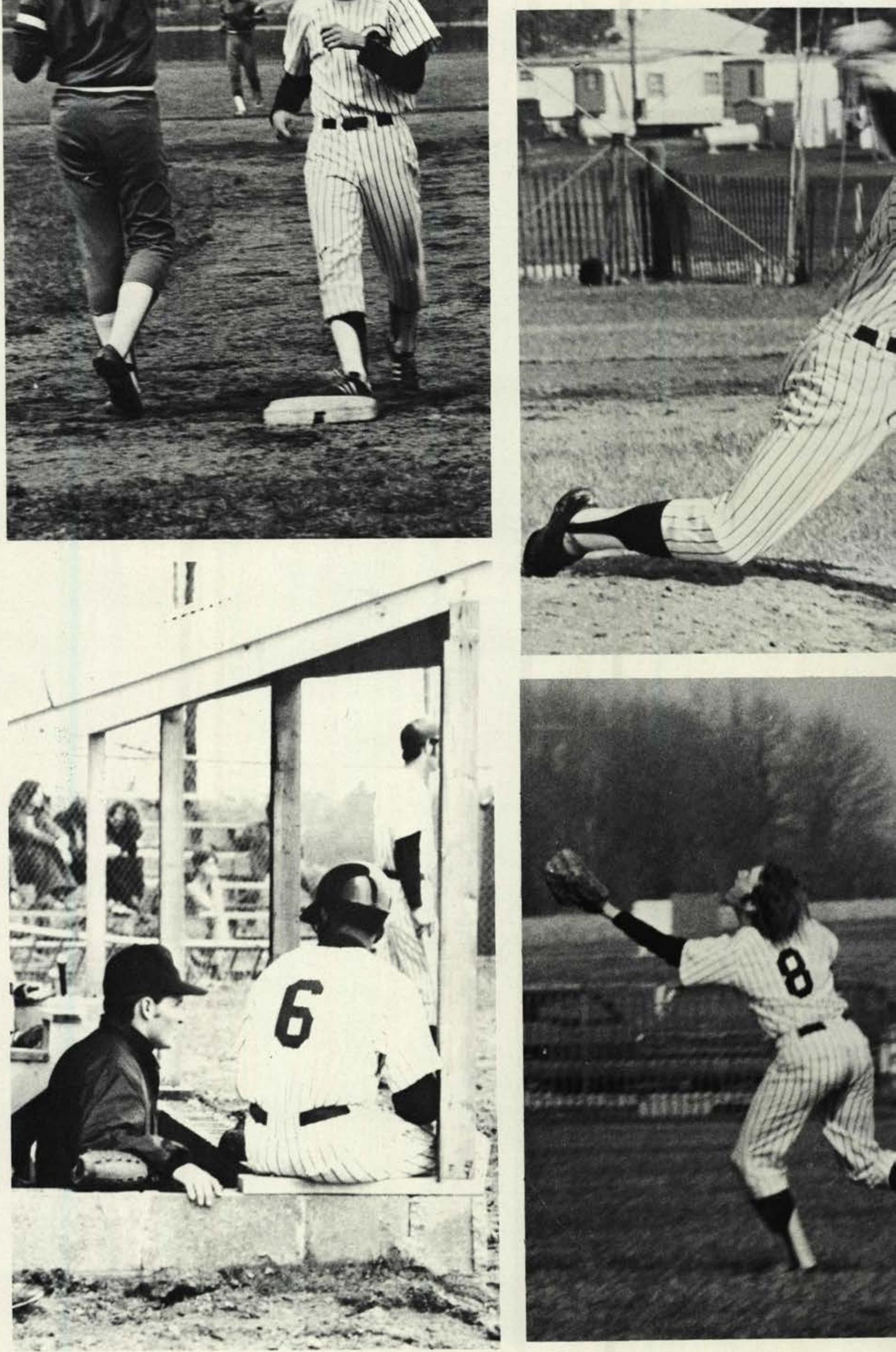


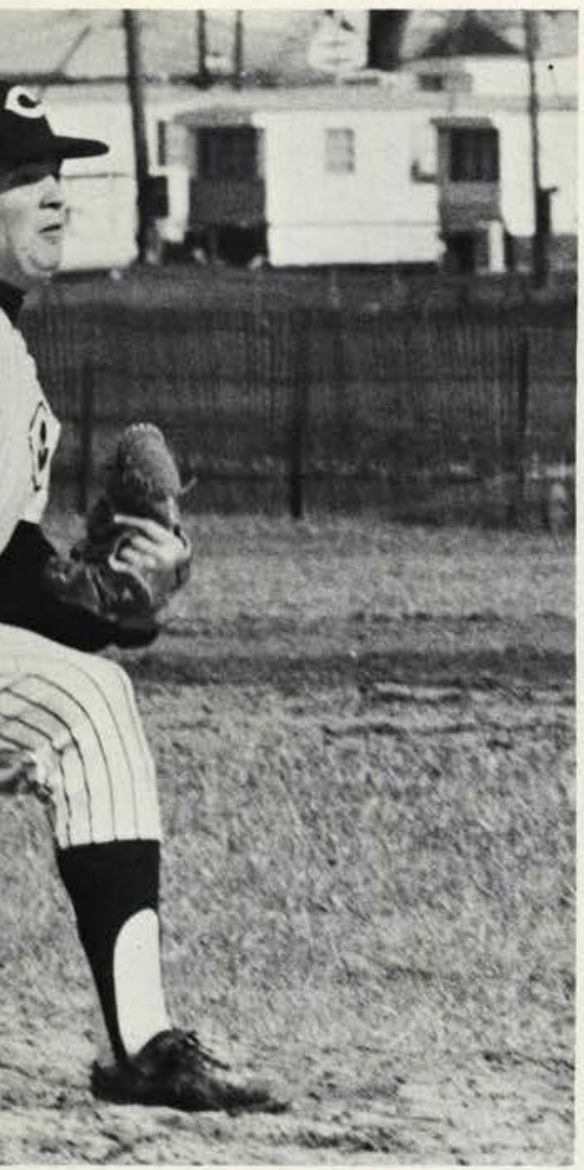

FRONT: Jerry Griswold, Dennis Lowstetter, Jack Browning, Tim Buhr, Joe Bartemus, Don Myers, Dave Soliday, Clancy Parker, Wynn Gerber, BACK: Coach Berry, Joel Woodcock, Jim Smith, Tim Kaufman, Dan Coomes, Tom Williams, Curt Bugno, Dave Johnston, Al Halladay, Rod Hansen, Jon Lawhead, Tom Burnett.
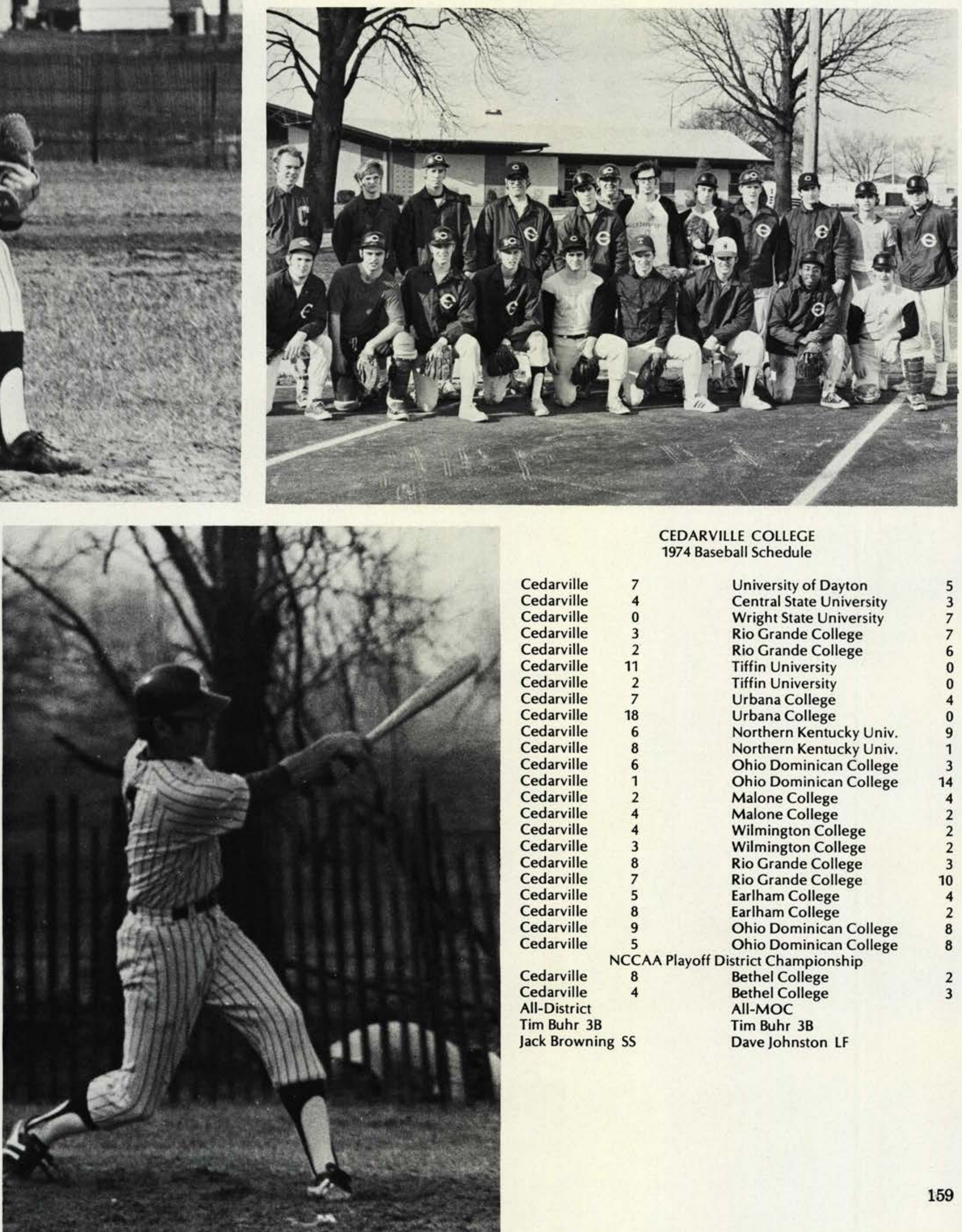

CEDARVILLE COLLEGE

1974 Baseball Schedule

$\begin{array}{lrlr}\text { Cedarville } & 7 & \text { University of Dayton } & 5 \\ \text { Cedarville } & 4 & \text { Central State University } & 3 \\ \text { Cedarville } & 0 & \text { Wright State University } & 7 \\ \text { Cedarville } & 3 & \text { Rio Grande College } & 7 \\ \text { Cedarville } & 2 & \text { Rio Grande College } & 6 \\ \text { Cedarville } & 11 & \text { Tiffin University } & 0 \\ \text { Cedarville } & 2 & \text { Tiffin University } & 0 \\ \text { Cedarville } & 7 & \text { Urbana College } & 4 \\ \text { Cedarville } & 18 & \text { Urbana College } & 0 \\ \text { Cedarville } & 6 & \text { Northern Kentucky Univ. } & 9 \\ \text { Cedarville } & 8 & \text { Northern Kentucky Univ. } & 1 \\ \text { Cedarville } & 6 & \text { Ohio Dominican College } & 3 \\ \text { Cedarville } & 1 & \text { Ohio Dominican College } & 14 \\ \text { Cedarville } & 2 & \text { Malone College } & 4 \\ \text { Cedarville } & 4 & \text { Malone College } & 2 \\ \text { Cedarville } & 4 & \text { Wilmington College } & 2 \\ \text { Cedarville } & 3 & \text { Wilmington College } & 2 \\ \text { Cedarville } & 8 & \text { Rio Grande College } & 3 \\ \text { Cedarville } & 7 & \text { Rio Grande College } & 10 \\ \text { Cedarville } & 5 & \text { Earlham College } & 4 \\ \text { Cedarville } & 8 & \text { Earlham College } & 2 \\ \text { Cedarville } & 9 & \text { Ohio Dominican College } & 8 \\ \text { Cedarville } & 5 & \text { Ohio Dominican College } & 8 \\ \text { Cedarville } & \text { NCCAA Playoff District Championship } & \\ \text { Cedarville } & 8 & \text { Bethel College } & 2 \\ \text { Cllatile } & \text { Bethel College } & 3\end{array}$

Cedarville

Tim Buhr 3B

Tim Buhr 3B

Jack Browning SS

Dave Johnston LF 

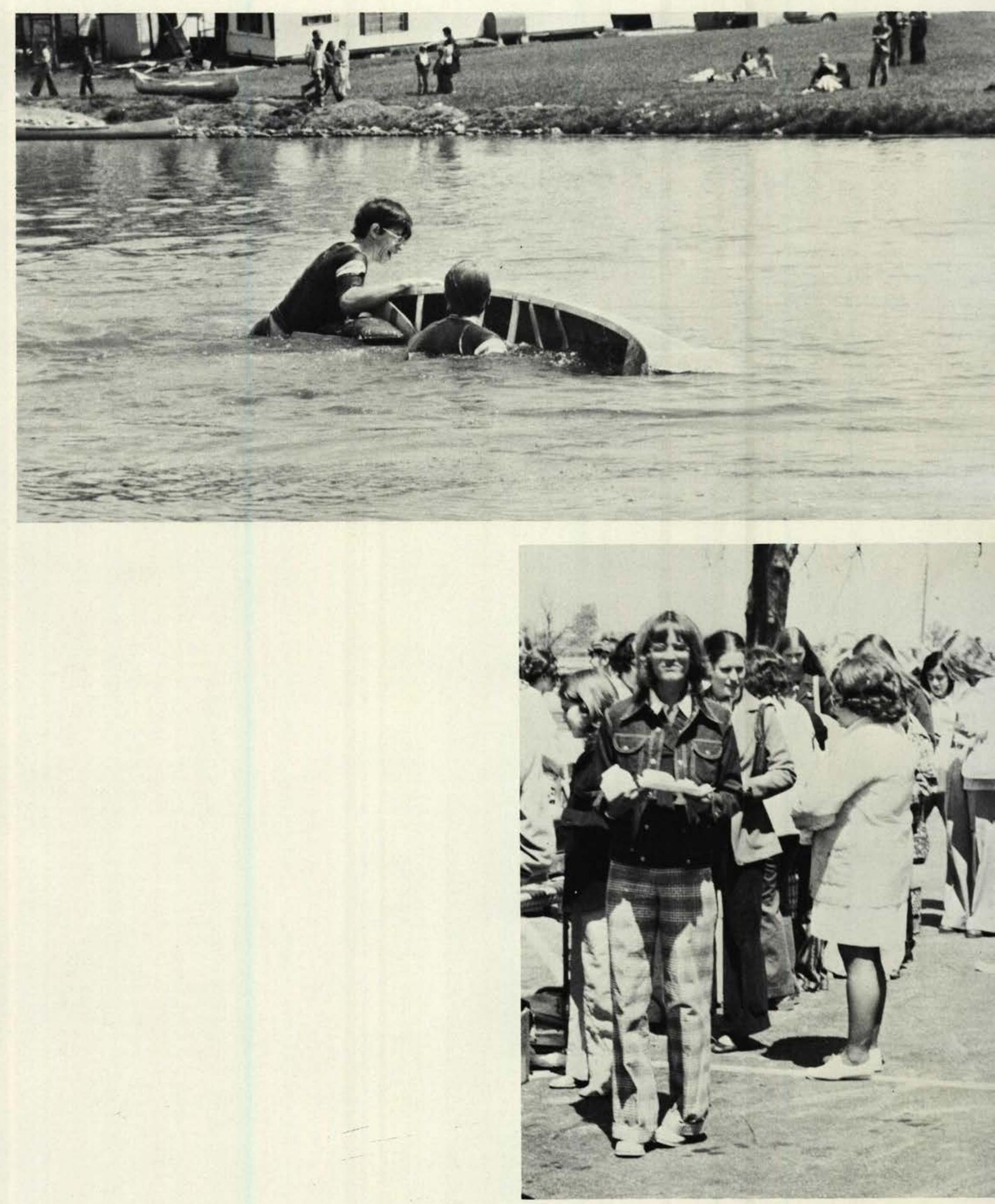


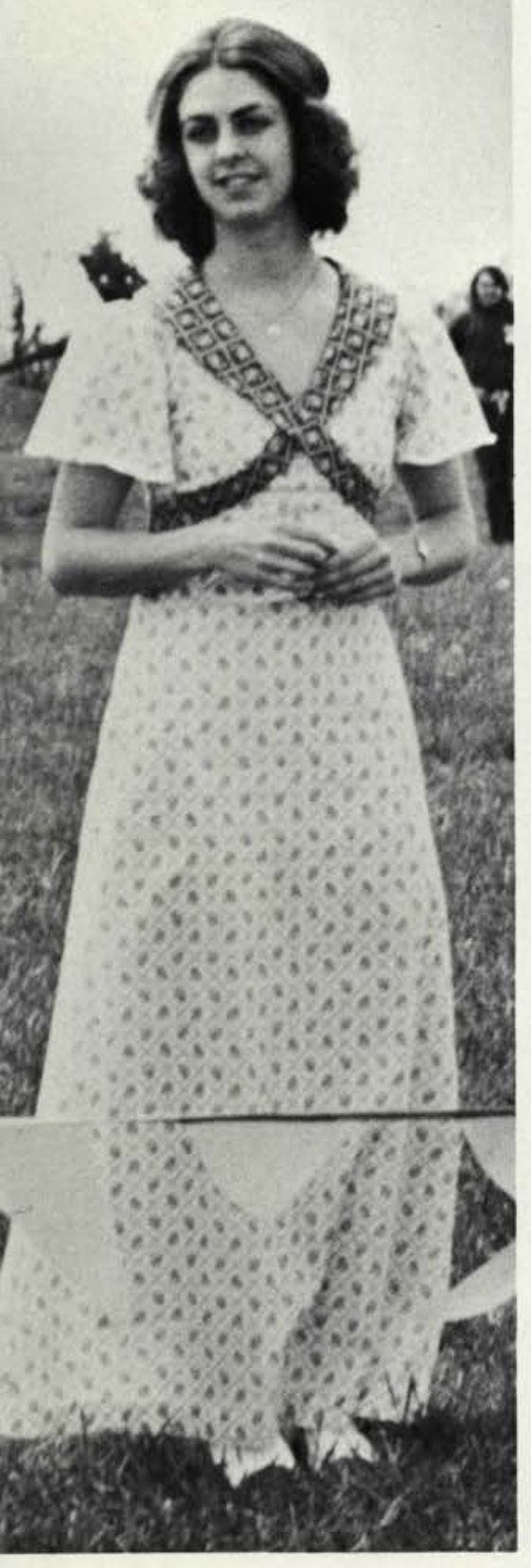

\section{Cedar Day}

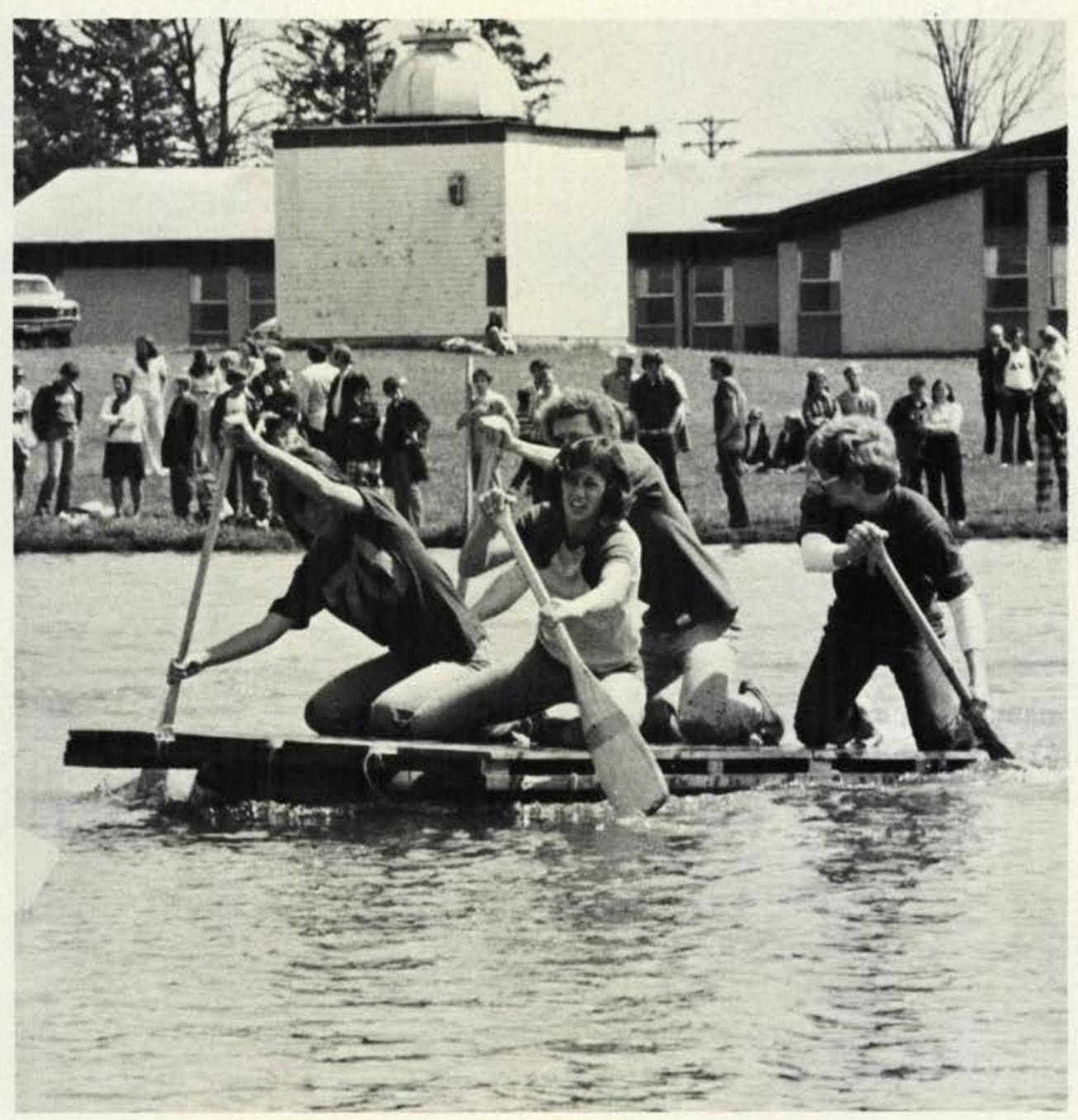

Hin

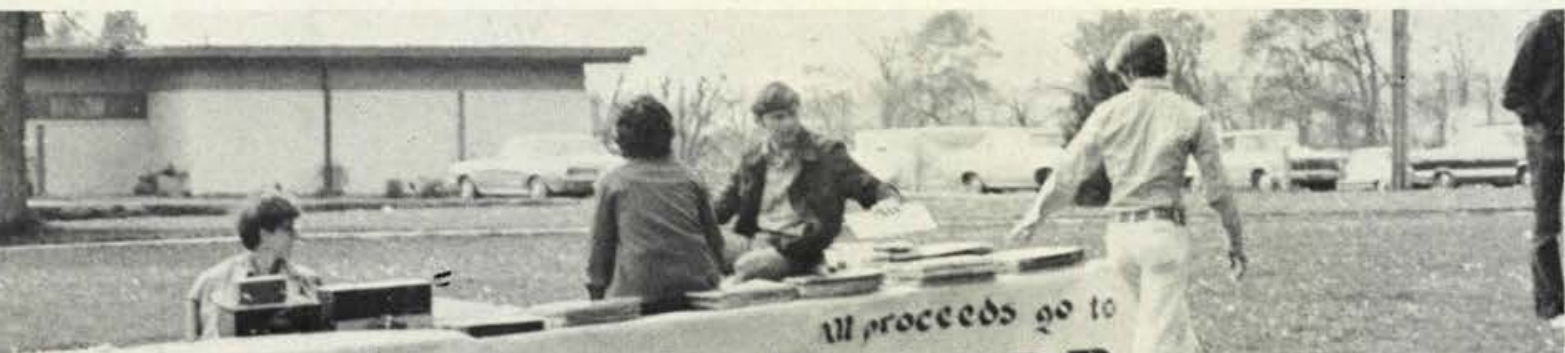

(1) Rasto club of c.c.

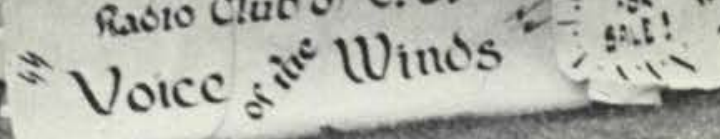




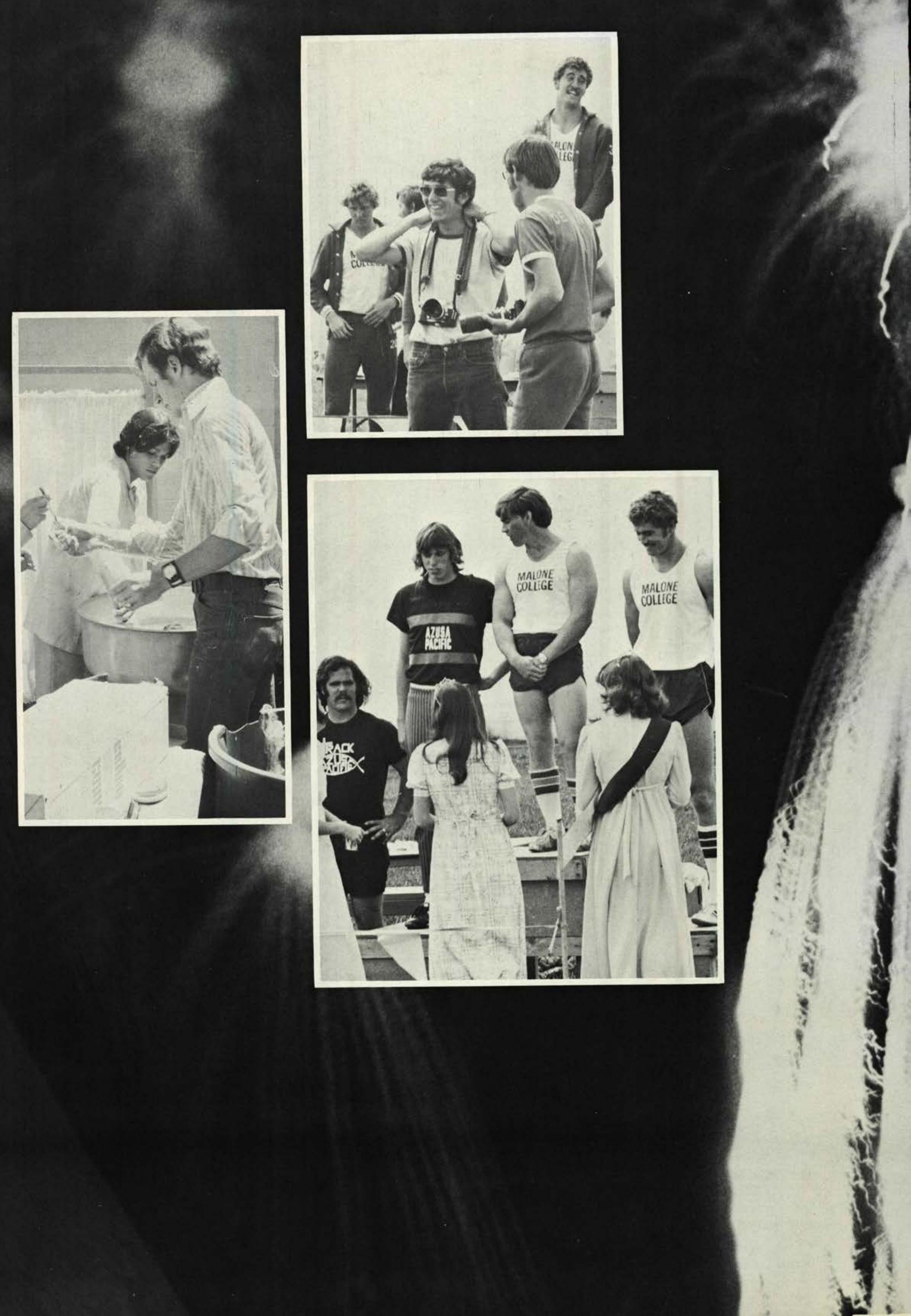



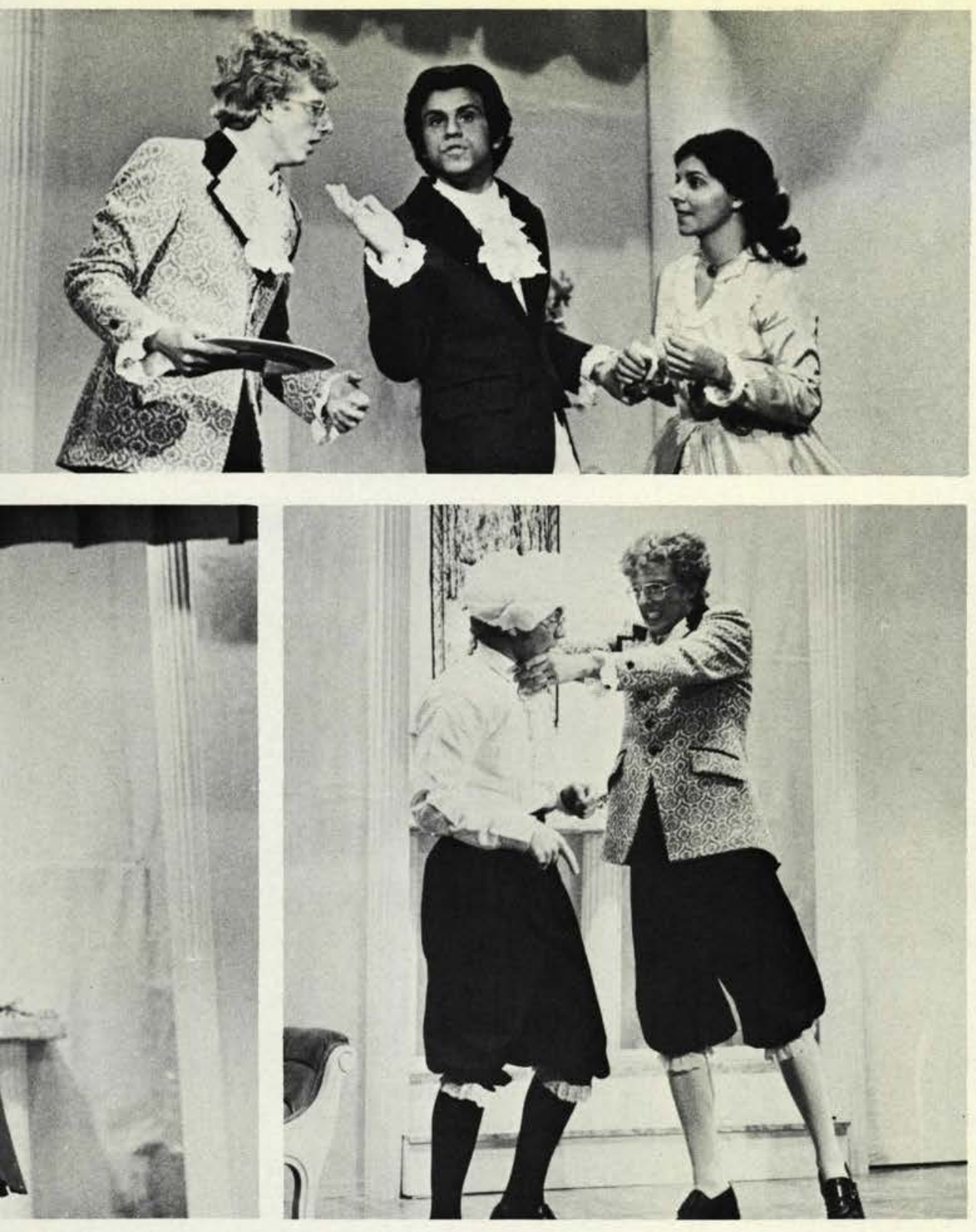

\section{Spring Play: The Miser}

Director: Mrs. Miriam Maddox

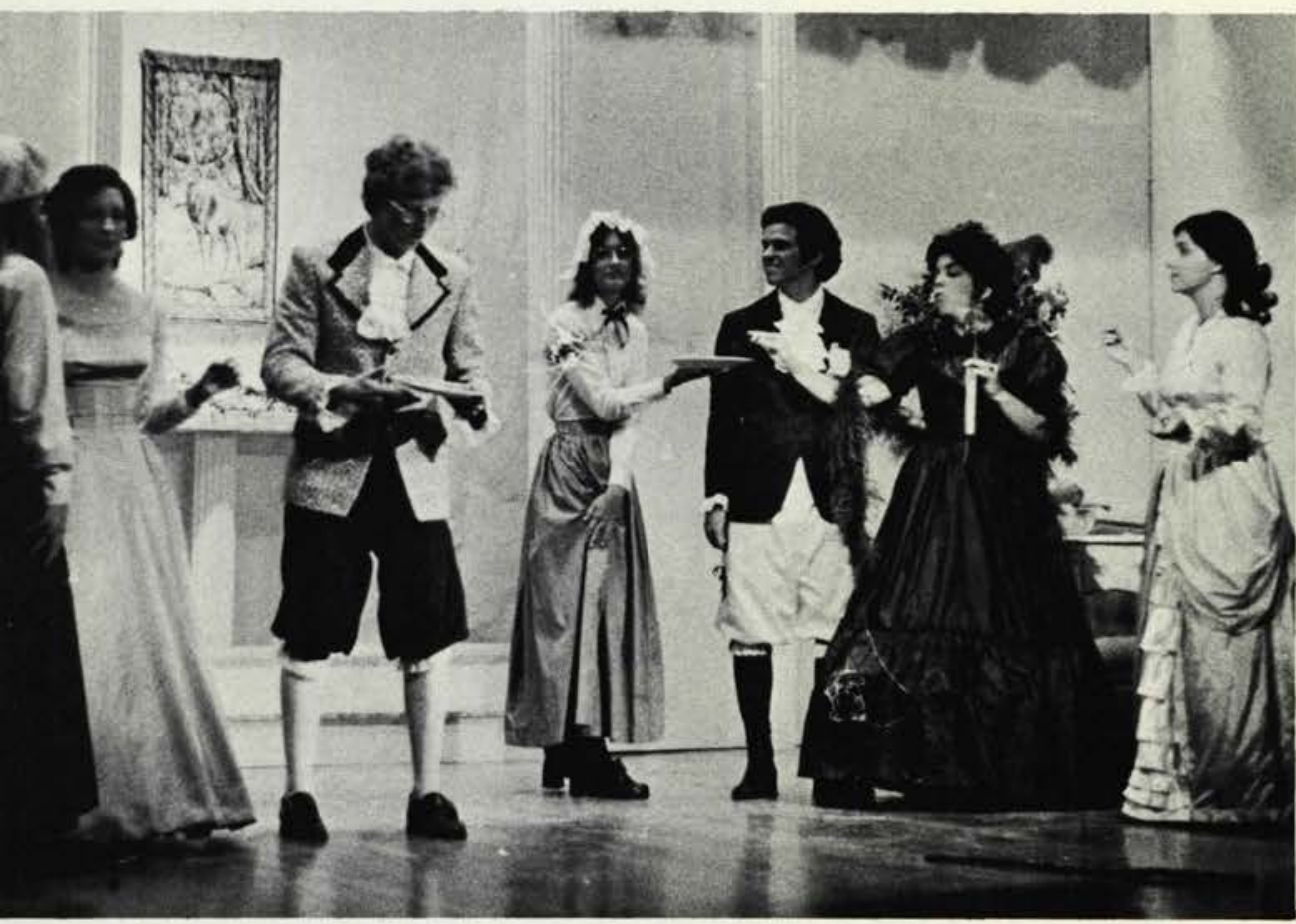




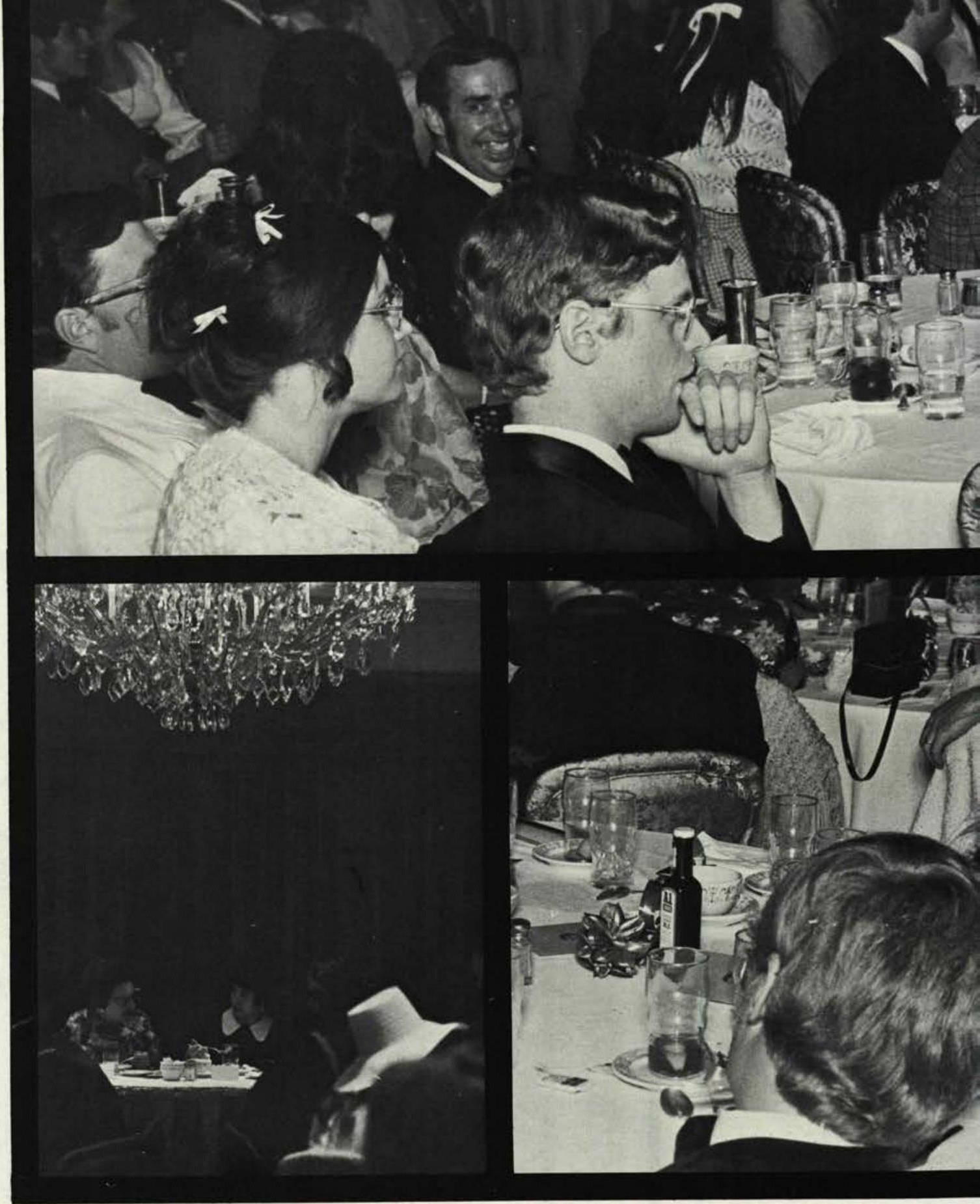

\section{Aufwiedersehen}

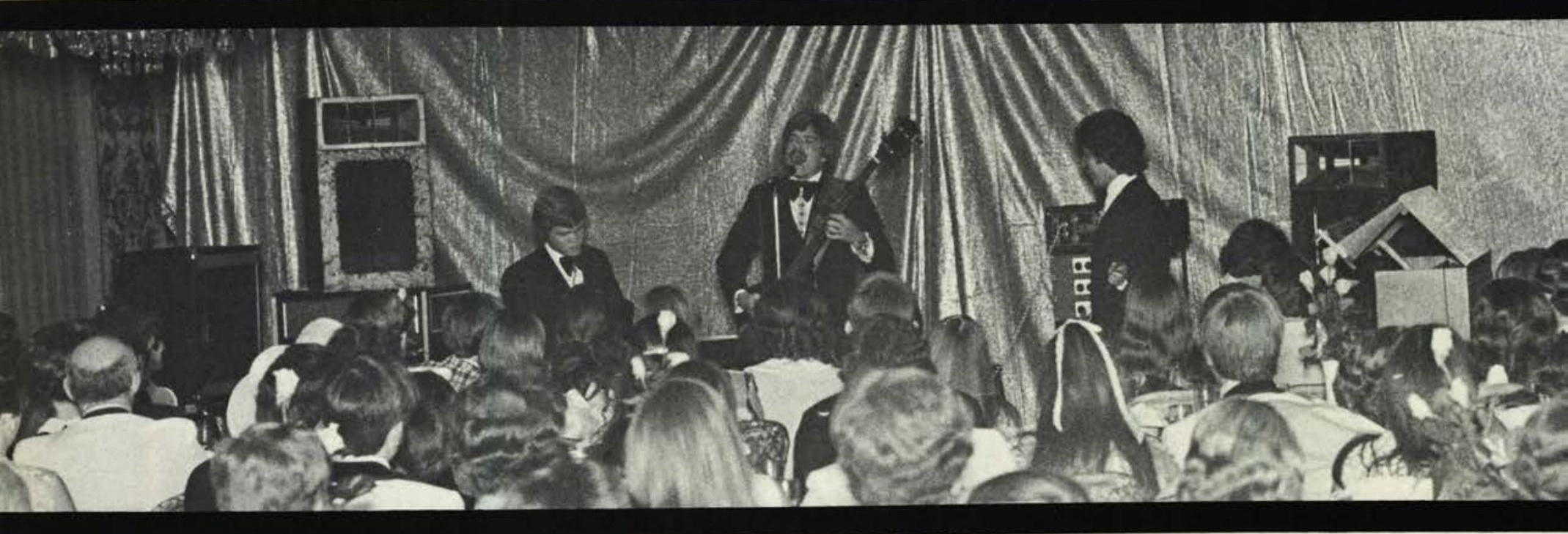



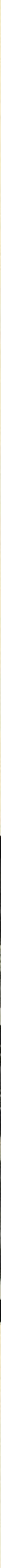


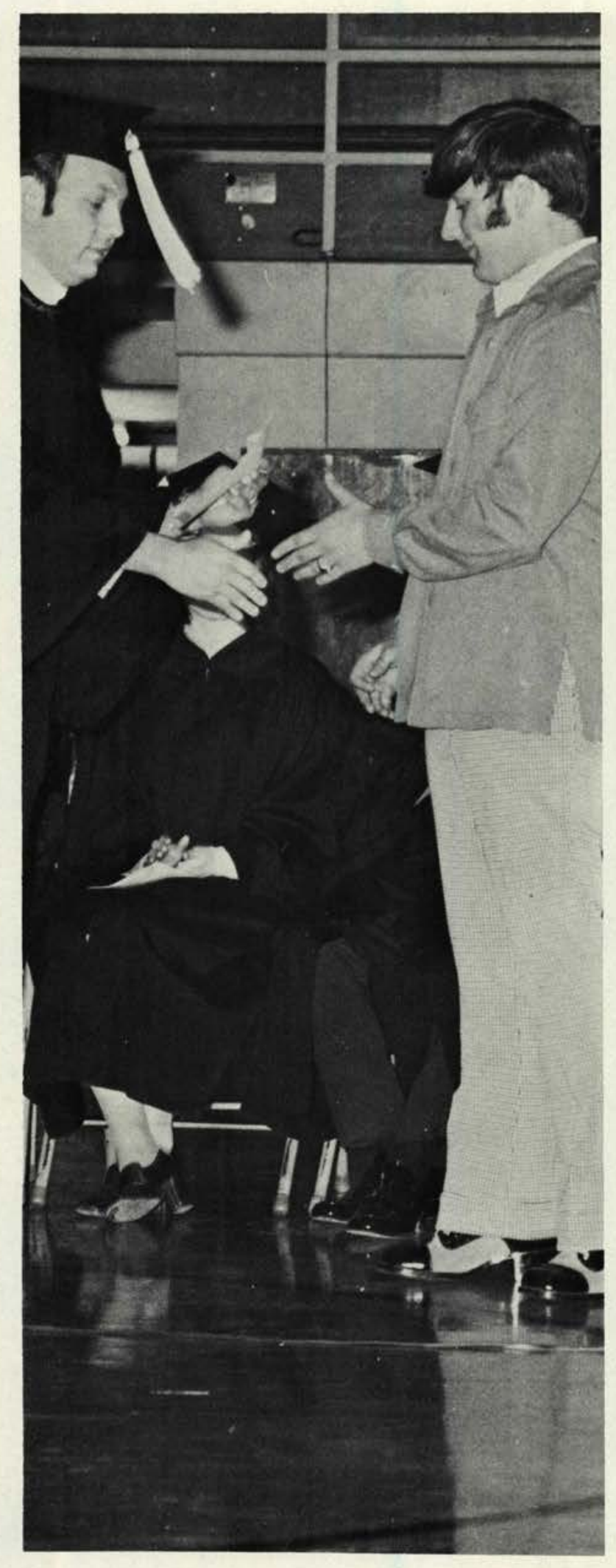

\section{A Day of Honors}

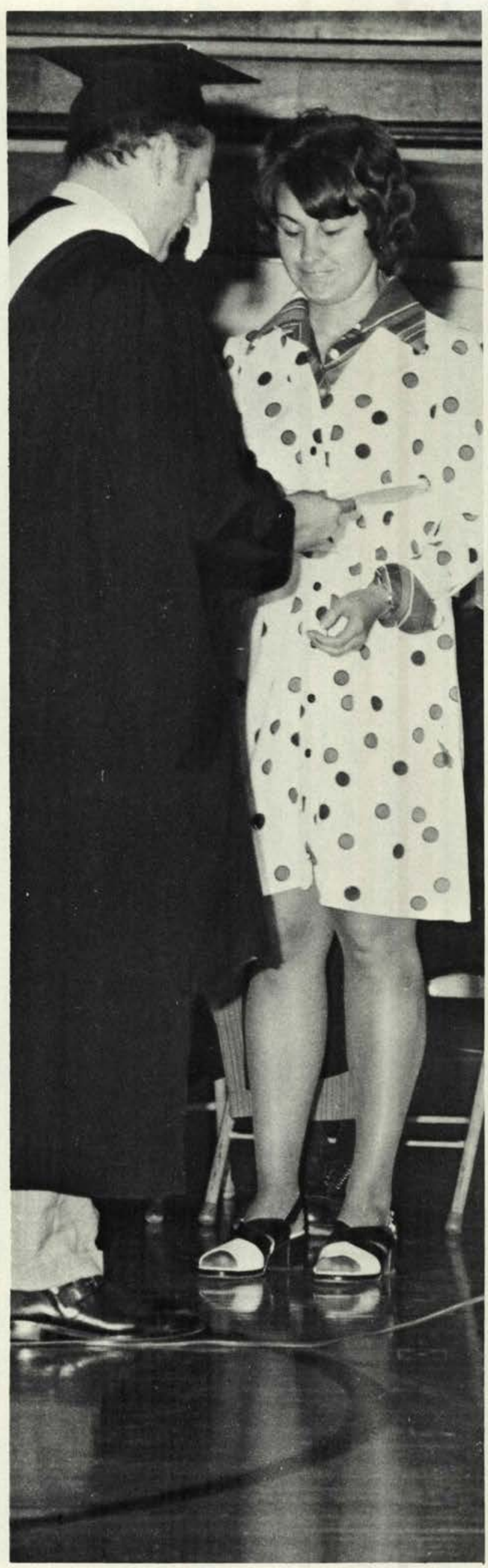




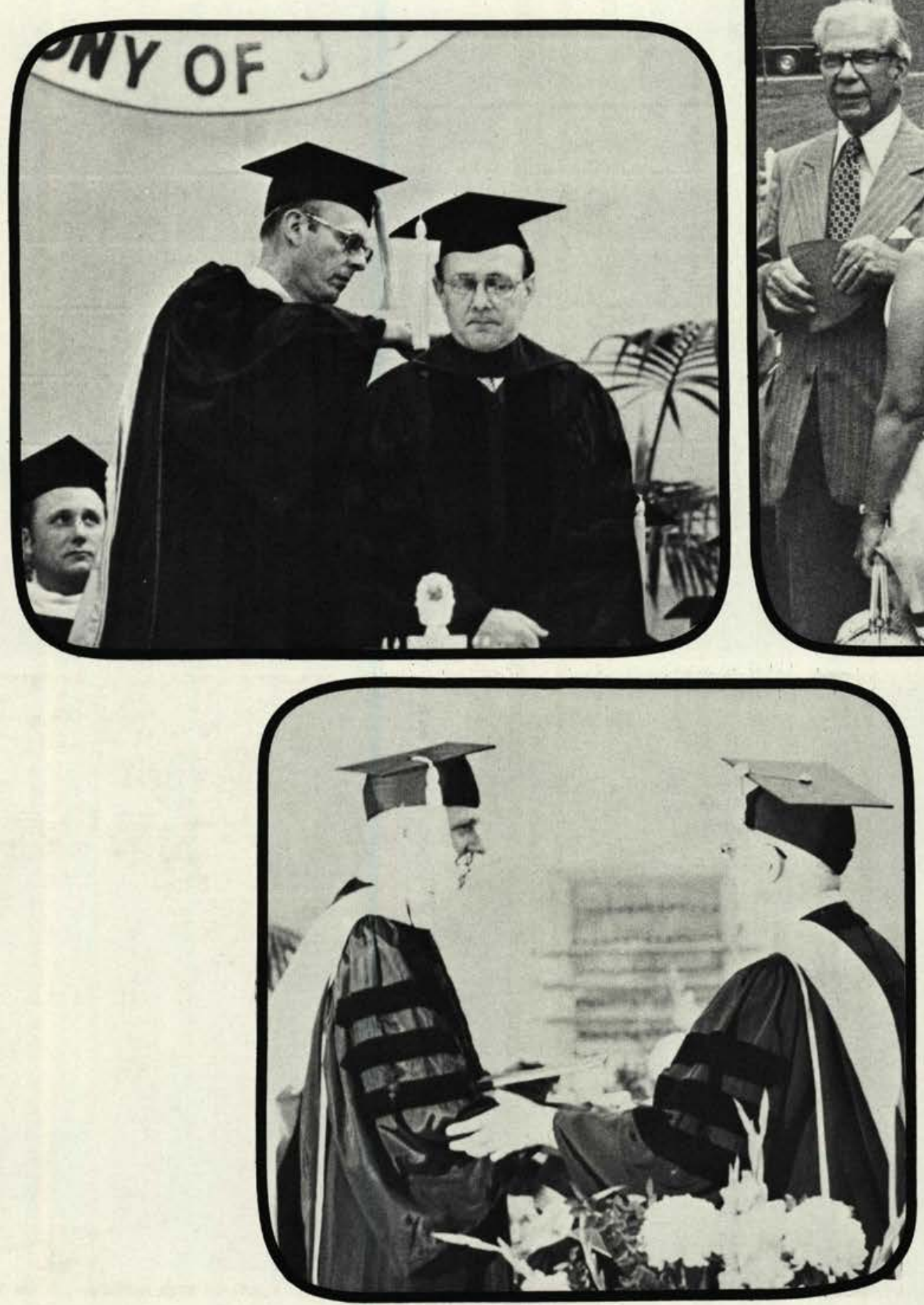




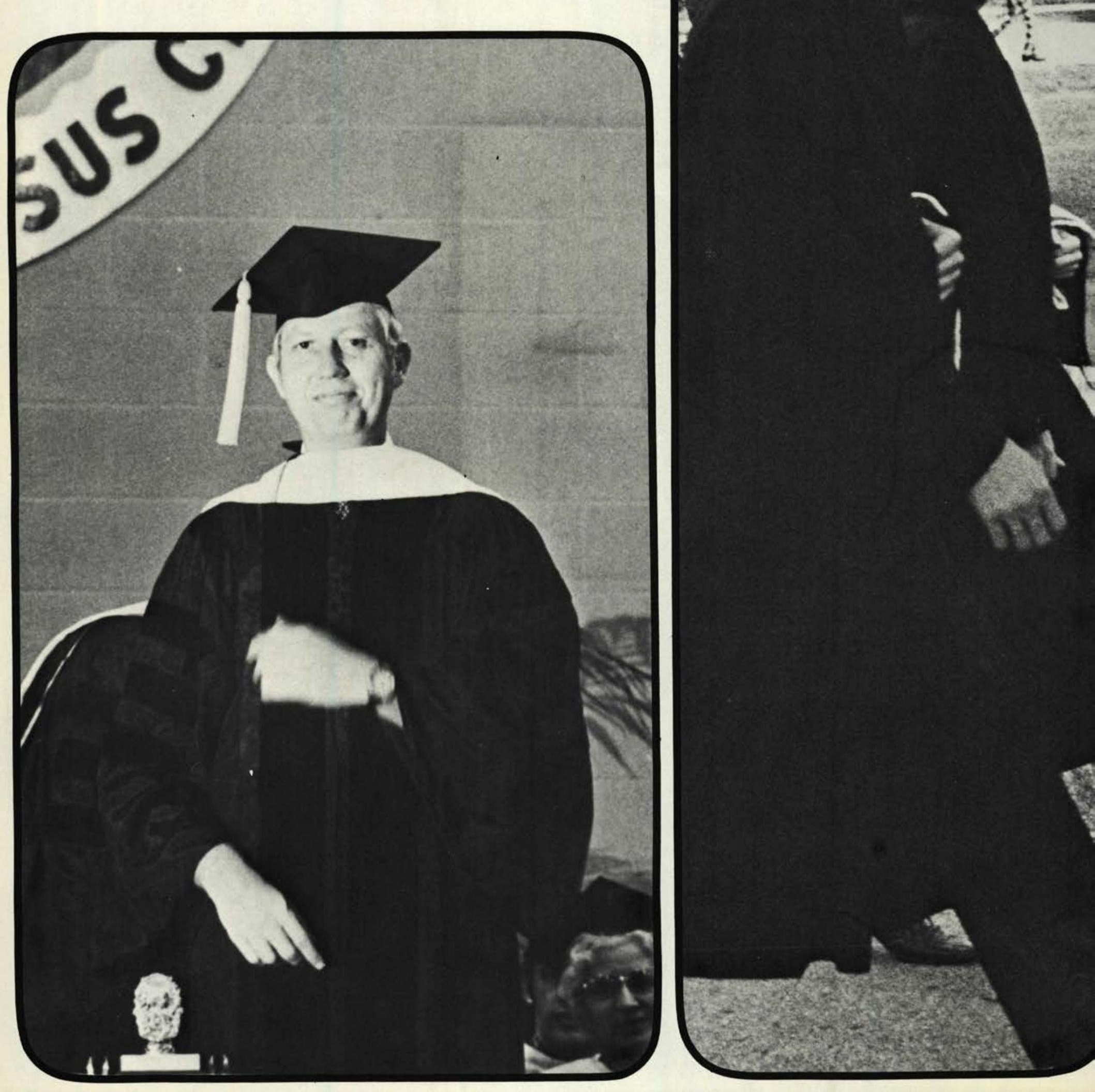




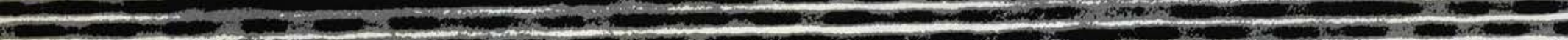

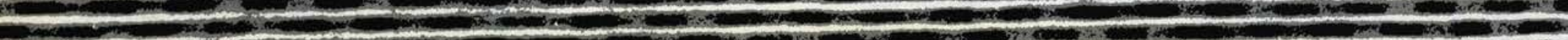

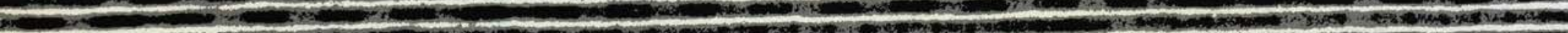

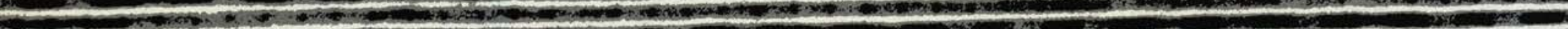
20

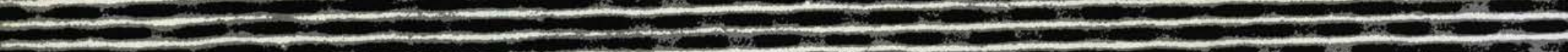

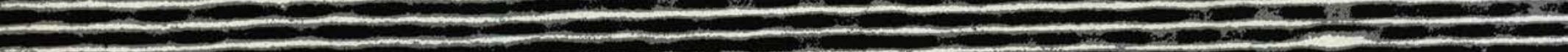

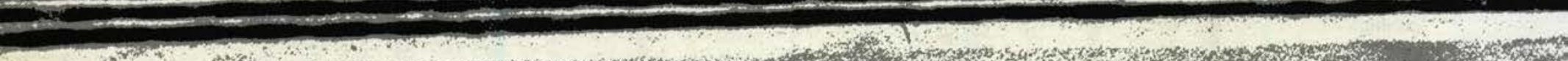

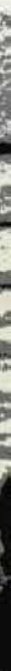
$-20=$

$=$

(a)

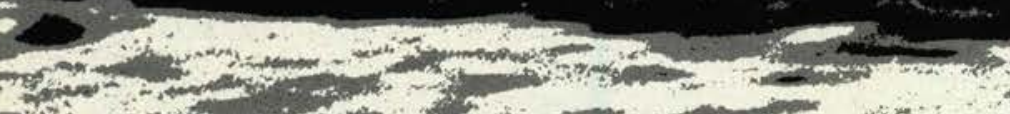

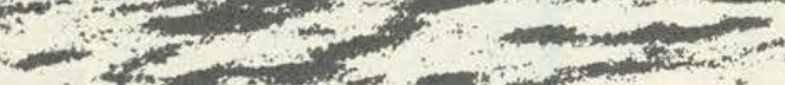

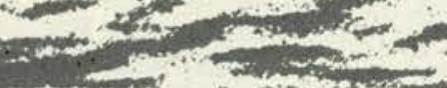
$10 . \quad x^{2}$

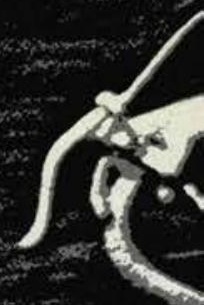

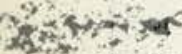



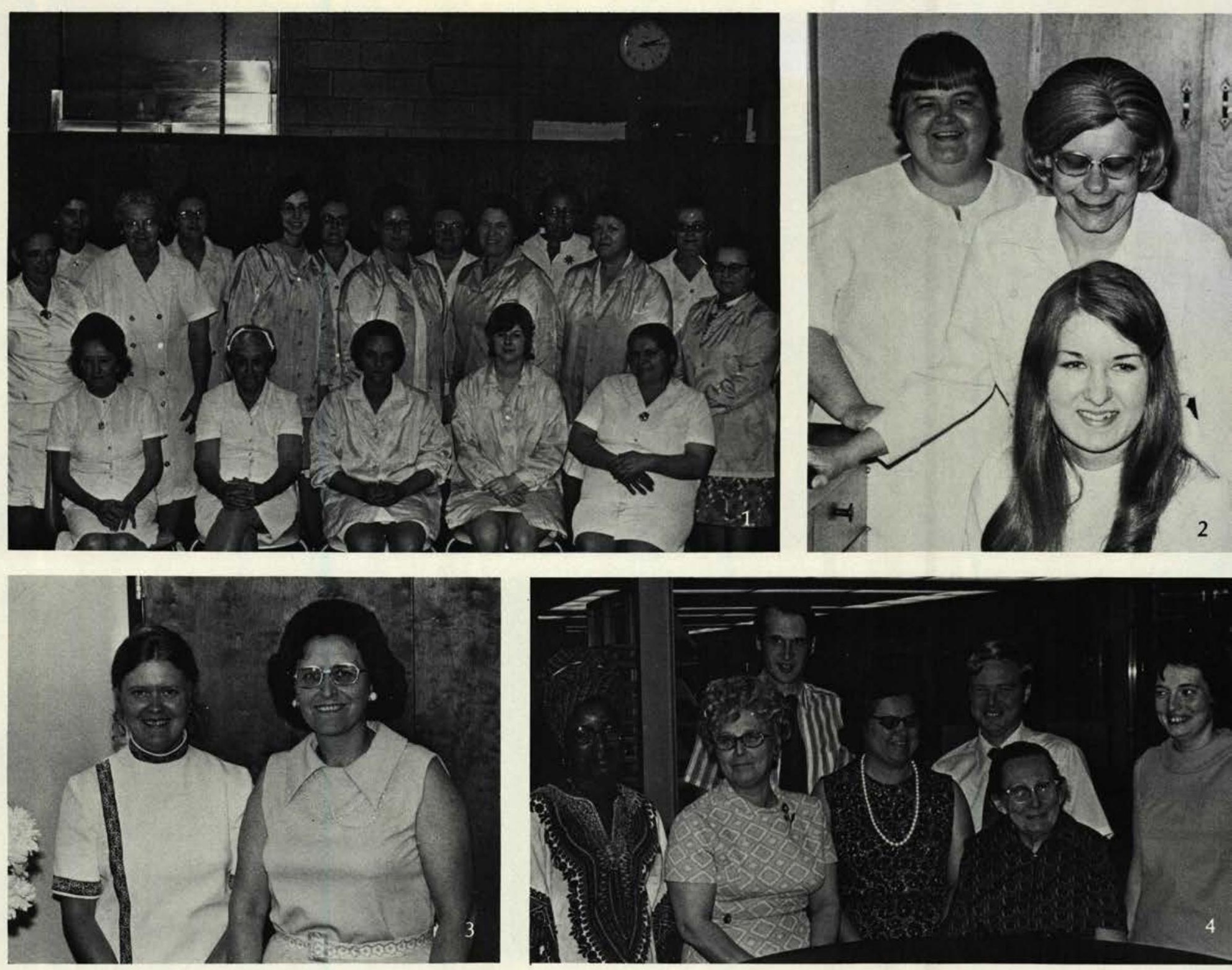

1. Cafeteria

2. Health Service

3. Secretaries to the Dir. of Development, Mrs. Hague, and to the Academic Dean, Mrs. Greenwood.

4. Library, full time

5. Secretary to the President, Mrs. Monroe

6. Secretary for the Education Office, Elaine Howard.

7. Resident Advisors
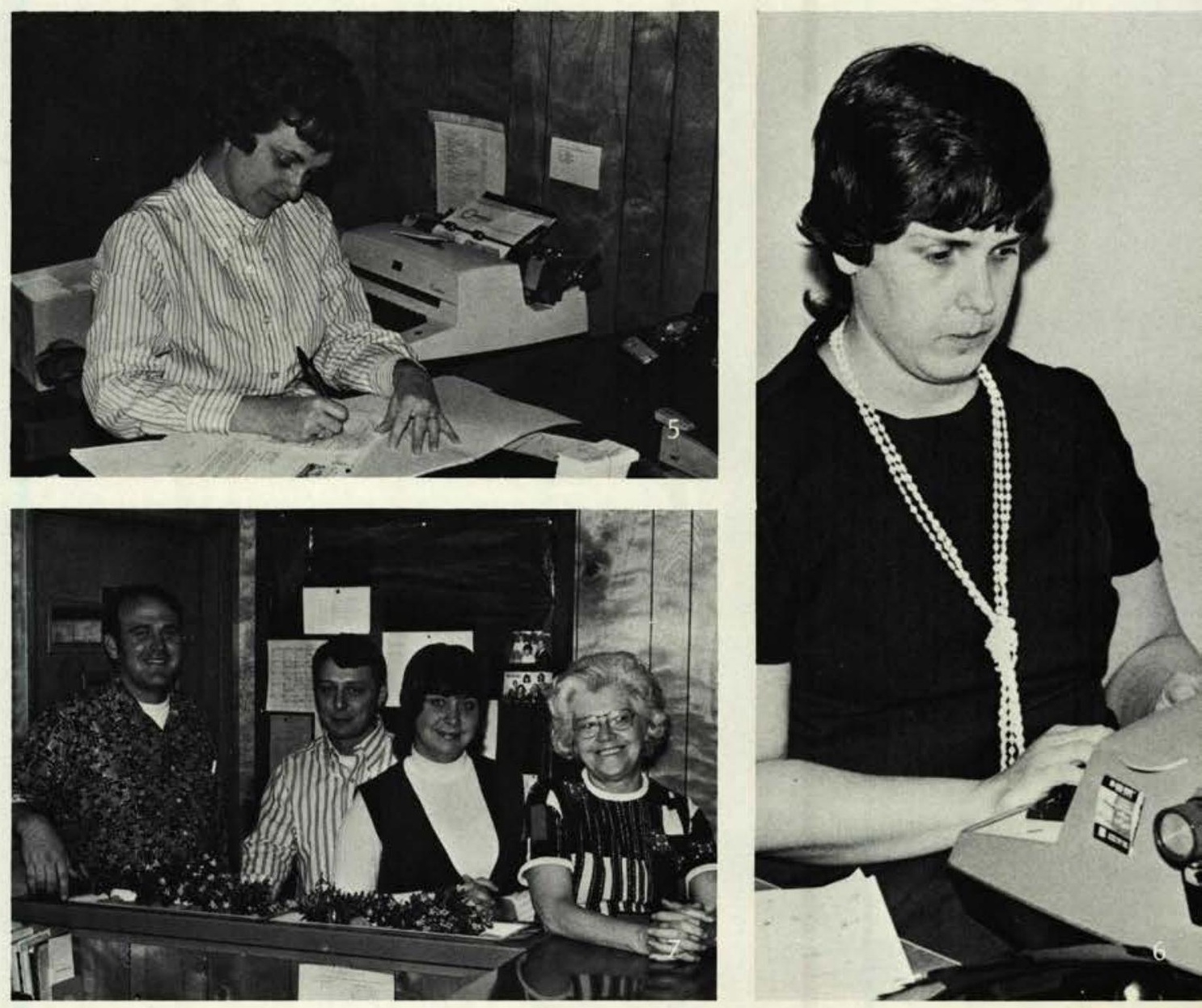

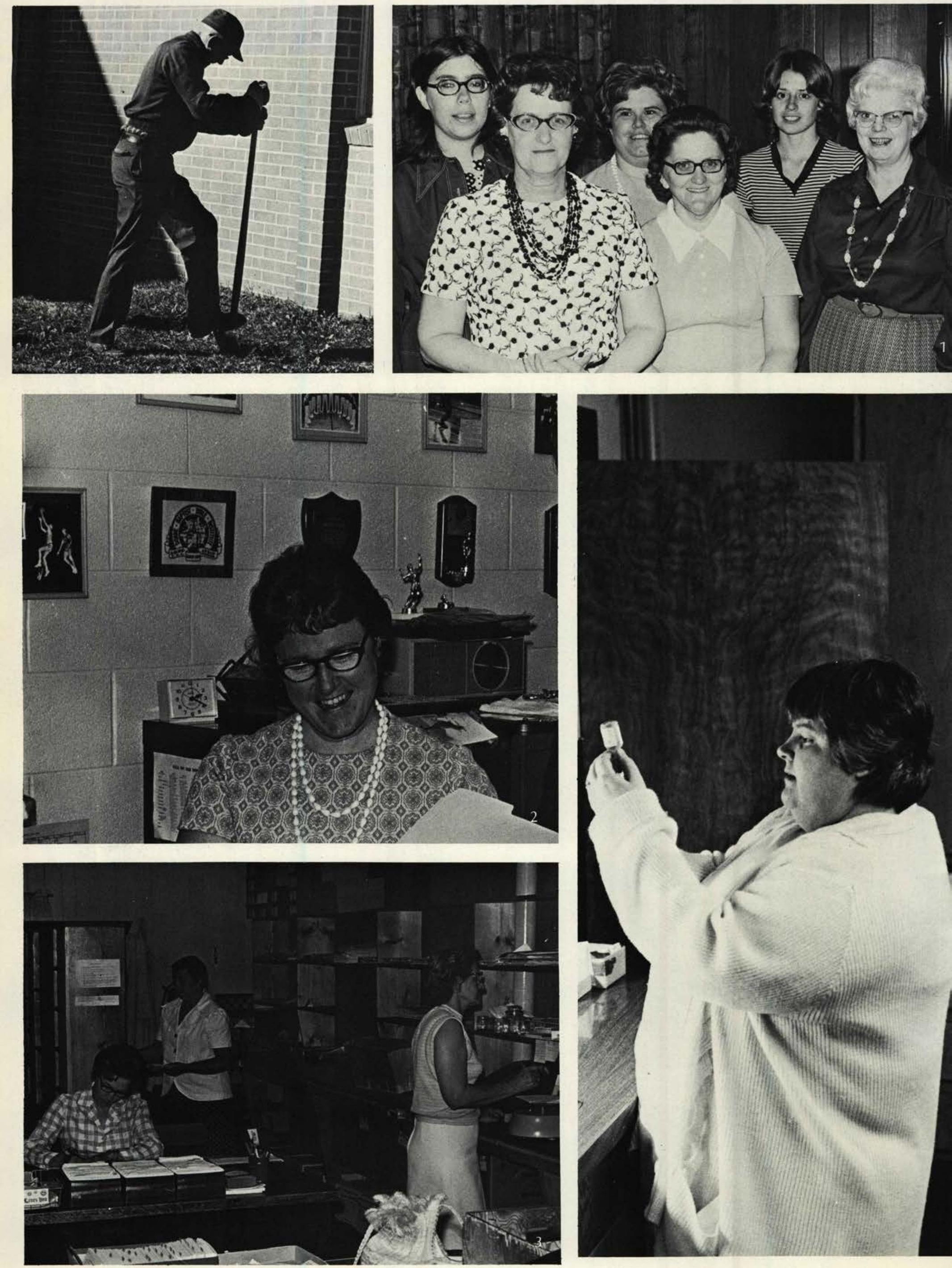



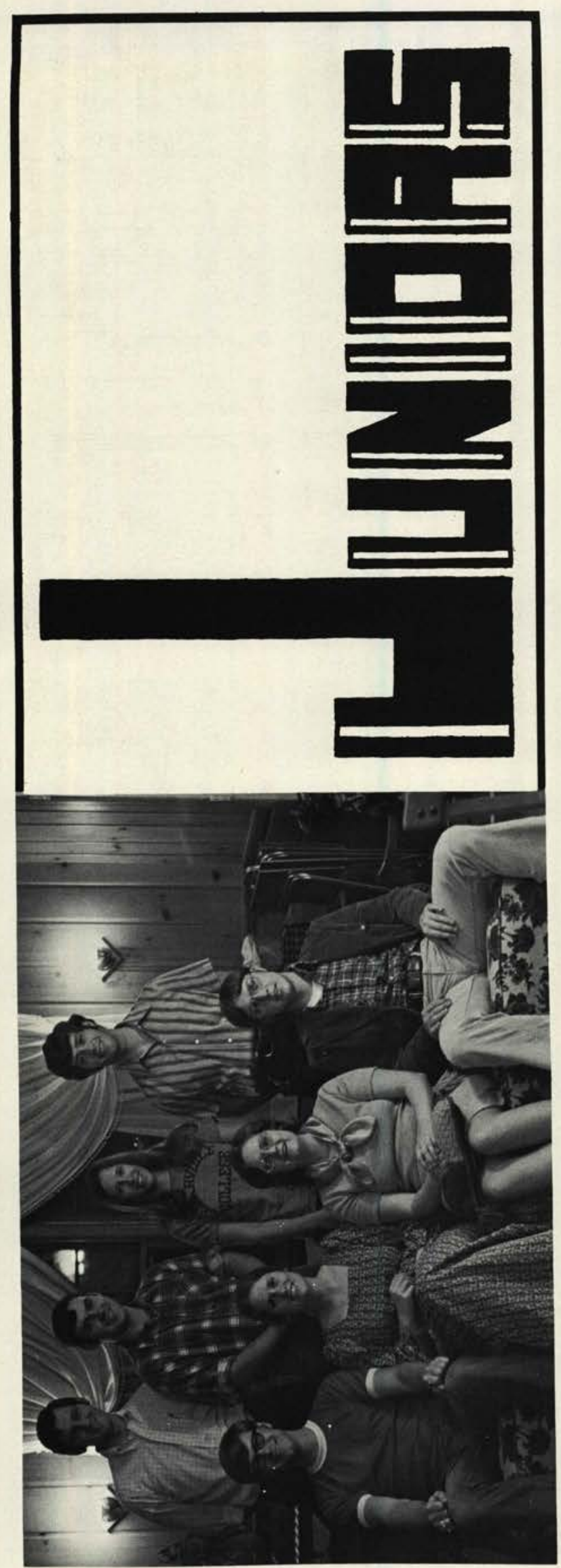

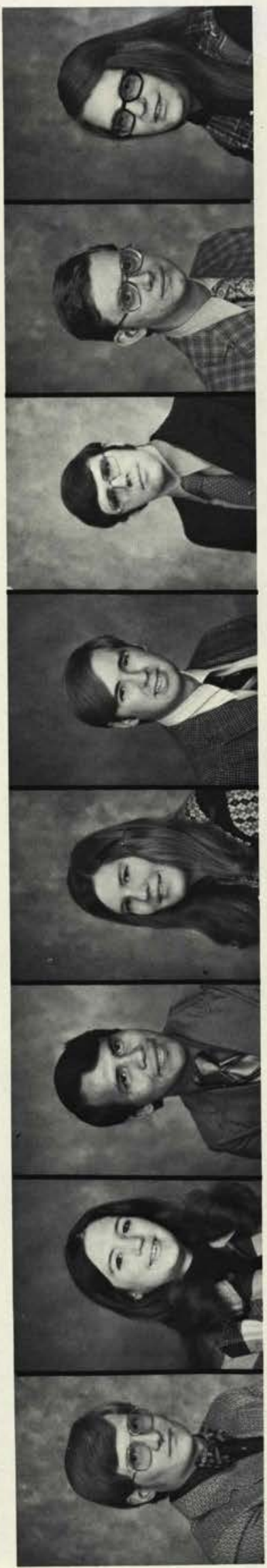
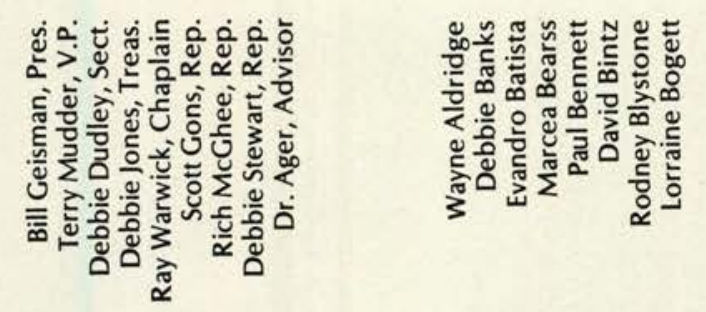
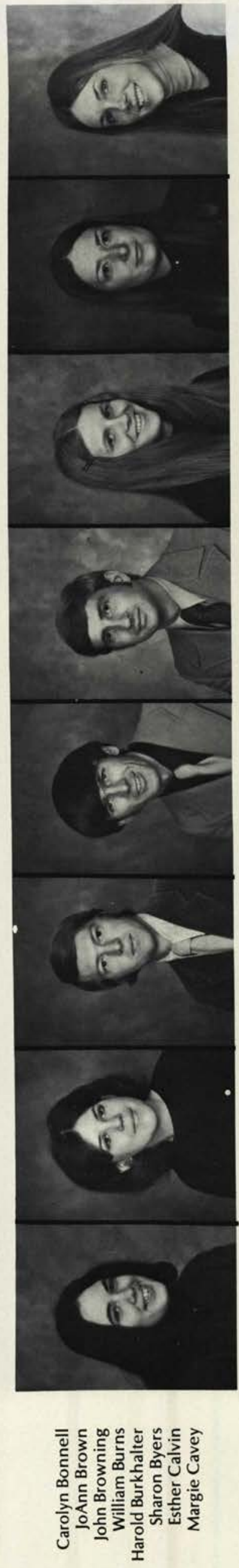

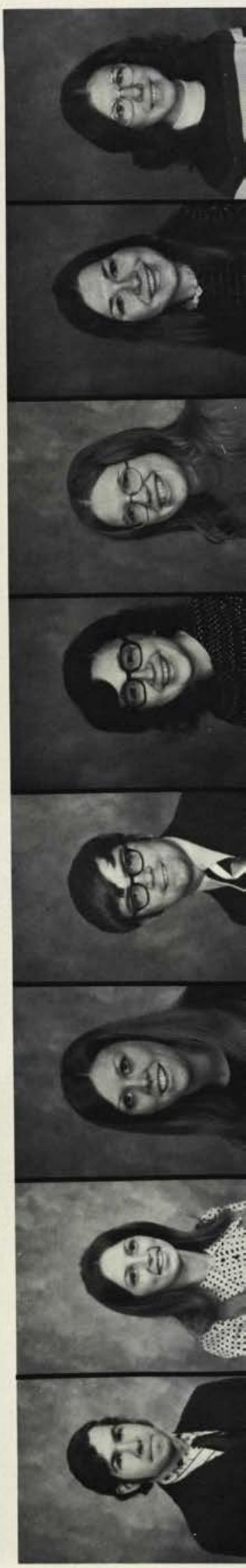



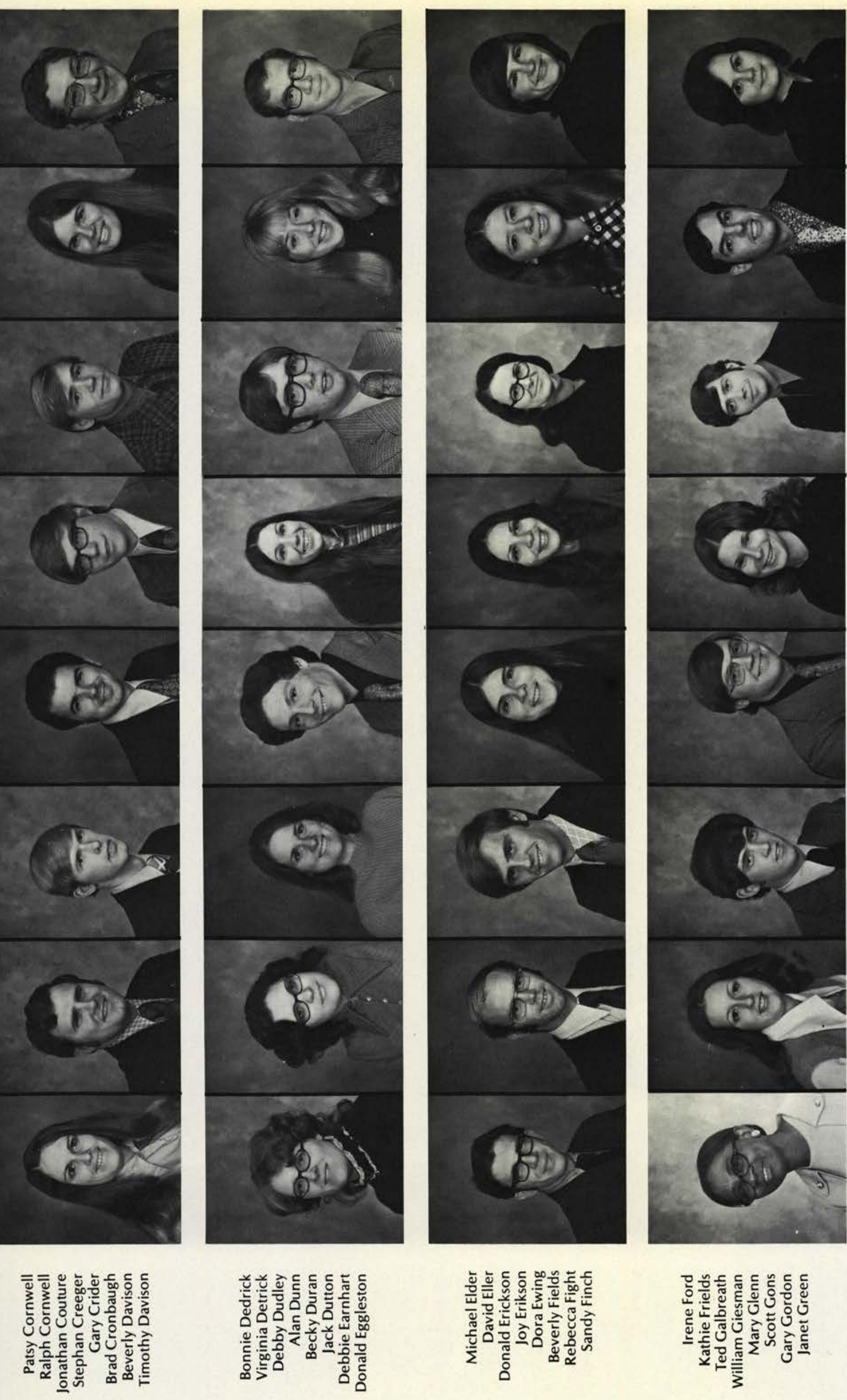

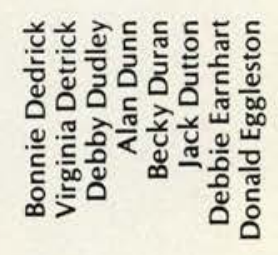

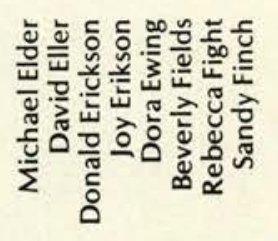

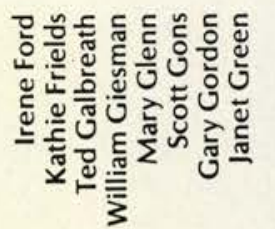



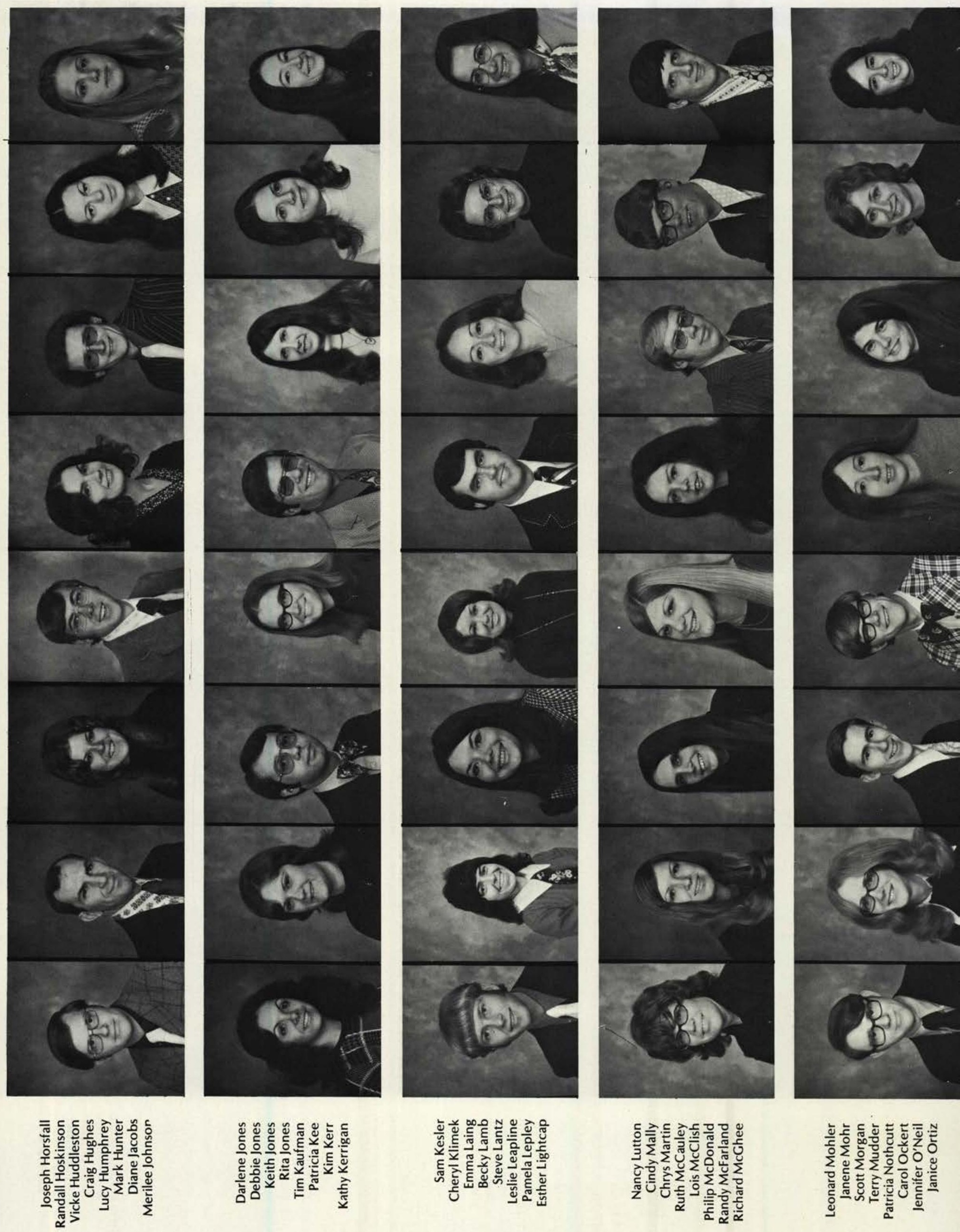

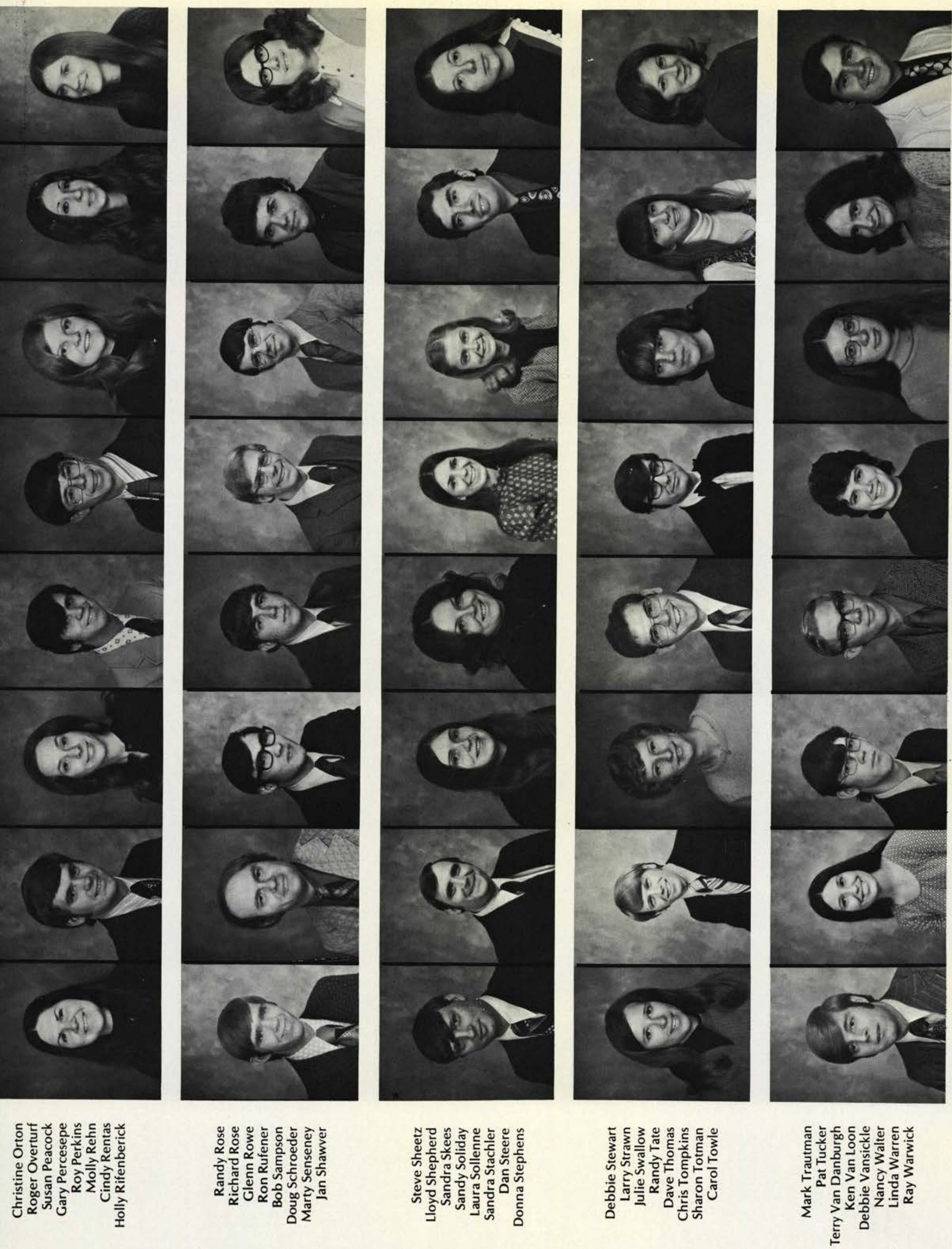


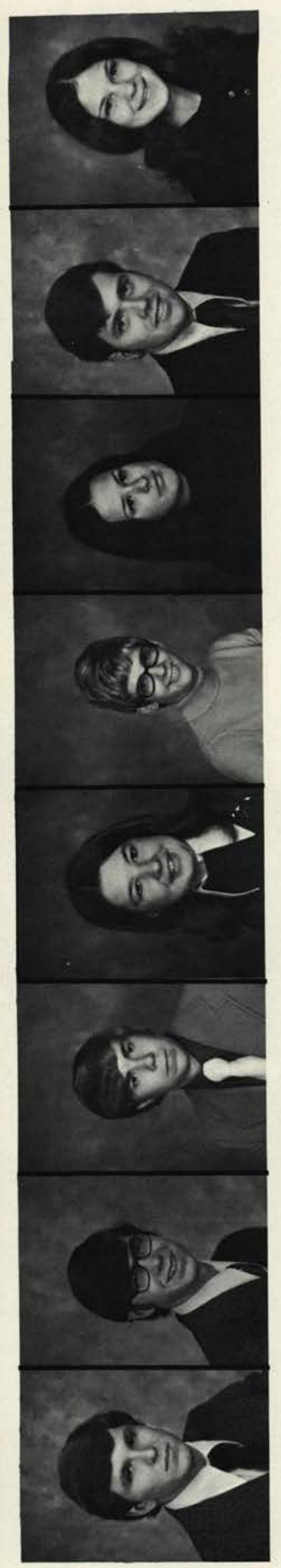

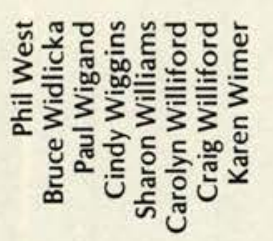
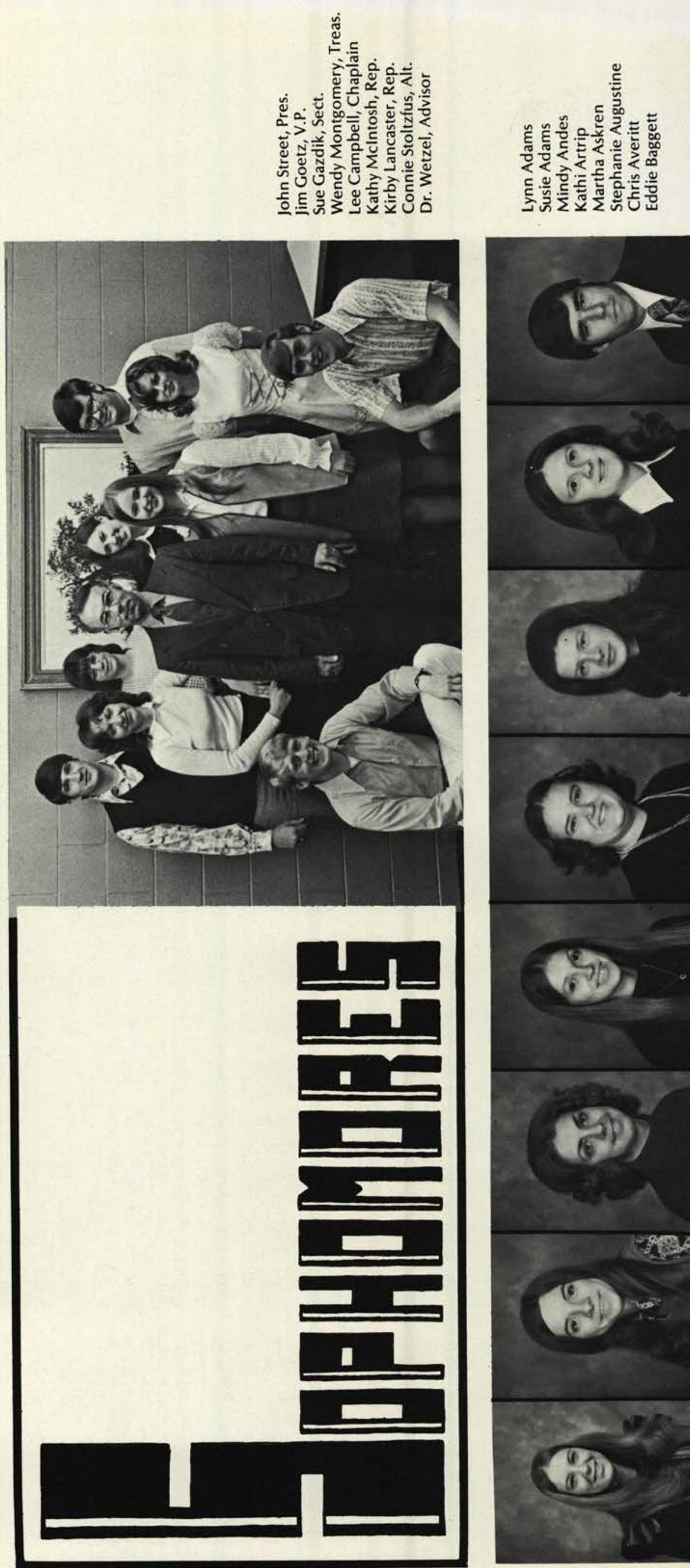


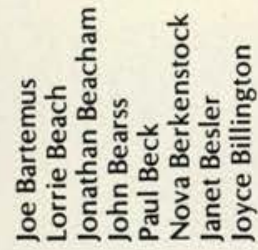

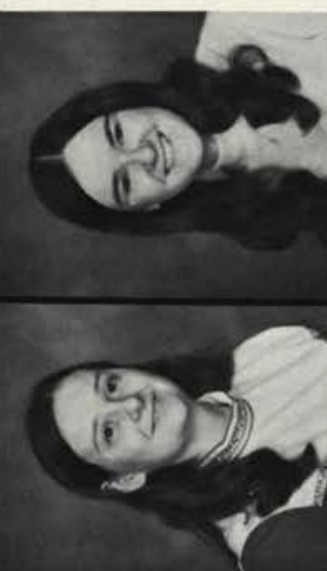

(a)

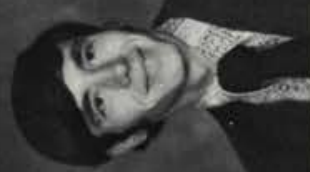

(5)

(6)

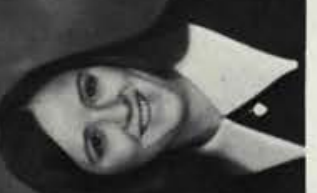

(iv)

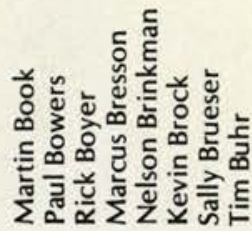

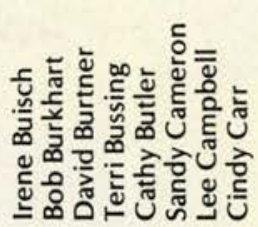

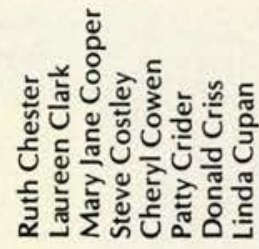

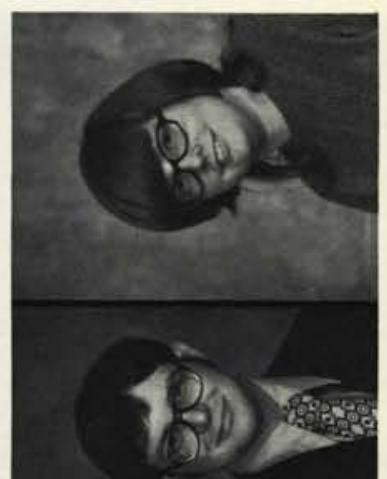

(8)

(8)
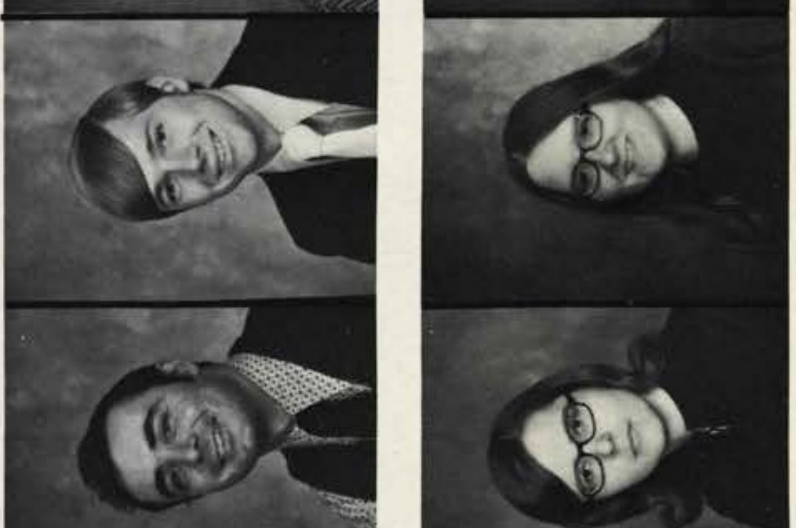

(i)

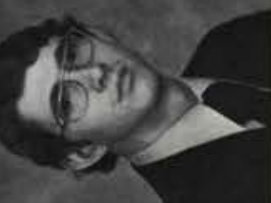

(i) 2
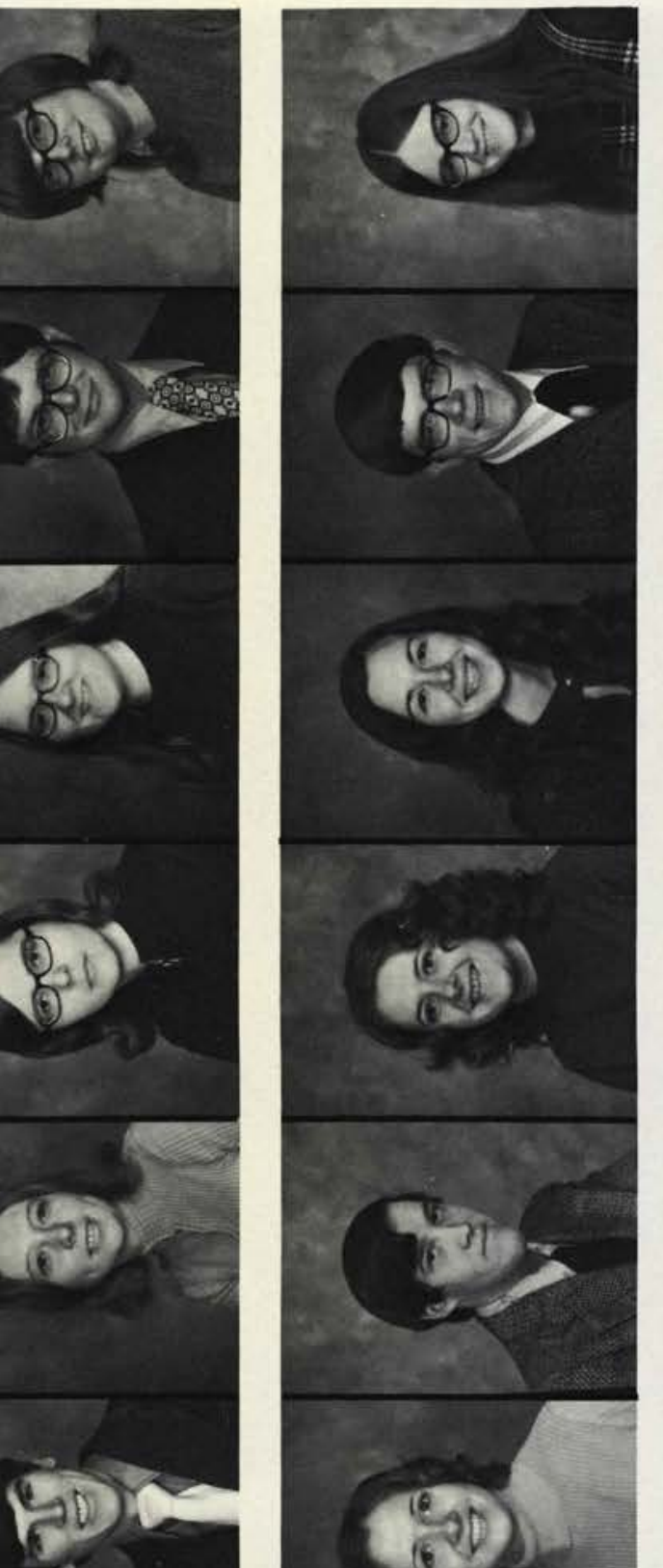

(62).

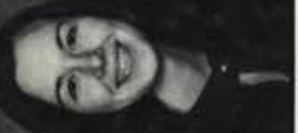

(6)
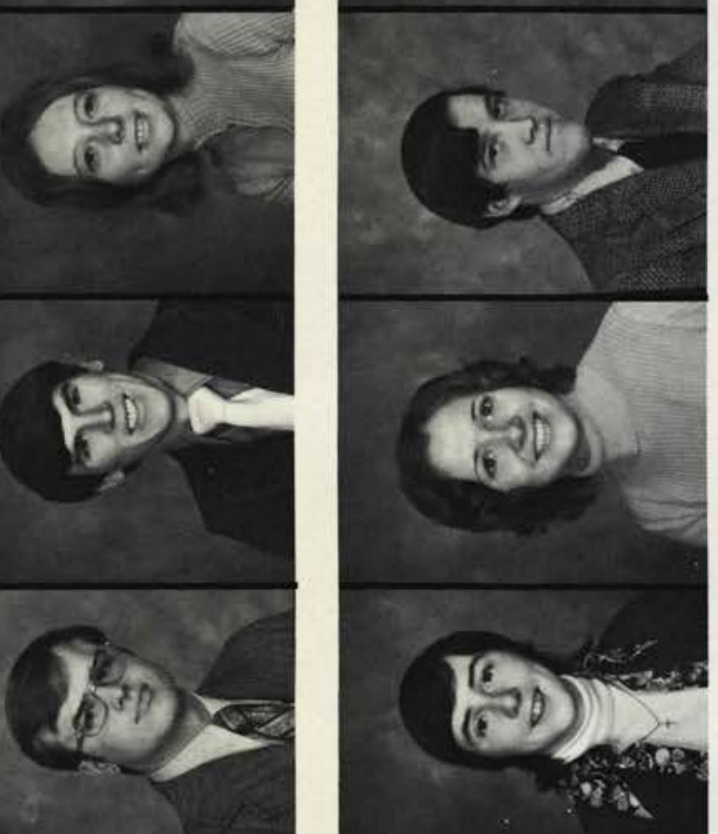

(2i) ) [11
(5)
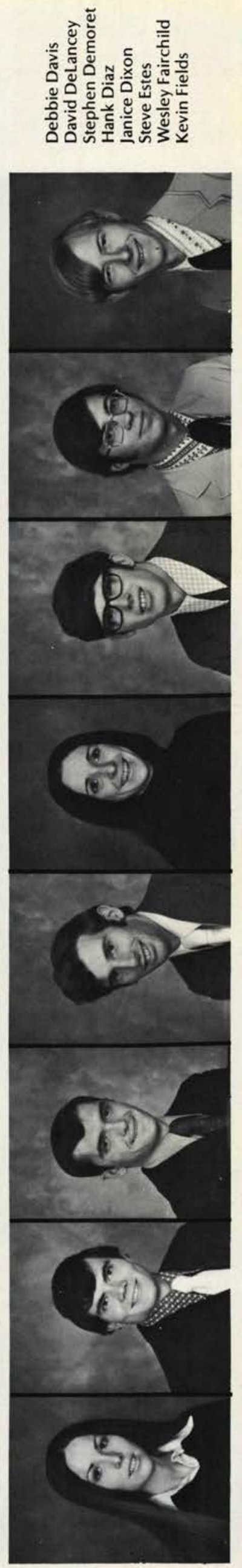


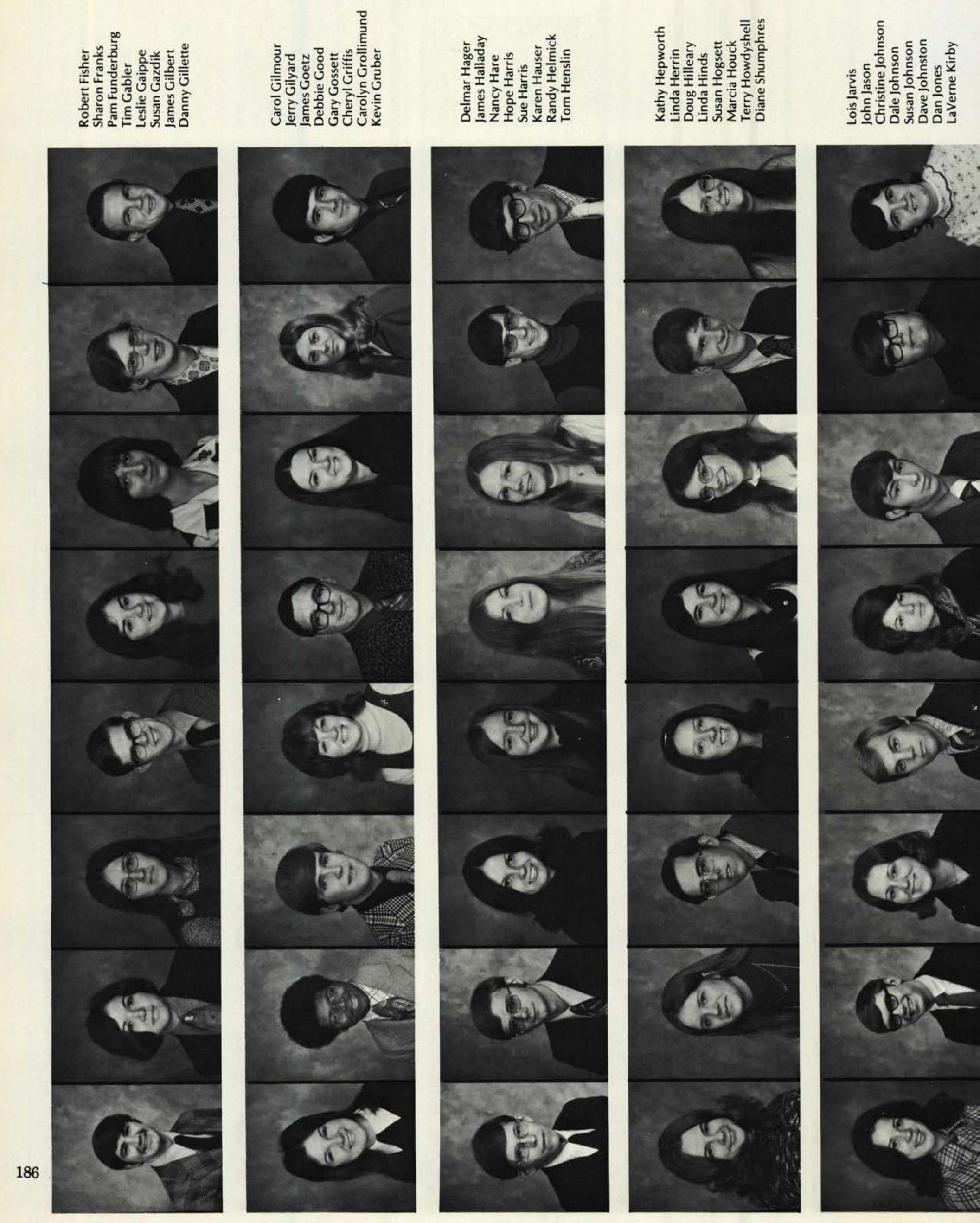




\section{范

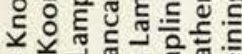

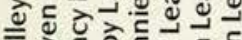

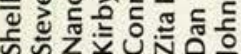

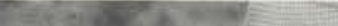 \\ (D)

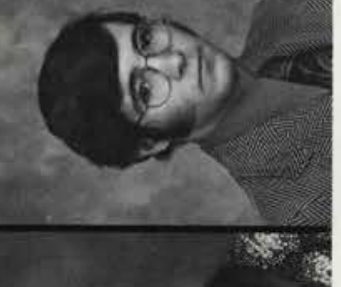

$\Rightarrow D^{2}$

3).

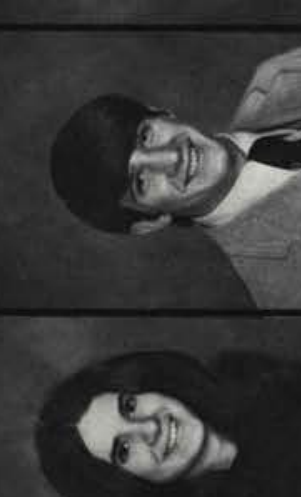

S.

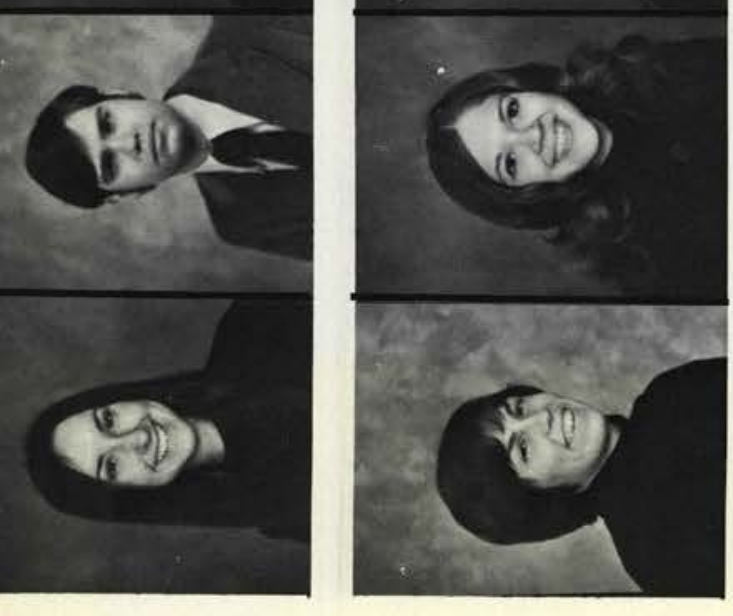

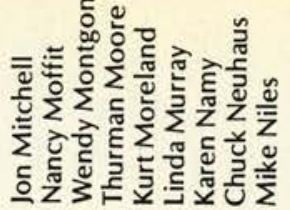

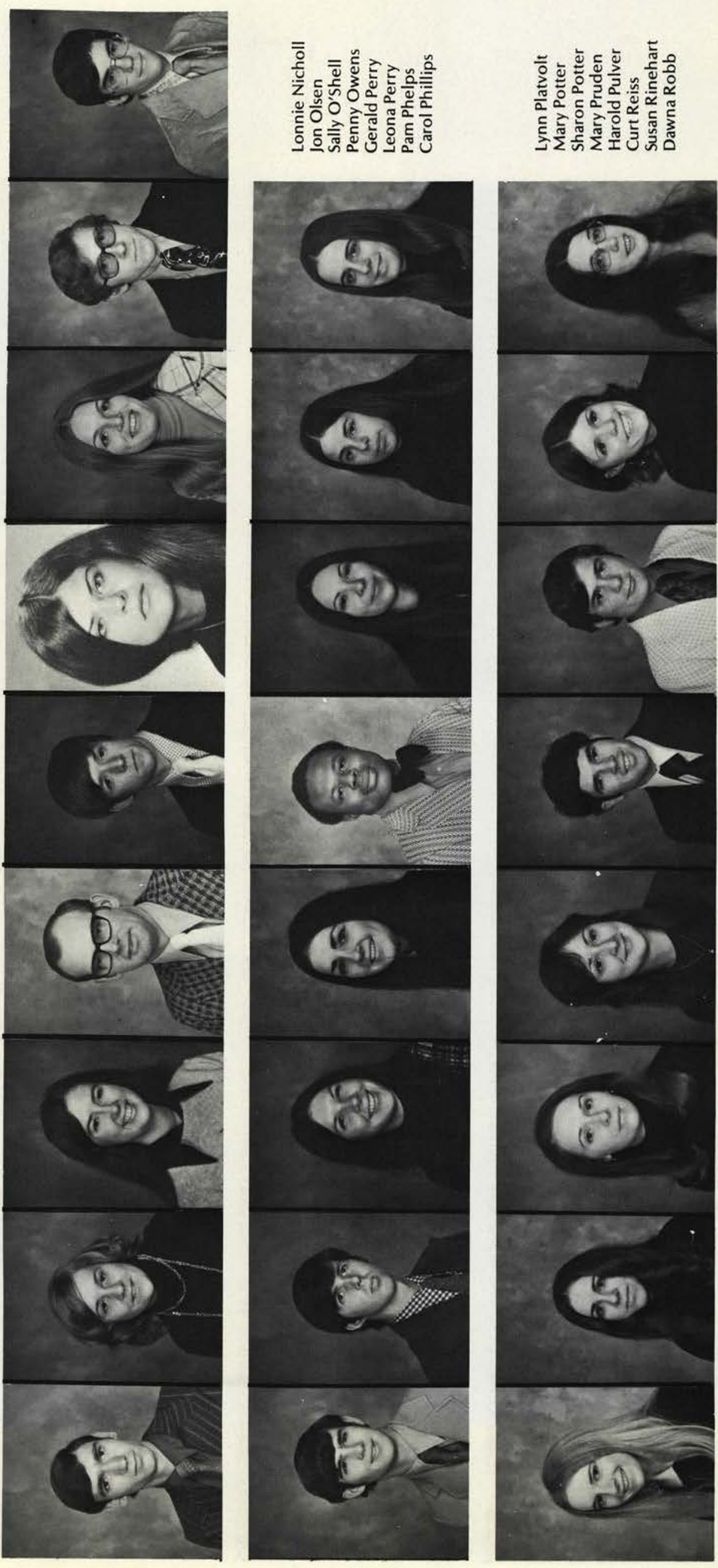



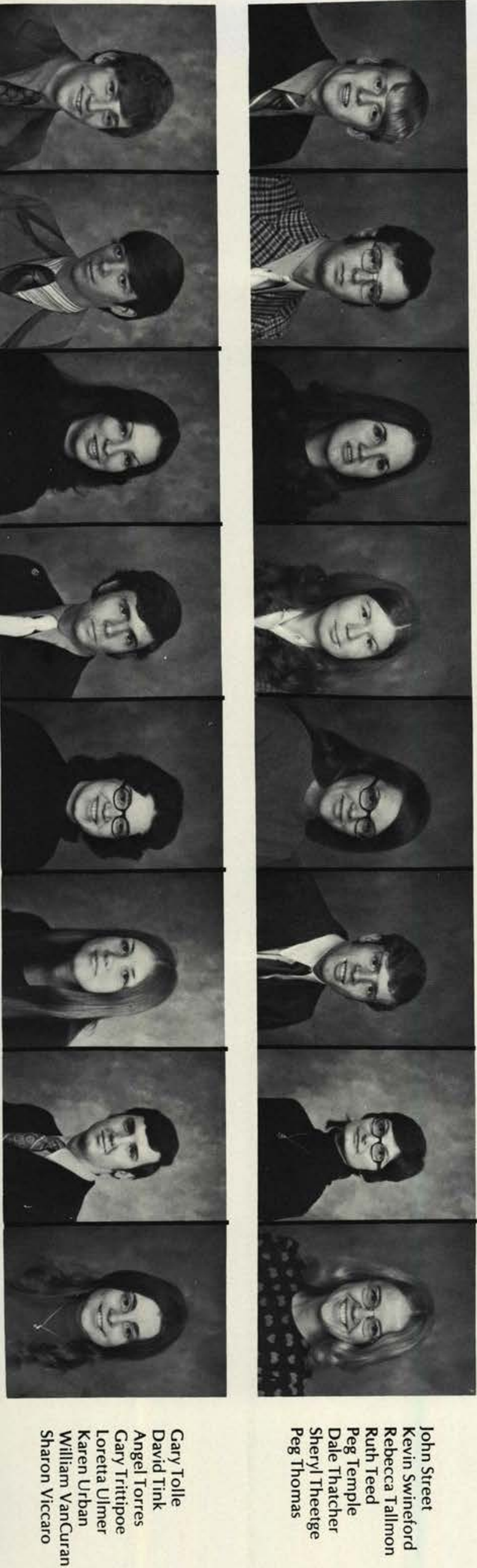
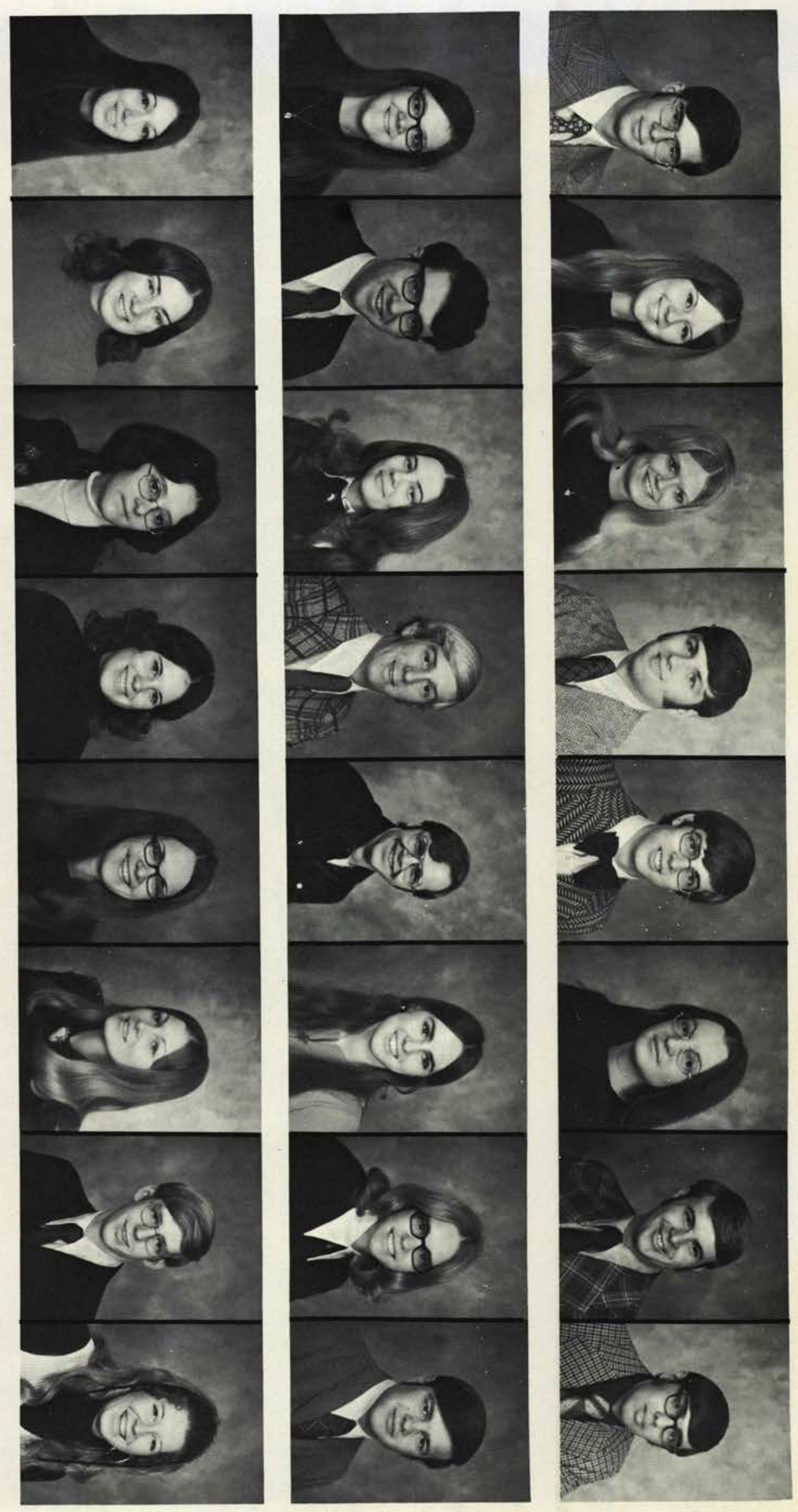


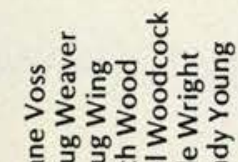

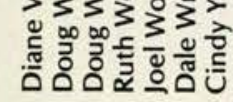

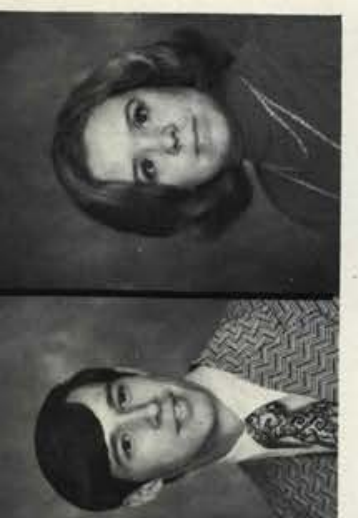

(1)

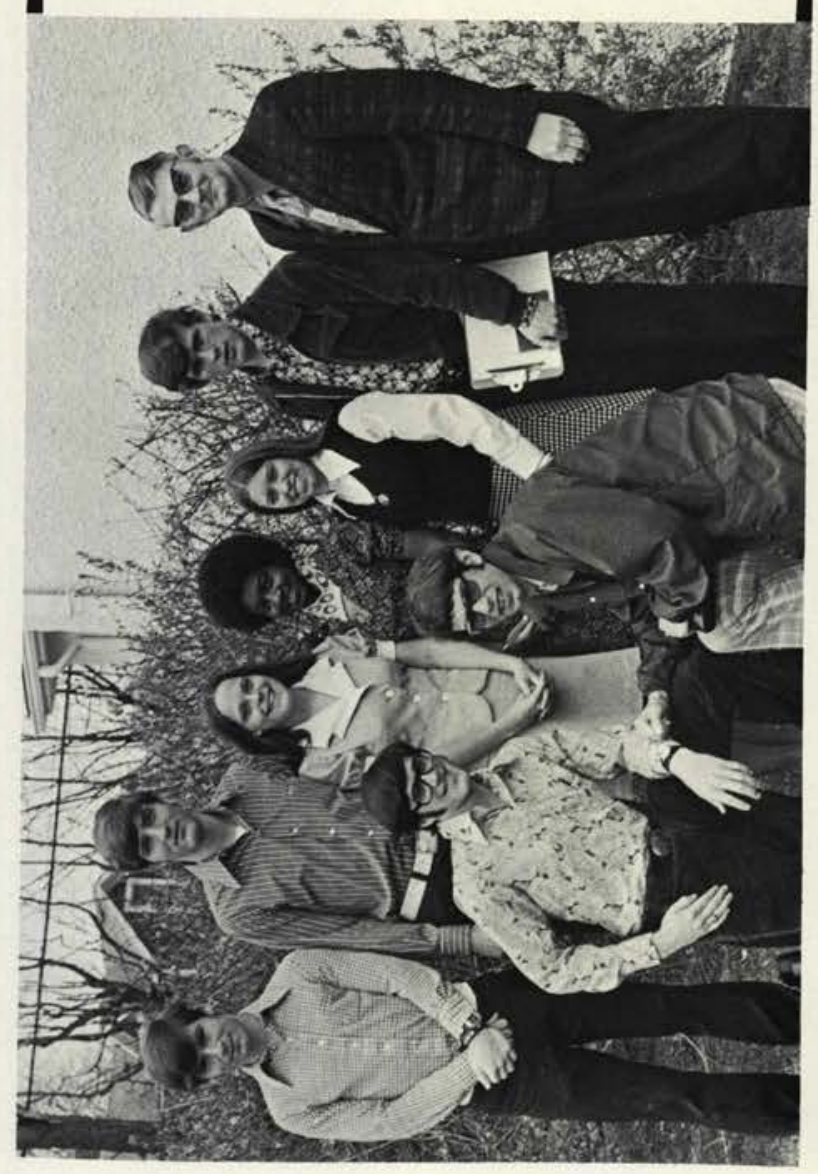

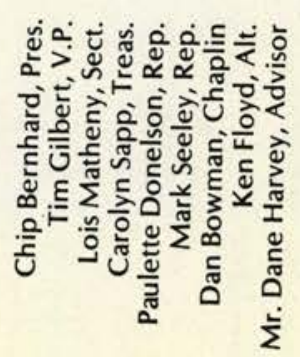

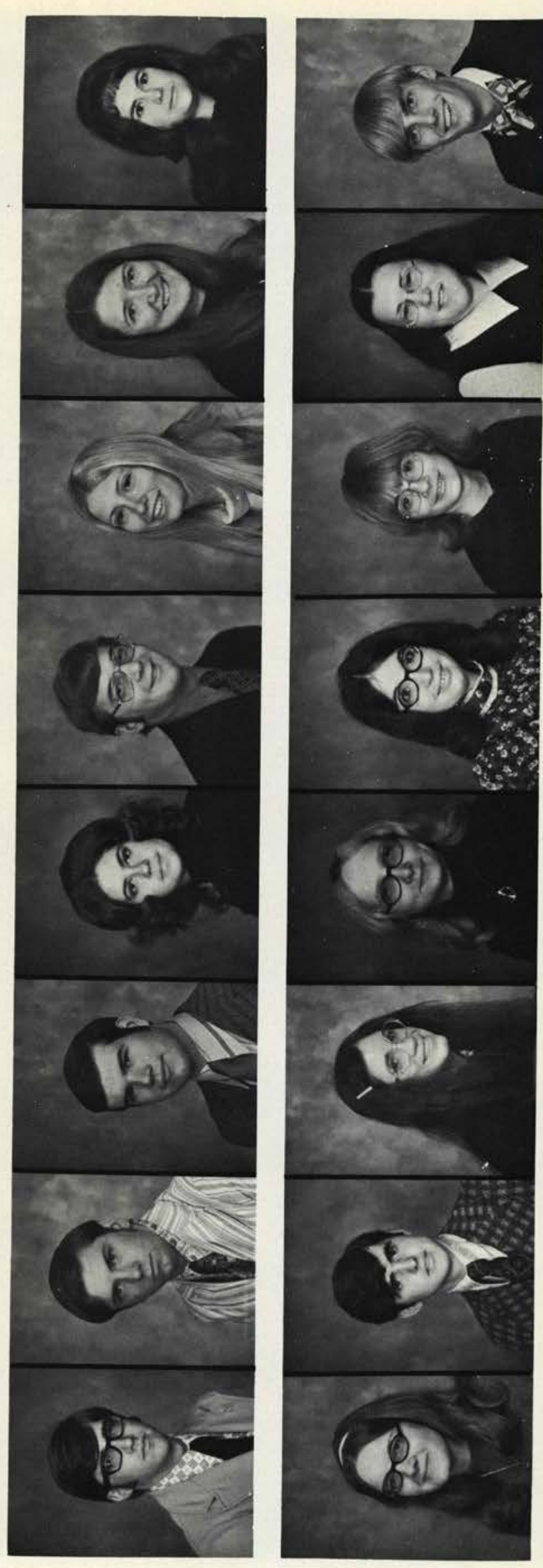

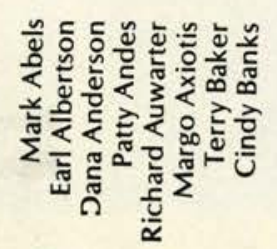

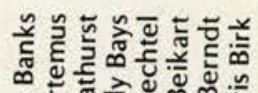

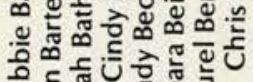

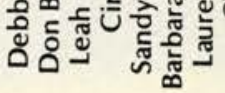




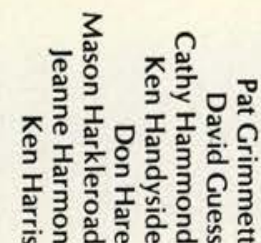

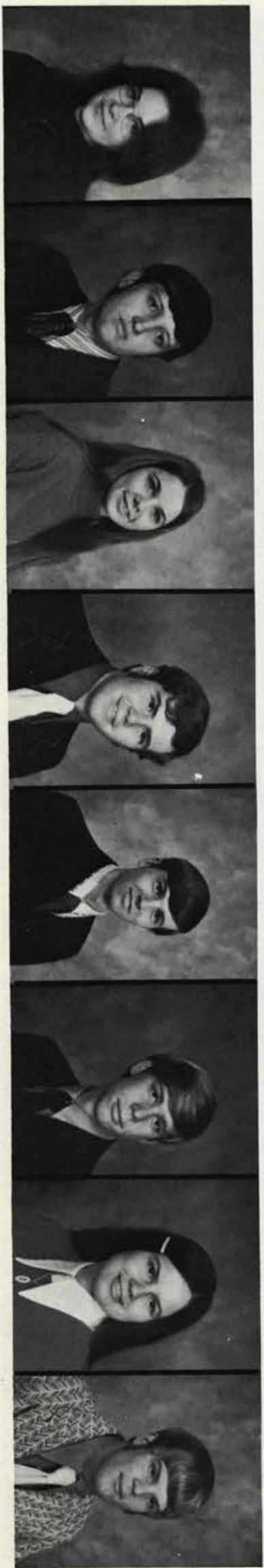

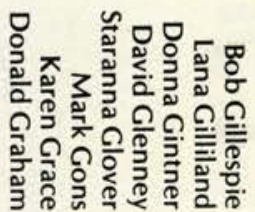
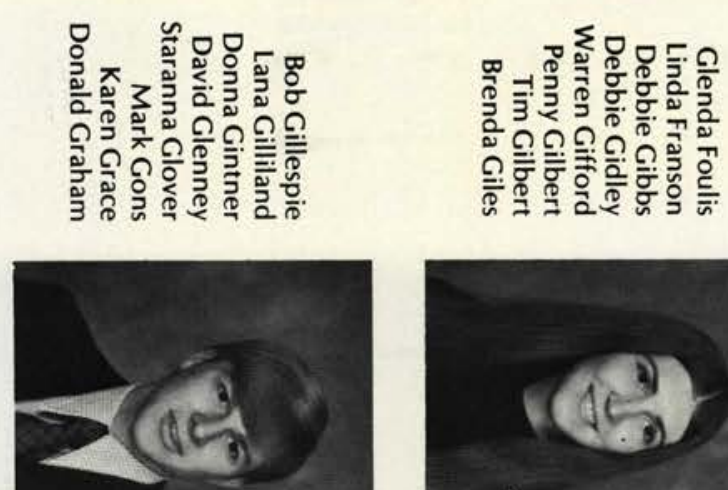

$\therefore \quad(40)$

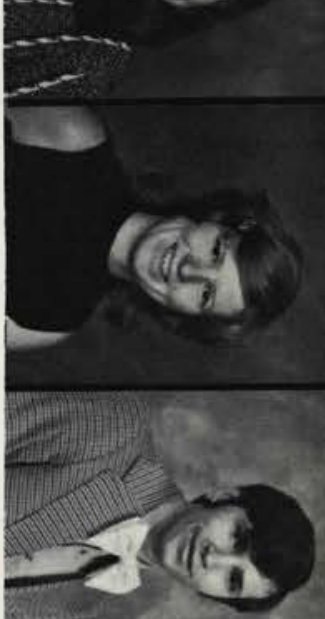

(बत)

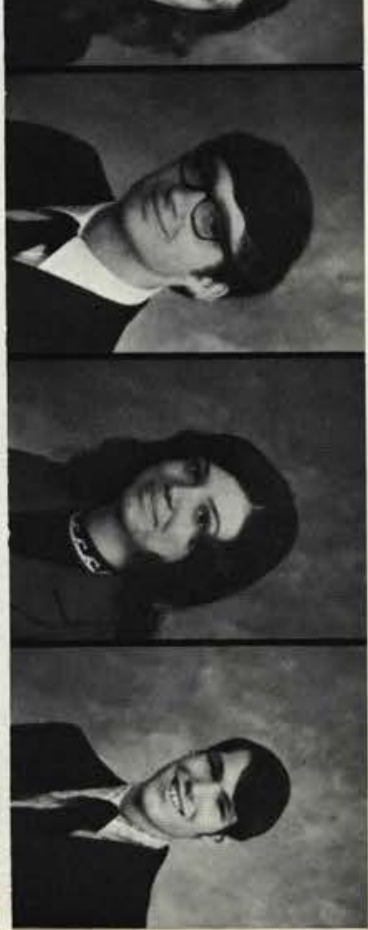

$\left.y(5)^{1}\right)$

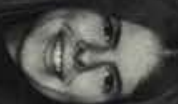

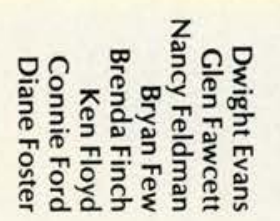
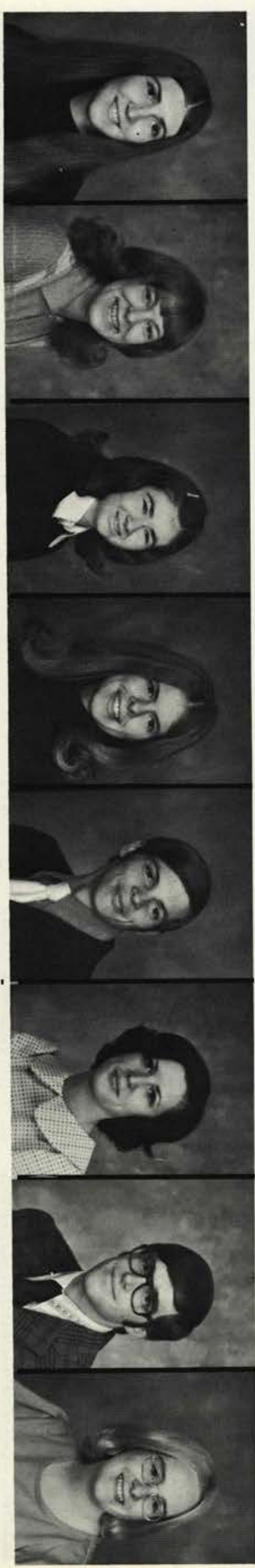

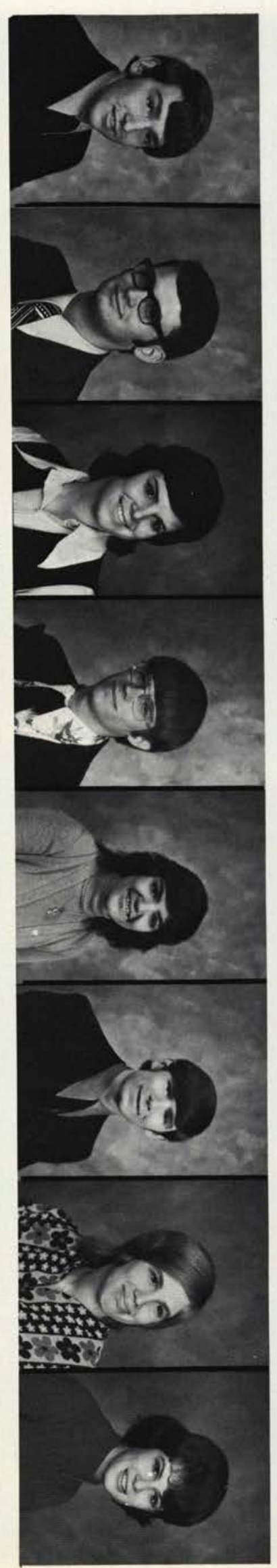

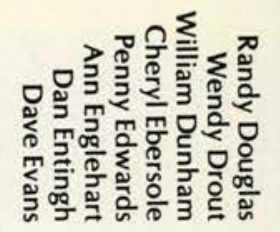

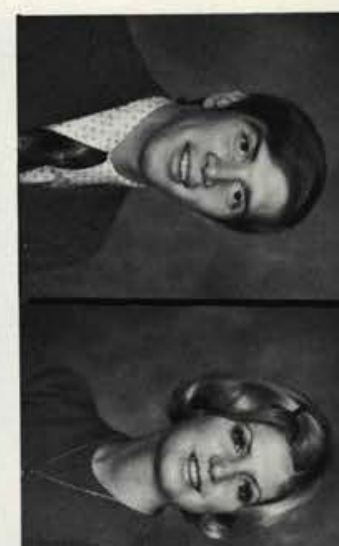

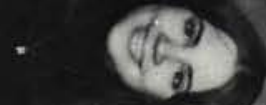

$\Rightarrow\left(\frac{13}{3}\right)$
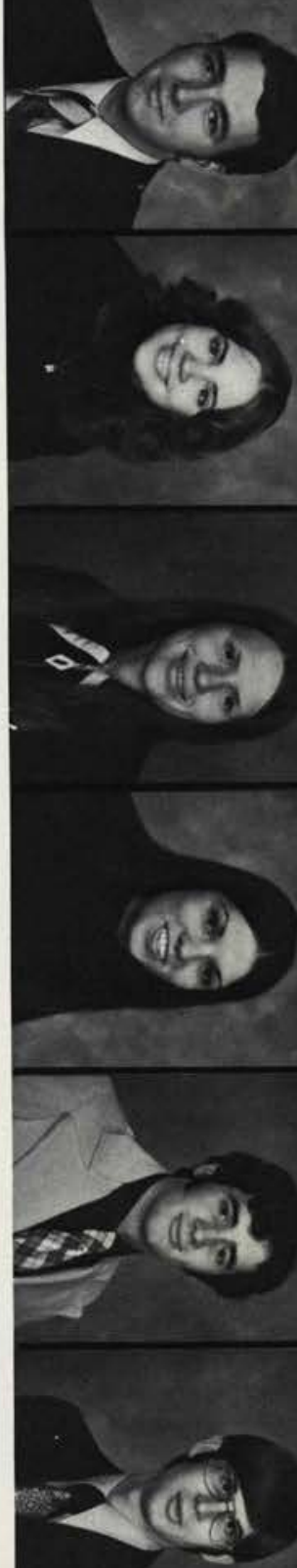


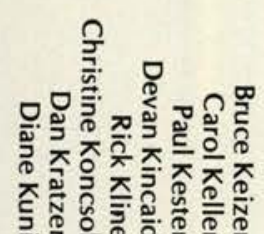

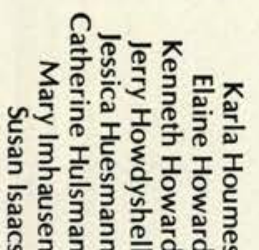

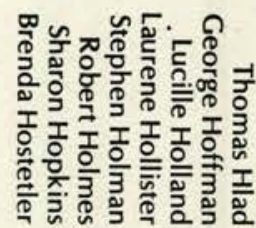

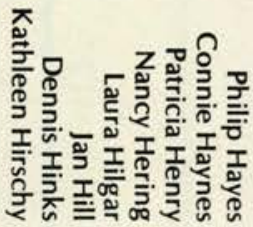

$\operatorname{loc} 35^{3}$
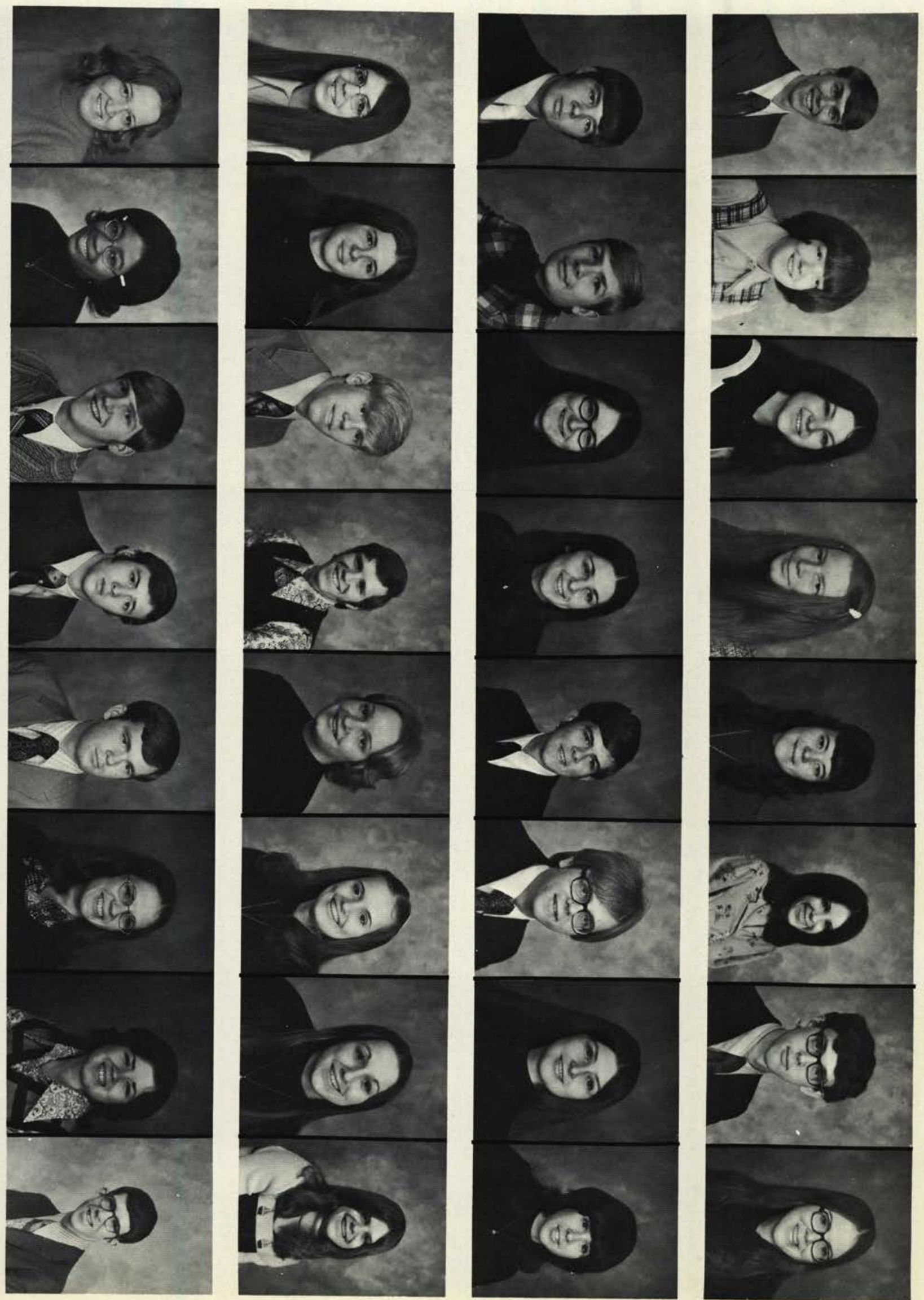

तब

(1)

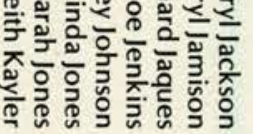

-

(a)

(ब)

(ब)
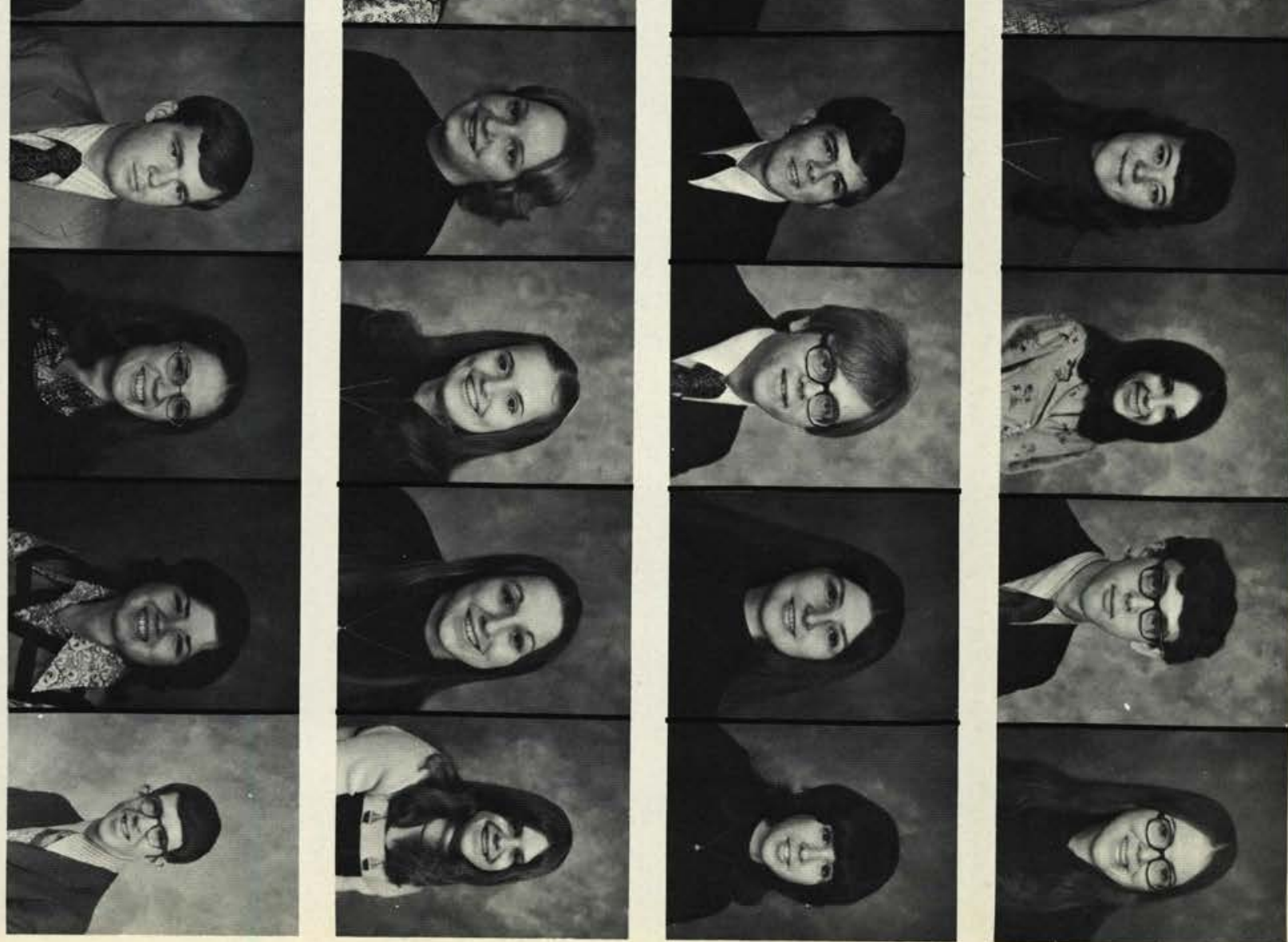

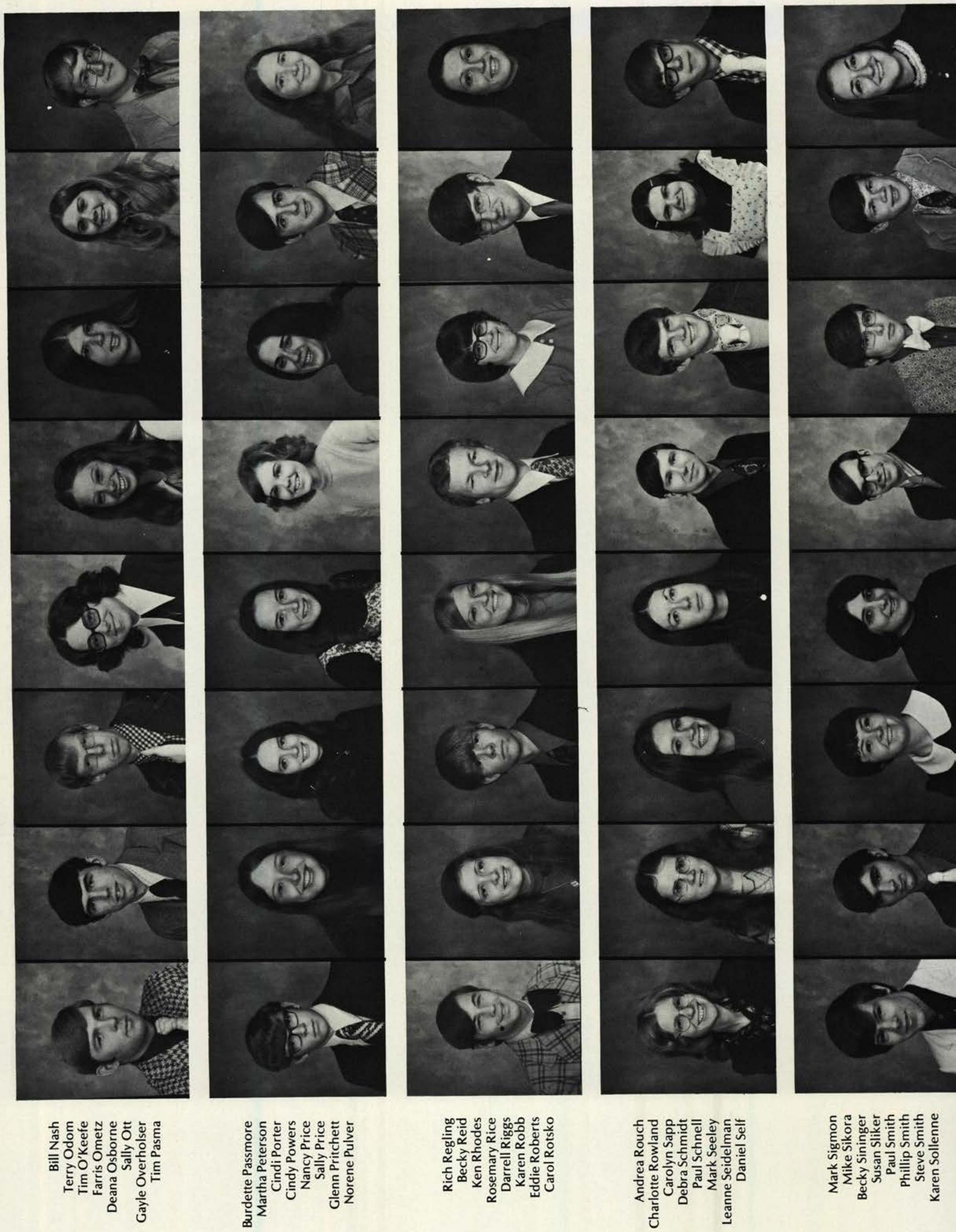

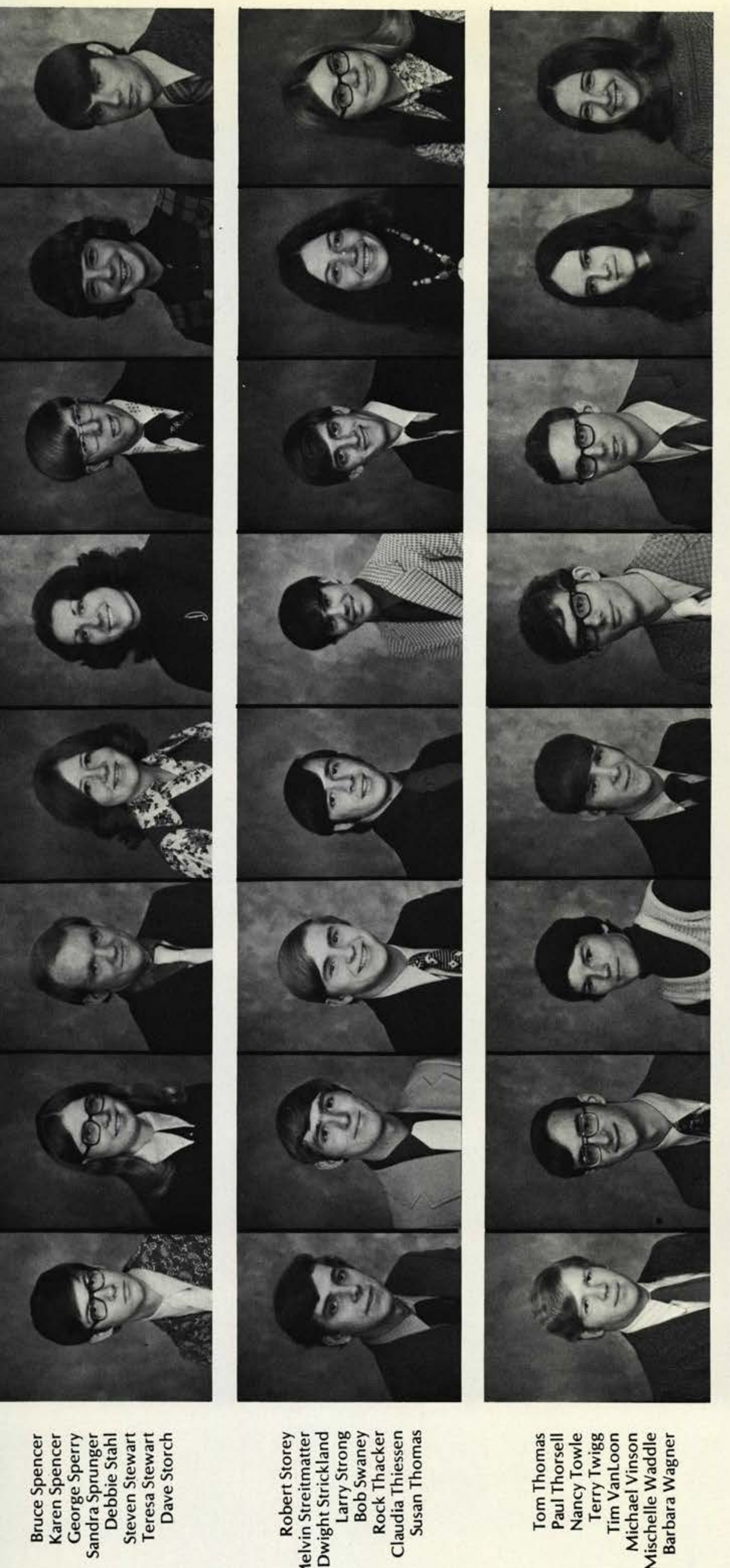

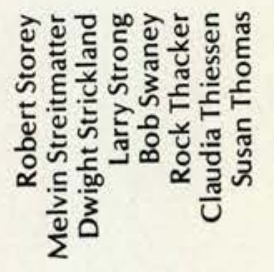

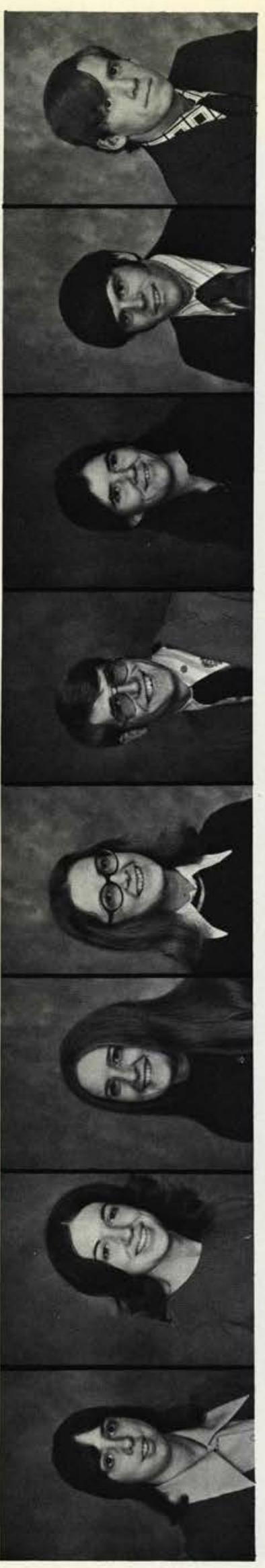
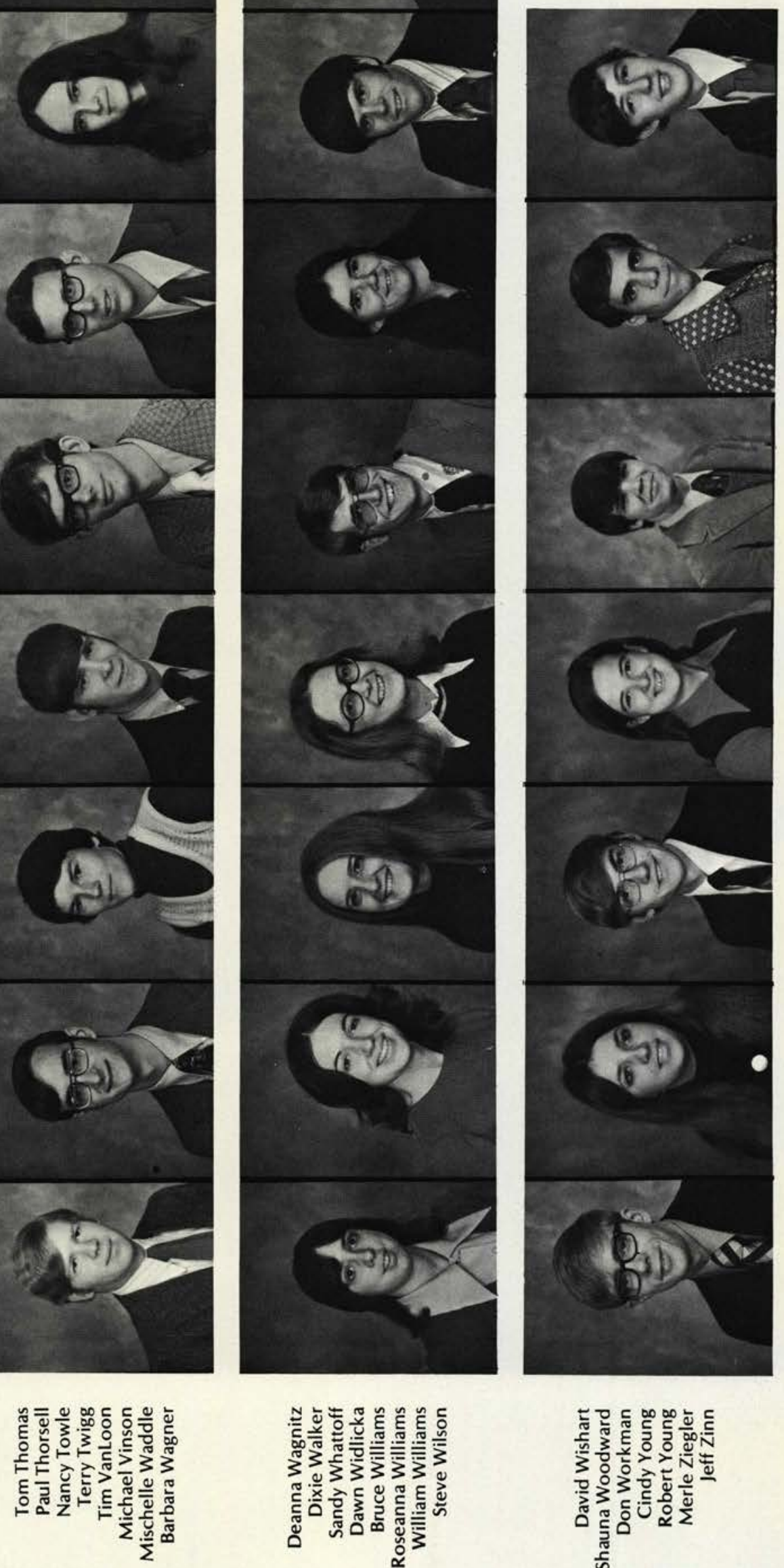

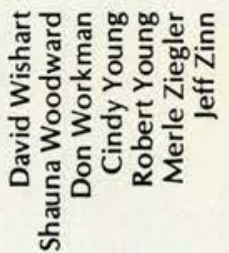




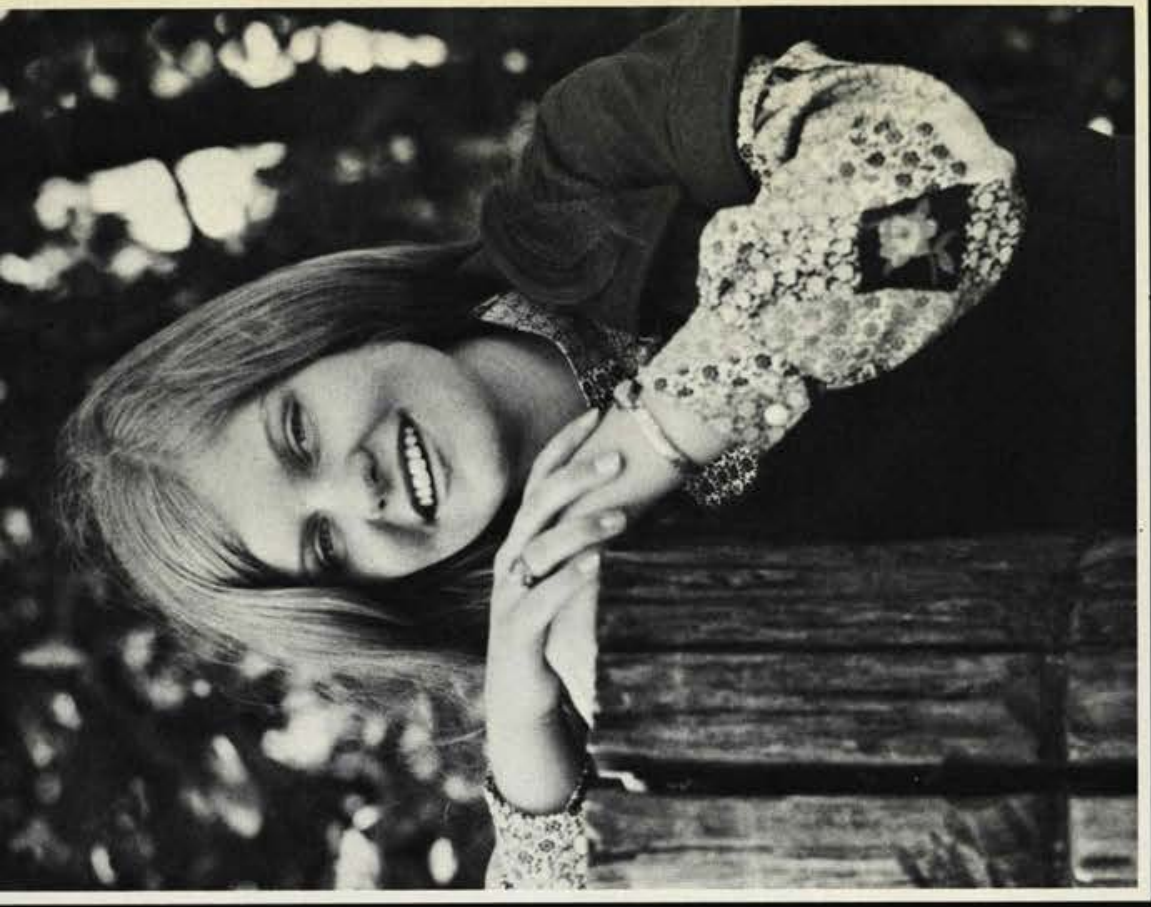

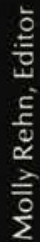

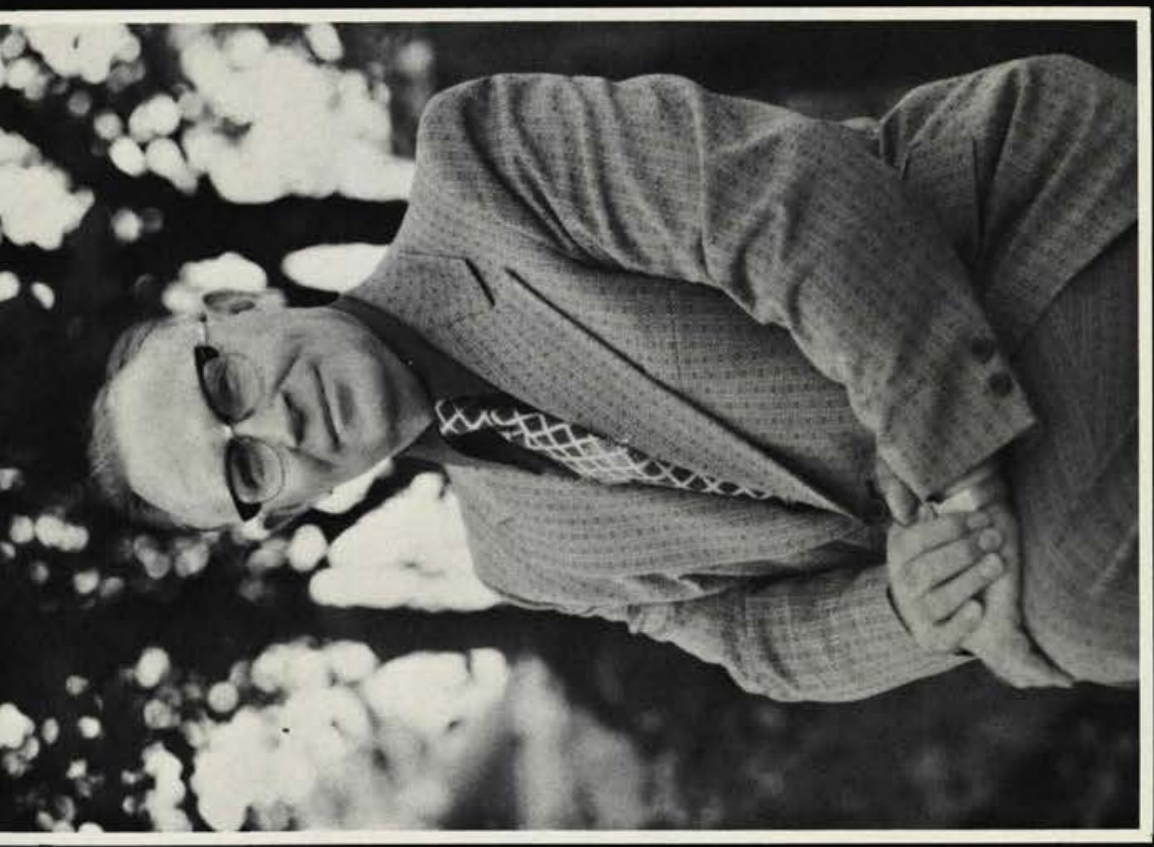

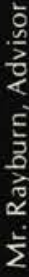

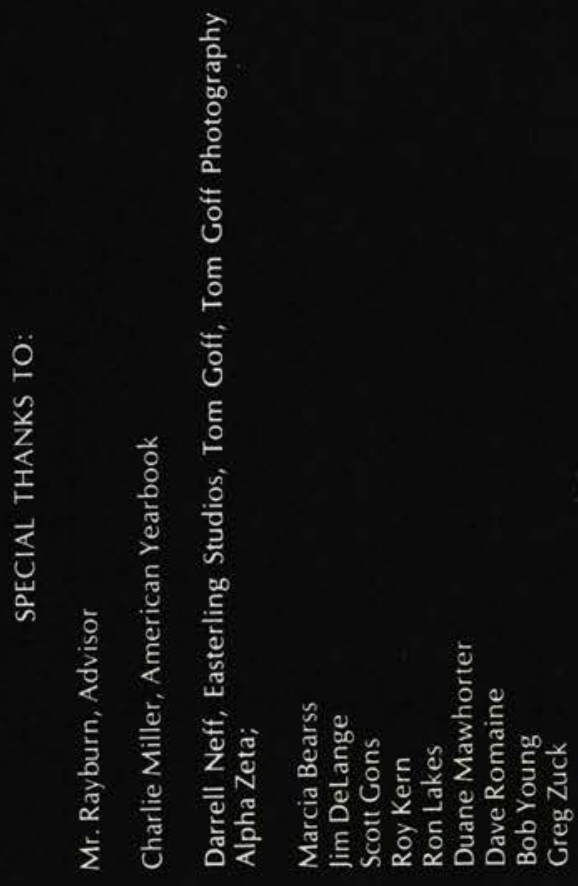


Leach's Laundromat

24 Hour Self-Service

in

Cedarville, Ohio

E. Xenia Stree

Behind Firehouse

Phone: 766-5186

\section{Duff's Smorgasboard}

37 W. Main St.

Xenia, Ohio

Lunch 11-3

Dinner $4-8$

$\$ 1.50$

Sun. and Hol. 11-8

$\$ 2.00$

$\$ 2.00$

\section{Wheelersburg \\ Baptist Church}

Wheelersburg, Ohio 45694

CONGRATULATES THE GRADUATES

OF THE CLASS OF 1974

"May God's Best Be Yours"

Pastor: Dick Sumner

Our Students at Cedarville:

Chyrs Martin and Shauna Woodard

\title{
CLINTONVILLE BAPTIST \\ $\mathrm{CHURCH}$
}

\author{
35 Oakland Park Avenue \\ Columbus, Ohio 43214 \\ Pastor George Hattenfield \\ CONGRATULATIONS TO THE \\ CLASS OF 1974
}




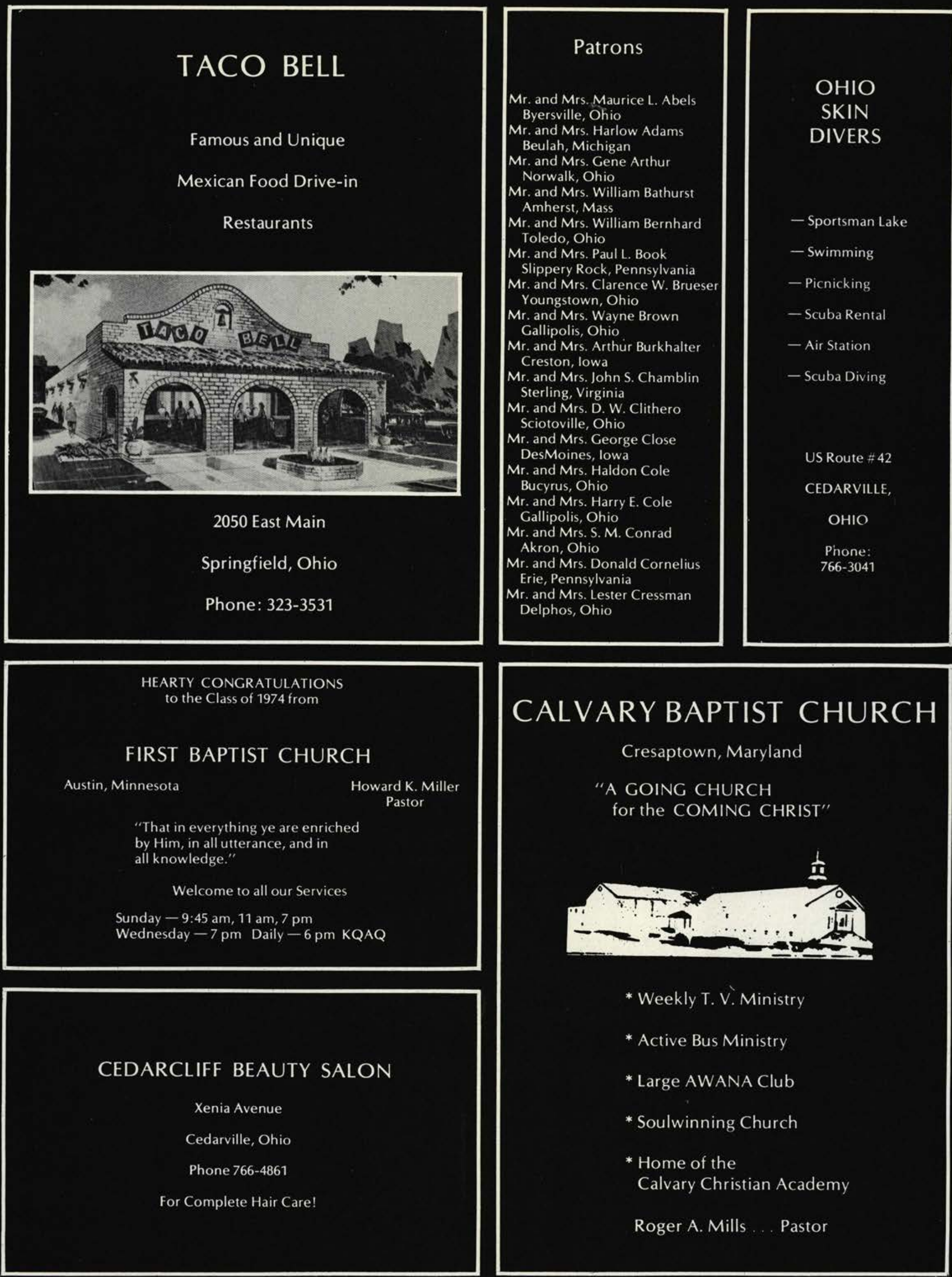


Hasser Barber Shop

255 Xenia Ave. - Yellowsprings

Complete Hair Care

- Razor cuts and

$$
\text { styling }
$$

Call for appointment Phone 767-8171
CONGRATULATIONS TO THE CLASS OF 1974

PROSPECT PARK BAPTIST CHURCH

1100 Harrison Avenue

Council Bluffs, lowa

Rev. Michael D. Scott, Pastor

"Preaching and teaching the Old

Book, the Precious Blood, the

New Birth and the Blessed Hope"

\section{GRACE BAPTIST CHURCH}

1100 S. Fairfield Street

Lombard, Illinois 60148
Rev. Everett Hawbaker, Pastor

Ronald Kilian, Youth \& Music Director

Our congratulations to the

graduating class of 1974 . Our desire

for each of you is that you may be a faithful

servant of Jesus Christ.

\section{PARENT PATRON}

Mr. and Mrs. Loyd Fight

Leavenworth, Kansas

Mr. and Mrs. John W. Floyd

Springfield, Ohio

Mr. and Mrs. Howard Foster

Ft. Wayne, Indiana

Mr. and Mrs. James H. Gabriel

Chicago, Illinois

Mrs. C. G. Gaippe

Parma, Ohio

Mr. William Gilmour

Niles, Ohio

Mr. and Mrs. James M. Gossett

Akron, Ohio

Mr. and Mrs. George Graham

Toledo, Ohio

Rev. and Mrs. Donald Grollimund

Princeton, Indiana

Mr. Homer Hale

East Alton, Illinois

Dr. and Mrs. D. C. Hambrick

Delaware, Ohio

Mr. and Mrs. Henry Hauser

Berea, Ohio

Mr. and Mrs. Richard Henslin

Dodge Center, Minnesota

Rev. and Mrs. Donald R. Hinks

Gettysburg, Pennsylvania

Mr. and Mrs. Thomas Holley

Twinsburg, Ohio

Mr. and Mrs. Calvin W. Hollister

Mobile, Alabama

Mr. and Mrs. Charles L. Hunter

Mentone, Indiana

\section{First Baptist Church}

266 Washington Ave.

Elyria, Ohio, 44035

(216) $322-5455$

Best wishes to the

graduating Class of 1974.

Home of Elyria Christian Academy

Pastor Willis R. Hall 


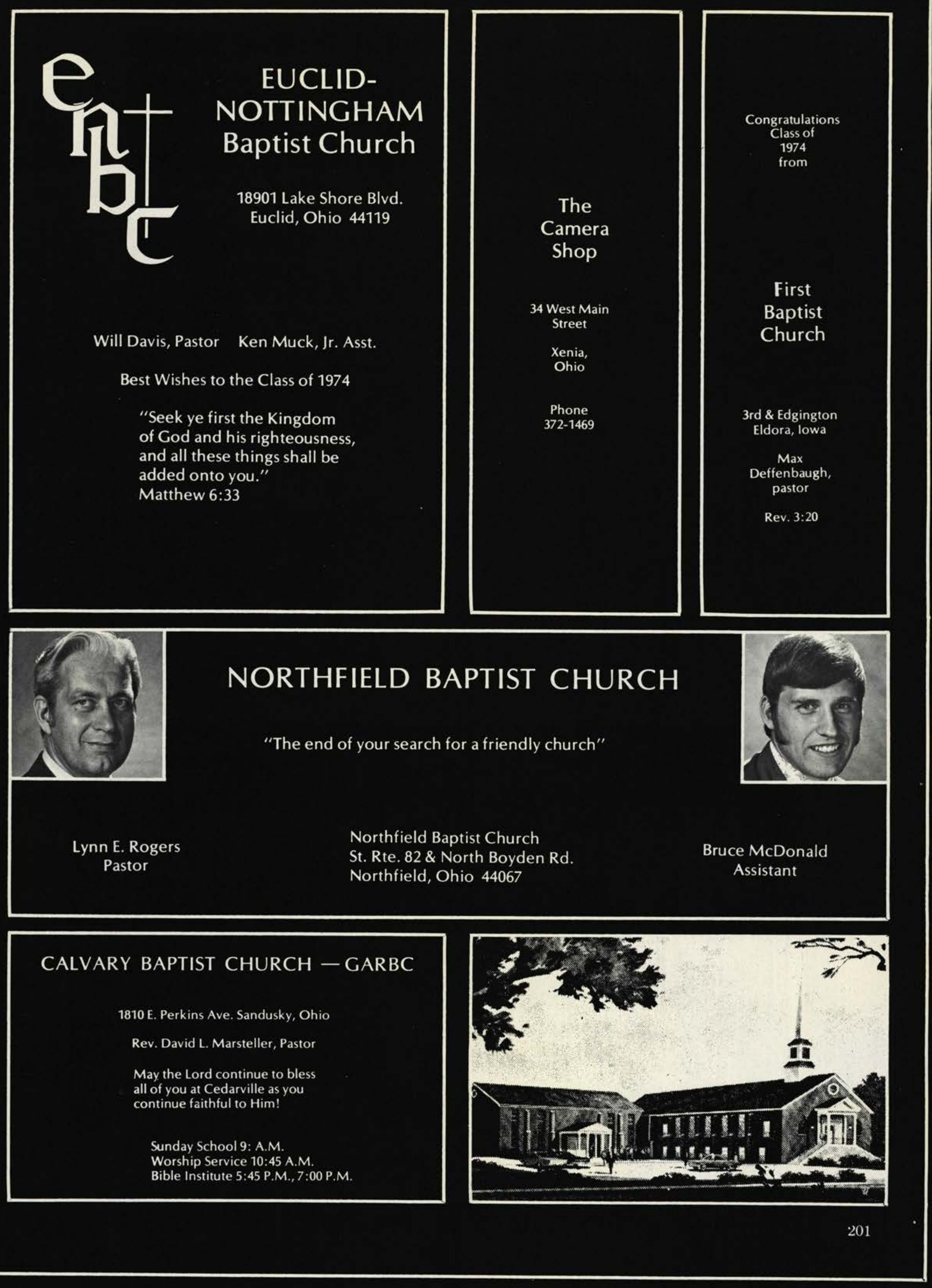


Calvary Baptist Church

Owassa, Michigan

CONGRATULATIONS

Mike

Encouragement to

Lucy, Laurie,

and Duane

Pastor Edward Mawhorter

FIRST BAPTIST CHURCH OF SILVIS

"The All Family Church"

11019 th Ave. Silvis, Illinois

Rev. John Lineberry, pastor

"The End of Your Search for a Friendly Church"

GREETINGS GEORGEANNA AND MARGO AXIOTIS AND CINDI PORTER

from the fine folk back at

First Baptist Church. The

Lord's blessings upon you.

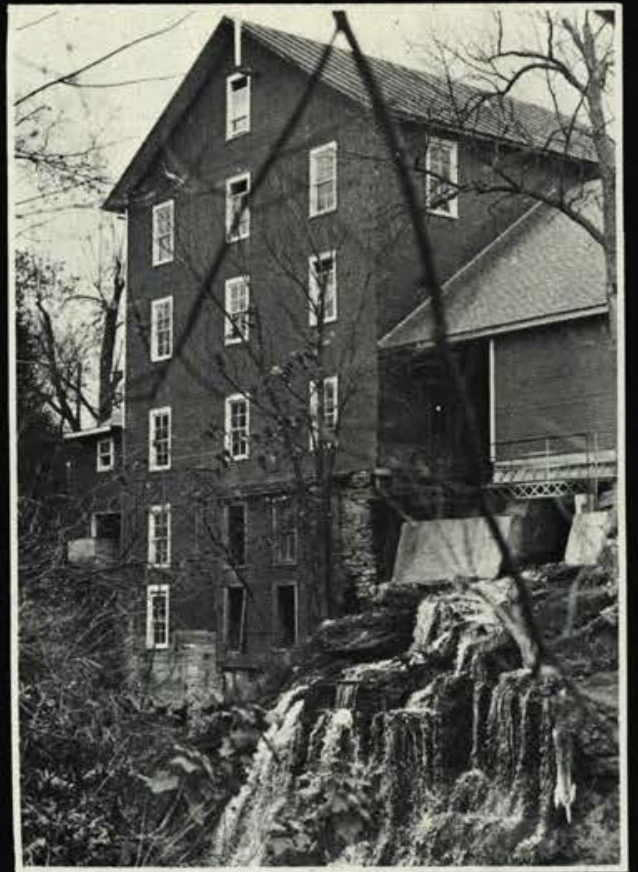

Clifton

Mills

Inc.

Est. 1869

Am.

Largest

Water

Powered

Grist

Mill

\section{MIAMI DEPOSIT BANK}

"Here to Serve You"

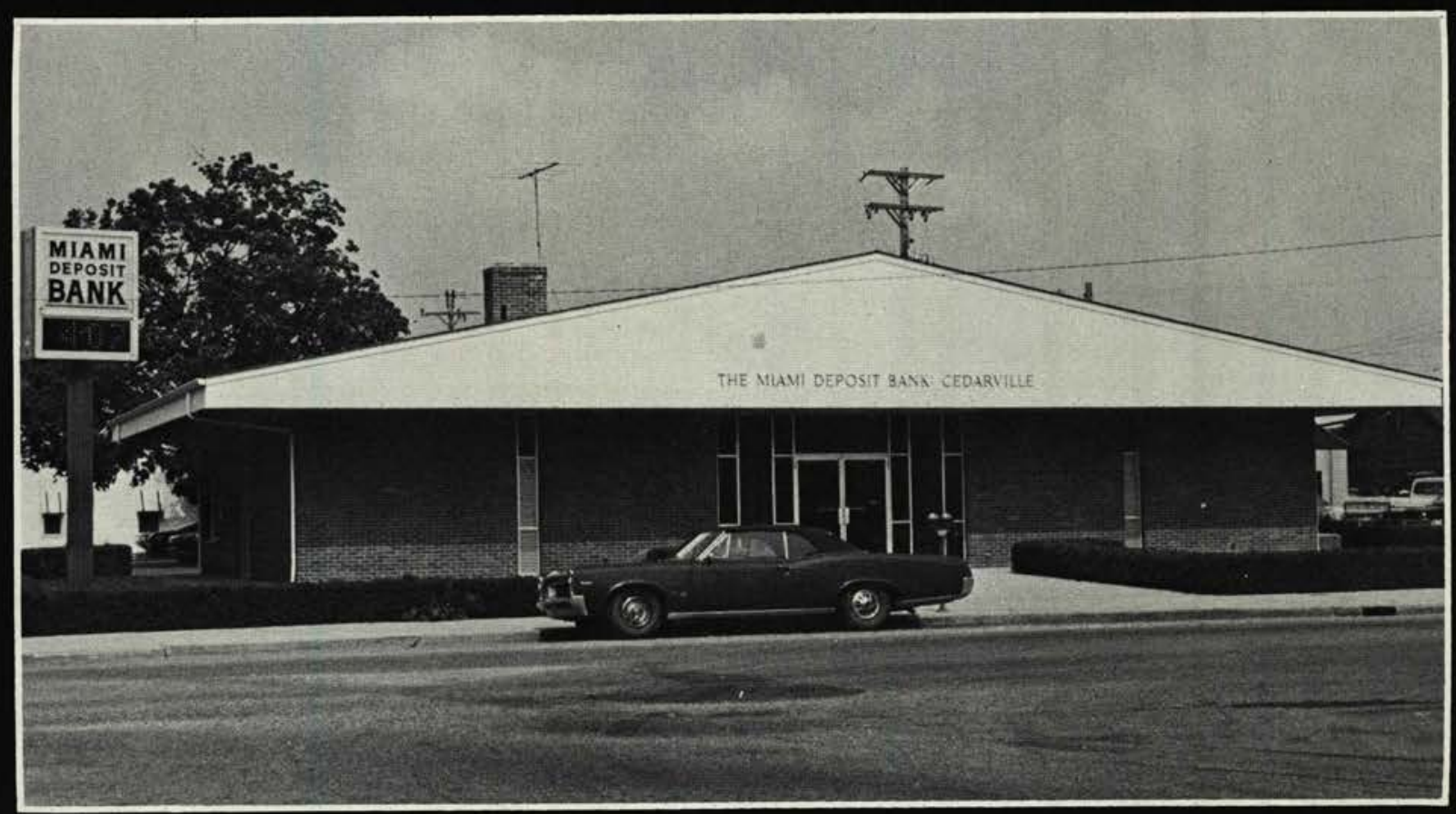

Member of F.D.I.C.

Cedarville, Ohio

Yellow Springs, Ohio

Phone: 766-3611 


\section{Southgate Baptist Church}

2111 South Center Boulevard

Springfield, Ohio

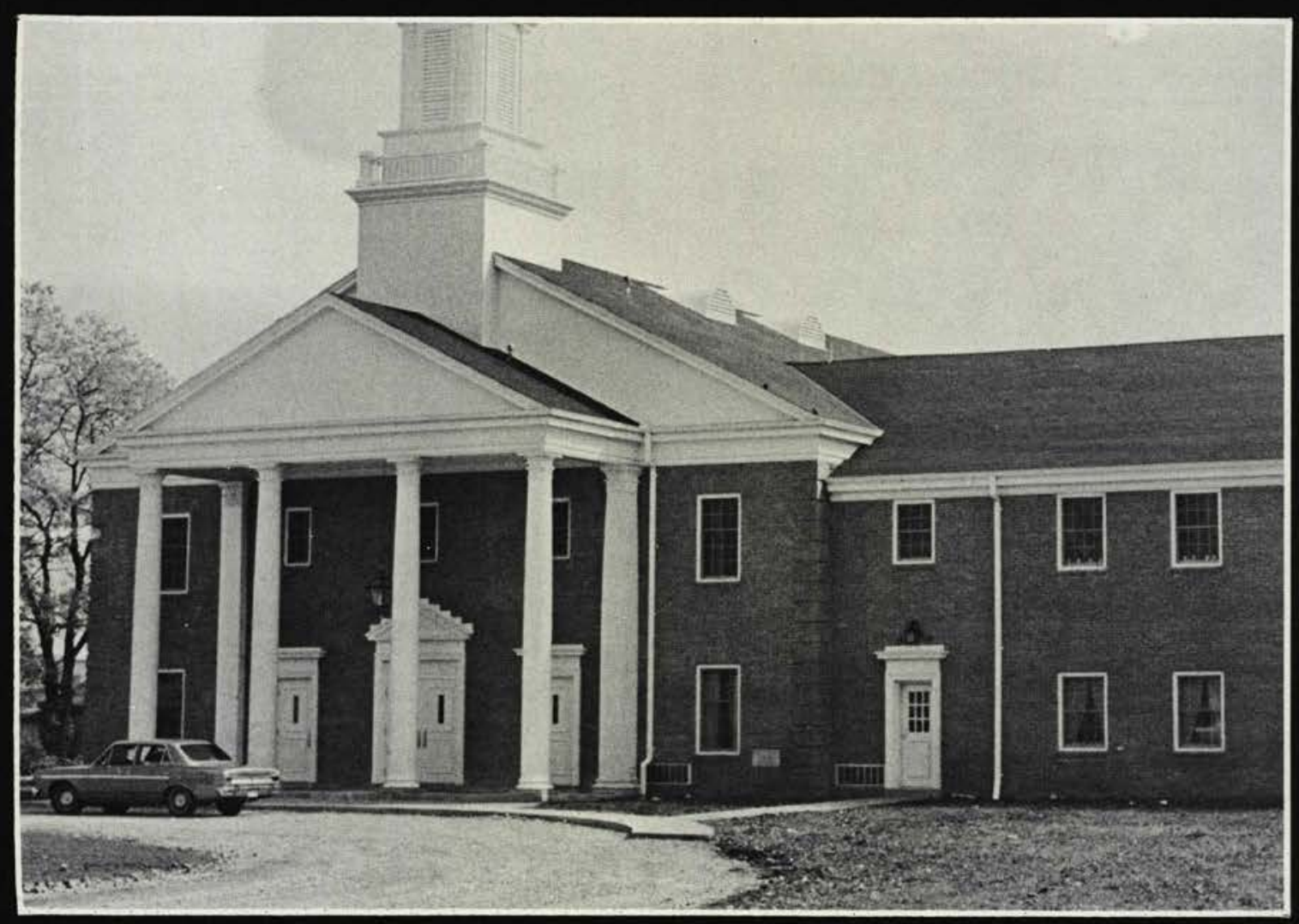

Congratulations Class of 1974

"And God said to man, behold the fear of the Lord that is wisdom; and to depart from evil is understanding."

Job $28: 28$ 


\title{
FIRST BAPTIST CHURCH
}

1001 N. Lincoln Street

Creston, lowa 50801

Norman Hoag, Pastor

Randall Cook, Youth Pastor

Our Program

Sunday School

9:45 AM

classes for all ages

Morning Worship

broadcast over KSIB

Young Peoples Hour

EvingServices

Prayer Meeting (Wednesday)

\author{
11:00 AM \\ 6:30 PM. \\ $7: 30$ PM \\ 8:00 PM
}

Best of Wishes to

the Senior

Graduates

Our Students at Cedarville:

Harold Burkhalter

Carol Johnson

Becky Tallmon

Nancy Tallmon
CEDARVILLE LIMESTONE

Company

in
Cedarville
Ohio
Crushed
Limestone,
Sand \&
Gravel

766-2351

Day 426-2108

\section{PARENT PATRONS}

Mr. and Mrs. Vincent Imbro

Carmel, Indiana

Mrs. Mary Jackson

Cleveland, Ohio

Mr. and Mrs. Richard C. Jaques

Toledo, Ohio

Mr. and Mrs. Russell Jenkins

McLean, Virginia

Mr. and Mrs. Bernard W. Johnson

Lapeer, Michigan

Mr. and Mrs. Harold Jarvis

Rohnert Park, California

Mr. and Mrs. Edwin Johnson

Medinah, Illinois

Mr. and Mrs. Robert E. Jones

Wellan, lowa

Mrs. Demona Jordan

Indianapolis, Indiana

Mr. and Mrs. Dillion Karsian

Hackensack, New Jersey

Mr. and Mrs. Paul Kayler

New Lebanon, Ohio

Mrs. Norma Keller

Springfield, Ohio

Mr. and Mrs. Neil Kennedy

Camillus, New York

Mr. and Mrs. Peter Kerrigan

Hackensack, New Jersey

Mr. and Mrs. William G. Kesler

Olney, Illinois

Mr. and Mrs. Richard Kincaid

Perry Ohio

Mr. and Mrs. Edward Kinzig

Dayton, Ohio

\section{The Chapel of Prayer Baptist Church}

\author{
"On \\ Chapel \\ Hill" \\ 1811 Brittain Road \\ Akron, Ohio 44310 \\ CONGRATULATIONS to \\ the Class of \\ 1974
}

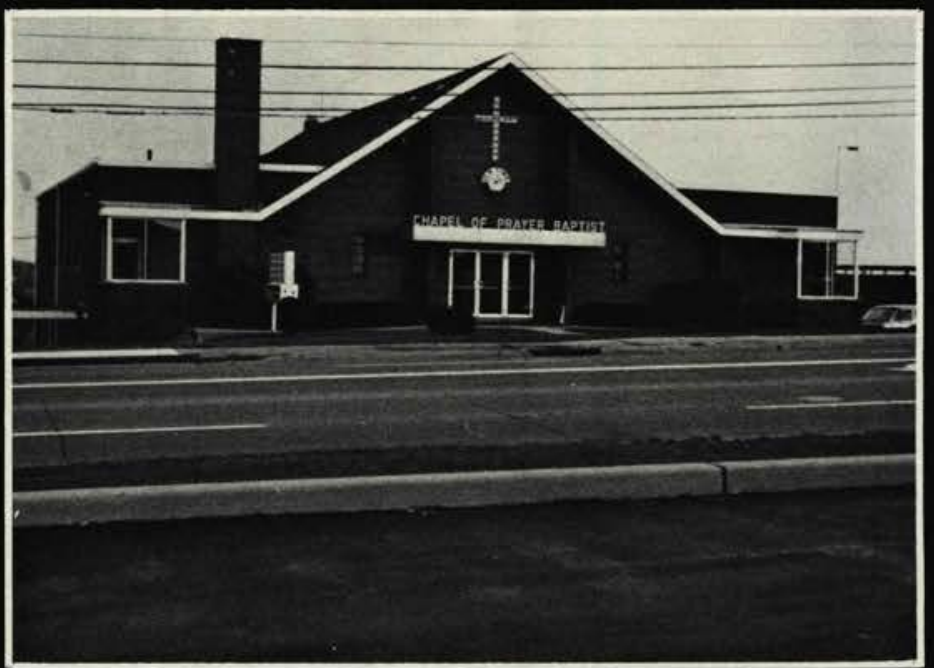




\section{Bethel Baptist Church}

1704 Springdale Road

Cherry Hill, New Jersey

Pastor: Dr. Carl F. Elgena

Director of Youth and Education:

Mr. Daniel Pascucci

Phone 609/424-2331

CONGRATULATIONS

TO THE

CLASS

OF

1974

\section{Chaplin Cleaners}

- Laundry service

- Shoe repair

49 North Main Street

Cedarville, Ohio

766-3871

\section{PARENT PATRONS}

Mr. and Mrs. Lad Rodriguez

Gary, Indiana

Mr. and Mrs. Ernes B. Rogers

Byesville, Ohio

Dr. and Mrs. Jon H. Rouch

Warren, Pennsylvania

Mr. and Mrs. Herman C. Salomon

Hamburg, New York

Mr. and Mrs. Thomas Sapp

Cleveland, Ohio

Mr. and Mrs. G. A. Saunders

Port Jervis, New York

Mr. and Mrs. Quinten Seeley

Medina, Ohio

Mr. and Mrs. William Seidelman, Sr.

Tacoma, Washington

Mr. and Mrs. Lawrence Seigneur

Stryker, Ohio

Mr. and Mrs. Donald E. Shawver

Lorain, Ohio

Mr. and Mrs. R. Gary Sheppard

Haddon Heights, New Jersey

Mr. and Mrs. William F. Sims

Waterloo, lowa

Mr. and Mrs. Floyd W. Sliker

Worthington, Pennsylvania

Mr. and Mrs. Chester A. Smith, Jr.

Wheelersburg, Ohio

Dr. Robert D. Smith

Lowell, Indiana

Mr. and Mrs. Russell T. Smith

Cincinnati, Ohio

Mr. and Mrs. Roger Spencer

Middlefield, Ohio

\section{PARENT PATRONS}

Mr. and Mrs. Stanley Kirby

Prospect, Ohio

Mr. and Mrs. W. H. Kuni

Atchison, Kansas

Mr. and Mrs. James E. LaBelle

Akron, Ohio

Mr. and Mrs. Andrew Lampir is

Sault Ste. Marie, Michigan

Dr. and Mrs. R. K. Lancaster

Batavia, Ohio

Mr. Raymond P. Lee Jr.

Henry, Illinois

Mr. Harold L, Lewright, Jr.

Eagle Grove, lowa

Mr. and Mrs. Don Linden

Overbrook, Kansas

Mr. and Mrs. Christian Lochel

Levittown, Pennsylvania

Mr. and Mrs. Vern Longacre

Fostoria, Ohio

Mr. and Mrs. Gerald Lowis

Sheffield Lane, Ohio

Mr. and Mrs. Melvin Lowrey

Piscataway, New Jersey

Mr. and Mrs. Dean W. Luers

Riverside, lowa

Mr. and Mrs. Nicholas G. Luketic

Hammond, Indiana

Mr. and Mrs. Burchard H. Lyon

Sherman, New York

Mr. Harold Madeen

Jamestown, New York

Mr. and Mrs. Vincent J. Mahl

LaGrange, Ohio
Mr. and Mrs. William T. Mapel Lima, Ohio

Mr. and Mrs. Thomas McClish Sharon, Pennsylvania

Mrs. Anna May Miller

Lebanon, Indiana

C. M. Miller

Saginaw, Michigan

Mr. and Mrs. David J. Moreland

LaVale, Maryland

Mrs. Elsie W. Morgan

Endicott, New York

Mr. and Mrs. Melvin Moreley

Edna, Kansas

Mr. and Mrs. Henry Mudder

Hastings, Minnesota

Mrs. Joyce Nicholl

Springfield, Ohio

Mr. Ray C. Owens

Chesapeake, Virginia

Mr. and Mrs. Larry C. Park

Hazel Park, Michigan

Mr. and Mrs. James A. Potter

Jersey Shore, Pennsylvania

Ltc. and Mrs. Donald C. Porter Rock Island, Illinois

Mr. and Mrs. James Reed

Ogleslry, Illinois

Mr. and Mrs. Irwin M. Rehn

Boonton, New Jersey

Mr. and Mrs. Claude B. Rice

Troy, Ohio

Mr. and Mrs. Louis H. Ricker

Tonawanda, New York 


\section{FAMOUS SPORTING GOODS}

"Specializing in the Finest in Team Equipment"

64 E. Main Phone:

372-3461

Xenia, Ohio
Tuesday, Wednesday, Saturday 9 to $5: 30$

Monday, Thursday, Friday 9 to $8: 30$
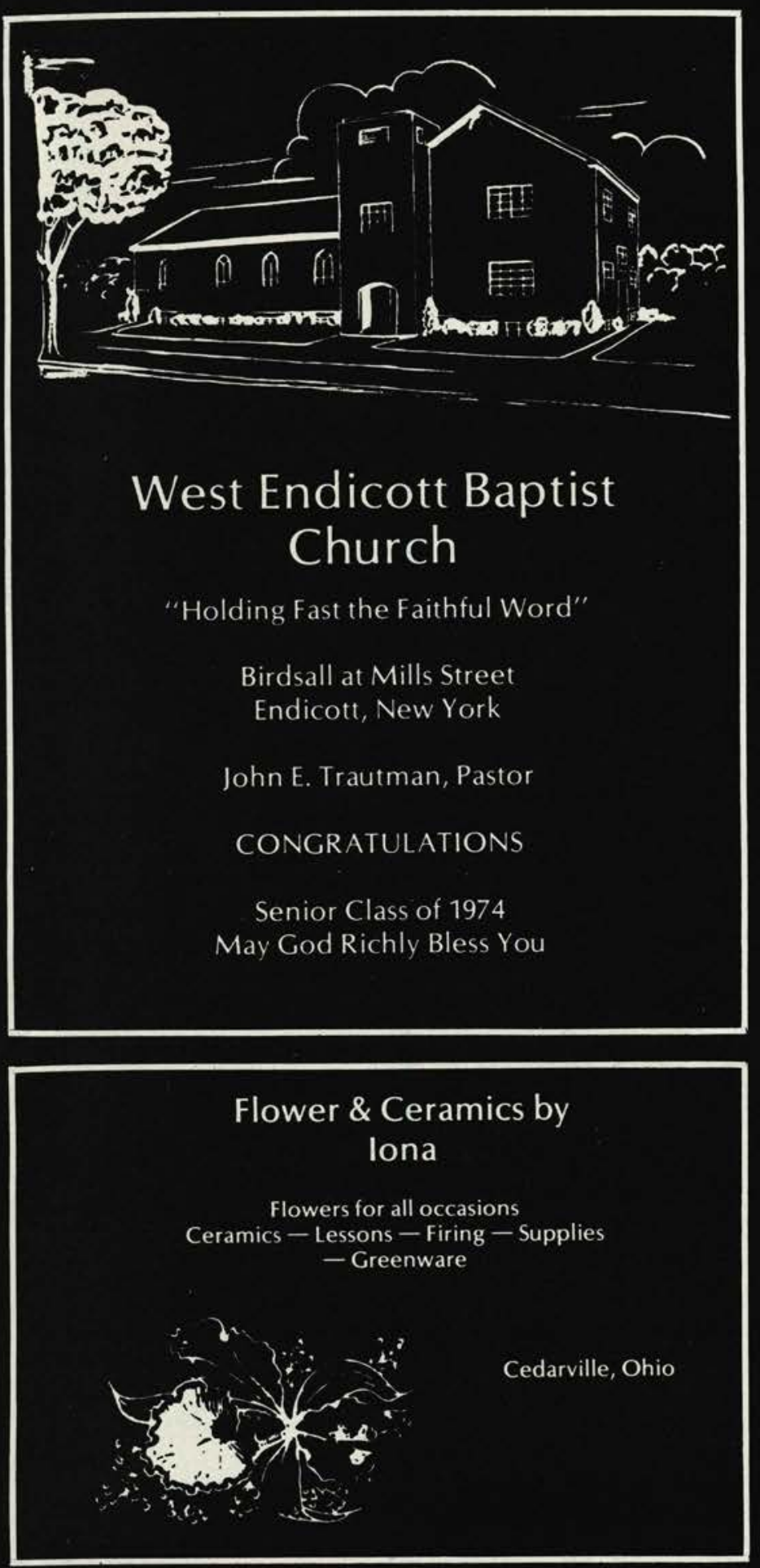

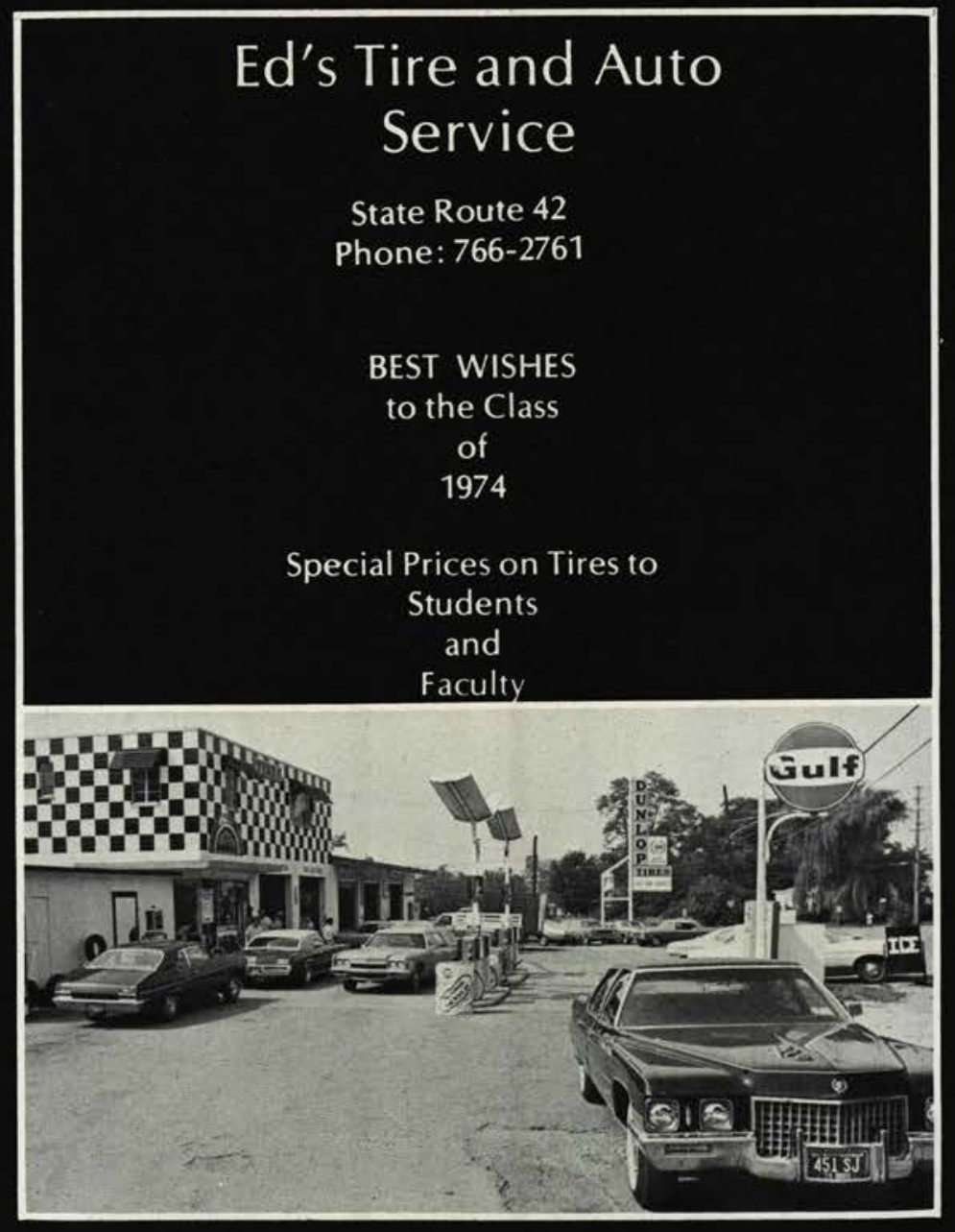

First Baptist Church

"Preaching the Word of God in Bremen and to the uttermost parts for 35 years." (This

is our

35 th

anniver-

sary.)

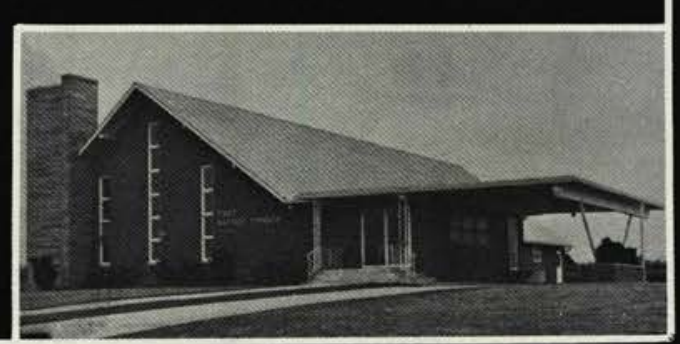




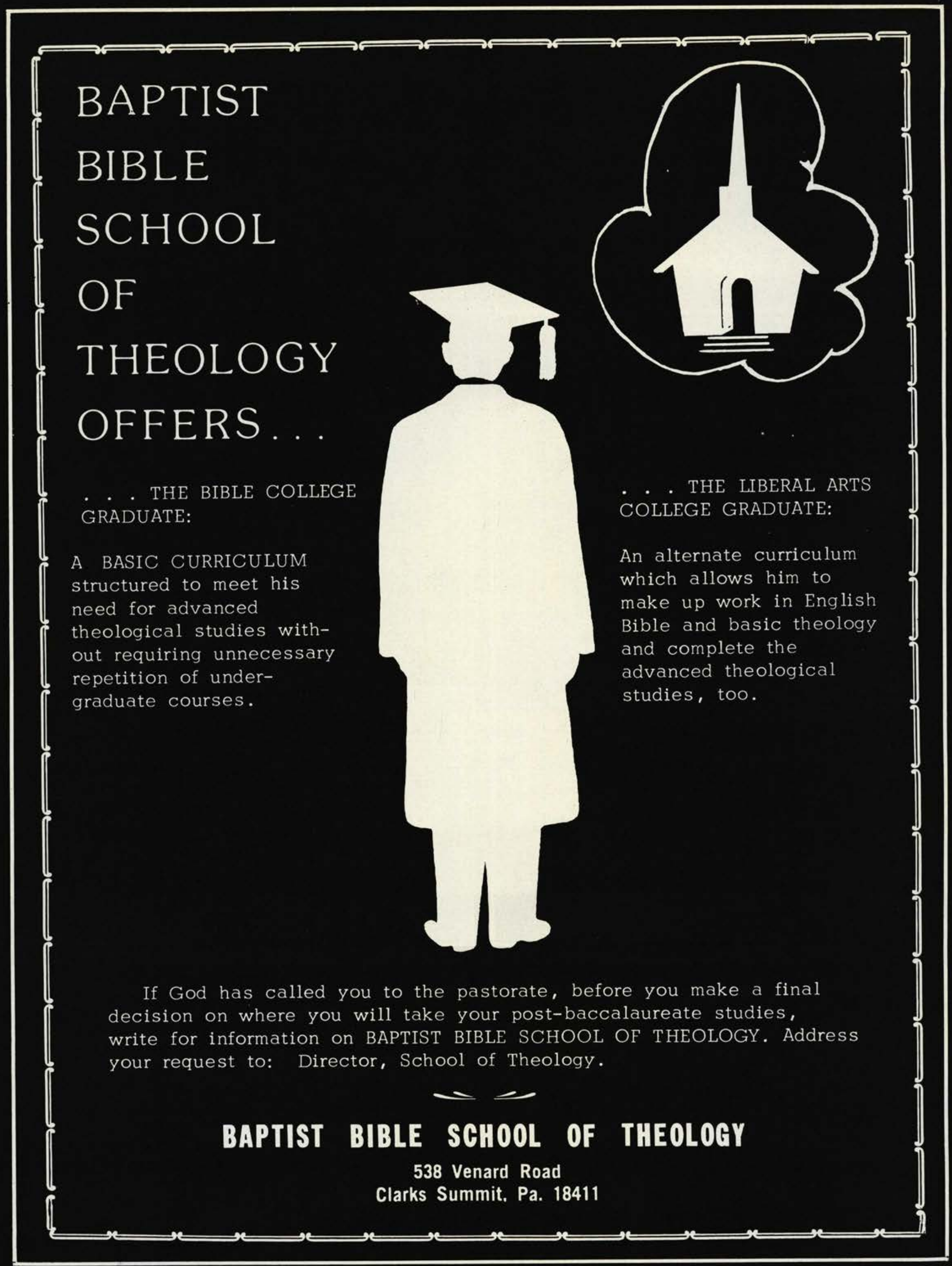




\section{North Baptist Church}

Ralph Gruenburg, Pastor

Christian Day School K-5

AWANA Shepherds Class

"Put on the Whole Armor"

$$
\text { Eph. 6:11 }
$$

2052 St. Paul St.

Rochester, N. Y. 14621

(716) 266-7008

\section{First Baptist Church}

$$
114 \text { - S. Maple }
$$

Pana, Illinois 62557

"May God use the graduates of

Cedarville to spread the good news of

salvation around the world."

Rev. Gerald Thurber, Pastor

Rev. Donald Sauser, Assis't.

\section{Xenia Red Barn}

Home of the Barnbuster

and Country Fried

Chicken

537 West Main Street

Xenia, Ohio

or the

Word of God and testimony of Jesus Christ

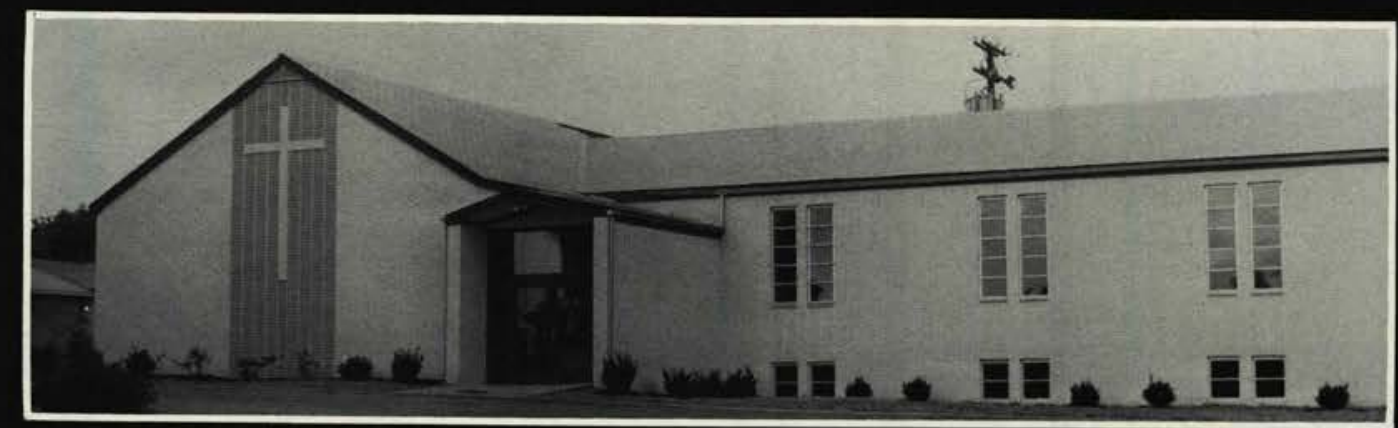

\section{Calvary Baptist Church}

Corner South Sixth Street and Race Ave.

Byesville, Ohio

Kenneth L. Pugh - Pastor

Sunday School

Worship Service

Youth Groups

Evening Service

Wednesday Service
9:30 AM
10:30 AM
6:15 PM
7:30 PM
$7: 30$ PM

Students at C. C.

Christine Malernee

Mark Fehrman

Mark Ables

Rex Rogers 


\section{FAITH BAPTIST CHURCH}

Russ Road

Joseph Godwin, Pastor
Greenville, Ohio

Thomas Bowman

Minister of Youth

\begin{tabular}{ll}
\multicolumn{2}{c}{ Our students attending Cedarville - } \\
Stephanie Augustine & Tim Gilbert \\
Sherri Burns & Kevin Gruber \\
Marty Cossins & Steve Gruber
\end{tabular}

Thresea Stewart

Also Mr. and Mrs. Dewan Engle, members of our church, are serving as dorm parents.

College Hill

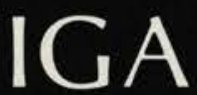

Cedarville,

Ohio
Mr and Mrs, Clifford Criss

Mansfield, Ohio

Mr. Wayne R. Crum

Medina, Ohio

Mr. and Mrs. Robert Cuenin

Amherst, Ohio

Mr. Solon P. Darnell

Royal Oak, Michigan

Mr. and Mrs. H. Richard Davison

Arcanum, Ohio

Mrs. Irene Dedrick

Dryden, New York

Mr. and Mrs. Eugene DeLand

Dresden, New York

Mr. and Mrs L eon De Mars

Columbus, Ohio

Mr: and Mrs. Wilbert Donalson

Hyannis, Massachusetis

Mr. and Mrs. William D. Dudles

Vestal, New York

Rev. Russell F. Fbersole, Ir

North Caldwell. New Jersed

Mr and Mrs. LeRov Ehnis

Brooklyn, Michigan

Mr. and Mrs. Paul tnglehart

Holly, Michigan

Mr and Mrs. Jack trickson

Hobart. Indiana

Mr. and Mrs. Walter H. C. Erikson

Palmer, Massachusetis

Mr. and Mrs Talmage Essex

Dayton, Ohio

Mr and Mrs. D L Feldman

Ankeny, lowa
Congratulations and God's

blessing upon the

graduating Class of 1974

\section{Calvary Baptist Church}

W. Southern and Tibbatts

Covington, Kentucky 41015

Galen C. Call, Pastor 


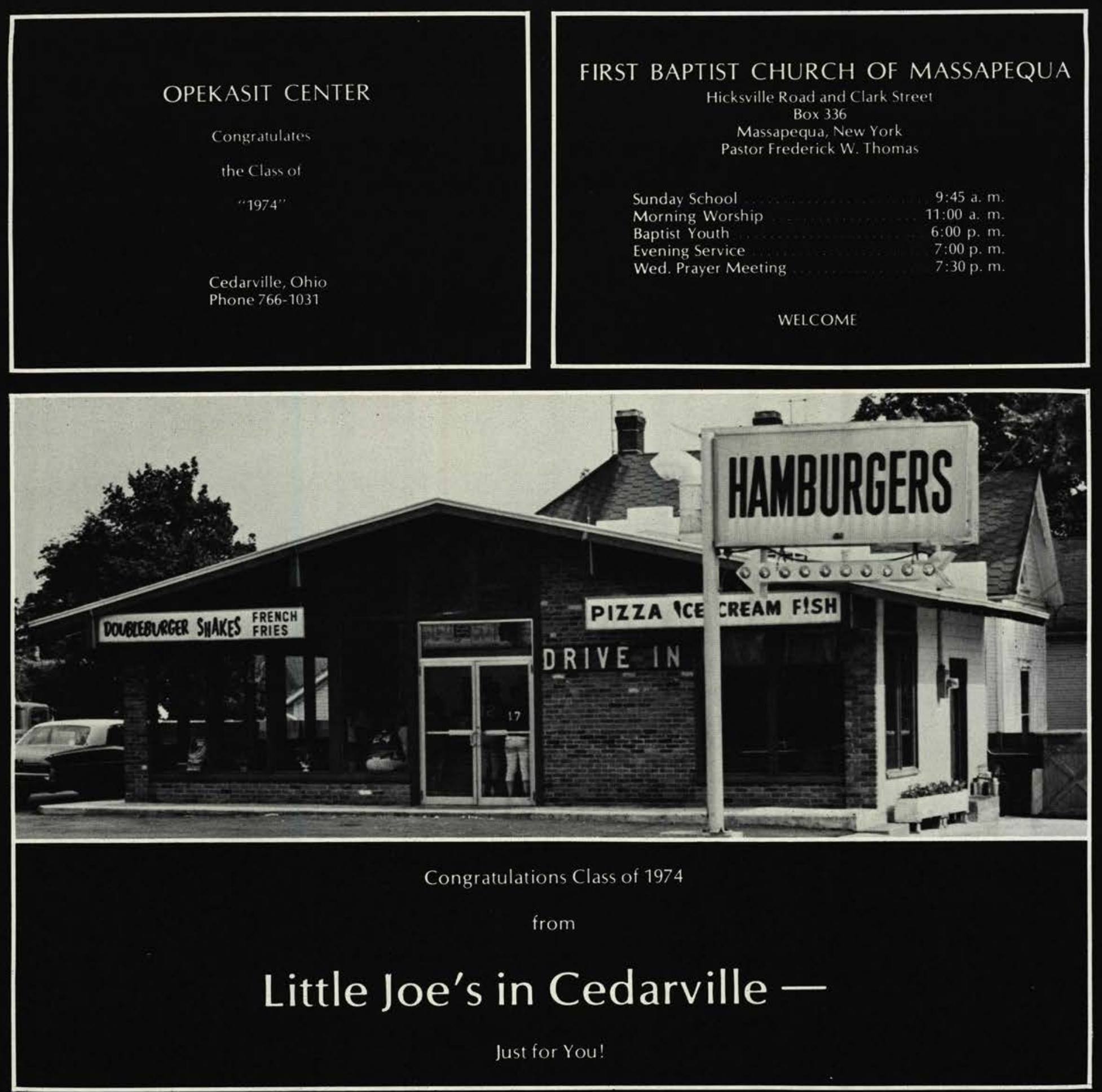

\section{HUNTSBURG BAPTIST CHURCH}

16401 Mayfield Rd.

Huntsburg, Ohio 44046

Ben H. Garlich, Pastor

"FOR THE WORD OF GOD AND

THE TESTIMONY OF JESUS CHRIST" 


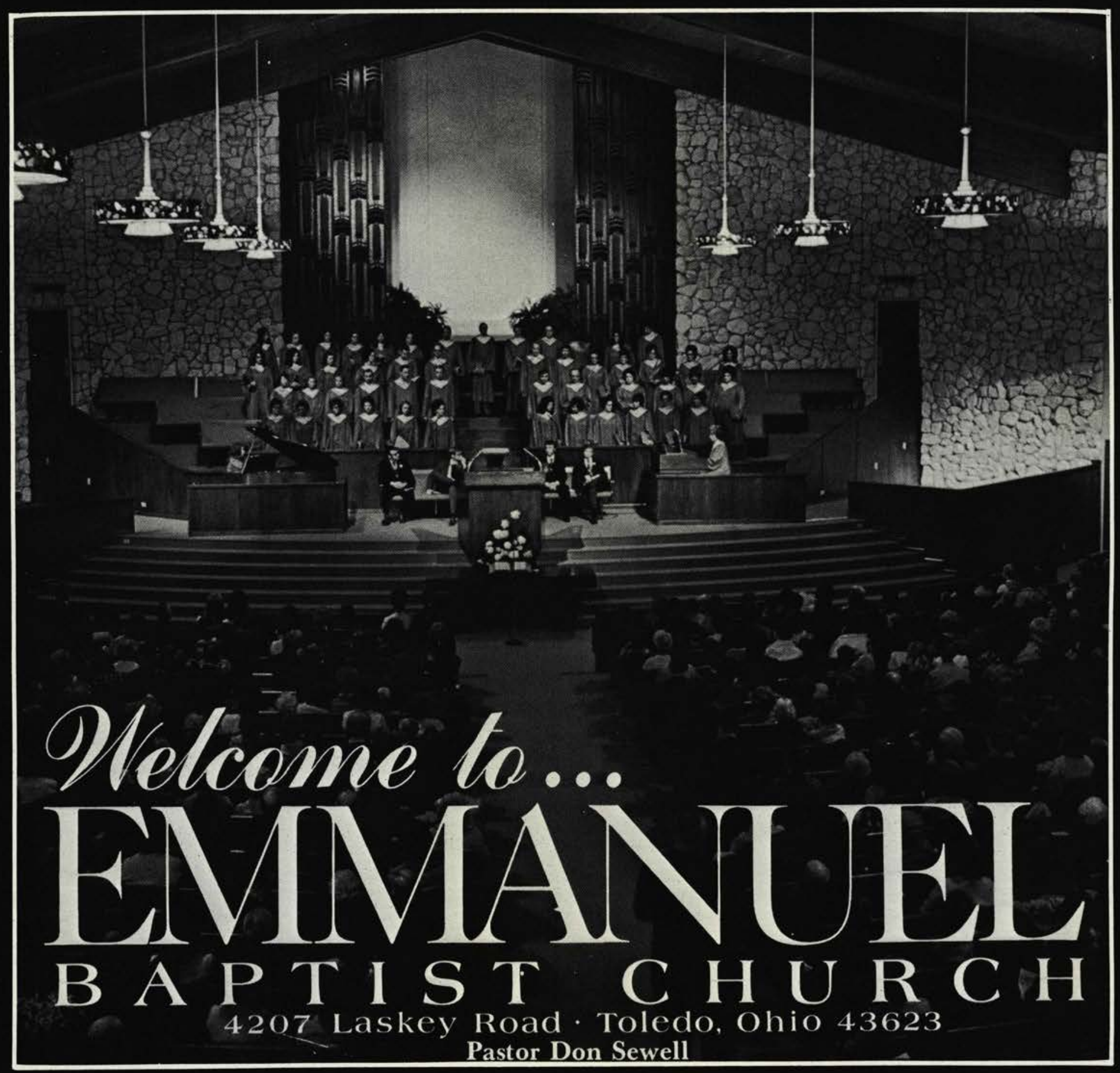




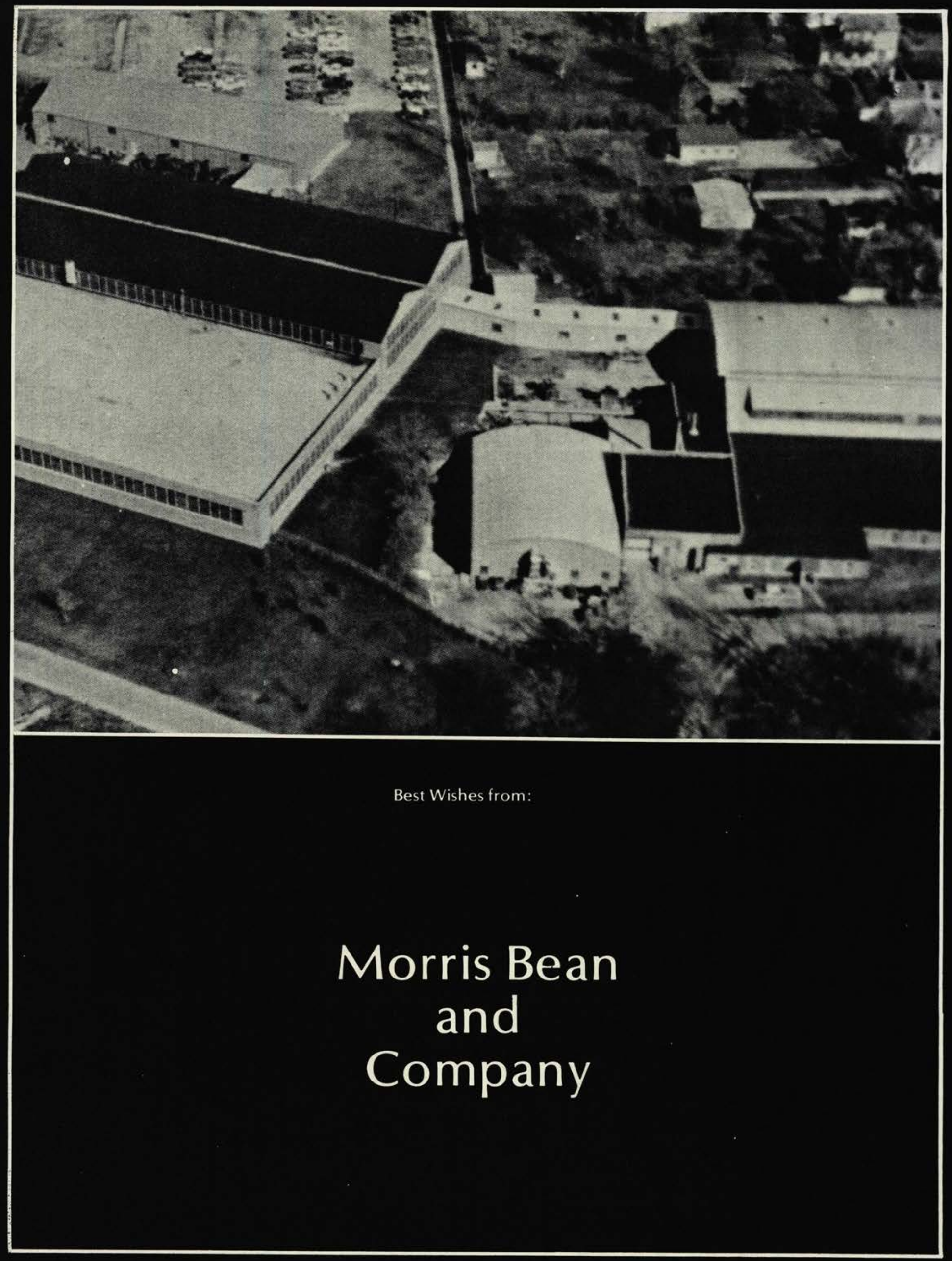



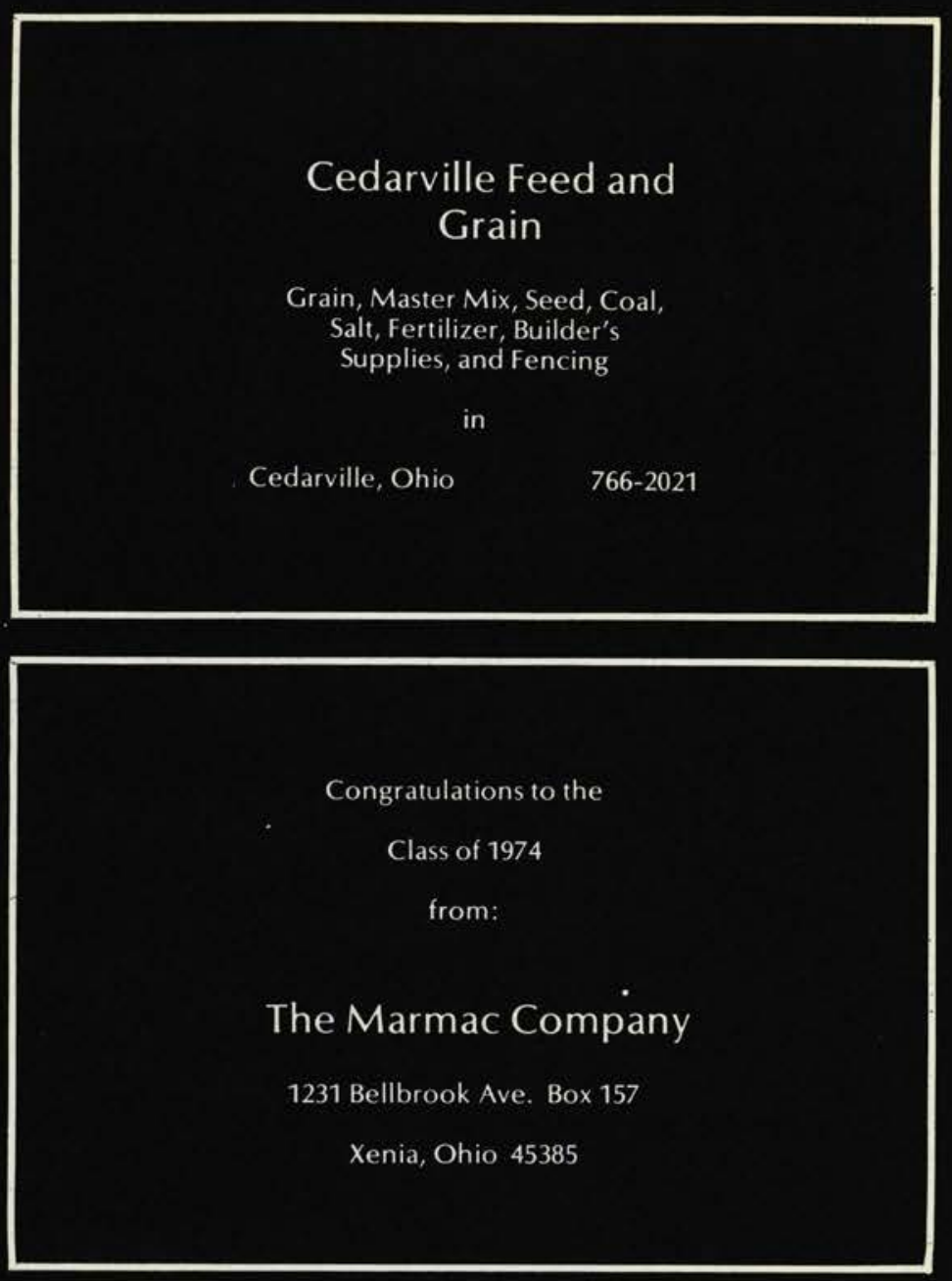

\section{Cedarville Federal} Savings \& Loan

Telephone 513-766-2141

Cedarville, Ohio 45314

Congratulations

to the

Class of 74

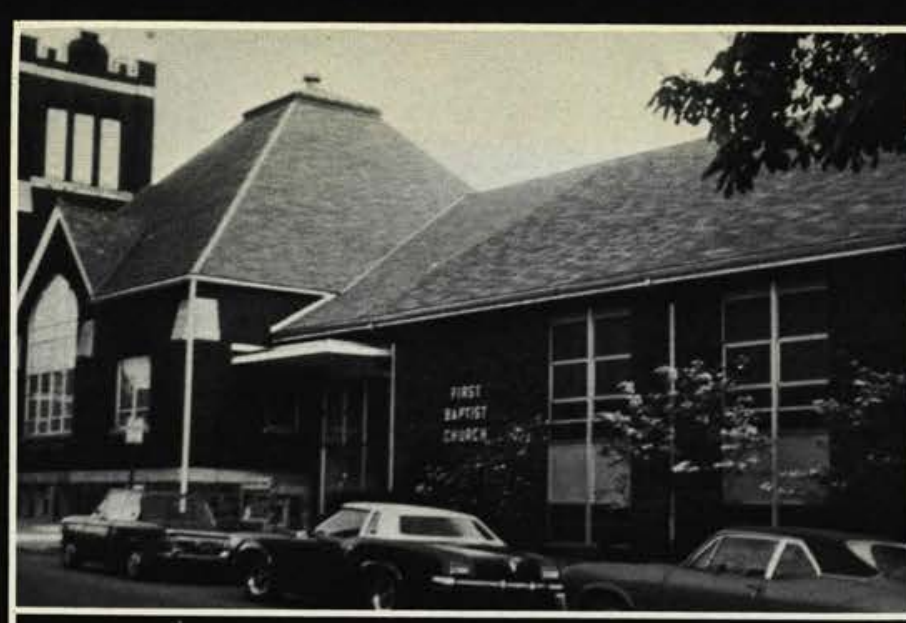

FIRST BAPTIST CHURCH

Russell R. Camp, Pastor

221 New Castle Street

Butler, Pa. 16001

Phone (412) 287-7241

Church Bible School

Morning Worship

9:45 A. M.

Training Groups

Evening Worship

Midweek Prayer

6:30 P. M

7:30 P. M.

7:30 P. M.

Best Wishes

from:

Immanuel

Baptist

Church

3417 Palmetto

Street

Columbus

Ohio

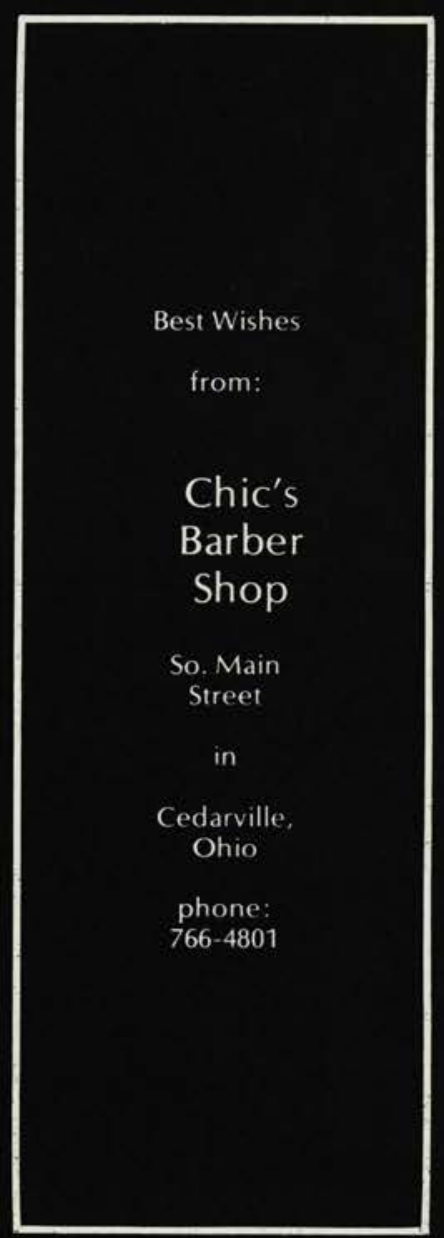




\section{PARENT PATRONS}

Mr. and Mrs. L. F. Sperry

Newtown Square, Pennsylvania

Mr. and Mrs. Maynard Stevens

Lindley, New York

Mr. and Mrs. Wayne N. Storch

Joliet, Illinois

Mr. and Mrs. Keith Steitmatter

Rensselaer, Indiana

Mr. and Mrs. Frew W. Strong

Utica, Michigan

Mr. Ralph Tallmon

Creston, lowa

Rev. and Mrs. Frederick W. Thomas

Massepequa, New York

Mr. and Mrs. Oshel R. Thomas

Lexington, Ohio

Mr. and Mrs. Paul W. Thorsell

Hudson, Ohio

Mr. and Mrs. H. C. Trautman

Avon Lake, Ohio

Mr. Floyd Vos

Muskegon, Michigan

Mr. Ben Waggoner

Brownsburg, Indiana

Mr. Carl B. Ward

Horseheads, New York

Mrs. Gail E. White

Springfield, Ohio

Mr. William E. Williams

Columbus, Ohio

Mr. Orin D. Wright

Athens, Ohio

Mr. and Mrs. Robert A. Wolf

St. Petersburg, Florida

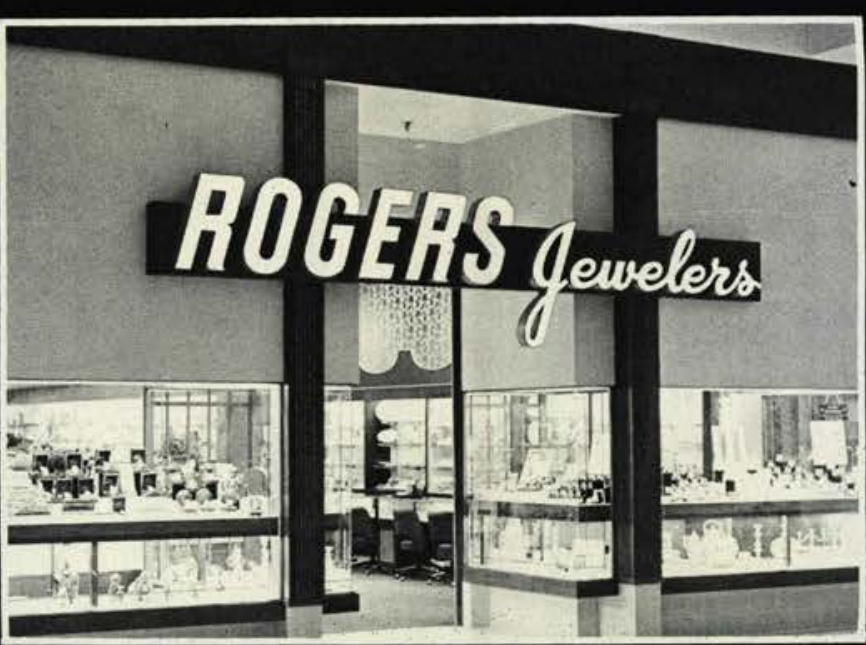

\section{Roger's Jewelry Co.}

\section{Authorized "Keepsake" Diamond Jewelers}

Fine Watches Jewelry Gift items

2 Locations to Serve You

6E. Main St. - Downtown Upper Valley Mall

\section{CALVARY BAPTIST $\mathrm{CHURCH}$}

58 West Harbine Avenue

Xenia, Ohio 45385

College Students Always Welcome

"A little Church with a Great God"

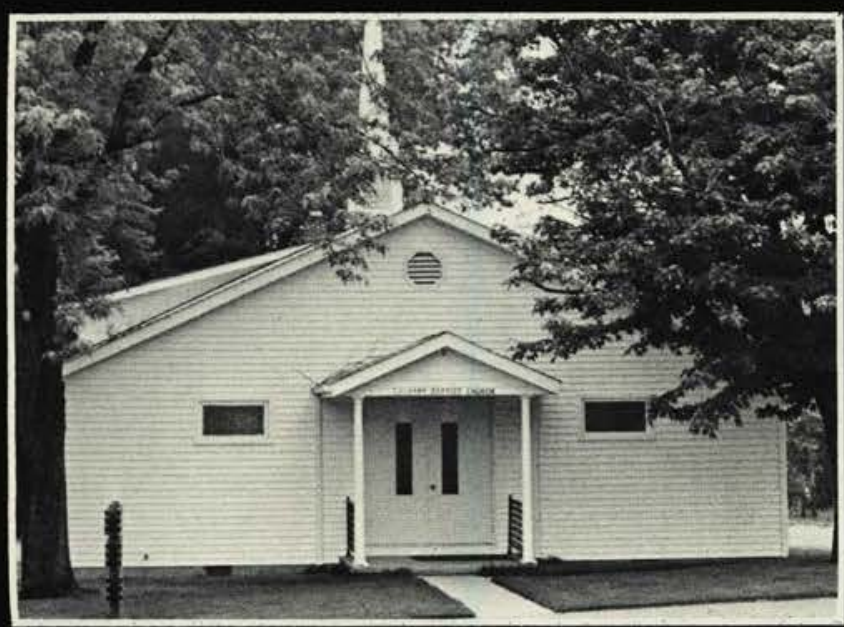




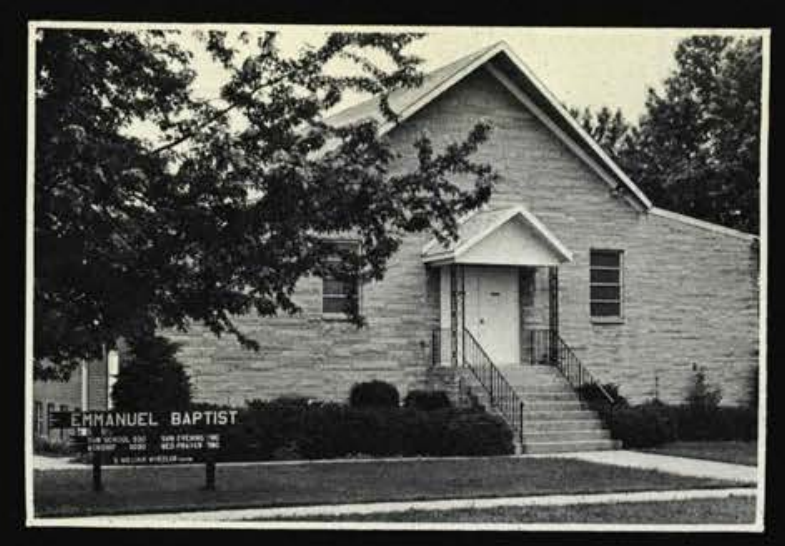

\section{Emmanuel Baptist Church}

1120 South Detroit Street

Xenia, Ohio 45382

\section{WAYNE'S SUPER VALU}

10 North Main Street

Cedarville, Ohio 45314

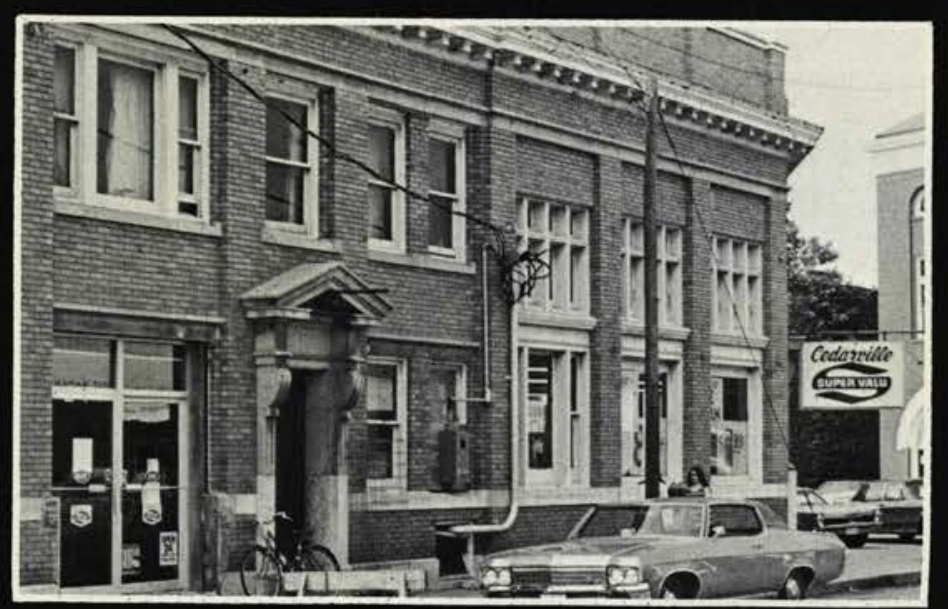

Congratulations to

the Class of

1974 


\section{VILLAGE RESTAURANT}

"In the Heat of

Cedarville"

Complete Food Service

Phone 766-5318

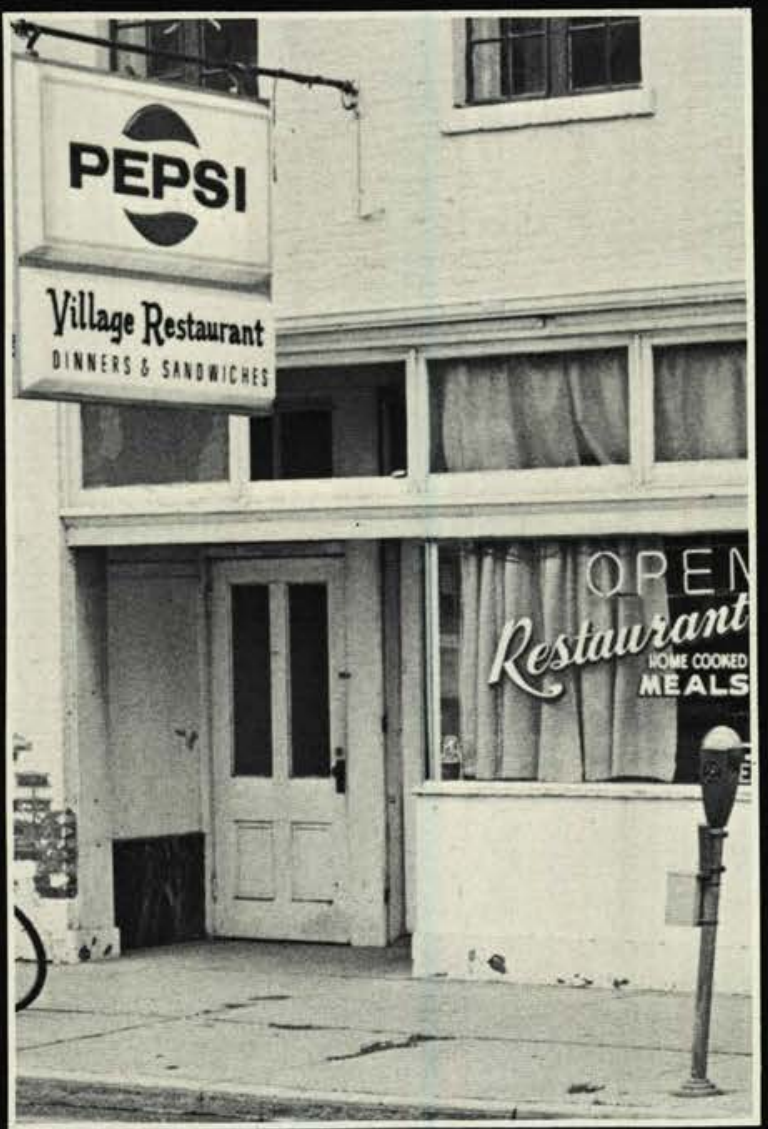

\section{PATRONS}

Mr. Gordon Wood

Mrs. Mamie Wood

Akron, Ohio

Rev. and Mrs. Edwin Wyman Sarasota, Florida

Mr. and Mrs. Charles Yasenka Broadview Heights, Ohio

Mr. and Mrs. Donald Young Hillsdale, Illinois

Braun Jewelers

Xenia, Ohio

Kennedy Korners

Xenia, Ohio

Rich Jewelers

Xenia, Ohio

A WARM WELCOME AWAITS YOU AT NORWOOD BAPTIST CHURCH 2037 Courtland Ave. Cincinnati, Oh. Phone - 513-611-7927

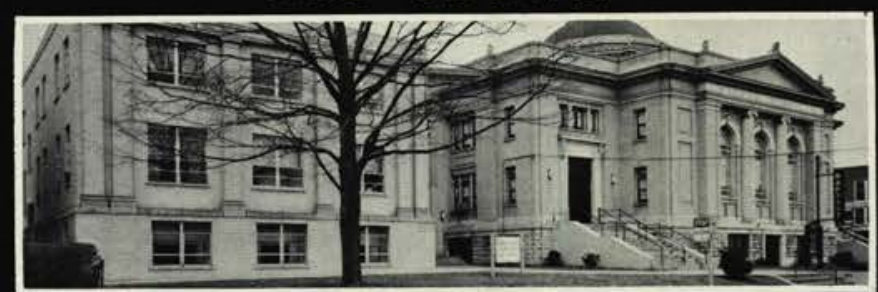

Pastor Albert Tassell

Services

Sun. 9:30 am Bible School 10:30 am Worship Service 6:15 pm Youth Groups 7:00 pm Gospel Service

Wed. 7:30 pm Prayer Meeting

Mon. thru Fri.

8:30 am Christian School and Kindergarten

Our Congratulations to all 1974 graduates! 


\section{HILLTOP SUNOCO}

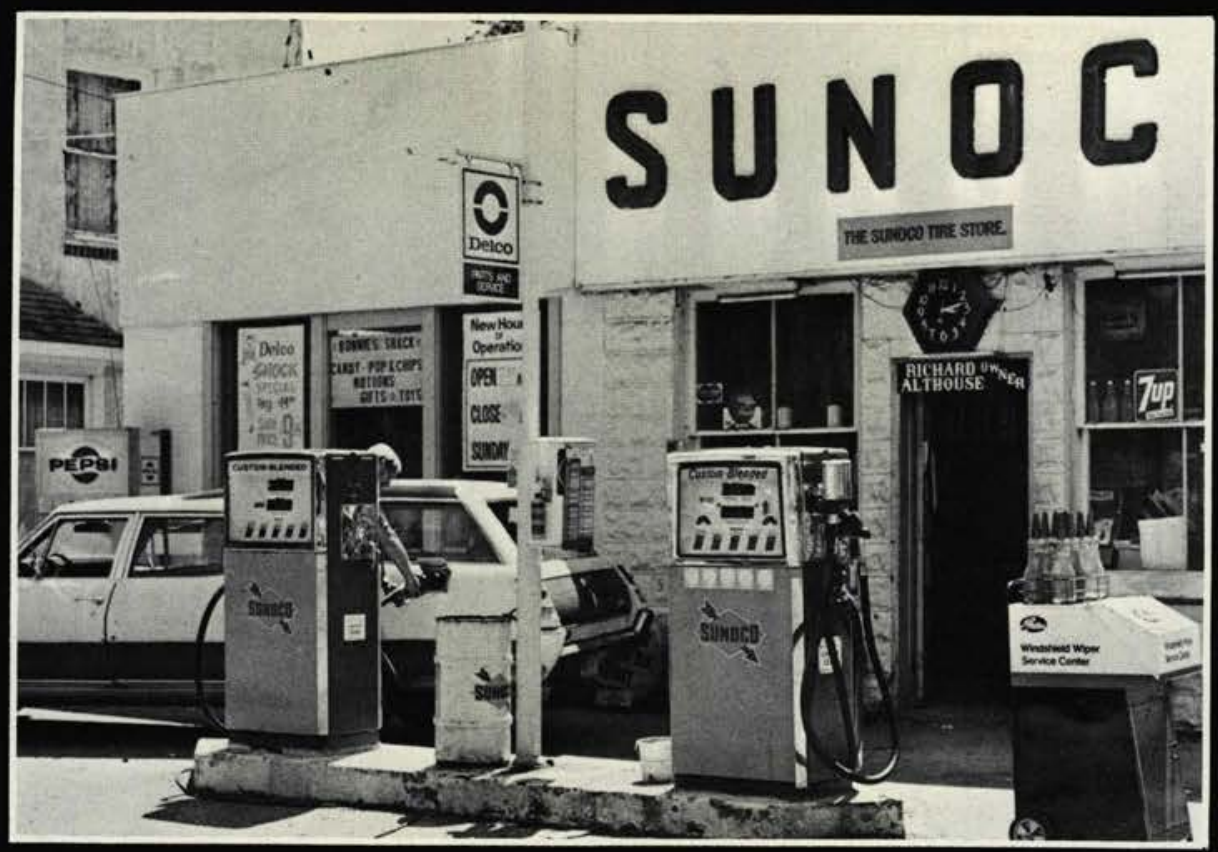

Will Give Free Red, White \& Blue Pen With Signing of Annual

\section{GERALD INSURANCE}

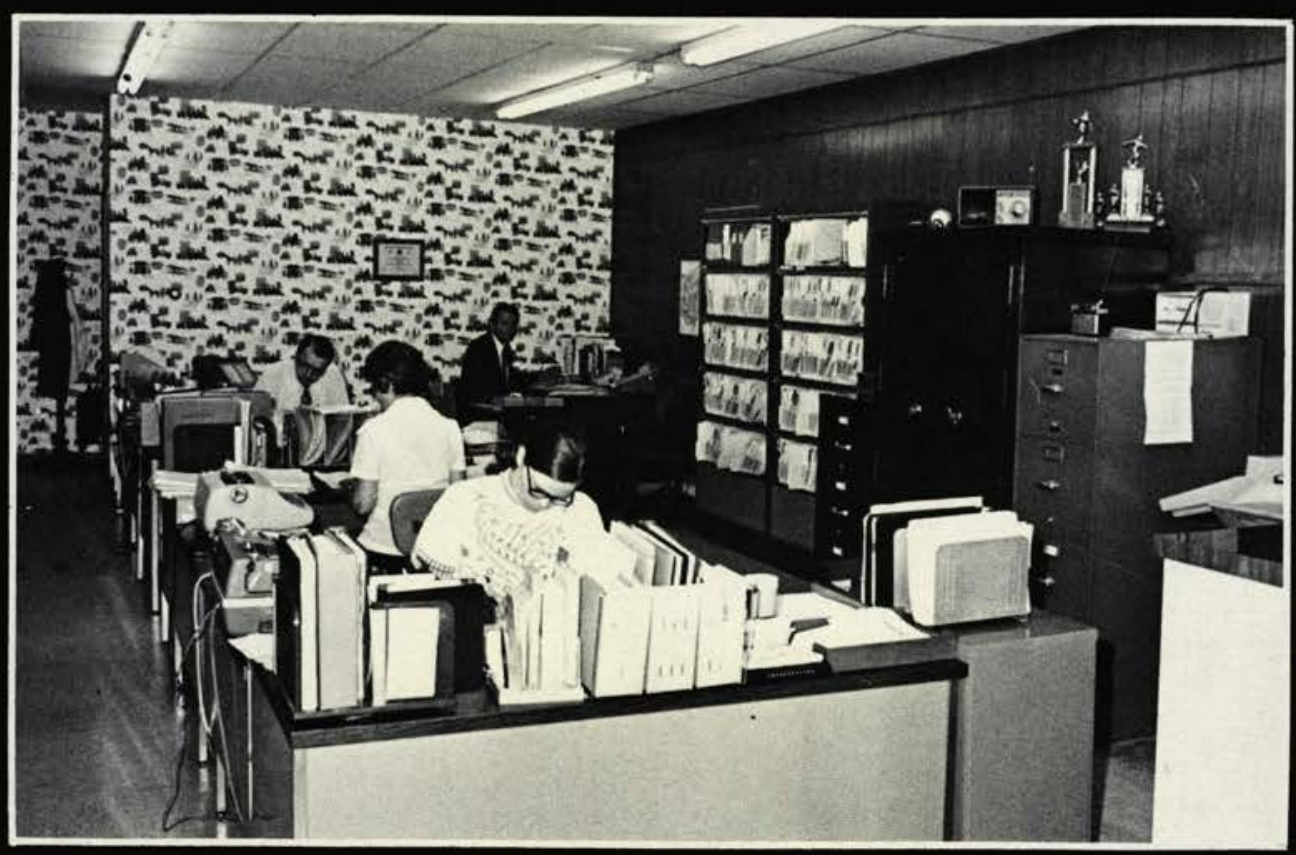

ivech Wat ge

Jamestown, Ohio 
Abbas, Robert, 98

Abels, Mark, 31, 189

Adamowich, Daniel, 56, 138

Adams, Beth, 184

Adams, Kenneth, 56

Adams, Sharon, 184

Ager, Merlin, 96

Albertson, Clayton, 56

Albertson, Earl, 113, 189

Aldridge, C. Wayne, 180

Allen, Larry

Alpha Chi, 113

Alpha Mu Chi, 127

Amenold, Tamara

Anassi, Demitreas

Anderson, Dana, 189

Anderson, Lyle, 97

Andes, Mindy, 25, 28, 184, 189

Armstrong, Mead, 94

Arthur, Dinah, 27, 30, 56, 87

Andes, Patrice

Artrip, Kathi, 15, 184, 137, 136

Askern, Martha, 139, 184

Augustine, Stephanie, 184

Auwarter, Laurie, 87

Auwarter, Richard, 189

Averitt, Christine, 87, 184

Avery, Raymond, 87

Axiotis, Georgeanna, 28, 32, 48, 56, 84

Axiotis, Margo, 17, 43, 189

Baggett, Edward, 194

Baker, Jeanne

Baker, Terry, 189

Baldridge, Elmer

Ballard, Stanley, 98

Band, 89

Banks, Cindy, 189, 138

Banks, Deborah, 189

Banks, Deborah L., 180, 84

Banks, Penelope

Barber, Dennis, 57

Barker, Timothy, 56, 113, 87, 138

Barlow, Floyd

Barnes, Susan

Barnett, Larry, 112, 113

Bartemus, Donald, 189

Bartemus, Joseph, 37, 36, 159

Bartemus, Sharon, 57

Barthurst, Leah, 189

Batista, Evandro, 180

Battaglia, John, 36

Battaglia, Michael

Baumann, Donald, 98

Bays, Cindy, 189

Beach, Lorraine

Beacham, James, $179 ; 87$

Beacham, Jonathan, 113, 87

Bearss, John

Bearss, Marcia, 180

Beaujean, William, 56

Beavers, Craig

Bechtel, Ronald, 57, 84

Bechtel, Sandra, 189

Beck, Paul, 24

Beikert, Barbara, 189

Bell, Eugene

Bentson, Gale

Bennett, Kelsey, 57

Bennett, Paul, 180

Bergen, Harmon, 95

Berkenstock, Nova

Berndt, Laurel, 189

Bernhard, William, 137, 111, 189

Berry, Paul, 36, 96, 159

Besler, Janet, 139

Billington, Joyce

Bintz, David, 180

Birk, Chris, 189

Bleeker, Marlene, 22

Blystone, Rodney, 28, 180

Bodenmiller, Bonny, 57

Bogert, Lorraine, 31, 180

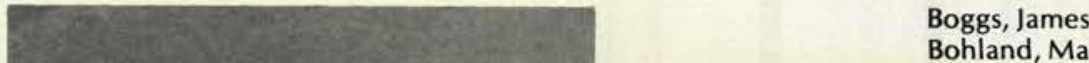

Bohland, Mark, 57

Bollback, Larry, 30, 36

Bonnell, Carolyn, 180

Book, Martin, 110

Book, Mitchell, 130

Bowen, Jeff, 19, 22, 111, 137

Bower, Lorraine, 179

Bowers, Paul, 28, 136, 146

Bowman, Daniel, 28, 31, 130, 189

Bowser, Valerie

Boyce, Michael, 57

Boyer, Rick

Bradford, Cheryl Huff, 58

Brady, Mark

Brentlinger, John

Bresson, Mark, 135

Briggs, Frederick

Brinkman, Nelson

Britt, William

Brock, Gary, 58

Brock, Kevin, 8, 19

Brooks, Catherine

Brown, Elaine, 117

Brown, JoAnn, 180

Browning, John, 159, 180

Browning, Sara, 138

Brueser, Sally, 133

Budnik, Paul

Bugno, Curt, 159

Buhr, Tim, 159

Buisch, Irene, 138, 139

Burkett, Robert, 104

Burkhalter, Harold, 25, 135, 180

Burkhard, Kathy, 58

Burkhart, Robert, 34, 113

Burnett, Thomas, 24, 159

Burns, Sherry, 57, 116

Burns, William, 180

Burrell, Brad, 147, 179

Burt, Howard

Burtner, David

Burtner, Pamela, 28

Busho, Bonnie, 58

Busho, Craig, 58

Bussing, Terri

Butcher, Debra, 28

Butler, Cathy, 87

Byers, Deborah, 58, 141

Byers, Joleen, 58

Byers, Sharon, 141, 180

Cagwin, Daniel

Callan, Donald, 30, 97

Callison, Ellen, 59

Calvin, Esther, 126, 180

Cameron, Sandra, 139

Campbell, Lee, 31, 136

Campbell, Barbara, 58

Carl, Janette

Carlton, Ken, 59, 133

Carlton, Linda, 84

Carpenter, James

Carpenter, Shirley, 59

Carr, Cindy, 19

Carr, Janet, 28

Carr, Patricia

Carson, Billie

Carter, Bertha, 43, 60

Casagrand, Fred

Cavey, John, 36

Cavey, Margareta, 9, 112, 126, 180

Cayton, Marlin, 113

Chambers, James

Chamblin, Sherry, 28

Chapman, David

Chester, Ruth

Choir, 84

Chrestensen, Karen, 59, 137

Clapp, David

Clark, James, 180

Clark, Laureen, 42, 43, 116

Clark, Marilee

Clark, Ronald, 131 
Clark, Russell, 30

Clark, Teresa, 180

Cleckner, Anne

Cleckner, Nancy, 59

Clithero, Connie

Cline, Jackie, 59

Cline, Judy, 126, 127, 180

Cline, Vicki

Close, Pamela

Colburn, Karen

Cole, Andrew, 34, 113

Cole, David, 113

Cole, Mark

Collison, Ellen

Commins, Dan, 180

Commins, Jackie, 180

Conley, James

Conrad, Deborah, 139, 180, 196

Cook, Linda, 180

Cook, Thomas, 36, 38

Coomes, Dan, 110, 159

Cooper, Barbara, 28, 30, 135

Cooper, Mary Jane

Cope, Merilee, 180

Cornelius, Linda

Cornwell, Ralph, 181

Cornwell, Patsy, 181

Cossins, Martin, 132

Costley, Stephen

Cotnoir, David, 59

Couch, Linda

Couture, Jonathan, 181

Cowell, Sheri 138, 126

Cowen, Cheryl, 139

Crady, Dennis

Craig, Diane, 59

Crane, Ronald

Creeger, Stephen, 181

Cressman, Sherrill, 19, 60, 116

Crider, Gary, 181

Crider, Patty

Criss, Donald, 84,87

Cronbaugh, Brad, 130, 135, 137, 181

Cronbaugh, Brian

Crum, Rhonda, 117

Cuenin, Eric, 60, 155

Cunagin, James

Cupan, Linda

Curlis, Larry

Dalton, Kathleen, 60

Danielson, Craig

Darnell, David

Davis, Deborah

Davis, Donald, 113

Davis, Jerry

Davis, William

Davison, Beverly 19, 28, 31, 127, 181

Davison, Tim, 181

Dear, Debra, 19, 25, 30, 48, 60, 77

Dear, Nancy

DeBruine, Joyce, 60

Decker, Diane

Decker, Lenore

Dedrick, Bonnie, 126, 181, 196

DeLancey, David, 36, 137, 155

Deland, Douglas, 19, 60, 138

DeLange, James, 113

DeMars, Denise, 42

Demoret, Stephen

Demos, Carol, 61

DePriest, Dana

DePriest, Roger

Dersham, Paula

Detrick, Virginia, 126, 181, 196

Detwiler, Tim, 146

Diaz, Henry, 36, 38, 39, 113, 138

Dilcher, Harry, 61

Dillsworth, Deborah, 126, 136

Dispennette, Carl

Dixon, Janice, 87, 136

Dixon, Patsy, 96

Dodson, Irma, 96

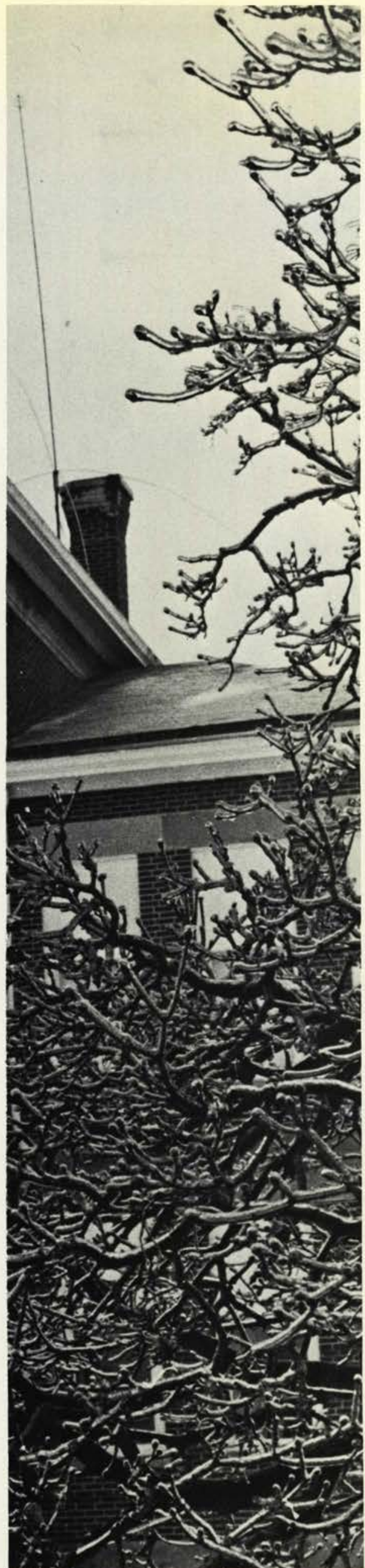

Dolph, Charles, 61

Dolph, Michael

Donalson, Paulette, 19, 130, 189

Donnelly, Laurie

Douglass, Diana, 30, 61

Douglas, Jim, 84, 113

Douglass, Randy, 113, 132

Dritsas, Ellie, 137

Drout, Wendy

Dudley, Deborah, 15, 181

Dunham, William

Dunn, Alan, 28, 181

Duran, Becky, 181

Dutton, Jack, 181

Earnhart, Deborah, 139, 181

Earnhart, Mark

Eberling, Jayne, 48, 54, 61, 126

Ebersole, Cheryl, 126

Eckstein, Michael

Edlund, Alan, 155

Edwards, Denise, 61, 116

Edwards, Jane

Edwards, Leslie

Edwards, Penny

Eggleston, Donald, 19, 30, 155, 181

Ehnis, Daniel, 33, 61

Eickhoff, Baron

Eissens, John, 110, 111

Elder, Michael, 181

Elkin, Cindy, 179

Eller, David, 131, 181

Elliot, Charles, 113, 138

Ellison, Gene

Elmore, Austin, 98

Emerson, Ruth, 31, 61, 127

Englehart, Ann, 141

Entingh, Daniel

Erickson, Don

Erikson, Joy, 43, 117, 181

Essex, Wayne

Estes, Dan, 18, 19, 31, 36, 62, 84, 113

Estes, Stephen, 15, 19, 84, 137

Evans, Dave

Evens, Dwight

Everhart, Rebecca

Ewing, Dora, 15, 28, 31, 138, 181

Ewing, Robert

Fair, James, 137

Fairchild, Wesley

Fawcett, Glenn, 113

Fehrman, Mark

Feldman, Nancy

Fenton, William

Few, Bryan

Few, Dennis

Fields, Beverly, 181

Fields, Kevin, 146, 147

Fight, Becky, 131, 181

Filson, Earl

Finch, Brenda, 138

Finch, Sandy, 42, 116, 181

Fisher, Jean, 94

Fisher, Michael, 22

Fisher, Robert, 28, 131

Flatter, Debbie

Floyd, Ken, 189

Ford, Connie, 117

Ford, Irene, 181

Foster, Diane, 137

Foulis, Glenda

Founds, Garrett

Francis, Steve, 34, 62, 112, 113

Francisco, Paul

Frank, Dwayne, 96

Franks, Sharon, 84, 146, 147

Franson, Linda

Frauenknecht, Marianne, 62, 42, 116

Frederick, David, 84

Frye, Bert, 99

Fields, Kathie, 181

Fry, George

Funderburg, Pam

Gabler, Tim, 138, 139 
Gabriel, James

Gaines, Keith

Gaippe, Leslie

Galbreath, Theodore, 147, 181

Gates, Donald, 62

Gates, John

Gathany, Paul, 95

Gazdik, Sharon, 19, 48, 62, 138

Gazdik, Susan, 136

Gerber, Wynn, 159

Gerver, Richard

Gibbons, Tawanna

Gibbs, Debra

Gidley, David, 105

Gidley, Deborah, 84, 87

Gierhart, Deborah

Giesel, Chris

Giesman, William, 19, 28, 136, 181

Gifford, Theodore, 19, 62

Gifford, Warren

Gilbert, James

Gilbert, Pennie

Gilbert, Tim, 189

Giles, Brenda

Gillespie, Robert, 28

Gillette, Danny

Gilliland, Lana

Gilmour, Carol

Gilyard, Jerry, 33, 34, 112, 113, 135

Ginter, Donna

Glenn, Mary, 28, 136, 181

Glenney, David

Glover, Staranna, 131, 138, 141

Godby, Louis

Goetz, Jim, 19, 112, 113

Gons, Mark

Gons, W. Scott, 19, 113

Gons, Mark

Gons, W. Scott, 19, 181

Good, Debbie, 31

Goodlander, Carolyn, 62

Gordan, Gary, 181

Gossett, Gary, 139

Grabianowski, Tim, 113

Grace, Karen

Graham, Donald

Gray, George

Green, Harold, 105

Green, Janet, 137, 181

Green, Mark

Green, Sharon, 139, 1

Greenwood, Edward, 96

Grier, James, 94

Griffeth, Mary

Griffeth, Sarah, 62

Griffeth, Walter, 99

Griffeths, Wayne

Griffis, Cheryl, 87

Grimmett, Pat

Griswold, Jerry, 63, 159

Grollimund, Carolyn

Gromacki, Robert, 94

Grosh, Jennifer, 126

Grosh, Jodi, 126

Grosh, Ronald, 95

Gruber, Kevin

Gruber, Steve, 63

Gruenberg, Ruth, 19, 181, 196

Guess, David

Haffey, Deborah, 95

Hager, Delmar

Hager, Tim

Hale, Suzanne, 63, 136

Halladay, Allen, 181, 159

Halladay, James

Halsey, Joseph, 99

Hambrick, Bruce

Hammond, Cathy

Hancock, Tim, 181

Handyside, Kenneth

Hansen, Rod, 63, 159

Hansen, Sherlyn, 63, 127

Hare, Donald, 36

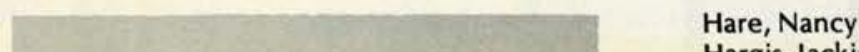

Hargis, Jackie, 63

Harkleroad, Mason

Harmon, Donald, 63

Harmon, Jeanne

Harriman, Joel, 28

Harriman, Sarah

Harris, Barbara

Harris, Hope, 126

Harris, Kenneth

Harris, Sue

Harrison, Cynthia

Harvey, Dane, 87, 98

Hauser, Karen

Hawbaker, James, 31, 112, 113

Hayes, Carolyn, 28, 31, 133, 136, 181

Hayes, Philip, 192, 196

Haynes, Connie, 192

Helling, Michael

Helmick, Larry, 98

Helmick, Randy

Henriques, Teresa, 22

Henry, Michael

Henry, Pat, 28, 192

Henslin, Tom, 131

Hepworth, Kathy, 87, 135

Hering, Nancy, 192

Herrin, Linda, 126

Heslip, Cheryl, 30, 42, 135, 181

Hickey, David, 30, 111

Hilgar, Laura, 131, 192

Hill, Jan, 192

Hilleary, Douglas, 39, 138

Hinds, Linda, 30

Hinks, Dennis, 147, 192

Hintz, James, 113

Hirschy, Kathleen, 192

Hlad, Thomas, 36, 192

Hockenberry, Grace

Hoffman, George, 155, 192

Hogsett, Susan

Holland, Lucille, 192

Holley, Terry, 36, 63

Hollingsworth, Ron

Hollister, Laurene, 192

Holman, Stephen, 28, 141, 192

Holmes, Robert, 192

Holt, Debbie, 64

Holwerda, Sandra, 19, 42, 116, 181

Holzworth, Holly, 64

Hook, Valerie, 43

Hopewell, Tom, 137, 181

Hopkins, Sharon, 131, 192

Horsfall, Joseph, 192

Horton, Harold

Hoskinson, Randy, 182

Hostetler, Brenda, 192

Hostetler, Debbie, 64

Houch, Marcia, 28

Houmes, Karla, 192

Howard, Elaine, 138, 192

Howard, Kenneth, 25, 31, 36, 37, 38, 113, 192

Howdyshell, Terry, 130, 192

Howell, Kathleen, 64, 84

Huddleston, Vicki, 182

Hudson, Douglas, 64

Huesmann, Jessica, 192

Hughes, Clyde, 64

Hughes, Craig, 182

Hulsman, Catherine, 192

Humphres, Diane, 133

Humphrey, Lucille, 182

Hunter, Mark, 182

Hutchison, John

Imbro, Becky

Imhausen, Mary, 192

Isaacs, Susan, 126, 139, 192

Jackson, Cheryl, 87, 192

Jacobs, Diane, 18, 19, 137, 182

Jacobs, Steven

Jamison, Cheryl, 192

Jacques, Rick, 31, 192

Jarvis, Lois, 136 
Jason, John

Jeffords, Barbara, 179

Jeffords, John, 84, 179

Jenkin, Holly, 64, 48

Jenkins, April, 64

Jenkins, Joe, 36, 132, 192

Jeremiah, James T., 15, 102

Jeremiah, Maryalyce, 97, 116

Jewell, Darla, 30, 48, 65

Johns, Sharon, 65

Johnson, Brenda

Johnson, Carol, 65

Johnson, Christine

Johnson, Clifford, 103

Johnson, Dale

Johnson, Doris, 33, 65

Johnson, Merilee, 19, 182

Johnson, Susan, 28, 130

Johnson, Wesley, 28, 192, 155

Johnston, David, 159

Jones, Daniel, 84

Jones, Darlene, 127, 182

Jones, Deborah, 27, 30, 116, 137, 182

Jones, Keith, 182

Jones, Linda, 192

Jones, Pamela, 65

Jones, Rick, 55

Jones, Rita, 14, 139, 182

Jones, Sarah, 192

Jordan, Vicki, 116, 117

Judge, Timothy

Jutton, Ronald, 65

Kammeyer, Vicki, 65, 87

Kappa, Delta Chi, 125

Karsian, Kathy, 65, 137

Kaufman, Tim, 30, 137, 159, 182

Kayler, Keith, 192

Kearney, June, 42, 43, 97

Kee, Pat, 182

Keizer, Bruce, 192

Keller, Carol, 84, 192

Kemble, Becky, 30, 66, 84

Kennedy, Karen, 66, 131

Kern, Roy, 104

Kerr, Kim, 182

Kerrigan, Kathy, 182

Kester, Paul, 36, 192

Kesler, Sam, 113, 182, 155

Keysor, Loretta

Killian, Lawrence, 99

Kincaid, Devan, 55, 192

King, Elvin, 97

Kinnibrugh, Jerry, 66

Kirby, Linden, 27, 48, 55, 66, 77

Kirby, LaVerne

Kistler, Jeff, 19, 18, 66

Klett, Bruce, 19, 87

Klimek, Cheryl, 182

Kline, Rick, 192

Knapp, Gary

Knowles, Shelley, 135, 138

Knowles, Tom, 26

Koncsol, Christine, 192

Konya, Alex

Koon, Steven, 28, 137

Knatzer, Dan, 192

Kraus, Mary

Kuni, Diane, 192

Kwak, Thomas

LaBelle, David, 193

Laing, Emma, 126, 182

Lakes, Ronald

Lamb, Amy

Lamb, Connie

Lamb, Becky, 25, 30, 31, 28, 48, 112, 182

Lampiris, Nancy

Lancaster, Kathy, 19, 193

Lancaster, Kirby, 54, 135

Landis, Kevin

Lantz, Steve, 84, 182

Larson, Stephen

Lautzenheiser, Rick, 132, 139

Lawlor, George, 94

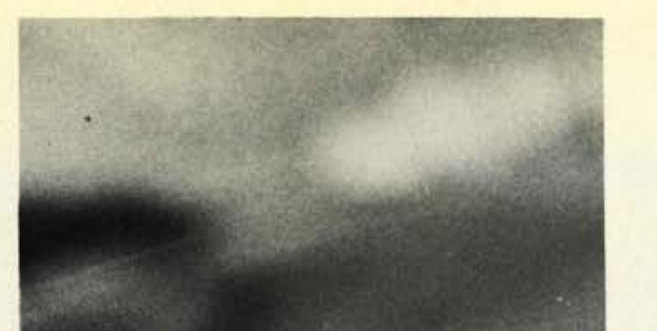

Lawhead, Jonathan, 159

Layne, Nanci

Leapline, Leslie, 126, 182

Leapline, Zita

Leatherman, Daniel

Lee, Brenda, 192

Lee, George, 34, 113

Lee, Timothy, 66

Leeke, Debra, 193

Leninger, John

Leisure, Mia, 193

Lemmen, Calvin, 193

Lepley, Pamela, 182, 117, 139

Lewis, Deena, 193

Lewis, Jeff

Lewis, Linda

Lewis, Martha

Lewright, Nancy, 57

Lichty, Dan

Lightrap, Esther, 28, 31, 48, 55, 136, 182

Lillback, Peter, 67, 176

Lilley, Charles

Linden, Rick

Linger, Penelope, 133, 193, 196, 139

Litfin, Melinda

Litts, Roberta, 135

Lo, Lawrence, 97

Loach, Barbara, 28, 193

Lochel, Nancy

Logsdan, 141

Long, Janet, 19, 48, 55, 67, 77

Longacre, Dave

Loper, Beth, 16, 193

Lotz, Ruth, 193

Loudenslager, Tim

Lowis, Michael, 193

Lowrey, Randy, 193

Lowstetter, Dennis, 67, 159

Luers, Norman, 87, 193

Luketic, Barrett, 19, 36, 37, 48, 67

Lutton, Nancy, 182

Lyon, Noreen

Mack, Roma, 132

Maddox, Miriam, 95

Madsen, Brenda, 193

Madsen, Karen

Mahl, Robert, 18, 19, 26, 67

Mains, Carol Jo

Malernee, Christine, 126

Mally, Cindy, 84, 182

Mangum, Brenda, 193

Manwaring, Alice, 193

Mapel, Linda, 67

Mariage, Michael, 193

Marshall, David

Marshall, Deitra, 132

Marshall, Susanne, 193

Martin, Carol, 138, 141

Martin, Chrys, 31, 182

Martin, Kirk, 155

Martin, Wayne, 193

Matheny, Lois, 28, 189, 193

Matson, David, 84, 97

Mawhorter, Michael, 30, 67

Mayo, Linda, 84, 193

McCall, Susan, 87, 193

McCallister, Steve, 68

McCann, Sandra, 193

McCann, Tawn

McCarty, Diana, 68

McCauley, Ruth, 182

McClish, Lois, 182

McCorkle, Gene

McCormick, William, 68

McCracken, Rich, 193

McDonald, Cleveland, 99

McDonald, Phil, 30, 182

McFarland, Randy, 146, 182

McGee, Gail, 31, 130

McGhee, Dean, 135, 193

McGhee, Richard, 19, 182

McGinnis, Sam, 110

McGolderick, James, 99 
McIntosh, Kathy, 15, 19

McIntosh, Richard, 94

McIntosh, Rick, 68

McKanna, Donald, 68

McMillen, Philip, 36, 68

McNamara, Lois

McNeely, Janice

McNiece, Wendy, 193

Me aker, Michelle, 135, 193

Meenah, Chris, 68

Melford, Bob, 84

Meloy, Richard, 68

Melton, Douglas

Metrell, Cindy

Merrill, Eunice, 43, 138, 193

Milam, John

Millard, Victor

Miller, Alice, 28, 130, 193

Miller, David, 193

Miller, Ronald, 193

Miller, Tim, 193

Millikan, Steve, 69, 155

Miracle, 197

Mitchell, David

Mitchell, Jonathan, 36, 113

Mitchell, Lynn, 30, 69, 77

Mitchell, Rosemarie

Mitchell, Sally, 69

Moffit, Nancy

Mohat, Robert, 146, 147, 193

Mohat, William, 193

Mohler, Leonard, 182

Mohr, Janene, 126, 182

Monroe, Allen, 87, 99

Monroe, Robert, 87, 97

Montgomery, Wendy, 84, 126, 137

Monts, James, 193

Moore, Elaine, 84, 193

Moore, Ray

Moore, Thomas

Moreland, Kurt

Morgan, Scott, 131, 182

Morley, Carla, 130, 135, 193

Muckley, Steven

Mudder, Terrance, 135, 182

Mueller, Sharon, 193

Muggleworth, Dale

Mulder, Karen, 69

Murger, Jean

Murdoch, J. Murray, 99, 155

Murray, Linda, 28

Murray, Marguerite

Myers, Jon, 110, 159

Nabring, Ranelle, 69

Nabring, Thales

Namy, Karen, 133

Namy, Keith, 55, 193

Nash, Bill, 146, 147, 194

Nelson, Sonia

Neuhaus, Charles, 155

Newman, Carolym, 69

Nicholas, David, 19, 69

Nicholas, Luann, 69

Nichols, Ken, 105, 189

Nicholl, Lonnie

Nickell, William

Niles, Michael, 146

Northcutt, Patsy, 127, 182

Ockert, Carol, 133, 139, 182

Odom, Terry, 194

O'Keefe, Tim, 130, 137, 194

Olsen, Jonathan, 131

Ometz, Farris, 194

O'Neil, Jennifer, 42, 43, 182

Orchestra

Orihood, Sally, 42, 43, 116

Ortiz, Janice, 136, 182

Orton, Christine, 132, 183

Orwick, Ann

Osborne, Deana, 139, 194

Osborne, Linda, 42, 90

O'Shell, Sally, 137

Ott, Sally, 194

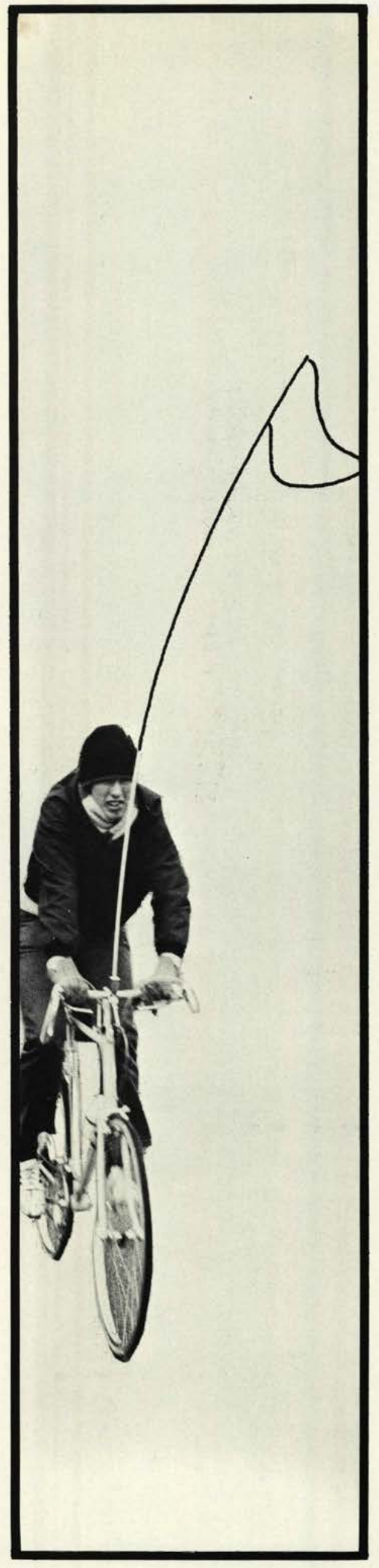

Otto, Barton, 70

Overholt, Becky, 70

Overholt, Steven, 18, 19, 70

Overturf, Roger, 183

Owens, Penny

Pape, Mark

Park, Kathleen

Parker, Clarence, 28, 30, 31, 70, 132, 159

Parker, Gloria, 70

Pasma, Tim, 28, 194

Passmore, Burdette, 194

Patterson, Charles

Pauley, Linda, 70

Pauling, Ted

Payne, Jack, 98

Payne, John

Peacock, Sue, 135, 183

PEMM 44

Percesepe, Gary, 183

Perkins, Roy, 113, 183

Perry, Gerald

Perry, Leona

Peterson, Martha, 194

Phelps, Pamela

Phillips, Carol, 135

Phillips, Harry

Phillips, Henry, 104

Phipps, James, 77, 95

Pi Delta, 15

Pi Sig, 151

Pierce, Linda

Platvolt, Lynn

Porter, Cindy, 133, 194

Portz, Gale, 70

Potter, Mary

Potter, Sharon

Potter, William, 30, 71, 110

Powers, Cindy, 84, 133, 194

Price, Nancy, 17, 84, 133, 194, 196

Price, Sally, 133, 194

Printy, Beațrice, 104, 127, 176

Pritchett, Glenn, 194

Profant, Richard, 36, 37, 71

Pruden, Mary, 28, 31, 126

Pulver, Harold, 138, 141

Pulver, Norene, 194

Rice, Rosemary, 194

Richards, Lori, 87

Rickard, Donald, 19, 102

Ricker, Rose

Riedel, Daniel

Riess, Curtis

Rifenberich, Holly

Riggs, Darrell, 194

Riggs, Jack, 94

Rinehart, Susan

Ritchie, Nancy, 71

Ritchie, Pat

Ritenburg, Dan, 84

Riter, William, 95

Robb, Dawna, 28, 43

Robb, Karen, 194

Roberts, Chris

Roberts, Eddie, 194

Robertson, Rod, 135

Robison, Rodney

Roby, Laúra, 117

Rodriguez, Laura, 71, 139, 141

Rogers, Rex, 48, 71

Rohrbach, Judy

Romaine, David, 19, 71

Romaine, Pamela

Rose, Randy, 136, 183

Rose, Richard, 183

Rosecrans, Daniel, 146

Rose, Mary

Rost, Jeffery, 130

Rothberg, James

Rothbuer, Alan

Rotsko, Carol, 194

Rouch, Andrea, 84, 194

Rowe, Glenn, 183

Rowland, Charlotte, 28, 194 
Rowland, Deborah

Rufener, Ron, 34, 113, 183

Rupert, Sandra

Rush, Delores

Salomon, Fay, 72

Sampson, Robert, 19, 34, 113, 183

Samson, Deborah

Sanders, Kenneth, 72

Sapp, Carolyn, 25, 189, 194

Saunders, Susan, 72

Schench, Herschel

Schmidt, Debra, 194

Schnell, Paul, 194

Schroeder, Douglas, 19, 28, 30, 32, 113, 183

Schroeder, Ronald, 72, 130, 137

Scott, Jack, 98, 127

Scott, Kathy, 72

Seaman, James, 94

Seaman, Lila, 96

Seeley, Mark, 19, 113, 146, 147, 189,194

Seevers, Stanley, 104

Seidelman, Leanne, 194

Seigneur, Dean

Seigneur, Renee, 72

Selden, Susan, 15, 28, 72

Self, Daniel, 194

Senseney, Martha, 183

Severs, Linda, 19, 72

Seymour, Robert

Sharp, Cathy, 73

Shaw, Richard, 73

Shawver, Jan, 183

Sheetz, Steven, 113, 183

Shepherd, Lloyd, 183

Sheperd, Wayne, 147

Sheppard, Charles, 18, 73

Shugars, Dean, 73

Sigma Delta Kappa, 132

Sigmon, Mark, 84, 194

Sikora, Michael, 194

Simons, John

Sims, Kevin, 73, 87, 112, 113, 130

Sine, April

Sininger, Becky, 194, 138

Skees, Sandra, 183

Skees, Stephen

Sliker, Susan, 194

Slocum, Sue

Slusher, David

Smart, Maynard, 84

Smith, Carol, 126

Smith, Gregory, 73

Smith, James, 159

Smith, Larry

Smith, Michael

Smith, Michael T.

Smith, Pat G., 126

Smith, Pat J., 139

Smith, Paul, 135, 141, 194

Smith, Phillip, 131, 194

Smith, Roger, 34, 113

Smith, Steven Ri.

Smith, Steven Ru., 111, 194

Smith, Teresa

Sneary, Joyce

Sneller, Deborah, 73, 126

Soliday, David, 159

Soliday, Sandra, 84, 183

Sollenne, Karen, 194

Sollene, Laura, 127, 131, 183

Spaulding, Ellen, 28, 31, 97, 136

Spencer, Bruce, 110, 195

Spencer, Debbie

Spencer, Edward, 96

Spencer, Karen, 131, 195

Spencer, Stephen, 73

Sperry, George, 28, 195

Spink, Stephen, 74

Spinks, Jackie, 28, 74, 77, 136

Sprunger, Sandra, 195

St. Clair, Jeff, 19, 74, 132, 146

St. Clair, Kenneth, 102

St. Denis, J. Wayne

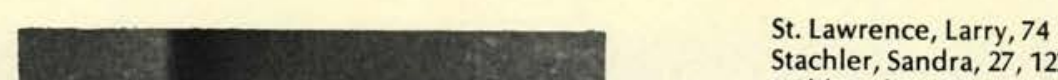

Stachler, Sandra, 27, 127, 183

Stahl, Debra, 195

Stauffer, Sue

Steenburg, Karen

Steere, Daniel, 183

Stemen, Daniel, 19, 74

Stephens, Donna, 183

Stevens, Linda

Stewart, Deborah, 19, 43, 87, 183

Stewart, Steven, 195

Stewart, Teresa, 133, 195

Stoltzfus, Connie

Stone, Lewis, 33, 34, 137, 136

Stone, Ruth

Stone, Sarah, 74

Storch, David, 195

Storey, Robert, 195

Strawn, Kathy, 30, 74

Strawn, Larry, 27, 183

Street, John 28, 31, 137, 147

Streitmatter, Melvin, 195

Strickland, Dwight, 195

Strong, Larry, 113, 195

Strychalski, Mary

Sullivan, Linda

Sutherland, Jeff

Swallow, Julia, 183

Swaney, Robert, 195

Swineford, Kevin

Taft, Ellen, 28, 84, 112, 130, 137

Tallmon, Nancy, 19, 74

Tallmon, Rebecca

Tate, Randy, 183

Tawney, E. David

Taylor, Cheryl, 74

Taylor, Christine, 138

Taylor, Nancy

Taylor, Phillip

Teed, Ruth, 87

Temple, Peg, 132

Thacker, Zachary, 111, 195

Thatcher, Dale, 110

Theetge, Sheryl

Thiessen, Claudia, 195

Thomas, David, 193

Thomas, John

Thomas, Keith

Thomas, Martin

Thomas, Margaret, 4

Thomas, Philip

Thomas, Ron, 136

Thomas, Susan, 195

Thomas, Thomas, 84, 195

Thompson, Mary

Thorsell, Paul, 138, 195

Timthaleans, 53

Tink, David

Tolle, Gary

Tompkins, Christine, 43, 183

Tompkins, Kathleen, 75

Torres, Angel

Totman, Sharon, 138, 183

Towle, Carol 30, 84, 183

Towle, Nancy, 43, 116, 195

Trautman, Mark, 183

Treachler, Dann, 87

Trittipoe, Gary, 36

Trivett, Janet

True, David, 28, 31, 32, 75, 77, 84, 113, 137

Trueblood, Gaylynn, 5

Tucker, Newton

Tucker, Patricia, 183

Tudor, Sonny, 111

Turner, Lee, 103

Turnbull, Elizabeth

Turner, James

Twigg, Dennis, 19, 27, 28, 30, 75, 77, 113,

136,137

Twigg, Terry, 195

UEWAA, 45

Ulmer, Loretta

Unthank, Joseph 
Urban, Karen

Uzzel, Rodney

Van Denburgh, Terry, 183

Van Loon, Kenneth, 183

Van Loon, Timothy, 84, 195

Vansickle, Debbie 139, 146, 147, 183, 196

Varsity C, 120

Viccaro, Sharon

Vinson, Michael, 195

Vos, Diane, 189

Waddle, Mischelle, 195

Waggoner, Bryan

Wagner, Barbara, 195

Wagner, Peggy, 25, 28, 48, 75, 87

Wagnitz, Deanna, 84, 195

Walborn, John, 84

Waldo, James, 17

Walker, David

Walker, Dick, 105

Walker, Dixie, 195

Walker, Nathan, 31, 113

Walker, Scott, 31

Wall, Joyce, 75,126

Walter, Nancy, 30, 137, 183

Ward, Debra, 84

Warren, Linda, 183

Warwick, Raymond, 28, 31, 183

Waters, Dennis

Watson, Ric, 110

WCDR, 146

Weah, Joseph, 30, 75

Weaver, Doug, 30, 132, 189

Weber, Rachel

Webster, Greg, 75, 110

Weeks, Rodney

Welch, Claudia, 75

West, Philip, 131, 136, 184

Wetzel, Daniel E., 99

Wetzel, Philip

Whatoff, Sandy, 195

Whispering Cedars, 148

White, Linda

White, Susan

White, Robert, 103

Widlicka, Bruce, 184

Widlicka, Dawn, 195

Wigand, Paul, 184

Wiggins, Cindy, 43, 114, 184

Wilcoxon, Donald

Will, Brenda, 76, 77, 133

Willan, Lenny

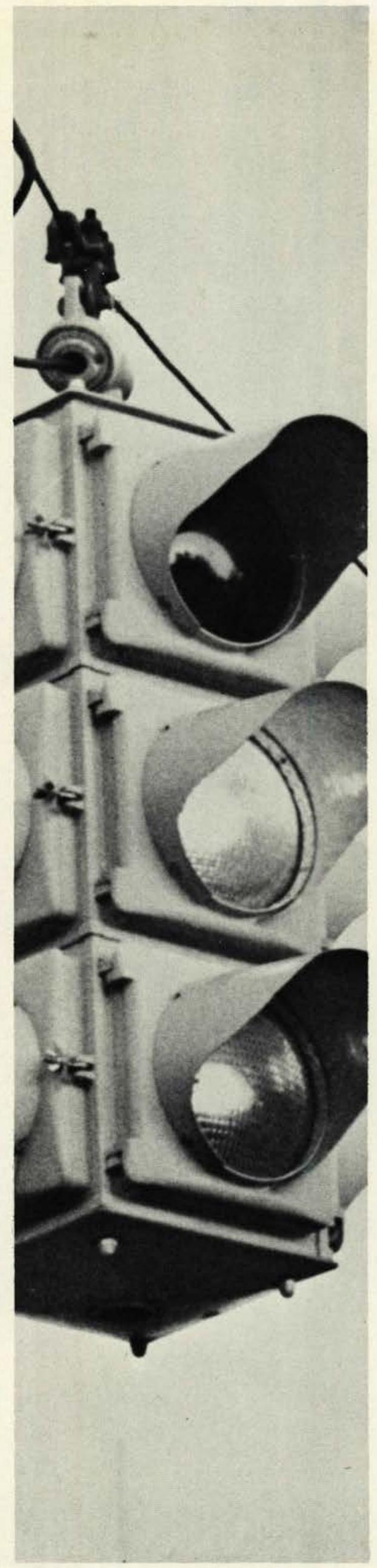

Williams, Bruce, 113, 195

Williams, Pamela, 76

Williams, Roseanna, 195

Williams, Thomas, 159

Williams, R. Wayne

Williams, Sharon, 184

Williams, William, 195, 155

Williamson, David

Williford, Carolyn, 184

Williford, Craig, 26

Wilsey, Mike

Wilson, Douglas, 84

Wilson, Gerald, 84

Wilson, P. Steven, 28, 195

Wimer, Karen, 112, 184

Wimer, Kenneth

Wineberg, Thomas

Wing, Douglas, 84, 131, 189

Wishart, David, 24, 195

Wolfe, Jeffery, 34, 31, 76, 113

Wolf, Kevin, 76

Wolf, Richard

Wolff, Howard, 76

Wood, Deborah, 76, 133

Wood, Doris, 184

Wood, Mary, 184

Wood, Ruth, 189

Woodcock, Joel, 159, 189

Woodward, Shauna, 195

Woolard, Dale, 130, 137, 139

Work, Ivan, 76

Work, Wendy, 76

Workman, Donald, 195

Worley, Daryle, 98

Wright, Dale, 189

Wyman, Jonathan, 36

Wymer, William, 77, 132

Yasenka, Charles

Yeaton, Donald, 77, 132

Yost, Donald, 179

Young, Alan

Young, Cindy J., 43, 117, 195

Young, Cindy E., 189

Young, Janet, 19, 77

Young, Robert, 84, 195

Young, Steve, 19, 110, 131, 137, 184

Ziegler, Beverly, 30, 184

Ziegler, Merle, 36, 38, 39, 195

Zinn, Jeff, 195

Zuck, Greg 


\section{Alpha Zeta}

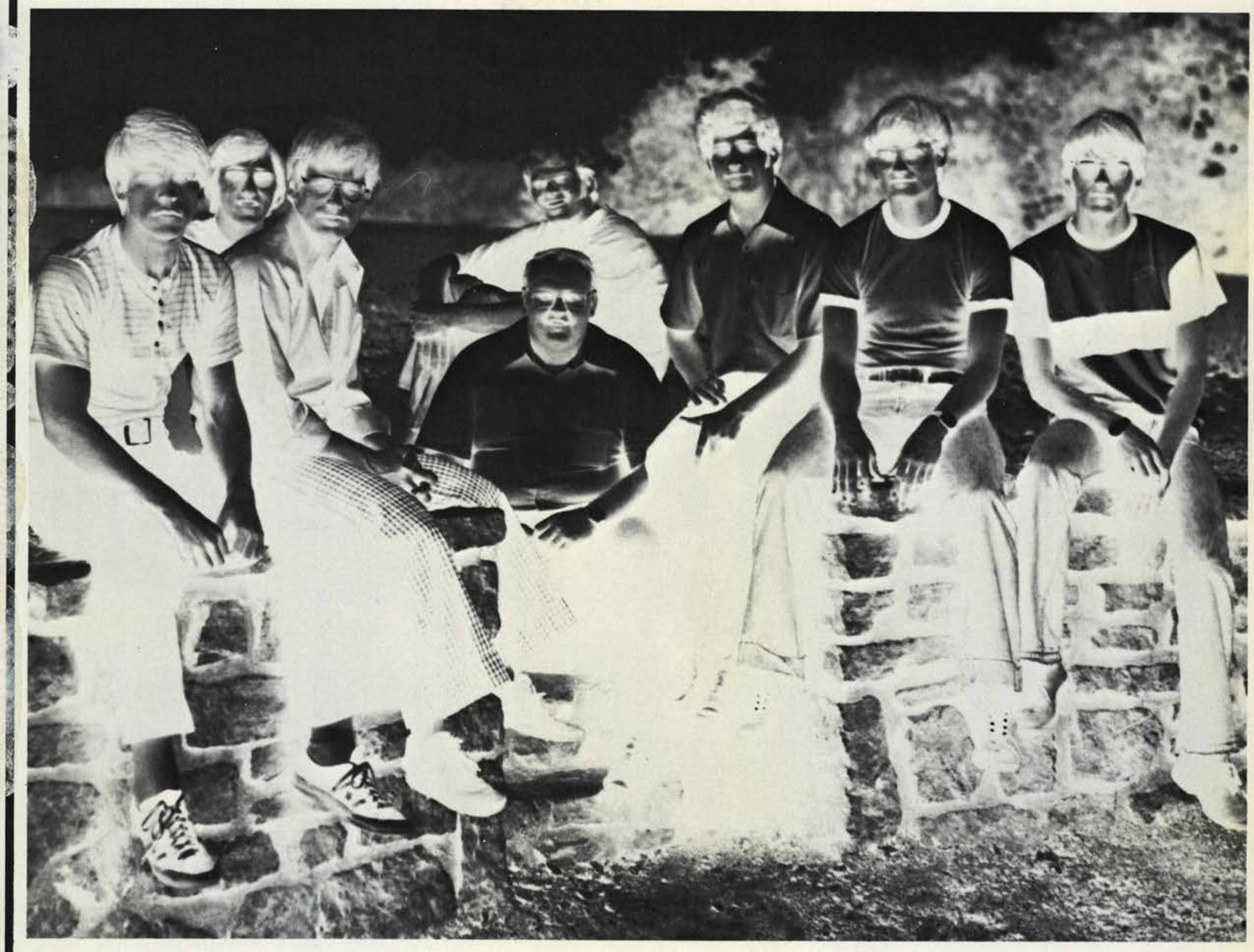

Bob Young

Greg Zuck

Dave Romaine

Duane Mawhorter

Roy Kern, Adviser

Scott Gons

Dale Mugglesworth

Jim Delange

NOT PICTURED:

Marcea Bearss

Dan Steman

Glen Pritchett 


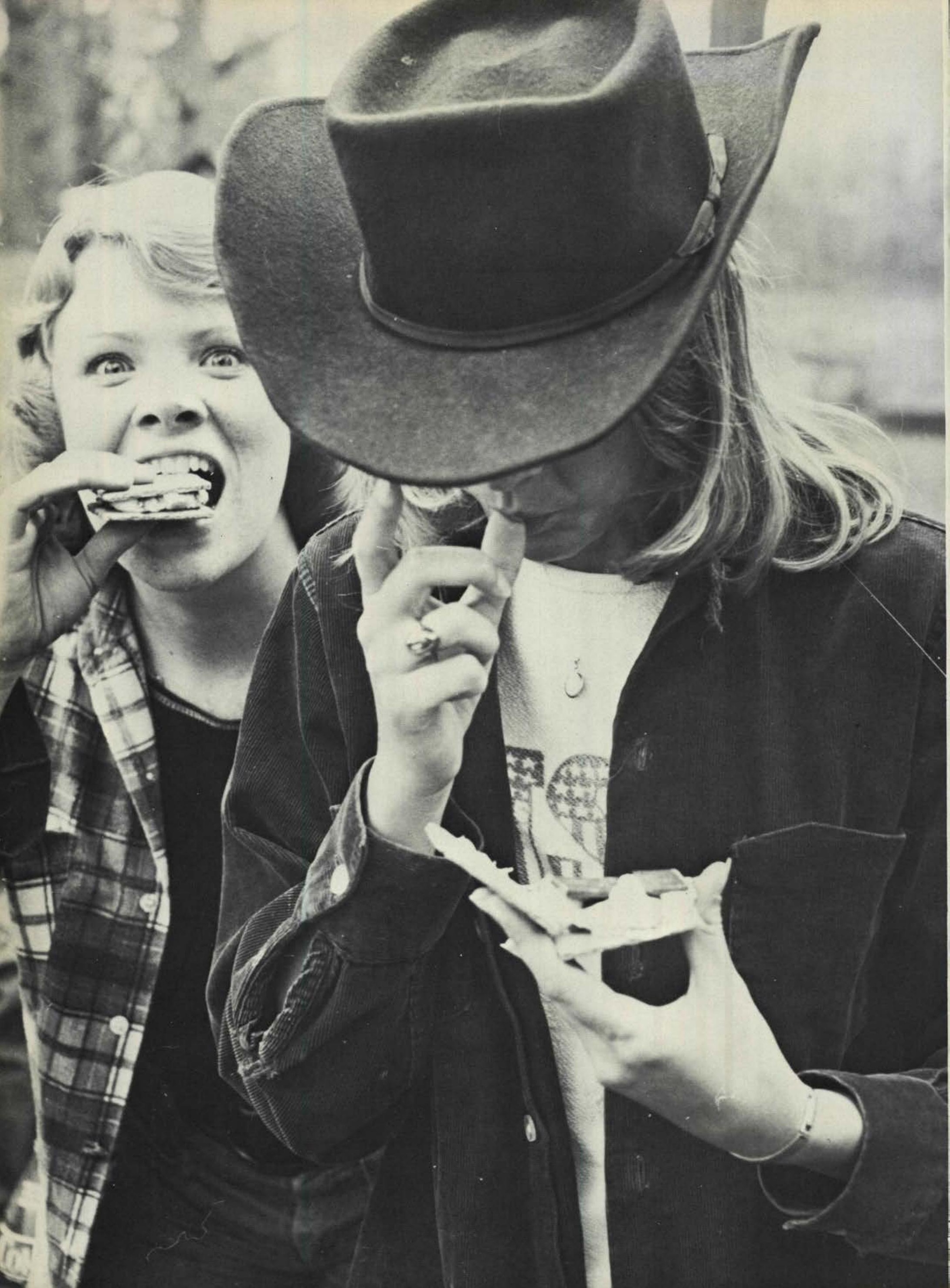




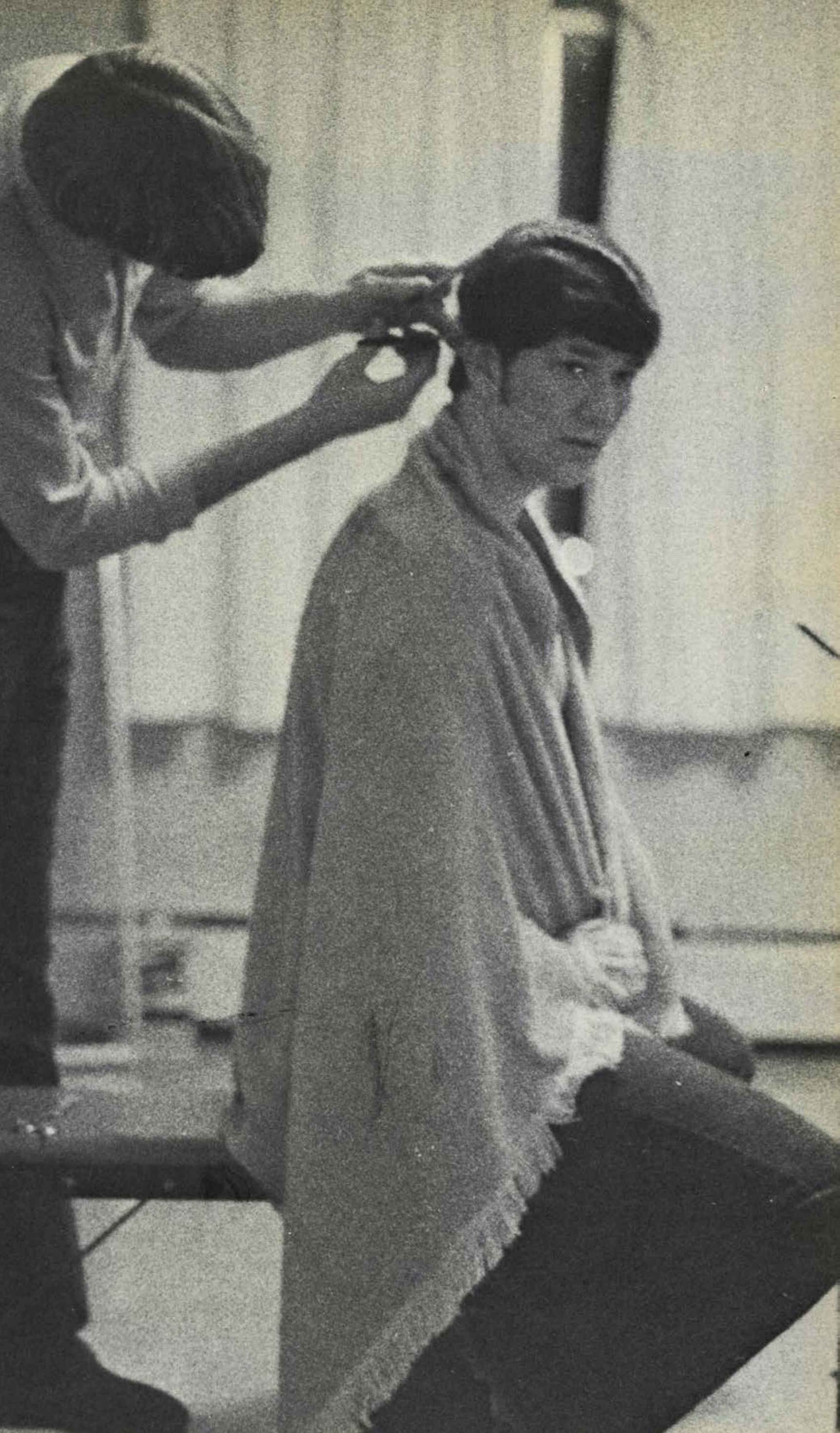


agenge

$604 \times$

co. 0.561

(n) arear 8

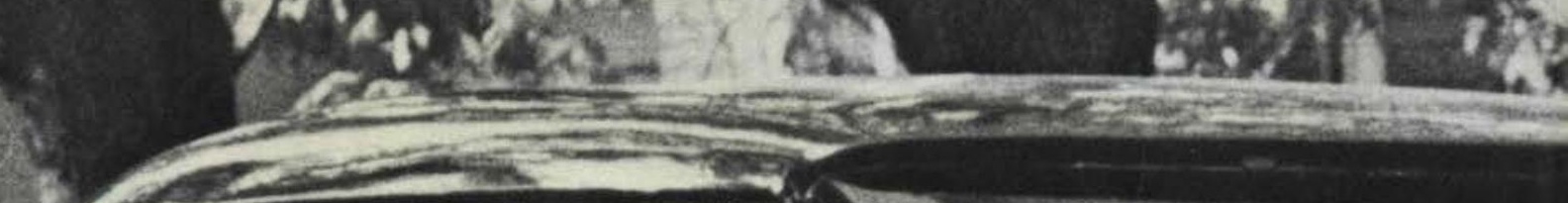

Ifeth

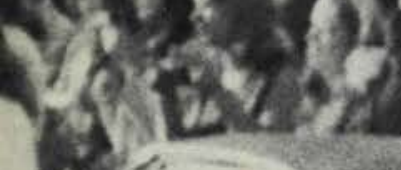

Q. $12^{2}$

$\approx$
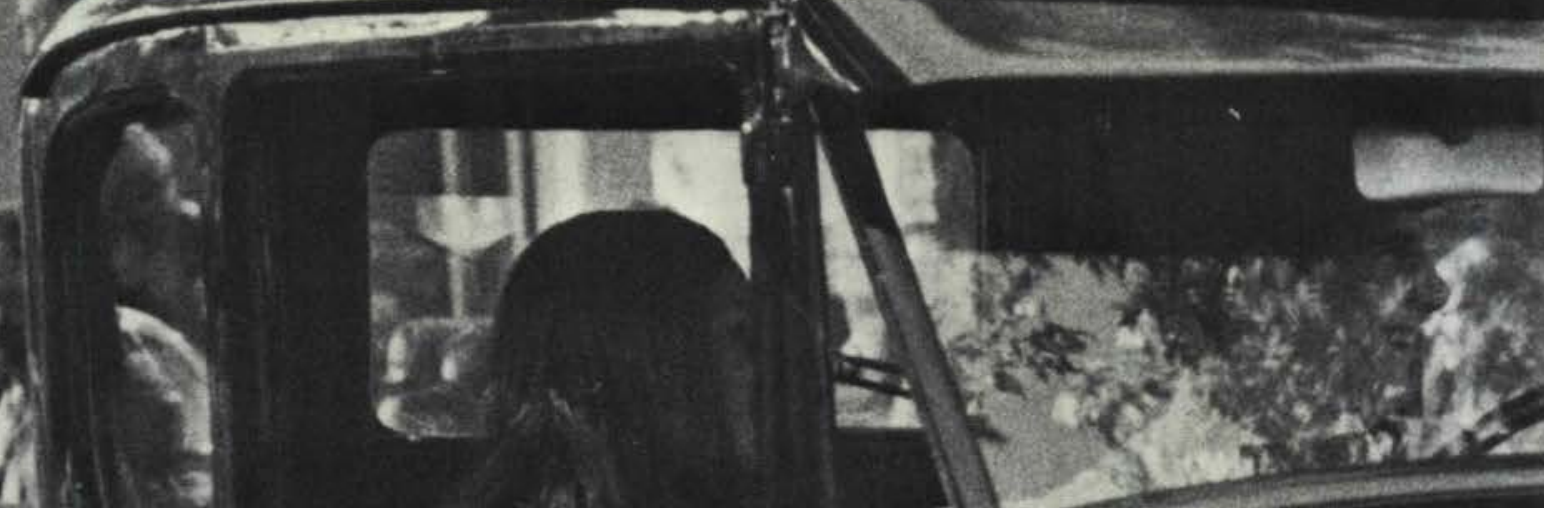

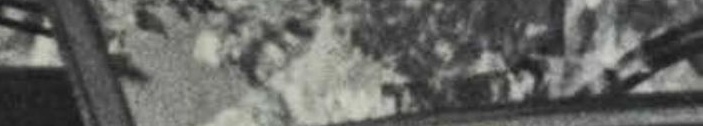

3

\section{at}

(⿻)
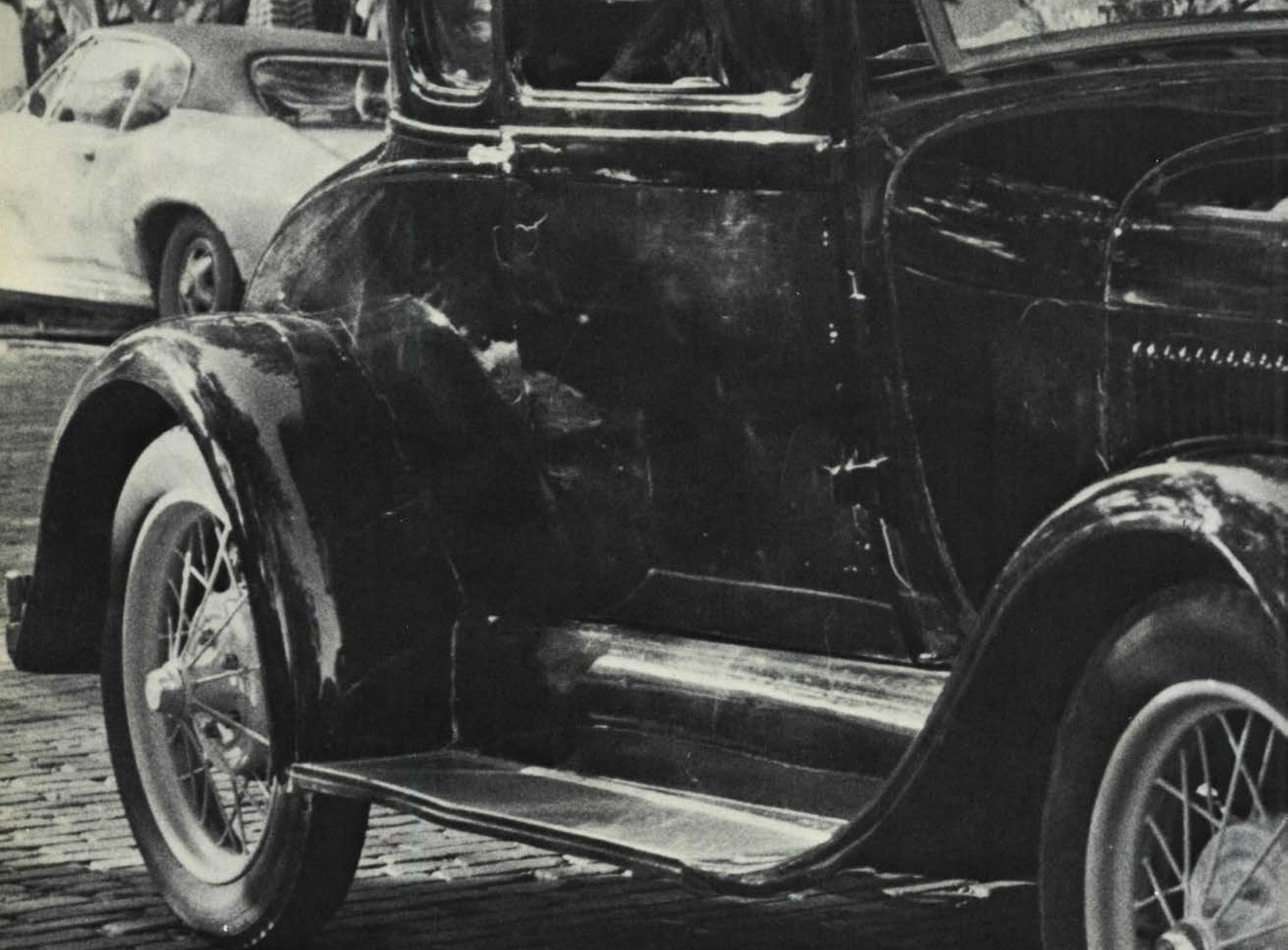

1

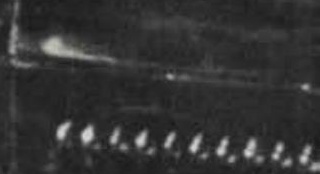

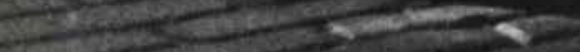

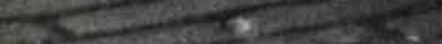

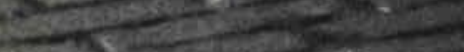

estem
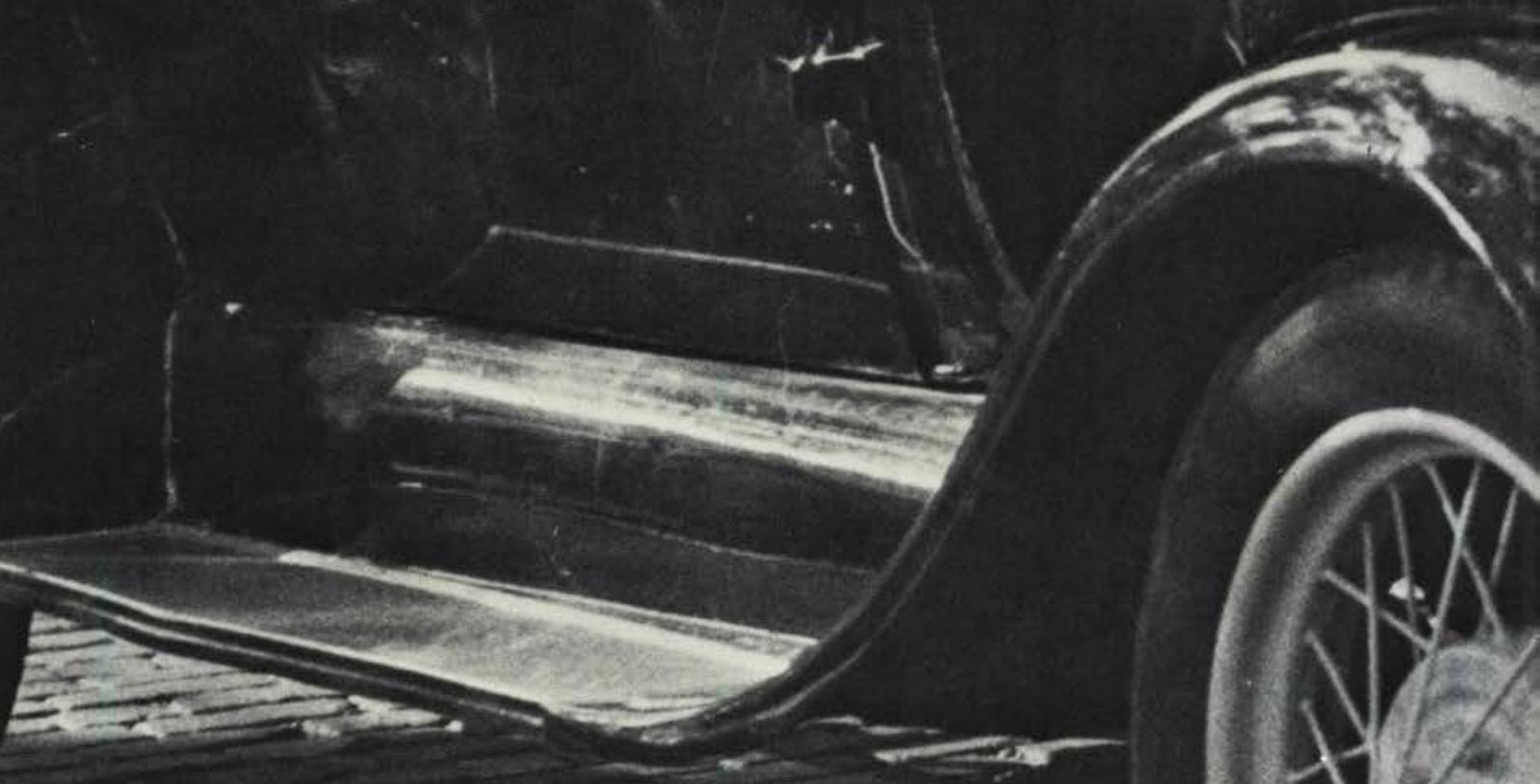

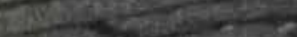

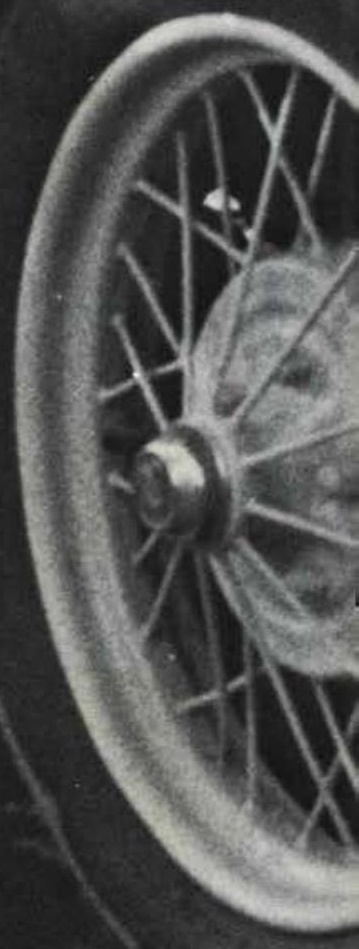




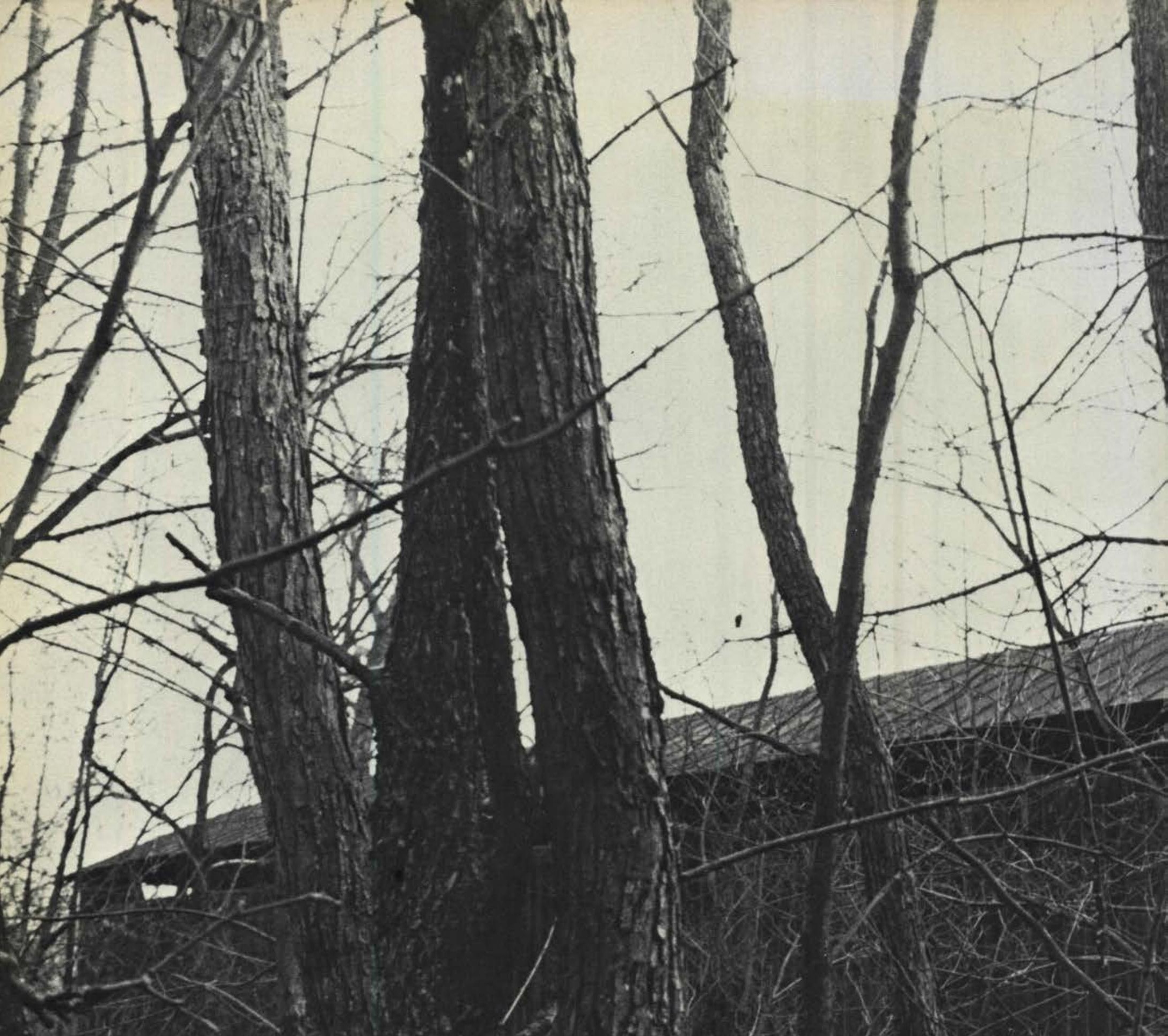

(I)

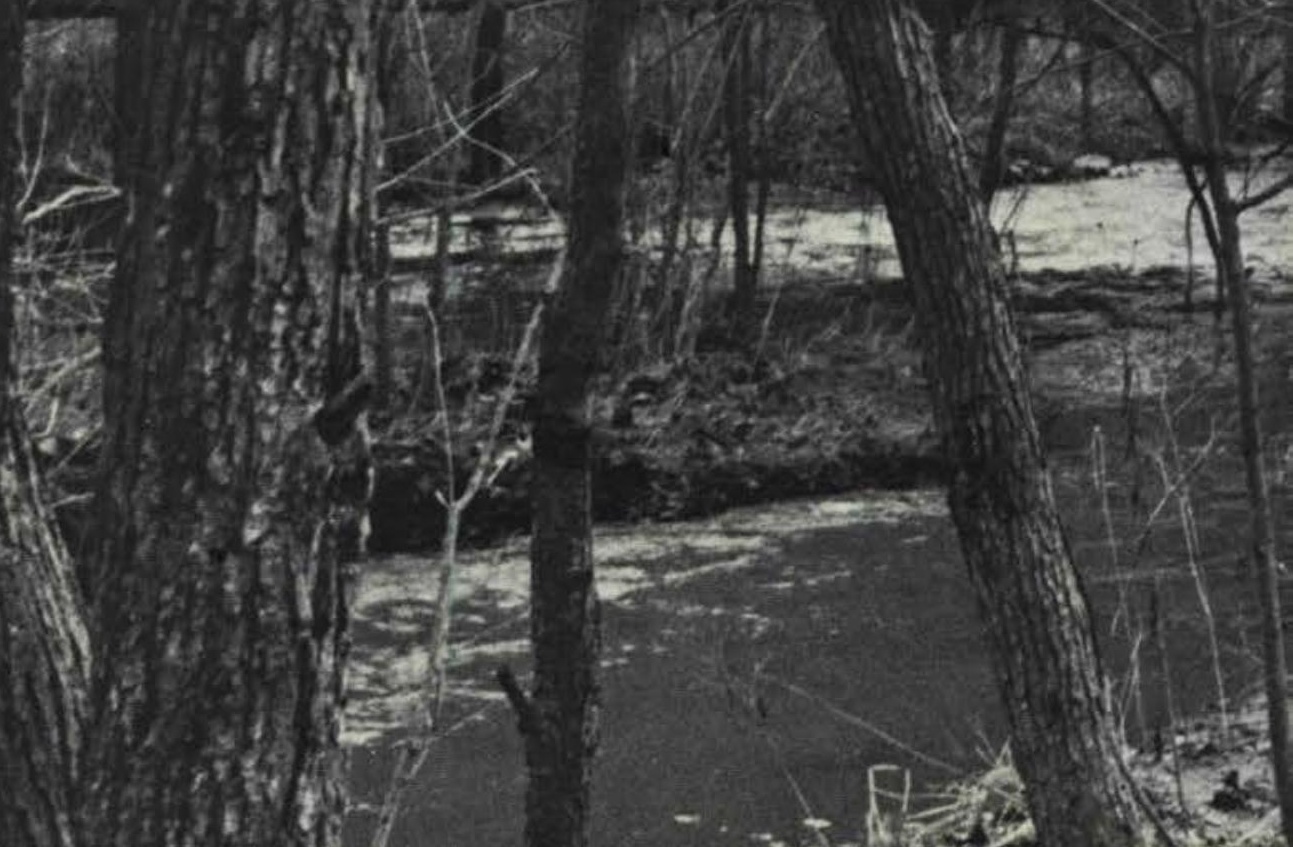




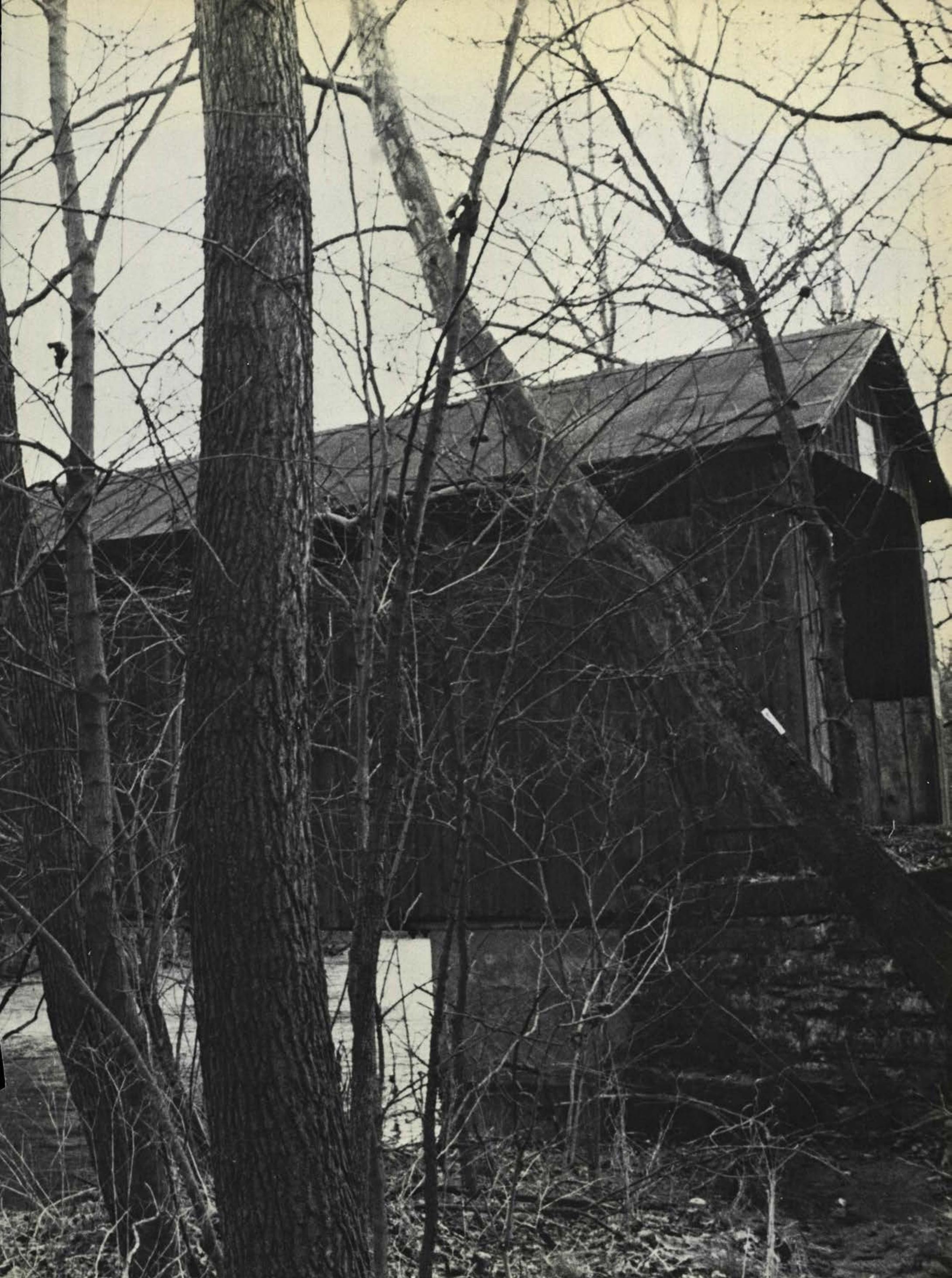




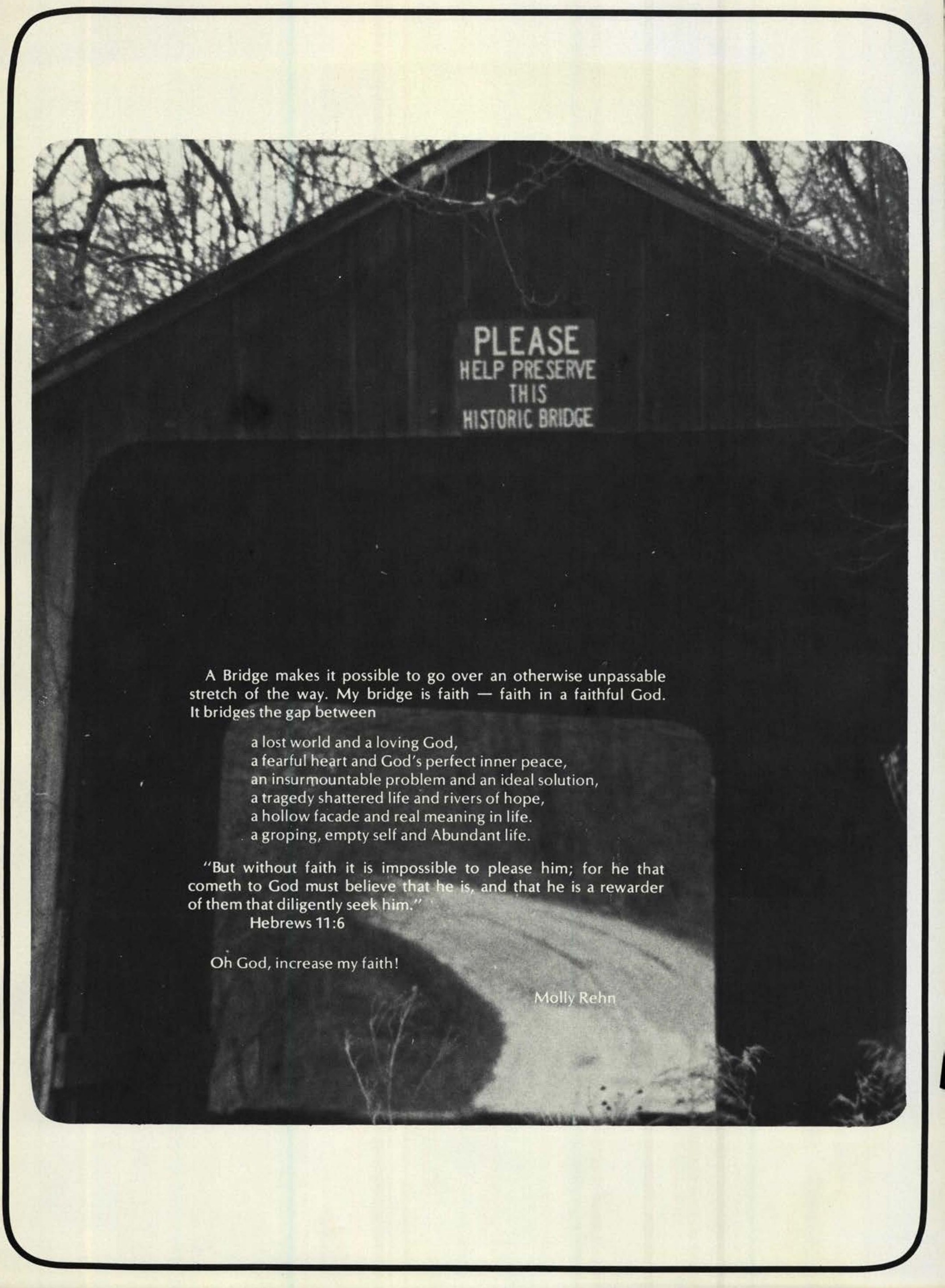




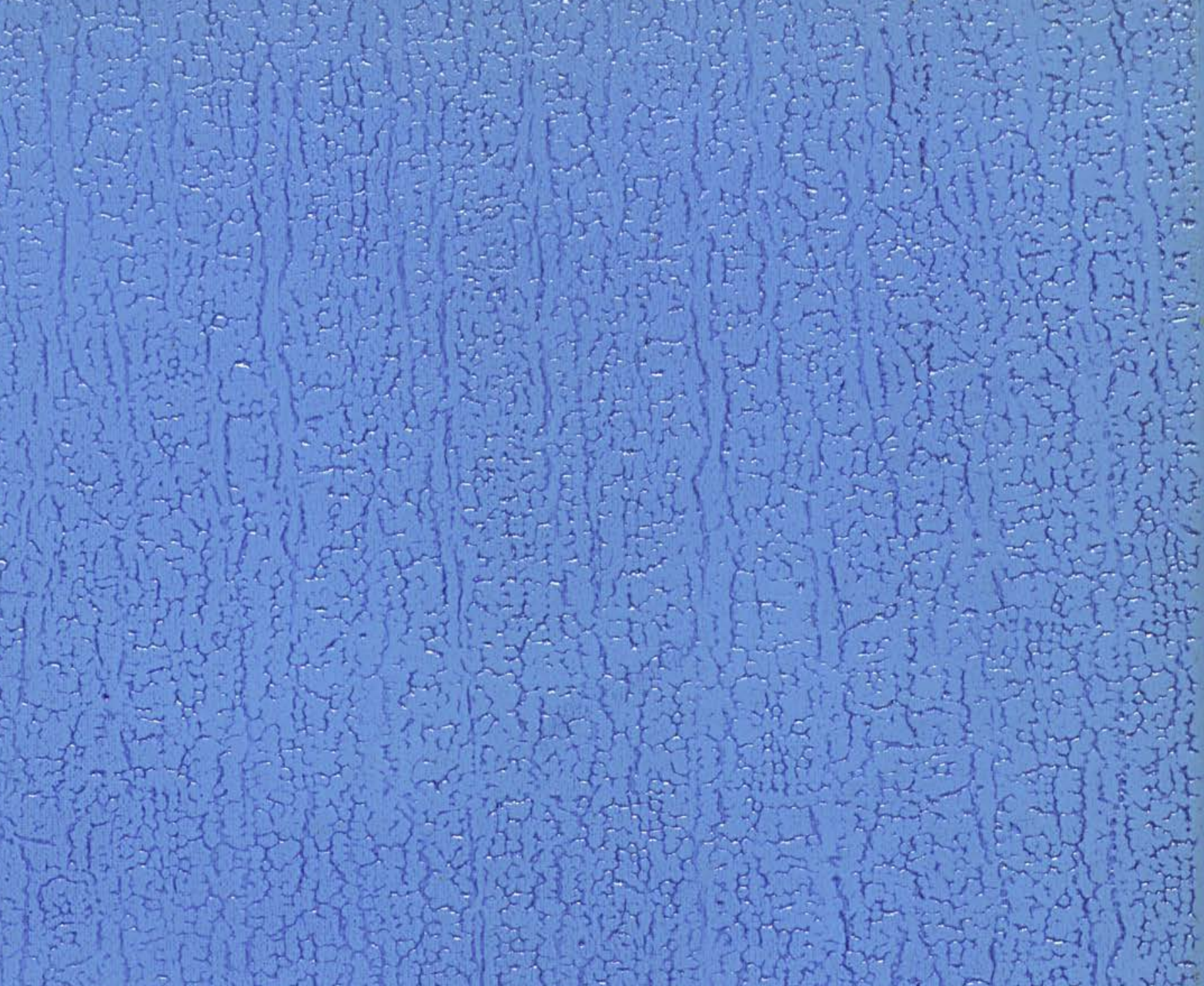

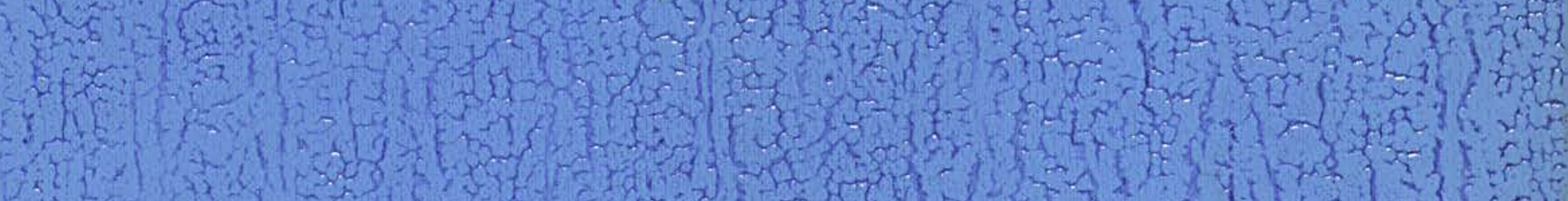
With x. H the

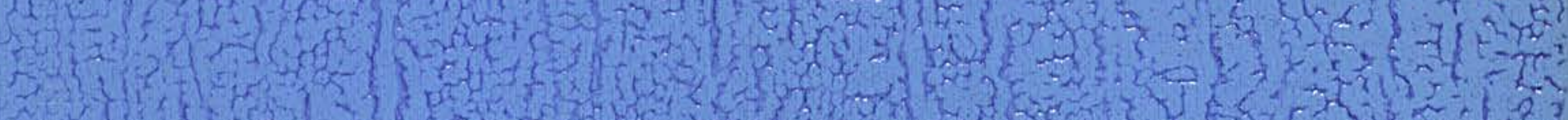

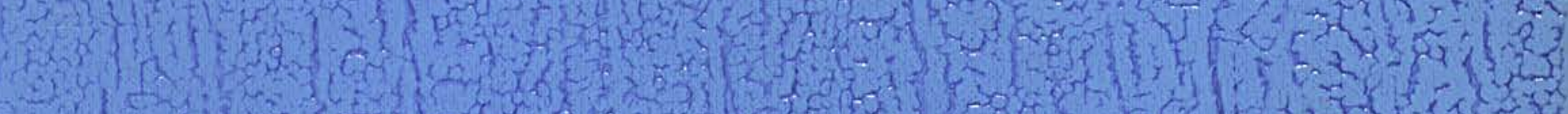

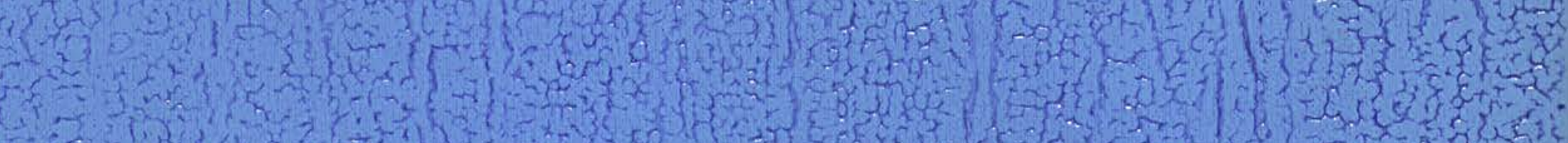
and

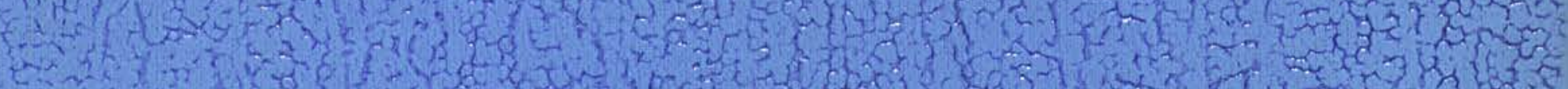

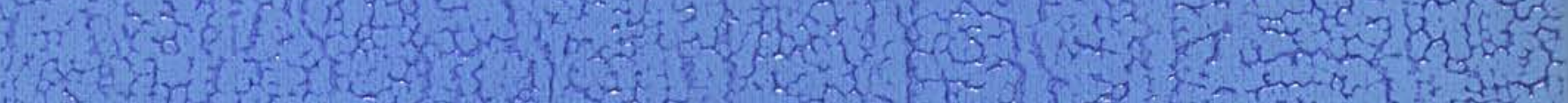
2. (6) 1007

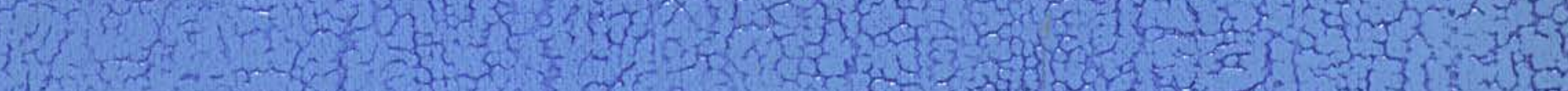
(1) (1) 2. int 